Florida International University FIU Digital Commons

$11-2-2015$

\title{
The Influence of the Proximal Amide Hydrogen Bonds and the Proximal Helix Dipole on the Catalytic Activity of Chloroperoxidase
}

armando d. pardillo

Florida International University, apard001@fiu.edu

DOI: $10.25148 /$ etd.FIDC000164

Follow this and additional works at: https://digitalcommons.fiu.edu/etd

Part of the Physical Chemistry Commons

\section{Recommended Citation}

pardillo, armando d., "The Influence of the Proximal Amide Hydrogen Bonds and the Proximal Helix Dipole on the Catalytic Activity of Chloroperoxidase" (2015). FIU Electronic Theses and Dissertations. 2270.

https://digitalcommons.fiu.edu/etd/2270 


\section{FLORIDA INTERNATIONAL UNIVERSITY}

Miami, Florida

THE INFLUENCE OF THE PROXIMAL AMIDE HYDROGEN BONDS AND THE PROXIMAL HELIX DIPOLE ON THE CATALYTIC ACTIVITY OF CHLOROPEROXIDASE

A dissertation submitted in partial fulfillment of

the requirements for the degree of

DOCTOR OF PHILOSOPHY

in

CHEMISTRY

by

Armando D. Pardillo 
To: Dean Michael R. Heithaus

College of Arts and Sciences

This dissertation, written by Armando D. Pardillo, and entitled The Influence of the Proximal Amide Hydrogen Bonds and the Proximal Helix Dipole on the Catalytic Activity of Chloroperoxidase, having been approved in respect to style and intellectual content, is referred to you for judgment.

We have read this dissertation and recommend that it be approved.

$\begin{array}{r}\hline \text { Alexander Mebel } \\ \hline \text { Xiaotang Wang } \\ \hline \text { Premstantinos Kavallieratos Chapagain } \\ \hline \text { David Chatfield, Major Professor }\end{array}$

Date of Defense: November 3, 2015

The dissertation of Armando D. Pardillo is approved.

Dean Michael R. Heithaus College of Arts and Sciences

Dean Lakshmi N. Reddi University Graduate School

Florida International University, 2015 
(C) Copyright 2015 by Armando D. Pardillo

All rights reserved. 


\section{DEDICATION}

I dedicate this dissertation to my parents. Their constant love and support has helped keep my morale up during difficult times. Their example and past sacrifices have motivated me to become a better man. 


\section{ACKNOWLEDGMENTS}

I would first like to thank my committee members, who have all made themselves available to me. Dr. Xiaotang Wang's knowledge and insight on CPO has been indispensable, especially in acquiring obscure information not easily obtained from a literature search. Dr. Alexander Mebel’s technical advice, especially his recommendations regarding obtaining accurate electronic states saved me days of work. Dr. Prem Chapagain's alternate perspective on molecular mechanics methodologies has been most helpful, and I am grateful for all the questions he was able to answer for me. Dr. Konstantinos Kavallieratos, as my random committee member and who was least familiar with my subject area and/or methodology, was very helpful with the way I presented my data. It is easy to lose one's self in assumptions known to an in-group, and Dr. Kavallieratos helped keep my explanations clear and well defined. I would especially like to thank Dr. David Chatfield, who has been with me every step of the way. He has always listened to my input and given me substantial freedom with my project. I have enjoyed our discussions and am grateful for the constant support and guidance. Aside from what I have gained under his scientific mentorship, I have learned a great deal about writing, the English language, and grammar. I learn at least one new word every time I meet with him.

Aside from my committee, I would also like to thank everyone from the Chatfield/Mebel Lab in OE-101. Dr. Alexander Morozov has answered so many questions for me, I don’t even know where to begin. Thank you for all your help. Dr. Cassian D’Cunha, Dr. Alexander Landera, Dr. Adeel Jamal, and Joao Ribeiro; thank you 
all for our interesting and entertaining discussions. I would also like to thank Dr. Xiaotang's student, Elena Shersher, with whom I have engaged in many conversations about CPO mutants and their catalytic activitives. I have made many friends at FIU, many of whom I have mentioned above. Thank you all for the great memories and making this experience enjoyable. 
ABSTRACT OF THE DISSERTATION

THE INFLUENCE OF THE PROXIMAL AMIDE HYDROGEN BONDS AND THE PROXIMAL HELIX DIPOLE ON THE CATALYTIC ACTIVITY OF

\title{
CHLOROPEROXIDASE
}

by

\author{
Armando D. Pardillo
}

Florida International University, 2015

Miami, Florida

Professor David Chatfield, Major Professor

Chloroperoxidase (CPO) is a heme-thiolate protein with exceptional versatility and great potential as a biocatalyst. The CPO reactive species, Compound I ( Cpd I) is of particular interest, as well as the Cytochrome P450 (P450) -type monoxygenase catalytic activity, which has significant biotechnological potential. Proximal hydrogen bonding of the axial sulfur with the backbone amides $(\mathrm{NH} \bullet \bullet \mathrm{S})$ is a conserved feature of hemethiolate enzymes. In $\mathrm{CPO}$, the effect of $\mathrm{NH} \cdots$ S bonds is amplified by the dipole moment of the proximal helix. The role of the proximal region has been disputed as to whether it simply protects the axial sulfur, or whether it additionally influences catalysis via modulation of the push effect.

The objective of the research presented herein is two-fold. First, the influence of the $\mathrm{NH} \cdots \mathrm{S}$ bonds on Cpd I formation is determined by obtaining the reaction coordinate, starting from a peroxide bound heme, for two model systems (one with proximal residues providing $\mathrm{NH} \bullet \cdot \mathrm{S}$ bonds and one without) and comparing the results. Secondly, the influence of the proximal region on the epoxidation of cis- $\beta$-methylsterene is obtained in 
a manner similar to the first objective. However, the reaction coordinate begins with a Cpd I-CBMS complex and the proximal contribution is extended to include the influence of the proximal helix dipole.

Our findings show that the proximal region stabilizes Cpd 0 relative to all other minima and reduces the barrier for Cpd 0’s formation. The stability of protonated Compound 0 is reduced, favoring a hybrid homo-heterolytic relative to a classic heterolytic mechanism for O-O bond scission. Additionally, the proximal region significantly enhances CPO's reactivity; the $\mathrm{C}_{\beta}-\mathrm{O}$ bond barrier is stabilized, while $\mathrm{C}_{\alpha}$-O$\mathrm{C}_{\beta}$ ring closure becomes barrierless. The stabilization of the reaction barrier correlates with increased electron density transfer to residues of the proximal pocket and involves a change in the electron transfer mechanism. These results can be traced to a reduction in the pKa of the heme-bound substrate and an increase in oxidation potential, a result of the proximal region reducing the push effect. 


\section{TABLE OF CONTENTS}

CHAPTER

PAGE

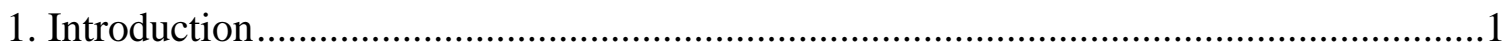

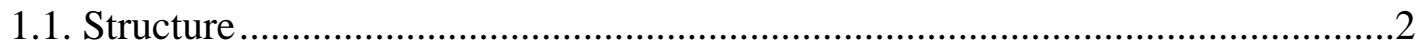

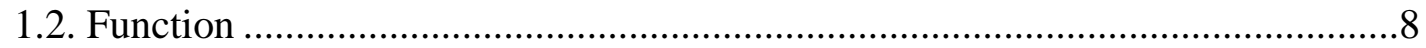

1.2.1. Halogenation - CPO's signature function …………….............................10

1.2.2. Peroxidations......................................................................................... 11

1.2.3. Two-electron oxidations .....................................................................12

1.2.3.1. Epoxidations ..................................................................................13

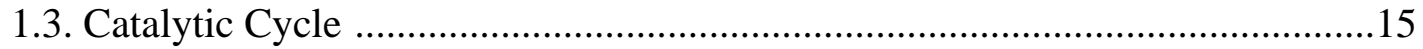

1.4. Mechanism of Cpd I Formation..................................................................16

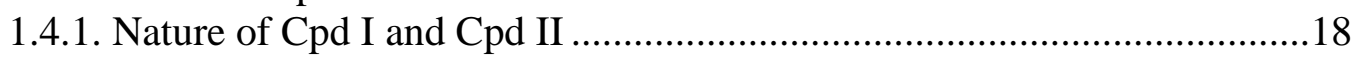

1.4.2. Structural Factors influencing Cpd I formation ........................................20

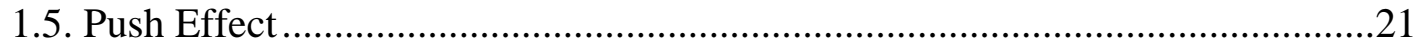

1.5.1. Proximal H-bond influence on Push Effect .............................................22

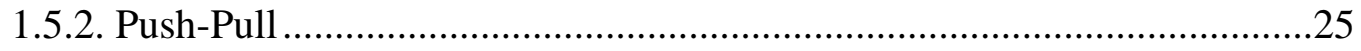

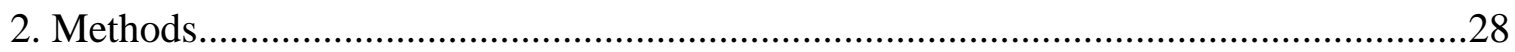

2.1. Quantum Mechanical Methods ..................................................................29

2.1.1. Ab-initio Methods ..................................................................................

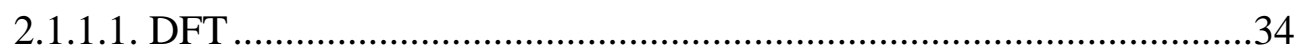

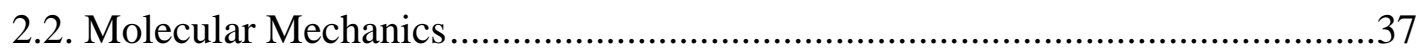

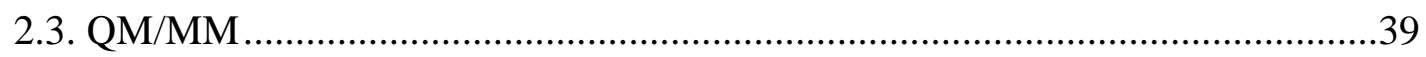

2.4. Software and Analytical Tools ......................................................................42

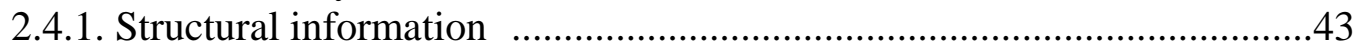

2.4.2. Population Analysis ..............................................................................4

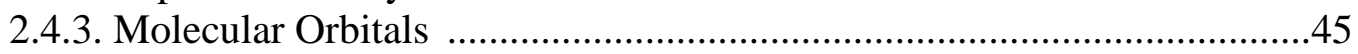

2.4.4. Molecular Mechanics Software ..............................................................46

3. Influence of the Proximal Pocket Hydrogen Bonds on the Mechanism of

Chloroperoxidase Compound I Formation ...............................................................4

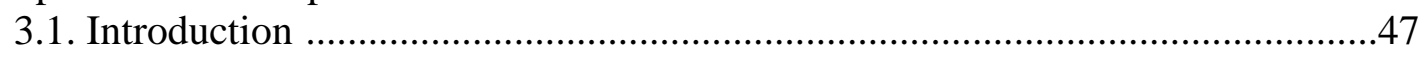

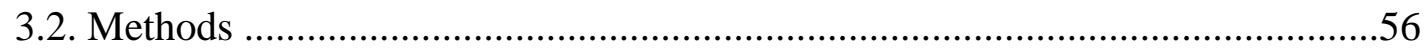

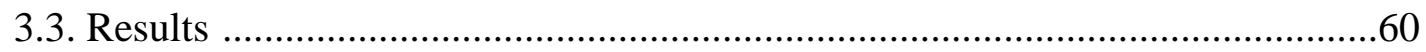

3.3.1. Reaction from FeHOOH to Cpd 0 ……………….................................60

3.3.2. Reaction from Cpd 0 to Cpd I..............................................................64

3.3.3. Quartet surface .....................................................................................

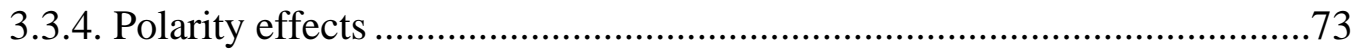

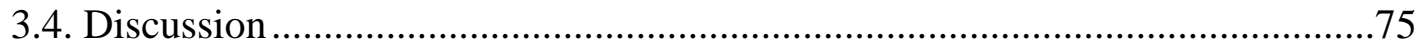

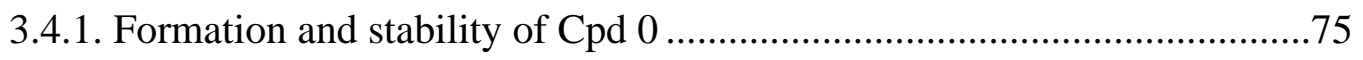

3.4.2. Stability of Cpd I .................................................................................

3.4.3. Mechanism of O-O bond scission............................................................76

3.4.4. Distal-side hydrogen bonding ....................................................................78 
3.4.5. Quartet Potential Energy Surface...............................................................79

3.5. Conclusion ..............................................................................................

4. Chloroperoxidase-Catalyzed Epoxidation of Cis- $\beta$-Methylstyrene: $\mathrm{NH} \cdots \mathrm{S}$

hydrogen bonds and Proximal Helix Dipole Change the Catalytic Mechanism and

Significantly Stabilize the Reaction Barrier .......................................................................82

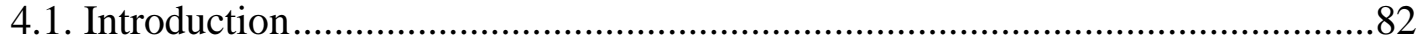

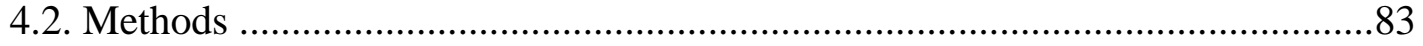

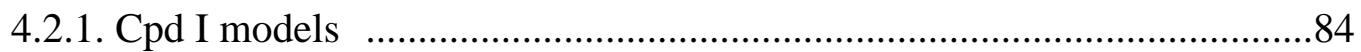

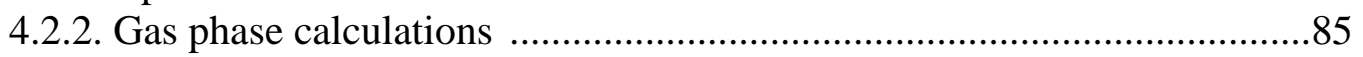

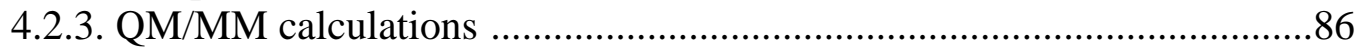

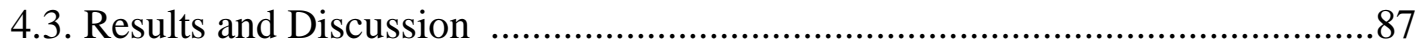

4.3.1. Potential Energy Surfaces of CBMS Epoxidation ......................................88

4.3.2. Cpd I orbitals .......................................................................................91

4.3.3. Electron transfer mechanisms on the doublet spin surface of Cpd I-A ....92

4.3.4. Electron transfer mechanisms on the doublet spin surface of Cpd I-B ....95

4.3.5. Electron transfer mechanisms on the quartet spin surface ........................98

4.3.6. Separating $\mathrm{NH} \bullet \bullet \mathrm{S}$ and helix effects....................................................100

4.3.7. Protein and solvent effect ....................................................................101

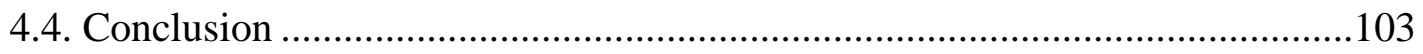

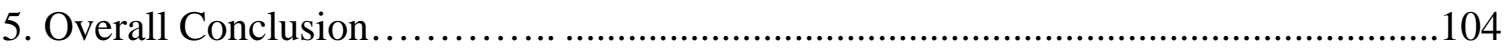

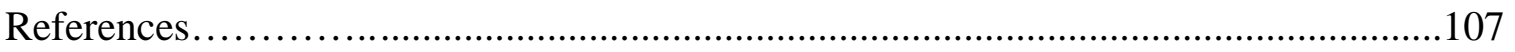

Appendices...................................................................................................122

VITA 


\section{LIST OF TABLES}

TABLE

PAGE

Table 1: Comparison of P450cam and CPO proximal pocket NH•••S hydrogen bonds.. 51

Table 2a: Interatomic distances for model B .........................................................62

Table 2b: Difference between interatomic distances for models B and A .......................62

Table 3: Natural Charge and Spin Density for Stationary Points ..................................63

Table 4: Energetic data for the portion of the PES from Cpd 0 to TS3 ..........................69

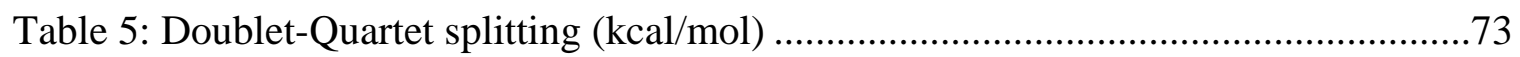

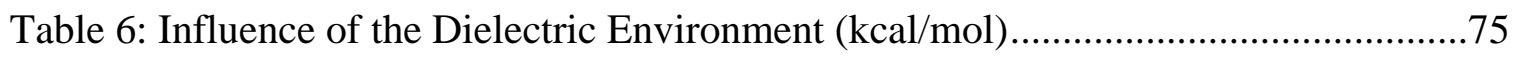

Table 7. Barriers (kcal/mol)/Charge Transfer to the proximal moiety. .........................90

Table 8. Natural Group spin densities/Charges of the optimized structures on the doublet PES surfaces.

Table 9. Natural Group spin densities/Charges and bond lengths ( $\AA$ ) of the optimized

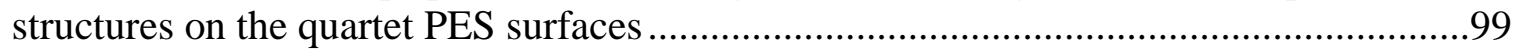

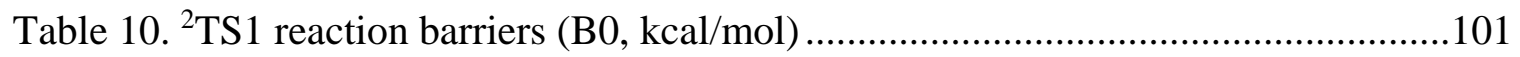

Table 11. ${ }^{2}$ TS1-CPO/B reaction barriers (B0) broken into specific contributions $(\mathrm{kcal} / \mathrm{mol})$. 


\section{LIST OF FIGURES}

FIGURE

PAGE

Figure 1: Crystal Structure of Chloroperoxidase (PDB Code: 1CPO) ..............................

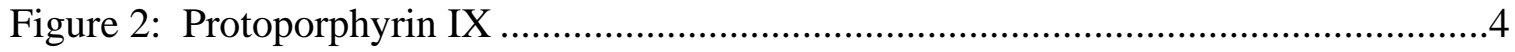

Figure 3: Slice through surface representation of chloroperoxidase .............................6

Figure 4: Stereoimage of Manganese binding site.................................................

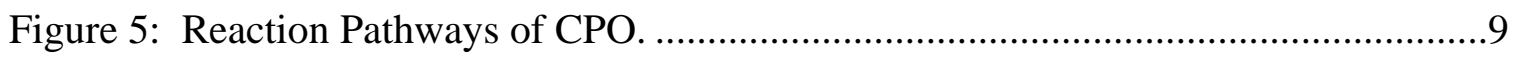

Figure 6: Cholorperoxidase Catalytic Reaction Cycle ..............................................15

Figure 7: Possible Binding Conformations of FeHOOH .............................................17

Figure 8: Formation of Cpd II via one-electron oxidation of Cpd I. ..............................20

Figure 9: Relative orientations of the proximal helix to the heme in P450cam (parallel)

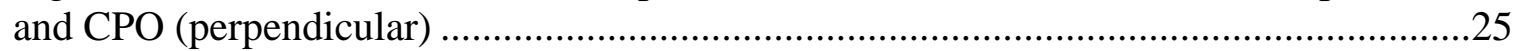

Figure 10: Partitioning of the system into the MM, QM, and boundary regions ..............41

Figure 11: a. Notation for substrate oxygens. b. Notation for ring nitrogens used by Shaik et al. c. Model System.

Figure 12: Reaction profile for the FeHOOH $\rightarrow$ Cpd I transformation on the doublet surface, for the model system with (model B) and without (model A) a proximal region present. The first transition state was only optimized with the B1 basis set, so the energy value denoted with the * represents the B3//B1 level of theory. The PES's with and without ZPE corrections are represented by solid and dashed lines, respectively..........54

Figure 13: Reaction profile for the FeHOOH $\rightarrow$ Cpd I transformation on the quartet surface, for the model system with (model B) and without (model A) a proximal region present. The three transition states are in fact near transition states, and are thus denoted with an asterix. The PES's with and without ZPE corrections are represented by solid and dashed lines, respectively. The zero of energy is taken as the energy of ${ }^{2} \mathrm{FeHOOH}$

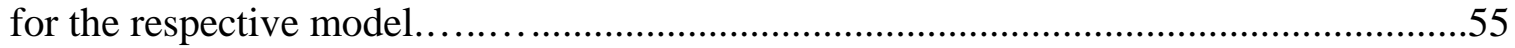

Figure 14: Structures for FeHOOH, TS1, and Cpd 0 for model B................................61

Figure 15: Structures for Cpd 0 through Cpd I for model B........................................65 
Figure 16: Bare-thiolate (A) and CPO-like (B) proximal pocket models of Cpd I. ..

Figure 17: The doublet and quartet potential energy surfaces (B0/B1 in kcal/mol) for 1S2R epoxidation of CBMS

Figure 18: Key Kohn-Sham molecular orbitals of Cpd I (doublet) involved in the epoxidation reaction.

Figure 19: Optimized structures ( ${ }^{2} \mathrm{I}^{*}-\mathrm{A}$ is not a stationary point) for Cpd I-A catalyzed epoxidation on the doublet spin surface

Figure 20 a: $\pi_{y z}^{*}$ radical attack on $\mathrm{C}=\mathrm{C}$ bond; b: $\mathrm{C}_{\alpha}$ radical corresponds to KS HOMO in the alpha manifold of ${ }^{2} \mathrm{I}^{*} \mathrm{~A}$; c: second highest occupied orbital in the alpha manifold of ${ }^{2} \mathrm{I}-\mathrm{A}$ is a mixture of $\varphi_{C \alpha}$ and $\varphi_{a_{2 u}+\sigma_{S}}$.

Figure 21: Optimized structures $\left({ }^{2} \mathrm{I}^{*}-\mathrm{B}\right.$ is not a stationary point) for Cpd I-B catalyzed epoxidation on the doublet spin surface.

Figure 22: A+NH•••S model. 101 


\title{
LIST OF ABBREVIATIONS
}

\author{
$\underline{\text { ABBREVIATION FULL NAME }}$ \\ BPO BROMOPEROXIDASE \\ CBMS CIS- $\beta$-METHYLSTYRENE \\ CCP CYTOCHROME C PEROXIDASE \\ CNDO COMPLETE NEGLECT OF DIFFERENTIAL OVERLAP \\ CPO CHLOROPEROXIDASE \\ DA DALTONS \\ DFT DENSITY FUNCTIONAL THEORY \\ DHP DEHALOPEROXIDASE \\ ECP $\quad$ EFFECTIVE CORE POTENTIAL \\ ESP ELECTROSTATIC POTENTIAL \\ GGA GENERALIZED GRADIENT APPROXIMATION \\ GTO GAUSSIAN TYPE ORBITALS \\ HF HARTREE FOCK \\ INDO INTERMEDIATE NEGLECT OF DIFFERENTIAL OVERLAP \\ IPO IODOPEROXIDASE




\begin{tabular}{ll} 
KS & KOHN-SHAM \\
LCAO & LINEAR COMBINATION OF ATOMIC ORBITALS \\
LDA & LOCAL DENSITY APPROXIMATION \\
LYP & LEE-YANG-PARR \\
MCD & MAGNETIC CIRCULAR DICHROISM \\
MCD & MONOCHLORODIMEDON \\
MD & MOLECULAR DYNAMICS \\
MM & POLEULAR MECHANICS \\
POS & NOLECULAR ORBITAL \\
PAT & NATURAL ATOMIC ORBITALS \\
NAO & NATUAL BOND ORBITALS \\
NBO & NEGLECT OF DIATOMIC DIFFERENTIAL OVERLAP \\
NDDO & \\
\hline
\end{tabular}




$\begin{array}{ll}\text { QM } & \text { QUANTUM MECHANICS } \\ \text { QM/MM } & \text { QUANTUM MECHANICS/MOLECULAR MECHANICS } \\ \text { ROHF } & \text { RESTRICTED OPEN-SHELL HARTREE-FOCK } \\ \text { RHF } & \text { RESTRICTED HARTREE-FOCK } \\ \text { SCF } & \text { SELF CONSISTENT FIELD } \\ \text { STO } & \text { SLATER TYPE ORBITAL } \\ \text { UHF } & \text { UNRISTRICTED HARTEE-FOCK } \\ \text { VDW } & \text { VAN DER WAALS }\end{array}$




\section{Introduction}

Chloroperoxidase (CPO), an enzyme produced by the marine fungus Caldariomyces fumago, is a versatile protein belonging to the heme-thiolate family of proteins. Hemethiolate proteins contain a protoheme prosthetic group with an axially bound thiolate anion of cysteine, and are one of the major hemoprotein groups found in living organisms. ${ }^{1}$ Other major hemoprotein groups inclue heme-imidazole (histidine), heme heme-phenolate (tyrosine), and heme-thioether (methionine). Hemes are one of the most widely used metalloporphyrins throughout the biosphere, as they are capable of shuttling electrons between proteins, transporting and storing $\mathrm{O}_{2}$, and are involved in enzymatic catalysis. ${ }^{2}$ Heme enzymes are able to catalyze both oxidative and reductive chemistry. However, since CPO utilizes the heme prosthetic group to catalyze oxidative reactions, our attention will be on oxidative chemistry. Heme enzyme oxidants can be classified broadly as either oxygenases (use $\mathrm{O}_{2}$ to oxidize substrates) or peroxidases (use $\mathrm{H}_{2} \mathrm{O}_{2}$ to oxidize substrates). Each substrate poses biological challenges. Although $\mathrm{O}_{2}$ is highly reactive, it is paramagnetic and thus difficult to activate since most biological molecules have paired spins. ${ }^{1}$ A reaction between $\mathrm{O}_{2}$ and these molecules is spin forbidden, and thus the utilization of a transition metal, namely the Fe of the heme, helps to reduce this barrier by reducing $\mathrm{O}_{2}$ to allow for $\mathrm{O}-\mathrm{O}$ cleavage. The use of $\mathrm{H}_{2} \mathrm{O}_{2}$ as an oxidant can be problematic, as the reaction between transition metals and $\mathrm{H}_{2} \mathrm{O}_{2}$ can generate highly reactive hydroxyl radicals when the O-O bond is cleaved homolytically. These hydroxyl radicals can then react with the enzyme's reactive site in a destructive manner. To avoid this, the heterolytic pathway is generally preferred, leading to production of $\mathrm{H}_{2} \mathrm{O}$ with the remaining oxygen bound to the iron. 
Chloroperoxidase is an extracellular fungal heme-enzyme that belongs to the

peroxidase family. Peroxidases are typically between 30,000-40,000 Da in size, and have a single heme connected to the protein via Fe-His ligation. However, CPO is atypical in this regard, as Cys29 rather than a histidine is ligated to the heme. Structurally, CPO is a heme-thiolate enzyme with a peroxide-like distal pocket: the distal pocket is polar and contains an acid-base catalyst, although the catalyst is Glu rather than the typical His. Like cytochrome P450 (P450) and nitic oxide synthase (NOS), CPO possesses amide hydrogen bonds to the sulfur of the heme thiolate. As for P450s and peroxidases, CPOs helical content is approximately $50 \%$ of the overall structure; however, the overall topology does not resemble these heme proteins. ${ }^{3}$ In regard to functionality, among the several reactions that CPO is capable of catalyzing are peroxidase-, P450-, and catalasetype reactions, in addition to the enzyme's signature reaction, chlorination (or more generally, halogenation). For these reasons, an attempt to classify chloroperoxidase simply by structure or function seems to do a disservice to the protein. However, it has most often been referred to as a peroxidase/P450 hybrid.

\subsection{Structure}

The crystal structure of CPO was solved in 1995 and elucidated many features that had been previously predicted, as well as others that were unknown. The crystal structure contains 299 residues, which is 52 less than the cDNA sequence. ${ }^{3}$ The original DNA sequence indicates that the complete CPO gene encodes for 373 amino acid residues. ${ }^{4}$ It was determined that the 373-residue long polypeptide chain undergoes two proteolytic 
cleavages: one removes the 20 -residue signal peptide from the $\mathrm{N}$ terminus and the other removes 52 residues from the $\mathrm{C}$ terminus. There are at least two other post-translational modifications, one of which is glycosylation and the other is cyclization of the N-terminal Glu. ${ }^{5}$ Chloroperoxidase is comprised of eight $\alpha$-helixes segments (A-H), three short $3_{10}$ helices (C', D' and G') and a short antiparallel $\beta$ sheet (see Fig. 1). ${ }^{3}$
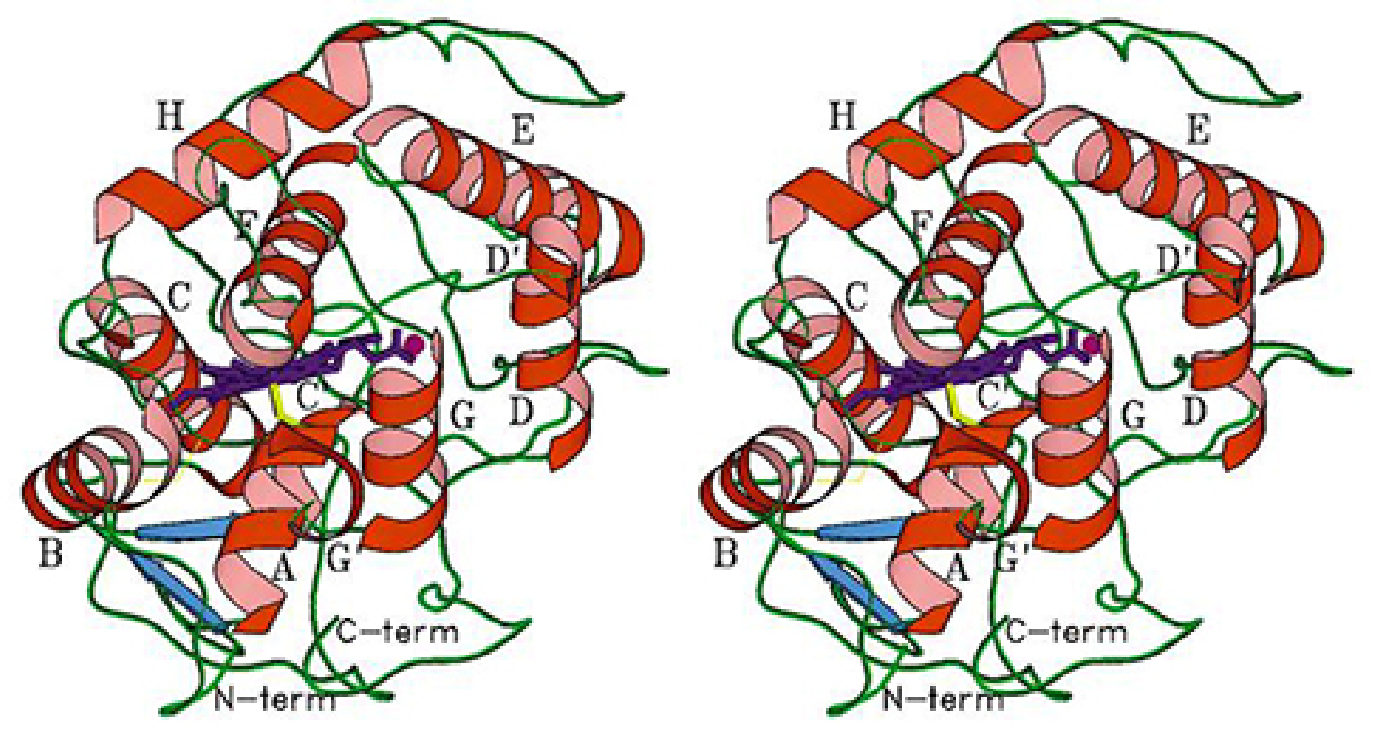

Figure 1: Crystal Structure of Chloroperoxidase (PDB Code: 1CPO) ${ }^{3}$.

The glycosylation of CPO is fairly extensive and involves both $\mathrm{N}$ - and O-linked glycosyl chains. The first CPO crystal structure obtained by Poulos et al. ${ }^{3}$ revealed two major isozymes; however, the crystal structure of form A was obtained to a higher resolution than that of form B and is thus the form more heavily studied by Poulos et al. Form A's molecular weight is $19 \%$ carbohydrate, accounting for 14 glycosylation sites and a total of 21 sugar groups. ${ }^{3}$ Three N-linked glycosyl chains are located on asparagine 
residues (Asn12, Asn 93, and Asn 216), and the remaining 11 O-linked glycosyl chains are located on serine and threonine residues located on the $\mathrm{C}$ terminal. ${ }^{3}$

The prosthetic group of $\mathrm{CPO}$ is a heme $\mathrm{b}$, an iron ligated to protoporphyrin IX. Protoporphyrin IX is made up of four pyrrole rings that are interconnected by methane bridges. There are four methyl substituents bound in the 1, 3, 5, and 8 positions, two vinyl groups bound in the 2 and 4 positions, and two propionates in positions 6 and 7 (see Fig. 2). The heme of CPO is bound to the apoprotein via the axial ligand, Cys29, and held in place by hydrophopic and hydrogen bonding interactions.

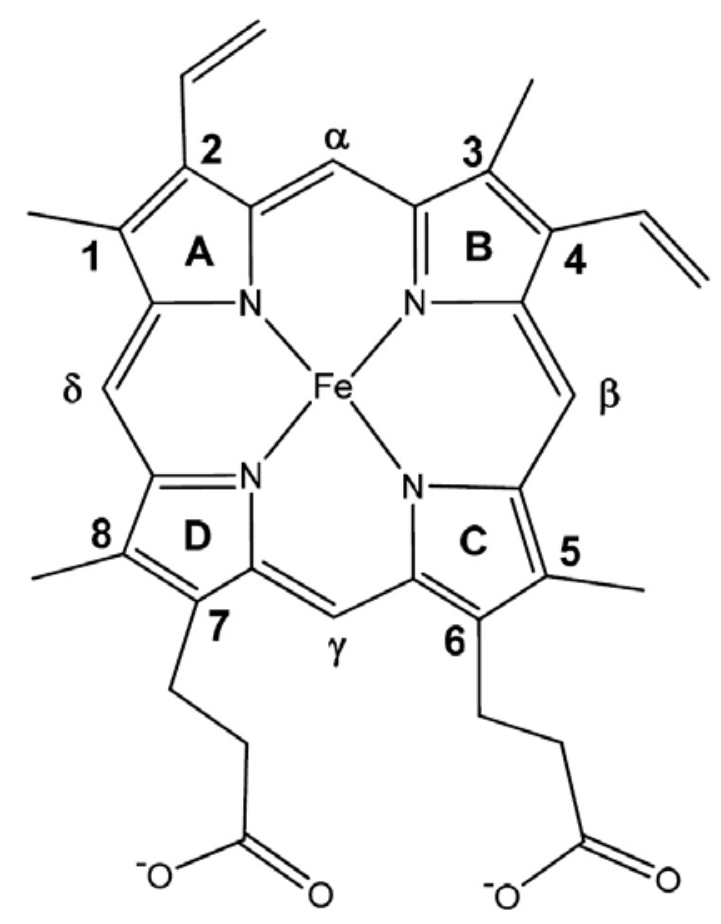

\section{Figure 2: Protoporphyrin IX}

Proximal helix A (residues 29-38), which contains Cys29, is oriented nearly perpendicular to the heme plane. This orientation is an interesting contrast to P450, in which the corresponding helix is oriented nearly parallel to the heme plane. The significance of the helix orientation will be discussed further in Chapter 1, Section 5 , the 
"push effect”. Helix A’s orientation is stabilized by hydrogen bonding interactions between Asn33 and Ala27, and between Asp37 and Arg26. The sulfur of Cys29 has two hydrogen bonding interactions with the amides of Ala31 and Leu32. The roles of the $\mathrm{NH} \cdots$ S hydrogen bonds in P450, as well as in NOS and CPO, have been postulated to be passive (protection of the axial sulfur; prevention of the formation of the deactivated species P420, as well as the prevention of the interaction with diatomics), ${ }^{7-9}$ as well as active (modulation of the "push effect" and the fine tuning of Fe-S bond covalency) ${ }^{8,10-12}$ and is the primary subject of this dissertation.

As previously mentioned, the distal pocket is polar, which is typical of peroxidases. The distal helix, Helix F, contains Glu183, which plays the role of acid-base catalyst for the formation of the reactive species, Cpd I. The imidazole side-chain nitrogens of His105 form hydrogen bonds with the carboxylate side chain of Glu183 and the backbone carbonyl oxygen of Asp106. This set of hydrogen bonds influences the effective pKa of the Glu183 carboxylate side chain, which participates directly in the formation of Cpd I. Additionally, His105 participates in a proton-shuttle during the acidbase catalysis of Cpd I. Molecular dynamics (MD) simulations ${ }^{13}$ have shown that the distal pocket changes character from polar (peroxidase-like) to hydrophobic (P450-like) during the epoxidation of cis- $\beta$-methylstyrene, with Glu183 being displaced out of the pocket and the distal cavity's volume being greatly increased. The results from these simulations corrected the prior assumption of an inflexible distal pocket, an assumption based on highly constrained molecular modeling studies using X-ray crystallographic data, ${ }^{14}$ Additionally, the expansion of the distal pocket explains prior experimental findings that disruption of the proton shuttle in a Glu183His mutant leads to an increase 
in the favorability of the epoxidation reaction over other CPO catalytic reactions. ${ }^{15}$ Access to the distal pocket is limited to two channels, referred to as the narrow and wide channels (see Fig. 3) ${ }^{16}$ The narrow channel is suggested to be responsible for providing halides access to the heme active site. This suggestion is supported by a halide binding site found at the surface of the protein and near the narrow channel, as well as two halide binding sites on the other end of the channel. ${ }^{16}$ Although the wide channel is without halide binding sites, this does not necessarily mean that halides are incapable of passing through the wide channel. However, the wide channel is more likely responsible for providing access to larger substrates that undergo 2-electron oxidizations. Experimental work has shown that epoxidation of substrates that exceed a 10-linear-carbon-atom chain are unable to access the heme, probably due to the width restriction of the channels. ${ }^{17,18}$

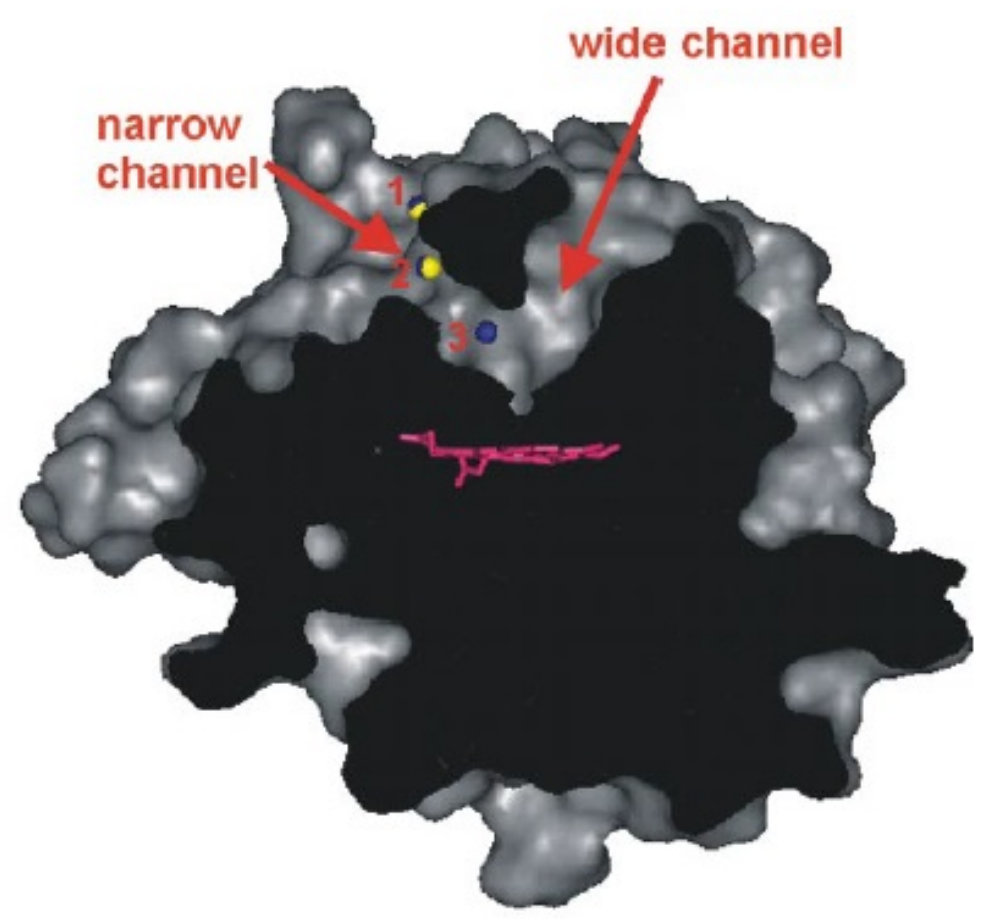

Figure 3: Slice through surface representation of chloroperoxidase. Heme is shown in magenta, and the narrow and wide channels are marked by arrows. The yellow and blue spheres are the bromide and iodide binding sites, respectively. 
An additional feature of CPO is a manganese(II) ion that is coordinated to a heme-propionate and to residues Glu104, His105, and Ser108, as well as two water ligands in an octahedral geometry (see Fig. 4). Early work indicated that the activity of the enzyme is not altered by the absence of the manganese(II) ion. ${ }^{3}$ However, it was recently shown that removal of the $\mathrm{Mn}^{2+}$ decreases overall catalytic activity. ${ }^{19}$ The $\mathrm{Mn}^{2+}$ ion in CPO was found to hold together the active domain (see Fig. 4), maintaining the preferred conformation, as shown by UV-Vis absorption spectroscopy. Spectra obtained from circular dichroism indicates a reduction in CPO's $\alpha$-helical character (uncoiling) when the $\mathrm{Mn}^{2+}$ ion is removed. ${ }^{19}$

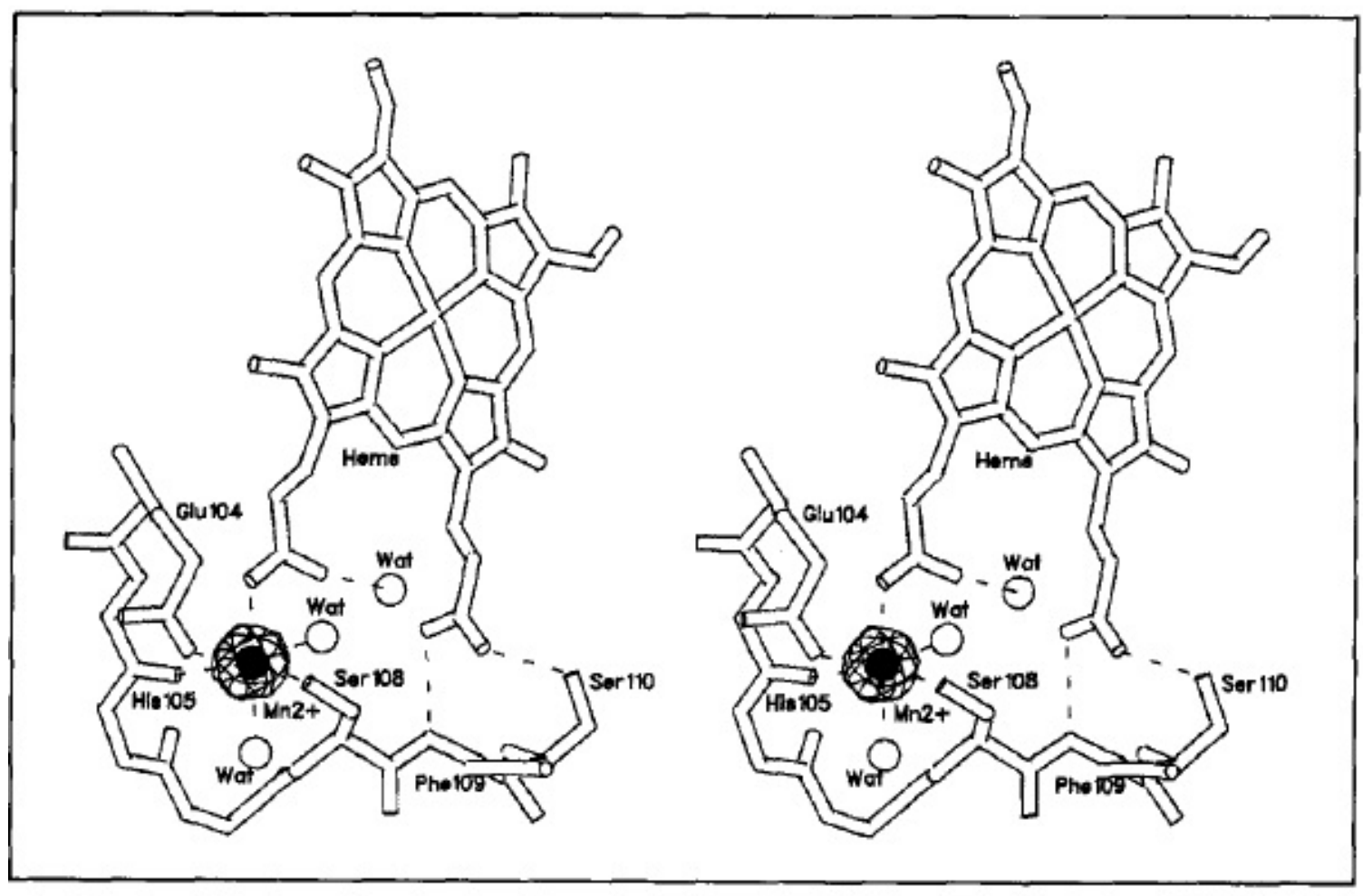

Figure 4: Stereoimage of Manganese binding site. 


\subsection{Function}

Chloroperoxidase is the most versatile heme protein currently known, capable of catalyzing a wide range of reactions. ${ }^{20}$ Chloroperoxidase's signature function is the utilization of chloride, bromide, and iodide ions in the halogenation of organic substrates. ${ }^{21-23}$ At the time of the discovery of these catalytic reactions, the biological mechanism for the formation of the carbon-chlorine bond was unknown, and CPO provided an opportunity to study this reaction. Since that time, continued research on chloroperoxidase has revealed not only a versatile protein whose study contributes to the understanding of hemethiolate proteins generally, but also a potential biocatalyst capable of producing chiral epoxide products for pharmaceutical synthesis.

Chloroperoxidase is capable of dehydrogenation, a characteristic of classical peroxidase activity; the dismutation of hydrogen peroxide, a characteristic of catalase activity; and the monooxygenation (hydroxylation, sulfoxidation, and epoxidation) of organic molecules, a characteristic of monooxygenases. The signature function (halogenation) is relatively specialized but is also observed in other haloperoxidases such as myeloperoxidase MPO, iodoperoxidase (IPO), and bromoperoxidase (BPO). Interestingly, CPO is also capable of dehalogenations, similarly to dehaloperoxidase (DHP). Additionally, the breadth of CPO's catalytic diversity is not limited to reaction type, but also to the broad range of substrate moieties and sizes CPO is capable of interacting with. This diversity will be discussed below.

In the preceding paragraph, various reactions were classified by the protein family characteristically responsible for performing their respective catalytic function. The purpose of this classification is to illustrate the many "faces" of CPO. For a more detailed 
discussion, especially one focused on the relationship between structure and function, it is helpful to organize the reactions into three types: the halogenation reaction, which is mediated via a diffusible intermediate; one-electron oxidations (peroxidations); and twoelectron oxidations (see Fig. 5).

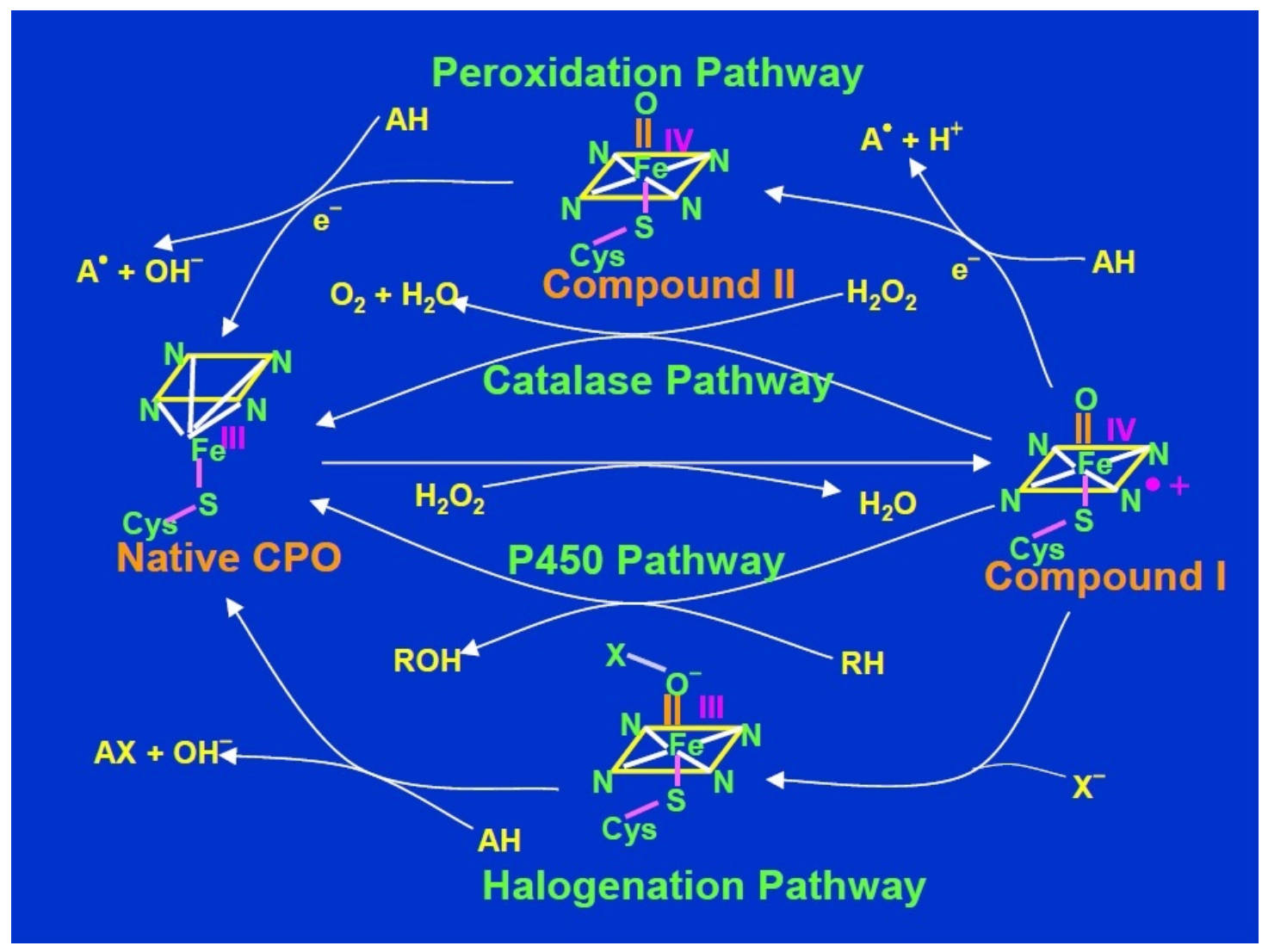

Figure 5: Reaction Pathways of CPO. The Catalase and P450 pathways are both two electron oxidations. ${ }^{24}$ 


\subsubsection{Halogenation - CPO’s signature function}

The mechanism of CPO-catalyzed halogenation is still not completely understood. In 2006, Manoj made significant contributions in elucidating the chlorination reaction, providing experimental results that support the earlier hypothesis that electrophilic chlorinating intermediates are produced in the active site of CPO and subsequently diffuse out of the protein to chlorinate organic substrates in free solution. ${ }^{17}$ It is speculated that a chloride $\left(\mathrm{Cl}^{-}\right)$ion binds to the oxygen of Cpd I and is oxidized to a chloride cation $\left(\mathrm{Cl}^{+}\right)$to form $\mathrm{Fe}(\mathrm{III})-\mathrm{O}-\mathrm{Cl}$, which may then react with another chloride ion to form $\mathrm{Cl}_{2}$, or release $\mathrm{ClO}^{-}$or $\mathrm{HClO}$ (via protonation) into the surroundings. However, the identity of the halogenating intermediate is still not known. Since chlorination occurs in free solution, substrates to be chlorinated are not size-restricted as they would be if the catalytic activity occurred in the distal pocket. Generally, any organic molecule that can undergo chlorination is a potential target for synthesis via CPO. Exceptions to this general rule include molecules that interfere with protein function, such as azide and carbon monoxide (CO). Azide binds to the heme center, preventing the formation of chlorinating intermediates, and CO deactivates $\mathrm{CPO}$, forming the P420 analog.

It is reasonable to assume that CPO-catalyzed bromination and iodination also occur via diffusible intermediates. Rates obtained from optical assays demonstrate that bromination of monochlorodimedon (MCD) occurs at twice the rate of chlorination. ${ }^{22}$ Iodization rates for MCD are difficult to obtain, as the formation of iodine ( $\left.\mathrm{I}_{2}\right)$ obscures the absorbance readings of dimedon, and thus the rate of iodine formation was obtained 
instead. The resultant rate is 1.5 times the rate of MCD chlorination. ${ }^{22}$ Fluorination is not catalyzed by CPO, as fluoride ions compete with hydrogen peroxide and halogen ion binding sites, and are thus an inhibitors of catalysis. ${ }^{22}$

\subsubsection{Peroxidations}

It has been suggested that single-electron oxidations (include hydrogen abstractions) occur at the surface of the enzyme, where surface residues obtain the oxidative equivalent of Cpd I through an electron channel. ${ }^{25}$ This suggestion is supported by the finding that peroxidative substrates do not display the Michaelis-Menten kinetics observed in two electron oxidations ${ }^{26,27}$ (which occur in the distal pocket), or zeroth-order kinetics as observed in chlorinations ${ }^{17,26,27}$ (which depend on the formation of chlorinating intermediates in the distal pocket). Furthermore, the $\mathrm{pH}$ profiles of different peroxidative substrates show optimal activity at different $\mathrm{pH}$ ranges, ${ }^{25}$ suggesting that substrate binding occurs with residues possessing different pKas. Chloroperoxidase-catalyzed peroxidation has been observed for large substrates, such as 2,2'-azino-bis(3ethylbenzthiazoline-6-sulfonic acid) and $\mathrm{N}, \mathrm{N}, \mathrm{N}^{\prime}, \mathrm{N}^{\prime}$-tetrabenzylphenylene diamine. As

previously mentioned, molecules with 10 or more linear carbon atoms are incapable of reaching the active site because of the narrowness of the channels. The full range of substrates capable of undergoing peroxidation by CPO has not been well studied, as CPO's peroxidative ability is smaller than that of other peroxidases. 


\subsubsection{Two-electron oxidations}

Two-electron oxidations catalyzed by CPO include the oxidation of alcohols to aldehydes, ${ }^{28}$ aldehydes to carboxylic acids, ${ }^{29}$ and dealkylations of alkyl amines. ${ }^{30}$ Additionally, there are stereoselective monooxygenations which include hydroxylation, ${ }^{31,}$ ${ }^{32}$ epoxidation, ${ }^{33-39}$ and sulfoxidation..$^{40,41}$ The monoxygenations/P-450 type oxidations have significant biotechnological potential and have garnered a significant amount of attention. Since P-450 type reactions take place in the distal pocket of CPO, steric interactions from particular residue side chains, rather than electronic effects, are likely to be the main contributing factor to stereoselectivity. Molecular dynamics simulations of cis- $\beta$-methylstyrene/CPO-Cpd I complexes found that enantiomeric product ratios are likely to be the result of steric interactions with the hydrophobic core (residues Phe103, Ile179, Val182, and Phe186). ${ }^{42}$ Additionally, substrates are limited to those capable of entering through the two channels (carbon-atom chains of nine atoms or fewer) that lead to the heme center.

The P-450 type reactions insert oxygen into a substrate via a single two-electron oxidation. However, CPO is also capable of performing two-electron oxidations in a stepwise manner. An example of this is the dehalogenation reaction. Introduction of 2,4,6trichlorophenol (TCP) to a steady-state CPO-Cpd I quickly reduced Cpd I to Cpd II. ${ }^{43}$ Once all of the $\mathrm{H}_{2} \mathrm{O}_{2}$ was consumed, Cpd II was returned to the resting state. Without an organic substrate, Cpd I will slowly be reduced to Cpd II, and then to the ferric state ( 75 seconds). However, if TCP is introduced to a steady-state Cpd II (which can remain 
unaltered for $\sim 10$ seconds), the ferric resting state will be obtained relatively quickly ( 2 seconds). ${ }^{43}$

Another variation on the 2-electron oxidation motif is CPO's catalase activity. Catalases decompose hydrogen peroxide into $\mathrm{H}_{2} \mathrm{O}$ and $\mathrm{O}_{2}$. The decomposition mechanism occurs via two steps in CPO. The first step is the formation of Cpd I, which is a 2-electron reduction of $\mathrm{H}_{2} \mathrm{O}_{2}$. Chloroperoxidase “abstracts” an oxygen from $\mathrm{H}_{2} \mathrm{O}_{2}$, producing $\mathrm{H}_{2} \mathrm{O}$ and Cpd I. The second step is a 2-electron oxidation, where Cpd I “inserts" an oxygen into a second $\mathrm{H}_{2} \mathrm{O}_{2}$ to produce $\mathrm{O}_{2}$ and $\mathrm{H}_{2} \mathrm{O}$. The words "abstracts" and "inserts" are given in quotations because the "abstraction" involves a complex rearrangement (discussed in the following section), and the "insertion” has not been mechanistically resolved. However, the net result can be considered an abstraction and an insertion.

\subsubsection{Epoxidations}

Chloroperoxidase has attracted much attention as a promising template for the rational design of synthetic biocatalysts. Chloroperoxidase is capable of producing enantiopure chiral synthons that can be used in the production of pharmacological compounds. Of particular interest to our group is the epoxidation of olefinic substrates. The Chatfield research group has focused on the epoxidation of $c i s-\beta$-methylstyrene (CBMS), ${ }^{13,42}$ as CPO is capable of catalyzing this substrate to an enantiomeric excess of 96\%, making CBMS an excellent substrate for determining the structural features responsible for 
enantioselectivity. ${ }^{39}$ Additionally, CBMS has been used in prior experimental and computational studies. ${ }^{14}$

A study by Gross et al. determined that the epoxidation of olefins by the perchlorato-bound model of Cpd I proceeds in two kinetic steps. ${ }^{44}$ First, there is an intermolecular transfer of an electron from the substrate to the oxyferryl moiety. This is followed by an intramolecular transfer of an electron from the iron to the porphyrin. The two-step mechanism was confirmed by theoretical DFT studies on the heme-thiolate $\left[(\mathrm{SH})^{-}-\mathrm{Fe}^{4+} \mathrm{O}^{2-}\left(\mathrm{N}_{4} \mathrm{C}_{20} \mathrm{H}_{12}\right)^{-}\right]$Cpd I species. ${ }^{45-48}$ More specifically, the reaction starts with an oxyferryl radical attack on the $\mathrm{C}=\mathrm{C} \pi$ bond, forming a $\mathrm{C}-\mathrm{O}$ bond, followed by $\mathrm{C}-\mathrm{O}-\mathrm{C}$ ring closure. The reaction can proceed on nearly degenerate low- and high-spin surfaces that possses distinct pathways with competing radical and cationic electronic states. The barrier for the formation of the first $\mathrm{C}-\mathrm{O}$ bond (rate limiting step) correlates with the energy of the olefin $\pi$-bond, ${ }^{48}$ while the barrier for the formation of the second C-O bond determines the lifetime of the reaction intermediate..$^{45-47}$ 


\subsection{Catalytic Cycle}

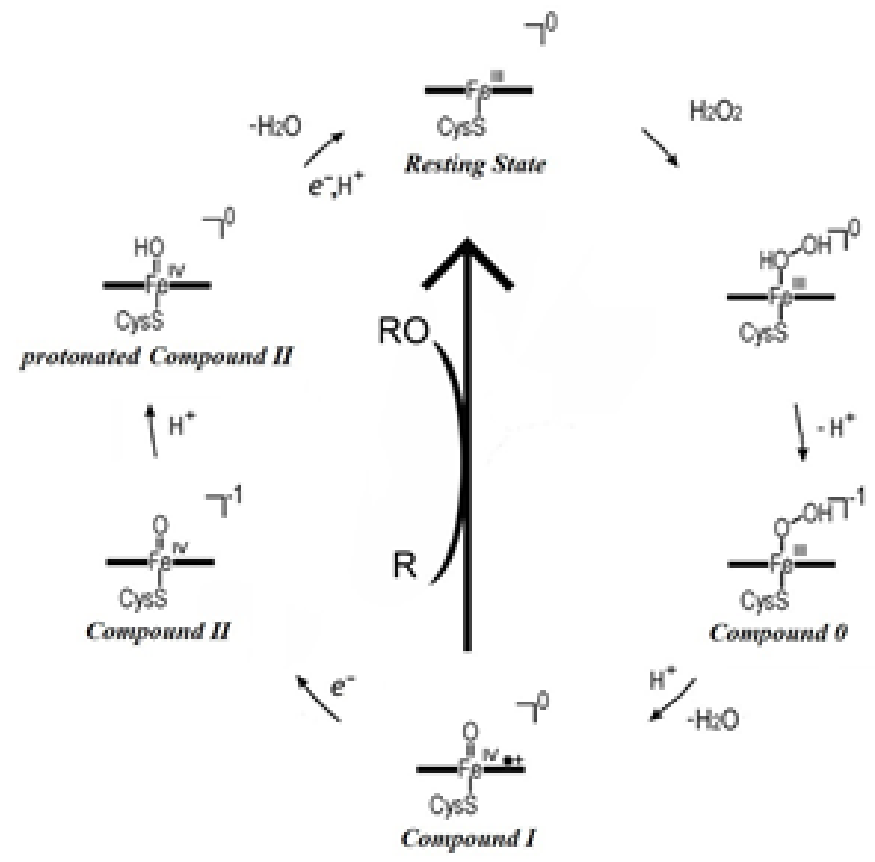

Figure 6: Cholorperoxidase Catalytic Reaction Cycle. The thick horizontal lines represent the porphyrin, so that they together with the Fe-Cys designate the heme with proximally bound cysteine. $R$ represents the substrate and $R O$ represents the oxygen insertion product.

The resting state of chloroperoxidase is found to be the high-spin, ferric form. ${ }^{49-51}$ This resting state is pentacoordinated; however, it is possible for water to bind to the resting state and convert the high spin state to a low spin state. The binding of $\mathrm{H}_{2} \mathrm{O}$ and the change in spin state were deduced from X-ray structures of substrate-free CPO ${ }^{16}$ and supported by additional observations that include conversion of high to low spin upon an increase in $\mathrm{pH}$ (to $\mathrm{pH}$ 6.0), as well as a change in temperature (from ambient conditions to $77 \mathrm{~K}) .{ }^{51,52}$ The typical activating substrate of $\mathrm{CPO}$ is hydrogen peroxide $\left(\mathrm{H}_{2} \mathrm{O}_{2}\right)$, although other alkyl peroxides are capable of converting the resting state into the catalytic form (Cpd I). The oxygen bound to the heme iron $\left(\mathrm{O}_{\text {prox }}\right)$ is deprotonated, 
producting Cpd 0. Subsequently, the distal oxygen $\left(\mathrm{O}_{\text {dist }}\right)$ is protonated, releasing a water molecule to form Cpd I. Compound I possesses an iron oxidized to Fe(IV) and a porphyrin oxidized to a cationic $\pi$ radical. From here, Cpd I can either oxidize a substrate via an oxygen insertion reaction and return to the resting state, or perform a peroxidation and form Cpd II. Compoundd II is structurally almost identical to Cpd I, except that the porphyrin no longer possesses the radical. Compound II receives a proton to form a ferryl hydroxide, and then receives another proton to release a water molecule and return to the resting state.

\subsection{Mechanism of Cpd I formation}

Focusing on $\mathrm{H}_{2} \mathrm{O}_{2}$ as the catalytic substrate, an oxygen atom of $\mathrm{H}_{2} \mathrm{O}_{2}$ binds to Fe, forming $\mathrm{Fe}(\mathrm{III}) \mathrm{HOOH}$. There are two possible binding conformations for $\mathrm{H}_{2} \mathrm{O}_{2}$ in the distal pocket that differ in the hydrogen bonding interactions of Glu183 and the substrate (See Fig. 7). Glutamic acid 183 can hydrogen bond with either the hydrogen of the proximal oxygen or the hydrogen of the distal oxygen of the substrate. A computational study by Shaik et al. has demonstrated that hydrogen bonding to the distal moiety will not lead to formation of the catalytic species, Cpd I. ${ }^{53}$ Hydrogen bonding must be with the proximal moiety, which allows the proton bound to $\mathrm{O}_{\text {prox }}$ to be abstracted and form the stable intermediate Cpd 0. Formation of Cpd 0 begins when a hydrogen that is covalently bonded to $\mathrm{O}_{\text {prox }}$ is transferred to a carboxylate oxygen of Glu183, and initially remains hydrogen bonded to $\mathrm{O}_{\text {prox. }}$ From our findings, the conformation in which Glu183 hydrogen bonds to the proximal oxygen of Cpd 0 (which we call Cpd $0_{\text {prox }}$ ) does not 
possess a local minimum, and is thus unstable (Shaik and coworkers did however, find a metastable Cpd $0_{\text {prox }}$, which they named Cpd 0). ${ }^{53}$ Instead, deprotonation of $\mathrm{O}_{\text {prox }}$ occurs concomitantly with a swivel of the -OOH moiety about the z-axis of the heme plane, centered on the iron, leading to a conformation with Glu183 and Odist sharing the hydrogen bond.

\section{Type 1}

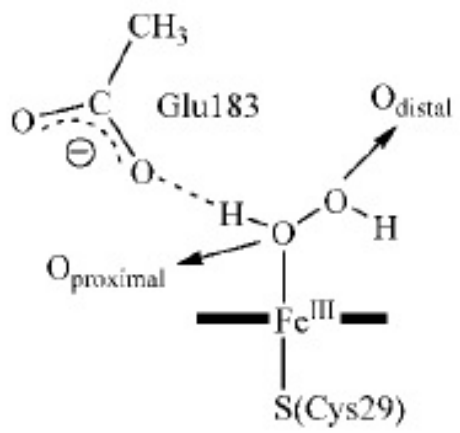

FeHOOH-1

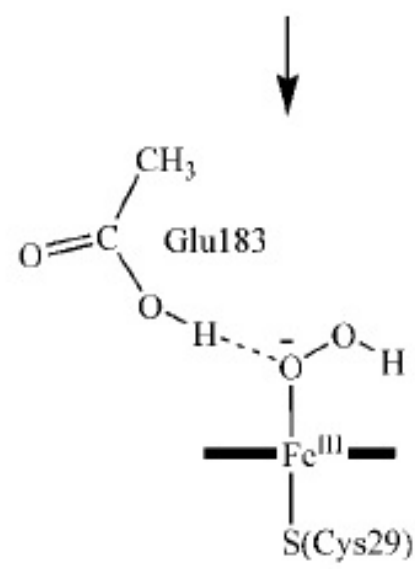

FeOOH-1
Type 2

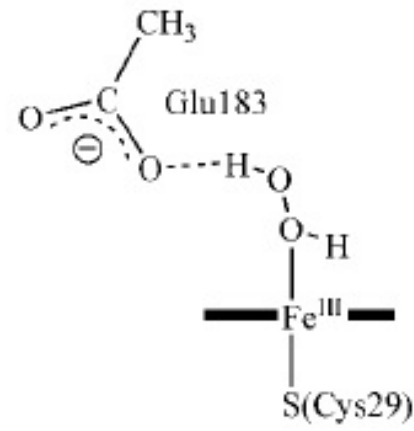

FeHOOH-2

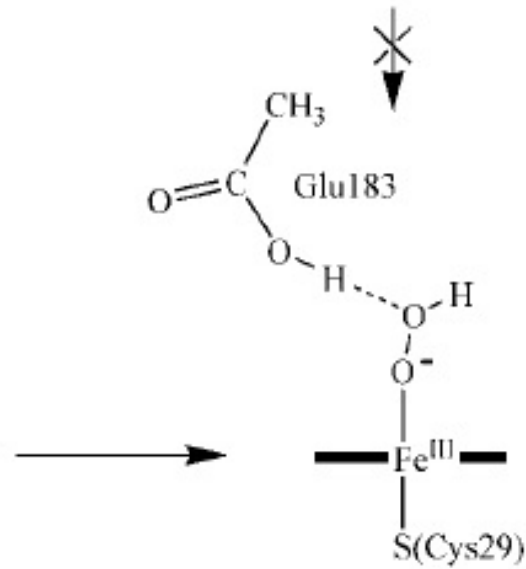

FeOOH-1 $1_{\text {rearr }}$

${ }^{a}$ The horizontal bold line represents the porphyrin ring.

Figure 7: Possible Binding Conformations of FeHOOH ${ }^{53}$ 
The stable Cpd 0 conformation thereby formed has $\mathrm{O}_{\text {dist }}$ hydrogen bonded to the protonated-Glu183 (for the remainder of this chapter, this conformation is simply referred to as Cpd 0), allowing Glu183 to easily protonate $\mathrm{O}_{\text {dist, }}$, leading to the release of an $\mathrm{H}_{2} \mathrm{O}$ molecule upon O-O scission. The aforementioned process, in which a distal residue performs the duty of acid-base catalyst to deprotonate and protonate a bound peroxide, followed by loss of $\mathrm{H}_{2} \mathrm{O}$, resulting in Cpd I formation corresponds to the Poulos-Kraut mechanism of Cpd I formation. ${ }^{54}$ The details of the Poulos-Kraut mechanism are still debated, in particular whether the protonation mechanism occurs in a stepwise fashion, with the formation of a stable protonated Compound 0 intermediate (protCpd0) preceding heterolytic O-O scission, or in a single step with $\mathrm{O}-\mathrm{O}$ bond scission occurring concomitantly with proton coupled electron transfer (PCET).$^{53}$ In the latter case, PCET is the second part of a hybrid homo-heterolytic process, where O-O scission is initially homolytic, but becomes heterolytic as the proton is transferred to the distal oxygen. These issues are at the heart of Chapter 3 and will be addressed in the Results section of that chapter. Regardless of the mechanism, the product is the catalytically active species, Cpd I, and a water molecule.

\subsubsection{Nature of Cpd I and Cpd II}

As mentioned above, Cpd I possesses an iron oxidized to Fe(IV) and a porphyrin oxidized to incorporate a cationic $\pi$ radical. These structural determinations were made prior to the first crystal structures; magnetic susceptibility and Mossbauer studies showed both the iron and the porphyrin to be oxidized, the former to Fe(IV). ${ }^{55,56}$ For some 
peroxidases, such as Cytochrome c Peroxidase (CCP), the moiety oxidized to possess a cation radical is an amino acid side chain such as tryptophan, rather than the porphyrin ring. ${ }^{56,57}$ However, $\mathrm{CPO}$ is not among these. The location of the radical is typically determined by EPR, and the $\mathrm{Fe}(\mathrm{IV})=\mathrm{O}$ double bond description was determined from Resonance Raman studies ${ }^{58-60}$ and XAFS. ${ }^{61-63}$ Initially, there was confusion because these studies were in disagreement with early crystal structures, which indicated that the Fe-O bond was a single bond and the $\mathrm{O}$ was protonated $[\mathrm{Fe}(\mathrm{IV})-\mathrm{OH}] .^{64-67}$ The confusion was determined to be caused by the generation of hydrated electrons (caused by X-rays) that can reduce metal centers ${ }^{68}$ and is currently a well known and manageable issue.

As has been discussed, Cpd I is capable of one- and two-electron oxidations. The two electron oxidations result in CPO returning directy to the ferric resting state, and the one electron oxidations result in the reduction of Cpd I to Compound II (Cpd II). In Cpd II, the majority of the occupancy of the gained electron is on the porphyrin, and thus there is no longer a delocalized cationic radical on the porphyrin ring. Initially, Cpd II is structurally nearly identical to Cpd I, but Cpd II may become protonated due to its greater basicity (see Fig. 8). Protonation occurs at the oxyferryl oxygen, resulting in a ferryl hydroxide. These results have been found experimentally and supported computationally. ${ }^{69,70}$ This iron(IV) hydroxide will accept a proton from either Glu183 or a water molecule in the distal pocket, leading to the production of another water molecule and the reduction of Cpd II to the ferric resting state. 


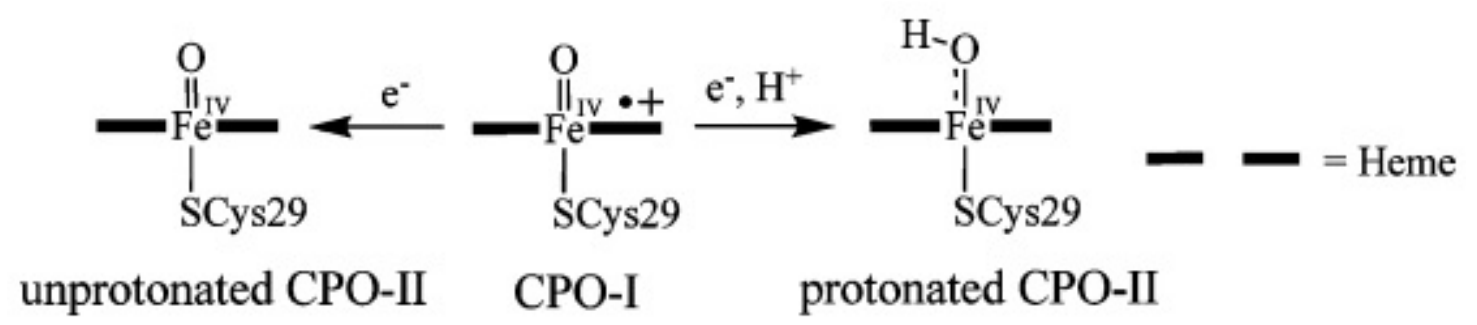

Figure 8: Formation of Cpd II via one-electron oxidation of Cpd I, possibly also with protonation. ${ }^{69}$

\subsubsection{Structural Factors influencing Cpd I formation}

Efficient catalytic activity depends on external factors such as substrate concentrations and environmental $\mathrm{pH}$, and is reliant on internal factors related to structure and function. Herein, we address the influence of protein structure on catalytic activity, particularly via modulation of the distal ligand's pKa and of the redox potential of Cpd I. In CPO, these factors are balanced to achieve the enzyme's particular catalytic characteristics.

The formation of the catalytic species, Cpd I, is dependent on the pKa of the distal ligand. If the pKa of FeHOOH (peroxide bound ferric heme) is too large, the barrier for deprotonation will be high and the rate Cpd 0 formation will be slow. Conversely if the pKa is too small, Cpd 0 will be difficult to protonate, and the rate of conversion of Cpd 0 to Cpd I, which is has been proposed to be the rate-limiting step in Cpd I formation in P450 and peroxidases, ${ }^{53}$ will be slow. Additionally, a proton source is required to facilitate O-O scission.

The redox potential of Cpd I is also important for efficient catalytic function, i.e., for the oxidation of natural substrates. The redox potential must be large enough not just 
to perform this function, but to do so at a sufficiently fast rate. Since substrate oxidation returns Cpd I to the ferric resting state, a fast rate translates into a high turnover rate, and thus an efficient enzyme. Experimental findings have shown that heme redox potentials have an inverse correlation with the negative charge on the proximal ligand, resulting in an inverse correlation with distal ligand basicity. ${ }^{12}$ Thus, the factors that facilitate the rate limiting step of Cpd I formation hamper catalytic function, and vice versa. Interestingly, then, $\mathrm{CPO}$ possesses particular structural motifs that balance the trade-off between substrate pKa and heme redox potentials.

\subsection{Push Effect}

As mentioned above, the rate limiting step in Cpd I formation for peroxidases and P-450 is O-O bond scission. This step is facilitated by an electron "push" from a polarizable proximal ligand, leading to a transfer of electron density to the heme iron, as suggested by Dawson. ${ }^{71,72}$ Manifestations of the push effect have been observed experimentally, ${ }^{73}$ and include an increased basicity of distal ligands, a decreased affinity of anionic ligands to Fe(III)porphyrins, and a decreased redox potential. ${ }^{74,75}$ Recently, a consensus has emerged $^{76}$ that the "push" of the proximal thiolate serves as a balance between the redox potential of the heme active center and the basicity of the distal ligand. ${ }^{10,77,78}$ Additionally, the thiolate "push" promotes the O-O bond scission of Compound 0 by increasing the basicity of the distal oxygen. ${ }^{10,78}$ As previously mentioned, CPO is unique among peroxidases for having a proximal cysteine bound to the heme as opposed to the more common histidine. Cysteine provides a stronger push effect than histidine, and thus 
CPO has a stronger push effect than other peroxidases. As can be seen from model studies, cysteine imparts the heme iron with a smaller reduction potential and produces substrates with a higher pKa, relative to histidine. ${ }^{79}$

It was proposed by Yoshioka et al. that in P-450, and thus by analogy also in other heme-thiolate proteins, an increase in $\pi$-electron donation from the proximal thiolate into the iron, rather than $\sigma$-electron donation, is responsible for promoting $\mathrm{O}-\mathrm{O}$ bond heterolysis of the substrate. ${ }^{80}$ In contrast, an increase in $\sigma$-electron donation from the

proximal thiolate into the $\mathrm{d}_{\mathrm{z}}{ }^{2}$ orbital results in a weakening of the trans Fe-substrate bond, and is known as the "trans influence." ${ }^{~} 1,82$ The trans influence was observed upon removal of $\mathrm{NH} \cdots$. S hydrogen bonds (by mutation) responsible for tuning down $\sigma$-electron donation. In the Cpd 0 species, strong $\sigma$-electron donation from the axial thiolate to the iron $\mathrm{d}_{\mathrm{z}}^{2}$ orbital should favor the release of the trans-OOH ligand of Cpd 0. In addition to obtaining mutants without the $\mathrm{NH} \bullet$ S hydrogen bonds responsible for regulating $\sigma$ electron donation (Q360L and Q360P) via mutagenesis, Yoshioka et al. also studied the L358P mutant, which removes the hydrogen that interacts with the $\mathrm{p}_{\pi}$-orbital of the proximal sulfur. ${ }^{80}$ The experimentally observed result is an increase of $\pi$-electron donation from the thiolate ligand through the iron $\mathrm{d}_{\mathrm{xz} / \mathrm{yz}}$ orbital.

\subsubsection{Proximal H-bond influence on Push Effect}

Although the push effect is beneficial for reducing the barrier for the rate limiting step of Cpd I formation, it also reduces the redox potential, impairing Cpd I's ability to perform oxidations. Additionally, the push effect hampers deprotonation steps found in 
peroxidative Cpd I formation, e.g., FeHOOH deprotonation. P-450 and CPO both possess a proximal cysteine, which produces a push effect stronger than that of proximal histidine. One view of the literature suggests that the sulfur/backbone-amide hydrogen bonds present in $\mathrm{P}-450$ and $\mathrm{CPO}$ serve to reduce the strong push effect. The most extensively characterized cytochrome P450, cytochrome P450cam (P450cam), possesses three such hydrogen bonds, which include one strong hydrogen bond (from Gly359), and two weak hydrogen bonds (from Leu358 and Gln360) ${ }^{83}$ Although CPO has one fewer hydrogen bond than P450cam, both hydrogen bonds (from Ala31 and Leu32) are strong in character. ${ }^{3}$ For specific details on the relative strengths of the hydrogen bonding interactions, see Table 3 of Chapter 3.

As has been previously mentioned, $\mathrm{NH} \bullet \bullet$ S hydrogen bonds are capable of tuning down electron donation from the sulfur to the iron moiety, reducing the push effect. The push effect is observed in Cpd I formation in a CPO model system, where the pKa of the substrate is increased, thus reducing the deprotonation barrier and increasing the protonation barrier (Chapter 3). Results from model studies analyzing the effect of $\mathrm{NH} \cdots \mathrm{S}$ hydrogen bonding on a $(\mathrm{SH})^{-}$thiolate indicate that $\mathrm{NH} \cdots \mathrm{S}$ hydrogen bonds influence hydroxylation versus epoxidation chemoselectivity, as well as the relative ordering of the cationic and radical epoxidation reaction pathways. ${ }^{46,}{ }^{47}$. Compound I models with (SH)$)^{-} \mathrm{Cl}^{-}$, imidazole, and $\mathrm{TyrO}^{-}-\mathrm{ArgH}^{+}$proximal ligands ${ }^{84}$ show an inverse correlation between the epoxidation barrier and the electron affinity. ${ }^{84}$ The inverse correlation suggests that the electropositive environment of the thiolate ligand created by $\mathrm{NH} \cdots \mathrm{S}$ bonding should favor the epoxidation reaction. Experimental work on P450st supports this suggestion ${ }^{85}$; however, the opposite effect was observed in the case of 
P450cam. ${ }^{86}$ As the effect of the $\mathrm{NH} \cdots$. S hydrogen bonds in the model study was estimated by adding two ammonia molecules and performing single-point calculations, it would seem that a more accurate representation of the unique variations contained in heme-thiolate proteins is necessary to understand how the $\mathrm{NH} \cdots \mathrm{S}$ hydrogen bonds modulate the mechanism of the epoxidation reaction.

The anionic character of the proximal thiolate ligand in CPO and P450 is modulated not just by the $\mathrm{NH} \cdots \mathrm{S}$ hydrogen bonding, by also by the electropositive environment of the $\mathrm{N}$-terminus of the proximal $\alpha$-helix. ${ }^{3,83}$ Experimental studies of heme-thiolate model complexes have shown that the proximal helix increases the $\mathrm{Fe}^{+3 /+2}$ redox potential by $130 \mathrm{mV}$ for a CPO model and by $70 \mathrm{mV}$ for a P450cam model. ${ }^{87}$ The increase in redox potential is more pronounced in CPO than in $\mathrm{P} 450$ because CPO's proximal helix is oriented almost perpendicular to the heme plane, while the proximal helix of CPO is oriented almost perpendicular to the heme plane. The perpendicular orientation allows CPO's helix backbone dipole moment to provide the strongest effect possible on the Fe-S coordination (Fig. 9). The Cys-Pro-Ala fragment present in the CPO model cooperates with the $\mathrm{NH} \cdots \mathrm{S}$ hydrogen bonds to stabilize the R-helical conformation, as opposed to the P-450 model fragment (Cys-Leu-Ala) which partially breaks the R-helix at the $\mathrm{N}$ terminus. The experimental data of Ueno et al. ${ }^{87}$ described above suggest that heme-thiolate enzymes are able to regulate the proximal pocket effect by adjusting the proximal helix orientation. Recent work by our group (Chapter 4), where the epoxidation of CBMS is studied with a model system representative of the CPO active site, including the $\mathrm{NH} \cdots \mathrm{S}$ hydrogen bonds and proximal helix, has shown that the $\mathrm{NH} \cdots$. S hydrogen bonds together with the proximal helix, increases the redox potential of 
Cpd I. The increase in redox potential is evident from the reduction of the barrier for the rate limiting step, and supports the suggestion that the proximal helix amplifies the $\mathrm{NH} \bullet \bullet$ S effect.
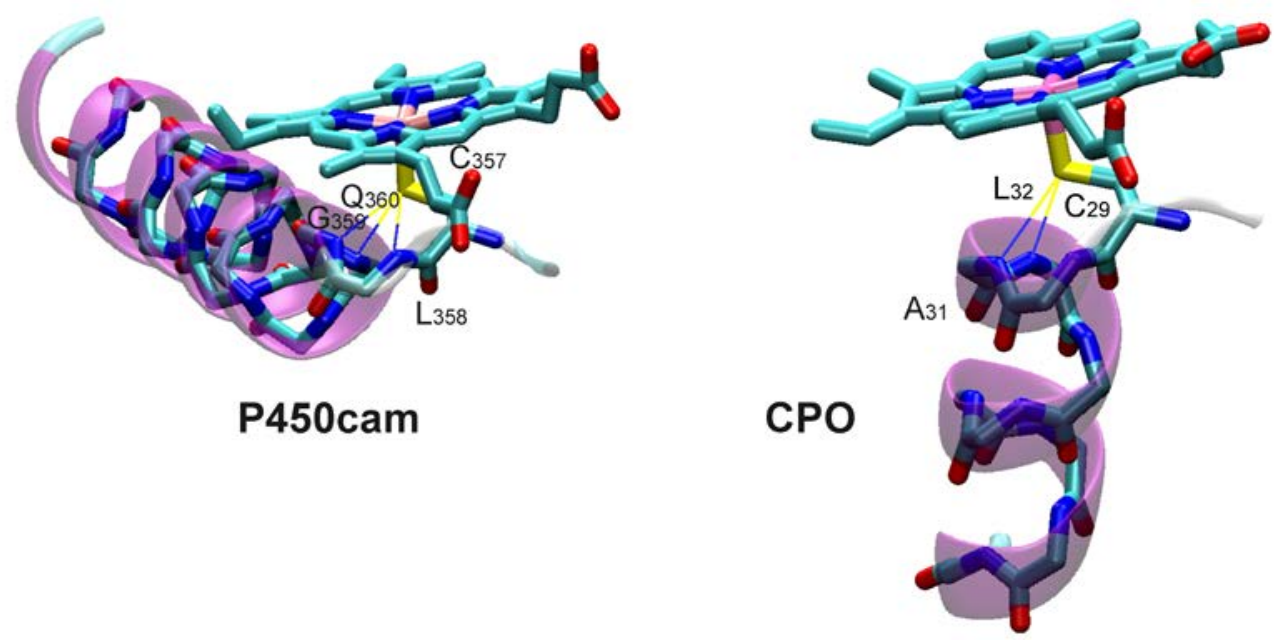

Figure 9: Relative orientations of the proximal helix to the heme in P450cam (parallel) and CPO (perpendicular).

\subsubsection{Push-Pull}

The "push" concept was later expanded to a "push-pull” concept, where in addition to the aforementioned "push" from the distal ligand, polar distal residues were suggested to aid in O-O bond scission by polarizing the O-O moiety via a "pull." ${ }^{\text {88 }}$ Early experimental evidence, in which the mutation of the proximal His to Glu (H157E) or Gln (H175Q) in cytochrome C peroxidase resulted in roughly the same rate of Cpd I formation as in the wild type, supported the hypothesis that the push effect was of minor significance. ${ }^{89}$ Furthermore, the "pull" was considered to be the major driver of Cpd I formation, as 
supported by the mutation of the distal histidine to leucine (H52L), which reduced the rate of Cpd I conversion by 5 orders of magnitude. ${ }^{90}$ Additionally, structural and sitedirected mutagenesis studies have demonstrated that proximal-imidazole-containing peroxidases are capable of cleaving the $\mathrm{O}-\mathrm{O}$ bond with the pull effect (exerted by the particular hydrogen bond pattern present in the distal pocket) alone. ${ }^{12,91}$

In P-450 and peroxidases, Cpd 0’s distal oxygen is protonated prior to O-O scission. Since Cpd 0 already possesses a proton on the distal oxygen, an additional proton produces an $\mathrm{H}_{2} \mathrm{O}$ moiety. Water is an excellent leaving group that facilitates $\mathrm{O}-\mathrm{O}$ scission. On the other hand, P-450 and nitric oxide synthase (NOS) do not possess an efficient hydrogen bonding environment in the distal pocket capable of producing a pull effect. In light of this, Dawson postulated that the push effect alone was sufficient to cleave the O-O bond. ${ }^{71}$ It was later determined from kinetic isotope effect measurements that two protons are required to form Cpd I from the reduced ferrous dioxygen complex $\left[\mathrm{Fe}(\mathrm{II})-\mathrm{O}_{2}\right]^{92}$ The ease with which protonation occurs is a push effect "manifestation"; however, the proton source is elusive. The Poulos-Kraut mechanism proposes that for peroxidase-catalyzed heterolytic cleavage, an acid-base catalyst is required. ${ }^{54}$ A similar mechanism is likely to occur in P-450 and NOS. Although the distal pocket of P-450 is relatively nonpolar, it does contain a polar residue (Asp251) oriented away from the heme, that forms an internal ion pair with Arg186. ${ }^{93}$ In this orientation, Asp251 cannot participate as an acid-base catalyst. However, crystal structures obtained for the wild type dioxygen-bound ferrous form [Fe(II)- $\left.\mathrm{O}_{2}\right]$ of P-450 showed that water molecules in the distal pocket form part of a proton relay system to the dioxygen substrate. ${ }^{94}$ Additionally, since it has long been known that Asp251 and Thr252 are important for 
dioxygen activation, crystal structures of those mutants were obtained (D251N and T252A), ${ }^{94}$ as well as computational studies that model Asp251 and Thr252 in a water relay with Cpd $0,{ }^{95}$ have been conducted. The results of these studies suggest that for P450, protonation of the $\mathrm{Fe}(\mathrm{II})-\mathrm{O}_{2}$ to form $\mathrm{Cpd} 0$, as well as the subsequent protonation of Cpd 0 are feasible.

In view of the issues discussed in the literature, which includes the push effect tuning down the redox potential, the push effect increasing the pKa of substrates, and the reduction of the push effect by the $\mathrm{NH} \cdots$. S hydrogen bonds and the proximal helix dipole, we have undertaken the task of determining the extent as to which the proximal region reduces the push effect and thus influences the catalytic activity of chloroperoxidase. In chapter 3 of this dissertation, the influence of the $\mathrm{NH} \cdots$ S hydrogen bonds on the formation of Cpd I will be addressed. In chapter 4, the focus will be on CPO-Cpd I epoxidation of CBMS, and will address the influence of the $\mathrm{NH} \cdots$ S hydrogen bonds and the proximal helix dipole on this mechanism. 


\section{Methods}

Although experimental methods have been useful in obtaining a significant amount of information related to $\mathrm{CPO}$, there are many questions that cannot be answered with current experimental tools. Of these questions, the ones of particular interest to our research group are mechanistic in nature. More specifically, we wish to understand the structure/function relationship of CPO in regard to the mechanisms of Cpd I formation, as well as the mechanisms behind the stereoselectivity of CPO-catalyzed epoxidation reactions leading to chiral epoxides. To shed light on the aforementioned issues, we have chosen a computational approach, varying our methodology with respect to the particular question we wish to resolve. Ideally, ab-initio methods are the best choice, as they are capable of converging to the exact solution of the Schrödinger equation for a particular system and are thus very accurate. However, in view of the cost of these methods, which includes memory, disk space, and time, an alternate method that employs approximations may be a better choice. When designing a model system, the computational chemist is wise to consider not only whether the system contains enough information to accurately answer a particular question, but also whether there is any unnecessary information that can be removed to reduce the computational cost. Below, various methods will be discussed, and their applications addressed. 


\subsection{Quantum Mechanical Methods}

Quantum mechanical methods can be divided into two categories: ab-initio and semiempirical. Ab-initio, in the context of computational chemistry, refers to the fundamental physical principles or "first principles" that are utilized to solve the Schrödinger equation for a many-body system. The Hartree-Fock (HF) theory lays the groundwork for other ab-initio methods. Hartree-Fock theory produces a wave function that, when operated on by the Hamiltonian, produces an expectation value with the lowest possible energy for a single-determinantal wave function for a particular basis set. ${ }^{96}$ Semi-empirical methods are also based on a HF formalism; however, they make approximations from empirical measurements. All modern semi-empirical methodologies ignore core electrons when forming the secular determinant, some neglect differential overlap to varying degrees (Complete Neglect of Differential Overlap [CNDO], Intermediate Neglect of Differential overlap [INDO], neglect of diatomic differential overlap [NDDO], etc.), while other methodologies modify the potential of mean force between atoms. The general principle for method developers is to replace time-consuming computational steps such as multidimensional integrals with parameters for the purpose of obtaining experimental agreement at a cheap computational cost. As semi-empirical methods are not utilized in this dissertation, they are outside of our scope and will not be discussed. 


\subsubsection{Ab-initio Methods}

The Hartree Fock Self-Consistent Field method provides a stepping stone for more sophisticated theories that aim to produce an accurate solution to the Schrödinger equation. Hartree developed the iterative "self consistent field” (SCF) method that allowed the construction of one-electron operators $h$ from "guessed" trial wave functions $(\psi) .{ }^{97}$ By solving each differential equation for the one-electron Schrödinger equation $\left(h_{i} \psi_{i}=\varepsilon i \psi_{i}\right)$, a new set of $\psi$ is obtained. The new and more accurate set of $\psi$ is treated as a new guess, and the procedure is repeated until the difference between the new and preceding sets of $\psi$ falls within a desired threshold. Fock extended the SCF procedure to Slater determinantal wave functions, ${ }^{98,}, 99$ as the prior formalism did not include exchange effects with the coulombic repulsion. ${ }^{96}$ The Hartree molecular orbitals, which could be individually determined as eigenfunctions of a set of one-electron operators, now include the interaction of each electron with the static field of all the other electrons (HF MO). The method was later expanded by Roothaan, allowing the HF calculations to be carried out using basis sets (set of $\mathrm{N}$ exact wavefunctions $\varphi$ utilized in linear combination) to represent the MOs. ${ }^{100}$ Since approaching the HF limit requires an infinite basis set and is thus impractical, much work has gone into identifying the minimum number of computationally efficient and chemically useful basis functions necessary to come close to the HF limit.

Although the Slater determinantal wave functions are quite accurate, the general four-index integral used to solve the MOs lacks an analytical solution when the basis functions are Slater-type orbitals (STOs). Since a numerical solution is expensive, an 
alternative was provided by Boys, who changed the radial decay of the STOs from $\mathrm{e}^{-\mathrm{r}}$ to $\mathrm{e}^{-(\mathrm{r} \wedge 2)} \cdot{ }^{101}$ The functions proposed by Boys have the form of a Gaussian (Gaussian-type orbitals; GTOs) and lead to integrals that can be solved analytically. However, GTOs produce s functions that are smooth and differentiable near the nucleus, and have a radial decay that is too rapid. To solve this issue, a linear combination of GTOs (referred to as 'primitive Gaussians') is chosen that mimicks an STO, thus providing accuracy to a computationally efficient and fast method. It has been found that the best balance between speed and accuracy is three primitive Gaussians used in linear combination. The basis set is referred to as STO-3G, which is a Slater-type orbital approximated by 3 primitives. The STO-3G basis set is a "minimal" basis set, meaning that there is only one basis function for each type of orbital core through valence. A way to increase flexibility is to increase the number of basis functions while using the same number of primitives. For example, instead of having 1 basis function (known as a 'single- $\zeta$ ' basis set) defined by 3 primitives, it is possible to have 2 basis functions (known as a 'double- $\zeta$ ' basis set) or more, where one function is defined by 2 primitives and the other is defined by 1 primitive. Increasing the number of basis functions in this manner has little effect on core electrons, but may be significant for valence electrons, which are subject to chemical bonding. To manage these issues, a split-valence basis set is employed, in which the core orbitals are represented by a single- $\zeta$ basis set, and the valence electrons are split into many functions. Using the 6-31G basis set as an example of the notation scheme, the first number (six) refers to the number of primitive Gaussians used in the contracted core functions, and the numbers after the hyphen refer to the number of primitives used in the valence functions. Since there are two numbers after the hyphen (a three and a one), it is 
double- $\zeta$. If there were three numbers, then it would be triple- $\zeta$, and so on. Although this methodology works well for describing atoms, there are times when the HF calculation, even while utilizing an infinite basis set, will produce a minimum-energy structure with an erroneous geometry.

The utilization of atom-centered basis functions from the core up to the valence functions is at times inadequate for representing particular geometries. For example, using only s and p functions in the determination of ammonia's geometry will result in a planar structure instead of the correct pyramidal structure. In order to add mathematical flexibility, basis functions corresponding to one-quantum-number-higher angular momentum than that of the valence electrons are added for each atom. In the example of ammonia $\left(\mathrm{NH}_{3}\right)$, nitrogen will contain an additional function ' $\mathrm{d}$ ', and the hydrogens will contain an addition function ' $\mathrm{p}$ '. In basis set notation, a star ' $*$ ' is added to denote that $\mathrm{d}$ functions have been added to polarize p functions, and two stars $(* *)$ indicate that in addition to the $\mathrm{d}$ functions added to polarize $\mathrm{p}$ functions, $\mathrm{p}$ functions have also been added to polarize s functions. Using the prior example of the 6-31G basis set, including polarization functions to the basis set would be expressed as 6-31G* and 6-31G**.

Another augmentation used to better represent the properties of a chemical system is the addition of diffuse functions. Diffuse functions have small exponents that allow a weakly bound electron to be further removed from the rest of the electron density. These functions are useful in accurately representing excited states, the highest MOs of anions, and calculating acidities and electron affinities. ${ }^{96}$ In the Pople family of basis sets, diffuse functions are represented by a '+', such as in $6-31+\mathrm{G}^{*}$. A single + indicates that heavy atoms are augmented with one s and one p diffuse function, and two 
successive pluses (eg., 6-31++G**) indicates that in addition to the augmentation on heavy atoms, diffuse s functions are added to hydrogens as well.

The model system studied in this dissertation contains two particular characteristics not easily resolved with the HF methodology as it has been developed in this dissertation thus far. First, the model system contains a very heavy element (Fe). Very heavy elements contain many electrons, and thus contribute significantly to the computational cost. Fortunately, the majority of the electrons are located in the core, and can be represented by an effective core potential (ECP). The ECP represents the combination of the nuclear and electronic core, and may be adjusted to include everything up to the outermost valence shell, or may not include the outermost core when the sub-valence shell is considered chemically significant. The second characteristic is related to the spin state of our system, for which we have modeled both the doublet and the quartet. The methods discussed thus far have been for a restricted hartree fock approach (RHF; closed shell systems), and thus assume that all electrons are paired (singlet). To solve this issue, an unrestricted hartree fock (UHF) method is used. Restricted open shell hartree fock (ROHF) methods solve the unpaired issue by not multiplying the density matrix for singly occupied orbitals by two. However, the problem with this method is that it fails to account for spin polarization in doubly occupied orbitals. To solve this issue, UHF treats the $\alpha$ and $\beta$ orbitals individually when the MOs are constructed. Although spin polarization is desirable, allowing the $\alpha$ and $\beta$ spins to have different wave functions produces a total wave function that is not an eigenfunction of the total spin angular momentum operator $\left(\hat{S}^{2}\right)$, resulting in a mixing of other spin 
states (spin contamination). The degree of contamination varies from system to system, and should be evaluated.

The final issue that should be discussed before proceeding to the next section is dynamical correlation. Dynamical correlation refers to the correlated motion of each electron with every other electron, which the HF approximation ignores. Instead, in the HF approximation, each electron interacts with all of the other electrons simultaneously as a static electric field. ${ }^{96}$ There are many ab initio methods that are capable of incorporating dynamical electron correlation, but discussion of them is outside the scope of the dissertation. Density functional theory (DFT), the QM method employed to solve the issues at the heart of this dissertation, does not address this issue directly. Instead, DFT utilizes the Kohn-Sham methodology ${ }^{102}$ discussed in the section below.

\subsubsection{DFT}

Wave functions are difficult to work with, as they are complex, antisymmetric [for fermions]; have $3 \mathrm{~N}$ spatial coordinates [where $\mathrm{N}$ is \# of electrons] and $\mathrm{N}$ spin components; and difficult to interpret (probability densities raised to the one-half power). Density functional theory allows for the determination of the energy and other properties of a system by utilizing a physical observable, the electron density. The scaling (dependence of computer time on $\mathrm{N}$ ) behavior of DFT is no worse than $\mathrm{N}^{3}$, where $\mathrm{N}$ is the number of Kohn-Sham orbital basis functions. Density functional theory scales better than HF theory by at least a factor of $\mathrm{N}$, and much better than other methods that include electron correlation. ${ }^{96}$ 
A wavefunction contains all the information about a system, but can the electron density be utilized in the same manner? The Hohenberg-Kohn existence theorem, which proves (via reductio ad absurdum) that the ground-state density determines the external potential, and thus the Hamiltonian, answers this question with an emphatic yes! ${ }^{103}$ However, the exact form of the density functional is not known, and approximate forms of the exchange-correlation energy are used. DFT thus yields an exact solution to an approximate problem. In addition to the Hohenberg-Kohn existence theorem, the Hohenberg-Kohn variational theorem shows that the density obeys a variational principle. Thus, starting with an appropriate initial density guess, an energy can be obtained that is greater than or equal to the true ground-state energy (true in the sense that the approximate system can be solved exactly).$^{103}$ All that is needed is a methodology for obtaining improved "guesses" of the density, as well as a way around calculating the energy expectation value of the Hamiltonian, which requires solving the Schrödinger equation.

Kohn and Sham simplified the Hamiltonian by expressing it as a sum of oneelectron operators (non-interacting system of electrons). ${ }^{102}$ By taking the fictitious system that has a ground-state density equal to some real system (interacting electrons), the energy functional can be divided into components for analysis. The components are the kinetic energy of the non-interacting electrons, the classical electron-electron repulsion, the correction to the kinetic energy to account for electron-electon interaction, and all the non-classical corrections to the electron-electron repulsion. ${ }^{96}$ The last two components, the corrections to the kinetic energy and non-classical electron-electron 
repulsions, are combined into a single term referred to as the exchange correlation energy $\left(E_{x c}\right)$. In addition, the correction to the classical self-interaction energy is included in this term.

In principle, DFT is an ab initio method as there are no approximations and Hohenberg and Kohn proved that a functional for the density must exist. However, in practice DFT is semi-empirical, as the form of the functional of the density is not known. Instead, approximations are used for both the exchange and the correlation. The functional used in our work is the B3LYP hybrid functional, where the "B" represents the Becke88 ${ }^{104}$ generalized gradient approximation (GGA) exchange functional, "LYP” represents the Lee, Yang, and Parr GGA correlation functional, ${ }^{105}$ and the " 3 ” represents the three parameters (a, b, and c) Becke used to fit a similar functional (B3PW91 to the G1 ${ }^{106}$ set of molecules. ${ }^{107,108}$ More specifically, the functionals were fit to the atomization energies, ionization potentials, proton affinities, and total atomic energies.

Although it is not evident in the name, the B3LYP hybrid functional employs the contribution of the Hartree-Fock exact exchange functional to the energy $\left(E_{x}{ }^{H F}\right)$ as well as the contribution of the local density approximation (LDA) to the energy with respect to both exchange and correlation $\left(\mathrm{Ex}_{\mathrm{x}}{ }^{\mathrm{LDA}}\right.$ and $\left.\mathrm{Ec}^{\mathrm{LDA}}\right)$.

$E_{X C}{ }^{B 3 L Y P}=(1-a) E_{X}{ }^{L D A}+a E_{X}{ }^{H F}+b \Delta E_{X}{ }^{B}+(1-c) E_{C}{ }^{L D A}+c E_{C}{ }^{L Y P}{ }^{109}$ where A, B, and C were optimized to $0.20,0.72$, and 0.81 , respectively. 


\subsection{Molecular Mechanics}

Computational QM methods have proven themselves useful in solving a variety of chemical questions. However, QM methods are computationally expensive, and thus are unable to treat large model systems and/or long-time dynamics, even when utilizing the fastest semi-empirical methods. To address these issues, classical mechanics can be employed to calculate and model the various physical properties of a system, although chemical properties generally cannot be directly addressed. In molecular mechanics (MM) methods, also referred to as force-field methods, atoms (or in the case of coarsegrained methods, groups of atoms) are represented as hard spheres with given mass, radius, charge, and polarizability, and bonds are represented as springs with an equilibrium bond distance and spring constant. The physical values of the aforementioned properties are obtained from experiment and quantum mechanical calculations. In order to calculate the potential energy surface (PES) of a system, the contribution to the energy of the all the bond stretching, bond angle bending, dihedral torsion, electrostatic interactions (Coulomb potential), van der Waals interactions, as well as coupling terms are taken into account. The exact functional form of each contributor to the PES varies with choice of force field. In this dissertation, we have chosen the CHARMM22 force field, ${ }^{110}$ with which our group has prior experience. Additionally, we have included CMAP corrections, ${ }^{111}$ as they improve the dynamical and structural properties of proteins. ${ }^{112}$

A force field consists of a functional form that defines how the energies and forces of each particle in a system are calculated. Additionally, a force field contains a set 
of parameters that defines how the relative positions of atoms determine the energy. The general scheme is given by the following expression. ${ }^{109}$

$$
\begin{aligned}
& U(\vec{R})=\sum_{\text {bonds }} K_{b}\left(\left(b-b_{0}\right)^{2}+\sum_{U B} K_{U B}\left(S-S_{0}\right)^{2}+\sum_{\text {angles }} K_{\theta}\left(\left(\theta-\theta_{0}\right)^{2}\right.\right. \\
& +\sum_{\text {dihedrals }} K_{\chi}\left((1+\cos (n \chi-\delta))+\sum_{\text {impropers }} K_{i m p}\left(\varphi-\varphi_{0}\right)^{2}\right. \\
& +\sum_{\substack{\text { nonbonded } \\
\text { Lennard Jones }}} \varepsilon\left[\left(\frac{R_{\min _{i j}}}{r_{i j}}\right)^{12}-\left(\frac{R_{\text {min }_{i j}}}{r_{i j}}\right)^{6}\right]+\sum_{\begin{array}{c}
\text { nonbonded } \\
\text { electrostatic }
\end{array}} \frac{q_{i} q_{j}}{\epsilon_{1} r_{i j}}
\end{aligned}
$$

$K_{\mathrm{b}}, K_{\mathrm{UB}}, K_{\theta}, K_{\chi}$, and $K_{\mathrm{imp}}$ are the bond, Urey-Bradley, angle, dihedral angle, and improper dihedral angle force constants, respectively; $b, S, \theta$, $\chi$, and $\varphi$ are the bond length, Urey-Bradley 1,3-distance, bond angle, dihedral angle, and improper torsion angle, respectively, with the subscript zero representing the equilibrium values for the individual terms. The non-bonded interactions are made up of the Lennard-Jones 6-12 term and the coulombic term. In the Lennard-Jones 6-12 term, $\varepsilon$ is the Lennard-Jones well depth, $R_{\min }$ is the distance at the Lennard-Jones minimum, and $r_{i j}$ is the distance between atoms $i$ and $j$. In the coulombic term, $q$ is the partial atomic charge, $\epsilon_{1}$ is the effective dielectric constant, and $r_{i j}$ is the distance between atoms $i$ and $j .{ }^{110}$

In the CHARMM22 force field, the intermolecular parameters, which include the Coulomb and Lennard-Jones parameter, are chosen from prior CHARMM force fields or ab-initio calculations on rigid monomers. These values are used to determine the intramolecular parameters, which include the bond length, Urey-Bradley, bond angle, and improper dihedral angle terms, whose initial values were chosen from structural and vibrational data. Using the improved intramolecular parameters, the intermolecular 
parameters are then improved, and the procedure is repeated until convergence is

obtained. The Urey-Bradley and improper dihedral terms are not obvious in their utility and warrant mention. The Urey-Bradley term is a harmonic term in the distance between atoms 1 and 3 in some of the angle terms, and is utilized on a case-by-case basis during the final determination of vibrational spectra, as it provides a more accurate representation of in-plane deformations and is capable of separating symmetric and asymmetric bond stretching modes. ${ }^{110}$ The improper dihedral term is necessary for maintaining proper geometry and chirality.

Molecular mechanics has great utility in finding equilibrium geometries and conformations, as well as in running dynamics. The major advantage MM has over QM is computational speed, making calculations on large model systems and/or long dynamics runs are possible. Where molecular mechanics falls short is in the inability to describe the electronic environment, and thus descriptions of bonding, chemical reactivity and chemical selectivity are unattainable. In order to make use of the advantages molecular mechanics and quantum mechanics provide, the two are combined into a hybrid quantum mechanics/molecular mechanics (QM/MM) multiscale method.

\subsection{QM/MM}

Hybrid quantum mechanics/molecular mechanics (QM/MM) methods are capable of simulating chemical reactions in large systems. For example, a catalytic reaction occurring in the active region of a protein can be modeled in the presence of the entire protein, as well as including the solvent environment. The entire system is partitioned 
into an inner region treated quantum-mechanically, and an outer region treated with molecular mechanics. The difficulty in this type of system lies in calculating the interactions between the two regions, as they strongly interact, and thus the energy of the entire system cannot be treated simply as a sum of the energies of the two regions. To properly account for the interaction between two regions that were treated at different levels of theory, a region that separates the two regions (designated the boundary region) must be well defined and carefully treated. There are a variety of schemes for expressing the entire energy of the system, as well as electrostatic and van der Waals interactions between the two regions, and the bonded interactions between the two regions. For the work in this paper, an additive scheme was chosen for expressing the energy of the system, the electrostatic embedding scheme was used to describe the coulombic interactions between the two regions, and the covalent bonds across the boundary are treated with a link-atom scheme, where the dangling bond is capped with a hydrogen atom. Since van der Waals interactions between the two systems are only significant for the atoms at the boundary, only pairs of atoms, where one atom is from the QM region and one atom is from the MM region, are considered. These van der Waals interactions are treated at the MM level; however, to avoid spurious electrostatic interactions, MM atom groups closer than $2 \AA$ to the hydrogen link atoms are removed. 


\section{Boundary \\ Inner subsystem \\ QM \\ Outer subsystem \\ MM}

Figure 10: Partitioning of the system into the MM, QM, and boundary regions. ${ }^{113}$

The entire energy of the sytem $\left[\operatorname{EQM}_{\mathrm{QMM}}(\mathbf{S})\right]$ in the additive scheme is the sum of the outer region treated at the $\mathrm{MM}$ level $\left[\operatorname{EmM}_{\mathrm{MM}}(\mathbf{O})\right]$, the inner region and the link atoms treated at the QM level $\left[\mathrm{E}_{\mathrm{QM}}(\mathbf{I}+\mathbf{L})\right]$, and the interaction between the two regions $\left[\mathrm{E}_{\mathrm{QM}}\right.$ $\operatorname{MM}(\mathbf{I}, \mathbf{O})]$, where $\left[\operatorname{EQM}_{\mathrm{QM}} \mathrm{MM}(\mathbf{I}, \mathbf{O})\right]$ is the sum of the electrostatic, van der Waals and bonded interactions between QM and MM atoms. ${ }^{113}$

$$
E_{Q M / M M}(S)=E_{M M}(O)+E_{Q M}(I+L)+E_{Q M-M M}(I, O)
$$

Since the link atoms are not part of the real system, a correction to the energy is justified and often expressed as $-\left[E_{M M}(L)+E_{M M}(I, L)\right]$.

$$
E_{Q M / M M}(S)=E_{M M}(O)+E_{Q M}(I+L)+E_{Q M-M M}(I, O)-\left[E_{M M}(L)+E_{M M}(I, L)\right]
$$

However, the use of this correction is often omitted, as it is unclear if the energy of the model is improved. In the case of our system, the correction was deemed unnecessary as 
our interest is not in absolute energies, but rather comparative models. The contributions of the link atoms cancel out when comparing our respective model systems.

As mentioned above, the coulombic interactions are treated using an electrostatic embedding scheme. In this scheme, the QM-MM electrostatic interaction is treated at the QM level, where the MM point charges are incorporated as one-electron terms in the QM Hamiltonian.

$$
H_{Q M / M M}=\sum_{a \in M M} \sum_{i \in Q M}\left(H_{v d w}^{a i}+H_{e l e}^{a i}\right)
$$

This scheme is obviously much more accurate and costly than the simpler mechanical embedding scheme, where QM-MM interactions are treated as MM-MM electrostatics. Additionally, the electronic structure of the QM region can adapt to changes in the MM environment and become polarized.

\subsection{Software and Analytical Tools}

Obtaining data from computational methods is often as simple as reading the output file generated during a computational run. However, there are times when additional software is required to interpret data. Herein, we will discuss the various types of data generated and the software required to obtain that data.

Our research group has utilized two particular software packages for obtaining the potential energy surface (PES) of the quantum mechanical systems. These two programs are NWChem $6.3^{114}$ and Gaussian 09. ${ }^{115}$ NWChem has the benefit of being open-source and scaleable to thousands of processors, while Gaussian is well known for being a 
robust QM package. Therefore, for the time-consuming computational jobs, NWChem was the program of choice, and Gaussian was used to obtain the single point energies of the stationary points obtained in NWChem. The energy of the system is easily obtained from the output files of both programs. Other forms of data must be specifically requested from the input file, or analyzed from the output file using an external program. For example, if the Mulliken spin density and Mulliken charge are required, the request must be specified in the input file of NWChem (Gaussian provides this data automatically). Additionally, if the geometry(ies) of a computational run requires analysis for the interpretation of a reaction coordinate, the generated outputted geometries of a “scan” (successive geometry optimizations along a reaction coordinate, where an internal coordinate is constrained and all other coordinates are allowed to relax) can be visualized using a program such as Molden 5.0. ${ }^{116}$

\subsubsection{Structural information}

We have made use of two programs for analyzing structural data. These programs are VMD 1.9.2 and Molden 5.0. ${ }^{116,117}$ The VMD program works well with files obtained from the protein data bank (PDB; files have extension .pdb). The VMD program can interpret geometric data such as bonds, angles, and dihedrals; add hydrogens to structures obtained from X-ray crystallography; and adjust the way a structure is represented by changing colors, adjusting the lighting, and altering the representations of the atoms (e.g., ball and stick models, licorice, ribbons, etc.) Molden is not as flexible as VMD, but it is a lightweight program that can interpret data quickly. It is most useful when visualizing 
output coordinates (file extension .xyz). As with VMD, bonds, angles, and dihedrals can be obtained.

\subsubsection{Population Analysis}

Population analysis refers to the study of charge distributions. It is useful for analyzing the electronic properties of molecular systems. To determine the partial atomic charges and spin densities on atoms or moieties of interest, there are a few choice analytical methods available. The Mulliken scheme (Mulliken population analysis; MPA) is quite common, as it is available in most molecular modeling software programs. ${ }^{118-121}$ Mulliken population analysis is computationally cheap, and works well when comparing changes in partial charge between different geometries. Mulliken population analysis is derived from the linear combination of atomic orbitals (LCAO) and is thus derived from the wave function, and the electrons are partitioned according to the degree to which the atomic orbitals’ contribute to the overall wave function. It is intuitive and generally sensible that electrons associated with only a single basis function belong to a single atom, and thus the Mulliken approach gives full occupancy of these electrons to a single atom (this simplicity becomes problematic when using diffuse functions). Determining to what degree a shared electron belongs to particular atom is difficult and Mulliken suggested evenly distributing these electrons, resulting in one of the disadvantages of the Mulliken method; the difference in electronegativities between atoms in a molecule is undeterminable. An additional disadvantage of MPA is that it varies significantly when 
different basis sets are used. When non-orthonormal basis sets are used, it is possible for the individual basis functions to have occupation numbers greater than $1 .{ }^{122}$

An alternative method to MPA is Natural Population Analysis (NPA), which uses orthonormal natural atomic orbitals (NAOs). ${ }^{123}$ The NAOs are derived by diagonalization of the localized block of the full density matrix of a molecule for basis functions on a particular atom. In other words, NAOs are composed of basis sets of single atoms. A benefit of this method is that atomic partial charges converge to a stable value as the size of the basis set is increased, unlike MPA. However, NPA charges tend to be the largest in magnitude amongst population analysis methods.

\subsubsection{Molecular Orbitals}

An additional tool for the analysis of a chemical system is the visualization of molecular orbitals. Since our QM method of choice is DFT, the MOs that are visualized are KohnSham MOs. The Gaussian output gives the occupancy of MOs, and the contribution of AOs to each MO. In order to visualize the MOs, it is necessary to use a stand-alone utility called "cubegen”, is part of the Gaussian 09 package. ${ }^{115}$ Cubegen produces a cube file from a Gaussian checkpoint file, and contains volumetric data that can be read by Gaussview 3.0. ${ }^{124}$ Gaussview is then used to visualize the MOs. A visual representation of MOs is useful because distortions to the MOs in response to changes in nuclear structure (primarily a result of changes in the contributions of AOs) can be analyzed and compared. In the case of a reaction profile, it is possible to observe the movement of electrons as a reaction proceeds. However, this can be very complicated, as some MOs 
span a large number of atoms and the exact AO-composition of the MOs is not provided by the software.

\subsubsection{Molecular Mechanics Software}

The aforementioned CHARMM force fields are available with the CHARMM software package ${ }^{125}$ The CHARMM software package is capable of utilizing other force fields, and has been developed primarily for studying biomolecular structures. However, CHARMM is useful for a wide range of many-particle systems. In our work, CHARMM was used to "fit” a QM model system into the full enzyme environment. The QM atoms were assigned electrostatic potential (ESP) charges and CHARMM van der Waals parameters. The MM region of the apo-protein was optimized while the QM region remained fixed. Finally, the QM/MM method was used to obtain the energy of the system using the previously described additive scheme. Further details are given in Chapter 4, which describes calculations using a QM/MM method. CHARMM is capable of significantly more, such as performing dynamics, conformational and path sampling, the construction of models, as well as a variety of analytical techniques. 


\section{Influence of the Proximal Pocket Hydrogen Bonds on the Mechanism of Chloroperoxidase Compound I Formation}

Reproduced with permission from Pardillo, A. D.; Morozov, A. N.; Chatfield, D. C., Proximal Pocket Hydrogen Bonds Significantly Influence the Mechanism of Chloroperoxidase Compound I Formation, J. Phys. Chem. B, 2015, 119, pp 12590-602, Copyright 2015 American Chemical Society.

\subsection{Introduction}

Chloroperoxidase (CPO), an enzyme isolated from the marine fungus Caldariomyces fumago, is a heme-thiolate protein with exceptional versatility. The signature function of CPO is to utilize chloride, bromide, and iodide ions in the halogenation of organic substrates. ${ }^{21-23} \mathrm{CPO}$ is also capable of dehydrogenations, which are characteristic of classical heme peroxidases, ${ }^{126,127}$ the dismutation of hydrogen peroxide, which is characteristic of catalase, ${ }^{126,128}$ and monooxygenation of many organic molecules, which is characteristic of monooxygenase. ${ }^{74}$ Like peroxidases, CPO forms its reactive species, Compound I (Cpd I) and Compound II (Cpd II), using a suitable peroxide as a substrate, and has a polar/charged residue in the distal pocket to assist as a general acid-base in the formation of Cpd I (Glu183; see Scheme 1 for the mechanism of formation of Cpd I and the notation for the various species to be discussed). However, like P450, CPO uses a cysteine-thiolate as the proximal heme ligand, and the distal binding pocket can be made 
hydrophobic by displacement of the Glu183 side chain. ${ }^{13,42}$ CPO can thus be considered a P450-peroxidase hybrid.

The strong electron-donating axial thiolate ligand has been proposed to facilitate O-O bond scission of the ferric hydroperoxide intermediate, Compound 0 (Cpd 0; see Scheme 1), an influence termed the "push effect” by Dawson. ${ }^{71,88}$ The push effect appears to operate by raising the pKa of Cpd 0 , favoring protonation of the distal oxygen (see Fig. 11a for definition of proximal and distal oxygens), ${ }^{80}$ leading to facile O-O heterolysis as suggested by Harris \& Loew. ${ }^{78}$ The push effect does not appear to substantially weaken the O-O bond prior to protonation. Density functional theory (DFT) studies on cytochrome P450 calibrated with sulfur K-edge XAS measurements show that the axial thiolate does not weaken the $\mathrm{O}-\mathrm{O}$ bond relative to a neutral imidazole ligand. Based on experimental findings, Yoshioka et al. proposed more specifically that a $\pi$ electron donation by the axial thiolate promotes protonation of the distal oxygen, leading to facile O-O heterolysis, an influence they termed the " $\pi$-push effect." 80

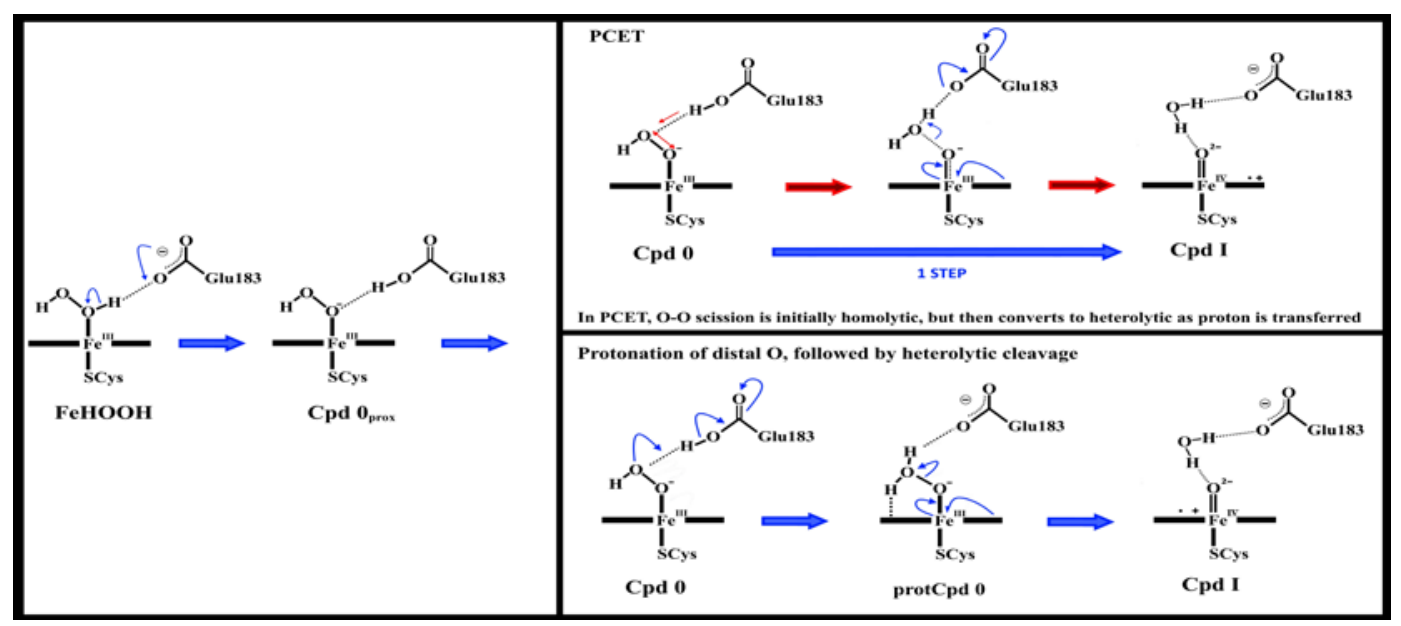

Scheme 1: Reaction mechanism of Cpd I formation from the heme-peroxide complex; PCET and classic heterolytic. Cpd II is not illustrated, as it is not part of our study. Cpd II has the same structure as Cpd I, with the exception that the cationic radical delocalized about the heme is no longer present, as a result of a one-electron reduction of Cpd I. 


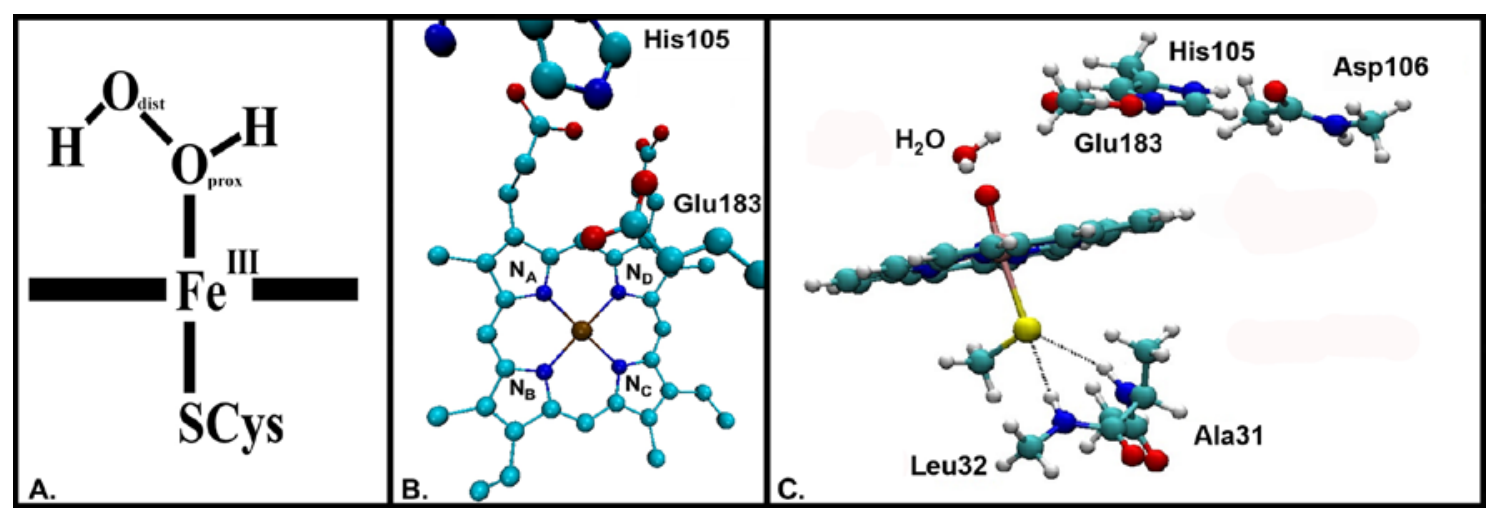

Figure 11: a. Notation for substrate oxygens. b. Notation for ring nitrogens used by Shaik et al. ${ }^{53}$ The perspective is looking "down" from the distal pocket. His105 can be seen extending from the heme in the same direction as the heme propionate side chains. c. Model system (Cpd I arbitrarily chosen) with proximal region included and $\mathrm{NH} \bullet \bullet \mathrm{S}$ hydrogen bonds shown as dotted lines.

A more controversial issue and the subject of this paper is the influence of hydrogen bonds to the axial sulfur on the push effect, in particular for the formation of Cpd I. In both P450 and CPO, several hydrogen bonds extend to the thiolate sulfur from the polypeptide amides that surround it ( $\mathrm{NH} \bullet \bullet$ S hydrogen bonds). P450cam possesses three such hydrogen bonds, one of which is strong (from Gly359), while two are weak (from Leu358 and Gln360). ${ }^{83}$ CPO possesses two such hydrogen bonds (from Ala31 and Leu32), and both are strong. ${ }^{3}$ Table 1 compares the geometries of the P450cam and CPO $\mathrm{NH} \bullet \bullet S$ hydrogen bonds in crystal structures [the P450cam structures (2cpp ${ }^{83}$ and $\left.1 \mathrm{dz} 4^{129}\right)$ are of the ferric resting state and the CPO structure $\left(2 \mathrm{~J}_{5} \mathrm{M}^{130}\right)$ is of Cpd 0 ; hydrogens were placed using the Visual Molecular Dynamics software program, ${ }^{117}$ with $\mathrm{N}-\mathrm{H}$ distances of $1.06 \AA$ ]. A range of roles for the $\mathrm{NH} \bullet \bullet S$ hydrogen bonds has been postulated, from passive (protecting the axial sulfur) to active (modulating $\mathrm{O}-\mathrm{O}$ bond scission in Cpd 0). In particular, it has been proposed that in P450, the function of $\mathrm{NH} \bullet$ • S hydrogen bonds is mainly to prevent the proximal cysteine from being 
protonated, which leads to the deactivated species P420, or reacting with diatomics. ${ }^{7-9}$ On the other hand, Poulos suggested that the $\mathrm{NH} \cdots$ S hydrogen bonds modulate the push effect of the thiolate ligand. ${ }^{12}$ Computational studies on model systems demonstrated the $\mathrm{NH} \bullet \bullet$ S hydrogen bonds shorten and strengthen the Fe-S bond. ${ }^{131,132}$ Computational studies on model compounds showed that $\mathrm{NH} \cdots$. S hydrogen bonds influence the regioselectivity of the P450-catalyzed oxidation of propene by differential alteration of the barriers for epoxidation and hydroxylation, ${ }^{46}$ as well as increase the rate-determining barrier for the NOS-catalyzed formation of nitric oxide by $2.0 \mathrm{kcal} / \mathrm{mol} .{ }^{133}$ It has been suggested that the $\mathrm{NH} \cdots$. S hydrogen bonds are responsible for fine tuning Fe-S bond covalency. ${ }^{8,10,11}$ Work on arene-thiolate compounds, ${ }^{134,}, 135$ alkane thiolate compounds, ${ }^{136}$ and tri- and tetra-peptide-heme-thiolate complexes, ${ }^{137}$ all found that the $\mathrm{NH} \cdots$ S hydrogen bonds increased the reduction potential of the heme, although Ueno et al. found a smaller increase when the P450 proximal environment was mimicked most closely. ${ }^{131}$ Studies of heme redox potentials along a series of P450cam mutants showed that as the number of hydrogen bonds to the sulfur of the axial cysteinate ligand, Cys357, decreased, the reduction potential decreased ${ }^{80}$ However, the changes were modest compared to those seen for some of the model compounds, possibly because these mutants removed the weak (from Leu358 and Gln360) but not the strong (from Gly359) hydrogen bonds. The strong $\mathrm{NH} \cdots$ S hydrogen bond in P450 was estimated to tune the reduction potential up by $>200 \mathrm{mV}$ and to tune the anisotropic $\pi$ covalency down, lowering the rotational barriers of the proximally bound oxygen intermediates and allowing for reorientation towards conformations that enhance reactivity, on the basis of sulfur K-edge X-ray absorption spectroscopy experiments coupled with DFT calculations. ${ }^{10}$ The sensitivity of 
calculated Fe-S bond $\pi$-covalencies to the presence of $\mathrm{NH} \bullet \bullet$ S hydrogen bonds in small model systems indicated that the NH•••S hydrogen bonds may influence the bond strength. ${ }^{10,11}$ At the same time, small shifts of the $\mathrm{S}(\sigma) \rightarrow \mathrm{Fe}(\mathrm{III})$ charge transfer (CT) transitions obtained via magnetic circular dichroism (MCD) spectroscopy in P450 model systems with five-coordinate high-spin ferric hemes show that removal of the hydrogen bonds results in only minor effects on the properties of the Fe-S bond, and is thus not significant for the fine tuning of Fe-S bond covalency. ${ }^{7,8}$ But again, this work was restricted to mutants that removed only the weak hydrogen bonds. On balance, although there has been some thought to the contrary, the preponderance of work on P450 indicates that strong, but not weak, hydrogen bonds to the axial sulfur influence the redox potential and the Fe-S bond covalency to a significant extent, and thus are likely to exert a significant influence on the formation of Cpd I. One would expect an even greater effect in CPO in view of the stronger hydrogen bonding to the proximal sulfur. For this reason, we set out to directly calculate the influence of amide hydrogen bonds to the axial sulfur on the formation of Cpd I in CPO.

\section{Table 1: Comparison of P450cam and CPO proximal pocket $\mathrm{NH} \bullet \bullet$ S hydrogen bonds.}

\begin{tabular}{|c|c|c|c|c|}
\hline & \multicolumn{4}{|c|}{ Residue $r(\mathrm{~N} \cdots \mathrm{S}) \mathrm{r}(\mathrm{S} \cdots \mathrm{H}) \Theta(\mathrm{N}-\mathrm{H}-\mathrm{S})$} \\
\hline \multirow[t]{5}{*}{ P450 (2cpp) } & \multirow{4}{*}{$\begin{array}{l}\text { Leu358 } \\
\text { Gly359 } \\
\text { Gln360 }\end{array}$} & \multirow[b]{2}{*}{3.57} & \multirow[b]{2}{*}{3.63} & \multirow{2}{*}{78.40} \\
\hline & & & & \\
\hline & & 3.25 & 2.60 & 121.40 \\
\hline & & 3.40 & 3.21 & 92.10 \\
\hline & & & & \\
\hline \multirow{3}{*}{$\mathrm{P} 450$ (1dz4) } & Leu358 & 3.53 & 3.53 & 81.44 \\
\hline & Gly359 & 3.31 & 2.58 & 129.40 \\
\hline & Gln 360 & 3.27 & 3.06 & 93.10 \\
\hline \multirow[t]{3}{*}{ CPO (2J5M) } & & & & \\
\hline & Ala31 & 3.41 & & 126.20 \\
\hline & Leu32 & 3.46 & 2.44 & 175.86 \\
\hline
\end{tabular}

Distances $r(\AA)$ and angles $\theta\left(^{\circ}\right)$ are given for selected $\mathrm{x}$-ray structures (pdb entries given). 
Shaik et al. performed calculations on the conversion of ferric CPO with hydrogen peroxide bound to the heme (FeHOOH) to Cpd I, using a DFT-based QM/ MM approach on the full enzyme. ${ }^{53,138}$ The preferred pathway from FeHOOH to Cpd I was found to proceed on the doublet surface through Cpd $0 .{ }^{53}$ The mechanism begins with the abstraction of a proton from the proximal oxygen, $\mathrm{O}_{\text {prox }}$, of the FeHOOH complex, leading to Cpd $0_{\text {prox }}$, a Compound 0 conformer possessing a hydrogen bond between $\mathrm{O}_{\text {prox }}$ and the Glu183 side-chain carboxylate. Reorientation of the hydrogen bond to extend to the distal oxygen, $\mathrm{O}_{\text {dist }}$, rather than $\mathrm{O}_{\text {prox, }}$ yields a more stable conformer that we designate Cpd 0 (Scheme 1). ${ }^{1} \quad$ In the next step, protonation of $O_{\text {dist }}$ by neutral Glu183 and O-O bond scission occur, with water departing. The O-O bond scission step was found to occur via a hybrid homo-heterolytic mechanism, beginning in a homolytic manner but converting to heterolytic when an electron and a proton are transferred simultaneously [proton coupled electron transfer (PCET)]. The nature of O-O bond cleavage during the formation of Cpd I may vary from enzyme to enzyme and is actively debated. ${ }^{139-141}$ Scheme 1 shows both the hybrid homo-heterolytic and the classic heterolytic mechanisms. For the latter, protonation of Cpd 0 leads to a protonated intermediate, protCpd0, prior to electron transfer and departure of water. The experimental and theoretical results of Dey et al. for P450cam ${ }^{11}$ suggest that the explicit inclusion of proximal $\mathrm{NH} \cdots \mathrm{S}$ hydrogen bonds in a quantum mechanical computational model is necessary to reproduce the experimental results.

\footnotetext{
${ }^{1}$ Shaik et al. labeled the conformers differently, calling the former (our Cpd $0_{\text {prox}}$ ) Cpd 0 and the latter (our Cpd 0) Cpd 0 rearr. We have chosen the alternative labeling because, in our systems, generally only the conformer with a hydrogen bond to $\mathrm{O}_{\text {dist }}$ is stable, and thus it is most straightforward to use the simpler label, Cpd 0, for this conformer.[33]
} 
The work of Shaik et al. on the formation of Cpd I in CPO demonstrated that the enzyme environment has a significant influence. ${ }^{53,138}$ However, the particular influence of the $\mathrm{NH} \cdots$ S hydrogen bonds has not been identified separately from the overall influence of the enzyme environment. In view of the theory that the push effect is crucial for $\mathrm{O}-\mathrm{O}$ scission ${ }^{73}$ and thus a critical driving force for the formation and reactivity of Cpd I, as well as that the second coordination sphere, especially hydrogen bonding to the axial ligand, may modulate the strength of the push effect, we have set out to determine the influence of the $\mathrm{NH} \cdots$ S hydrogen bonds on the formation of CPO Cpd I. To do so, we isolated the influence of the $\mathrm{NH} \cdots$ S hydrogen bonds from other influences by building a model of the active site (vide infra), and we performed calculations of the formation of Cpd I with and without the proximal region present. The results have implications for the role of $\mathrm{NH} \cdots \mathrm{S}$ hydrogen bonds in $\mathrm{CPO}$ and also in other heme thiolate proteins such as P450 and nitric oxide synthase (NOS).

On the basis of our results, we are able to address the following questions: What influence do the $\mathrm{NH} \cdots \mathrm{S}$ hydrogen bonds have on the formation and stability of Cpd 0 and Cpd I? Do the NH»••S hydrogen bonds affect the preference for a hybrid homoheterolytic or a classic heterolytic process for O-O bond scission? We find that overall barriers for transformation of the peroxide bound heme (FeHOOH) into Cpd I are similar with and without the proximal region; however, Cpd 0 is formed more easily (barrier reduced by $7.7 \mathrm{kcal} / \mathrm{mol}$; Fig. 12) and is a more stable intermediate with the proximal region present. We find that the $\mathrm{NH} \bullet$ S hydrogen bonds modulate the favorability of a hybrid homo-heterolytic mechanism by stabilizing Cpd 0 and destabilizing protCpd0. Overall, the $\mathrm{NH} \cdots \mathrm{S}$ hydrogen bonds appear to fine tune reactivity by reducing the pKa of 


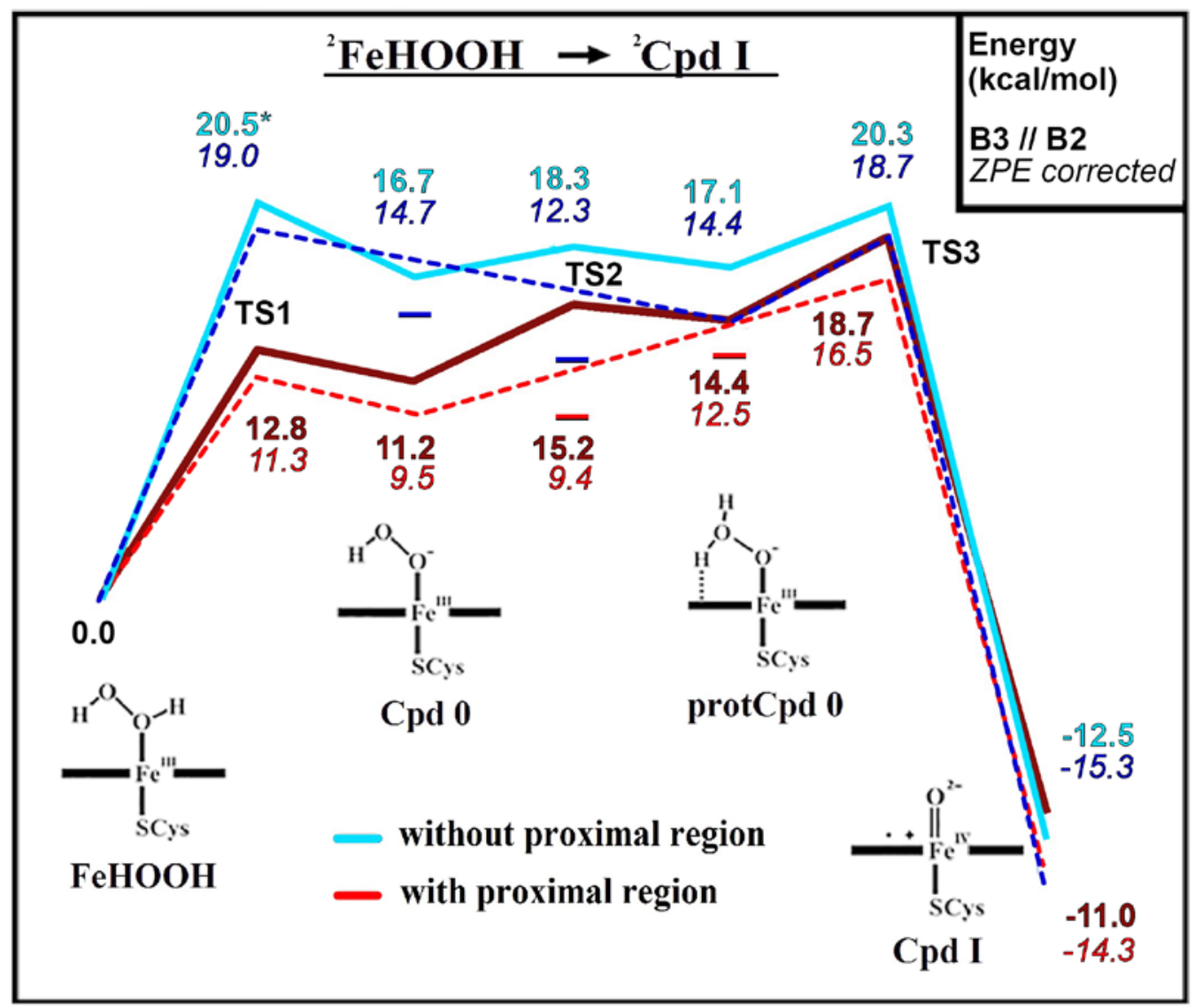

Figure 12: Reaction profile for the FeHOOH $\rightarrow$ Cpd I transformation on the doublet surface, for the model system with (model B) and without (model A) a proximal region present. The first transition state was only optimized with the B1 basis set, so the energy value denoted with the * represents the B3//B1 level of theory. The PES's with and without ZPE corrections are represented by solid and dashed lines, respectively.

the heme-bound peroxide, thereby decreasing the barrier for mechanistic steps which involve deprotonation of the peroxide substrate and increasing the barrier when protonation of the substrate is involved. Interestingly, it was observed that protCpd0 is stabilized by a distal hydrogen bonding network in which the distal oxygen forms hydrogen bonds with a porphyrin ring nitrogen and with the carboxylate of Glu183. The destabilization of protCpd0 upon inclusion of the proximal region derives in part from a weakening of this hydrogen bonding network. The aforementioned findings, which 
pertain to the doublet surface, are also obtained on the higher-energy quartet surface (Fig.

13).

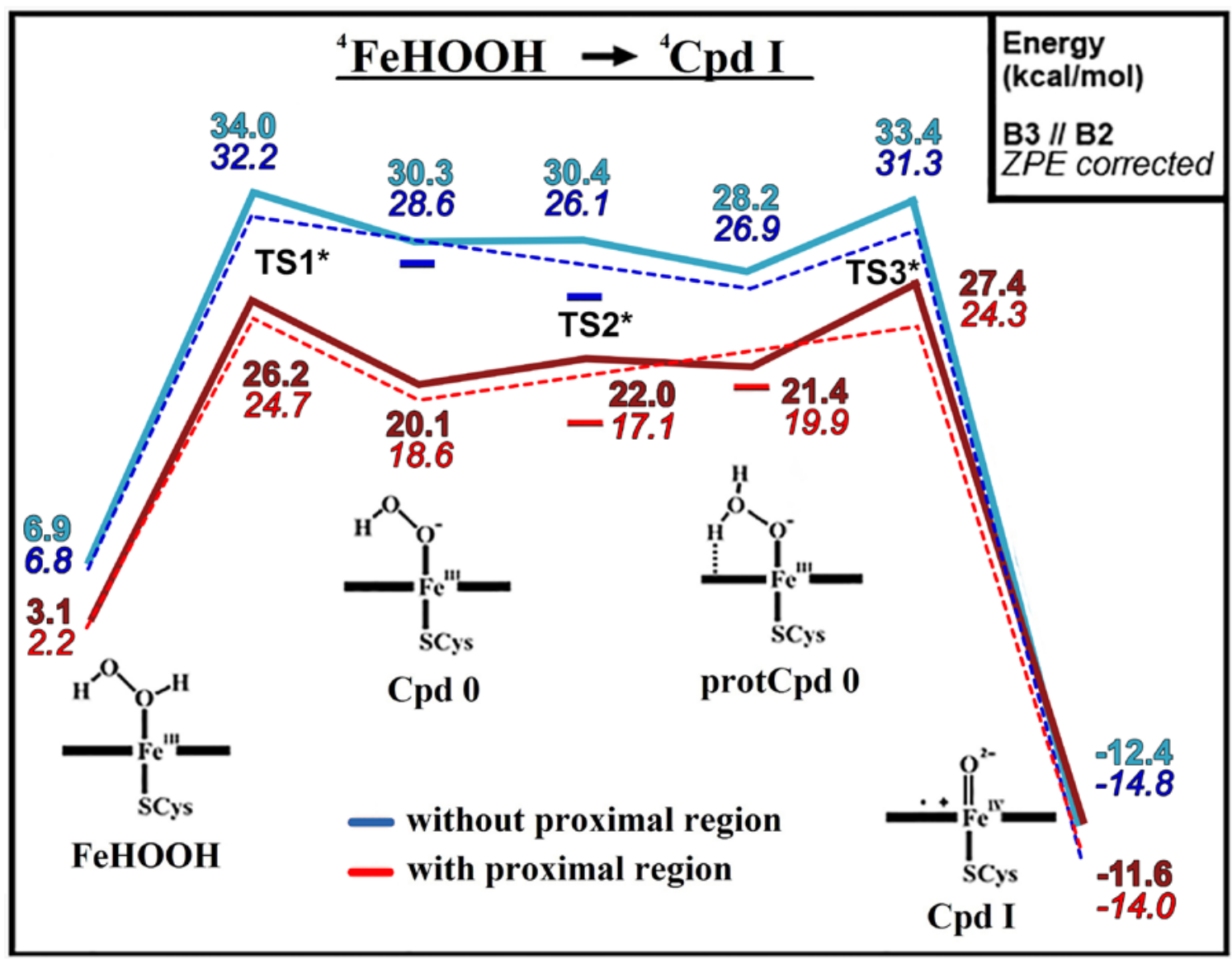

Figure 13: Reaction profile for the FeHOOH $\rightarrow$ Cpd I transformation on the quartet surface, for the model system with (model B) and without (model A) a proximal region present. The three transition states are in fact near transition states, and are thus denoted with an asterix. The PES's with and without ZPE corrections are represented by solid and dashed lines, respectively. The zero of energy is taken as the energy of ${ }^{2} \mathrm{FeHOOH}$ for the respective model. 


\subsection{Methods}

The active region of chloroperoxidase is represented by a model system containing a heme (without the vinyl and propionate side chains) with a proximally bound $\mathrm{SCH}_{3}$ (representing Cys29), and distal pocket residues Glu183 (modeled as $\mathrm{CH}_{3} \mathrm{COO}-$ ), His105 (modeled as 4-methylimidazole), and Asp106 (modeled as the backbone structure $\mathrm{CH}_{3} \mathrm{CONHCH}_{3}$ ). In crystal structures ${ }^{3,12,130}$ and MD simulations, ${ }^{13,14,42,142}$ the imidazole side-chain nitrogens of His105 form hydrogen bonds with the carboxylate side chain of Glu183 and the backbone carbonyl oxygen of Asp106. This set of hydrogen bonds influences the effective pKa of the Glu183 carboxylate side chain, which participates directly in the formation of Cpd I. For this reason, we considered inclusion of all of these moieties in the QM model system essential. Exclusion of any of them alters the reaction profile significantly (data now shown). A comparative model system which adds the proximal residues Pro30, Ala31, and Leu32 was then built for the purpose of determining the influence of proximal-pocket amide-sulfur hydrogen bonds on Cpd I formation (Fig. 11c). For later reference, we will refer to the systems without and with the proximal residues as models $\mathrm{A}$ and $\mathrm{B}$, respectively. The relative orientation of the aforementioned residues with respect to the heme was obtained from a crystal structure of the Cpd 0 species found in the protein data bank (PDB code $2 \mathrm{~J}^{2} \mathrm{M}^{130}$ ). This orientation was maintained with constraints, using ghost atoms, without interfering with Glu183 and His105 side chain flexibility. To evaluate the importance of the strength of the amidesulfur hydrogen bonds, a set of model B structures was created in which the hydrogen bond distances between the amides and the Cys-S were constrained to different values. In 
the crystal structure, the N-S distances for the amide hydrogen bonds from residues Ala31 and Leu32 are $3.41 \AA$ and $3.46 \AA$, respectively. This pair of distances was varied from 3.11, 3.16 $\AA$ to 3.61, $3.66 \AA$ in increments of $0.1 \AA$ for the segment of the reaction mechanism from Cpd 0 to Cpd I. Reaction profiles were then obtained while maintaining these constraints.

QM calculations were performed using NWChem $6.3^{114}$ for geometry optimizations and Gaussian $09^{115}$ for single point energy calculations of the stationary points. Stability checks were performed to ensure correctness of the wave functions. The hybrid DFT functional B3LYP ${ }^{104,105,107,108}$ and a double- $\zeta$ basis set augmented with polarization functions (6-31G(d)) and a LANL2DZ pseudopotential ${ }^{143,144}$ on the iron (LACVP; basis set B1) were used for geometry optimizations of model B in which the amide-sulfur hydrogen bond distances were varied from their crystal-structure values. All other geometry optimizations were performed with addition of diffuse functions (631+G(d)) on all atoms except Fe (basis set B2). For the stationary points obtained with B2, single point energies were calculated using the Wachter's all electron basis set ${ }^{145}$ augmented with diffuse $d$ and polarization $f$ functions for the iron, and the 6-31++G(d,p) basis set for the rest of the atoms (basis set B3). Values discussed in the paper will be at the highest level of theory (B3//B2), except when the discussion involves modulation of the NH $\cdots$ S hydrogen bond distances, which will be presented with LACVP (B1) results. However, transition state 1 (TS1) was not optimized with basis set B2, as this structure was quite problematic to obtain using basis set B1, due to the very flat potential energy surface (PES). 
As mentioned earlier, prior calculations showed the doublet surface to be preferred for Cpd I formation. ${ }^{53}$ We obtained the quartet surface in addition to the doublet surface, though, for two reasons: First, our model system does not contain the full enzyme environment, which could potentially be responsible for stabilizing the doublet surface relative to the quartet surface. Second, the doublet and quartet states of Cpd I are nearly degenerate. ${ }^{53}$ For these reasons, a study of the proximal hydrogen bond influence on the doublet/quartet splitting in Cpd I is warranted.

The reaction coordinate was determined by starting with an optimized Cpd I heme-thiolate, adding the distal residues of model system A together with the aforementioned ghost atoms and constraints, and optimizing to allow for side-chain flexibily. Next, protCpd0 was obtained by introducing $\mathrm{H}_{2} \mathrm{O}$ to the system and performing a relaxed geometry scan with the $\mathrm{H}_{2} \mathrm{O}$ approaching the oxygen of Cpd I. All stationary points for the full reaction profile were then obtained via a series of restrained optimizations, following the reaction coordinate in reverse from protCpd0 to the peroxide bound species (FeHOOH). All approximate stationary points obtained with restrained optimization were subjected to full optimization. To check for hysteresis effects, the barrier for each step was also calculated in the forward direction. No hysteresis effects were observed. The relative orientations of the distal-pocket residues with respect to each other and to the heme were well maintained, with moderate side-chain flexibility, across all species along the reaction coordinate (see overlaid structures in Fig. A1).

The method for obtaining the transition states was abbreviated for the quartet surface, as the computational demands were high and this surface was not the main focus of our study. Once it became apparent that all quartet stationary points (with the 
exception of Cpd I) were significantly higher in energy than their respective doublet counterparts, the time consuming calculations necessary for obtaining fully optimized transition states was avoided. Instead, near transition state structures were obtained with the restrained optimization procedure described above. The structural and energetic differences between the approximate and fully optimized transition states were small for the doublet surface, so we expect the quartet transition state energies reported to represent quite accurate lower bounds.

Minimum energy paths were obtained by following the intrinsic reaction coordinate (IRC) ${ }^{146}$ in the forward and reverse directions, beginning with each transition state. In the resulting reaction profile (Fig. 12) and throughout this paper, FeHOOH is taken as the zero of energy unless otherwise stated. At the B1 level, frequency calculations were performed on all stationary points with the exception of the unbound ferric resting state. From these calculations, zero point corrections to the energy (ZPE) were obtained. Mulliken charges and spin densities were analyzed to verify the electronic structures of the reaction endpoints and intermediates. Mulliken analysis of electronic aspects of the reaction was supplemented with Natural Population Analysis (NPA) ${ }^{123}$, as NPA suffers less than Mulliken analysis from sensitivity to basis set size, especially upon addition of diffuse functions. 


\subsection{Results}

Below we first discuss the PES without ZPE corrections, followed by discussion of the ZPE corrections, for each stage of the reaction. This facilitates comparison with previous calculations in the literature that did not include ZPE corrections. ${ }^{53}$ All data discussed regard the doublet surface unless otherwise expressly stated.

\subsubsection{Reaction from FeHOOH to Cpd 0}

Starting with model A, we introduce hydrogen peroxide to the heme system to form the bound complex, FeHOOH. The orientation of $\mathrm{HOOH}$ is stabilized by two hydrogenbonding interactions, one between $\mathrm{O}_{\text {prox }}$ and the Glu183 carboxylate, and the other between $\mathrm{O}_{\text {dist }}$ and atom $\mathrm{N}_{\mathrm{B}}$ of the porphyrin ring [porph $\left(\mathrm{N}_{\mathrm{B}}\right)$; see Fig. 11b]. Because the pKa's of a heme-bound peroxide and glutamatic acid are similar, ${ }^{147}$ it is very difficult to deprotonate $\mathrm{O}_{\text {prox }}$ and create a Cpd 0 conformer in which $\mathrm{O}_{\text {prox }}$ is hydrogen-bonded to a neutral Glu183 (Cpd 0 prox $)$. One can create Cpd $0_{\text {prox }}$ using constraints, but it is not stable; removal of the constraints yields FeHOOH upon subsequent optimization. Apparently the heme thiolate raises the pKa of the bound $\mathrm{H}_{2} \mathrm{O}_{2}$, preventing stabilization of Cpd 0 prox. Instead, deprotonation occurs via an angle bending mode with concomitant torsion of the N-Fe-O-O dihedral, leading directly to Cpd 0, the conformer with an $\mathrm{O}_{\text {dist }}-\mathrm{Glu} 183$ 
hydrogen bond $\left(\Delta \mathrm{E}^{\ddagger}=20.5 \mathrm{kcal} / \mathrm{mol}\right){ }^{2}$ At the transition state, rotation of the $-\mathrm{OOH}$ moiety about the Fe-O axis breaks the hydrogen-bonding interaction of porph( $\left.\mathrm{N}_{\mathrm{B}}\right)$ with Odist, leading to an orientation of the substrate that allows for a favorable OdistGlu183(O) hydrogen bonding interaction. This stabilizes the proton on Glu183 and maintains it at a far enough distance from $\mathrm{O}_{\text {prox }}$ to disfavor reprotonation (Fig. 14), with a reverse barrier of $3.8 \mathrm{kcal} / \mathrm{mol}$. The deprotonation of FeHOOH by Glu183 to form Cpd 0
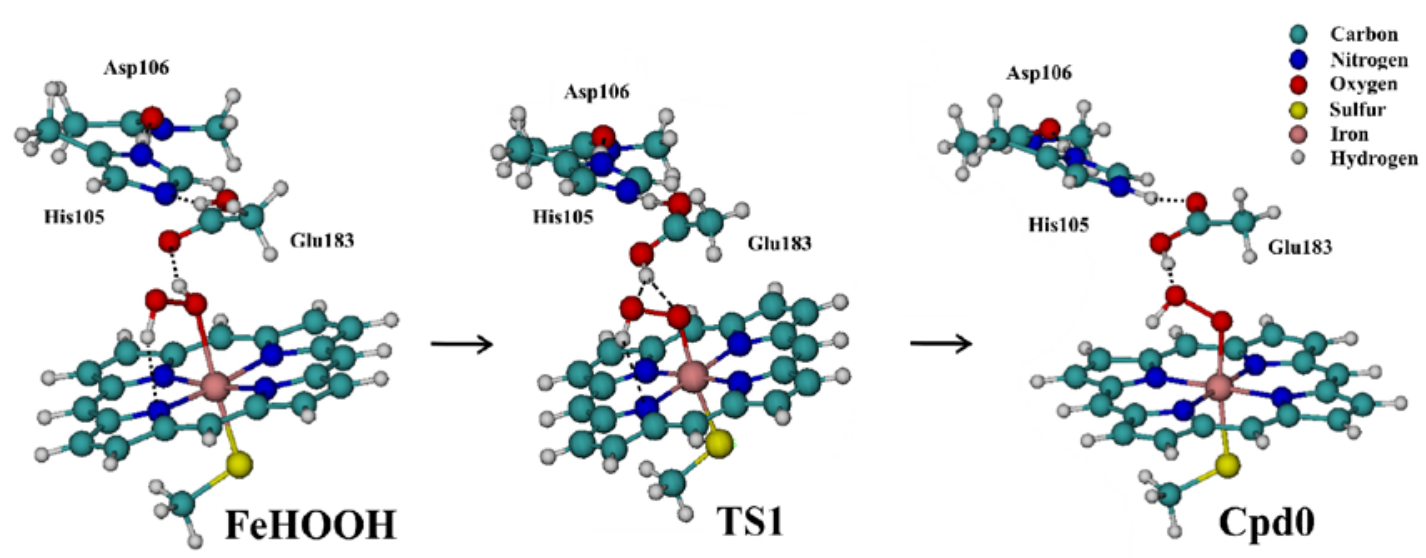

Figure 14: Structures for FeHOOH, TS1, and Cpd 0 for model B. Those for model $A$ are very similar. See Tables $2 a$ and $2 b$ for key distance data. The proximal region has been removed for simplicity. See Figure 11c for depiction of the proximal region.

is endothermic, with Cpd 0 lying $16.7 \mathrm{kcal} / \mathrm{mol}$ higher in energy. It is interesting to note that it is unlikely that Glu183 would be able to deprotonate FeHOOH if Odist were hydrogen bonded to $\operatorname{porph}\left(\mathrm{N}_{\mathrm{A}}\right)$ rather than to porph $\left(\mathrm{N}_{\mathrm{B}}\right)$, as the substrate would have an orientation lacking favorable Glu183(O)-O $\mathrm{O}_{\text {prox }}$ hydrogen bonds.

Inclusion of proximal residues 29-32 (model B) causes significant change to the energetics of the reaction from FeHOOH to Cpd 0. In FeHOOH, there is a decrease in the

${ }^{2}$ All energetic values provided were obtained with the B3//B2 basis set for each species. The exception is TS1, for which the B3//B1 basis set was used, as B2 optimizations were unfeasible for this saddle point. 
charge on the sulfur (natural charge of 0.01 without proximal region present vs -0.09 with it), an increase in the charge on the iron (natural charge of 0.84 vs 0.87 ), weakening of the Fe-S bond (2.215 vs $2.249 \AA$ ), and strengthening of the Fe-O bond (2.290 vs $2.190 \AA$ ) for the $\mathrm{FeHOOH}$ species (see Tables 2a and $2 \mathrm{~b}$ for bond distances). Thus there is a weakening of the normal trans influence, suggesting that the thiolate's push effect is reduced by the NH $\cdots$ S hydrogen bonds introduced with Ala31 and Leu32. The barrier for Table 2a: Interatomic distances for model B

\begin{tabular}{|c|c|c|c|c|c|c|c|c|}
\hline distances in $\AA$ & Fe+HOOH & FeHOOH & TS1 $^{1}$ & CpdO & TS2 & protCpdO & TS3 & Cpd I \\
\hline Fe-S & 2.239 & 2.249 & 2.364 & 2.367 & 2.326 & 2.314 & 2.370 & 2.663 \\
Fe-O & - & 2.190 & 1.882 & 1.904 & 1.983 & 1.970 & 1.764 & 1.619 \\
O-O & 1.466 & 1.451 & 1.467 & 1.471 & 1.494 & 1.500 & 1.720 & - \\
Fe-N(avg.) & 2.025 & 2.031 & 2.031 & 2.037 & 2.033 & 2.031 & 2.029 & 2.030 \\
\hline
\end{tabular}

Table 2b: Difference between interatomic distances for models $B$ and $A$

\begin{tabular}{|c|c|c|c|c|c|c|c|c|}
\hline distances $^{2}$ in $\AA$ & $\mathrm{Fe}+\mathrm{HOOH}$ & $\mathrm{FeHOOH}$ & $\mathrm{TS}^{1}$ & $\mathrm{Cpd0}$ & $\mathrm{TS} 2$ & protCpd0 & TS3 & Cpd I \\
\hline $\mathrm{Fe}-\mathrm{S}$ & 0.024 & 0.034 & 0.048 & 0.068 & 0.053 & 0.057 & 0.068 & 0.079 \\
$\mathrm{Fe}-\mathrm{O}$ & - & -0.100 & -0.028 & -0.043 & -0.017 & -0.060 & -0.014 & -0.002 \\
$\mathrm{O}-\mathrm{O}$ & - & -0.002 & -0.009 & -0.009 & -0.002 & -0.004 & -0.056 & - \\
$\mathrm{Fe}-\mathrm{N}$ (avg.) & -0.009 & 0.008 & -0.001 & -0.002 & -0.004 & -0.004 & -0.007 & -0.005 \\
\hline
\end{tabular}

${ }^{1}$ TS1 distances obtained with B1 basis set. ${ }^{2}$ Distance is model B minus model A. deprotonation of $\mathrm{O}_{\text {prox }}$ to yield Cpd 0 is reduced by $7.7 \mathrm{kcal} / \mathrm{mol}$, from 20.5 to 12.8 $\mathrm{kcal} / \mathrm{mol}$, with respect to model A. This can be explained as a consequence of a reduction in the pKa of the heme-bound peroxide substrate, also attributable to a reduced push effect. In model B, as in model A, deprotonation of FeHOOH leads directly to a Cpd 0 conformer possessing an Odist-Glu183 hydrogen bond, via an angle bending mode with concomitant torsion of the N-Fe-O-O dihedral (Fig. 14). The proximal region stabilizes Cpd 0 by $5.4 \mathrm{kcal} / \mathrm{mol}$ (difference between model A and model B energies of Cpd 0 relative to $\mathrm{FeHOOH}$ ). However, unlike for model A, it was also possible to obtain a

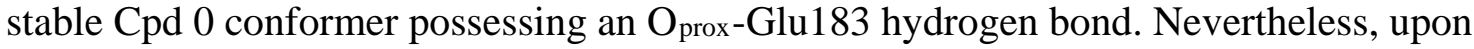
optimizing a near-transition-state conformation for the rearrangement of the hydrogen 
bond from $\mathrm{O}_{\text {prox }}$ to $\mathrm{O}_{\text {dist, }}$, the transition state was found to be lower in energy than the

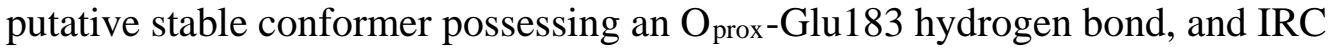
calculations towards that conformer led directly to FeHOOH. This region of the PES is very flat, and thus small perturbations of a barely stable structure led to an alternate and energetically more favorable reaction coordinate where a stable Cpd 0 conformer with Oprox-Glu183 hydrogen bonding is not found.

The charges and spin densities for FeHOOH, TS1, and Cpd 0 are in agreement with prior calculations, confirming the correctness of the electronic states. ${ }^{139}$ Natural charges and spin densities are given in Table 3. Mulliken values, which as mentioned earlier are more sensitive to basis set size, are given in Tables S5-S8 for comparison with prior work. Trends in the Mulliken and natural values are generally similar, with for example the Mulliken spin density on iron being 0.1 larger than the natural value across all species. Changes in the charges and spin densities reflect the progress of reaction in

\section{Table 3: Natural Charge and Spin Density for Stationary Points}

\begin{tabular}{|c|c|c|c|c|c|c|c|c|c|c|c|c|}
\hline \multirow[b]{2}{*}{$\begin{array}{c}\text { Basis Set } \\
\text { B3//B2 }\end{array}$} & \multicolumn{6}{|c|}{ Natural Charge } & \multicolumn{6}{|c|}{ Natural Spin } \\
\hline & $\mathrm{Fe}$ & $\mathrm{S}$ & $\begin{array}{c}\text { porphyrin } \\
+\mathrm{SCH}_{3}\end{array}$ & $\mathrm{O}_{\text {prox }}$ & $\mathrm{O}_{\text {dist }}$ & $\begin{array}{c}\text { substrate } \\
\text { or } \mathrm{H}_{2} \mathrm{O} \\
\end{array}$ & $\mathrm{Fe}$ & $\mathrm{S}$ & $\begin{array}{c}\text { porphyrin } \\
+\mathrm{SCH}_{3}\end{array}$ & $\mathrm{O}_{\text {prox }}$ & $\mathrm{O}_{\text {dist }}$ & $\begin{array}{c}\text { substrate } \\
\text { or } \mathrm{H}_{2} \mathrm{O}\end{array}$ \\
\hline $\mathrm{Fe}$ & 0.99 & -0.10 & -0.96 & N/A & N/A & N/A & 1.11 & -0.03 & -0.11 & N/A & N/A & N/A \\
\hline $\mathrm{Fe}$ & 0.95 & -0.01 & -0.95 & N/A & N/A & N/A & 1.07 & 0.00 & -0.07 & N/A & N/A & N/A \\
\hline $\mathrm{FeHOOH}$ & 0.87 & -0.09 & -0.96 & -0.49 & -0.47 & 0.07 & 0.94 & 0.07 & 0.05 & 0.01 & 0.00 & 0.01 \\
\hline $\mathrm{FeHOOH}$ & 0.84 & 0.01 & -0.93 & -0.51 & -0.47 & 0.05 & 0.91 & 0.11 & 0.09 & 0.00 & 0.00 & 0.01 \\
\hline TS1 $*$ & 0.90 & -0.29 & -1.27 & -0.47 & -0.53 & -0.50 & 0.91 & 0.03 & -0.02 & 0.10 & 0.01 & 0.11 \\
\hline TS1 * & 0.85 & -0.19 & -1.24 & -0.49 & -0.54 & -0.55 & 0.89 & 0.08 & 0.01 & 0.09 & 0.01 & 0.09 \\
\hline CpdOrearr & 0.90 & -0.25 & -1.23 & -0.46 & -0.55 & -0.51 & 0.89 & 0.04 & 0.01 & 0.10 & 0.00 & 0.10 \\
\hline CpdOrearr & 0.85 & -0.13 & -1.18 & -0.49 & -0.56 & -0.54 & 0.85 & 0.11 & 0.06 & 0.09 & 0.00 & 0.08 \\
\hline TS2 & 0.88 & -0.18 & -1.05 & -0.45 & -0.52 & -0.43 & 0.92 & 0.06 & 0.03 & 0.05 & 0.00 & 0.05 \\
\hline TS2 & 0.84 & -0.09 & -1.08 & -0.47 & -0.53 & -0.47 & 0.87 & 0.12 & 0.08 & 0.06 & 0.00 & 0.05 \\
\hline protCpdo & 0.88 & -0.18 & -1.05 & -0.45 & -0.52 & 0.11 & 0.92 & 0.06 & 0.03 & 0.05 & 0.00 & 0.01 \\
\hline protCpdo & 0.83 & -0.06 & -1.00 & -0.47 & -0.52 & 0.09 & 0.87 & 0.13 & 0.09 & 0.04 & 0.00 & 0.04 \\
\hline TS3 & 0.89 & -0.20 & -0.98 & -0.36 & -0.65 & 0.06 & 1.01 & 0.03 & -0.02 & 0.08 & -0.08 & 0.01 \\
\hline TS3 & 0.85 & -0.08 & -0.90 & -0.40 & -0.69 & -0.02 & 1.05 & 0.11 & 0.05 & 0.01 & -0.12 & -0.10 \\
\hline Cpd I & 1.01 & -0.07 & -0.48 & -0.49 & -1.02 & -0.02 & 1.15 & -0.58 & -1.03 & 0.89 & 0.01 & 0.01 \\
\hline Cpd I & 0.98 & 0.09 & -0.48 & -0.50 & -1.03 & -0.02 & 1.13 & -0.74 & -1.03 & 0.90 & 0.00 & 0.00 \\
\hline
\end{tabular}

Species highlighted in gray are for model $\mathrm{A}$; those not highlighted are for model B. *TS1 was obtained only at the B3//B1 level. 
understandable ways. For example, the natural spin densities on $\mathrm{Fe}$ and $\mathrm{O}_{\text {prox }}$ decrease and increase, respectively, as Cpd 0 is formed, from values of 0.94 and 0.01 (FeHOOH, model B) to 0.89 and 0.10 (Cpd 0, model B), reflecting strengthening of Fe-O bonding. Comparison of models A and B reveals that $\mathrm{NH} \bullet \bullet$ S hydrogen bonding induces charge polarization of the heme-thiolate, giving more negative charge on $\mathrm{S}$ and more positive charge on $\mathrm{Fe}$ and $\mathrm{O}_{\text {prox }}$ across all species, as has been noted previously. ${ }^{131,148}$

The ZPE corrections alter the picture developed so far only slightly. The energy of Compound 0 is reduced, relative to $\mathrm{FeHOOH}$, by 2.0 and $1.7 \mathrm{kcal} / \mathrm{mol}$ for models $\mathrm{A}$ and $\mathrm{B}$, respectively. The stabilization of Cpd 0 due to the proximal region is thus 5.2 with ZPE included vs $5.4 \mathrm{kcal} / \mathrm{mol}$ without. With ZPE, the barrier to formation of Cpd 0 is reduced by $1.5 \mathrm{kcal} / \mathrm{mol}$ for both models. The barrier reduction due to inclusion of the proximal region is thus $7.6 \mathrm{kcal} / \mathrm{mol}$ with $\mathrm{ZPE}$ included vs $7.7 \mathrm{kcal} / \mathrm{mol}$ without.

\subsubsection{Reaction from Cpd 0 to Cpd I}

We initially sought a transition state for model A analogous to the one found for the hybrid homo-heterolytic process identified in calculations on the full enzyme, ${ }^{53,138}$ using a reaction coordinate that combined the protonation and $\mathrm{O}-\mathrm{O}$ bond scission steps. However, we were unsuccessful after repeated attempts. Instead, Cpd 0 was transformed into Cpd I via a two-step, classic heterolytic mechanism: protonation of the distal oxygen leads to a stable protCpd0 intermediate, followed by electron transfer and O-O bond scission as $\mathrm{H}_{2} \mathrm{O}$ departs; see Fig. 15. However, the barrier for protonation of Cpd 0 is small, $1.5 \mathrm{kcal} / \mathrm{mol}$, and the reverse barrier converting protCpd0 back to Cpd 0 is even 
smaller, $1.1 \mathrm{kcal} / \mathrm{mol}$. Thus protCpd0 is only marginally stable, and small environmental influences could conceivably remove its stability altogether, yielding a one-step, concerted process of transformation of Cpd 0 into Cpd I. From protCpd0, the barrier for bond scission to yield Cpd I is also modest at $3.2 \mathrm{kcal} / \mathrm{mol}$.

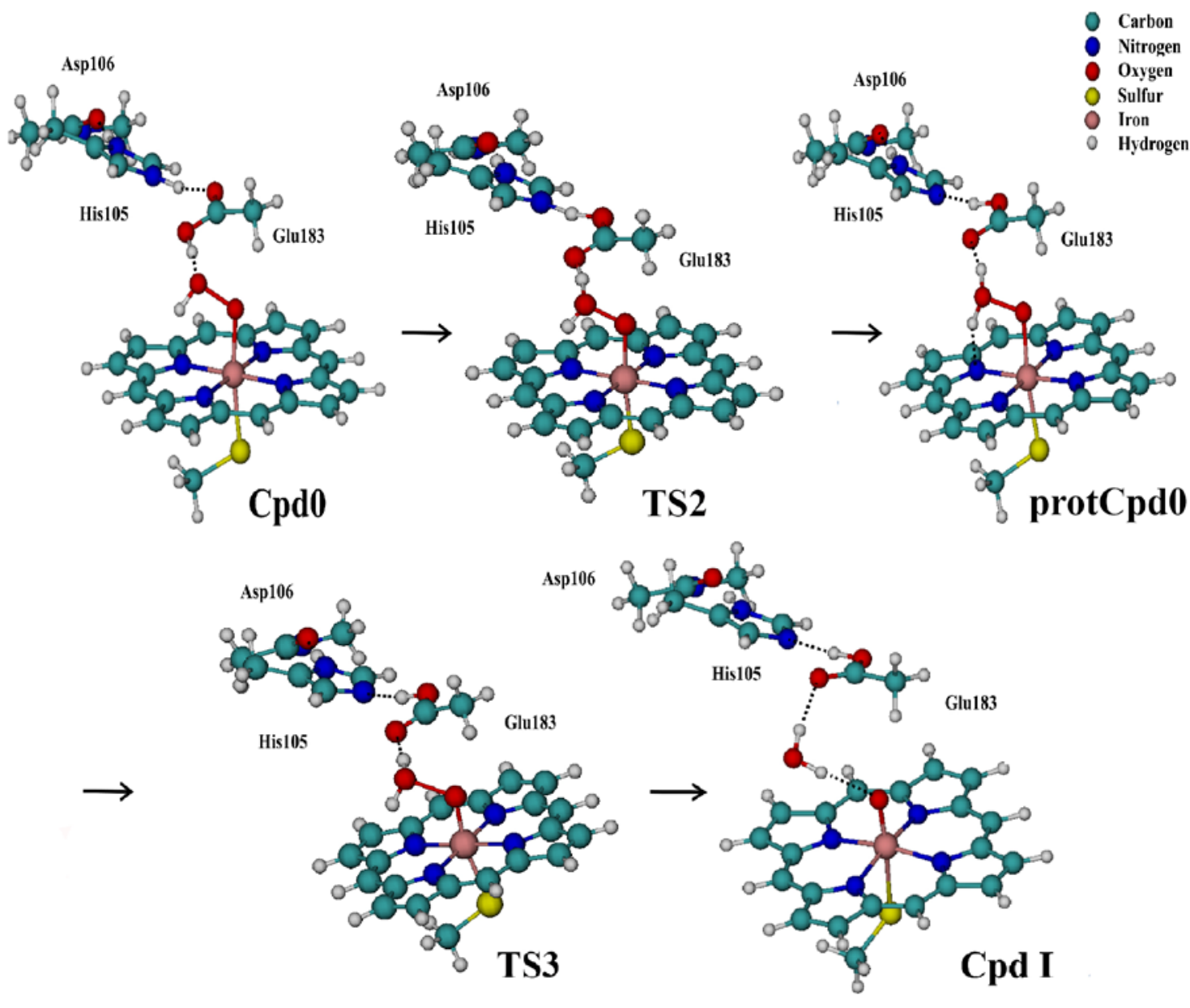

Figure 15: Structures for Cpd 0 through Cpd I for model B. Those for model A are very similar. See Tables $2 a$ and $2 b$ for key distance data. The proximal region has been removed for simplicity. See Figure 1c for depiction of the proximal region.

With the proximal region present (model B), a classic heterolytic mechanism is still obtained, but with several differences from model A. The barrier for protonation of Cpd 0 increases from 1.5 to $4.0 \mathrm{kcal} / \mathrm{mol}$. The formation of protCpd0 from Cpd 0 
becomes more endothermic by $2.7 \mathrm{kcal} / \mathrm{mol}$ and the barrier for $\mathrm{O}-\mathrm{O}$ bond scission increases from 3.2 to $4.3 \mathrm{kcal} / \mathrm{mol}$, leading to a net $3.8 \mathrm{kcal} / \mathrm{mol}$ increase in the barrier for transformation of Cpd 0 into Cpd I. Furthermore protCpd0, marginally stable without the proximal region, becomes even less stable upon inclusion of the proximal region, as the reverse barrier leading back to Cpd 0 is reduced from 1.1 to $0.8 \mathrm{kcal} / \mathrm{mol}$. Apparently, then, inclusion of the proximal region reduces the relative favorability of a classic heterolytic over a hybrid homo-heterolytic mechanism. Because the $\mathrm{NH} \bullet$ S hydrogen bonds reduce the push effect, these findings support the hypothesis that the push effect makes protonation of the Cpd 0 more favorable, leading to facile O-O heterolysis. ${ }^{78,80}$ Without ZPE corrections to the PES, then, our model B mechanism is different from the mechanism deduced from QM/MM calculations on the full enzyme..$^{53}$ In the QM/MM work, proton transfer occurred after incipient homolytic O-O bond cleavage. The bond-cleavage transition state was later than for model B [O-O distance of 1.816 vs $1.720 \AA$ (Table 2a)] and had a ferryl iron rather than a ferric iron (vide infra). The cleavage process became heterolytic only after this transition state. Electron transfer to the departing $\mathrm{H}_{2} \mathrm{O}$ occurred directly from the iron, which converted briefly to the perferryl (5+) oxidation state before a further electron transfer from the porph/SCH 3 moiety returned it to the ferryl state to give Cpd I.

When ZPE corrections are included, the barrier separating Cpd 0 and protCpd0 disappears altogether for both models A and B: the energy of the TS2 structure is below that of both Cpd 0 and protCpd0. For model A, protCpd0 has an energy very slightly below $(0.4 \mathrm{kcal} / \mathrm{mol})$ that of Cpd 0 . This is indicative of a very flat PES, with facile interchange of the proton between Glu183(O) and $\mathrm{O}_{\text {dist. }}$ For model B there is a 3.0 
$\mathrm{kcal} / \mathrm{mol}$ difference between the ZPE-corrected energies of Cpd $0(9.5 \mathrm{kcal} / \mathrm{mol})$ and protCpd0 (12.5 kcal $/ \mathrm{mol})$. Compound 0 is clearly favored, and there is no evidence that protCpd0 is a stable intermediate after correcting for ZPE. This is consistent with the QM/MM calculations on the full enzyme, which yielded a one-step, concerted process for the transformation of Cpd 0 into Cpd I. ${ }^{53}$ The model B calculations thus demonstrate that the $\mathrm{NH} \cdots \mathrm{S}$ hydrogen bonds increase the favorability of such a process over a classic heterolytic mechanism. Because the stable intermediate is protCpd0 for model A but Cpd 0 for model $\mathrm{B}$, the $\mathrm{NH} \cdots$ S hydrogen bonds effectively increase the barrier separating the intermediate and Cpd I by $2.6 \mathrm{kcal} / \mathrm{mol}$ (from $4.3 \mathrm{kcal} / \mathrm{mol}$ for model A to $7.0 \mathrm{kcal} / \mathrm{mol}$ for model B).

Interatomic distance data (Tables 2a and 2b), as well as the charges and spin densities (Table 3, S2, and S3), provide insight into this mechanism. We begin with discussion of model A. In the overall neutral system, a natural charge of -1.00 is distributed over the porphyrin ring and methyl thiolate (porph/SCH 3 , Table 3) in the protCpd0 species. The porph/SCH 3 moiety has a very small total spin density (0.09). The value of the Mulliken spin density on the iron, 0.96 , indicates a ferric iron $^{34}$ (the natural spin density is 0.83 ). The short $\mathrm{O}-\mathrm{O}$ distance, $1.504 \AA$, indicates that cleavage has not begun. As protCpd0 distorts to TS3, there is transfer of negative charge from $\mathrm{O}_{\text {prox }}$ to Odist, consistent with incipient heterolytic cleavage as the $\mathrm{O}-\mathrm{O}$ bond distance increases to $1.776 \AA$; at TS3, the iron remains ferric. After TS3 is passed, an electron is transferred out of the porph/SCH 3 moiety, as indicated by the charge and spin density on that moiety in Cpd I (-0.48 and -1.03, respectively). Simultaneously, the $\mathrm{OH}_{2}{ }^{+}$moiety accepts an electron and departs as neutral $\mathrm{H}_{2} \mathrm{O}$ [natural charge decreases from 0.56 (protCpd0) to 
0.42 (TS3) to 0.00 (Cpd I)]. The charge on $\mathrm{O}_{\text {prox }}$ returns to roughly its value in protCpd0. During the transformation from TS3 to Cpd I, the spin density on Oprox increases from 0.01 to 0.90 , and an increase in the natural spin density on the iron to 1.13 [the Mulliken values are 1.26 (B1) and 1.39 (B3//B2)] indicates a ferryl (4+) iron in Cpd I. This is consistent with the orbital description of doublet Cpd I discussed elsewhere, in which there are three unpaired electrons, distributed as follows: one alpha electron in a $\pi_{\mathrm{xz}}$ orbital [formed from $\mathrm{O}\left(\mathrm{p}_{\mathrm{x}}\right)$ and $\mathrm{Fe}\left(\mathrm{d}_{\mathrm{xz}}\right)$ orbitals], one alpha electron in an $\mathrm{Fe}\left(\mathrm{d}_{\mathrm{yz}}\right)$ orbital, and one beta electron in an a2u-like orbital delocalized over the porph/SCH3 moiety. ${ }^{131}$, $139,149,150$

Trends in the charges and spin densities are similar for model B. However, inclusion of the proximal region (model B) decreases the charge on the sulfur and increases the charge on the iron across all species. Smaller increases in the charge on $\mathrm{O}_{\text {prox }}$ and even smaller increases on $\mathrm{O}_{\text {dist }}$ are also observed. The proximal region also decreases the magnitude of the spin density on the sulfur atom in Cpd I, indicating that the $\mathrm{NH} \cdots$ S hydrogen bonds cause the porphyrin/sulphur based radical to be localized to a greater extent on the porphyrin ring. This is consistent with prior computational work comparing a bare Cpd I heme-methylthiolate to a QM/MM P450 Cpd I model, ${ }^{148}$ in which the protein environment shifted the occupancy of the radical from the sulfur to the heme (the sulfur spin density was changed from -0.728 to -0.349 , close to Rapid FreezeQuench ENDOR experimental results on CPO in which the Cpd I sulfur spin density maximum is $-0.23^{128}$ ). Our results show that the $\mathrm{NH} \cdots$ S hydrogen bonds contribute significantly to this shift (sulfur spin density changed from -0.786 to -0.605 ). It is important to note that, although it has been suggested that using -SH as the proximal 
thiolate ligand provides better agreement with DFT/MM results than using $-\mathrm{SCH}_{3}$ or even the full cysteine ligand, ${ }^{151}$ we chose to use $\mathrm{SCH}_{3}$. We made this choice because, although -SH gives a more realistic delocalization of the Cpd I radical (\%porphyrin occupancy vs \%sulfur occupancy), the methyl thiolate more accurately represents the proper geometry of the proximal thiolate (Fe-S-C angle of $\sim 109^{\circ}$ as opposed to Fe-S-H angle of $\left.\sim 98^{\circ}\right)^{150}$ and thus more accurately represents the proper hybridization of the $\mathrm{s}$ and p orbitals. Given the postulate that a $\pi$-push effect influences reactivity, ${ }^{80}$ enforcing this geometry may be important so that $\mathrm{S}(\mathrm{p})-\mathrm{Fe}(\mathrm{d})$ orbital overlap is maintained.

We also investigated the relationship between the strength of the NH・•S hydrogen bonds and the stability of the protCpd0 intermediate. The residues of the proximal region were incrementally displaced relative to the proximal sulfur to adjust the length of the $\mathrm{NH} \cdots \mathrm{S}$ hydrogen bonds while approximately maintaining their orientation, and the portion of the reaction profile for the transformation of Cpd 0 into Cpd I was calculated. Tests show that the B1 level of theory correctly reproduces trends in the barrier heights (Fig. A2), so B1 was used to reduce computer time for these repetitive calculations. The results are shown in Table 4; for conciseness, the notation 3.X is used to indicate that the pair of backbone amide(N)-sulfur distances is 3.X1 $\AA$ for $\operatorname{AlaN}(\mathrm{H}) \bullet \cdot \mathrm{S}$

Table 4: Energetic data for the portion of the PES from Cpd 0 to TS3

\begin{tabular}{|c|c|c|c|}
\hline $\mathbf{N H} \cdots \mathbf{S ~ d i s t a n c e}$ & $\boldsymbol{\Delta E}_{\mathbf{p}}{ }^{\mathbf{T S} 2}$ & $\mathbf{\Delta E}_{\mathbf{d p}}{ }^{\mathbf{T S 2}}$ & $\mathbf{\Delta E}_{\mathbf{s c i}}{ }^{\mathbf{T S} 3}$ \\
\hline $\mathbf{3 . 1} \AA$ & 4.38 & 1.18 & 5.50 \\
$\mathbf{3 . 2} \AA$ & 4.30 & 0.96 & 5.20 \\
$\mathbf{3 . 3} \AA$ & 4.21 & 0.98 & 5.16 \\
$\mathbf{3 . 4} \AA$ & 4.11 & 1.03 & 5.11 \\
$\mathbf{3 . 5} \AA$ & 4.00 & 1.07 & 5.05 \\
$\mathbf{3 . 6} \AA$ & 3.88 & 1.14 & 5.03 \\
$\mathbf{M o d e l} \mathbf{A}$ & 2.05 & 2.81 & 4.71 \\
\hline
\end{tabular}

$\Delta \mathbf{E}_{\mathbf{p}}{ }^{\mathrm{TS} 2}$ is the barrier for protonation of $\mathbf{C p d} 0\left(\Delta \mathbf{E}_{\mathbf{p}}{ }^{\mathrm{TS} 2}=\mathbf{E}^{\mathrm{TS} 2}-\mathbf{E}^{\mathrm{Cpd} 0}\right), \Delta \mathbf{E}_{\mathrm{dp}}{ }^{\mathrm{TS2} 2}$ is the barrier for deprotonation of protCpd0 $\left(\Delta \mathbf{E}_{\mathrm{dp}}{ }^{\mathrm{TS} 2}=\mathbf{E}^{\mathrm{TS} 2}-\mathrm{E}^{\text {protCpdo }}\right)$, and $\Delta \mathbf{E}_{\text {sci }}{ }^{\mathrm{TS3}}$ is the barrier for $\mathrm{O}-\mathrm{O}$ bond scission to form $\mathrm{Cpd} \mathrm{I}\left(\Delta \mathbf{E}_{\mathrm{sci}}{ }^{\mathrm{TS}}=\mathrm{E}^{\mathrm{TS} 3}{ }_{-}\right.$

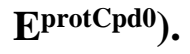


and 3.X6 $\AA$ for LeuN(H) $\bullet \cdot$ S. It was found that the barrier for protonation of Cpd 0 is directly correlated with hydrogen bond strength, while the stability of protCpd0 (as judged from $\Delta \mathrm{E}_{\mathrm{dp}}^{\mathrm{TS} 2}$, the barrier for deprotonation of protCpd0) is inversely correlated with the hydrogen bond strength. ${ }^{3}$ While the changes in barrier heights might appear modest, assuming a simple Arrhenius relationship leads to the prediction that the $\mathrm{NH} \cdots \mathrm{S}$ hydrogen bonds decrease the rate of protonation of Cpd 0 by a factor of up to 49 and increase the rate of deprotonation of protCpd0 by a factor of up to 22 at $300 \mathrm{~K}$ for the range of structures given in Table 4. The rate of transformation of protCpd0 into Cpd I is increased by a factor of up to 3.7. These results reinforce the observation that the $\mathrm{NH} \cdots \mathrm{S}$ hydrogen bonds contribute to the preference of full-enzyme models of CPO for hybrid homo-heterolytic rather than classic heterolytic O-O bond scission.

The proximal region influences some key bond lengths (Table 2a and 2b). Inclusion of the proximal region weakens the Fe-S bond and strengthens the Fe-O bond for the Cpd 0, protCpd0, and Cpd I species, as it does for FeHOOH. The O-O bond is slightly shortened by inclusion of the proximal region in all species. The effect is about an order of magnitude smaller than the effects on the Fe-S and Fe-O bonds. These observations are reinforced by corresponding trends as the $\mathrm{NH} \cdots$ S hydrogen bond distances are varied (Table 19).

We conclude this section with two remarks concerning hydrogen bonding in the distal pocket. The first pertains to two hydrogen bonds that stabilize protCpd0, one

\footnotetext{
${ }^{3}$ The reverse barrier for the shortest hydrogen bond (S-N distance of $3.1 \AA$ ) deviated from this trend due to a methyl rotation in the proximal region of the protCpd0 species that helped to stabilize the structure.
} 
between $\mathrm{O}_{\text {dist }}\left(\right.$ donor) and porph $\left(\mathrm{N}_{\mathrm{A}}\right)$, and another between $\mathrm{O}_{\text {dist }}$ (donor) and the Glu183 carboxylate. Inclusion of the proximal region (model B vs model A) weakens the $\mathrm{O}_{\text {dist }}(\mathrm{H})$-porph $\left(\mathrm{N}_{\mathrm{A}}\right)$ hydrogen bond (the $\mathrm{N}-\mathrm{H}$ distance is increased from $1.749 \AA$ to 1.762 , and the $\mathrm{N}-\mathrm{H}-\mathrm{O}$ angle is reduced from $151.9^{\circ}$ to $\left.149.9^{\circ}\right)$, while strengthening the $\mathrm{O}_{\text {dist }}(\mathrm{H})$ OGlu183 hydrogen bond ( $\mathrm{O}_{\text {Glu183-H }} \mathrm{H}$ distance decreased by $0.05 \AA$ ), pulling the $\mathrm{O}_{\text {dist }} \mathrm{H}_{2}$ moiety away from $\mathrm{N}_{\mathrm{A}}$ and yielding a process closer to hybrid homo-heterolytic.

The second remark concerns the importance of Asp106. In initial attempts to obtain a reaction profile for the transformation of Cpd 0 into Cpd I, the model A system was built without Asp106. In the full enzyme, His105 is doubly protonated, with the imidazole nitrogens forming hydrogen bonds with the Glu183 carboxylate and the backbone carbonyl oxygen of Asp106. We maintained this His105 protonation in the model systems, but without Asp106, obtaining the reaction profile in reverse to test for hysteresis was problematic. Although Cpd 0 is a stable stationary point, obtaining it beginning from protCpd0 required a complicated, two dimensional constrained optimization, probably because the pKa of His105 is unrealistically low without the stabilizing influence of Asp106. When Asp106 was included in the system, hysteresis effects and the need to use multiple constraints to obtain the reaction profile disappeared. We conclude that Asp106 is an essential part of a "proton shuttle triad" consisting of Asp106, His105, and Glu183, and that inclusion of Asp106 in the QM region during modeling may be essential for obtaining correct reaction profiles. 


\subsubsection{Quartet surface}

The quartet PES lies at higher energy than the doublet PES. The doublet-quartet splittings are in the range $12-14 \mathrm{kcal} / \mathrm{mol}$ for model A except at the $\mathrm{FeHOOH}$ and Cpd I endpoints (Table 5). For model B, the splittings are reduced by $\sim 5-7 \mathrm{kcal} / \mathrm{mol}$ for all stationary points except TS1 and Cpd I, for which the splittings are nearly the same for both models. In Fig. 13, the quartet PES is depicted referenced to the ${ }^{2} \mathrm{FeHOOH}$ zero of energy for the respective model, A or B. The quartet PES is somewhat flatter than the doublet PES in the Cpd 0-to-protCpd0 region but is otherwise quite similar for the two models. Thus the influence of the NH•••S hydrogen bonds on the quartet PES mimics that on the doublet PES. As on the doublet PES, there is a small barrier to protonation of Cpd 0 that disappears once the ZPE is included, for both models. The mechanism on the quartet surface is also best described as classic heterolytic for model A but hybrid homoheterolytic for model B. The peroxide and iron interact only weakly in the ${ }^{4} \mathrm{FeHOOH}$ binding complex, leading to a large Fe-O distance $(2.726 \AA$ and $2.681 \AA$ for models $\mathrm{A}$ and $B$ respectively), due to a $\pi^{*}\left[\mathrm{~d}_{\mathrm{z}}{ }^{2}(\mathrm{Fe})-\mathrm{p}(\mathrm{O})\right]$ antibonding interaction. The doublet-quartet splittings are qualitatively similar to those obtained in QM/MM calculations on a fullenzyme model of $\mathrm{CPO}^{53}$ except for the ${ }^{4} \mathrm{FeHOOH}$ binding complex, which is more tightly bound in the earlier calculations (Fe-O distance $0.212 \AA$ shorter and energy 7.1 $\mathrm{kcal} / \mathrm{mol}$ lower than in model B). This difference is probably due our use of a larger basis set, which included polarization and diffuse functions on all atoms during geometry optimization. 
Table 5: Doublet-Quartet splitting (kcal/mol)*

\begin{tabular}{|l|r|r|}
\hline & Model A & Model B \\
\hline FeHOOH & 6.8 & 2.2 \\
TS1 & 13.2 & 13.4 \\
Cpd 0 & 13.9 & 9.1 \\
TS2 & 13.8 & 7.7 \\
protCpd0 & 12.5 & 7.4 \\
TS3 & 12.6 & 7.8 \\
Cpd I & 0.5 & 0.3 \\
\hline
\end{tabular}

*Quartet energy minus doublet energy, B3//B2 level, including ZPE calculated at B1 level. Absolute energies of all stationary points are given in the SI.

\subsubsection{Polarity effects}

The influence of the portion of the protein environment omitted from the model systems was estimated by employing an implicit solvation model with a dielectric constant representative of the protein interior. The in vacuo calculations described thus far effectively use a delectric constant of unity. To represent the protein interior, the implicit solvation model PCM ${ }^{152,153}$ was employed using a dielectric constant of $\varepsilon=5.7$ and a scaled van der Waals radius for the solute-solvent boundary $(\alpha=1.1)$. Single-point energies were calculated with the B1 basis set at the B1-optimized geometries for the doublet surface, for both models A and B. All energies were reduced relative to the gas phase, with the greatest effect being on Cpd 0.

Inclusion of the increased-dielectric environment reduces the barrier and reaction energy for Cpd 0 formation. For models A and B, TS1 is reduced by 12.3 and 10.0 $\mathrm{kcal} / \mathrm{mol}$, respectively (Table 6). The contribution of the $\mathrm{NH} \bullet \bullet \mathrm{S}$ hydrogen bonds to the reduction of the TS1 barrier is decreased by $2.3 \mathrm{kcal} / \mathrm{mol}$ (from 6.2 to $3.9 \mathrm{kcal} / \mathrm{mol}$ ). For 
model B, inclusion of the dielectric environment produces a Cpd 0 that is more stable than FeHOOH by $1.7 \mathrm{kcal} / \mathrm{mol}$. The deprotonation of FeHOOH for model B with the increased-dielectric environment closely resembles the results obtained with QM/MM work. $^{53}$ It is reasonable to expect a stable Cpd $0_{\text {prox }}$ intermediate for both models $A$ and B when the dielectric environment is included.

Although the increased-dielectric environment reduces the deprotonation barrier of Cpd 0, it increases the protonation barrier of Cpd 0. For model B, the energy of the transition state leading to protCpd 0 is reduced by $3.3 \mathrm{kcal} / \mathrm{mol}$; however, since the dielectric environment significantly stabilizes Cpd 0 by $10.0 \mathrm{kcal} / \mathrm{mol}$, the overall effect is an increase in the protonation barrier (by $6.7 \mathrm{kcal} / \mathrm{mol}$ ). ProtCpd0 is no longer a stable intermediate in either model system, even without inclusion of the ZPE corrections. Since protCpd 0 is no longer stable, we conclude that both the increased-dielectric environment and the $\mathrm{NH} \bullet \bullet$ S hydrogen bonds contribute to the preference for a single-step conversion of Cpd 0 into Cpd I. The barrier for the Cpd 0 to Cpd I step is significantly increased, and is primarily attributed to the significant stabilization of Cpd 0. Overall, the effect of $\mathrm{NH} \bullet$ S hydrogen bonds on the reaction profile in the higher-dielectric environment is similar that in gas phase even though the reaction profile looks quite different because of the large stabilization of Cpd 0 relative to other species. 
Table 6: Influence of the Dielectric Environment (kcal/mol)

\begin{tabular}{|l|ccccccc|}
\hline & FeHOOH & TS1 & Cpd0 & TS2 & protCpd0 & TS3 & Cpd I \\
\hline Model A - NoPCM & 0.0 & 18.0 & 14.2 & 16.2 & 13.4 & 18.1 & -10.0 \\
Model A - PCM & 0.0 & 5.7 & 2.2 & 10.2 & 11.9 & 17.0 & -13.2 \\
\hline Model B - NoPCM & 0.0 & 11.9 & 8.3 & 12.4 & 11.4 & 16.5 & -7.7 \\
Model B - PCM & 0.0 & 1.9 & -1.7 & 9.1 & 10.4 & 15.8 & -11.8 \\
\hline
\end{tabular}

\subsection{Discussion}

\subsubsection{Formation and stability of Cpd 0}

The proximal region causes a substantial increase in the stability of Cpd 0 and a reduction of the barrier to its formation. The energy of Cpd 0 is 14.7 and $9.5 \mathrm{kcal} / \mathrm{mol}$ for models A and B, respectively, with ZPE included. The corresponding barriers for formation of Cpd 0 are 19.0 and $11.3 \mathrm{kcal} / \mathrm{mol}$ (Figure 12 ). On this basis, the $\mathrm{NH} \bullet \bullet \mathrm{S}$ hydrogen bonds are predicted to increase the rate of the forward reaction to form Cpd 0 by a factor of $3.8 \mathrm{x}$ $10^{5}$ at $300 \mathrm{~K}$. These differences are mainly a pKa effect resulting from a reduction of the bound peroxide's pKa by the $\mathrm{NH} \bullet \bullet$ S hydrogen bonds. Overall, the proximal region reduces the relative energy and the barrier to formation of Cpd 0 by over 30\%, enabling more facile formation of Cpd 0 and increasing its concentration in reaction mixtures.

\subsubsection{Stability of Cpd I}

The proximal region impacts the stability of Cpd I, an important factor for the reactivity of the enzyme that has been related to the Cpd 0/Cpd I relative energy ( $\left.E_{C p d 0}-E_{C p d I}\right)$. Here 
we discuss energies without ZPE corrections for comparison with literature values for other enzymes. Inclusion of the proximal region raises the energy of Cpd I by 1.5 $\mathrm{kcal} / \mathrm{mol}$ and lowers the energy of Cpd 0 by $5.4 \mathrm{kcal} / \mathrm{mol}$, resulting in a $6.9 \mathrm{kcal} / \mathrm{mol}$ reduction (from 29.2 to $22.2 \mathrm{kcal} / \mathrm{mol}$ ) in the Cpd 0/Cpd I relative energy. The model B value is near the upper end of the range of values obtained in QM/MM calculations on a set of structures selected from MD simulations of the entire enzyme (15.0 to 23.8 $\mathrm{kcal} / \mathrm{mol}) .{ }^{138}$ By comparison, Cpd 0/Cpd I relative energies of $27 \mathrm{kcal} / \mathrm{mol}$ and 8-10 $\mathrm{kcal} / \mathrm{mol}$ have been calculated for horseradish peroxidase (HRP) ${ }^{140,154}$ and P450cam, ${ }^{141}$ respectively. Experimentally, the stability of Cpd I in horseradish peroxidase (HRP), $\mathrm{CPO}$, and $\mathrm{P} 450$ follows the order $\mathrm{HRP}>\mathrm{CPO}>\mathrm{P} 450$. Thus the proximal region reduces the Cpd 0/Cpd I relative energy, primarily by stabilizing Cpd 0 (as noted previously by Lai et al. ${ }^{138}$ ), and is needed to give a correct ordering of the stabilities of Cpd I in the three enzymes when model B is taken to represent CPO.

\subsubsection{Mechanism of $\mathrm{O}-\mathrm{O}$ bond scission}

In HRP, CPO, and P450cam, calculations have found a heterolytic mechanism for O-O bond scission, in contrast to NOS and heme oxygenase (HO), which exhibit homolytic cleavage mechanisms. Even among the heterolytic mechanisms, a variety of subtypes has been observed. In P450cam, protonation of the distal oxygen to yield a stable intermediate prior to electron transfer and scission of the O-O bond (classic heterolytic mechanism) has been calculated for one channel of the reaction, the Glu366 channel. In full-enzyme calculations on HRP and CPO, a hybrid homo-heterolytic has been observed. 
The Asp251 channel of P450cam yielded homolytic cleavage to give an $\mathrm{OH}$ radical hydrogen bonded to Cpd I, followed by proton and electron transfer to convert the $\mathrm{OH}$ radical to water.

Our calculations yield a classic heterolytic process for O-O bond scission for model A, with protonation of Cpd 0 preceding O-O bond scission. Protonation of Cpd 0 is facile, as the barrier separating the nearly isoenergetic species Cpd 0 and protCpd0 is small and disappears upon inclusion of ZPE. Model B disfavors protonation of Cpd 0, presumably because the $\mathrm{NH} \cdots$ S hydrogen bonds raise the pKa of protCpd0. With ZPE, protCpd0 lies $3.0 \mathrm{kcal} / \mathrm{mol}$ higher than Cpd 0 with no intervening barrier, so transformation of Cpd 0 into Cpd I will occur in a single, concerted step. These results indicate that the preference of $\mathrm{CPO}$ for a hybrid homo-heterolytic rather than a classic heterolytic mechanism for $\mathrm{O}-\mathrm{O}$ bond cleavage ${ }^{53,138}$ is at least in part due to the influence of the $\mathrm{NH} \cdots$ S hydrogen bonds.

The importance of the axial ligand for promoting the protonation of a distal ligand was also observed in computational studies of model systems related to P450nor, a fungal nitric oxide reductase. ${ }^{155-157}$ The model studies showed that the "push effect" of the axial thiolate renders the ferrous heme-HNO species sufficiently basic to accept an additional proton and form the key catalytic species “Intermediate I," while an analogous complex with an axial imidazole will not yield a stable protonated intermediate. In that P450nor study, the identity of the axial ligand (thiolate vs imidazole) was key. In CPO, we have shown that even the environment of an axial thiolate can alter a distal ligand's pKa enough to affect the catalytic mechanism. 
It is likely that the pKa of the catalytic protonating residue, Glu183 in the case of $\mathrm{CPO}$, is important in determining the nature of the $\mathrm{O}-\mathrm{O}$ bond cleavage mechanism. The formation of a fully-cleaved $\mathrm{OH}$ radical prior to proton and electron transfer in the Asp251 channel of P450cam may be attributable to the relatively high pKa of Thr252. Even in the Glu366 channel of P450cam, in which a protCpd0 intermediate can be located, the barrier is very high ( $26 \mathrm{kcal} / \mathrm{mol})$ and not competitive with a homolytic mechanism. The lower pKa of Glu183 in CPO may be responsible for protonation of $\mathrm{O}_{\text {dist }}$ prior to completion of O-O bond scission, giving a hybrid homo-heterolytic mechanism in the full-enzyme calculations and in model B, and a classic heterolytic mechanism in model A.

\subsubsection{Distal-side hydrogen bonding}

The distal oxygen forms two hydrogen bonds that are probably critical for the stability of protCpd0, as noted in the Results. One of these, with the Glu183 carboxylate, was present in prior full-enzyme calculations of the O-O bond scission step of CPO. ${ }^{53}$ The other, with porph $\left(\mathrm{N}_{\mathrm{B}}\right)$, was not, although such a hydrogen bond has been noted before in similar contexts. ${ }^{141}$ It is interesting to note that the transition state obtained for the O-O bond cleavage step in the full-enzyme calculations is very similar to protCpd0, with the acidic proton of Glu183 almost completely transferred to the distal oxygen while the O-O bond undergoes scission ( $\mathrm{O}_{\text {dist }}-\mathrm{H}$ distance of $1.059 \AA \AA$ vs $1.045 \AA$ for protCpd0 in our model B). This suggests that protCpd0 was just barely unstable, and one can speculate that the presence of a hydrogen bond with a porphyrin ring nitrogen or a water molecule might 
provide stability. Furthermore, since the strength of distal-side hydrogen bonding is sensitive to modulation of the heme bound substrate's pKa by the NH $\cdots$ S hydrogen bonds in the proximal region, there appears to be a co-dependency of the distal- and proximal-side hydrogen bond networks, as predicted by Poulos. ${ }^{12}$

\subsubsection{Quartet Potential Energy Surface}

The influence of the proximal region on the quartet surface mirrors that of the doublet surface. As on the doublet surface, the barrier for Cpd 0 formation is reduced by the $\mathrm{NH} \bullet$ S hydrogen bonds ( 2.9 vs $7.7 \mathrm{kcal} / \mathrm{mol}$, quartet vs doublet), as is the Cpd 0/Cpd I relative energy (10.8 vs $6.2 \mathrm{kcal} / \mathrm{mol}$, with ZPE included). Again, model A proceeds via a classic heterolytic mechanism, and inclusion of $\mathrm{NH} \cdots$ S hydrogen bonds and ZPE correction leads to the preference for a hybrid homo-heterolytic mechanism for O-O cleavage. The probability of a spin-forbidden transition between the doublet and quartet surfaces during the formation of Cpd I is small because the two surfaces do not cross, and the energy difference is 7+ kcal/mol (B3//B2 level, ZPE-corrected) for all stationary points for the transformation of FeHOOH to Cpd I except for the reaction endpoints.

\subsection{Conclusion}

DFT calculations of the chloroperoxidase models presented here demonstrate the significant influence amide hydrogen bonds to the sulfur atom of the proximal cysteine 
residue have on regulating the mechanism of Cpd I formation. On the doublet surface, which is energetically preferred, these hydrogen bonding interactions reduce the pKa of the bound peroxide substrate, stabilizing the Cpd 0 intermediate by $5.2 \mathrm{kcal} / \mathrm{mol}$ relative to $\mathrm{FeHOOH}$ and thereby providing a thermodynamic driving force for facile deprotonation of FeHOOH. The barrier for formation of Cpd 0 is stabilized by 7.7 $\mathrm{kcal} / \mathrm{mol}$. Comparable results are found on the quartet surface.

Our model active-site system lacking $\mathrm{NH} \cdots$. S hydrogen bonds manifests a classic heterolytic mechanism for O-O bond scission. Facile protonation of Cpd 0 leads to the nearly isoenergetic species protCpd0, after which electron transfer and departure of water give Cpd I. The addition of four proximal-side residues to the model to create the native $\mathrm{NH} \cdots \mathrm{S}$ hydrogen bonds destabilizes protCpd0. Cleavage of the O-O bond then occurs in a single, concerted step consistent with the hybrid homo-heterolytic process identified in QM/MM calculations on the full enzyme. ${ }^{53}$ Thus, by stabilizing Cpd 0 and destabilizing protCpd0, the NH$\cdots$ S hydrogen bonds increase the favorability of a hybrid homoheterolytic relative to a classic heterolytic mechanism for O-O bond scission.

Furthermore, because the $\mathrm{NH} \cdots$ S hydrogen bonds reduce the push effect, these findings support the hypothesis that the push effect makes protonation of Cpd 0 more favorable, leading to facile $\mathrm{O}-\mathrm{O}$ heterolysis. ${ }^{78,} 80$

Altogether, we find that the hydrogen bond networks on the proximal and distal sides of the heme function together to modulate the mechanism of reaction, as suggested by Poulos. ${ }^{12}$ These results confirm and extend long-standing theories that the $\mathrm{NH} \cdots \mathrm{S}$ hydrogen bonds in heme thiolate proteins influence reactivity by reducing the thiolate "push effect”. Additionally, the influence of the $\mathrm{NH} \cdots$ S hydrogen bonds may be 
augmented by the dipole of the alpha helix present in the proximal pocket. As was

mentioned by Ueno et al., ${ }^{87}$ the $\alpha$-helical conformation coupled with $\mathrm{NH} \cdots$. S hydrogen bond is one of the effects thought to cause a positive shift of the FeIII/FeII redox potential in the native folded proteins. This could be studied using a model system with the full helix represented and would be an interesting topic for future research.

\section{Additional Data}

The following data is given in Appendix A: Superposition of optimized structures, energies of stationary points, zero-point energies, imaginary frequencies of first-order saddle points, Mulliken charges and spins, influence of basis set on relative energies, influence of $\mathrm{NH} \cdots \mathrm{S}$ hydrogen bond distance on selected interatomic distances, and Cartesian coordinates of stationary points. 


\section{The Influence of the NH•••S hydrogen bonds and Proximal Helix Dipole on the Catalytic Mechanism of the Chloroperoxidase-Catalyzed Epoxidation of Cis- $\beta$ - Methylstyrene}

Reproduced in part with permission from Morozov, A. N.; Pardillo, A. D.;

Chatfield, D. C., Chloroperoxidase-Catalyzed Epoxidation of Cis- $\beta$-Methylstyrene: NHS Hydrogen Bonds And Proximal Helix Dipole Change The Catalytic Mechanism And Significantly Lower The Reaction Barrier, J. Phys. Chem. B, 2015, 119, pp 14350-63 Copyright 2015 American Chemical Society.

\subsection{Introduction}

The findings presented in Chapter 3 show that NH•••S hydrogen bonds in heme thiolate proteins reduce the thiolate push effect. The reduction of the push effect is evident from the reduction of the pKa in the heme-bound substrates of the model systems. Herein, we continue our analysis of the $\mathrm{NH} \bullet \bullet$ S hydrogen bond contribution to the reduction of the “push effect”, now focusing our attention on Cpd I catalyzed epoxidation of cis- $\beta$ methylstyrene. Additionally, we extend our model system to include the proximal helix dipole contribution to the reduction of the "push effect". The results show that the combined influence of the $\mathrm{NH} \bullet \bullet S$ hydrogen bonds and the proximal helix dipole reduce the barrier for the rate limiting step by about $\sim 4.6 \mathrm{kcal} / \mathrm{mol}$, while $\mathrm{C}_{\alpha}-\mathrm{O}-\mathrm{C}_{\beta}$ ring closure becomes barrierless. We have determined, as a lower bound, that $\sim 1 / 3$ of this barrier reduction can be attributed to the dipole moment of the proximal helix, and the remainder 
to the NH•••S hydrogen bonds. The stabilization of the reaction barrier correlates with an increased electron density transfer to residues of the proximal pocket, resulting in an increased redox potential of Cpd I. The effect is most pronounced on the doublet spin surface and involves a change in the electron transfer mechanism. In addition to our findings, we have established a minimal representation of the proximal region required for small model systems to realistically mimic the active site in CPO, as the full-enzyme, QM/MM calculation yields essentially the same result as our small model system.

\subsection{Methods}

DFT calculations of Cpd I-catalyzed epoxidation of cis- $\beta$-methylstyrene (CBMS) were carried out. Cpd I was modeled as an $\mathrm{R}^{-}-\mathrm{Fe}^{4+} \mathrm{O}^{2-}\left(\mathrm{N}_{4} \mathrm{C}_{20} \mathrm{H}_{12}\right)^{-}$moiety having a heme without the vinyl and propionate side chains, with $\mathrm{R}^{-}=\left(\mathrm{SCH}_{3}\right)^{-}$in model $\mathrm{A}(\mathrm{Cpd} \mathrm{I-B})$ and $\mathrm{R}^{-}=\mathrm{CH}_{3}-\mathrm{NH}-$ Asn-Leu-Ala-Pro-Cys-Pro-Ala-CO-CHз in model B (Cpd I-B). The Cpd IA model represents Cpd I with a bare thiolate ligand (Fig. 16 A), and the Cpd I-B model represents Cpd I with the proximal pocket, where the thiolate ligand is the deprotonated side chain of Cys29 and the proximal apoprotein environment includes residues 27-33 of CPO (Fig. 16 B). In the Cpd I-B model, the proximal region was restricted to residues 2733 due to the limits of our computer resources; however, these residues are the most extended representation of the proximal pocket of CPO within our limitations. Residues 27-33 include the proximal amino acid residues with $\mathrm{C}_{\alpha}$ atoms within $8 \AA$ of the proximal sulfur. The steric environment of the axial sulfur, the $\mathrm{A}_{31}$ :NH-C29:S, and $\mathrm{L}_{32}: \mathrm{NH}-\mathrm{C}_{29}: \mathrm{S}$ hydrogen bonds, the electrostatic environment provided by the $\mathrm{N}$-terminus 
of the proximal $\alpha$-helix, and the dipole moment imposed on the active center by the $\mathrm{C}_{29}: \mathrm{CO}-\mathrm{N}_{33}: \mathrm{NH}$ and $\mathrm{P}_{30}: \mathrm{CO}-\mathrm{A}_{34}: \mathrm{NH}$ backbone hydrogen bonds of the proximal helix are all represented. Due to our limitations, residues 35 to 38 of the proximal helix were not included, and thus our calculations provide a lower bound to the actual effect of the backbone dipole moment of the proximal helix.

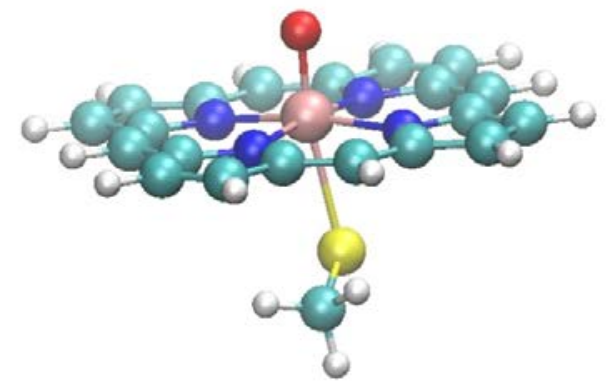

A

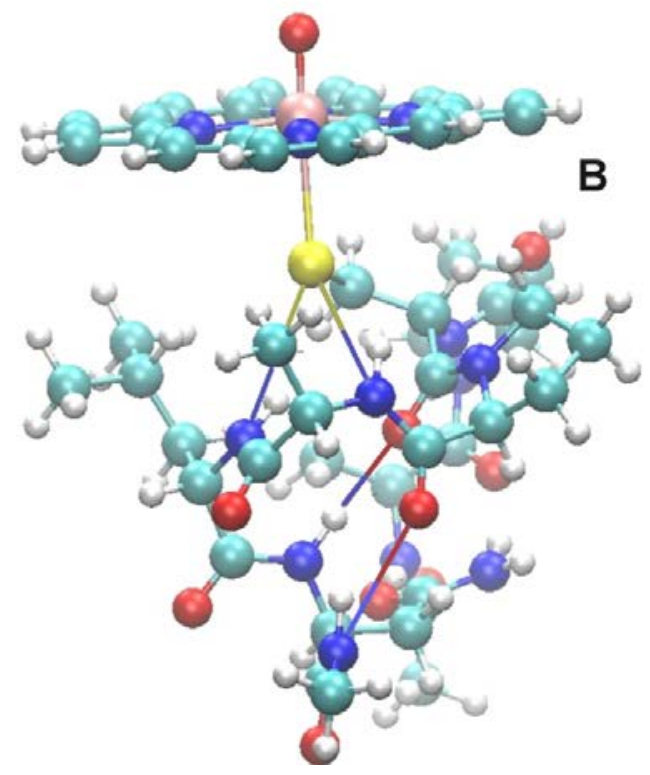

Fig. 16 Bare-thiolate (A) and CPO-like (B) proximal pocket models of Cpd I.

\subsubsection{Cpd I models}

All calculations on the 43-atom Cpd I-A species (Fig. 16 A) were performed without geometric constraints. All transition state structures have one imaginary normal mode frequency while all stable structures have only positive, real frequencies. All calculations on the 141-atom Cpd I-B species (Fig. 16 B) were performed using geometric constraints on the proximal peptide fragment to maintain backbone and side-chain hydrogen bonds, 
backbone $\varphi, \psi$ dihedrals, and the orientation of the proximal helix relative to the heme moiety as in the crystal structure of CPO (PDB code $1 \mathrm{CPO}) .{ }^{3}$ The stationary points have normal modes with imaginary frequencies caused by the applied constraints. The axial sulfur/amide backbone hydrogen bonds were not constrained. The Cartesian coordinates of all structures presented are given in Supplementary Information (SI) Section A. The details of the applied constraints are given in SI Section B1.

\subsubsection{Gas phase calculations}

Unrestricted DFT calculations were performed using the B3LYP ${ }^{105,} 107$ hybrid density functional (UB3LYP). No symmetry restrictions were used in the calculations. Geometry optimizations and numerical frequency calculations were carried out with the LANL2DZ ECP double- $\zeta$ basis set for $\mathrm{Fe}^{144}$ and the $6-31 \mathrm{G}^{*}$ basis set for $\mathrm{H}, \mathrm{C}, \mathrm{N}, \mathrm{O},{ }^{158}$ and $\mathrm{S}^{159}$ atoms (basis set B0) using NWChem 6.1 software. ${ }^{114}$ The stability of the density functions for the optimized structures was checked with the B0 basis set using Gaussian09 software. ${ }^{115}$ Energy refining was performed with the LANL2TZ+ ECP triple- $\zeta$ basis set for $\mathrm{Fe}^{160}$ and 6-311++G** basis set for $\mathrm{H}, \mathrm{C}, \mathrm{N}, \mathrm{O},{ }^{161}$ and $\mathrm{S}^{162}$ atoms (basis set $\mathrm{B} 1$ ) using Gaussian-09. Natural Population Analysis ${ }^{163}$ (NPA) of spin/charge densities and Natural Bond Orbital ${ }^{164}$ (NBO) analysis of bonding patterns were carried out using NBO 6.0 software. ${ }^{165}$ 


\subsection{3. $\mathrm{QM} / \mathrm{MM}$ calculations}

The results of our previous molecular dynamics (MD) simulations of a reactant and a transition-state complex (the latter was parameterized using the Q2MM methodology ${ }^{166}$ ) of the CPO-catalyzed 1S2R epoxidation of $\mathrm{CBMS}^{13,42}$ on the doublet spin surface were used to estimate the effect of the protein and solvent beyond those captured by model B. A snapshot of CPO solvated in a truncated octahedron of about 7000 TIP3P ${ }^{167}$ water molecules from the MD simulation of the transition state ${ }^{13}$ was used as the initial structure for the QM/MM setup. The Cpd I-B/CBMS gas phase optimized structures were embedded into this CPO snapshot to replace the corresponding portion of the snapshot structure and ultimately become the QM region of QM/MM calculations. The internal coordinate facility of the CHARMM software package ${ }^{125}$ was used to do this. The systems thus obtained were then minimized at the MM level of theory using the CHARM22 force field ${ }^{110}$ and CHARMM software. During these minimizations i) atoms in the region later to be treated at the QM level (“QM atoms”) were kept fixed; ii) QM atoms were assigned point charges obtained from the gas phase QM calculations using the ESP facility of NWChem; iii) the solvent and protein atoms were fixed if they were more than $6 \AA$ apart from a QM atom. After the minimizations, the dangling bonds at the QM/MM boundary were saturated with hydrogen atoms. MM atom groups closer than 2 $\AA$ to these hydrogen atoms were removed to avoid spurious electrostatic interactions. In the QM/MM systems prepared in this manner, the QM portions had exactly the same coordinates as in the corresponding gas phase optimized Cpd I-B/CBMS structures. This allowed gas-phase molecular orbitals to be used as the initial guess for the QM/MM 
calculations. The latter were carried out at the UB3LYP/B0 level of theory for the QM portion of the calculation and CHARM22 for the MM portion, using an electrostatic/Van der Waals embedding scheme. ${ }^{113}$ Only single-point energy calculations were performed with this QM/MM approach.

\subsection{Results and Discussion}

For both low- and high-spin surfaces, the observed stabilization of the rate-limiting barrier for CPO-Cpd I catalyzed epoxidation of CBMS correlates with an increased electron density transfer to the proximal pocket upon crossing of the $\mathrm{C}_{\beta}-\mathrm{O}$ transition-state barrier. Natural analysis ${ }^{163,164}$ reveals an intricate difference between Cpd I-A and Cpd IB epoxidation along the low-spin surface. The Cpd I-A oxyferryl $\pi^{*}$ radical attack on the $\mathrm{C}=\mathrm{C}$ double bond initiates release of an electron having the same spin as the thiolateporphyrin radical, as opposed to Cpd I-B, where the same mechanistic step results in the release of an electron having the opposite spin as the thiolate-porphyrin radical. In Cpd I-A, this step reduces $\mathrm{Fe}^{\mathrm{IV}}$ to $\mathrm{Fe}^{\mathrm{III}}$ and is the first and rate limiting kinetic event, followed by a second barrier, where transfer of an electron to the thiolate-porphyrin orbital occurs with formation of the $\mathrm{C}_{\alpha}-\mathrm{O}-\mathrm{C}_{\beta}$ ring. Since the release of the electron has the opposite spin as the thiolate-porphyrin radical in Cpd I-B, this electron is directly transferred to the thiolate porphyrin. This step is followed by a barrierless $\mathrm{C}_{\alpha}-\mathrm{O}-\mathrm{C}_{\beta}$ ring closure concomitant with the $\mathrm{Fe}^{\mathrm{IV}} / \mathrm{Fe}^{\mathrm{III}}$ reduction. The change in the electron transfer mechanisms

derives from the proximal pocket's stabilization of the doubly occupied thiolate- 
porphyrin $\mathrm{a}_{2 \mathrm{u}}+$ ఐbrbital relative to the oxyferryl $\pi^{*}$ orbitals in the Cpd I-B model. On the quartet spin surface, the electron transfer mechanism is the same for both Cpd I-A and Cpd I-B, where initial electron transfer is to the oxyferryl azu+ §orbital. However, $\mathrm{C}_{\alpha-}$ O- $\mathrm{C}_{\beta}$ ring closure and $\mathrm{Fe}^{\mathrm{IV}} / \mathrm{Fe}^{\mathrm{III}}$ reduction on the high-spin surface for Cpd I-A possesses a barrier similar to the low-spin surface. QM/MM calculations were carried out to confirm the reported effect in the full-enzyme environment.

\subsubsection{Potential Energy Surfaces of CBMS Epoxidation}

Formation of the 1S2R epoxide enantiomer is the main channel for CPO-catalyzed epoxidation of CBMS. ${ }^{39}$ The calculated UB3LYP/B1//B0 doublet and quartet potential energy surfaces (PES) for 1S2R epoxidation of CBMS by model species Cpd I-A and Cpd I-B are shown in Fig. 17. The results include zero point energy corrections calculated at the UB3LYP/B0 level. For both models, the reactant state (R) is Cpd I + CBMS bound complex. The reactant states on the low- and high-spin surfaces are nearly degenerate. In the case of Cpd I-A catalyzed epoxidation, both high- and low-spin surfaces were found to have an intermediate state (I) with the beta-carbon covalently bonded to the distal oxygen and the $\mathrm{C}_{\alpha}-\mathrm{O}-\mathrm{C}_{\beta}$ ring not yet formed. On both spin surfaces, I is separated from $\mathrm{E}$ by a relatively small barrier (TS2) for $\mathrm{C}_{\alpha}-\mathrm{O}-\mathrm{C}_{\beta}$ ring closure. In the case of Cpd I-B catalyzed epoxidation, TS1 is converted into E in a barrierless manner on both the doublet and quartet spin surfaces. For the rest of this chapter, abbreviations will be used to designate the stationary points; for example, ${ }^{2}$ TS1-A designates TS1 on the doublet spin surface for Cpd I-A-catalyzed epoxidation of CBMS. 


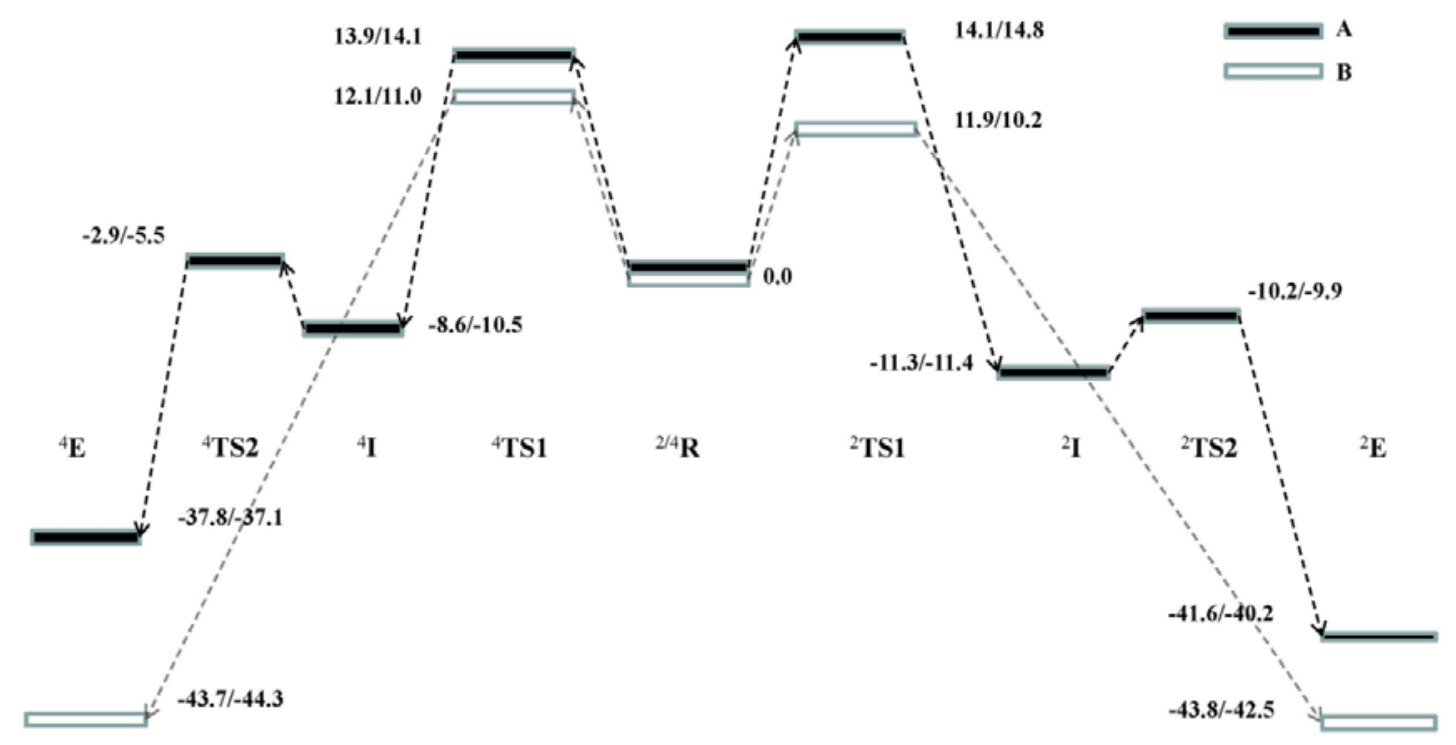

Fig. 17 The doublet and quartet potential energy surfaces (B0/B1 in $\mathrm{kcal} / \mathrm{mol})$ for 1S2R epoxidation of CBMS.

The calculations show that, on both spin surfaces, the rate-limiting TS1 barrier is significantly stabilized by the proximal helix dipole and $\mathrm{NH} \cdots \mathrm{S}$ bonds, by $-4.6 \mathrm{kcal} / \mathrm{mol}$ and $-3.1 \mathrm{kcal} / \mathrm{mol}$ for the doublet and quartet surfaces, respectively, while electron charge transfer $\left(\mathrm{CT}=\right.$ Natural Charge ${ }_{\mathrm{TS}}$-Natural Charge $\left._{\mathrm{R}}\right)$ to the proximal moiety in transition state TS1 is increased (Table 7). The stabilization of TS1 is stronger on the doublet spin surface, which correlates with a large increase of CT to the proximal moiety (primarily to the sulfur), -0.10 e and -0.06 e for the doublet and quartet surfaces, respectively. At the same time, the spin densities of, the $\mathrm{O}$ and $\mathrm{Fe}$ atoms and of the $\mathrm{C}_{\alpha} \mathrm{H}$ atom pair indicate that, on the doublet spin surface, the Cpd I-A and Cpd I-B species have different mechanisms of electron transfer (Table 8). We shall elucidate this difference in terms of occupancies of molecular orbitals involved in the reaction. 
Table 7. Barriers (kcal/mol)/Charge Transfer to the proximal moiety.

\begin{tabular}{|l|l|l|}
\hline & doublet & quartet \\
\hline TS1-A & $14.8 /-0.03$ & $14.1 /-0.02$ \\
\hline TS1-B & $10.2 /-0.13$ & $11.0 /-0.08$ \\
\hline
\end{tabular}

Table 8. Natural Group spin densities/Charges of the optimized structures on the doublet spin PES surfaces.

\begin{tabular}{|c|c|c|c|c|c|c|c|c|c|}
\hline & \multicolumn{8}{|c|}{ Natural Spin Densities/ Natural Atomic Charges } \\
\hline & & S-R & Por & $\mathrm{Fe}$ & $\mathrm{O}$ & $\mathrm{C}_{\beta} \mathrm{H}$ & $\mathrm{C}_{a} \mathrm{H}$ & $\mathrm{R}_{1}^{\dagger}$ & $\mathrm{R}_{2}^{\dagger}$ \\
\hline \multirow{3}{*}{${ }^{2} \mathbf{R}$} & A & $\begin{array}{l}- \\
0.75 / \\
-0.05\end{array}$ & $\begin{array}{l}-0.30 / \\
-0.49\end{array}$ & $\begin{array}{l}1.10 / \\
0.91\end{array}$ & $\begin{array}{l}0.95 / \\
-0.37\end{array}$ & $\begin{array}{l}0.00 / \\
0.06\end{array}$ & $\begin{array}{c}0.00 / \\
-0.03\end{array}$ & $\begin{array}{c}0.00 / \\
-0.05\end{array}$ & $\begin{array}{l}0.00 / \\
0.02\end{array}$ \\
\hline & B & $\begin{array}{l}- \\
0.60 / \\
-0.27\end{array}$ & $\begin{array}{l}-0.50 / \\
-0.32\end{array}$ & $\begin{array}{l}1.15 / \\
0.95\end{array}$ & $\begin{array}{l}0.95 / \\
-0.36\end{array}$ & $\begin{array}{l}0.00 / \\
0.06\end{array}$ & $\begin{array}{c}0.00 / \\
-0.03\end{array}$ & $\begin{array}{c}0.00 / \\
-0.05\end{array}$ & $\begin{array}{l}0.00 / \\
0.02\end{array}$ \\
\hline & $\begin{array}{l}\text { CP } \\
\mathbf{O}\end{array}$ & $\begin{array}{l}- \\
0.50 / \\
-0.38 \\
\end{array}$ & $\begin{array}{l}-0.58 / \\
-0.22\end{array}$ & $\begin{array}{l}1.12 / \\
0.95\end{array}$ & $\begin{array}{l}0.96 / \\
-0.35 \\
\end{array}$ & $\begin{array}{l}0.00 / \\
0.03\end{array}$ & $\begin{array}{c}0.00 / \\
-0.01\end{array}$ & $\begin{array}{r}0.00 / \\
-0.04\end{array}$ & $\begin{array}{l}0.00 / \\
0.02\end{array}$ \\
\hline \multirow{3}{*}{${ }^{2}$ TS1 } & A & $\begin{array}{l}- \\
0.70 / \\
-0.08 \\
\end{array}$ & $\begin{array}{l}-0.30 / \\
-0.58\end{array}$ & $\begin{array}{l}0.95 / \\
0.90\end{array}$ & $\begin{array}{r}0.75 / \\
-0.44 \\
\end{array}$ & $\begin{array}{l}- \\
0.10 / \\
0.15\end{array}$ & $\begin{array}{l}0.30 / \\
0.04\end{array}$ & $\begin{array}{c}0.10 / \\
-0.02\end{array}$ & $\begin{array}{l}0.00 / \\
0.03\end{array}$ \\
\hline & B & $\begin{array}{l}- \\
0.30 / \\
-0.40\end{array}$ & $\begin{array}{l}-0.25 / \\
-0.51\end{array}$ & $\begin{array}{l}1.40 / \\
0.96\end{array}$ & $\begin{array}{l}0.50 / \\
-0.42 \\
\end{array}$ & $\begin{array}{l}- \\
0.05 / \\
0.18\end{array}$ & $\begin{array}{l}- \\
0.20 / \\
0.08\end{array}$ & $\begin{array}{l}- \\
0.10 / \\
0.07\end{array}$ & $\begin{array}{l}0.00 / \\
0.04\end{array}$ \\
\hline & $\begin{array}{l}\text { CP } \\
\mathbf{O}\end{array}$ & $\begin{array}{l}- \\
0.23 / \\
-0.48 \\
\end{array}$ & $\begin{array}{l}-0.22 / \\
-0.49\end{array}$ & $\begin{array}{l}1.40 / \\
0.98\end{array}$ & $\begin{array}{r}0.45 / \\
-0.42 \\
\end{array}$ & $\begin{array}{l}- \\
0.06 / \\
0.19\end{array}$ & $\begin{array}{l}- \\
0.21 / \\
0.09 \\
\end{array}$ & $\begin{array}{l}- \\
0.13 / \\
0.08 \\
\end{array}$ & $\begin{array}{l}0.00 / \\
0.05\end{array}$ \\
\hline \multirow[t]{2}{*}{${ }^{2} \mathbf{I}^{*}$} & A & $\begin{array}{l}- \\
0.60 / \\
-0.09 \\
\end{array}$ & $\begin{array}{l}-0.30 / \\
-0.60\end{array}$ & $\begin{array}{l}0.95 / \\
0.94\end{array}$ & $\begin{array}{r}0.20 / \\
-0.65 \\
\end{array}$ & $\begin{array}{l}0.00 / \\
0.27\end{array}$ & $\begin{array}{l}0.55 / \\
0.13\end{array}$ & $\begin{array}{c}0.25 / \\
-0.01\end{array}$ & $\begin{array}{l}0.00 / \\
0.02\end{array}$ \\
\hline & $\mathbf{B}$ & $\begin{array}{l}- \\
0.05 / \\
-0.43 \\
\end{array}$ & $\begin{array}{l}-0.05 / \\
-0.67\end{array}$ & $\begin{array}{l}1.20 / \\
0.97\end{array}$ & $\begin{array}{r}0.25 / \\
-0.54 \\
\end{array}$ & $\begin{array}{l}0.00 / \\
0.27\end{array}$ & $\begin{array}{l}- \\
0.25 / \\
0.23 \\
\end{array}$ & $\begin{array}{l}- \\
0.10 / \\
0.14 \\
\end{array}$ & $\begin{array}{l}0.00 / \\
0.04\end{array}$ \\
\hline${ }^{2} \mathbf{I}$ & A & $\begin{array}{r}0.00 / \\
-0.22 \\
\end{array}$ & $\begin{array}{l}-0.25 / \\
-0.71\end{array}$ & $\begin{array}{l}0.99 / \\
0.88\end{array}$ & $\begin{array}{l}- \\
0.05 / \\
-0.73 \\
\end{array}$ & $\begin{array}{l}0.00 / \\
0.33\end{array}$ & $\begin{array}{l}0.25 / \\
0.24\end{array}$ & $\begin{array}{r}0.10 / \\
0.165 \\
\end{array}$ & $\begin{array}{r}0.00 / \\
-0.05\end{array}$ \\
\hline${ }^{2} \mathbf{T S} 2$ & A & $\begin{array}{l}0.25 / \\
-0.17\end{array}$ & $\begin{array}{c}0.10 / \\
-0.69\end{array}$ & $\begin{array}{l}0.85 / \\
0.86\end{array}$ & $\begin{array}{l}0.15 / \\
-0.66 \\
\end{array}$ & $\begin{array}{l}0.00 / \\
0.28\end{array}$ & $\begin{array}{l}- \\
0.25 / \\
0.26\end{array}$ & $\begin{array}{l}- \\
0.10 / \\
-0.02\end{array}$ & $\begin{array}{l}0.00 / \\
0.01\end{array}$ \\
\hline \multirow[t]{2}{*}{${ }^{2} \mathbf{E}$} & A & $\begin{array}{r}0.05 / \\
-0.12 \\
\end{array}$ & $\begin{array}{l}-0.05 / \\
-0.83\end{array}$ & $\begin{array}{l}1.00 / \\
0.86\end{array}$ & $\begin{array}{r}0.00 / \\
-0.56 \\
\end{array}$ & $\begin{array}{l}0.00 / \\
0.33\end{array}$ & $\begin{array}{l}0.00 / \\
0.32\end{array}$ & $\begin{array}{c}0.00 / \\
-0.02\end{array}$ & $\begin{array}{l}0.00 / \\
0.02\end{array}$ \\
\hline & B & $\begin{array}{l}- \\
0.05 / \\
-0.27\end{array}$ & $\begin{array}{l}-0.05 / \\
-0.77\end{array}$ & $\begin{array}{l}1.10 / \\
0.94\end{array}$ & $\begin{array}{l}0.00 / \\
-0.56\end{array}$ & $\begin{array}{l}0.00 / \\
0.33\end{array}$ & $\begin{array}{l}0.00 / \\
0.32\end{array}$ & $\begin{array}{c}0.00 / \\
-0.02\end{array}$ & $\begin{array}{l}0.00 / \\
0.03\end{array}$ \\
\hline
\end{tabular}

${ }^{\dagger} \mathbf{R}_{1}$ - benzylic group of CBMS; $\mathbf{R}_{2}$ - methyl group of CBMS. *Not a true intermediate. 


\subsubsection{Cpd I orbitals}

The key Kohn-Sham (KS) molecular orbitals involved in the epoxidation reaction are the oxygen 2p-, iron 3d-based orbitals and the thiolate-porhyrin $\mathrm{a}_{2 u}+\sigma \mathrm{s}$ orbital. The occupancies of these orbitals, as shown in Fig. 18, describe the experimentally established doublet tri-radical ground state of the Cpd I species of CPO, ${ }^{128,}{ }^{168}$ i. e. $\left(\delta_{x^{2}-y^{2}}\right)^{2}\left(\pi_{x z}^{*}\right)^{1}\left(\pi_{y z}^{*}\right)^{1}\left(\varphi_{a_{2 u}+\sigma_{S}}\right)^{1}$. The highest doubly occupied orbital is the nonbonding $\delta$-type iron $d_{\mathrm{x}^{2}-y^{2}}$-based orbital. Two singly occupied $\pi^{*}$ orbitals are the antibonding combinations of the iron $d_{x z}\left(d_{y z}\right)$ with the oxygen $p_{x}\left(p_{y}\right)$ atomic orbitals. The singly occupied thiolate-porphyrin orbital mixes the porphyrin a2u orbital with the sulfur $\sigma$-type orbital. The virtual orbitals are antibonding: the $\sigma_{z^{2}}^{*}$, which involves interaction of the iron $d_{z^{2}}$ orbital with the oxygen and sulfur $\sigma$-type orbitals, and the $\delta_{x y}^{*}$, which involves interaction of the iron $d_{x y}$ orbital with the nitrogen orbitals. The $\pi_{x z}^{*}$ and $\pi_{y z}^{*}$ electrons of Cpd I are ferromagnetically coupled with each other, i. e., $\pi_{x z}^{*}, \pi_{y z}^{*} S=1$, and antiferromagnetically coupled with the $\varphi_{a_{2 u}+\sigma_{S}}$ electron, i.e., ${ }^{\varphi_{2 u}+\sigma_{S}} S_{z}=-1 / 2$ when $\pi_{x z}^{*}, \pi_{y z}^{*} S_{z}=1$, so that ${ }^{C p d I} S=1 / 2$. For the rest of this chapter, the ferromagnetic and antiferromagnetic relationship of the $\varphi_{a_{2 u}+\sigma_{S}}$ electron to the $\pi_{x z}^{*}, \pi_{y z}^{*}$ electrons is referred as belonging to alpha and beta electron manifolds, respectively. 


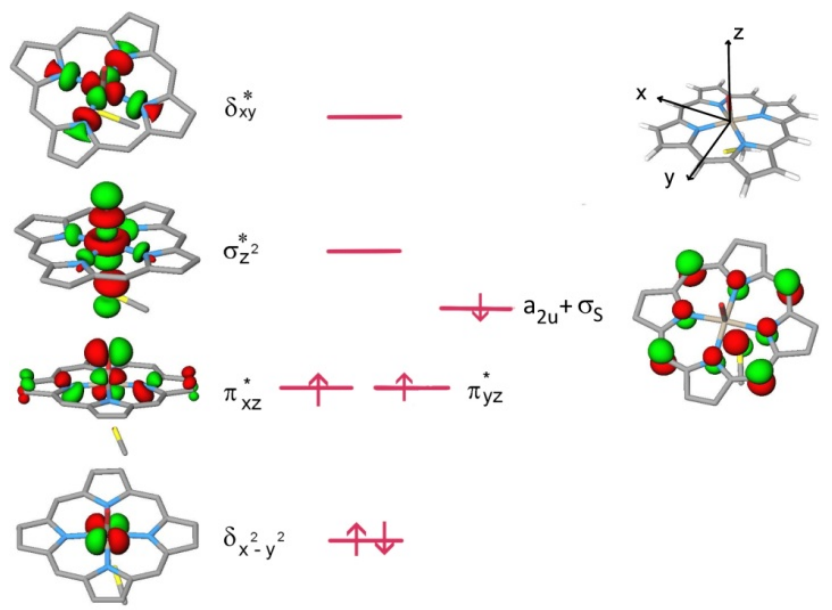

Figure 18 Key Kohn-Sham molecular orbitals of Cpd I (doublet) involved in the epoxidation reaction.

\subsubsection{Electron transfer mechanisms on the doublet spin surface of Cpd I-A}

In the ${ }^{2} \mathrm{R}-\mathrm{A}$ state the methyl group of CBMS interacts weakly with the oxygen atom of the heme (Fig. 19). The epoxidation reaction starts with the Fe-O $\pi_{y z}^{*}$ (in our system of coordinate) radical attack on the $\pi$ orbital of the $\mathrm{C}_{\alpha}=\mathrm{C}_{\beta}$ double bond of CBMS (Fig. 20a), whereby the $\mathrm{C}_{\alpha}=\mathrm{C}_{\beta}$ double bond is converted into a $\mathrm{C}_{\alpha}-\mathrm{C}_{\beta}$ single bond and the $\mathrm{C}_{\beta}-\mathrm{O}$ bond is formed. Formally, $\mathrm{C}_{\beta}-\mathrm{O}$ bond formation is described as a one electron reduction of the heme-thiolate moiety in which the electron is transferred from the olefin $\pi$ bond to either the $\pi_{y z}^{*}$ or the $\varphi_{a_{2 u}+\sigma_{S}}$ orbital. NBO analysis of ${ }^{2}$ TS1-A revealed that the $\pi$ orbital of the substrate $C_{\alpha}=C_{\beta}$ double bond donates the beta electron to the incipient $C_{\beta}-\mathrm{O}$ bond while the alpha electron remains localized in the $\pi$ bond. Since the $\varphi_{a_{2 u}+\sigma_{S}}$ orbital is already occupied by a beta electron, the electron from the olefin $\pi$ bond is formally transferred to $\pi_{y z}^{*}$ orbital (Scheme 2). The resulting $\left(\pi_{x z}^{*}\right)^{1}\left(\pi_{y z}^{*}\right)^{2}\left(\varphi_{a_{2 u}+\sigma_{S}}\right)^{1}\left(\varphi_{C \alpha}\right)^{1}$ 
triradical state is readily evidenced by the spin densities of the ${ }^{2} \mathrm{I}^{*}$-A structure (Table 8). ${ }^{2} \mathrm{I}^{*}$-A follows ${ }^{2} \mathrm{TS} 1-\mathrm{A}$ and has the $\mathrm{C}_{\beta}-\mathrm{O}$ bond almost formed, but it is not a true intermediate yet. Relative to ${ }^{2} \mathrm{R}-\mathrm{A},{ }^{2} \mathrm{I}^{*}$-A has the O-Fe bond length increased $\sim 0.08 \AA$ and the Fe-S bond length decreased $\sim 0.07 \AA$ (Fig.5). ${ }^{2} \mathrm{I}^{*}$-A is characterized by a buildup of alpha spin density on the substrate's $\mathrm{C}_{\alpha}$ carbon, with corresponding reductions on the oxygen and iron atoms, while the thiolate-porphyrin beta spin density is little changed (Table 8). $\left(\varphi_{C \alpha}\right)^{1}$ corresponds to the KS HOMO in the alpha manifold of ${ }^{2} \mathrm{I}^{*}$-A (Fig. 20b). The true intermediate state ${ }^{2} \mathrm{I}-\mathrm{A}$ is reached when ${ }^{2} \mathrm{I}^{*}$-A's benzylic group is rearranged to have a weak C-H-O interaction (Fig. 19). Interestingly, this rearrangement is paralleled by a further $\sim 0.15 \AA$ stretching of the O-Fe bond and by $\sim 0.18 \AA$ shrinking of the Fe-S bond while the spin density changes indicate a delocalization of the substrate's alpha radical from the $\mathrm{C}_{\alpha}$ carbon into the heme moiety (Table 8). For styrene epoxidation by Cpd I, a transfer of the $\varphi_{C \alpha}$ electron to either the $\sigma_{z^{2}}^{*}$ or the $\delta_{x y}^{*}$ orbital was proposed by Kumar et al. to describe the possible electronic structures of the carbocationic intermediate. ${ }^{47}$ For the case of CBMS epoxidation by Cpd I-A on the doublet spin surface, we found that both the $\sigma_{z^{2}}^{*}$ and $\delta_{x y}^{*}$ KS orbitals remain virtual in the intermediate state ${ }^{2} \mathrm{I}$-A. It follows that the only opportunity left for the substrate's alpha electron is to be delocalized into the $\varphi_{a_{2 u}+\sigma_{S}}$ orbital. Indeed, the second highest occupied orbital in the alpha manifold of ${ }^{2} \mathrm{I}-\mathrm{A}$ is a mixture of $\varphi_{C \alpha}$ and $\varphi_{a_{2 u}+\sigma_{S}}$ (Fig. 20c). Thus the ${ }^{2} \mathrm{I}-\mathrm{A}$ structure has a character quite similar to the product state ${ }^{2} \mathrm{E}$-A, although the $\mathrm{C}_{\alpha}-\mathrm{O}$ bond is not formed yet. Taking into account that ${ }^{2} \mathrm{I}-\mathrm{A}$ has significant spin density left both on the porphyrin moiety and on the $\mathrm{C}_{\alpha}$ carbon (Table 8), we propose that the intermediate 
state ${ }^{2}$ I-A has mixed $\left(\pi_{x z}^{*}\right)^{1}\left(\pi_{y z}^{*}\right)^{2}\left(\varphi_{a_{2 u}+\sigma_{S}}\right)^{1}\left(\varphi_{C \alpha}\right)^{1}:\left(\pi_{x z}^{*}\right)^{1}\left(\pi_{y z}^{*}\right)^{2}\left(\varphi_{a_{2 u}+\sigma_{S}}\right)^{2}$ character (Scheme 2). Consequently one would expect a small barrier for the last stage of the reaction, which should lead to the second reduction of the heme-thiolate moiety to reach the $\left(\pi_{x z}^{*}\right)^{1}\left(\pi_{y z}^{*}\right)^{2}\left(\varphi_{a_{z u}+\sigma_{S}}\right)^{2}$ resting state and the epoxide product. In accord with this expectation, the calculations showed that the ${ }^{2} \mathrm{TS} 2-\mathrm{A}$ barrier is $1.5 \mathrm{kcal} / \mathrm{mol}$ (Fig.3). In ${ }^{2} \mathrm{TS} 2-\mathrm{A}$, the $\mathrm{C}_{\alpha}-\mathrm{O}$ distance is just $\sim 0.17 \AA$ shorter than in ${ }^{2} \mathrm{I}-\mathrm{A}$ (Fig. 19). The resting-state electronic structure $\left(\pi_{x z}^{*}\right)^{1}\left(\pi_{y z}^{*}\right)^{2}\left(\varphi_{a_{2 u}+\sigma_{S}}\right)^{2}$ of the heme-thiolate moiety (Scheme 2) was confirmed by an analysis of KS orbitals.

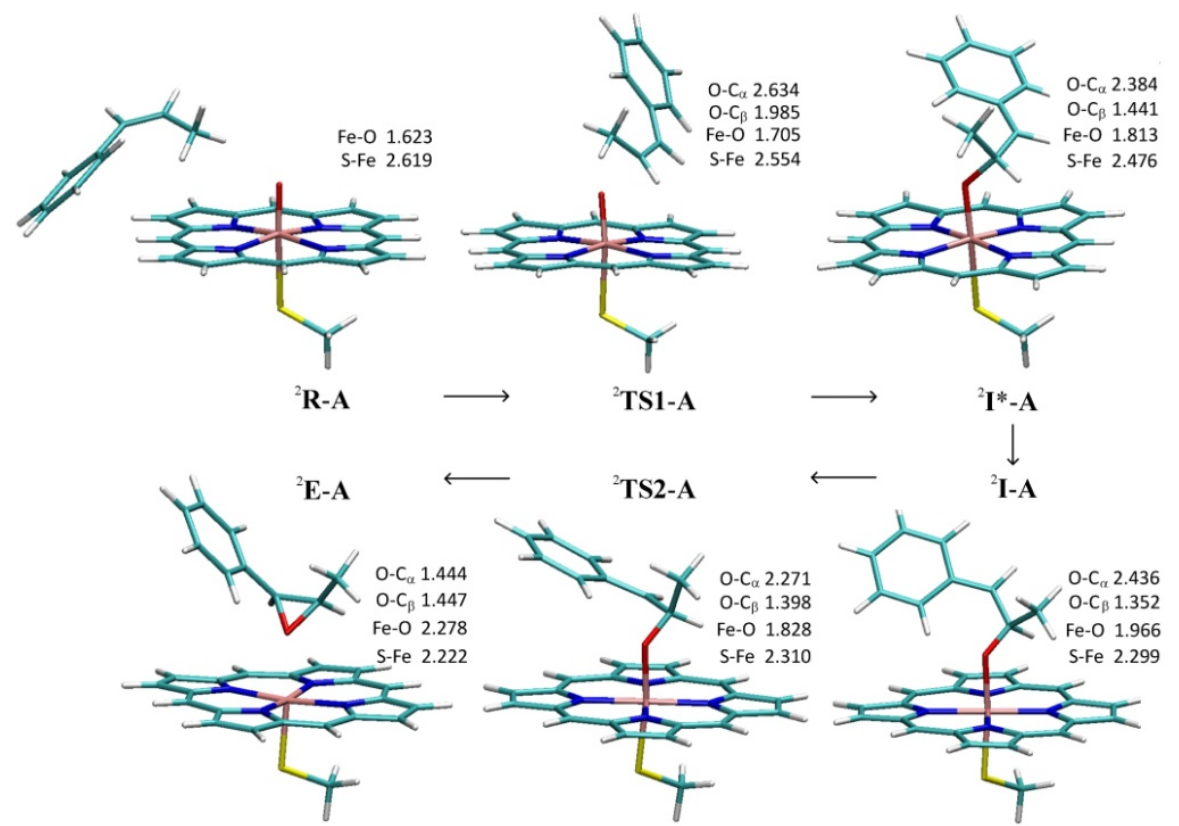

Figure 19: Optimized structures ( ${ }^{2} I^{*}-\mathrm{A}$ is not a stationary point) for Cpd I-A catalyzed epoxidation on the doublet spin surface. Key bond parameters are given in Å. 

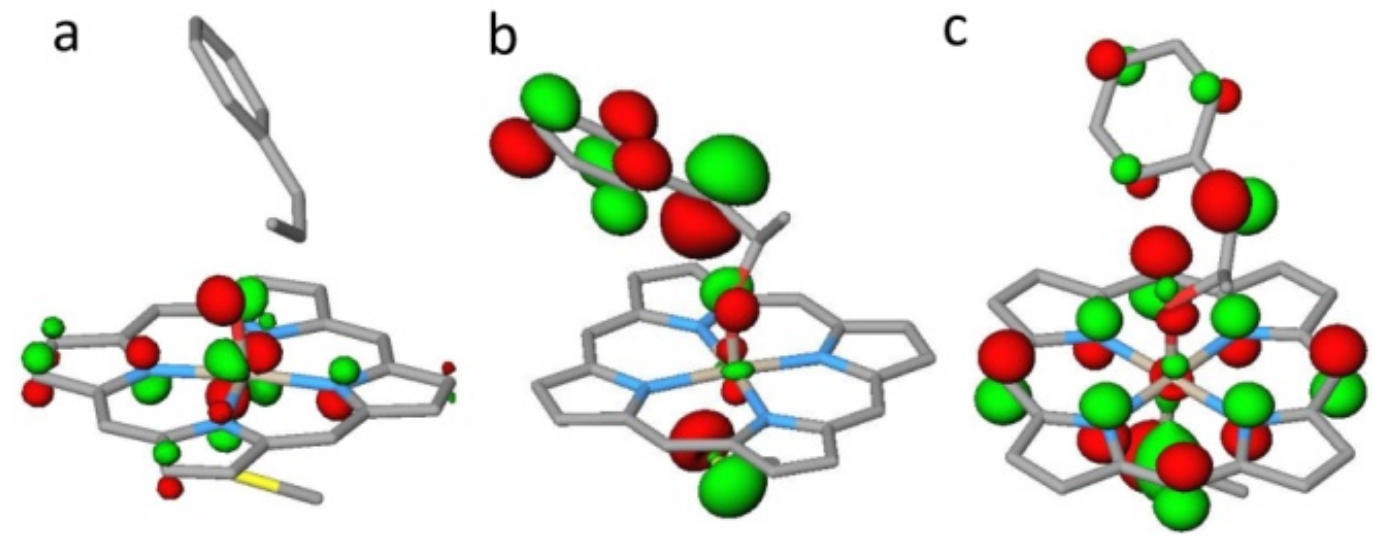

Figure. 20 a: $\pi_{y z}^{*}$ radical attack on $C=C$ bond; $b$ : $C_{a}$ radical corresponds to $\mathrm{KS}$ HOMO in the alpha manifold of ${ }^{2} \mathrm{I}^{*}$-A; c: second highest occupied orbital in the alpha manifold of ${ }^{2} I-A$ is a mixture of $\varphi_{C \alpha}$ and $\varphi_{a_{2 u}+\sigma_{S}}$.
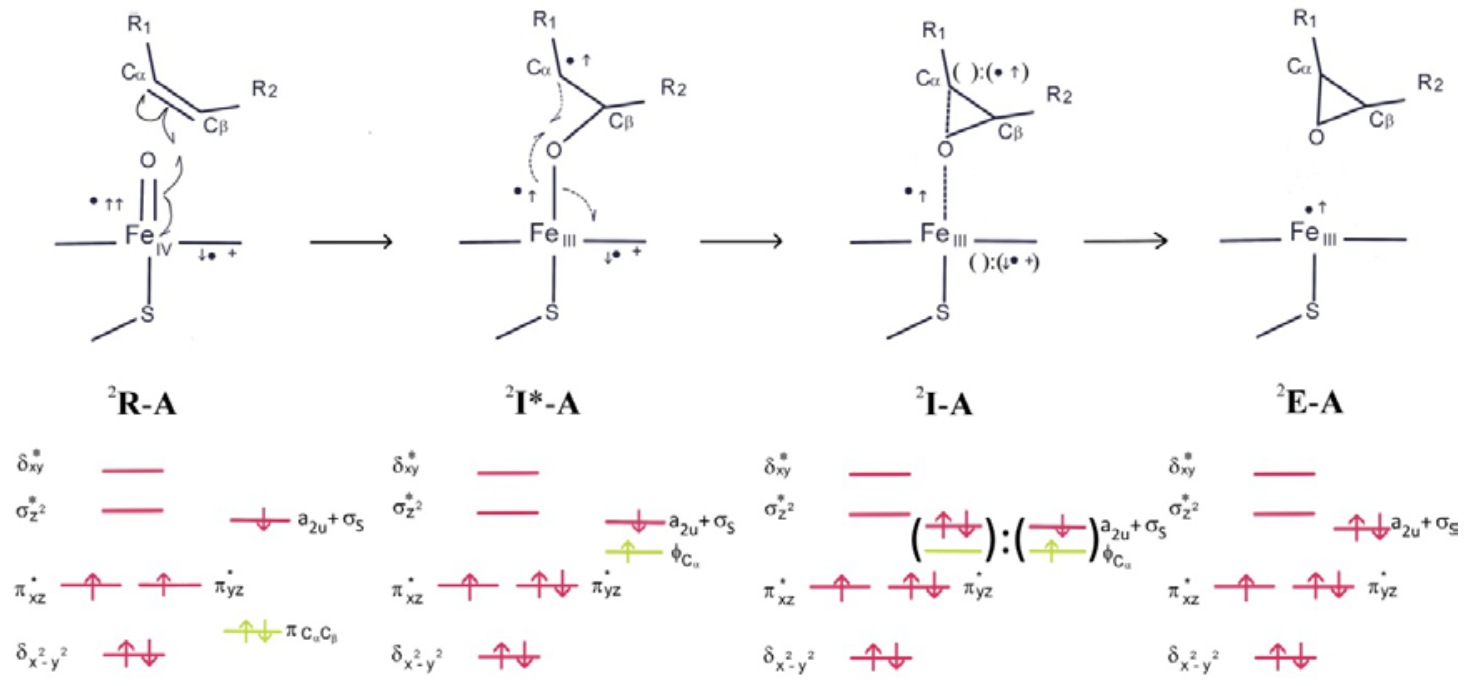

Scheme 2. Cpd I-A catalyzed epoxidation on the doublet spin surface.

\subsubsection{Electron transfer mechanisms on the doublet spin surface of Cpd I-B}

In reactant state ${ }^{2} \mathrm{R}-\mathrm{B}$, similarly to ${ }^{2} \mathrm{R}-\mathrm{A}$, the methyl group of CBMS has a steric contact with the oxygen atom of Cpd I-B (Fig. 21). Compared to ${ }^{2} \mathrm{R}-\mathrm{A},{ }^{2} \mathrm{R}-\mathrm{B}$ has a S-Fe bond 
$0.16 \AA$ longer while the distribution of the $\varphi_{a_{2 u}+\sigma_{S}}$ radical is shifted from the sulfur to the porphyrin by $\sim 0.15$ e (Fig. 21, Table 8). The dipole moment of the proximal helix and

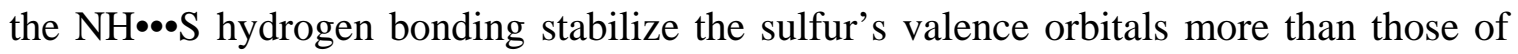
the iron and porphyrin, causing a decrease in the contribution of $\sigma_{S}$ to $\varphi_{a_{2 u}+\sigma_{S}}$. The proximal pocket also results in weaker mixing of the $\sigma_{S}$ and $\pi_{S}$ with the iron d orbitals, leading to the weakening of the S-Fe bond. In transition state ${ }^{2}$ TS1-B (Fig. 21), the substrate's orientation is appropriate for $\pi_{x z}^{*}$ radical attack on the $\pi$ orbital of the $\mathrm{C}_{\alpha}=\mathrm{C}_{\beta}$ double bond. NBO analysis of ${ }^{2}$ TS1-B showed that the $\pi$ orbital of the substrate's $C_{\alpha}=C_{\beta}$ double bond donates its alpha electron to the incipient $\mathrm{C}_{\beta}-\mathrm{O}$ bond while the beta electron remains localized in the $\pi$ bond. Consequently the alpha electron from the olefin $\pi$ bond is formally transferred to the $\varphi_{a_{2 u}+\sigma_{S}}$ orbital (Scheme 3). It follows that the proximal pocket stabilizes the electrons in the $\varphi_{a_{2 u}+\sigma_{S}}$ orbital relative to those in the $\pi_{x z}^{*} / \pi_{y z}^{*}$ orbitals. This is likely the result of interplay between the decreased push of the proximal thiolate and the exchange interactions of the transferred electron. The $\left(\pi_{x z}^{*}\right)^{1}\left(\pi_{y z}^{*}\right)^{1}\left(\varphi_{a_{2 u}+\sigma_{S}}\right)^{2}\left(\varphi_{C \alpha}\right)^{1}$ triradical state is consistent with the spin densities of the ${ }^{2} \mathrm{I}^{*}$-B structure. ${ }^{2} \mathrm{I}^{*}$-B has the beta spin density on the substrate's $\mathrm{C}_{\alpha}$ carbon while the beta spin density on the porphyrin and sulfur has almost vanished (Table 8). The $\left(\pi_{x z}^{*}\right)^{1}\left(\pi_{y z}^{*}\right)^{1}\left(\varphi_{a_{2 u}+\sigma_{S}}\right)^{2}\left(\varphi_{C \alpha}\right)^{1}$ triradical state of ${ }^{2}{ }^{*}$-B was also confirmed by analysis of the KS orbitals. The last stage of Cpd I-B catalyzed epoxidation of CBMS is reduction of $\mathrm{Fe}^{\mathrm{IV}}$ to $\mathrm{Fe}^{\mathrm{III}}$ (Scheme 3). Formally this is described as electron transfer from the $\varphi_{C \alpha}$ to the $\pi_{x z}^{*}$ orbital. Reduction of $\mathrm{Fe}^{\mathrm{IV}}$ to $\mathrm{Fe}^{\mathrm{III}}$ follows ${ }^{2} \mathrm{I}^{*}$ - $\mathrm{B}$ in a barrierless manner to reach the $\left(\pi_{x z}^{*}\right)^{2}\left(\pi_{y z}^{*}\right)^{1}\left(\varphi_{a_{2 u}+\sigma_{S}}\right)^{2}$ resting state of the heme-thiolate moiety and the epoxide 
product. The resting state electronic structure $\left(\pi_{x z}^{*}\right)^{2}\left(\pi_{y z}^{*}\right)^{1}\left(\varphi_{a_{2 u}+\sigma_{S}}\right)^{2}$ of the hemethiolate moiety (Scheme 3) was confirmed by an analysis of the KS orbitals.

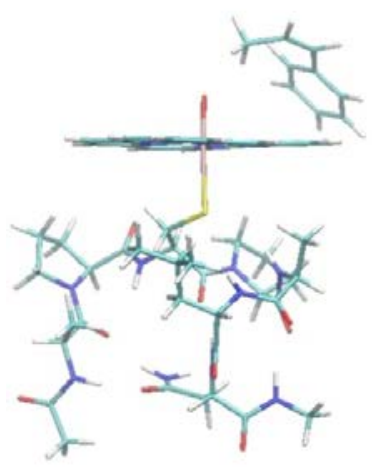

Fe-O 1.619

S-Fe 2.776

${ }^{2} \mathbf{R}-\mathbf{B}$
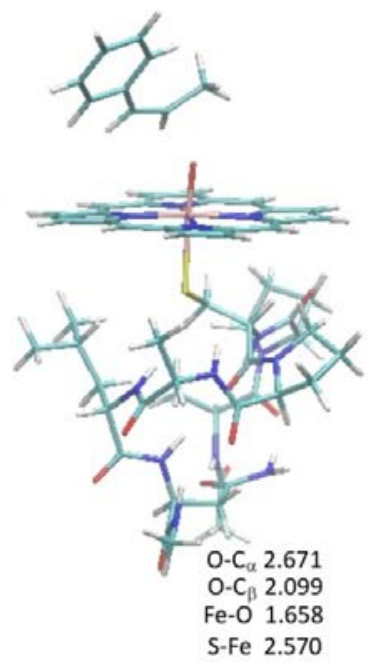

${ }^{2}$ TS1-B $\longrightarrow$
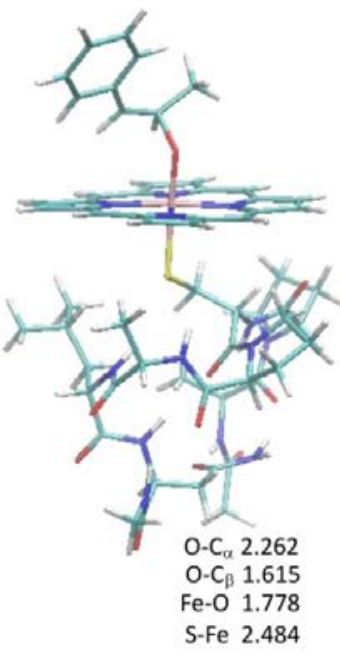

${ }^{2} \mathbf{I}^{*}-\mathbf{B}$

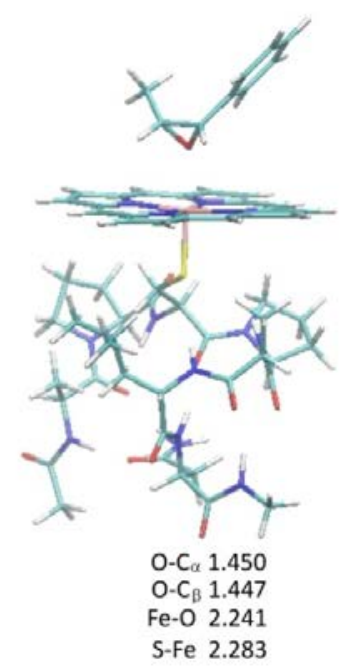

${ }^{2}$ E-B

Figure. 21 Optimized structures $\left({ }^{2} I^{*}-B\right.$ is not a stationary point) for Cpd I-B catalyzed epoxidation on the doublet spin surface. Key bond parameters are given in $\AA$.

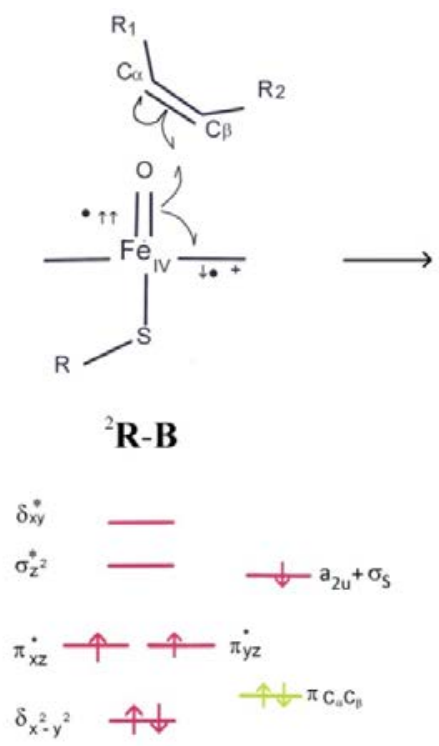

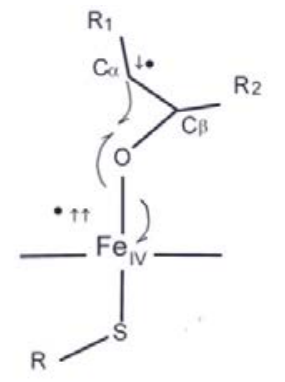

${ }^{2} \mathbf{I}^{*}-\mathbf{B}$
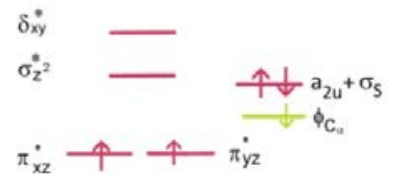

$\delta_{x^{2}-y^{2}}^{\uparrow} \uparrow \downarrow$
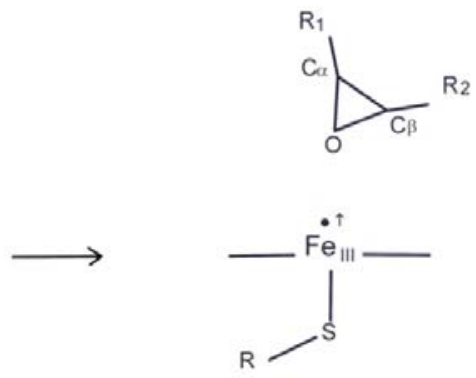

${ }^{2} \mathbf{E}-\mathbf{B}$

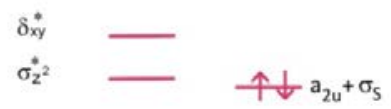

$\pi_{x z} \uparrow \downarrow \uparrow \pi_{y z}$

$\delta_{x^{2}-y^{2}} \uparrow \downarrow$

Scheme 3. Cpd I-B catalyzed epoxidation on the doublet spin surface. 


\subsubsection{Electron transfer mechanisms on the quartet spin surface}

The effect of CPO's proximal moiety on the reactant state is similar on the quartet and doublet spin surfaces. The S-Fe bond is $\sim 0.13 \AA$ longer in ${ }^{4} \mathrm{R}$-B than in ${ }^{4} \mathrm{R}$-A (doublet surface: $\sim 0.16 \AA$ ). In ${ }^{4} \mathrm{R}-\mathrm{B}$, the distribution of the $\varphi_{a_{2 u}+\sigma_{S}}$ radical is shifted from sulfur to porphyrin by $\sim 0.15$ e (Table 9) (doublet surface: $~ 0.15$ e). Electronic structure analysis revealed, however, that the electron transfer mechanism is the same for the Cpd I-A and the Cpd I-B models on the quartet surface, rather different as on the doublet surface. In the rate limiting event on the quartet surface, the $\pi$ orbital of the $\mathrm{C}_{\alpha}=\mathrm{C}_{\beta}$ double bond donates its beta electron to the newly formed $\mathrm{C}_{\beta}-\mathrm{O}$ bond; for both models this electron is formally transferred to the $\varphi_{a_{2 u}+\sigma_{S}}$ orbital (Scheme 4). Analysis of the spin densities (Table 9) and KS orbitals clearly confirms that ${ }^{4} \mathrm{I}$ has a $\left(\pi_{x z}^{*}\right)^{1}\left(\pi_{y z}^{*}\right)^{1}\left(\varphi_{a_{2 u}+\sigma_{S}}\right)^{2}\left(\varphi_{C \alpha}\right)^{1}$ triradical state. In the second step of the reaction, the $\mathrm{C}_{\alpha}$ radical is formally transferred to the $\sigma_{z^{2}}^{*}$ orbital and the $\mathrm{C}_{\alpha}-\mathrm{O}-\mathrm{C}_{\beta}$ ring is formed. This costs $\sim 5 \mathrm{kcal} / \mathrm{mol}$ in the case of Cpd I-A. For Cpd I-B it is a barrierless step, a result of the stabilization of the $\sigma_{z^{2}}^{*}$ orbital by the proximal pocket. For both models the resting state electronic structure of the hemethiolate moiety, $\left(\pi_{x z}^{*}\right)^{1}\left(\pi_{y z}^{*}\right)^{1}\left(\sigma_{z^{2}}^{*}\right)^{1}\left(\varphi_{a_{2 u}+\sigma_{S}}\right)^{2}$ (Scheme 4), was manifested by the spin densities (Table 9) and confirmed by an analysis of KS orbitals. 
Table 9. Natural Group spin densities/Charges and bond lengths ( $\AA$ ) of the optimized structures on the quartet spin PES surfaces.

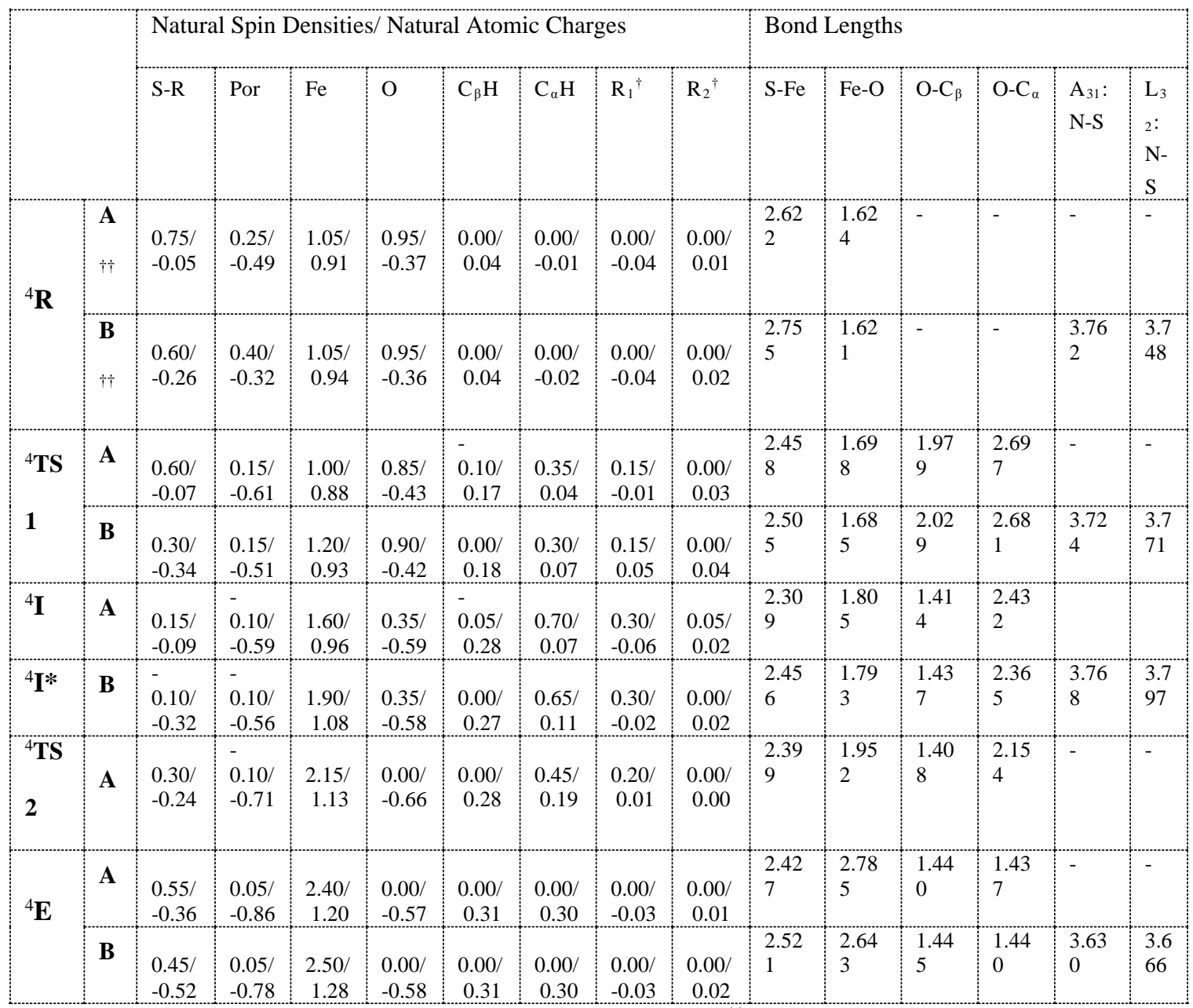

${ }^{\dagger} R_{1}$ - benzylic group of CBMS; $R_{2}$ - methyl group of CBMS. ${ }^{+\dagger} A$ - Cpd I-A, B - Cpd I-B. * Not a true intermediate. 


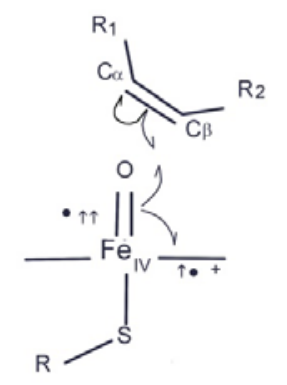

${ }^{4} \mathbf{R}$

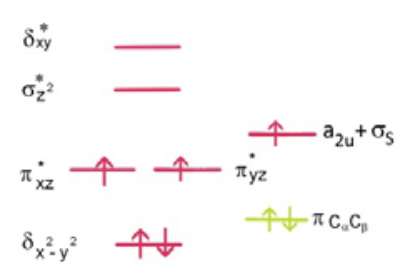

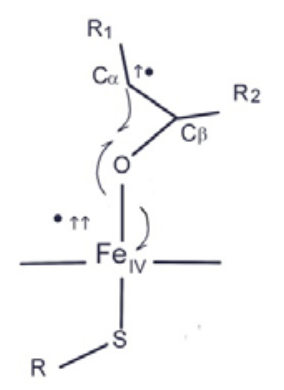

${ }^{4} \mathbf{I}$

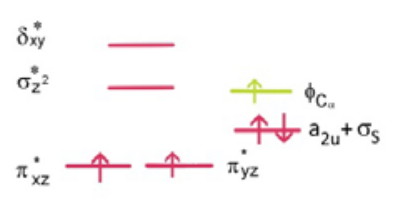

$\delta_{x^{2} \cdot y^{2}} \uparrow \downarrow$
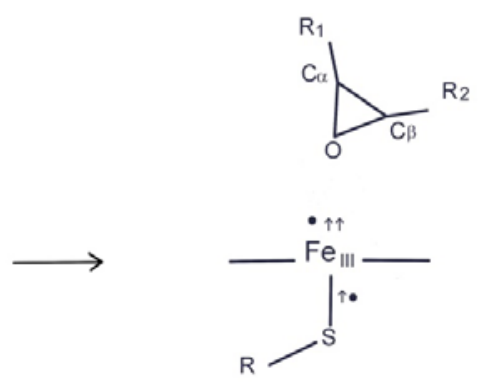

${ }^{4} \mathbf{E}$

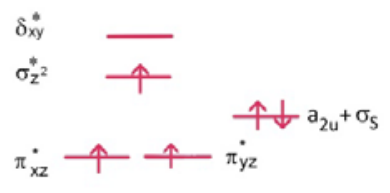

$\delta_{x^{2}-y^{2}} \uparrow \downarrow$

Scheme 4. Cpd I-A/B catalyzed epoxidation on the quartet spin surface

\subsubsection{Separating $\mathrm{NH} \cdots$ S and helix effects}

Having calculated the energetic effect of CPO's proximal pocket (Fig. 17) on the CPOcatalyzed epoxidation of CBMS, it is interesting to separate the effect of the proximal amide bonds from that of the proximal helix. For this purpose model A was modified by adding a blocked alanine dipeptide (model $\mathrm{A}+\mathrm{NH} \cdots \mathrm{S}$ ) to mimic the $\mathrm{A}_{31}: \mathrm{NH}-\mathrm{C}_{29}: \mathrm{S}$ and $\mathrm{L}_{32}: \mathrm{NH}-\mathrm{C}_{29}$ :S hydrogen bonds (Fig. 22). The orientation of the blocked alanine dipeptide was constrained to reproduce the orientations of the proximal $\mathrm{NH} \cdots$ S hydrogen bonds in the $1 \mathrm{CPO} x$-ray structure. ${ }^{3}$ The details of the applied constraints are given in SI Section B2. The reactant and the rate limiting TS1 stationary points were optimized and frequency calculations were performed for the doublet spin state. The calculations showed (Table 10) that $\sim 1 / 3$ of total barrier lowering is from the helix dipole and $\sim 2 / 3$ is 
from the $\mathrm{A}_{31}: \mathrm{NH}-\mathrm{C}_{29}: \mathrm{S}$ and $\mathrm{L}_{32}: \mathrm{NH}-\mathrm{C}_{29}: \mathrm{S}$ hydrogen bonds. It should be noted that, since model B is a partial representation of the proximal helix, this estimation gives a lower bound for the proximal helix effect.

Table 10. ${ }^{2}$ TS1 reaction barriers (B0, kcal $\left./ \mathrm{mol}\right)$.

\begin{tabular}{|l|l|l|l|}
\hline & A & A+NH$\cdots$ S & B \\
\hline${ }^{2}$ TS1 & 14.1 & 12.7 & 11.9 \\
\hline
\end{tabular}

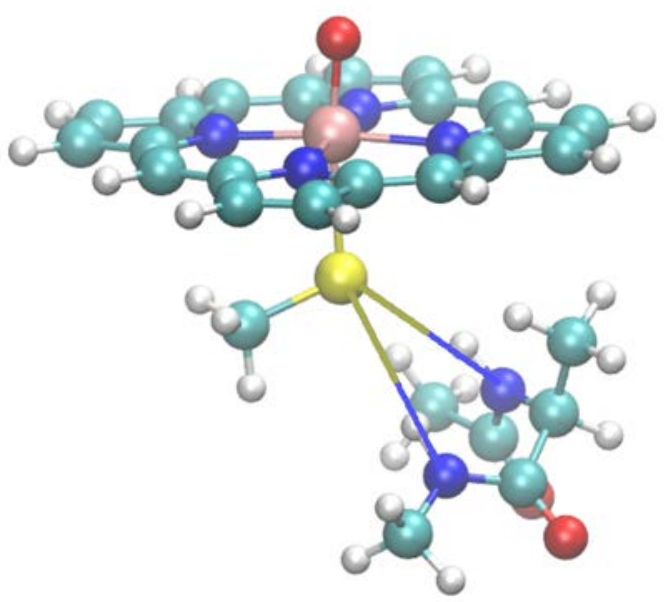

Figure 22: A+NH॰••S model.

\subsubsection{Protein and solvent effect}

The effect of the full enzyme and solvent, in addition to the portion already included in model $\mathrm{B}$, was estimated for the doublet reactant binding complex $\left({ }^{2} \mathrm{R}-\mathrm{CPO}\right)$ and $\mathrm{C}_{\beta}-\mathrm{O}$ transition state $\left({ }^{2} \mathrm{TS} 1-\mathrm{CPO}\right)$ on the basis of $\mathrm{QM} / \mathrm{MM}$ calculations. The QM-optimized ${ }^{2}$ TS1-B structure neatly replaced the corresponding portion of the Q2MM-optimized 
CPO/CBMS transition state structure without unfavorable steric interactions with the distal pocket (see Methods). The ${ }^{2} \mathrm{R}-\mathrm{B}$ structure, however, did not "fit" directly because of unfavorable interactions between the substrate, CBMS, and the hydrophobic core (V67, $\mathrm{L}_{70}, \mathrm{~A}_{71}, \mathrm{~F}_{103}, \mathrm{I}_{179}, \mathrm{~V}_{182}, \mathrm{~F}_{186}, \mathrm{I}_{234}$, and $\left.\mathrm{V}_{237}\right),{ }^{13,42}$ and thus had to be re-optimized with additional constraints to force CBMS to adopt the orientation of the free energy minimum of the solvated CPO/CBMS binding complex at room temperature. ${ }^{13}$ The details of the applied constraints are given in the SI Section B2. The modified ${ }^{2} \mathrm{R}-\mathrm{B}$ structure thus created “fitted” into the full enzyme to constitute ${ }^{2} \mathrm{R}-\mathrm{CPO}$ 's QM region, without steric clashes. This is consistent with our previous results, which found that the steric interactions of CBMS in the distal pocket of CPO favor the transition sate over the binding complex. ${ }^{13,42}$ This is a manifestation of one of the general principles of enzyme catalysis. $^{169}$

Table 11. ${ }^{2}$ TS1-CPO/B reaction barriers (B0) broken into specific contributions (kcal/mol).

\begin{tabular}{|l|l|l|l|l|l|}
\hline & QM/MM & QM & $\begin{array}{l}\text { QM/MM } \\
\text { Electrostatic }\end{array}$ & $\begin{array}{l}\text { QM/MM } \\
\text { VdW }\end{array}$ & MM \\
\hline CPO & 11.5 & 9.7 & 4.0 & -1 & -1.2 \\
\hline B & - & 11.9 & - & - & - \\
\hline
\end{tabular}

Table 11 presents the ${ }^{2} \mathrm{TS} 1-\mathrm{CPO}$ reaction barrier broken into specific contributions. The decrease of the QM part of the ${ }^{2} \mathrm{TS} 1-\mathrm{CPO}$ barrier relative to that of ${ }^{2} \mathrm{TS} 1-\mathrm{B}$ is the result of the above-described reorientation of the substrate by the distal pocket's hydrophobic core in the reactant structure. Overall, the effects of the enzyme/solvent environment (beyond the effects of the proximal pocket already included in model B) nearly cancel each other out, so that the ${ }^{2} \mathrm{TS} 1-\mathrm{CPO}$ barrier is almost the same 
as the barrier of ${ }^{2} \mathrm{TS} 1-\mathrm{B}$ (11.5 vs $11.9 \mathrm{kcal} / \mathrm{mol}$, Table 11$)$. The natural spin densities obtained from the QM/MM calculations on ${ }^{2} \mathrm{TS} 1-\mathrm{CPO}$ (Table 8) showed that the electron transfer mechanism in the full enzyme environment is the same as that for model B. This demonstrates that the proximal region effects quantified for model B remain significant in the full enzyme environment.

We also attempted to carry out QM/MM calculations for the quartet spin surface using the MM part of the full-enzyme structure as for the doublet, as no such structure was available for the quartet state. This resulted in spurious MM contributions to ${ }^{4} \mathrm{TS} 1-$ CPO barrier, which indicates that the apoenzyme structure may be altered in the quartet state. Investigation of this matter is beyond the scope of this paper.

\subsection{Conclusion}

The results of this study demonstrate that the $\mathrm{NH} \bullet \bullet \mathrm{S}$ hydrogen bonds and the proximal helix dipole tune the catalytic reactivity of CPO, which is consistent with the previously reported DFT calculations of P450-catalyzed epoxidations. ${ }^{45-47}$ In the case of the CPOcatalyzed epoxidation of CBMS, the proximal pocket reduces the rate-limiting barrier, which is for an electron transfer from an olefin $\pi$ bond to the heme-thiolate moiety, by $\sim 4.6 \mathrm{kcal} / \mathrm{mol}$ relative to a bare thiolate Cpd I. About $1 / 3$ of the decrease is due to the dipole moment of the proximal helix, while the rest is due to the effect of the $\mathrm{NH} \bullet \bullet \mathrm{S}$ hydrogen bonds. On the doublet spin surface, the electronic effects of CPO's proximal pocket alter the preferred electron transfer mechanism of the epoxidation reaction, which involves the interplay between the relative stabilities of the doubly occupied thiolate- 
porphyrin $\mathrm{a}_{2 \mathrm{u}}+\sigma_{\mathrm{s}}$ and the oxyferryl $\pi^{*}$ orbitals. This shows that the bare thiolate heme model is not sufficient to reproduce the mechanism of CPO-catalyzed epoxidation. Our results suggest that the C-P-A-L and C-L-G-X peptide fragments are the minimal realistic models for theoretical calculations of CPO- and P450-catalyzed epoxidations, respectively. Using these fragments allows a good representation of the experimental geometries, of radical delocalization in Cpd I, and of tuning of the push effect by the proximal pocket, as well as capturing a significant portion of the proximal pocket effect on the reaction barrier for epoxidation.

\section{Overall Conclusion}

The goal of this work was to determine to what extent the proximal amide hydrogen bonds and the proximal helix dipole influence the catalytic activity of chloroperoxidase. The initial stage of investigation was on the formation of the catalytic species, Cpd I, focusing exclusively on the influence of the amide hydrogen bonds. The research was then expanded to study the Cpd I-catalyzed epoxidation of CBMS, and the proximal region of the model system was enlarged to include the influence of the proximal helix dipole. It was determined that the $\mathrm{NH} \cdots$ • S hydrogen bonds reduce the push effect, thereby regulating the mechanism of Cpd I formation and increasing epoxidation activity.

Additionally, the proximal helix dipole augments the influence of the $\mathrm{NH} \cdots$ S hydrogen bonds by significantly contributing to the increase in epoxidation activity (approximately 1/3 contribution). 
For Cpd I formation, the proximal NH•••S hydrogen bonds stabilize the Cpd 0 intermediate relative to all other species and reduce the barrier for Cpd 0’s formation from FeHOOH. Additionally, the protCpd 0 species is destabilized, increasing the favorability of a hybrid homo-heterolytic relative to a classic heterolytic mechanism for O-O bond scission. The modulation of the overall mechanism for Cpd I formation is a result of the proximal $\mathrm{NH} \bullet \bullet S$ hydrogen bonds reducing the push effect, which in turn decreases the pKa of the distally bound heme substrate.

In regards to the catalytic capability of Cpd I, the proximal $\mathrm{NH} \bullet \bullet$ S hydrogen bonds in conjunction with the proximal helix dipole favor a higher electron affinity at the expense of the proton affinity of Cpd I. In the case of CBMS epoxidation, the proximal pocket reduces the rate-limiting barrier for an electron transfer from an olefin $\pi$ bond to the heme-thiolate moiety ( $\mathrm{C}_{\beta}-\mathrm{O}$ bond barrier), and the $\mathrm{C}_{\alpha}-\mathrm{O}-\mathrm{C}_{\beta}$ ring closure becomes barrierless. The process occurs via a change in the electron transfer mechanism, which derives from the proximal pocket stabilizing the thiolate-porphyrin $\mathrm{a}_{2 \mathrm{u}}+\sigma \mathrm{s}$ orbital relative to the oxyferryl $\pi^{*}$ orbitals.

In 1996, Poulos suggested that the hydrogen bond networks on the proximal and distal sides of the heme function together to modulate the mechanism of reaction. ${ }^{12}$ In work published by Harris and Loew in 1998, as well as in work published by Yoshioka in 2002, it was hypothesized that the push effect makes protonation of Cpd 0 more favorable, leading to facile O-O heterolysis. ${ }^{78,80}$ Experimental data by Ueno et al.in 1998 suggested that the proximal helix may amplify the $\mathrm{NH} \bullet \bullet$ S effect and thus be of significant importance for the catalysis in heme-thiolate enzymes. ${ }^{87}$ Our work confirms and extends all of these long-standing theories. 
Having confirmed the significance of the proximal $\mathrm{NH} \cdots$ S hydrogen bonds and the proximal helix dipole on catalytic activity, the next logical step in continuing this work is to perform simulations of CPO mutants for whose catalytic activity has been determined. Of particular interest are mutants R26A and N33A, which have both lost hydrogen bonding interactions responsible for stabilizing the orientation of the proximal helix. Interestingly, both of these mutants have increased epoxidation and peroxidation activity however, they posses no chlorination activity and little to no catalase activity. The increased epoxidation activity is counterintuitive, since the orientation of the proximal helix is important for increasing the redox potential, and should be investigated. 
References

1. Omura, T., Heme-thiolate proteins. Biochem. Biophys. Res. Commun. 2005, 338, 404-409.

2. Poulos, T. L., Heme enzyme structure and function. Chem. Rev. (Washington, DC, U. S.) 2014, 114, 3919-3962.

3. Sundaramoorthy, M.; Terner, J.; Poulos, T. L., The crystal structure of chloroperoxidase: a heme peroxidase-cytochrome P450 functional hybrid. Structure (London) 1995, 3, 1367-77.

4. Fang, G. H.; Kenigsberg, P.; Axley, M. J.; Nuell, M.; Hager, L. P., Cloning and sequencing of chloroperoxidase cDNA. Nucleic Acids Res. 1986, 14, 8061-71.

5. Kenigsberg, P.; Fang, G. H.; Hager, L. P., Post-translational modifications of chloroperoxidase from caldariomyces fumago. Arch. Biochem. Biophys. 1987, 254, 409-15.

6. Collins, D. P. Spectroscopic characterization of the reaction intermediates of the heme-thiolate proteins of cytochrome P450cam and Caldariomyces fumago chloroperoxidase and investingation of the structure and kinetics of hemecontaining proteins using magnetic circular dichroism and rapid-scan, stopped-flow spectroscopy. Copyright (C) 2015 American Chemical Society (ACS). All Rights Reserved., 2012.

7. Lehnert, N., Elucidating second coordination sphere effects in heme proteins using low-temperature magnetic circular dichroism spectroscopy. J. Inorg. Biochem. 2012, 110, 83-93.

8. Galinato, M. G. I.; Spolitak, T.; Ballou, D. P.; Lehnert, N., Elucidating the role of the proximal cysteine hydrogen-bonding network in ferric cytochrome P450cam and corresponding mutants using magnetic circular dichroism spectroscopy. Biochemistry 2011, 50, 1053-1069.

9. Sabat, J.; Stuehr, D. J.; Yeh, S.-R.; Rousseau, D. L., Characterization of the proximal ligand in the P420 form of inducible nitric oxide synthase. J. Am. Chem. Soc. 2009, 131, 12186-12192.

10. Dey, A.; Jiang, Y.; Ortiz de Montellano, P.; Hodgson, K. O.; Hedman, B.; Solomon, E. I., S K-edge XAS and DFT calculations on cytochrome P450: covalent and ionic contributions to the cysteine-fe bond and their contribution to reactivity. J. Am. Chem. Soc. 2009, 131, 7869-7878. 
11. Dey, A.; Okamura, T.; Ueyama, N.; Hedman, B.; Hodgson, K. O.; Solomon, E. I., Sulfur K-Edge XAS and DFT calculations on P450 model complexes: effects of hydrogen bonding on electronic structure and redox potentials. J. Am. Chem. Soc. 2005, 127, 12046-12053.

12. Poulos, T. L., The role of the proximal ligand in heme enzymes. JBIC, J. Biol. Inorg. Chem. 1996, 1, 356-359.

13. Morozov, A. N.; Chatfield, D. C., Chloroperoxidase-catalyzed epoxidation of cis- $\beta$ methylstyrene: distal pocket flexibility tunes catalytic reactivity. J. Phys. Chem. B 2012, 116, 12905-12914.

14. Sundaramoorthy, M.; Terner, J.; Poulos, T. L., Stereochemistry of the chloroperoxidase active site: crystallographic and molecular-modeling studies. Chem. Biol. 1998, 5, 461-473.

15. Yi, X.; Conesa, A.; Punt, P. J.; Hager, L. P., Examining the role of glutamic acid 183 in chloroperoxidase catalysis. J. Biol. Chem. 2003, 278, 13855-13859.

16. Kuehnel, K.; Blankenfeldt, W.; Terner, J.; Schlichting, I., Crystal structures of chloroperoxidase with its bound substrates and complexed with formate, acetate, and nitrate. J. Biol. Chem. 2006, 281, 23990-23998.

17. Manoj, K. M., Chlorinations catalyzed by chloroperoxidase occur via diffusible intermediate(s) and the reaction components play multiple roles in the overall process. Biochim. Biophys. Acta, Proteins Proteomics 2006, 1764, 1325-1339.

18. Lakner, F. J.; Cain, K. P.; Hager, L. P., Enantioselective Epoxidation of $\omega$-Bromo2-methyl-1-alkenes Catalyzed by Chloroperoxidase. Effect of chain length on selectivity and efficiency. J. Am. Chem. Soc. 1997, 119, 443-444.

19. Li, H.-y.; Jiang, Y.-c.; Hu, M.-c.; Li, S.-n.; Zhai, Q.-g., Relationship between Mn2+ in the domain of active center of chloroperoxidase and enzyme catalytic performance. Gaodeng Хиехіао Ниахие Хиеbаo 2013, 34, 875-880.

20. Yi, X.; Mroczko, M.; Manoj, K. M.; Wang, X.; Hager, L. P., Replacement of the proximal heme thiolate ligand in chloroperoxidase with a histidine residue. Proc. Natl. Acad. Sci. U. S. A. 1999, 96, 12412-12417.

21. Shaw, P. D.; Hager, L. P., An enzymic chlorination reaction. J. Am. Chem. Soc. 1959, 81, 1011-12.

22. Hager, L. P.; Morris, D. R.; Brown, F. S.; Eberwein, H., Chloroperoxidase. II. Utilization of halogen anions. J. Biol. Chem. 1966, 241, 1769-77.

23. Morris, D. R.; Hager, L. P., Chloroperoxidase. I. Isolation and properties of the crystalline glycoprotein. J. Biol. Chem. 1966, 241, 1763-8. 
24. Wang, X., Special topics in biochemistry lecture notes, Lecture 8, Slide 13

In 2010.

25. Manoj, K. M.; Hager, L. P., Chloroperoxidase, a janus enzyme. Biochemistry 2008, 47, 2997-3003.

26. Manoj, K. M.; Yi, X.; Rai, G. P.; Hager, L. P., A Kinetic epoxidation assay for chloroperoxidase. Biochem. Biophys. Res. Commun. 1999, 266, 301-303.

27. Manoj, K. M.; Hager, L. P., Utilization of peroxide and its relevance in oxygen insertion reactions catalyzed by chloroperoxidase. Biochim. Biophys. Acta, Protein Struct. Mol. Enzymol. 2001, 1547, 408-417.

28. Geigert, J.; Dalietos, D. J.; Neidleman, S. L.; Lee, T. D.; Wadsworth, J., Peroxide oxidation of primary alcohols to aldehydes by chloroperoxidase catalysis. Biochem Biophys Res Commun 1983, 114, 1104-8.

29. Van Deurzen, M. P. J.; Van Rantwijk, F.; Sheldon, R. A., Chloroperoxidasecatalyzed oxidation of 5-(hydroxymethyl)furfural. J. Carbohydr. Chem. 1997, 16, 299-309.

30. Kedderis, G. L.; Koop, D. R.; Hollenberg, P. F., N-demethylation reactions catalyzed by chloroperoxidase. J. Biol. Chem. 1980, 255, 10174-82.

31. Toy, P. H.; Newcomb, M.; Hager, L. P., Hypersensitive radical probe studies of chloroperoxidase-catalyzed hydroxylation reactions. Chem Res Toxicol 1998, 11, 816-23.

32. Miller, V. P.; Tschirret-Guth, R. A.; Ortiz, d. M. P. R., Chloroperoxidase-catalyzed benzylic hydroxylation. Arch Biochem Biophys 1995, 319, 333-40.

33. Manoj, K. M.; Lakner, F. J.; Hager, L. P., Epoxidation of indene by chloroperoxidase. J. Mol. Catal. B: Enzym. 2000, 9, 107-111.

34. Hu, S.; Hager, L. P., Asymmetric epoxidation of functionalized cis-olefins catalyzed by chloroperoxidase. Tetrahedron Lett. 1999, 40, 1641-1644.

35. Lakner, F. J.; Hager, L. P., Chloroperoxidase-mediated asymmetric epoxidation. Synthesis of (R)-dimethyl 2-methylaziridine-1,2-dicarboxylate - a potential methylamino acid synthon. Tetrahedron: Asymmetry 1997, 8, 3547-3550.

36. Lakner, F. J.; Hager, L. P., Chloroperoxidase as enantioselective epoxidation catalyst: an efficient synthesis of (R)-(-)-mevalonolactone. J. Org. Chem. 1996, 61, 3923-3925. 
37. Hager, L. P.; Allain, E. J. Stereoselective epoxidation of alkenes by chloroperoxidase. US5358860A, 1994.

38. Colonna, S.; Gaggero, N.; Casella, L.; Carrea, G.; Pasta, P., Enantioselective epoxidation of styrene derivatives by chloroperoxidase catalysis. Tetrahedron: Asymmetry 1993, 4, 1325-30.

39. Allain, E. J.; Hager, L. P.; Deng, L.; Jacobsen, E. N., Highly enantioselective epoxidation of disubstituted alkenes with hydrogen peroxide catalyzed by chloroperoxidase. J. Am. Chem. Soc. 1993, 115, 4415-16.

40. Fu, H.; Kondo, H.; Ichikawa, Y.; Look, G. C.; Wong, C. H., Chloroperoxidasecatalyzed asymmetric synthesis: enantioselective reactions of chiral hydroperoxides with sulfides and bromohydroxylation of glycals. J. Org. Chem. 1992, 57, 7265-70.

41. Colonna, S.; Gaggero, N.; Manfredi, A.; Casella, L.; Gullotti, M., Asymmetric oxidation of sulfides, catalyzed by chloroperoxidase. J. Chem. Soc., Chem. Commun. 1988, 1451-2.

42. Morozov, A. N.; D'Cunha, C.; Alvarez, C. A.; Chatfield, D. C., Enantiospecificity of chloroperoxidase-catalyzed epoxidation: biased molecular dynamics study of a cis- $\beta$-methylstyrene/chloroperoxidase-compound I complex. Biophys. J. 2011, 100, 1066-1075.

43. Osborne, R. L.; Coggins, M. K.; Terner, J.; Dawson, J. H., Caldariomyces fumago chloroperoxidase catalyzes the oxidative dehalogenation of chlorophenols by a mechanism involving two one-electron steps. J. Am. Chem. Soc. 2007, 129, 1483814839 .

44. Gross, Z.; Nimri, S.; Barzilay, C. M.; Simkhovich, L., Reaction profile of the last step in cytochrome P-450 catalysis revealed by studies of model complexes. JBIC, J. Biol. Inorg. Chem. 1997, 2, 492-506.

45. de Visser, S. P.; Ogliaro, F.; Harris, N.; Shaik, S., Multi-state epoxidation of ethene by cytochrome P450: a quantum chemical study. J. Am. Chem. Soc. 2001, 123, 3037-3047.

46. de Visser, S. P.; Ogliaro, F.; Sharma, P. K.; Shaik, S., What factors affect the regioselectivity of oxidation by cytochrome P450? A DFT study of allylic hydroxylation and double bond epoxidation in a model reaction. J. Am. Chem. Soc. 2002, 124, 11809-11826.

47. Kumar, D.; de Visser, S. P.; Shaik, S., multistate reactivity in styrene epoxidation by compound I to cytochrome P450: mechanisms of products and side products formation. Chem. - Eur. J. 2005, 11, 2825-2835. 
48. Kumar, D.; Karamzadeh, B.; Sastry, G. N.; de Visser, S. P., What factors influence the rate constant of substrate epoxidation by compound I of cytochrome P450 and analogous iron(IV)-oxo oxidants? J. Am. Chem. Soc. 2010, 132, 7656-7667.

49. Champion, P. M.; Chiang, R.; Munck, E.; Debrunner, P.; Hager, L. P., Mossbauer investigations of high-spin ferrous heme proteins. II. Chloroperoxidase, horseradish peroxidase, and hemoglobin. Biochemistry 1975, 14, 4159-66.

50. Remba, R. D.; Champion, P. M.; Fitchen, D. B.; Chiang, R.; Hager, L. P., Resonance Raman investigations of chloroperoxidase, horseradish peroxidase, and cytochrome c using Soret band laser excitation. Biochemistry 1979, 18,2280-90.

51. Hollenberg, P. F.; Hager, L. P.; Blumberg, W. E.; Peisach, J., An electron paramagnetic resonance study of the high and low spin forms of chloroperoxidase. J. Biol. Chem. 1980, 255, 4801-7.

52. Lambeir, A. M.; Dunford, H. B., A kinetic and spectral study of the alkaline transitions of chloroperoxidase. Arch. Biochem. Biophys. 1983, 220, 549-56.

53. Chen, H.; Hirao, H.; Derat, E.; Schlichting, I.; Shaik, S., Quantum mechanical/molecular mechanical study on the mechanisms of compound I formation in the catalytic cycle of chloroperoxidase: an overview on heme enzymes. J. Phys. Chem. B 2008, 112, 9490-9500.

54. Poulos, T. L.; Kraut, J., The stereochemistry of peroxidase catalysis. J. Biol. Chem. 1980, 255, 8199-205.

55. Fajer, J.; Borg, D. C.; Forman, A.; Dolphin, D.; Felton, R. H., pi-cation radicals and dications of metalloporphyrins. J Am Chem Soc 1970, 92, 3451-9.

56. Dolphin, D.; Forman, A.; Borg, D. C.; Fajer, J.; Felton, R. H., Compounds I of catalase and horse radish peroxidase: $\pi$-cation radicals. Proc. Nat. Acad. Sci. U. S. 1971, 68, 614-18.

57. Coulson, A. F.; Yonetani, T., Oxidation of cytochrome c peroxidase with hydrogen peroxide: identification of the "endogenous donor". Biochem Biophys Res Commun 1972, 49, 391-8.

58. Hashimoto, S.; Tatsuno, Y.; Kitagawa, T., Resonance Raman evidence for the presence of the FeIV=O bond in horseradish peroxidase compound II. Proc. Jpn. Acad., Ser. B 1984, 60, 345-8.

59. Sitter, A. J.; Reczek, C. M.; Terner, J., Observation of the FeIV=O stretching vibration of ferryl myoglobin by resonance Raman spectroscopy. Biochim. Biophys. Acta, Protein Struct. Mol. Enzymol. 1985, 828, 229-35. 
60. Reczek, C. M.; Sitter, A. J.; Terner, J., Resonance Raman characterization of heme iron(IV)=oxygen groups of intermediates of yeast cytochrome c peroxidase and lactoperoxidase. J. Mol. Struct. 1989, 214, 27-41.

61. Chance, B.; Powers, L.; Ching, Y.; Poulos, T.; Schonbaum, G. R.; Yamazaki, I.; Paul, K. G., X-ray absorption studies of intermediates in peroxidase activity. Arch. Biochem. Biophys. 1984, 235, 596-611.

62. Penner-Hahn, J. E.; Smith Eble, K.; McMurry, T. J.; Renner, M.; Balch, A. L.; Groves, J. T.; Dawson, J. H.; Hodgson, K. O., Structural characterization of horseradish peroxidase using EXAFS spectroscopy. Evidence for $\mathrm{Fe}=\mathrm{O}$ ligation in compounds I and II. J. Am. Chem. Soc. 1986, 108, 7819-25.

63. Chance, M.; Powers, L.; Poulos, T.; Chance, B., Cytochrome c peroxidase compound ES is identical with horseradish peroxidase compound I in iron-ligand distances. Biochemistry 1986, 25, 1266-70.

64. Fulop, V.; Phizackerley, R. P.; Soltis, S. M.; Clifton, I. J.; Wakatsuki, S.; Erman, J.; Hajdu, J.; Edwards, S. L., Laue diffraction study on the structure of cytochrome c peroxidase compound I. Structure 1994, 2, 201-8.

65. Hersleth, H. P.; Dalhus, B.; Gorbitz, C. H.; Andersson, K. K., An iron hydroxide moiety in the $1.35 \AA$ resolution structure of hydrogen peroxide derived myoglobin compound II at pH 5.2. JBIC, J. Biol. Inorg. Chem. 2002, 7, 299-304.

66. Murshudov, G. N.; Grebenko, A. I.; Brannigan, J. A.; Antson, A. A.; Barynin, V. V.; Dodson, G. G.; Dauter, Z.; Wilson, K. S.; Melik-Adamyan, W. R., The structures of micrococcus lysodeikticus catalase, its ferryl intermediate (compound II) and NADPH complex. Acta Crystallogr., Sect. D: Biol. Crystallogr. 2002, D58, 1972-1982.

67. Bonagura, C. A.; Bhaskar, B.; Shimizu, H.; Li, H.; Sundaramoorthy, M.; McRee, D. E.; Goodin, D. B.; Poulos, T. L., High-resolution crystal structures and spectroscopy of native and compound I cytochrome c peroxidase. Biochemistry 2003, 42, 5600-5608.

68. Hersleth, H.-P.; Hsiao, Y.-W.; Ryde, U.; Gorbitz, C. H.; Andersson, K. K., The influence of $\mathrm{x}$-rays on the structural studies of peroxide-derived myoglobin intermediates. Chem. Biodiversity 2008, 5, 2067-2089.

69. Lai, W.; Chen, H.; Shaik, S., What kinds of ferryl species exist for compound II of chloroperoxidase? A dialog of theory with experiment. J. Phys. Chem. B 2009, 113, 7912-7917. 
70. Stone, K. L.; Hoffart, L. M.; Behan, R. K.; Krebs, C.; Green, M. T., Evidence for two ferryl species in chloroperoxidase compound II. J. Am. Chem. Soc. 2006, 128, 6147-6153.

71. Dawson, J. H.; Holm, R. H.; Trudell, J. R.; Barth, G.; Linder, R. E.; Bunnenberg, E.; Djerassi, C.; Tang, S. C., Magnetic circular dichroism studies. 43. Oxidized cytochrome P-450. Magnetic circular dichroism evidence for thiolate ligation in the substrate-bound form. Implications for the catalytic mechanism. J. Am. Chem. Soc. 1976, 98, 3707-9.

72. Sono, M.; Andersson, L. A.; Dawson, J. H., Sulfur donor ligand binding to ferric cytochrome P-450-CAM and myoglobin. Ultraviolet-visible absorption magnetic circular dichroism, and electron paramagnetic resonance spectroscopic investigation of the complexes. J. Biol. Chem. 1982, 257, 8308-20.

73. Ogliaro, F.; de Visser, S. P.; Shaik, S., The 'push' effect of the thiolate ligand in cytochrome P450: a theoretical gauging. J. Inorg. Biochem. 2002, 91, 554-567.

74. Dawson, J. H.; Sono, M., Cytochrome P-450 and chloroperoxidase: thiolate-ligated heme enzymes. Spectroscopic determination of their active-site structures and mechanistic implications of thiolate ligation. Chem. Rev. 1987, 87, 1255-76.

75. Sono, M.; Roach, M. P.; Coulter, E. D.; Dawson, J. H., Heme-containing oxygenases. Chem. Rev. (Washington, D. C.) 1996, 96, 2841-2887.

76. Groves, J. T., Enzymatic C-H bond activation Using push to get pull. Nat. Chem. 2014, 6, 89-91.

77. Green, M. T.; Dawson, J. H.; Gray, H. B., Oxoiron(IV) in Chloroperoxidase compound II is basic: implications for P450 chemistry. Science (Washington, DC, U. S.) 2004, 304, 1653-1656.

78. Harris, D. L.; Loew, G. H., Theoretical investigation of the proton assisted pathway to formation of cytochrome P450 compound I. J. Am. Chem. Soc. 1998, 120, (35), 8941-8948.

79. Rydberg, P.; Sigfridsson, E.; Ryde, U., On the role of the axial ligand in heme proteins: a theoretical study. JBIC, J. Biol. Inorg. Chem. 2004, 9, 203-223.

80. Yoshioka, S.; Tosha, T.; Takahashi, S.; Ishimori, K.; Hori, H.; Morishima, I., Roles of the proximal hydrogen bonding network in cytochrome P450cam-catalyzed oxygenation. J. Am. Chem. Soc. 2002, 124, 14571-14579.

81. Collman, J. P.; Hegedus, L. S.; Norton, J. R.; Finke, R. G., Organometallic chemistry of transition metals: Principles and Use, Pt. 2. Mir: 1989; p 397. 
82. Collman, J. P.; Hegedus, L., Principles and applications of organotransition metal chemistry. University Science Books: 1980; p 715.

83. Poulos, T. L.; Finzel, B. C.; Howard, A. J., High-resolution crystal structure of cytochrome P450cam. J. Mol. Biol. 1987, 195, 687-700.

84. Wang, R.; De Visser, S. P., How does the push/pull effect of the axial ligand influence the catalytic properties of Compound I of catalase and cytochrome P450? J. Inorg. Biochem. 2007, 101, 1464-1472.

85. Matsumura, H.; Wakatabi, M.; Omi, S.; Ohtaki, A.; Nakamura, N.; Yohda, M.; Ohno, H., Modulation of redox potential and alteration in reactivity via the peroxide shunt pathway by mutation of cytochrome P450 around the proximal heme ligand. Biochemistry 2008, 47, 4834-4842.

86. Yoshioka, S.; Takahashi, S.; Ishimori, K.; Morishima, I., Roles of the axial push effect in cytochrome P450cam studied with the site-directed mutagenesis at the heme proximal site. J. Inorg. Biochem. 2000, 81, 141-151.

87. Ueno, T.; Kousumi, Y.; Yoshizawa-Kumagaye, K.; Nakajima, K.; Ueyama, N.; Okamura, T.-a.; Nakamura, A., Role of $\alpha$-helix conformation cooperating with $\mathrm{NH} \cdots \mathrm{S}$ hydrogen bond in the active site of cytochrome P-450 and chloroperoxidase: synthesis and properties of [MIII(OEP)(Cys-Helical Peptide)] (M = Fe and Ga). J. Am. Chem. Soc. 1998, 120, 12264-12273.

88. Dawson, J. H., Probing structure-function relations in heme-containing oxygenases and peroxidases. Science (Washington, D. C., 1883-) 1988, 240, 433-9.

89. Choudhury, K.; Sundaramoorthy, M.; Hickman, A.; Yonetani, T.; Woehl, E.; Dunn, M. F.; Poulos, T. L., Role of the proximal ligand in peroxidase catalysis. Crystallographic, kinetic, and spectral studies of cytochrome c peroxidase proximal ligand mutants. J Biol Chem 1994, 269, 20239-49.

90. Erman, J. E.; Vitello, L. B.; Miller, M. A.; Shaw, A.; Brown, K. A.; Kraut, J., Histidine 52 is a critical residue for rapid formation of cytochrome c peroxidase compound I. Biochemistry 1993, 32, 9798-806.

91. Ortiz de Montellano, P. R.; Editor, Cytochrome P450: structure, mechanism, and biochemistry, Second Edition. Plenum: 1995; p 630.

92. Aikens, J.; Sligar, S. G., Kinetic solvent isotope effects during oxygen activation by cytochrome P-450cam. J. Am. Chem. Soc. 1994, 116, 1143-4.

93. Poulos, T. L.; Finzel, B. C.; Gunsalus, I. C.; Wagner, G. C.; Kraut, J., The 2.6- $\AA$ crystal structure of pseudomonas putida cytochrome P-450 J. Biol. Chem. 1985, 260, 16122-30. 
94. Nagano, S.; Poulos, T. L., Crystallographic study on the dioxygen complex of wildtype and mutant cytochrome P 450cam: implications for the dioxygen activation mechanism. J. Biol. Chem. 2005, 280, 31659-31663.

95. Kumar, D.; Hirao, H.; De Visser, S. P.; Zheng, J.; Wang, D.; Thiel, W.; Shaik, S., New features in the catalytic cycle of cytochrome P450 during the formation of compound I from compound 0. J. Phys. Chem. B 2005, 109, 19946-19951.

96. Cramer, C. J., Essentials of computational chemistry. 1st ed.; John Wiley \& Sons Ltd.: Baffins Lane, 2002; p 542.

97. Hartree, D. R., The wave mechanics of an atom with a non-coulomb central field. I. Theory and methods. Proc. Cambridge Philos. Soc. 1928, 24, 89-110.

98. Slater, J. V., HC, The theory of complex spectra. Phys. Rev. A: Gen. Phys. 1929, 34, 1293-1295.

99. Fock, V., "Self-consistent field" with interchange for sodium. Z. Phys. 1930, 62, 795-805.

100. Roothan, C. C. J., New developements in molecular orbital theory. Rev. Mod. Phys. 1951, 23.

101. Boys, S. F., Electronic Wave Functions. I. A general method of calculation for the stationary states of any molecular system. Proc. Roy. Soc. 1950, 200, 542-554.

102. Kohn, W. S., L. J., Self-consistent equations including exchange and correlation effects Phys. Rev. 1965, 140, A1133-A1138.

103. Hohenberg, P. K., W., Inhomogeneous electron gas. Phys. Rev. 1964, 136, B864B871.

104. Becke, A. D., Density-functional exchange-energy approximation with correct asymptotic behavior. Phys. Rev. A: Gen. Phys. 1988, 38, 3098-100.

105. Lee, C.; Yang, W.; Parr, R. G., Development of the Colle-Salvetti correlationenergy formula into a functional of the electron density. Phys. Rev. B: Condens. Matter 1988, 37, 785-9.

106. Grossman, J. C., Benchmark quantum Monte Carlo calculations. J. Chem. Phys. 2002, 117, 1434-1440.

107. Becke, A. D., Density-functional thermochemistry. III. The role of exact exchange. J. Chem. Phys. 1993, 98, 5648-52.

108. Becke, A. D., A new mixing of Hartree-Fock and local-density-functional theories. J. Chem. Phys. 1993, 98, 1372-7. 
109. Boresh, S. K., Jeffery; Miller, Benjamin T.; Singh, Rishi P.; Venable, Richard M.; Weidlich, Iwona; Woodcock, H. Lee; Wu, Xiongwu Charmmtutorial. http://www.charmmtutorial.org/index.php/The_Energy_Function\#The_force_field_ terms $(08 / 11 / 2015)$

110. MacKerell, A. D., Jr.; Bashford, D.; Bellott, M.; Dunbrack, R. L.; Evanseck, J. D.; Field, M. J.; Fischer, S.; Gao, J.; Guo, H.; Ha, S.; et al., All-atom empirical potential for molecular modeling and dynamics studies of proteins. J. Phys. Chem. B 1998, 102, 3586-3616.

111. MacKerell, A. D., Jr., Empirical force fields for biological macromolecules: overview and issues. J. Comput. Chem. 2004, 25, 1584-1604.

112. Buck, M.; Bouguet-Bonnet, S.; Pastor, R. W.; MacKerell, A. D., Jr., Importance of the CMAP correction to the CHARMM22 protein force field: dynamics of hen lysozyme. Biophys. J. 2006, 90, L36-L38.

113. Senn, H. M.; Thiel, W., QM/MM methods for biological systems. Top. Curr. Chem. 2007, 268, (Atomistic Approaches in Modern Biology), 173-290.

114. Valiev, M., E. J. Bylaska, N. Govind, K. Kowalski, T. P. Straatsma, H. J. J. Van Dam, D. Wang, J. Nieplocha, E. Apra, T. L. Windus, and W. A. de Jong, NWChem: A comprehensive and scalable open-source solution for large scale molecular simulations. Computer Physics Communications 2010, 181, 1477-1489.

115. Frisch, M. J.; Trucks, G. W.; Schlegel, H. B.; Scuseria, G. E.; Robb, M. A.; Cheeseman, J. R.; Scalmani, G.; Barone, V.; Mennucci, B.; Petersson, G. A., et al., Gaussian09 Revision D.01, Gaussian, Inc., Wallingford CT, 2009.

116. Schaftenaar, G. N., J.H., Molden: a pre- and post-processing program for molecular and electronic structures. J. Comput.Aided Mol. Design 2000, 14, 123-134.

117. Humphrey, W. D., A.; Schulten, K. , VMD - visual molecular dynamics. J. Molec. Graphics 1996, 14.1, 33-38.

118. Mulliken, R. S., Electronic population analysis on LCAO-MO molecular-wave functions. IV. Bonding and antibonding in LCAO and valence-bond theories. $J$. Chem. Phys. 1955, 23, 2343-6.

119. Mulliken, R. S., Electronic population analysis on LCAO-MO molecular-wave functions. III. Effects of hybridization on overlap and gross AO populations. $J$. Chem. Phys. 1955, 23, 2338-42.

120. Mulliken, R. S., Electronic population analysis on LCAO-MO [linear combination of atomic orbital-molecular orbital] molecular wave functions. II. Overlap populations, bond orders, and covalent bond energies. J. Chem. Phys. 1955, 23, 1841-6. 
121. Mulliken, R. S., Electronic population analysis on LCAO-MO [linear combination of atomic orbital-molecular orbital] molecular wave functions. I. J. Chem. Phys.

1955, 23, 1833-40.

122. Kubelka, J. Population Analysis. http://www.uwyo.edu/kubelkachem/population_analysis.pdf (September 7, 2015).

123. Reed, A. E.; Weinstock, R. B.; Weinhold, F., Natural population analysis. J. Chem. Phys. 1985, 83, 735-46.

124. Dennington, R. K., Todd; Millam, John GaussView, 5; Semichem Inc.: Shawnee Mission, KS, 2009.

125. Brooks, B. R.; Brooks, C. L., III; Mackerell, A. D., Jr.; Nilsson, L.; Petrella, R. J.; Roux, B.; Won, Y.; Archontis, G.; Bartels, C.; Boresch, S.; Caflisch, A.; et al., CHARMM: The biomolecular simulation program. J. Comput. Chem. 2009, 30, 1545-1614.

126. Libby, R. D.; Beachy, T. M.; Phipps, A. K., Quantitating direct chlorine transfer from enzyme to substrate in chloroperoxidase-catalyzed reactions. J. Biol. Chem. 1996, 271, 21820-21827.

127. Rutter, R.; Hager, L. P., The detection of two electron paramagnetic resonance radical signals associated with chloroperoxidase compound I. J. Biol. Chem. 1982, 257, 7958-61.

128. Kim, S. H.; Perera, R.; Hager, L. P.; Dawson, J. H.; Hoffman, B. M., Rapid freezequench endor study of chloroperoxidase compound I: the site of the radical. J. Am. Chem. Soc. 2006, 128, 5598-5599.

129. Schlichting, I.; Berendzen, J.; Chu, K.; Stock, A. M.; Maves, S. A.; Benson, D. E.; Sweet, R. M.; Ringe, D.; Petsko, G. A.; Sligar, S. G., The catalytic pathway of cytochrome P450cam at atomic resolution. Science (New York, N.Y.) 2000, 287, 1615-22.

130. Kuehnel, K.; Derat, E.; Terner, J.; Shaik, S.; Schlichting, I., Structure and quantum chemical characterization of chloroperoxidase compound 0 , a common reaction intermediate of diverse heme enzymes. Proc. Natl. Acad. Sci. U. S. A. 2007, 104, 99-104.

131. Ogliaro, F.; Cohen, S.; de Visser, S. P.; Shaik, S., Medium polarization and hydrogen bonding effects on compound I of cytochrome P450: what kind of a radical is it really? J. Am. Chem. Soc. 2000, 122, 12892-12893.

132. Ogliaro, F.; De Visser, S. P.; Cohen, S.; Kaneti, J.; Shaik, S., The experimentally elusive oxidant of cytochrome P450: a theoretical "trapping" defining more closely the "real" species. ChemBioChem 2001, 2, 848-851. 
133. de Visser, S. P.; Tan, L. S., Is the bound substrate in nitric oxide synthase protonated or neutral and what is the active oxidant that performs substrate hydroxylation? Journal of the American Chemical Society 2008, 130, 12961-12974.

134. Ueyama, N.; Nishikawa, N.; Yamada, Y.; Okamura, T.-a.; Nakamura, A., Cytochrome P-450 Model (Porphinato)(thiolato)iron(III) Complexes with single and double NH $\cdots$ S hydrogen bonds at the thiolate Site. J. Am. Chem. Soc. 1996, $118,12826-12827$.

135. Ueyama, N.; Nishikawa, N.; Yamada, Y.; Okamura, T.-A.; Oka, S.; Sakurai, H.; Nakamura, A., Synthesis and properties of octaethylporphinato(arenethiolato)FeIII complexes with intramolecular $\mathrm{NH} \cdots \mathrm{S}$ Hydrogen Bond: chemical function of the hydrogen bond. Inorg. Chem. 1998, 37, 2415-2421.

136. Suzuki, N.; Higuchi, T.; Urano, Y.; Kikuchi, K.; Uekusa, H.; Ohashi, Y.; Uchida, T.; Kitagawa, T.; Nagano, T., Novel iron porphyrin-alkanethiolate complex with intramolecular $\mathrm{NH} \cdots \mathrm{S}$ hydrogen bond: synthesis, spectroscopy, and reactivity. $J$. Am. Chem. Soc. 1999, 121, 11571-11572.

137. Ueno, T.; Nishikawa, N.; Moriyama, S.; Adachi, S.; Lee, K.; Okamura, T.-a.; Ueyama, N.; Nakamura, A., Role of the invariant peptide fragment forming $\mathrm{NH} \cdots \mathrm{S}$ hydrogen bonds in the active site of cytochrome P-450 and chloroperoxidase: synthesis and properties of cys-containing peptide $\mathrm{Fe}(\mathrm{III})$ and $\mathrm{Ga}(\mathrm{III})$ (Octaethylporphinato) complexes as models. Inorg. Chem. 1999, 38, 1199-1210.

138. Lai, W.; Chen, H.; Cho, K.-B.; Shaik, S., Effects of substrate, protein environment, and proximal ligand mutation on compound I and compound 0 of chloroperoxidase. J. Phys. Chem. A 2009, 113, 11763-11771.

139. Cho, K.-B.; Hirao, H.; Chen, H.; Carvajal, M. A.; Cohen, S.; Derat, E.; Thiel, W.; Shaik, S., Compound I in heme thiolate enzymes: a comparative QM/MM study. $J$. Phys. Chem. A 2008, 112, 13128-13138.

140. Derat, E.; Shaik, S., The Poulos-Kraut mechanism of compound I formation in horseradish peroxidase: a QM/MM Study. J. Phys. Chem. B 2006, 110, 1052610533.

141. Zheng, J.; Wang, D.; Thiel, W.; Shaik, S., QM/MM study of mechanisms for compound I formation in the catalytic cycle of cytochrome P450cam. J. Am. Chem. Soc. 2006, 128, 13204-13215.

142. Filizola, M.; Loew, G. H., Probing the role of protein environment in compound I formation of chloroperoxidase (CPO). J. Am. Chem. Soc. 2000, 122, 3599-3605. 
143. Friesner, R. A.; Murphy, R. B.; Beachy, M. D.; Ringnalda, M. N.; Pollard, W. T.; Dunietz, B. D.; Cao, Y., Correlated ab initio electronic structure calculations for large molecules. Journal of Physical Chemistry A 1999, 103, 1913-1928.

144. Hay, P. J.; Wadt, W. R., Ab initio effective core potentials for molecular calculations. Potentials for potassium to gold including the outermost core orbitals. J. Chem. Phys. 1985, 82, 299-310.

145. Wachters, A. J. H., Gaussian basis set for molecular wavefunctions containing third-row atoms. Journal of Chemical Physics 1970, 52, 1033-6.

146. Fukui, K., Formulation of the reaction coordinate. J. Phys. Chem. 1970, 74, 4161-3.

147. Jones, P.; Dunford, H. B., The mechanism of compound I formation revisited. J. Inorg. Biochem. 2005, 99, 2292-2298.

148. Schoeneboom, J. C.; Lin, H.; Reuter, N.; Thiel, W.; Cohen, S.; Ogliaro, F.; Shaik, S., The elusive oxidant species of cytochrome P450 enzymes: characterization by combined quantum mechanical/molecular mechanical (QM/MM) calculations. J Am Chem Soc 2002, 124, 8142-51.

149. Harris, D. L., High-valent intermediates of heme proteins and model compounds. Curr. Opin. Chem. Biol. 2001, 5, 724-735.

150. Ogliaro, F.; Cohen, S.; Filatov, M.; Harris, N.; Shaik, S., The high-valent compound of cytochrome P450: The nature of the Fe-S bond and the role of the thiolate ligand as an internal electron donor. Angew. Chem., Int. Ed. 2000, 39, 3851-3855.

151. Shaik, S.; Cohen, S.; Wang, Y.; Chen, H.; Kumar, D.; Thiel, W., P450 enzymes: their structure, reactivity, and selectivity-modeled by QM/MM calculations. Chem Rev 2010, 110, 949-1017.

152. Miertus, S.; Scrocco, E.; Tomasi, J., Electrostatic interaction of a solute with a continuum. A direct utilization of ab initio molecular potentials for the prevision of solvent effects. Chemical Physics 1981, 55, 117-29.

153. Miertus, S.; Tomasi, J., Approximate evaluations of the electrostatic free energy and internal energy changes in solution processes. Chemical Physics 1982, 65, 23945.

154. Derat, E.; Shaik, S.; Rovira, C.; Vidossich, P.; Alfonso-Prieto, M., The Effect of a Water Molecule on the Mechanism of Formation of Compound 0 in horseradish peroxidase. J. Am. Chem. Soc. 2007, 129, 6346-6347.

155. Daiber, A.; Nauser, T.; Takaya, N.; Kudo, T.; Weber, P.; Hultschig, C.; Shoun, H.; Ullrich, V., Isotope effects and intermediates in the reduction of NO by P450NOR. Journal of inorganic biochemistry 2002, 88, 343-352. 
156. Lehnert, N.; Praneeth, V. K. K.; Paulat, F., Electronic structure of iron(II)porphyrin nitroxyl complexes: molecular mechanism of fungal nitric oxide reductase (P450nor). Journal of Computational Chemistry 2006, 27, 1338-1351.

157. McQuarters, A. B.; Wirgau, N. E.; Lehnert, N., Model complexes of key intermediates in fungal cytochrome P450 nitric oxide reductase (P450nor). Current opinion in chemical biology 2014, 19, 82-89.

158. Hariharan, P. C.; Pople, J. A., Influence of polarization functions on MO hydrogenation energies. Theor. Chim. Acta 1973, 28, 213-22.

159. Francl, M. M.; Pietro, W. J.; Hehre, W. J.; Binkley, J. S.; Gordon, M. S.; DeFrees, D. J.; Pople, J. A., Self-consistent molecular orbital methods. XXIII. A polarizationtype basis set for second-row elements. J. Chem. Phys. 1982, 77, 3654-65.

160. Hay, P. J.; Wadt, W. R., Ab initio effective core potentials for molecular calculations. Potentials for the transition metal atoms scandium to mercury. $J$. Chem. Phys. 1985, 82, 270-83.

161. Krishnan, R.; Binkley, J. S.; Seeger, R.; Pople, J. A., Self-consistent molecular orbital methods. XX. A basis set for correlated wave functions. J. Chem. Phys. 1980, 72, 650-4.

162. McLean, A. D.; Chandler, G. S., Contracted gaussian basis sets for molecular calculations. I. Second row atoms, Z = 11-18. J. Chem. Phys. 1980, 72, 5639-48.

163. Foster, J. P.; Weinhold, F., Natural hybrid orbitals. J. Am. Chem. Soc. 1980, 102, 7211-18.

164. Reed, A. E.; Weinhold, F., Natural bond orbital analysis of near-Hartree-Fock water dimer. J. Chem. Phys. 1983, 78, 4066-73.

165. Glendening, E. D.; Landis, C. R.; Weinhold, F., NBO 6.0: Natural bond orbital analysis program. J. Comput. Chem. 2013, 34, 1429-1437.

166. Norrby, P. O., Selectivity in asymmetric synthesis from QM-guided molecular mechanics. J. Mol. Struct.: THEOCHEM 2000, 506, 9-16.

167. Jorgensen, W. L.; Chandrasekhar, J.; Madura, J. D.; Impey, R. W.; Klein, M. L., Comparison of simple potential functions for simulating liquid water. J. Chem. Phys. 1983, 79, 926-35.

168. Rutter, R.; Hager, L. P.; Dhonau, H.; Hendrich, M.; Valentine, M.; Debrunner, P., Chloroperoxidase compound I: electron paramagnetic resonance and Moessbauer studies. Biochemistry 1984, 23, 6809-16. 
169. Garcia-Viloca, M.; Gao, J.; Karplus, M.; Truhlar, D. G., How enzymes work: Analysis by modern rate theory and computer simulations. Science (Washington, DC, U. S.) 2004, 303, 186-195. 


\section{Appendices}

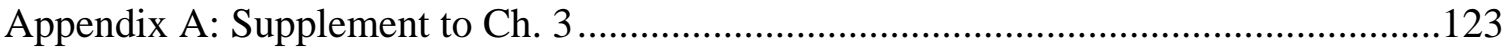

Figure A1: Superposition of all optimized structures ............................................123

Table A1: Energies of stationary points on doublet surface (in hartrees) ...................124

Table A2: Energies of stationary points on doublet surface (in $\mathrm{kcal} / \mathrm{mol}$ ).................125

Table A3: Energies of stationary points on quartet surface ...................................126

Table A4: Zero Point Energy Corrections (doublet) ...............................................126

Table A5: Zero Point Energy Corrections (quartet) ...............................................126

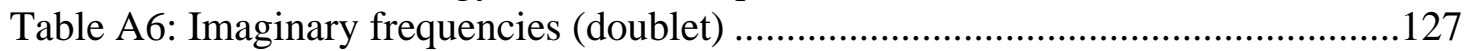

Table A7: Mulliken Charges, B1 level..............................................................127

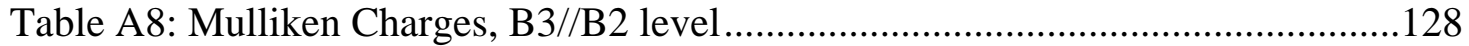

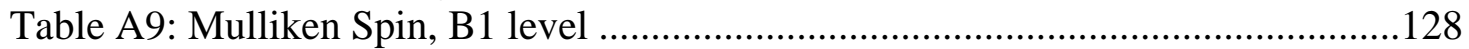

Table A10: Mulliken Spin, B3//B2 level...........................................................129

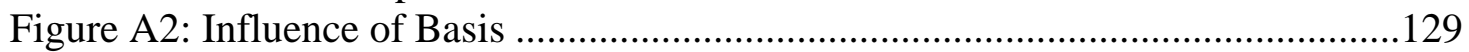

Table A11: Influence of $\mathrm{NH} \cdots \mathrm{S}$ hydrogen bond distance ...................................130

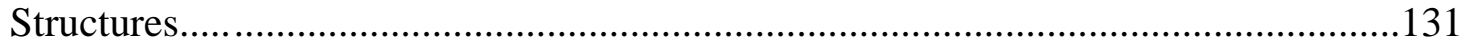

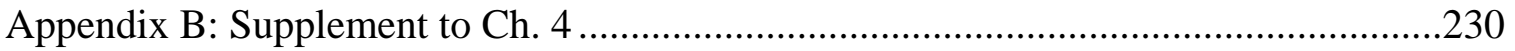

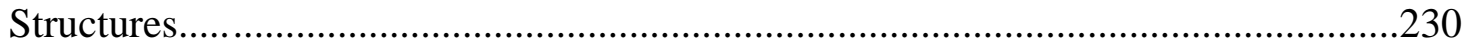

Appendix C: Computational and Submission Scripts...............................................279

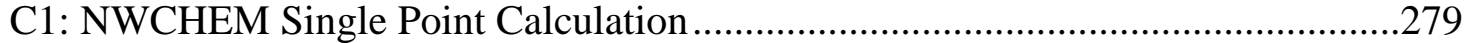

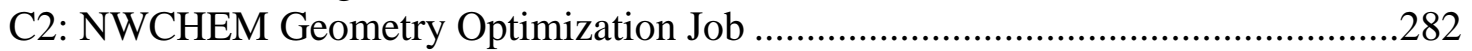

C3: NWCHEM Saddle Point Optimization............................................................285

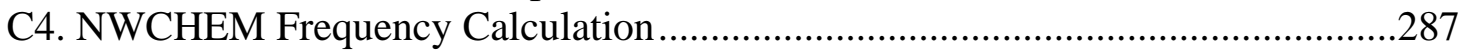

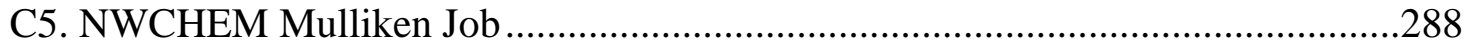

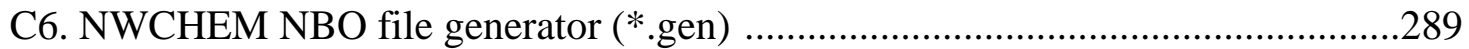

C7. Gaussian Single Point Calculation Job .............................................................290

C8. Continuation Gaussian Single Point Calculation Job .......................................293

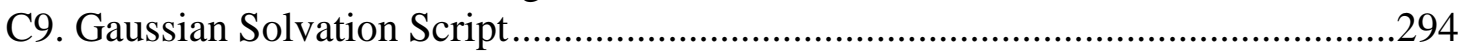

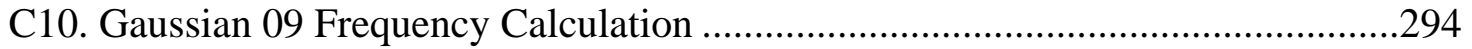

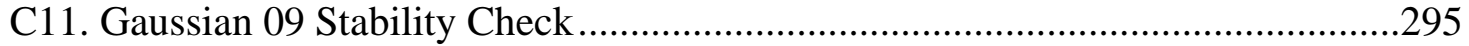

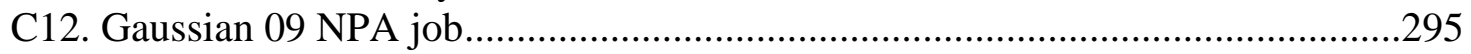

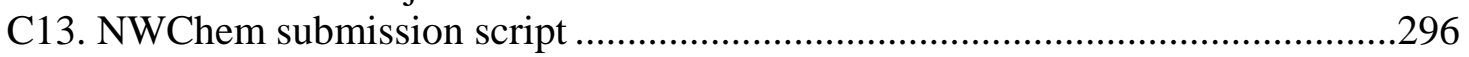

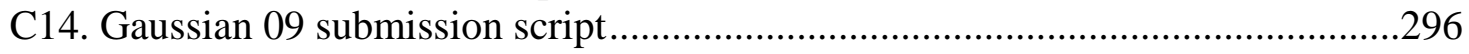

C15. CHARMM Topology File (selected segments of file) ....................................296

C16. CHARMM Parameter File (selected segments of file) ...................................298

C17. CHARMM Coordinate File (selected segments of file) ..................................300

C18. CHARMM Protein Structure File (selected segments of file) ..........................301

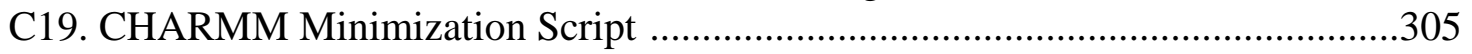

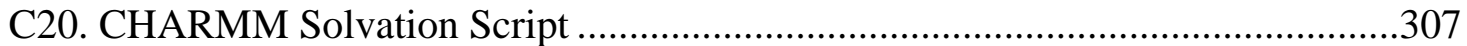

C21. CHARMM Dynamics - Heating Script.......................................................309

C22. CHARMM Dynamics - Equilibration Script .............................................311 


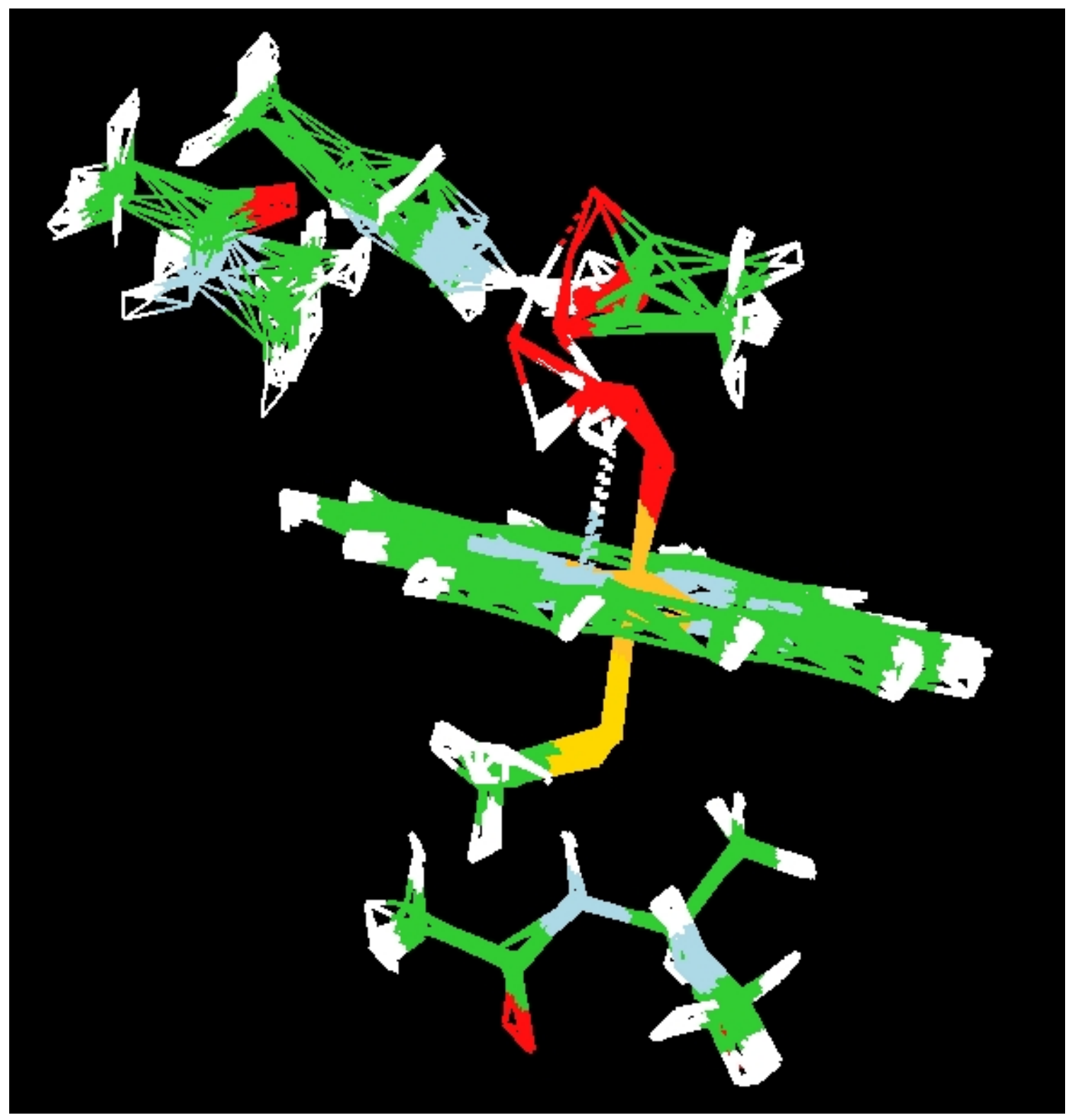

Figure A1. Superposition of all optimized structures obtained for both model A and model B at B3LYP/6-31G* plus LANL2DZ(Fe) level (16 species). This figure shows that proper constraints were utilized in order to maintain residues in the correct orientation relative to the heme while still allowing for side-chain flexibility. 
Table A1 : Energies of stationary points on the doublet surface (in hartrees)

\begin{tabular}{|c|c|c|c|c|c|c|c|c|}
\hline Hartrees & $\mathrm{Fe}+\mathrm{HOOH}$ & $\mathrm{FeHOOH}$ & TS1 & Cpdo & TS2 & protCpdo & TS3 & Cpdl \\
\hline \multicolumn{9}{|l|}{ NWCHEM Energetics } \\
\hline \multicolumn{9}{|l|}{ Basis Set B1 } \\
\hline Model A & -2444.651536 & -2444.680169 & -2444.651428 & -2444.657546 & -2444.654276 & -2444.658753 & -2444.651252 & -2444.696337 \\
\hline Model B @ 2.9 A & $\mathrm{N} / \mathrm{A}$ & N/A & $\mathrm{N} / \mathrm{A}$ & -2940.490924 & -2940.483682 & -2940.484859 & -2940.476963 & -2940.519592 \\
\hline Model B @ 3.0 A & N/A & $\mathrm{N} / \mathrm{A}$ & N/A & \begin{tabular}{|l|}
-2940.498779 \\
\end{tabular} & -2940.491644 & -2940.492103 & -2940.484749 & -2940.524813 \\
\hline Model B @ 3.1A & N/A & N/A & $\mathrm{N} / \mathrm{A}$ & \begin{tabular}{|l|}
-2940.50425 \\
\end{tabular} & -2940.497269 & -2940.499154 & -2940.490389 & -2940.529196 \\
\hline Model B @ 3.2A & N/A & N/A & N/A & \begin{tabular}{|l|}
-2940.507914 \\
\end{tabular} & -2940.501066 & -2940.502563 & -2940.494266 & -2940.532895 \\
\hline Model B @ 3.3 A & -2940.497986 & -2940.52305 & -2940.503655 & -2940.510185 & -2940.50347 & -2940.505042 & -2940.496814 & -2940.53547 \\
\hline Model B @ 3.4 A & -2940.499542 & -2940.524661 & -2940.505766 & -2940.511433 & -2940.504887 & -2940.506529 & -2940.498382 & -2940.537145 \\
\hline Model B @ 3.5 A & -2940.500319 & -2940.525575 & -2940.505696 & -2940.511922 & -2940.505552 & -2940.50725 & -2940.499199 & -2940.53812 \\
\hline Model B @ 3.6 A & N/A & N/A & N/A & \begin{tabular}{|l|}
-2940.511873 \\
\end{tabular} & -2940.505688 & -2940.50751 & -2940.49949 & -2940.538601 \\
\hline \multicolumn{9}{|l|}{ Basis Set B2 } \\
\hline Model A & -2444.737257 & -2444.762332 & N/A & -2444.734254 & -2444.729944 & -2444.73332 & -2444.7254 & -2444.778565 \\
\hline Model B & -2940.608338 & -2940.62702 & N/A & \begin{tabular}{|l|}
-2940.608117 \\
\end{tabular} & -2940.600017 & -2940.602256 & -2940.593618 & -2940.641142 \\
\hline Gaussian Energetics & $\mathrm{Fe}+\mathrm{HOOH}$ & $\mathrm{FeHOOH}$ & TS1 & Cpd0rearr & TS3 & protCpd0 & TS4 & Cpdl \\
\hline \multicolumn{9}{|l|}{ Basis Set B1 } \\
\hline Model A & -2444.653659 & -2444.679969 & -2444.651245 & -2444.657407 & -2444.654134 & -2444.658626 & -2444.651117 & -2444.695935 \\
\hline Model B & -2940.501656 & -2940.524479 & -2940.505574 & -2940.511215 & -2940.504675 & -2940.50635 & -2940.498176 & -2940.53669 \\
\hline \multicolumn{9}{|l|}{ Basis Set B2 } \\
\hline Model A & -2444.738858 & -2444.763214 & $\mathrm{~N} / \mathrm{A}$ & \begin{tabular}{|l|}
-2444.734757 \\
\end{tabular} & -2444.730448 & -2444.733828 & -2444.725886 & -2444.77906 \\
\hline Model B & -2940.610037 & -2940.627986 & $\mathrm{~N} / \mathrm{A}$ & -2940.609186 & -2940.60063 & -2940.603151 & -2940.594601 & -2940.641742 \\
\hline \multicolumn{9}{|l|}{ Basis Set B2//B3 } \\
\hline Model A & -3585.080854 & -3585.10383 & -3585.071156 & -3585.077285 & -3585.074842 & -3585.076549 & -3585.071437 & -3585.123784 \\
\hline Model B & -4080.972402 & -4080.988841 & -4080.968372 & -4080.970933 & -4080.964625 & -4080.965864 & -4080.959056 & -4081.006368 \\
\hline
\end{tabular}

The distance given in the left column for some Model B energies represents the H-S distances for the two $\mathrm{NH} \cdots \mathrm{S}$ hydrogen bonds, as defined in the paper. Energies are without zero-point energy. 
Table A2: Energies of Stationary points of the doublet surface (in $\mathrm{kcal} / \mathrm{mol}$ )

\begin{tabular}{|c|c|c|c|c|c|c|c|c|}
\hline Hartrees & $\mathrm{Fe}+\mathrm{HOOH}$ & FенООН & TS1* & Cpdo & TS2 & protCpdo & TS3 & Cpdl \\
\hline \multicolumn{9}{|l|}{ NWCHEM Energetics } \\
\hline \multicolumn{9}{|c|}{ Basis Set B1 (Full Mechanism) } \\
\hline Model A & 18.0 & 0.0 & 18.0 & 14.2 & 16.2 & 13.4 & 18.1 & -10.1 \\
\hline Model B@3.3Â & 15.7 & 0.0 & 12.2 & 8.1 & 12.3 & 11.3 & 16.5 & -7.8 \\
\hline Model B@3.4 A & 15.8 & 0.0 & 11.9 & 8.3 & 12.4 & 11.4 & 16.5 & -7.8 \\
\hline Model B@3.5A & 15.8 & 0.0 & 12.5 & 8.6 & 12.6 & 11.5 & 16.6 & -7.9 \\
\hline \multicolumn{9}{|c|}{ Basis Set B1 (Cpd 0 to Cpd I) } \\
\hline Model A & - & - & - & 0.00 & 2.05 & -0.76 & 3.95 & -24.34 \\
\hline Model B@2.9 A & - & - & - & 0.00 & 4.54 & 3.81 & 8.76 & -17.99 \\
\hline Model B@3.0A & - & - & - & 0.00 & 4.48 & 4.19 & 8.80 & -16.34 \\
\hline Model B@3.1 § & - & - & - & 0.00 & 4.38 & 3.20 & 8.70 & -15.65 \\
\hline Model B @ 3.2 A & - & - & - & 0.00 & 4.30 & 3.36 & 8.56 & -15.68 \\
\hline Model B@3.3 A & - & - & - & 0.00 & 4.21 & 3.23 & 8.39 & -15.87 \\
\hline Model B@3.4 A & - & - & - & 0.00 & 4.11 & 3.08 & 8.19 & -16.13 \\
\hline Model B@3.5 A & - & - & - & 0.00 & 4.00 & 2.93 & 7.98 & -16.44 \\
\hline Model B@3.6A & - & - & - & 0.00 & 3.88 & 2.74 & 7.77 & -16.77 \\
\hline \multicolumn{9}{|l|}{ Basis Set B2 } \\
\hline Model A & 15.7 & 0.0 & N/A & 17.6 & 20.3 & 18.2 & 23.2 & -10.2 \\
\hline Model B & 11.7 & 0.0 & N/A & 11.9 & 16.9 & 15.5 & 21.0 & -8.9 \\
\hline Gaussian Energetics & $\mathrm{Fe}+\mathrm{HOOH}$ & $\mathrm{FeHOOH}$ & TS1 & Cpd0 & TS2 & protCpdo & TS3 & Cpdl \\
\hline \multicolumn{9}{|l|}{ Basis Set B1 } \\
\hline Model A & 16.5 & 0.0 & 18.0 & 14.2 & 16.2 & 13.4 & 18.1 & -10.0 \\
\hline Model B & 14.3 & 0.0 & 11.9 & 8.3 & 12.4 & 11.4 & 16.5 & -7.7 \\
\hline \multicolumn{9}{|l|}{ Basis Set B2 } \\
\hline Model A & 15.3 & 0.0 & N/A & 17.9 & 20.6 & 18.4 & 23.4 & -9.9 \\
\hline Model B & 11.3 & 0.0 & N/A & 11.8 & 17.2 & 15.6 & 20.9 & -8.6 \\
\hline \multicolumn{9}{|l|}{ Basis Set B2/B3 } \\
\hline Model A & 14.4 & 0.0 & 20.5 & 16.7 & 18.2 & 17.1 & 20.3 & -12.5 \\
\hline Model B & 10.3 & 0.0 & 12.8 & 11.2 & 15.2 & 14.4 & 18.7 & -11.0 \\
\hline
\end{tabular}

The distance given in the left column for some Model B energies represents the H-S distances for the two $\mathrm{NH}^{\cdots} \mathrm{S}$ hydrogen bonds, as defined in the paper. Energies are without zero-point energy. 
Table A3: Energies of Stationary points on the quartet surface

\begin{tabular}{|c|c|c|c|c|c|c|c|c|}
\hline Hartrees & $\mathrm{Fe}+\mathrm{HOOH}$ & $\mathrm{FeHOOH}$ & $\mathrm{TS}^{*}$ & Cpdo & TS2 & protCpdo & TS3 & Cpdl \\
\hline \multicolumn{9}{|c|}{ NWCHEM Energetics } \\
\hline \multicolumn{9}{|c|}{ Basis Set B2 } \\
\hline Model A & -2444.733672 & -2444.74992 & -2444.70791 & -2444.712902 & -2444.710875 & -2444.715511 & -2444.706868 & -2444.778297 \\
\hline Model B & -2940.612755 & -2940.613045 & -2940.584174 & -2940.593372 & -2940.587675 & -2940.590428 & -2940.577814 & -2940.642165 \\
\hline \multicolumn{9}{|c|}{ Gaussian Energetics } \\
\hline \multicolumn{9}{|c|}{ Basis Set B2//B3 } \\
\hline Model A & -3585.079743 & -3585.09278 & -3585.049525 & -3585.055433 & -3585.055263 & -3585.058902 & -3585.050556 & -3585.123458 \\
\hline Model B & -4080.979021 & -4080.983842 & -4080.94697 & -4080.956702 & -4080.953758 & -4080.954751 & -4080.945059 & -4081.007253 \\
\hline $\mathrm{kcal} / \mathrm{mol}$ & $\mathrm{Fe}+\mathrm{HOOH}$ & $\mathrm{FeHOOH}$ & $\mathrm{TS}^{*}$ & Cpdo & TS2 & protCpd0 & TS3 & Cpdl \\
\hline \multicolumn{9}{|c|}{ NWCHEM Energetics } \\
\hline \multicolumn{9}{|c|}{ Basis Set B2 } \\
\hline Model A & 10.2 & 0.0 & 26.4 & 23.2 & 24.5 & 21.6 & 27.0 & -17.8 \\
\hline Model B & 0.2 & 0.0 & 18.1 & 12.3 & 15.9 & 14.2 & 22.1 & -18.3 \\
\hline \multicolumn{9}{|c|}{ Gaussian Energetics } \\
\hline \multicolumn{9}{|l|}{ Basis Set B2//B3 } \\
\hline Model A & 8.2 & 0.0 & 27.1 & 23.4 & 23.5 & 21.3 & 26.5 & -19.3 \\
\hline Model B & 3.0 & 0.0 & 23.1 & 17.0 & 18.9 & 18.3 & 24.3 & -14.7 \\
\hline
\end{tabular}

Table A4: Zero Point Energy corrections (doublet)

\begin{tabular}{|c|c|c|}
\hline \multirow{2}{*}{ FeHOOH } & \multicolumn{2}{|c|}{ ZPE correction $(\mathrm{kcal} / \mathrm{mol})$} \\
\cline { 2 - 3 } TS1 prox & Model A & Model B \\
Cpd0 & 384.9 & 503.4 \\
TS2 & 383.5 & 501.9 \\
protCpd0 & 379.1 & 501.7 \\
TS3 & 382.2 & 497.5 \\
Cpdl & 383.3 & 501.5 \\
\hline
\end{tabular}

Table A5: Zero Point Energy corrections (quartet)

\begin{tabular}{|c|c|c|}
\hline \multirow{2}{*}{ FeHOOH } & \multicolumn{2}{|c|}{ ZPE correction (kcal/mol) } \\
\cline { 2 - 3 } TS1 & Model A & Model B \\
Cpd0 & 384.8 & 502.4 \\
TS2 & 383.22 & 501.9 \\
protCpd0 & 380.6 & 501.9 \\
TS3 & 383.6 & 598.5 \\
Cpdl & 382.8 & 501.9 \\
\end{tabular}


Table A6: Imaginary frequencies obtained from saddle point optimizations (doublet).

\begin{tabular}{|c|cc|}
\hline & model & frequency $\left(\mathrm{cm}^{-1}\right)$ \\
\hline$\underline{\text { TS1 }}$ & Model A & -248.55 \\
& Model B & -200.69 \\
$\underline{\text { TS2 }}$ & & \\
& Model A & -817.23 \\
& Model B & -667.51 \\
& & \\
& Model A & -435.83 \\
& Model B & -442.68 \\
\hline
\end{tabular}

Optimizations and frequencies were performed at the B3LYP/6-31G* plus LANL2DZ(Fe).

Table A7: Mulliken Charges, B1 level

Mulliken Charges for B1 Basis Set

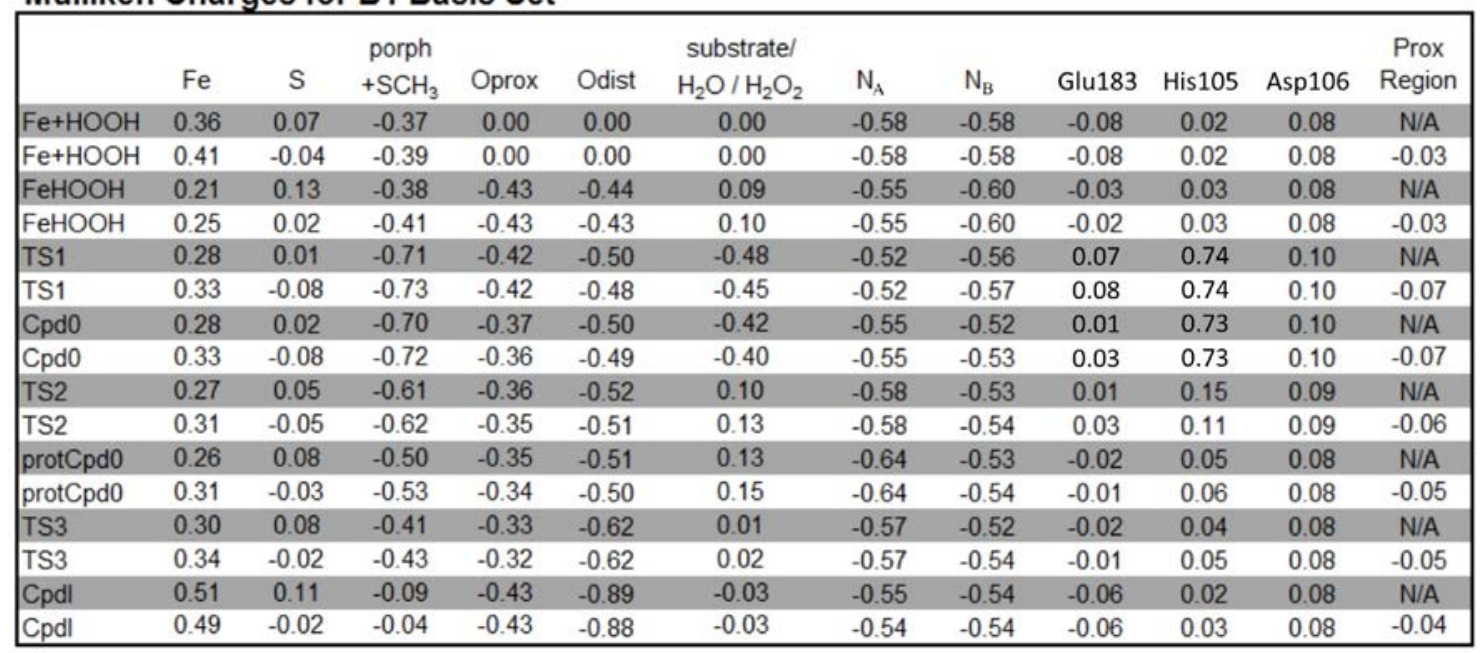

Species highlighted in grey are for model A; unhighlighted are model B. Prox region refers to Pro30, Ala31, and Leu32. * TS1 is optimized at B1 level. 
Table A8: Mulliken Charges, B3//B2 Level

Mulliken Charges for B3 // B2 Basis Set

\begin{tabular}{|c|c|c|c|c|c|c|c|c|c|c|c|c|c|c|}
\hline & $\mathrm{Fe}$ & $\mathrm{S}$ & $\begin{array}{c}\text { porph } \\
+\mathrm{SCH}_{3}\end{array}$ & Oprox & Odist & $\mathrm{H} 77$ & $\mathrm{H} 78$ & $\begin{array}{c}\text { substrate/ } \\
\mathrm{H}_{2} \mathrm{O} / \mathrm{H}_{2} \mathrm{O}_{2}\end{array}$ & $\mathrm{~N}_{A}$ & $\mathrm{~N}_{\mathrm{B}}$ & Glu183 & His 105 & Asp106 & $\begin{array}{c}\text { Proximal } \\
\text { Region }\end{array}$ \\
\hline $\mathrm{Fe}+\mathrm{HOOH}$ & 0.70 & -0.01 & -0.70 & 0.00 & 0.00 & 0.00 & 0.00 & 0.00 & 0.09 & -0.08 & -0.02 & -0.03 & 0.05 & N/A \\
\hline $\mathrm{Fe}+\mathrm{HOOH}$ & 1.10 & -0.85 & -1.24 & 0.00 & 0.00 & 0.00 & 0.00 & 0.00 & 0.10 & -0.07 & 0.00 & -0.04 & 0.05 & 0.13 \\
\hline $\mathrm{FeHOOH}$ & 0.42 & 0.35 & -0.88 & -0.24 & -0.53 & 0.61 & 0.57 & 0.42 & -0.20 & -0.54 & -0.67 & 0.01 & 0.05 & N/A \\
\hline $\mathrm{FeHOOH}$ & 0.87 & -0.40 & -1.64 & 0.05 & -0.42 & 0.45 & 0.43 & 0.51 & 0.24 & -0.58 & 0.15 & -0.02 & 0.04 & 0.09 \\
\hline TS1* & 0.84 & -0.05 & -1.60 & -0.16 & -0.43 & 0.43 & 0.48 & -0.16 & -0.04 & -0.18 & -0.03 & 0.89 & 0.06 & N/A \\
\hline TS1* & 1.29 & -0.84 & -2.38 & 0.00 & -0.46 & 0.44 & 0.48 & -0.02 & 0.07 & -0.35 & 0.05 & 0.89 & 0.07 & 0.12 \\
\hline Cpd0 & 0.74 & -0.05 & -1.44 & -0.25 & -0.34 & 0.39 & 0.50 & -0.20 & 0.10 & -0.17 & -0.16 & 0.24 & 0.07 & N/A \\
\hline Cpd0 & 0.88 & -0.70 & -1.80 & -0.14 & -0.34 & 0.40 & 0.50 & -0.07 & 0.29 & -0.22 & 0.07 & 0.86 & 0.07 & -0.01 \\
\hline TS2 & 0.79 & -0.01 & -1.35 & -0.21 & -0.39 & 0.40 & 0.56 & 0.36 & 0.00 & -0.19 & 0.04 & 0.09 & 0.06 & N/A \\
\hline TS2 & 1.13 & -0.78 & -1.79 & -0.12 & -0.42 & 0.40 & 0.57 & 0.42 & -0.03 & -0.22 & 0.12 & 0.04 & 0.06 & 0.02 \\
\hline protCpd0 & 0.96 & -0.01 & -1.39 & -0.15 & -0.42 & 0.39 & 0.52 & 0.34 & -0.23 & -0.20 & -0.03 & -0.02 & 0.04 & N/A \\
\hline protCpd0 & 1.26 & -0.79 & -1.84 & -0.10 & -0.42 & 0.37 & 0.54 & 0.40 & -0.14 & -0.24 & 0.12 & -0.02 & 0.05 & 0.03 \\
\hline TS3 & 0.40 & 0.05 & -0.90 & -0.04 & -0.45 & 0.41 & 0.48 & 0.41 & 0.20 & -0.16 & -0.02 & -0.03 & 0.04 & N/A \\
\hline TS3 & 0.65 & -0.72 & -1.40 & 0.09 & -0.42 & 0.43 & 0.50 & 0.60 & 0.30 & -0.19 & 0.13 & -0.03 & 0.05 & 0.00 \\
\hline CpdI & 0.48 & 0.16 & -0.36 & -0.21 & -0.74 & 0.38 & 0.40 & 0.05 & 0.50 & 0.00 & -0.32 & -0.02 & 0.05 & N/A \\
\hline CpdI & 0.87 & -0.37 & -0.83 & -0.17 & -0.73 & 0.38 & 0.41 & 0.06 & 0.55 & 0.01 & 0.03 & -0.02 & 0.05 & 0.01 \\
\hline
\end{tabular}

Species highlighted in grey are for model A; unhighlighted are model B. Prox region refers to Pro30, Ala31, and Leu32.

\section{Table A9: Mulliken Spin, B1 level}

Mulliken Spins for B1 Basis Set

\begin{tabular}{|c|c|c|c|c|c|c|c|c|c|c|c|c|}
\hline & $\mathrm{Fe}$ & $\mathrm{S}$ & $\begin{array}{c}\text { porph } \\
+\mathrm{SCH}_{3}\end{array}$ & Oprox & Odist & $\begin{array}{l}\text { substrate/ } \\
\mathrm{H}_{2} \mathrm{O} / \mathrm{H}_{2} \mathrm{O}_{2}\end{array}$ & $\mathrm{~N}_{\mathrm{A}}$ & $N_{B}$ & Glu183 & His105 & Asp106 & $\begin{array}{c}\text { Prox } \\
\text { Region }\end{array}$ \\
\hline $\mathrm{Fe}+\mathrm{HOOH}$ & 1.22 & -0.08 & -0.22 & 0.00 & 0.00 & 0.00 & -0.03 & -0.04 & 0.00 & 0.00 & 0.00 & N/A \\
\hline $\mathrm{Fe}+\mathrm{HOOH}$ & 1.27 & -0.11 & -0.26 & 0.00 & 0.00 & 0.00 & -0.04 & -0.04 & 0.00 & 0.00 & 0.00 & 0.00 \\
\hline $\mathrm{FeHOOH}$ & 1.03 & 0.05 & -0.03 & 0.00 & 0.00 & 0.00 & -0.02 & -0.02 & 0.00 & 0.00 & 0.00 & N/A \\
\hline $\mathrm{FeHOOH}$ & 1.07 & 0.00 & -0.07 & 0.00 & 0.00 & 0.00 & -0.02 & -0.02 & 0.00 & 0.00 & 0.00 & 0.00 \\
\hline TS1 & 0.92 & 0.08 & 0.00 & 0.08 & 0.01 & 0.08 & -0.01 & -0.01 & 0.00 & 0.00 & 0.00 & N/A \\
\hline TS1 & 0.95 & 0.02 & -0.04 & 0.09 & 0.01 & 0.09 & -0.01 & -0.02 & 0.00 & 0.00 & 0.00 & 0.00 \\
\hline Cpd0 & 0.93 & 0.08 & -0.01 & 0.08 & 0.00 & 0.08 & -0.01 & -0.01 & 0.00 & 0.00 & 0.00 & N/A \\
\hline Cpd0 & 0.96 & 0.02 & -0.06 & 0.09 & 0.00 & 0.09 & -0.01 & -0.02 & 0.00 & 0.00 & 0.00 & 0.00 \\
\hline TS2 & 0.96 & 0.10 & 0.01 & 0.03 & 0.00 & 0.03 & -0.01 & -0.01 & 0.00 & 0.00 & 0.00 & N/A \\
\hline TS2 & 1.01 & 0.02 & -0.06 & 0.05 & 0.00 & 0.05 & -0.02 & -0.02 & 0.00 & 0.00 & 0.00 & 0.00 \\
\hline protCpd0 & 0.96 & 0.10 & 0.01 & 0.03 & 0.00 & 0.03 & -0.01 & -0.01 & 0.00 & 0.00 & 0.00 & N/A \\
\hline protCpd0 & 1.02 & 0.02 & -0.05 & 0.04 & 0.00 & 0.03 & -0.01 & -0.02 & 0.00 & 0.00 & 0.00 & 0.00 \\
\hline TS3 & 1.06 & 0.08 & -0.02 & 0.03 & -0.08 & -0.04 & -0.02 & -0.02 & 0.00 & 0.00 & 0.00 & N/A \\
\hline TS3 & 1.14 & 0.00 & -0.10 & 0.05 & -0.10 & -0.04 & -0.02 & -0.02 & 0.00 & 0.00 & 0.00 & 0.00 \\
\hline Cpdl & 1.26 & -0.75 & -1.10 & 0.83 & 0.01 & 0.01 & -0.12 & -0.04 & 0.00 & 0.00 & 0.00 & N/A \\
\hline Cpdl & 1.26 & -0.53 & -1.09 & 0.83 & 0.01 & 0.01 & -0.16 & -0.08 & 0.00 & 0.00 & 0.00 & -0.02 \\
\hline
\end{tabular}

Species highlighted in grey are for model A; unhighlighted are Model B. Prox Region refers to Pro30, Ala31, and Leu32 
Table A10: Mulliken Spin, B3//B2 level

\begin{tabular}{|c|c|c|c|c|c|c|c|c|c|c|c|c|c|c|}
\hline & $\mathrm{Fe}$ & $\mathrm{S}$ & $\begin{array}{c}\text { porph } \\
+\mathrm{SCH}_{3}\end{array}$ & Oprox & Odist & $\mathrm{H} 77$ & $\mathrm{H} 78$ & $\begin{array}{c}\text { substrate/ } \\
\mathrm{H}_{2} \mathrm{O} / \mathrm{H}_{2} \mathrm{O}_{2}\end{array}$ & $\mathrm{~N}_{\mathrm{A}}$ & $\mathrm{N}_{\mathrm{B}}$ & Glu183 & His 105 & Asp106 & $\begin{array}{c}\text { Proximal } \\
\text { Region }\end{array}$ \\
\hline $\mathrm{Fe}+\mathrm{HOOH}$ & 1.09 & 0.02 & -0.09 & 0.00 & 0.00 & 0.00 & 0.00 & 0.00 & -0.03 & -0.03 & 0.00 & 0.00 & 0.00 & $\mathrm{~N} / \mathrm{A}$ \\
\hline $\mathrm{Fe}+\mathrm{HOOH}$ & 1.13 & 0.02 & -0.12 & 0.00 & 0.00 & 0.00 & 0.00 & 0.00 & -0.03 & -0.04 & 0.00 & 0.00 & 0.00 & -0.01 \\
\hline $\mathrm{FeHOOH}$ & 0.99 & 0.10 & 0.01 & 0.00 & 0.00 & 0.00 & 0.00 & 0.00 & -0.01 & -0.02 & 0.00 & 0.00 & 0.00 & $\mathrm{~N} / \mathrm{A}$ \\
\hline $\mathrm{FeHOOH}$ & 1.02 & 0.09 & -0.01 & 0.00 & 0.00 & 0.00 & 0.00 & 0.00 & -0.01 & -0.01 & 0.00 & 0.00 & 0.00 & -0.01 \\
\hline TS1* & 0.90 & 0.09 & 0.02 & 0.07 & 0.00 & 0.00 & 0.00 & 0.07 & 0.00 & -0.01 & 0.00 & 0.00 & 0.00 & N/A \\
\hline TS1* & 0.92 & 0.07 & 0.00 & 0.08 & 0.01 & 0.00 & 0.00 & 0.09 & 0.00 & 0.01 & 0.00 & 0.00 & 0.00 & 0.00 \\
\hline Cpd0 & 0.90 & 0.10 & 0.03 & 0.06 & 0.00 & 0.00 & 0.00 & 0.06 & -0.01 & -0.01 & 0.00 & 0.00 & 0.00 & N/A \\
\hline Cpd0 & 0.95 & 0.06 & -0.02 & 0.07 & 0.00 & 0.00 & 0.00 & 0.08 & -0.01 & -0.01 & 0.00 & 0.00 & 0.00 & -0.01 \\
\hline TS2 & 0.92 & 0.11 & 0.04 & 0.04 & 0.00 & 0.00 & 0.00 & 0.04 & -0.01 & -0.01 & 0.00 & 0.00 & 0.00 & N/A \\
\hline TS2 & 0.98 & 0.07 & -0.01 & 0.04 & 0.00 & 0.00 & 0.00 & 0.04 & -0.02 & -0.01 & 0.00 & 0.00 & 0.00 & -0.01 \\
\hline protCpd0 & 0.93 & 0.12 & 0.05 & 0.03 & 0.00 & 0.00 & 0.00 & 0.03 & -0.01 & -0.01 & 0.00 & 0.00 & 0.00 & N/A \\
\hline protCpd0 & 0.99 & 0.08 & -0.01 & 0.03 & 0.00 & 0.00 & 0.00 & 0.03 & -0.02 & -0.01 & 0.00 & 0.00 & 0.00 & -0.01 \\
\hline TS3 & 1.17 & 0.08 & -0.02 & -0.04 & -0.11 & 0.00 & 0.00 & -0.15 & -0.02 & -0.01 & 0.00 & 0.00 & 0.00 & N/A \\
\hline TS3 & 1.13 & 0.03 & -0.09 & 0.04 & -0.08 & 0.00 & 0.00 & -0.04 & -0.02 & -0.01 & 0.00 & 0.00 & 0.00 & -0.01 \\
\hline Cpdl & 1.39 & -0.79 & -1.17 & 0.78 & 0.00 & 0.00 & 0.00 & 0.00 & -0.07 & -0.10 & 0.00 & 0.00 & 0.00 & N/A \\
\hline Cpdl & 1.40 & -0.61 & -1.18 & 0.77 & 0.00 & 0.00 & 0.00 & 0.00 & -0.13 & -0.08 & 0.00 & 0.00 & 0.00 & 0.01 \\
\hline
\end{tabular}

Species highlighted in grey are for model A; unhighlighted are model B. Prox region refers to Pro30, Ala31, and Leu32. * TS1 is optimized at B1 level.

Figure A2. Influence of Basis Set

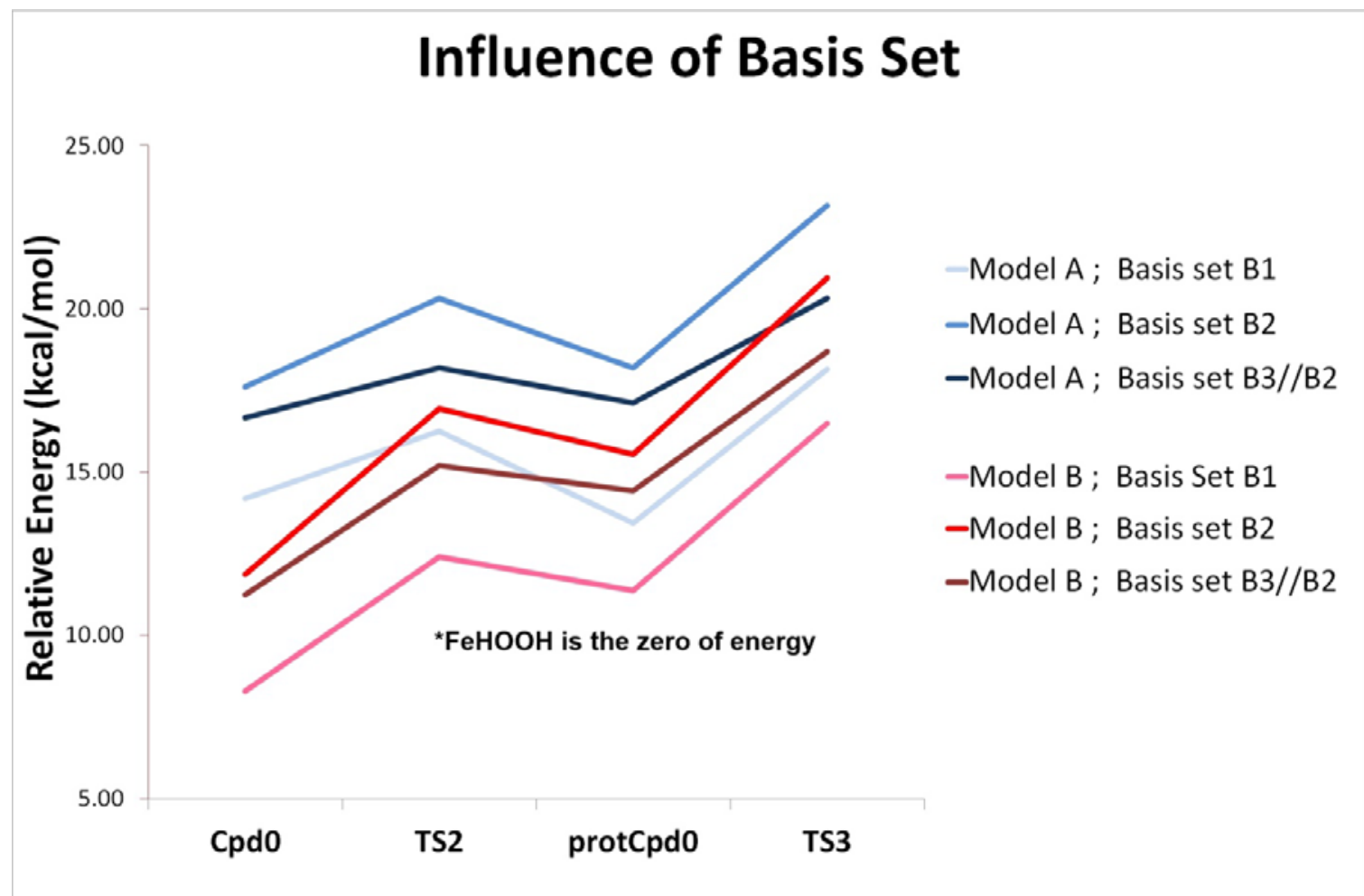

The zero of energy is taken as FeHOOH in all cases, as described in the paper. 
Table A11: Influence of $\mathrm{NH} \cdots$ S hydrogen bond distance $(\AA)$ on selected interatomic distances, including those involved in the trans influence.

\begin{tabular}{|l|ccccccc|}
\hline \multirow{2}{*}{$\begin{array}{c}\text { B3LYP 6-31G* } \\
\text { +LANL2DZ }\end{array}$} & \multicolumn{7}{|c|}{$\mathrm{NH}^{*} \mathrm{~S}$ hydrogen bond Distance } \\
\hline Fe-S & 3.1 & 3.2 & 3.3 & 3.4 & 3.5 & 3.6 & Model A \\
\hline FeHOOH & - & - & 2.256 & 2.251 & 2.247 & - & 2.222 \\
\cline { 2 - 8 } TS1 & - & - & 2.371 & 2.364 & 2.358 & - & 2.316 \\
Cpd0 & 2.388 & 2.380 & 2.372 & 2.367 & 2.360 & 2.355 & 2.313 \\
\cline { 2 - 8 } TS2 & 2.356 & 2.349 & 2.343 & 2.335 & 2.330 & 2.326 & 2.293 \\
\cline { 2 - 8 } protCpd0 & 2.334 & 2.326 & 2.320 & 2.314 & 2.309 & 2.304 & 2.269 \\
\cline { 2 - 8 } TS3 & 2.389 & 2.380 & 2.371 & 2.365 & 2.358 & 2.352 & 2.312 \\
\cline { 2 - 8 } Cpdl & 2.647 & 2.625 & 2.615 & 2.607 & 2.601 & 2.598 & 2.605 \\
\hline Fe-O & & & & & & & \\
\hline FeHOOH & - & - & 2.230 & 2.229 & 2.229 & - & 2.277 \\
\cline { 2 - 8 } TS1 & - & - & 1.875 & 1.882 & 1.89 & - & 1.910 \\
\cline { 2 - 8 } Cpd0 & 1.876 & 1.876 & 1.877 & 1.878 & 1.879 & 1.881 & 1.914 \\
\cline { 2 - 8 } TS2 & 1.928 & 1.927 & 1.927 & 1.926 & 1.926 & 1.927 & 1.953 \\
\cline { 2 - 8 } protCpd0 & 1.961 & 1.959 & 1.958 & 1.959 & 1.959 & 1.960 & 1.990 \\
\cline { 2 - 8 } TS3 & 1.741 & 1.741 & 1.741 & 1.742 & 1.742 & 1.743 & 1.762 \\
\cline { 2 - 8 } Cpdl & 1.627 & 1.627 & 1.627 & 1.627 & 1.627 & 1.627 & 1.626 \\
\hline $\mathrm{O}_{\text {prox-O }}$ dist & & & & & & & \\
\hline FeHOOH & - & - & 1.453 & 1.453 & 1.453 & - & 1.451 \\
TS1 & - & - & 1.469 & 1.467 & 1.468 & - & 1.476 \\
Cpd0 & 1.476 & 1.476 & 1.476 & 1.477 & 1.477 & 1.477 & 1.483 \\
\cline { 2 - 8 } TS2 & 1.492 & 1.492 & 1.492 & 1.493 & 1.493 & 1.493 & 1.494 \\
\cline { 2 - 7 } protCpd0 & 1.503 & 1.502 & 1.503 & 1.503 & 1.503 & 1.503 & 1.506 \\
\cline { 2 - 7 } TS3 & 1.778 & 1.778 & 1.778 & 1.778 & 1.778 & 1.779 & 1.773 \\
\hline
\end{tabular}




\section{Structures}

\section{Doublet; Basis set B1; Model A; Fe+HOOH}

$\begin{array}{lrrr}74 & & & \\ \mathrm{O} & -10.23775876 & 4.86717411 & -5.10559318 \\ \mathrm{C} & -10.79006742 & 5.81200037 & -4.53561869 \\ \mathrm{~N} & -11.91121001 & 5.63230593 & -3.78783177 \\ \mathrm{C} & -12.54123948 & 4.33606356 & -3.59470296 \\ \mathrm{C} & -10.25079946 & 7.23133917 & -4.65549405 \\ \mathrm{H} & -12.33407191 & 6.44091835 & -3.35520750 \\ \mathrm{H} & -13.56946369 & 4.34188583 & -3.97304861 \\ \mathrm{H} & -12.55331943 & 4.06110316 & -2.53405879 \\ \mathrm{H} & -11.95867684 & 3.60143595 & -4.15070464 \\ \mathrm{H} & -10.84846115 & 7.97733097 & -4.12278827 \\ \mathrm{H} & -10.20750318 & 7.49857408 & -5.71545939 \\ \mathrm{H} & -9.22795159 & 7.25392933 & -4.26661251 \\ \mathrm{C} & -4.17783890 & -1.63116949 & -0.83854219 \\ \mathrm{C} & -3.28299182 & -0.50794158 & -0.69059765 \\ \mathrm{~N} & -1.99252997 & -0.98077352 & -0.42685745 \\ \mathrm{C} & -2.08636974 & -2.36801222 & -0.39235392 \\ \mathrm{C} & -3.44991028 & -2.76636769 & -0.64981656 \\ \mathrm{C} & -3.69379614 & 0.83724414 & -0.81013044 \\ \mathrm{C} & -2.75037600 & 1.88944966 & -0.64306757 \\ \mathrm{~N} & -1.37896525 & 1.78535404 & -0.35899150 \\ \mathrm{C} & -0.88091846 & 3.09140572 & -0.29520127 \\ \mathrm{C} & -1.95863634 & 4.01614913 & -0.54701777 \\ \mathrm{C} & -3.09061228 & 3.28587556 & -0.76339509 \\ \mathrm{C} & 0.45552404 & 3.49642166 & -0.04179004 \\ \mathrm{C} & 1.49864201 & 2.56834923 & 0.14513982 \\ \mathrm{~N} & 1.39128361 & 1.17723184 & 0.14987877 \\ \mathrm{C} & 2.68409891 & 0.68671992 & 0.35410297 \\ \mathrm{C} & 3.59905266 & 1.79613413 & 0.47211813 \\ \mathrm{C} & 2.87582450 & 2.94479060 & 0.34959953 \\ \mathrm{Fe} & -0.33693285 & 0.10609056 & 0.06054112 \\ \mathrm{~S} & -0.76181761 & 0.01943449 & 2.24035543 \\ \mathrm{C} & -0.47062561 & 1.67982645 & 2.94543121 \\ \mathrm{C} & 3.09104703 & -0.66507144 & 0.42750153 \\ \mathrm{C} & 2.15863679 & -1.71307863 & 0.30163565 \\ \mathrm{~N} & 0.78633295 & -1.59899808 & 0.07642663 \\ \mathrm{C} & 0.29285543 & -2.89755266 & 0.04103790 \\ \mathrm{C} & 1.37353454 & -3.83458801 & 0.23532165\end{array}$




$\begin{array}{lrrr}\mathrm{C} & 2.51550017 & -3.10954668 & 0.38864285 \\ \mathrm{C} & -1.04252395 & -3.28662767 & -0.15952512 \\ \mathrm{H} & 4.13646932 & -0.89661761 & 0.58995977 \\ \mathrm{H} & 1.25875636 & -4.91141805 & 0.24223528 \\ \mathrm{H} & 4.66650119 & 1.69453027 & 0.62505017 \\ \mathrm{H} & 3.23513350 & 3.96603696 & 0.38043256 \\ \mathrm{H} & 0.68258479 & 4.55556860 & -0.02252259 \\ \mathrm{H} & -1.84940258 & 5.09356231 & -0.56972888 \\ \mathrm{H} & -4.08157400 & 3.65348739 & -0.99951214 \\ \mathrm{H} & -4.72423628 & 1.07313120 & -1.04171006 \\ \mathrm{H} & -5.23248949 & -1.54529260 & -1.06821917 \\ \mathrm{H} & -3.79169499 & -3.79317283 & -0.69111346 \\ \mathrm{H} & -1.28034133 & -4.34405490 & -0.15612068 \\ \mathrm{H} & 3.52240969 & -3.47499290 & 0.54850401 \\ \mathrm{H} & -0.71781764 & 1.61021473 & 4.01052044 \\ \mathrm{H} & 0.57440165 & 1.98274567 & 2.84477273 \\ \mathrm{H} & -1.11453861 & 2.43150686 & 2.48266569 \\ \mathrm{C} & -2.28548002 & -0.85852520 & -5.41369694 \\ \mathrm{C} & -3.19818935 & 0.34650816 & -5.55941382 \\ \mathrm{O} & -2.86104626 & 1.39406172 & -6.08470103 \\ \mathrm{O} & -4.40843985 & 0.11969514 & -5.04171826 \\ \mathrm{H} & -1.32955182 & -0.65794419 & -5.89953762 \\ \mathrm{H} & -2.75435907 & -1.74337297 & -5.85695174 \\ \mathrm{H} & -2.12210709 & -1.07324774 & -4.35159163 \\ \mathrm{~N} & -6.02119634 & 2.33170847 & -5.37544155 \\ \mathrm{C} & -5.53344679 & 3.47844470 & -5.96692433 \\ \mathrm{C} & -6.52864057 & 4.42374543 & -6.06310806 \\ \mathrm{~N} & -7.64855515 & 3.82495774 & -5.51032888 \\ \mathrm{C} & -7.29267225 & 2.57539983 & -5.11759940 \\ \mathrm{C} & -6.56134790 & 5.81538796 & -6.61262139 \\ \mathrm{H} & -6.86867046 & 6.54137015 & -5.84814515 \\ \mathrm{H} & -5.56715320 & 6.10335789 & -6.96563129 \\ \mathrm{H} & -7.25731501 & 5.90850556 & -7.45641238 \\ \mathrm{H} & -8.57645494 & 4.24545318 & -5.42388026 \\ \mathrm{H} & -7.98628685 & 1.88660391 & -4.65516964 \\ \mathrm{H} & -4.98510118 & 0.94102425 & -5.16546218 \\ & & & \\ \mathrm{H} & -4.50063232 & 3.52882563 & -6.28270195\end{array}$

\section{Doublet; Basis set B1; Model A; FeHOOH}

78

O $\quad-10.29405337 \quad 4.60148294 \quad-5.39415006$ 


\begin{tabular}{|c|c|c|c|}
\hline $\mathrm{C}$ & -11.10286674 & 5.48593869 & -5.09706261 \\
\hline $\mathrm{N}$ & -12.32991001 & 5.18692138 & -4.59650838 \\
\hline $\mathrm{C}$ & -12.79051208 & 3.82581192 & -4.37156007 \\
\hline $\mathrm{C}$ & -10.77003393 & 6.95879431 & -5.28747052 \\
\hline $\mathrm{H}$ & -12.96061800 & 5.94736036 & -4.38700322 \\
\hline $\mathrm{H}$ & -13.68125278 & 3.61119614 & -4.97229150 \\
\hline $\mathrm{H}$ & -13.02604020 & 3.66348853 & -3.31392814 \\
\hline $\mathrm{H}$ & -11.98662865 & 3.15271240 & -4.66949990 \\
\hline $\mathrm{H}$ & -11.58137482 & 7.63511027 & -5.00192175 \\
\hline $\mathrm{H}$ & -10.51897365 & 7.12670418 & -6.33906190 \\
\hline $\mathrm{H}$ & -9.88316269 & 7.19997756 & -4.69299166 \\
\hline $\mathrm{C}$ & -4.33179670 & -1.24645166 & -0.98692998 \\
\hline $\mathrm{C}$ & -3.28768349 & -0.25304974 & -0.90164472 \\
\hline $\mathrm{N}$ & -2.05896086 & -0.80438044 & -0.74996908 \\
\hline $\mathrm{C}$ & -2.30328652 & -2.14065488 & -0.74091278 \\
\hline $\mathrm{C}$ & -3.70741878 & -2.44482064 & -0.87363365 \\
\hline $\mathrm{C}$ & -3.48495072 & 1.08487413 & -1.00692709 \\
\hline $\mathrm{C}$ & -2.54956779 & 2.10889265 & -0.87356765 \\
\hline $\mathrm{N}$ & -1.20691885 & 1.96088052 & -0.64815032 \\
\hline $\mathrm{C}$ & -0.67756040 & 3.23269506 & -0.59891943 \\
\hline $\mathrm{C}$ & -1.72588371 & 4.20673108 & -0.78917671 \\
\hline $\mathrm{C}$ & -2.88214473 & 3.51111551 & -0.96879879 \\
\hline $\mathrm{C}$ & 0.66755376 & 3.53288721 & -0.40689240 \\
\hline $\mathrm{C}$ & 1.67301496 & 2.56419137 & -0.27923599 \\
\hline $\mathrm{N}$ & 1.48929770 & 1.18923475 & -0.36539487 \\
\hline $\mathrm{C}$ & 2.74374434 & 0.61196010 & -0.19285374 \\
\hline $\mathrm{C}$ & 3.72332516 & 1.65053721 & -0.00995690 \\
\hline $\mathrm{C}$ & 3.06679625 & 2.84788834 & -0.06170970 \\
\hline $\mathrm{Fe}$ & -0.28271085 & 0.20891924 & -0.45955309 \\
\hline $\mathrm{S}$ & -0.57888926 & 0.05076799 & 1.73727873 \\
\hline $\mathrm{C}$ & -0.06140636 & 1.61860284 & 2.52201307 \\
\hline $\mathrm{C}$ & 3.02666883 & -0.76064153 & -0.22081942 \\
\hline $\mathrm{C}$ & 2.03302653 & -1.74545598 & -0.37085018 \\
\hline $\mathrm{N}$ & 0.67033760 & -1.53530070 & -0.50952114 \\
\hline $\mathrm{C}$ & 0.07296961 & -2.77585321 & -0.57823623 \\
\hline $\mathrm{C}$ & 1.09159294 & -3.79840568 & -0.50432428 \\
\hline $\mathrm{C}$ & 2.29285845 & -3.16690148 & -0.37964022 \\
\hline $\mathrm{C}$ & -1.30526001 & -3.05718927 & -0.67416666 \\
\hline $\mathrm{H}$ & 4.05600402 & -1.08069539 & -0.10501647 \\
\hline $\mathrm{H}$ & 0.89764761 & -4.86320773 & -0.54252888 \\
\hline $\mathrm{H}$ & 4.78245828 & 1.47715735 & 0.13504730 \\
\hline $\mathrm{H}$ & 3.48507554 & 3.84242508 & 0.03200606 \\
\hline $\mathrm{H}$ & 0.96124555 & 4.57631427 & -0.35887700 \\
\hline $\mathrm{H}$ & -1.57596989 & 5.27911008 & -0.79780550 \\
\hline $\mathrm{H}$ & -3.87610919 & 3.89304661 & -1.16354589 \\
\hline
\end{tabular}




$\begin{array}{lrrr}\mathrm{H} & -4.50324249 & 1.41860317 & -1.19163428 \\ \mathrm{H} & -5.38290589 & -1.02723381 & -1.12064074 \\ \mathrm{H} & -4.12489841 & -3.44323415 & -0.89104622 \\ \mathrm{H} & -1.56977151 & -4.11097366 & -0.69340727 \\ \mathrm{H} & 3.27637298 & -3.61256067 & -0.29630608 \\ \mathrm{H} & -0.27875931 & 1.52088567 & 3.59098125 \\ \mathrm{H} & 1.00883251 & 1.80059697 & 2.39445144 \\ \mathrm{O} & -0.62207033 & 2.46852345 & 2.12464609 \\ \mathrm{C} & -0.14362095 & 0.29800341 & -2.73034075 \\ \mathrm{C} & -2.40883897 & -0.77679689 & -4.38026536 \\ \mathrm{O} & -3.01949519 & 0.58975853 & -4.22824706 \\ \mathrm{O} & -2.36030082 & 1.58705806 & -3.93866819 \\ \mathrm{H} & -4.32906344 & 0.58226141 & -4.41500092 \\ \mathrm{H} & -1.45934760 & -0.70369402 & -4.91506141 \\ \mathrm{H} & -3.08863547 & -1.45123495 & -4.90527122 \\ \mathrm{~N} & -2.18896566 & -1.19373926 & -3.39422699 \\ \mathrm{C} & -5.81166136 & 2.83342805 & -4.40317561 \\ \mathrm{C} & -5.37659582 & 3.97650446 & -5.03538135 \\ \mathrm{~N} & -6.44805773 & 4.71965134 & -5.46984277 \\ \mathrm{C} & -7.56493948 & 4.00404287 & -5.08221307 \\ \mathrm{C} & -7.13159447 & 2.88109546 & -4.45368797 \\ \mathrm{H} & -6.49212498 & 6.01741231 & -6.20829506 \\ \mathrm{H} & -7.01020119 & 6.80235294 & -5.64091144 \\ \mathrm{H} & -5.47324621 & 6.36571214 & -6.39903559 \\ \mathrm{H} & -6.99994172 & 5.92374934 & -7.17698838 \\ \mathrm{H} & -8.54052178 & 4.26841991 & -5.24607744 \\ \mathrm{H} & -7.80507014 & 2.13282831 & -4.05980702 \\ \mathrm{H} & -4.76181786 & 1.49079327 & -4.29361070 \\ \mathrm{O} & -4.32115728 & 4.18724206 & -5.14169878 \\ \mathrm{H} & 1.09578247 & 0.87503714 & -3.22358795 \\ \mathrm{H} & 1.48914596 & 1.13736017 & -2.36423065 \\ & -0.81724977 & 0.92813881 & -3.10108503\end{array}$

\section{Doublet; Basis set B1; Model A; Transition State 1}

$\begin{array}{llll}\mathrm{O} & -10.14878634 & 4.52209956 & -4.97710288 \\ \mathrm{C} & -10.89625578 & 5.42342368 & -4.56966681 \\ \mathrm{~N} & -12.05650975 & 5.15165442 & -3.92996922 \\ \mathrm{C} & -12.54229531 & 3.80225657 & -3.67797109 \\ \mathrm{C} & -10.54784860 & 6.88830233 & -4.74280918 \\ \mathrm{H} & -12.63527679 & 5.92744097 & -3.64110244\end{array}$




\begin{tabular}{|c|c|c|c|}
\hline $\mathrm{H}$ & -13.49833877 & 3.63306582 & -4.18462727 \\
\hline $\mathrm{H}$ & -12.67182672 & 3.63316389 & -2.60384052 \\
\hline $\mathrm{H}$ & -11.80191935 & 3.10413007 & -4.06781567 \\
\hline $\mathrm{H}$ & -11.32267852 & 7.56981196 & -4.38078098 \\
\hline $\mathrm{H}$ & -10.36805088 & 7.08746732 & -5.80364837 \\
\hline $\mathrm{H}$ & -9.61812897 & 7.09940629 & -4.20381673 \\
\hline $\mathrm{C}$ & -4.08718458 & -1.41206812 & -1.02123934 \\
\hline $\mathrm{C}$ & -3.08946958 & -0.36683752 & -0.92586047 \\
\hline $\mathrm{N}$ & -1.84377475 & -0.89600482 & -0.77265696 \\
\hline $\mathrm{C}$ & -2.02068742 & -2.24540292 & -0.75451898 \\
\hline $\mathrm{C}$ & -3.41764679 & -2.58929660 & -0.90557924 \\
\hline $\mathrm{C}$ & -3.36677215 & 0.98210960 & -0.96753429 \\
\hline $\mathrm{C}$ & -2.45016631 & 2.02755914 & -0.81251872 \\
\hline $\mathrm{N}$ & -1.09500255 & 1.90276719 & -0.65463498 \\
\hline $\mathrm{C}$ & -0.58124882 & 3.17250590 & -0.57546060 \\
\hline $\mathrm{C}$ & -1.65471451 & 4.13808559 & -0.67093265 \\
\hline $\mathrm{C}$ & -2.80886379 & 3.42952789 & -0.82119953 \\
\hline $\mathrm{C}$ & 0.77110607 & 3.49621748 & -0.42808265 \\
\hline $\mathrm{C}$ & 1.81195676 & 2.56367587 & -0.35469923 \\
\hline $\mathrm{N}$ & 1.67234501 & 1.19121748 & -0.44103292 \\
\hline $\mathrm{C}$ & 2.93878430 & 0.65837783 & -0.27800924 \\
\hline $\mathrm{C}$ & 3.89503486 & 1.72856682 & -0.11472320 \\
\hline $\mathrm{C}$ & 3.20237420 & 2.90212895 & -0.16206989 \\
\hline $\mathrm{Fe}$ & -0.09389663 & 0.16152615 & -0.58880033 \\
\hline S & -0.38301740 & 0.01740158 & 1.70485558 \\
\hline $\mathrm{C}$ & 0.16852401 & 1.57055842 & 2.50226024 \\
\hline $\mathrm{C}$ & 3.26764341 & -0.70422462 & -0.24369703 \\
\hline $\mathrm{C}$ & 2.29763681 & -1.72546253 & -0.34585436 \\
\hline $\mathrm{N}$ & 0.93244046 & -1.57119311 & -0.50232536 \\
\hline $\mathrm{C}$ & 0.37423299 & -2.82865484 & -0.51013311 \\
\hline $\mathrm{C}$ & 1.42570651 & -3.81667137 & -0.36901419 \\
\hline $\mathrm{C}$ & 2.60361833 & -3.14086614 & -0.27077558 \\
\hline $\mathrm{C}$ & -0.99427620 & -3.15337307 & -0.61809132 \\
\hline $\mathrm{H}$ & 4.30533160 & -0.98920327 & -0.11153449 \\
\hline $\mathrm{H}$ & 1.26606694 & -4.88803454 & -0.34629197 \\
\hline $\mathrm{H}$ & 4.95974723 & 1.58539056 & 0.02494227 \\
\hline $\mathrm{H}$ & 3.58528645 & 3.91122667 & -0.06928448 \\
\hline $\mathrm{H}$ & 1.03441240 & 4.54744957 & -0.36214160 \\
\hline $\mathrm{H}$ & -1.52463435 & 5.21278315 & -0.62632183 \\
\hline $\mathrm{H}$ & -3.81938335 & 3.80719276 & -0.92444529 \\
\hline $\mathrm{H}$ & -4.40730411 & 1.27169242 & -1.09290860 \\
\hline $\mathrm{H}$ & -5.15020713 & -1.24427105 & -1.14437526 \\
\hline $\mathrm{H}$ & -3.80880121 & -3.59916004 & -0.91701443 \\
\hline $\mathrm{H}$ & -1.24318737 & -4.21054213 & -0.60133162 \\
\hline $\mathrm{H}$ & 3.60013516 & -3.54904468 & -0.15210871 \\
\hline
\end{tabular}




$\begin{array}{lrrr}\mathrm{H} & -0.00121382 & 1.46885623 & 3.57982830 \\ \mathrm{H} & 1.23361307 & 1.75823505 & 2.33489639 \\ \mathrm{H} & -0.39997435 & 2.43375422 & 2.14173069 \\ \mathrm{O} & -0.09848280 & 0.11121203 & -2.49839319 \\ \mathrm{C} & -2.27209801 & -0.83370750 & -4.52332405 \\ \mathrm{C} & -2.95356970 & 0.47378633 & -4.29736419 \\ \mathrm{O} & -2.24569023 & 1.49609292 & -3.87100106 \\ \mathrm{O} & -4.15414270 & 0.60925073 & -4.57711915 \\ \mathrm{H} & -1.82605831 & -0.81473753 & -5.52691269 \\ \mathrm{H} & -3.00271723 & -1.64267154 & -4.48314410 \\ \mathrm{H} & -1.46120182 & -0.98285596 & -3.80384899 \\ \mathrm{~N} & -5.70760085 & 2.63259798 & -4.71667421 \\ \mathrm{C} & -5.30786389 & 3.77225524 & -5.36980942 \\ \mathrm{C} & -6.41829433 & 4.53906977 & -5.59925859 \\ \mathrm{~N} & -7.48404654 & 3.83347922 & -5.06589515 \\ \mathrm{C} & -7.02402552 & 2.68259632 & -4.54783313 \\ \mathrm{C} & -6.51032506 & 5.87594071 & -6.25228811 \\ \mathrm{H} & -6.87749787 & 6.64359852 & -5.56026091 \\ \mathrm{H} & -5.51977330 & 6.18252449 & -6.59636899 \\ \mathrm{H} & -7.18056889 & 5.86114702 & -7.11993421 \\ \mathrm{H} & -8.47268879 & 4.14002103 & -5.06664096 \\ \mathrm{H} & -7.62978841 & 1.92355595 & -4.07818128 \\ \mathrm{H} & -5.03065673 & 1.81083915 & -4.51121729 \\ \mathrm{H} & -4.27063483 & 3.94618097 & -5.61141544 \\ \mathrm{O} & 0.53399571 & 1.24903427 & -3.19433774 \\ \mathrm{H} & 1.16384478 & 1.54621812 & -2.51170822 \\ \mathrm{H} & -1.36596747 & 1.21532978 & -3.48826250\end{array}$

\section{Doublet; Basis set B1; Model A; Compound 0}

$\begin{array}{llll}\mathrm{O} & -10.20852097 & 4.65725355 & -5.33655635 \\ \mathrm{C} & -11.00144314 & 5.51510551 & -4.92201829 \\ \mathrm{~N} & -12.22575663 & 5.18519427 & -4.45087281 \\ \mathrm{C} & -12.73031788 & 3.82021202 & -4.40126764 \\ \mathrm{C} & -10.64154818 & 6.98775610 & -4.89536786 \\ \mathrm{H} & -12.83656190 & 5.92990293 & -4.14644796 \\ \mathrm{H} & -13.63239836 & 3.71831005 & -5.01370113 \\ \mathrm{H} & -12.96304738 & 3.53106657 & -3.37092487 \\ \mathrm{H} & -11.95401659 & 3.16431627 & -4.79458612 \\ \mathrm{H} & -11.45987745 & 7.63381704 & -4.56533524 \\ \mathrm{H} & -10.32942624 & 7.29492692 & -5.89804958\end{array}$




\begin{tabular}{|c|c|c|c|}
\hline $\mathrm{H}$ & -9.78928227 & 7.13399177 & -4.22315966 \\
\hline C & -4.16937583 & -1.43457475 & -0.81221556 \\
\hline C & -3.16781569 & -0.38908030 & -0.73116279 \\
\hline $\mathrm{N}$ & -1.92371556 & -0.94594767 & -0.58077860 \\
\hline $\mathrm{C}$ & -2.10480956 & -2.30281995 & -0.5594675 \\
\hline $\mathrm{C}$ & -3.51140103 & -2.61955671 & -0.6981921 \\
\hline $\mathrm{C}$ & -3.45604863 & 0.97468359 & -0.7831299 \\
\hline C & -2.50523304 & 1.99651091 & -0.6465050 \\
\hline $\mathrm{N}$ & -1.14363256 & 1.83221425 & -0.5142655 \\
\hline C & -0.59686981 & 3.09427565 & -0.41214374 \\
\hline C & -1.64677984 & 4.08388996 & -0.47930551 \\
\hline $\mathrm{C}$ & -2.82192732 & 3.40593615 & -0.6267715 \\
\hline $\mathrm{C}$ & 0.76950879 & 3.38090329 & -0.2923475 \\
\hline C & 1.79133842 & 2.42614196 & -0.24066543 \\
\hline $\mathrm{N}$ & 1.62872635 & 1.06197192 & -0.29421920 \\
\hline C & 2.88999024 & 0.51388777 & -0.16935706 \\
\hline $\mathrm{C}$ & 3.86996922 & 1.57239343 & -0.05472599 \\
\hline $\mathrm{C}$ & 3.19394199 & 2.75270765 & -0.09906546 \\
\hline $\mathrm{Fe}$ & -0.14910012 & 0.06244174 & -0.44108358 \\
\hline S & -0.31815959 & -0.09524587 & 1.86050795 \\
\hline $\mathrm{C}$ & -0.18450808 & 1.56902117 & 2.6119132 \\
\hline $\mathrm{C}$ & 3.20660474 & -0.84947507 & -0.1252686 \\
\hline $\mathrm{C}$ & 2.22008412 & -1.85921376 & -0.2071227 \\
\hline $\mathrm{N}$ & 0.85463221 & -1.69035138 & -0.3555969 \\
\hline $\mathrm{C}$ & 0.28294458 & -2.94271683 & -0.3438273 \\
\hline $\mathrm{C}$ & 1.32430533 & -3.94097499 & -0.2037475 \\
\hline $\mathrm{C}$ & 2.51026973 & -3.27696688 & -0.1218368 \\
\hline $\mathrm{C}$ & -1.09162864 & -3.24892793 & -0.4334999 \\
\hline $\mathrm{H}$ & 4.24276434 & -1.14632151 & -0.0100324 \\
\hline $\mathrm{H}$ & 1.15298190 & -5.01017309 & -0.1692490 \\
\hline $\mathrm{H}$ & 4.93615991 & 1.41298830 & 0.0515280 \\
\hline $\mathrm{H}$ & 3.59066481 & 3.75876693 & -0.0363427 \\
\hline $\mathrm{H}$ & 1.05818851 & 4.42592808 & -0.2342728 \\
\hline $\mathrm{H}$ & -1.49172763 & 5.15438686 & -0.4168655 \\
\hline $\mathrm{H}$ & -3.82291135 & 3.81333532 & -0.7071132 \\
\hline $\mathrm{H}$ & -4.49507473 & 1.26920410 & -0.8945547 \\
\hline $\mathrm{H}$ & -5.23269813 & -1.26292477 & -0.9291641 \\
\hline $\mathrm{H}$ & -3.92080357 & -3.62244310 & -0.7056991 \\
\hline $\mathrm{H}$ & -1.36943354 & -4.29787632 & -0.411032 \\
\hline $\mathrm{H}$ & 3.50318891 & -3.69496620 & -0.0073056 \\
\hline $\mathrm{H}$ & -0.25882372 & 1.44823634 & 3.6982120 \\
\hline $\mathrm{H}$ & 0.77646365 & 2.03887168 & 2.3801132 \\
\hline $\mathrm{H}$ & -0.98864120 & 2.23305210 & 2.2797870 \\
\hline $\mathrm{O}$ & -0.10003694 & -0.06880428 & -2.3501818 \\
\hline $\mathrm{C}$ & -2.44835026 & -0.75210330 & -4.5620380 \\
\hline
\end{tabular}




$\begin{array}{lrrr}\mathrm{C} & -3.20811046 & 0.52120806 & -4.36883956 \\ \mathrm{O} & -2.55806734 & 1.59475019 & -3.99344967 \\ \mathrm{O} & -4.42590733 & 0.57159712 & -4.61585018 \\ \mathrm{H} & -1.88202858 & -0.68216155 & -5.49992175 \\ \mathrm{H} & -3.13959215 & -1.59288880 & -4.62515839 \\ \mathrm{H} & -1.71870734 & -0.89656770 & -3.75403959 \\ \mathrm{~N} & -5.81446212 & 2.70961951 & -4.79243823 \\ \mathrm{C} & -5.34912109 & 3.90963499 & -5.27571048 \\ \mathrm{C} & -6.43169051 & 4.70683877 & -5.53873918 \\ \mathrm{~N} & -7.54624266 & 3.95736093 & -5.19609347 \\ \mathrm{C} & -7.14075241 & 2.75519646 & -4.75791420 \\ \mathrm{C} & -6.50078337 & 6.09096063 & -6.08845313 \\ \mathrm{H} & -6.98075085 & 6.78453653 & -5.38746291 \\ \mathrm{H} & -5.49182126 & 6.46121913 & -6.28573049 \\ \mathrm{H} & -7.06325055 & 6.12363917 & -7.02903756 \\ \mathrm{H} & -8.52913637 & 4.27039069 & -5.27308270 \\ \mathrm{H} & -7.79002535 & 1.95618797 & -4.43558012 \\ \mathrm{H} & -5.18594579 & 1.83425908 & -4.60184593 \\ \mathrm{H} & -4.29191256 & 4.09493495 & -5.38771373 \\ \mathrm{O} & -0.14323734 & 1.19452704 & -3.12620540 \\ \mathrm{H} & -0.03845867 & 1.85819582 & -2.41840012 \\ \mathrm{H} & -1.59235301 & 1.39192595 & -3.66300554\end{array}$

\section{Doublet; Basis set B1; Model A; Transition State 2}

$\begin{array}{llll}\mathrm{O} & -10.22100913 & 4.61464116 & -5.38483524 \\ \mathrm{C} & -11.03362158 & 5.43734800 & -4.94465381 \\ \mathrm{~N} & -12.24950555 & 5.05855709 & -4.48101427 \\ \mathrm{C} & -12.70899117 & 3.67774063 & -4.46640230 \\ \mathrm{C} & -10.71323927 & 6.92107003 & -4.89246466 \\ \mathrm{H} & -12.88029690 & 5.77534361 & -4.15166066 \\ \mathrm{H} & -13.60714447 & 3.56052400 & -5.08228711 \\ \mathrm{H} & -12.93210303 & 3.35364630 & -3.44415746 \\ \mathrm{H} & -11.91061122 & 3.05889076 & -4.87552401 \\ \mathrm{H} & -11.53611459 & 7.53606800 & -4.51675374 \\ \mathrm{H} & -10.44691866 & 7.25935328 & -5.89834677 \\ \mathrm{H} & -9.83991399 & 7.07306268 & -4.24977235 \\ \mathrm{C} & -4.20000481 & -1.36831111 & -0.77346258 \\ \mathrm{C} & -3.18210982 & -0.33847211 & -0.70160373 \\ \mathrm{~N} & -1.94492089 & -0.91428673 & -0.56087189 \\ \mathrm{C} & -2.14791536 & -2.26857165 & -0.53424640\end{array}$




\begin{tabular}{|c|c|c|c|}
\hline C & -3.56002410 & -2.56313255 & -0.66093908 \\
\hline $\mathrm{C}$ & -3.45315044 & 1.02913052 & -0.74666414 \\
\hline $\mathrm{C}$ & -2.48802062 & 2.03739939 & -0.61116934 \\
\hline $\mathrm{N}$ & -1.12314557 & 1.85261762 & -0.50293993 \\
\hline $\mathrm{C}$ & -0.55681803 & 3.10954499 & -0.39517229 \\
\hline $\mathrm{C}$ & -1.59486732 & 4.11050456 & -0.43456950 \\
\hline $\mathrm{C}$ & -2.78191514 & 3.44948189 & -0.57089469 \\
\hline $\mathrm{C}$ & 0.81651232 & 3.36997452 & -0.30986445 \\
\hline $\mathrm{C}$ & 1.82216300 & 2.39821950 & -0.27552489 \\
\hline $\mathrm{N}$ & 1.63775810 & 1.03613825 & -0.31628153 \\
\hline $\mathrm{C}$ & 2.89347143 & 0.47035440 & -0.20741084 \\
\hline $\mathrm{C}$ & 3.89048118 & 1.51469335 & -0.11707739 \\
\hline $\mathrm{C}$ & 3.23153925 & 2.70436616 & -0.16061410 \\
\hline $\mathrm{Fe}$ & -0.15486805 & 0.06532623 & -0.42985009 \\
\hline S & -0.29335882 & -0.09554590 & 1.85370762 \\
\hline $\mathrm{C}$ & -0.13319579 & 1.56660166 & 2.60290959 \\
\hline $\mathrm{C}$ & 3.19060323 & -0.89670103 & -0.15989372 \\
\hline $\mathrm{C}$ & 2.18705484 & -1.89052171 & -0.23002201 \\
\hline $\mathrm{N}$ & 0.82242626 & -1.70021125 & -0.37064219 \\
\hline $\mathrm{C}$ & 0.23073525 & -2.94448183 & -0.34232596 \\
\hline $\mathrm{C}$ & 1.25730021 & -3.95716762 & -0.20483825 \\
\hline $\mathrm{C}$ & 2.45444209 & -3.31156058 & -0.13819629 \\
\hline $\mathrm{C}$ & -1.14914999 & -3.23018150 & -0.41335794 \\
\hline $\mathrm{H}$ & 4.22303944 & -1.20937624 & -0.05481711 \\
\hline $\mathrm{H}$ & 1.06946994 & -5.02313429 & -0.16145929 \\
\hline $\mathrm{H}$ & 4.95568339 & 1.34000378 & -0.02739535 \\
\hline $\mathrm{H}$ & 3.64391913 & 3.70480557 & -0.11310548 \\
\hline $\mathrm{H}$ & 1.12501281 & 4.40951425 & -0.25723924 \\
\hline $\mathrm{H}$ & -1.42602758 & 5.17840902 & -0.36748929 \\
\hline $\mathrm{H}$ & -3.77722372 & 3.87239041 & -0.63506594 \\
\hline $\mathrm{H}$ & -4.48863217 & 1.33832259 & -0.84811663 \\
\hline $\mathrm{H}$ & -5.26118647 & -1.17975135 & -0.88199223 \\
\hline $\mathrm{H}$ & -3.98516017 & -3.55933817 & -0.66263435 \\
\hline $\mathrm{H}$ & -1.44298176 & -4.27436589 & -0.38193063 \\
\hline $\mathrm{H}$ & 3.44139985 & -3.74472122 & -0.02964454 \\
\hline $\mathrm{H}$ & -0.18664135 & 1.44106001 & 3.68968358 \\
\hline $\mathrm{H}$ & 0.82645685 & 2.02881542 & 2.35270297 \\
\hline $\mathrm{H}$ & -0.94004642 & 2.23547898 & 2.28870725 \\
\hline $\mathrm{O}$ & -0.15023777 & -0.04137157 & -2.37948880 \\
\hline $\mathrm{C}$ & -2.51989014 & -0.72184870 & -4.53676923 \\
\hline $\mathrm{C}$ & -3.17666555 & 0.60953904 & -4.35642868 \\
\hline $\mathrm{O}$ & -2.49769944 & 1.62076922 & -3.97574560 \\
\hline $\mathrm{O}$ & -4.41127686 & 0.68932688 & -4.64911351 \\
\hline $\mathrm{H}$ & -2.02888343 & -0.73282566 & -5.51878548 \\
\hline $\mathrm{H}$ & -3.26416062 & -1.51893105 & -4.51218484 \\
\hline
\end{tabular}




$\begin{array}{llll}\mathrm{H} & -1.74817494 & -0.87930642 & -3.77435519 \\ \mathrm{~N} & -5.74528156 & 2.76290086 & -4.80868608 \\ \mathrm{C} & -5.32184052 & 3.99445874 & -5.24973715 \\ \mathrm{C} & -6.41567886 & 4.77549713 & -5.52669196 \\ \mathrm{~N} & -7.51513070 & 3.98785577 & -5.23719851 \\ \mathrm{C} & -7.07035690 & 2.78496405 & -4.81604322 \\ \mathrm{C} & -6.48116330 & 6.18067384 & -6.02927190 \\ \mathrm{H} & -6.97068758 & 6.85254105 & -5.31283434 \\ \mathrm{H} & -5.46865303 & 6.55568130 & -6.19880219 \\ \mathrm{H} & -7.02927619 & 6.25066064 & -6.97687708 \\ \mathrm{H} & -8.50119237 & 4.26725626 & -5.33176233 \\ \mathrm{H} & -7.71484815 & 1.96569884 & -4.53461358 \\ \mathrm{H} & -4.98063392 & 1.73488160 & -4.63526887 \\ \mathrm{H} & -4.27068792 & 4.22859093 & -5.33260304 \\ \mathrm{O} & -0.24535044 & 1.26761164 & -3.09261148 \\ & & & \\ \mathrm{H} & -0.26055436 & 1.88472240 & -2.32872446 \\ \mathrm{H} & -1.41289010 & 1.37793089 & -3.57516244\end{array}$

\section{Doublet; Basis set B1; Model A; protonated Compound 0}

$\begin{array}{llll}\mathrm{O} & -10.30258578 & 4.67856907 & -5.35904159 \\ \mathrm{C} & -11.09709555 & 5.54051711 & -4.96950690 \\ \mathrm{~N} & -12.32676596 & 5.21163844 & -4.49677015 \\ \mathrm{C} & -12.80992680 & 3.84234804 & -4.40988445 \\ \mathrm{C} & -10.74182861 & 7.01941965 & -5.00671525 \\ \mathrm{H} & -12.94434275 & 5.95682234 & -4.20813840 \\ \mathrm{H} & -13.70631039 & 3.70563763 & -5.02471610 \\ \mathrm{H} & -13.04456251 & 3.57691692 & -3.37316769 \\ \mathrm{H} & -12.01895634 & 3.18972317 & -4.77926231 \\ \mathrm{H} & -11.54360503 & 7.67472998 & -4.65343426 \\ \mathrm{H} & -10.48582353 & 7.29165221 & -6.03502708 \\ \mathrm{H} & -9.85307254 & 7.18486900 & -4.38947583 \\ \mathrm{C} & -4.13411380 & -1.45276903 & -0.84993722 \\ \mathrm{C} & -3.12699738 & -0.41531902 & -0.75183334 \\ \mathrm{~N} & -1.88659712 & -0.97391264 & -0.58955234 \\ \mathrm{C} & -2.08116028 & -2.32985509 & -0.56662054 \\ \mathrm{C} & -3.48558869 & -2.64060482 & -0.72507006 \\ \mathrm{C} & -3.41799317 & 0.94569594 & -0.78040276 \\ \mathrm{C} & -2.48206352 & 1.97255115 & -0.62071216 \\ \mathrm{~N} & -1.10356492 & 1.81277943 & -0.53961125\end{array}$




\begin{tabular}{|c|c|c|c|}
\hline $\mathrm{C}$ & -0.56651482 & 3.08434010 & -0.37134063 \\
\hline $\mathrm{C}$ & -1.62663422 & 4.05445089 & -0.36503677 \\
\hline $\mathrm{C}$ & -2.80134391 & 3.37109873 & -0.52154086 \\
\hline $\mathrm{C}$ & 0.80017950 & 3.35352616 & -0.29987319 \\
\hline $\mathrm{C}$ & 1.81669835 & 2.39322365 & -0.26148215 \\
\hline $\mathrm{N}$ & 1.65210757 & 1.02915282 & -0.27747007 \\
\hline C & 2.91625852 & 0.48426968 & -0.16770642 \\
\hline $\mathrm{C}$ & 3.89873495 & 1.54303253 & -0.09675476 \\
\hline C & 3.22195233 & 2.72154874 & -0.15698128 \\
\hline $\mathrm{Fe}$ & -0.10955938 & 0.02349654 & -0.39733873 \\
\hline S & -0.28931379 & -0.13162494 & 1.85965021 \\
\hline $\mathrm{C}$ & -0.17309822 & 1.53655647 & 2.60218106 \\
\hline $\mathrm{C}$ & 3.22829512 & -0.87628810 & -0.11112262 \\
\hline $\mathrm{C}$ & 2.24126908 & -1.88767130 & -0.18187227 \\
\hline $\mathrm{N}$ & 0.87549850 & -1.71878796 & -0.3392990 \\
\hline $\mathrm{C}$ & 0.29868676 & -2.97116873 & -0.3168815 \\
\hline $\mathrm{C}$ & 1.33800428 & -3.96664865 & -0.16170573 \\
\hline $\mathrm{C}$ & 2.52587477 & -3.30374056 & -0.08232101 \\
\hline $\mathrm{C}$ & -1.07748743 & -3.27511866 & -0.4171633 \\
\hline $\mathrm{H}$ & 4.26446320 & -1.17522654 & -0.00203512 \\
\hline $\mathrm{H}$ & 1.16594045 & -5.03515771 & -0.11931843 \\
\hline $\mathrm{H}$ & 4.96654072 & 1.38493161 & -0.00943501 \\
\hline $\mathrm{H}$ & 3.61898531 & 3.72868576 & -0.1282722 \\
\hline $\mathrm{H}$ & 1.10051389 & 4.39503774 & -0.2385987 \\
\hline $\mathrm{H}$ & -1.48506701 & 5.12291074 & -0.2592096 \\
\hline $\mathrm{H}$ & -3.80492228 & 3.77565689 & -0.5652118 \\
\hline $\mathrm{H}$ & -4.45786670 & 1.23641557 & -0.8899080 \\
\hline $\mathrm{H}$ & -5.19288723 & -1.27133921 & -0.9860955 \\
\hline $\mathrm{H}$ & -3.89857151 & -3.64153618 & -0.7389642 \\
\hline $\mathrm{H}$ & -1.35629345 & -4.32343346 & -0.3892941 \\
\hline $\mathrm{H}$ & 3.51691853 & -3.72381662 & 0.0382933 \\
\hline $\mathrm{H}$ & -0.23075506 & 1.41094763 & 3.68847502 \\
\hline $\mathrm{H}$ & 0.77754477 & 2.01837439 & 2.35627534 \\
\hline $\mathrm{H}$ & -0.99391721 & 2.18435984 & 2.28085710 \\
\hline $\mathrm{O}$ & -0.01641621 & -0.05793650 & -2.3837066 \\
\hline $\mathrm{C}$ & -2.44930800 & -0.75625568 & -4.4904327 \\
\hline $\mathrm{C}$ & -3.12437579 & 0.57123793 & -4.32942848 \\
\hline $\mathrm{O}$ & -2.48078134 & 1.60834322 & -4.09570110 \\
\hline $\mathrm{O}$ & -4.42084056 & 0.53659646 & -4.4925695 \\
\hline $\mathrm{H}$ & -1.96624322 & -0.78235566 & -5.4757014 \\
\hline $\mathrm{H}$ & -3.16744830 & -1.57575042 & -4.432978 \\
\hline $\mathrm{H}$ & -1.66805965 & -0.87834017 & -3.7329166 \\
\hline $\mathrm{N}$ & -5.85005136 & 2.74831687 & -4.5921430 \\
\hline C & -5.40002721 & 3.94379935 & -5.1045819 \\
\hline C & -6.46453397 & 4.74153565 & -5.4480492 \\
\hline
\end{tabular}




$\begin{array}{llll}\mathrm{N} & -7.58883232 & 4.00415694 & -5.12718455 \\ \mathrm{C} & -7.16989578 & 2.81663863 & -4.62377130 \\ \mathrm{C} & -6.50310673 & 6.10958001 & -6.04449299 \\ \mathrm{H} & -7.00322627 & 6.83458517 & -5.38853849 \\ \mathrm{H} & -5.48346725 & 6.46635375 & -6.21412172 \\ \mathrm{H} & -7.02698892 & 6.12349955 & -7.00886746 \\ \mathrm{H} & -8.56205368 & 4.29913695 & -5.25425422 \\ \mathrm{H} & -7.84914343 & 2.04015388 & -4.30159412 \\ \mathrm{H} & -4.88530472 & 1.45601875 & -4.43620658 \\ \mathrm{H} & -4.34231678 & 4.14954410 & -5.19335681 \\ \mathrm{O} & -0.16862601 & 1.32189102 & -2.96669962 \\ \mathrm{H} & -0.48658814 & 1.78098830 & -2.12219548 \\ \mathrm{H} & -1.03380690 & 1.30808368 & -3.53524215\end{array}$

\section{Doublet; Basis set B1; Model A; Transition State 3}

$\begin{array}{llll}\mathrm{O} & -10.29502169 & 4.65039951 & -5.32708565 \\ \mathrm{C} & -11.08400442 & 5.52361861 & -4.95322411 \\ \mathrm{~N} & -12.31359863 & 5.21000193 & -4.46970198 \\ \mathrm{C} & -12.80143923 & 3.84477917 & -4.35164371 \\ \mathrm{C} & -10.72224987 & 6.99996490 & -5.02421361 \\ \mathrm{H} & -12.92695898 & 5.96363836 & -4.19425461 \\ \mathrm{H} & -13.70020070 & 3.69791006 & -4.96067982 \\ \mathrm{H} & -13.03383209 & 3.60265520 & -3.30871143 \\ \mathrm{H} & -12.01388468 & 3.18146820 & -4.70913663 \\ \mathrm{H} & -11.51921572 & 7.66650738 & -4.68111994 \\ \mathrm{H} & -10.47043327 & 7.24846557 & -6.05953465 \\ \mathrm{H} & -9.82945125 & 7.17441607 & -4.41539786 \\ \mathrm{C} & -4.16078811 & -1.41270942 & -0.81350263 \\ \mathrm{C} & -3.14885881 & -0.38181882 & -0.72963856 \\ \mathrm{~N} & -1.90758070 & -0.92132475 & -0.58354268 \\ \mathrm{C} & -2.10396050 & -2.26900498 & -0.55959712 \\ \mathrm{C} & -3.50577559 & -2.59602622 & -0.69773491 \\ \mathrm{C} & -3.40689719 & 0.96736993 & -0.76612116 \\ \mathrm{C} & -2.45417795 & 1.98345214 & -0.63063133 \\ \mathrm{~N} & -1.09170663 & 1.81956414 & -0.49014339 \\ \mathrm{C} & -0.54175587 & 3.08325781 & -0.40929530 \\ \mathrm{C} & -1.59023289 & 4.06985589 & -0.49640763 \\ \mathrm{C} & -2.76591678 & 3.39161876 & -0.63381865 \\ \mathrm{C} & 0.82333939 & 3.36740227 & -0.29691918 \\ \mathrm{C} & 1.84372646 & 2.43472835 & -0.21953640\end{array}$




\begin{tabular}{|c|c|c|c|}
\hline $\mathrm{N}$ & 1.66552795 & 1.07983234 & -0.25510637 \\
\hline C & 2.92322800 & 0.53663486 & -0.15376378 \\
\hline $\mathrm{C}$ & 3.91461108 & 1.58231493 & -0.05431024 \\
\hline C & 3.24430388 & 2.76450193 & -0.09599239 \\
\hline $\mathrm{Fe}$ & -0.11115221 & 0.09353647 & -0.43568407 \\
\hline S & -0.25519596 & -0.13320367 & 1.86095004 \\
\hline $\mathrm{C}$ & -0.28612265 & 1.53570855 & 2.61180359 \\
\hline $\mathrm{C}$ & 3.21486268 & -0.81597964 & -0.13710398 \\
\hline $\mathrm{C}$ & 2.23168819 & -1.82648513 & -0.21668765 \\
\hline $\mathrm{N}$ & 0.86658203 & -1.65373005 & -0.33974660 \\
\hline $\mathrm{C}$ & 0.28154538 & -2.89843104 & -0.33991081 \\
\hline $\mathrm{C}$ & 1.31907661 & -3.90098172 & -0.2243759 \\
\hline $\mathrm{C}$ & 2.51183112 & -3.24494110 & -0.15338850 \\
\hline $\mathrm{C}$ & -1.09620537 & -3.19015718 & -0.42826459 \\
\hline $\mathrm{H}$ & 4.24963188 & -1.12733055 & -0.0443210 \\
\hline $\mathrm{H}$ & 1.14256095 & -4.96925186 & -0.2004188 \\
\hline $\mathrm{H}$ & 4.97997736 & 1.41143835 & 0.03587044 \\
\hline $\mathrm{H}$ & 3.63975552 & 3.77123866 & -0.04740030 \\
\hline $\mathrm{H}$ & 1.10128963 & 4.41679523 & -0.26279604 \\
\hline $\mathrm{H}$ & -1.43349680 & 5.14077111 & -0.45996838 \\
\hline $\mathrm{H}$ & -3.76430357 & 3.79753966 & -0.7373265 \\
\hline $\mathrm{H}$ & -4.43752725 & 1.28497298 & -0.89218843 \\
\hline $\mathrm{H}$ & -5.22014341 & -1.22778200 & -0.9370488 \\
\hline $\mathrm{H}$ & -3.90696518 & -3.60156286 & -0.70507360 \\
\hline $\mathrm{H}$ & -1.36475211 & -4.24220245 & -0.40759416 \\
\hline $\mathrm{H}$ & 3.50286055 & -3.67137510 & -0.0586587 \\
\hline $\mathrm{H}$ & -0.33346158 & 1.39977323 & 3.69746687 \\
\hline $\mathrm{H}$ & 0.62034442 & 2.09903932 & 2.3713225 \\
\hline $\mathrm{H}$ & -1.15914193 & 2.11130709 & 2.29136849 \\
\hline $\mathrm{O}$ & 0.05931794 & -0.10669426 & -2.1783068 \\
\hline $\mathrm{C}$ & -2.45070822 & -0.81570803 & -4.4045767 \\
\hline $\mathrm{C}$ & -3.12672255 & 0.51709164 & -4.2438106 \\
\hline $\mathrm{O}$ & -2.49960145 & 1.56238792 & -4.02553907 \\
\hline $\mathrm{O}$ & -4.43027905 & 0.46748486 & -4.3932782 \\
\hline $\mathrm{H}$ & -1.99097719 & -0.85545207 & -5.4001554 \\
\hline $\mathrm{H}$ & -3.16694896 & -1.63409968 & -4.3170254 \\
\hline $\mathrm{H}$ & -1.65373134 & -0.92588551 & -3.6633617 \\
\hline $\mathrm{N}$ & -5.84605880 & 2.71658529 & -4.5300907 \\
\hline C & -5.39245117 & 3.89867915 & -5.0714807 \\
\hline $\mathrm{C}$ & -6.45420916 & 4.69472444 & -5.4291501 \\
\hline $\mathrm{N}$ & -7.58093733 & 3.96984505 & -5.0870277 \\
\hline $\mathrm{C}$ & -7.16520722 & 2.79193203 & -4.55853553 \\
\hline $\mathrm{C}$ & -6.50032767 & 6.04794470 & -6.0595749 \\
\hline $\mathrm{H}$ & -6.98881834 & 6.79017630 & -5.4141157 \\
\hline $\mathrm{H}$ & -5.48343796 & 6.39932953 & -6.2554720 \\
\hline
\end{tabular}




$\begin{array}{lrrr}\mathrm{H} & -7.04014304 & 6.03744259 & -7.01513349 \\ \mathrm{H} & -8.55288979 & 4.26550914 & -5.21910525 \\ \mathrm{H} & -7.84759615 & 2.02668267 & -4.21634595 \\ \mathrm{H} & -4.88149362 & 1.38725014 & -4.34983613 \\ \mathrm{H} & -4.33393396 & 4.09617468 & -5.16928129 \\ \mathrm{O} & 0.01467581 & 1.35373840 & -3.18332124 \\ \mathrm{H} & 0.02314251 & 1.99290258 & -2.44279637 \\ \mathrm{H} & -0.94588233 & 1.36061290 & -3.51809328\end{array}$

\section{Doublet; Basis set B1; Model A; Compound I}

$\begin{array}{llll}\mathrm{O} & -10.27731313 & 4.69594143 & -4.95975597 \\ \mathrm{C} & -10.95189345 & 5.58890520 & -4.43952250 \\ \mathrm{~N} & -12.12622634 & 5.31640749 & -3.81115859 \\ \mathrm{C} & -12.67830618 & 3.97576974 & -3.69944904 \\ \mathrm{C} & -10.50980166 & 7.04516957 & -4.49086349 \\ \mathrm{H} & -12.64955104 & 6.08534589 & -3.41773258 \\ \mathrm{H} & -13.65562338 & 3.91361602 & -4.19092946 \\ \mathrm{H} & -12.78861727 & 3.68507443 & -2.64877253 \\ \mathrm{H} & -11.98568816 & 3.29301912 & -4.19168485 \\ \mathrm{H} & -11.19966493 & 7.73387311 & -3.99367359 \\ \mathrm{H} & -10.40437611 & 7.34043875 & -5.53911970 \\ \mathrm{H} & -9.52440413 & 7.13103633 & -4.02251529 \\ \mathrm{C} & -3.90778566 & -1.70151204 & -1.00938894 \\ \mathrm{C} & -2.98395082 & -0.60404692 & -0.85302628 \\ \mathrm{~N} & -1.70344970 & -1.09272737 & -0.68786386 \\ \mathrm{C} & -1.80410742 & -2.46871074 & -0.73726392 \\ \mathrm{C} & -3.18228472 & -2.85100289 & -0.93099644 \\ \mathrm{C} & -3.36490835 & 0.74628730 & -0.85266324 \\ \mathrm{C} & -2.44472916 & 1.78407755 & -0.67210685 \\ \mathrm{~N} & -1.07178712 & 1.64951973 & -0.47635295 \\ \mathrm{C} & -0.56214076 & 2.93171446 & -0.37883477 \\ \mathrm{C} & -1.63884105 & 3.88703576 & -0.47754140 \\ \mathrm{C} & -2.78915824 & 3.18425532 & -0.66176763 \\ \mathrm{C} & 0.78367725 & 3.28373494 & -0.22357036 \\ \mathrm{C} & 1.84713756 & 2.38301385 & -0.18148983\end{array}$




\begin{tabular}{|c|c|c|c|}
\hline $\mathrm{N}$ & 1.74473279 & 1.01616082 & -0.24028205 \\
\hline $\mathrm{C}$ & 3.03832107 & 0.53642572 & -0.16297743 \\
\hline $\mathrm{C}$ & 3.96610542 & 1.63497566 & -0.04254642 \\
\hline C & 3.22905296 & 2.77869119 & -0.05253996 \\
\hline $\mathrm{Fe}$ & 0.03876150 & -0.03795747 & -0.61036574 \\
\hline S & -0.59485212 & 0.04517404 & 1.91524180 \\
\hline $\mathrm{C}$ & 0.46484073 & 1.25620781 & 2.75694590 \\
\hline $\mathrm{C}$ & 3.41114625 & -0.80331753 & -0.18150862 \\
\hline $\mathrm{C}$ & 2.47963580 & -1.85425634 & -0.29501976 \\
\hline $\mathrm{N}$ & 1.10763749 & -1.73501248 & -0.40619977 \\
\hline $\mathrm{C}$ & 0.60168683 & -3.01473761 & -0.48711227 \\
\hline $\mathrm{C}$ & 1.68416983 & -3.96622937 & -0.4125645 \\
\hline $\mathrm{C}$ & 2.83962277 & -3.25163607 & -0.29757417 \\
\hline $\mathrm{C}$ & -0.74962386 & -3.37708038 & -0.6297882 \\
\hline $\mathrm{H}$ & 4.46284214 & -1.05589392 & -0.1064471 \\
\hline $\mathrm{H}$ & 1.56205020 & -5.04152558 & -0.4479755 \\
\hline $\mathrm{H}$ & 5.04039131 & 1.52558685 & 0.03582742 \\
\hline $\mathrm{H}$ & 3.57159721 & 3.80347328 & 0.01363388 \\
\hline $\mathrm{H}$ & 1.01647968 & 4.34207893 & -0.17559639 \\
\hline $\mathrm{H}$ & -1.50944336 & 4.96094341 & -0.4355464 \\
\hline $\mathrm{H}$ & -3.79277116 & 3.56620757 & -0.7983526 \\
\hline $\mathrm{H}$ & -4.40759293 & 0.99537649 & -1.0093552 \\
\hline $\mathrm{H}$ & -4.97332412 & -1.58750483 & -1.1614750 \\
\hline $\mathrm{H}$ & -3.53253678 & -3.87303423 & -1.0021948 \\
\hline $\mathrm{H}$ & -0.98321137 & -4.43513556 & -0.6797445 \\
\hline $\mathrm{H}$ & 3.85294062 & -3.62519952 & -0.2190012 \\
\hline $\mathrm{H}$ & 0.20380862 & 1.28914492 & 3.81883960 \\
\hline $\mathrm{H}$ & 1.52328708 & 1.01419066 & $2.6307011<$ \\
\hline $\mathrm{H}$ & 0.28560859 & 2.24857101 & 2.32485878 \\
\hline $\mathrm{O}$ & 0.20503364 & 0.03091096 & -2.22667081 \\
\hline $\mathrm{C}$ & -2.21200862 & -0.81230877 & -4.8702856 \\
\hline $\mathrm{C}$ & -3.07180218 & 0.43649680 & -4.8634068 \\
\hline $\mathrm{O}$ & -2.62364256 & 1.56395777 & -5.0323645 \\
\hline $\mathrm{O}$ & -4.36390478 & 0.18812715 & -4.6658137 \\
\hline $\mathrm{H}$ & -1.71142212 & -0.89133600 & -5.8412251 \\
\hline $\mathrm{H}$ & -2.80338160 & -1.71052917 & -4.6848279 \\
\hline $\mathrm{H}$ & -1.43261007 & -0.71259349 & -4.1066492 \\
\hline $\mathrm{N}$ & -5.87839369 & 2.47679449 & -4.8412775 \\
\hline $\mathrm{C}$ & -5.42517287 & 3.66243370 & -5.3828374 \\
\hline $\mathrm{C}$ & -6.47432330 & 4.53544601 & -5.5609986 \\
\hline $\mathrm{N}$ & -7.59057740 & 3.85170388 & -5.1075659 \\
\hline $\mathrm{C}$ & -7.18175975 & 2.62686019 & -4.6926221 \\
\hline $\mathrm{C}$ & -6.57820428 & 5.91444247 & -6.1326082 \\
\hline $\mathrm{H}$ & -6.96675877 & 6.63658975 & -5.4019618 \\
\hline $\mathrm{H}$ & -5.59161716 & 6.26466556 & -6.4494295 \\
\hline
\end{tabular}




$\begin{array}{lrrr}\mathrm{H} & -7.23959715 & 5.94193657 & -7.00806151 \\ \mathrm{H} & -8.55052740 & 4.20426243 & -5.10223674 \\ \mathrm{H} & -7.86093895 & 1.88442756 & -4.29664380 \\ \mathrm{H} & -4.88183877 & 1.05949940 & -4.69828658 \\ \mathrm{H} & -4.37569239 & 3.79573041 & -5.60569890 \\ \mathrm{O} & -0.19821664 & 2.57658517 & -3.68671918 \\ \mathrm{H} & 0.09117785 & 1.74744230 & -3.26758854 \\ \mathrm{H} & -0.97907824 & 2.30415414 & -4.20300292\end{array}$

\section{Doublet; Basis set B1; Model B; Fe+HOOH}

96

$\begin{array}{llll}\mathrm{O} & -10.13146837 & 4.71973034 & -4.72350041 \\ \mathrm{C} & -10.68407198 & 5.69165271 & -4.20046680 \\ \mathrm{~N} & -11.76230848 & 5.54193177 & -3.38663291 \\ \mathrm{C} & -12.34891446 & 4.24962761 & -3.06916221 \\ \mathrm{C} & -10.19253925 & 7.11059095 & -4.45282246 \\ \mathrm{H} & -12.19053155 & 6.37092064 & -3.00029663 \\ \mathrm{H} & -13.38674952 & 4.19558646 & -3.41633076 \\ \mathrm{H} & -12.32421850 & 4.06498019 & -1.98949745 \\ \mathrm{H} & -11.76051175 & 3.48704757 & -3.57942524 \\ \mathrm{H} & -10.77807125 & 7.87867525 & -3.93847097 \\ \mathrm{H} & -10.21967136 & 7.30237174 & -5.52963163 \\ \mathrm{H} & -9.14940790 & 7.18502065 & -4.12997344 \\ \mathrm{C} & -4.03747678 & -1.69496100 & -0.99991067 \\ \mathrm{C} & -3.13189117 & -0.58526908 & -0.82847560 \\ \mathrm{~N} & -1.84678212 & -1.04225906 & -0.58978639 \\ \mathrm{C} & -1.94626694 & -2.41956420 & -0.59592024 \\ \mathrm{C} & -3.30565646 & -2.83055357 & -0.84845785 \\ \mathrm{C} & -3.51750420 & 0.74705247 & -0.91197012 \\ \mathrm{C} & -2.60868413 & 1.81407275 & -0.75725054 \\ \mathrm{~N} & -1.23886852 & 1.73107200 & -0.51051163 \\ \mathrm{C} & -0.77373749 & 3.04388917 & -0.43874705 \\ \mathrm{C} & -1.87100377 & 3.95223723 & -0.65086177 \\ \mathrm{C} & -2.99056598 & 3.20019330 & -0.85216230 \\ \mathrm{C} & 0.55418323 & 3.45339981 & -0.21439840 \\ \mathrm{C} & 1.61118604 & 2.54375962 & -0.06934629\end{array}$




\begin{tabular}{|c|c|c|c|}
\hline $\mathrm{N}$ & 1.51025309 & 1.15640009 & -0.07707268 \\
\hline C & 2.80806159 & 0.67842470 & 0.07894768 \\
\hline $\mathrm{C}$ & 3.72322151 & 1.78774375 & 0.18291790 \\
\hline C & 2.98872876 & 2.93215131 & 0.09673230 \\
\hline $\mathrm{Fe}$ & -0.19459510 & 0.06543119 & -0.13386902 \\
\hline S & -0.58263349 & -0.02864518 & 2.07607786 \\
\hline $\mathrm{C}$ & 0.00021084 & 1.53667007 & 2.82541062 \\
\hline $\mathrm{C}$ & 3.21026791 & -0.66719322 & 0.11795199 \\
\hline $\mathrm{C}$ & 2.29665566 & -1.72301548 & 0.00070312 \\
\hline $\mathrm{N}$ & 0.92216064 & -1.61446234 & -0.18872989 \\
\hline $\mathrm{C}$ & 0.44074076 & -2.91424634 & -0.23059657 \\
\hline $\mathrm{C}$ & 1.52944178 & -3.84655083 & -0.07766793 \\
\hline $\mathrm{C}$ & 2.66969795 & -3.11470480 & 0.05812577 \\
\hline $\mathrm{C}$ & -0.89278731 & -3.30450239 & -0.40597018 \\
\hline $\mathrm{H}$ & 4.26181007 & -0.89506518 & 0.24729708 \\
\hline $\mathrm{H}$ & 1.42078152 & -4.92375663 & -0.08354317 \\
\hline $\mathrm{H}$ & 4.79517215 & 1.68860727 & 0.29977064 \\
\hline $\mathrm{H}$ & 3.33895786 & 3.95629496 & 0.12708664 \\
\hline $\mathrm{H}$ & 0.77100878 & 4.51505047 & -0.1867477 \\
\hline $\mathrm{H}$ & -1.78130755 & 5.03132436 & -0.6600487 \\
\hline $\mathrm{H}$ & -3.99584609 & 3.54338364 & -1.0614745 \\
\hline $\mathrm{H}$ & -4.55201795 & 0.98395733 & -1.13004314 \\
\hline $\mathrm{H}$ & -5.09178546 & -1.59569211 & -1.22286089 \\
\hline $\mathrm{H}$ & -3.63460209 & -3.85977119 & -0.9141354 \\
\hline $\mathrm{H}$ & -1.10826727 & -4.36755770 & -0.4184080 \\
\hline $\mathrm{H}$ & 3.68348645 & -3.47256081 & 0.18724309 \\
\hline $\mathrm{H}$ & -0.31603376 & 1.52025386 & 3.87413508 \\
\hline $\mathrm{H}$ & 1.08861370 & 1.61486626 & 2.78324618 \\
\hline $\mathrm{H}$ & -0.44817654 & 2.40345167 & 2.33520218 \\
\hline $\mathrm{C}$ & -2.11513100 & -0.95152767 & -5.10252477 \\
\hline $\mathrm{C}$ & -3.02302918 & 0.25849227 & -5.25216266 \\
\hline $\mathrm{O}$ & -2.71984831 & 1.26293990 & -5.8720364 \\
\hline $\mathrm{O}$ & -4.19357509 & 0.08701598 & -4.6272909 \\
\hline $\mathrm{H}$ & -1.16810073 & -0.76457589 & -5.6106210 \\
\hline $\mathrm{H}$ & -2.59829936 & -1.83689639 & -5.529952 \\
\hline $\mathrm{H}$ & -1.93567966 & -1.15685566 & -4.0415789 \\
\hline $\mathrm{N}$ & -5.87036206 & 2.26766163 & -5.0308165 \\
\hline $\mathrm{C}$ & -5.45022926 & 3.37178908 & -5.7432374 \\
\hline $\mathrm{C}$ & -6.47174438 & 4.28860855 & -5.8502586 \\
\hline $\mathrm{N}$ & -7.53880460 & 3.71678085 & -5.1790273 \\
\hline $\mathrm{C}$ & -7.12899526 & 2.50969378 & -4.7116412 \\
\hline $\mathrm{C}$ & -6.55382468 & 5.63607118 & -6.5002112 \\
\hline $\mathrm{H}$ & -6.75257622 & 6.43402152 & -5.7719909 \\
\hline $\mathrm{H}$ & -5.60610839 & 5.86809613 & -6.9943245 \\
\hline $\mathrm{H}$ & -7.34566158 & 5.67802092 & -7.2590874 \\
\hline
\end{tabular}




$\begin{array}{lrrr}\mathrm{H} & -8.47039187 & 4.12457452 & -5.06843704 \\ \mathrm{H} & -7.77756485 & 1.84782363 & -4.15418051 \\ \mathrm{H} & -4.77801173 & 0.89673173 & -4.78006136 \\ \mathrm{H} & -4.44184420 & 3.41757827 & -6.13089735 \\ \mathrm{~N} & -0.57759802 & -1.95435012 & 4.95066220 \\ \mathrm{C} & 0.66123917 & -2.37611426 & 5.57525612 \\ \mathrm{C} & -1.78607488 & -2.48305625 & 5.30300965 \\ \mathrm{C} & -2.98580264 & -1.99449824 & 4.45653111 \\ \mathrm{~N} & -3.08818820 & -0.54502013 & 4.33077352 \\ \mathrm{C} & -3.43990535 & 0.25497629 & 5.38236264 \\ \mathrm{C} & -3.45337064 & 1.75098012 & 5.08501261 \\ \mathrm{C} & -3.02378075 & -2.68343069 & 3.08960824 \\ \mathrm{O} & -1.93184955 & -3.33587768 & 6.16983249 \\ \mathrm{O} & -3.73624254 & -0.18755714 & 6.48476373 \\ \mathrm{H} & -0.52485937 & -1.35014007 & 4.13963843 \\ \mathrm{H} & 1.30558726 & -2.91456495 & 4.86785783 \\ \mathrm{H} & 1.21766618 & -1.51723171 & 5.96997005 \\ \mathrm{H} & 0.40158697 & -3.04426530 & 6.39794602 \\ \mathrm{H} & -3.84891153 & -2.32080328 & 5.04433126 \\ \mathrm{H} & -2.16542823 & -2.41528707 & 2.46733314 \\ \mathrm{H} & -3.01508338 & -3.76868558 & 3.23305546 \\ \mathrm{H} & -3.93717970 & -2.41313971 & 2.54892868 \\ \mathrm{H} & -2.77667664 & -0.12513211 & 3.46236726 \\ \mathrm{H} & -3.25614727 & 1.99671190 & 4.03655675 \\ \mathrm{H} & -4.42952375 & 2.15522598 & 5.36953658 \\ \mathrm{H} & -2.70364563 & 2.24399469 & 5.71324609\end{array}$

\section{Doublet; Basis set B1; Model B; FeHOOH}

100

$\begin{array}{lccl}\mathrm{O} & -10.27708700 & 4.49546998 & -5.17994838 \\ \mathrm{C} & -11.10259823 & 5.36078734 & -4.87105973 \\ \mathrm{~N} & -12.32409659 & 5.03316780 & -4.37645351 \\ \mathrm{C} & -12.76239344 & 3.66109586 & -4.17337375 \\ \mathrm{C} & -10.79681172 & 6.84194599 & -5.03871064 \\ \mathrm{H} & -12.96835148 & 5.77938441 & -4.15713229 \\ \mathrm{H} & -13.65155787 & 3.44313113 & -4.77509187 \\ \mathrm{H} & -12.99208286 & 3.47721518 & -3.11802546 \\ \mathrm{H} & -11.94910971 & 3.00580601 & -4.48511702 \\ \mathrm{H} & -11.61689026 & 7.49903396 & -4.73407438 \\ \mathrm{H} & -10.55877804 & 7.03285538 & -6.08950504 \\ \mathrm{H} & -9.90873395 & 7.08821091 & -4.44821909\end{array}$




\begin{tabular}{|c|c|c|c|}
\hline $\mathrm{C}$ & -4.24624691 & -1.33075699 & -0.89914580 \\
\hline C & -3.20767717 & -0.33475028 & -0.78729114 \\
\hline $\mathrm{N}$ & -1.97687871 & -0.88384939 & -0.63779145 \\
\hline C & -2.21499911 & -2.22143452 & -0.6637950 \\
\hline $\mathrm{C}$ & -3.61666228 & -2.52884768 & -0.81103680 \\
\hline $\mathrm{C}$ & -3.40957730 & 1.00353394 & -0.8753292 \\
\hline $\mathrm{C}$ & -2.47682439 & 2.02840348 & -0.7345208 \\
\hline $\mathrm{N}$ & -1.13042484 & 1.88212072 & -0.5284152 \\
\hline $\mathrm{C}$ & -0.60495935 & 3.15564495 & -0.4684802 \\
\hline C & -1.65887098 & 4.12713518 & -0.6293577 \\
\hline $\mathrm{C}$ & -2.81506078 & 3.42953721 & -0.8044638 \\
\hline $\mathrm{C}$ & 0.74211136 & 3.45848879 & -0.29811869 \\
\hline $\mathrm{C}$ & 1.75271674 & 2.49194228 & -0.20312035 \\
\hline $\mathrm{N}$ & 1.57139573 & 1.11672680 & -0.29532867 \\
\hline $\mathrm{C}$ & 2.83101915 & 0.54270877 & -0.1498036 \\
\hline $\mathrm{C}$ & 3.81040004 & 1.58325356 & 0.0206786 \\
\hline $\mathrm{C}$ & 3.14907296 & 2.77859040 & -0.0109254 \\
\hline $\mathrm{Fe}$ & -0.20035162 & 0.13201885 & -0.37362003 \\
\hline S & -0.46184634 & -0.00427837 & 1.85762887 \\
\hline $\mathrm{C}$ & 0.27540144 & 1.47676989 & 2.64243131 \\
\hline $\mathrm{C}$ & 3.11701142 & -0.82889761 & -0.1891666 \\
\hline $\mathrm{C}$ & 2.12297897 & -1.81333356 & -0.3294554 \\
\hline $\mathrm{N}$ & 0.75719239 & -1.60503106 & -0.4467761 \\
\hline $\mathrm{C}$ & 0.16340453 & -2.84827559 & -0.5237771 \\
\hline $\mathrm{C}$ & 1.18532907 & -3.86717392 & -0.4724544 \\
\hline $\mathrm{C}$ & 2.38609888 & -3.23281480 & -0.3556452 \\
\hline $\mathrm{C}$ & -1.21277460 & -3.13463609 & -0.6158057 \\
\hline $\mathrm{H}$ & 4.14860831 & -1.14756959 & -0.0925820 \\
\hline $\mathrm{H}$ & 0.99416622 & -4.93192515 & -0.51945484 \\
\hline $\mathrm{H}$ & 4.87257768 & 1.41251491 & 0.14394352 \\
\hline $\mathrm{H}$ & 3.56575625 & 3.77377316 & 0.0813855 \\
\hline $\mathrm{H}$ & 1.03331040 & 4.50218117 & -0.24425599 \\
\hline $\mathrm{H}$ & -1.51335407 & 5.19998263 & -0.6235388 \\
\hline $\mathrm{H}$ & -3.81272369 & 3.81063001 & -0.9803871 \\
\hline $\mathrm{H}$ & -4.42932456 & 1.33636202 & -1.0523684 \\
\hline $\mathrm{H}$ & -5.29742625 & -1.11377794 & -1.0351351 \\
\hline $\mathrm{H}$ & -4.02917985 & -3.52837555 & -0.8535975 \\
\hline $\mathrm{H}$ & -1.47227312 & -4.18890568 & -0.6539411 \\
\hline $\mathrm{H}$ & 3.37181883 & -3.67609182 & -0.2892443 \\
\hline $\mathrm{H}$ & 0.04995589 & 1.41357442 & 3.7123775 \\
\hline $\mathrm{H}$ & 1.35941062 & 1.50082980 & 2.5102250 \\
\hline $\mathrm{H}$ & -0.15966955 & 2.39554998 & 2.2429439 \\
\hline $\mathrm{O}$ & -0.11086718 & 0.22173625 & -2.5987022 \\
\hline $\mathrm{C}$ & -2.36880205 & -0.81664983 & -4.282222 \\
\hline C & -2.96889640 & 0.54947371 & -4.092523 \\
\hline
\end{tabular}




\begin{tabular}{|c|c|c|c|}
\hline $\mathrm{O}$ & -2.29861573 & 1.53092345 & -3.77005099 \\
\hline $\mathrm{O}$ & -4.27543144 & 0.55911173 & -4.28285540 \\
\hline $\mathrm{H}$ & -1.42273692 & -0.73662146 & -4.82247498 \\
\hline $\mathrm{H}$ & -3.05605411 & -1.47311306 & -4.81986347 \\
\hline $\mathrm{H}$ & -2.14566566 & -1.26091670 & -3.30908541 \\
\hline $\mathrm{N}$ & -5.76818357 & 2.78754101 & -4.22189856 \\
\hline C & -5.35345463 & 3.94396650 & -4.84334953 \\
\hline $\mathrm{C}$ & -6.43788207 & 4.67689805 & -5.26230590 \\
\hline $\mathrm{N}$ & -7.54193578 & 3.94180779 & -4.8757602 \\
\hline $\mathrm{C}$ & -7.08950568 & 2.81735760 & -4.2641266 \\
\hline $\mathrm{C}$ & -6.49563791 & 5.98397722 & -5.9837647 \\
\hline $\mathrm{H}$ & -7.01758976 & 6.75719426 & -5.4041627 \\
\hline $\mathrm{H}$ & -5.48036832 & 6.34282710 & -6.1737758 \\
\hline $\mathrm{H}$ & -7.00670939 & 5.89885770 & -6.9514280 \\
\hline $\mathrm{H}$ & -8.52282967 & 4.19198663 & -5.0344507 \\
\hline $\mathrm{H}$ & -7.75026401 & 2.05499840 & -3.8759135 \\
\hline $\mathrm{H}$ & -4.71133563 & 1.46662116 & -4.14236162 \\
\hline $\mathrm{H}$ & -4.30218496 & 4.17201619 & -4.95489811 \\
\hline $\mathrm{O}$ & 1.12144701 & 0.77248227 & -3.13574791 \\
\hline $\mathrm{H}$ & 1.53728950 & 1.06086592 & -2.29551723 \\
\hline $\mathrm{H}$ & -0.79012668 & 0.85312698 & -2.96569065 \\
\hline $\mathrm{N}$ & -0.39503662 & -2.00727572 & 4.67811430 \\
\hline $\mathrm{C}$ & 0.85356454 & -2.51860167 & 5.20918719 \\
\hline C & -1.60672375 & -2.48059381 & 5.0922048 \\
\hline $\mathrm{C}$ & -2.82461404 & -1.90326917 & 4.3323063 \\
\hline $\mathrm{N}$ & -2.85244061 & -0.44748977 & $4.2485957 €$ \\
\hline $\mathrm{C}$ & -3.09570620 & 0.34026275 & 5.33886482 \\
\hline C & -3.01660628 & 1.84231157 & 5.0846058 \\
\hline $\mathrm{C}$ & -2.98348753 & -2.55358528 & 2.9559344 \\
\hline $\mathrm{O}$ & -1.74728427 & -3.34461355 & 5.9491776 \\
\hline $\mathrm{O}$ & -3.37425216 & -0.11388410 & 6.4416249 \\
\hline $\mathrm{H}$ & -0.35796189 & -1.40143722 & 3.8661291 \\
\hline $\mathrm{H}$ & 1.39809795 & -3.11180928 & 4.46234803 \\
\hline $\mathrm{H}$ & 1.50226450 & -1.70058174 & 5.54489903 \\
\hline $\mathrm{H}$ & 0.61091336 & -3.15792424 & 6.05954923 \\
\hline $\mathrm{H}$ & -3.66853403 & -2.19571282 & 4.9640650 \\
\hline $\mathrm{H}$ & -2.14689179 & -2.32265205 & 2.2913502 \\
\hline $\mathrm{H}$ & -3.03430018 & -3.64078678 & 3.0753060 \\
\hline $\mathrm{H}$ & -3.90735023 & -2.21378038 & 2.4758551 \\
\hline $\mathrm{H}$ & -2.52938609 & -0.02276040 & 3.3857859 \\
\hline $\mathrm{H}$ & -2.83804506 & 2.10588910 & 4.0372540 \\
\hline $\mathrm{H}$ & -3.95266501 & 2.30298795 & 5.4148718 \\
\hline $\mathrm{H}$ & -2.21401837 & 2.26346771 & 5.6995744 \\
\hline
\end{tabular}




\section{Doublet; Basis set B1; Model B; Transition State 1}

100

$\begin{array}{llll}\mathrm{O} & -10.13818606 & 4.54801001 & -4.91859294 \\ \mathrm{C} & -10.86146774 & 5.47197202 & -4.51632589 \\ \mathrm{~N} & -12.01505587 & 5.23646807 & -3.85223958 \\ \mathrm{C} & -12.52351438 & 3.90271232 & -3.56300949 \\ \mathrm{C} & -10.48732271 & 6.92601792 & -4.72025541 \\ \mathrm{H} & -12.57121985 & 6.02969448 & -3.56618544 \\ \mathrm{H} & -13.50549619 & 3.75535173 & -4.02439789 \\ \mathrm{H} & -12.60916723 & 3.74735847 & -2.48235413 \\ \mathrm{H} & -11.82020792 & 3.18109438 & -3.97784221 \\ \mathrm{H} & -11.24996499 & 7.62817534 & -4.37223056 \\ \mathrm{H} & -10.30482895 & 7.10028545 & -5.78496464 \\ \mathrm{H} & -9.55396854 & 7.13251974 & -4.18550006 \\ \mathrm{C} & -4.14658574 & -1.47953279 & -1.06058673 \\ \mathrm{C} & -3.16329650 & -0.42804106 & -0.91278077 \\ \mathrm{~N} & -1.91859734 & -0.94695050 & -0.71832590 \\ \mathrm{C} & -2.08515263 & -2.29823784 & -0.72449895 \\ \mathrm{C} & -3.47127081 & -2.65248945 & -0.93525371 \\ \mathrm{C} & -3.44886826 & 0.91766238 & -0.94758131 \\ \mathrm{C} & -2.54613315 & 1.97019824 & -0.75732379 \\ \mathrm{~N} & -1.19463776 & 1.85758116 & -0.56336283 \\ \mathrm{C} & -0.69351784 & 3.13222332 & -0.46881009 \\ \mathrm{C} & -1.77330244 & 4.08695944 & -0.58758038 \\ \mathrm{C} & -2.91674775 & 3.36831226 & -0.76952261 \\ \mathrm{C} & 0.65164600 & 3.46949306 & -0.28837268 \\ \mathrm{C} & 1.69717813 & 2.54219529 & -0.19368883 \\ \mathrm{~N} & 1.57197790 & 1.16625951 & -0.28493931 \\ \mathrm{C} & 2.83998903 & 0.64237144 & -0.09137142 \\ \mathrm{C} & 3.78125991 & 1.72090496 & 0.09591834 \\ \mathrm{C} & 3.07928221 & 2.88901011 & 0.03320541 \\ \mathrm{Fe} & -0.18351511 & 0.12610543 & -0.48819243 \\ \mathrm{~S} & -0.55434073 & 0.01240685 & 1.84356336 \\ \mathrm{C} & 0.19953834 & 1.47014757 & 2.66501806 \\ \mathrm{C} & 3.17852516 & -0.71947992 & -0.05193530 \\ \mathrm{C} & 2.21562663 & -1.74464187 & -0.17880670 \\ \mathrm{~N} & 0.85236847 & -1.59745699 & -0.36826614 \\ \mathrm{C} & 0.30249280 & -2.86026177 & -0.39524194 \\ \mathrm{C} & 1.35601332 & -3.84053822 & -0.22785652 \\ \mathrm{C} & 2.52685430 & -3.15752910 & -0.09861074 \\ & -1.05863440 & -3.19815563 & -0.55124270 \\ \mathrm{H} & & -0.99815286 & 0.10447386\end{array}$




\begin{tabular}{|c|c|c|c|}
\hline $\mathrm{H}$ & 1.20272841 & -4.91268147 & -0.21091554 \\
\hline $\mathrm{H}$ & 4.84343884 & 1.58763350 & 0.26118341 \\
\hline $\mathrm{H}$ & 3.45238330 & 3.90057342 & 0.13742253 \\
\hline $\mathrm{H}$ & 0.90522351 & 4.52220065 & -0.21386362 \\
\hline $\mathrm{H}$ & -1.65451594 & 5.16246211 & -0.53673369 \\
\hline $\mathrm{H}$ & -3.92724354 & 3.73727564 & -0.89881049 \\
\hline $\mathrm{H}$ & -4.48670366 & 1.20093474 & -1.10396851 \\
\hline $\mathrm{H}$ & -5.20532993 & -1.31912488 & -1.22218928 \\
\hline $\mathrm{H}$ & -3.85173198 & -3.66545485 & -0.97343660 \\
\hline $\mathrm{H}$ & -1.29774296 & -4.25729891 & -0.5484963 \\
\hline $\mathrm{H}$ & 3.52220364 & -3.56024568 & 0.04452724 \\
\hline $\mathrm{H}$ & 0.00810709 & 1.38980456 & 3.74083349 \\
\hline $\mathrm{H}$ & 1.28076197 & 1.50349507 & 2.50606015 \\
\hline $\mathrm{H}$ & -0.23937991 & 2.40376474 & 2.30204036 \\
\hline $\mathrm{O}$ & -0.14241603 & 0.10318809 & -2.36960188 \\
\hline $\mathrm{C}$ & -2.26230259 & -0.87587496 & -4.47923798 \\
\hline $\mathrm{C}$ & -2.95990768 & 0.42382525 & -4.2584538 \\
\hline $\mathrm{O}$ & -2.26538871 & 1.44060725 & -3.7907928 \\
\hline $\mathrm{O}$ & -4.14874916 & 0.56027797 & -4.5732708 \\
\hline $\mathrm{H}$ & -1.72863758 & -0.81920862 & -5.4374780 \\
\hline $\mathrm{H}$ & -2.99282864 & -1.68369442 & -4.5337532 \\
\hline $\mathrm{H}$ & -1.51410933 & -1.06145624 & -3.7038036 \\
\hline $\mathrm{N}$ & -5.73850869 & 2.57310649 & -4.7097423 \\
\hline $\mathrm{C}$ & -5.32772607 & 3.69261814 & -5.3905261 \\
\hline $\mathrm{C}$ & -6.42674331 & 4.47737849 & -5.6106717 \\
\hline $\mathrm{N}$ & -7.49590272 & 3.80301827 & -5.0439908 \\
\hline $\mathrm{C}$ & -7.05035654 & 2.65227200 & -4.5151886 \\
\hline $\mathrm{C}$ & -6.50579096 & 5.80536618 & -6.2811338 \\
\hline $\mathrm{H}$ & -6.84972834 & 6.58821748 & -5.5943377 \\
\hline $\mathrm{H}$ & -5.51580759 & 6.09084611 & -6.6444360 \\
\hline $\mathrm{H}$ & -7.18969329 & 5.79018115 & -7.1379812 \\
\hline $\mathrm{H}$ & -8.47896128 & 4.13089221 & $-5.030784 \subseteq$ \\
\hline $\mathrm{H}$ & -7.66205459 & 1.91451877 & -4.0199770 \\
\hline $\mathrm{H}$ & -5.07148603 & 1.75698961 & -4.49768330 \\
\hline $\mathrm{H}$ & -4.29240394 & 3.84101498 & -5.65562397 \\
\hline $\mathrm{O}$ & 0.56517341 & 1.20992539 & -3.0221872 \\
\hline $\mathrm{H}$ & 1.26157080 & 1.38924366 & -2.3619356 \\
\hline $\mathrm{H}$ & -1.40998531 & 1.14028633 & -3.3759841 \\
\hline $\mathrm{N}$ & -0.50540623 & -1.93024044 & 4.7063115 \\
\hline C & 0.74370640 & -2.36868728 & 5.2962899 \\
\hline $\mathrm{C}$ & -1.70494101 & -2.46232073 & 5.0792417 \\
\hline $\mathrm{C}$ & -2.91787639 & -1.96706821 & 4.2548835 \\
\hline $\mathrm{N}$ & -3.01934439 & -0.51667771 & 4.1396309 \\
\hline $\mathrm{C}$ & -3.35189482 & 0.27483581 & 5.2009899 \\
\hline $\mathrm{C}$ & -3.24683327 & 1.77535291 & 4.9420399 \\
\hline
\end{tabular}




$\begin{array}{llll}\mathrm{C} & -2.97719882 & -2.64739479 & 2.88442428 \\ \mathrm{O} & -1.83785502 & -3.31120465 & 5.95482083 \\ \mathrm{O} & -3.73130750 & -0.16534566 & 6.28098230 \\ \mathrm{H} & -0.46214927 & -1.35786922 & 3.86520035 \\ \mathrm{H} & 1.34108895 & -2.95758584 & 4.58708639 \\ \mathrm{H} & 1.34360829 & -1.51175866 & 5.62600426 \\ \mathrm{H} & 0.50073842 & -2.99291780 & 6.15790470 \\ \mathrm{H} & -3.77322726 & -2.29165089 & 4.85420920 \\ \mathrm{H} & -2.12812471 & -2.37326650 & 2.25348261 \\ \mathrm{H} & -2.96961529 & -3.73433744 & 3.01965820 \\ \mathrm{H} & -3.89684965 & -2.36780660 & 2.35935960 \\ \mathrm{H} & -2.56530611 & -0.09549410 & 3.33039226 \\ \mathrm{H} & -3.00522402 & 2.02750836 & 3.90494706 \\ \mathrm{H} & -4.19527224 & 2.24654082 & 5.21831787 \\ \mathrm{H} & -2.47394619 & 2.19257856 & 5.59692705\end{array}$

\section{Doublet; Basis set B1; Model B; Compound 0}

100

$\begin{array}{llll}\mathrm{O} & -10.20287761 & 4.64936010 & -5.23922912 \\ \mathrm{C} & -10.97550796 & 5.52606632 & -4.82386738 \\ \mathrm{~N} & -12.18703780 & 5.22173650 & -4.30687902 \\ \mathrm{C} & -12.70429954 & 3.86416625 & -4.20495793 \\ \mathrm{C} & -10.60188241 & 6.99478163 & -4.84469066 \\ \mathrm{H} & -12.78084241 & 5.98081048 & -4.00413211 \\ \mathrm{H} & -13.62781063 & 3.75779975 & -4.78360994 \\ \mathrm{H} & -12.90493505 & 3.60444771 & -3.16018418 \\ \mathrm{H} & -11.94927679 & 3.18921025 & -4.60726657 \\ \mathrm{H} & -11.40536090 & 7.65674927 & -4.50994471 \\ \mathrm{H} & -10.31377707 & 7.27393924 & -5.86266435 \\ \mathrm{H} & -9.73110143 & 7.15025871 & -4.19869987 \\ \mathrm{C} & -4.15888603 & -1.48429995 & -0.87853543 \\ \mathrm{C} & -3.17410500 & -0.42887731 & -0.75737141 \\ \mathrm{~N} & -1.92678309 & -0.97343859 & -0.57763247 \\ \mathrm{C} & -2.09260188 & -2.33209573 & -0.58156131 \\ \mathrm{C} & -3.48973734 & -2.66362694 & -0.76345265 \\ \mathrm{C} & -3.47651279 & 0.93142864 & -0.80996845 \\ \mathrm{C} & -2.53849359 & 1.96202176 & -0.65655641 \\ \mathrm{~N} & -1.17695379 & 1.81255554 & -0.50699753 \\ \mathrm{C} & -0.64586523 & 3.07922394 & -0.39440406 \\ \mathrm{C} & -1.70561538 & 4.05780526 & -0.47107641 \\ \mathrm{C} & -2.87072276 & 3.36768227 & -0.63575207 \\ \mathrm{C} & 0.71616638 & 3.38207613 & -0.25871884 \\ \mathrm{C} & 1.74667852 & 2.43895736 & -0.20159833\end{array}$




\begin{tabular}{|c|c|c|c|}
\hline $\mathrm{N}$ & 1.59726454 & 1.07127409 & -0.25107557 \\
\hline $\mathrm{C}$ & 2.86493497 & 0.53771729 & -0.11996280 \\
\hline $\mathrm{C}$ & 3.83209257 & 1.60636447 & -0.00434887 \\
\hline C & 3.14373262 & 2.77972599 & -0.05527355 \\
\hline $\mathrm{Fe}$ & -0.16617568 & 0.05476245 & -0.42701234 \\
\hline S & -0.44018610 & -0.04936342 & 1.92035398 \\
\hline $\mathrm{C}$ & 0.15953551 & 1.50513883 & 2.68852784 \\
\hline $\mathrm{C}$ & 3.19715486 & -0.82185595 & -0.07147495 \\
\hline $\mathrm{C}$ & 2.22109921 & -1.84047325 & -0.16136211 \\
\hline $\mathrm{N}$ & 0.85494586 & -1.68487098 & -0.31903296 \\
\hline $\mathrm{C}$ & 0.29693701 & -2.94466098 & -0.32385257 \\
\hline $\mathrm{C}$ & 1.34757393 & -3.93191919 & -0.17609211 \\
\hline $\mathrm{C}$ & 2.52484644 & -3.25562645 & -0.07862260 \\
\hline $\mathrm{C}$ & -1.07058236 & -3.26716657 & -0.44111848 \\
\hline $\mathrm{H}$ & 4.23562777 & -1.10774761 & 0.04854470 \\
\hline $\mathrm{H}$ & 1.18741969 & -5.00278011 & -0.14849161 \\
\hline $\mathrm{H}$ & 4.89929710 & 1.45864616 & 0.10662564 \\
\hline $\mathrm{H}$ & 3.52964101 & 3.78983346 & 0.00651274 \\
\hline $\mathrm{H}$ & 0.99217277 & 4.43013309 & -0.19782551 \\
\hline $\mathrm{H}$ & -1.56287296 & 5.12940971 & -0.40222688 \\
\hline $\mathrm{H}$ & -3.87495280 & 3.76408497 & -0.72694791 \\
\hline $\mathrm{H}$ & -4.51616940 & 1.21467496 & -0.94036616 \\
\hline $\mathrm{H}$ & -5.22034129 & -1.32409002 & -1.02315179 \\
\hline $\mathrm{H}$ & -3.88643008 & -3.67078525 & -0.79489729 \\
\hline $\mathrm{H}$ & -1.33548524 & -4.31936032 & -0.43440953 \\
\hline $\mathrm{H}$ & 3.52082486 & -3.66353481 & 0.04376109 \\
\hline $\mathrm{H}$ & 0.00770997 & 1.42765737 & 3.77070765 \\
\hline $\mathrm{H}$ & 1.22550800 & 1.65599951 & 2.49766107 \\
\hline $\mathrm{H}$ & -0.39036401 & 2.37571643 & 2.32003486 \\
\hline $\mathrm{O}$ & -0.09872938 & -0.08364618 & -2.29880453 \\
\hline $\mathrm{C}$ & -2.40236586 & -0.77394456 & -4.58081646 \\
\hline $\mathrm{C}$ & -3.17177834 & 0.49127607 & -4.36754350 \\
\hline $\mathrm{O}$ & -2.52604682 & 1.56862947 & -3.97944166 \\
\hline $\mathrm{O}$ & -4.38790871 & 0.53956430 & -4.60566110 \\
\hline $\mathrm{H}$ & -1.81176477 & -0.67540634 & -5.50080777 \\
\hline $\mathrm{H}$ & -3.08952938 & -1.61367676 & -4.68516371 \\
\hline $\mathrm{H}$ & -1.69386962 & -0.94118835 & -3.75967412 \\
\hline $\mathrm{N}$ & -5.81907156 & 2.67288443 & -4.78744023 \\
\hline $\mathrm{C}$ & -5.35737230 & 3.85626576 & -5.31372484 \\
\hline $\mathrm{C}$ & -6.44057755 & 4.65662203 & -5.56209896 \\
\hline $\mathrm{N}$ & -7.55062217 & 3.92609687 & -5.16760796 \\
\hline $\mathrm{C}$ & -7.14392179 & 2.73115657 & -4.71311403 \\
\hline $\mathrm{C}$ & -6.50922603 & 6.02892302 & -6.14044930 \\
\hline $\mathrm{H}$ & -6.96324756 & 6.74281044 & -5.44276386 \\
\hline $\mathrm{H}$ & -5.50191944 & 6.38297561 & -6.37252488 \\
\hline
\end{tabular}




$\begin{array}{lrrr}\mathrm{H} & -7.09616906 & 6.04689479 & -7.06628800 \\ \mathrm{H} & -8.53375202 & 4.24818855 & -5.22018177 \\ \mathrm{H} & -7.79015115 & 1.94667501 & -4.35144029 \\ \mathrm{H} & -5.19255642 & 1.81205120 & -4.59007570 \\ \mathrm{H} & -4.30277532 & 4.02936807 & -5.46261797 \\ \mathrm{O} & -0.07475178 & 1.16134412 & -3.09257348 \\ \mathrm{H} & 0.08436683 & 1.83673106 & -2.40661926 \\ \mathrm{H} & -1.57088327 & 1.37447513 & -3.65669161 \\ \mathrm{~N} & -0.26775566 & -1.97883232 & 4.78723685 \\ \mathrm{C} & 1.00488365 & -2.40931769 & 5.33077661 \\ \mathrm{C} & -1.45026943 & -2.51087289 & 5.20976066 \\ \mathrm{C} & -2.69726402 & -2.02223109 & 4.43381691 \\ \mathrm{~N} & -2.81166977 & -0.57342680 & 4.31399618 \\ \mathrm{C} & -3.10475007 & 0.22346875 & 5.38270724 \\ \mathrm{C} & -3.03929742 & 1.72301621 & 5.10577576 \\ \mathrm{C} & -2.81174308 & -2.71177766 & 3.07134925 \\ \mathrm{O} & -1.54624388 & -3.35927660 & 6.09078706 \\ \mathrm{O} & -3.42235104 & -0.21248630 & 6.48409656 \\ \mathrm{H} & -0.25844688 & -1.39846877 & 3.95073419 \\ \mathrm{H} & 1.58745405 & -2.97597428 & 4.59196330 \\ \mathrm{H} & 1.60344496 & -1.55035850 & 5.65817862 \\ \mathrm{H} & 0.79730841 & -3.05232620 & 6.18783285 \\ \mathrm{H} & -3.52545071 & -2.34712686 & 5.07017470 \\ \mathrm{H} & -1.99165448 & -2.43807163 & 2.40321765 \\ \mathrm{H} & -2.79355958 & -3.79762734 & 3.21390607 \\ \mathrm{H} & -3.75412629 & -2.43974850 & 2.58384571 \\ \mathrm{H} & -2.41011515 & -0.15645746 & 3.47585869 \\ \mathrm{H} & -2.86797986 & 1.96942173 & 4.05343622 \\ \mathrm{H} & -3.97584359 & 2.18163816 & 5.43778759 \\ \mathrm{H} & -2.23334096 & 2.15875463 & 5.70659219\end{array}$

\section{Doublet; Basis set B1; Model B; Transition State 2}

100

$\begin{array}{llll}\mathrm{O} & -10.24266886 & 4.60588757 & -5.40210140 \\ \mathrm{C} & -11.07074411 & 5.42728399 & -4.98956034 \\ \mathrm{~N} & -12.29246333 & 5.04392898 & -4.54471745 \\ \mathrm{C} & -12.73993385 & 3.65935332 & -4.52045862 \\ \mathrm{C} & -10.76358518 & 6.91474828 & -4.95354687 \\ \mathrm{H} & -12.93618835 & 5.75937821 & -4.23822730 \\ \mathrm{H} & -13.62415324 & 3.52549765 & -5.15291785 \\ \mathrm{H} & -12.98125465 & 3.34713146 & -3.49864914\end{array}$




\begin{tabular}{|c|c|c|c|}
\hline $\mathrm{H}$ & -11.92777291 & 3.04242443 & -4.90462940 \\
\hline $\mathrm{H}$ & -11.59706722 & 7.52797763 & -4.59884819 \\
\hline $\mathrm{H}$ & -10.48517700 & 7.24084365 & -5.96018769 \\
\hline $\mathrm{H}$ & -9.90112456 & 7.08303235 & -4.30040666 \\
\hline $\mathrm{C}$ & -4.22064798 & -1.38762525 & -0.80806200 \\
\hline C & -3.20910330 & -0.35697507 & -0.69844571 \\
\hline $\mathrm{N}$ & -1.97232877 & -0.93215408 & -0.53700135 \\
\hline $\mathrm{C}$ & -2.17265758 & -2.28681760 & -0.53962928 \\
\hline $\mathrm{C}$ & -3.57954333 & -2.58313269 & -0.70190719 \\
\hline $\mathrm{C}$ & -3.48302342 & 1.00965508 & -0.73849740 \\
\hline $\mathrm{C}$ & -2.52128950 & 2.01830005 & -0.58772961 \\
\hline $\mathrm{N}$ & -1.15602643 & 1.83617408 & -0.47127531 \\
\hline $\mathrm{C}$ & -0.59380618 & 3.09393320 & -0.35197807 \\
\hline $\mathrm{C}$ & -1.63410349 & 4.09196217 & -0.39096171 \\
\hline $\mathrm{C}$ & -2.81821727 & 3.42882418 & -0.53970433 \\
\hline C & 0.77918059 & 3.35653532 & -0.26298204 \\
\hline C & 1.78471577 & 2.38678543 & -0.23336396 \\
\hline $\mathrm{N}$ & 1.60109830 & 1.02275731 & -0.27215026 \\
\hline $\mathrm{C}$ & 2.85943668 & 0.45999864 & -0.16725509 \\
\hline $\mathrm{C}$ & 3.85369077 & 1.50577737 & -0.07991431 \\
\hline $\mathrm{C}$ & 3.19246001 & 2.69452224 & -0.12277240 \\
\hline $\mathrm{Fe}$ & -0.18714324 & 0.05158252 & -0.40242678 \\
\hline S & -0.39638469 & -0.06605711 & 1.92049252 \\
\hline $\mathrm{C}$ & 0.21732235 & 1.48662793 & 2.67812346 \\
\hline $\mathrm{C}$ & 3.16091578 & -0.90617801 & -0.12198470 \\
\hline $\mathrm{C}$ & 2.15830027 & -1.89947124 & -0.19831761 \\
\hline $\mathrm{N}$ & 0.79332026 & -1.70965113 & -0.34208760 \\
\hline $\mathrm{C}$ & 0.20397919 & -2.95698939 & -0.32683858 \\
\hline $\mathrm{C}$ & 1.23160152 & -3.96785215 & -0.18855909 \\
\hline $\mathrm{C}$ & 2.42675359 & -3.32043675 & -0.11230307 \\
\hline C & -1.17250518 & -3.24684174 & -0.41456479 \\
\hline $\mathrm{H}$ & 4.19369737 & -1.21718343 & -0.01770422 \\
\hline $\mathrm{H}$ & 1.04537665 & -5.03408309 & -0.15189538 \\
\hline $\mathrm{H}$ & 4.91926068 & 1.33309477 & 0.00682336 \\
\hline $\mathrm{H}$ & 3.60324462 & 3.69544990 & -0.07683832 \\
\hline $\mathrm{H}$ & 1.08585481 & 4.39620670 & -0.20720117 \\
\hline $\mathrm{H}$ & -1.46860710 & 5.15958896 & -0.31575904 \\
\hline $\mathrm{H}$ & -3.81379197 & 3.84962091 & -0.60891537 \\
\hline $\mathrm{H}$ & -4.51729503 & 1.31732213 & -0.85301053 \\
\hline $\mathrm{H}$ & -5.27947058 & -1.20043951 & -0.93749079 \\
\hline $\mathrm{H}$ & -4.00135482 & -3.58005107 & -0.72843737 \\
\hline $\mathrm{H}$ & -1.46333298 & -4.29191252 & -0.39806763 \\
\hline $\mathrm{H}$ & 3.41376281 & -3.75242977 & -0.00151766 \\
\hline $\mathrm{H}$ & 0.09085956 & 1.39820912 & 3.76247209 \\
\hline $\mathrm{H}$ & 1.27773816 & 1.64038964 & 2.46232653 \\
\hline
\end{tabular}




\begin{tabular}{|c|c|c|c|}
\hline $\mathrm{H}$ & -0.34523845 & 2.35682350 & 2.32944901 \\
\hline $\mathrm{O}$ & -0.18434007 & -0.05096303 & -2.32613975 \\
\hline $\mathrm{C}$ & -2.52328582 & -0.71863847 & -4.53240891 \\
\hline $\mathrm{C}$ & -3.16279107 & 0.61442985 & -4.31593717 \\
\hline $\mathrm{O}$ & -2.48455301 & 1.61319662 & -3.92241741 \\
\hline $\mathrm{O}$ & -4.40699902 & 0.69388132 & -4.59708756 \\
\hline $\mathrm{H}$ & -2.04720053 & -0.71641716 & -5.5216279 \\
\hline $\mathrm{H}$ & -3.27454898 & -1.50908902 & -4.5108926 \\
\hline $\mathrm{H}$ & -1.74475486 & -0.89886039 & -3.78354546 \\
\hline $\mathrm{N}$ & -5.75990242 & 2.78666568 & -4.71900046 \\
\hline C & -5.34030753 & 4.01203045 & -5.18185209 \\
\hline C & -6.43163371 & 4.78766579 & -5.48436085 \\
\hline $\mathrm{N}$ & -7.53184878 & 4.00494606 & -5.18882744 \\
\hline $\mathrm{C}$ & -7.08445485 & 2.81096305 & -4.73983409 \\
\hline $\mathrm{C}$ & -6.48936737 & 6.18409259 & -6.01108095 \\
\hline $\mathrm{H}$ & -6.98475085 & 6.86889983 & -5.3108048 \\
\hline $\mathrm{H}$ & -5.47443688 & 6.55490607 & -6.1759590 \\
\hline $\mathrm{H}$ & -7.02771307 & 6.24166946 & -6.96522375 \\
\hline $\mathrm{H}$ & -8.51722016 & 4.27882699 & -5.2988582 \\
\hline $\mathrm{H}$ & -7.73391962 & 1.99842704 & -4.44918584 \\
\hline $\mathrm{H}$ & -4.93465216 & 1.68699385 & -4.55288389 \\
\hline $\mathrm{H}$ & -4.28994273 & 4.25140277 & -5.2643692 \\
\hline $\mathrm{O}$ & -0.23548151 & 1.25672348 & -3.0437108 \\
\hline $\mathrm{H}$ & -0.23792128 & 1.88386897 & -2.2865706 \\
\hline $\mathrm{H}$ & -1.37365754 & 1.36854278 & -3.5157215 \\
\hline $\mathrm{N}$ & -0.19066478 & -2.02507856 & 4.7650512 \\
\hline $\mathrm{C}$ & 1.08639694 & -2.48708557 & 5.2715827 \\
\hline $\mathrm{C}$ & -1.37375252 & -2.53346275 & 5.2154645 \\
\hline $\mathrm{C}$ & -2.62947171 & -2.00900829 & 4.47840737 \\
\hline $\mathrm{N}$ & -2.71295859 & -0.55695535 & 4.37418449 \\
\hline $\mathrm{C}$ & -2.95809145 & 0.23634040 & 5.4584724 \\
\hline $\mathrm{C}$ & -2.89643282 & 1.73711371 & 5.1892370 \\
\hline $\mathrm{C}$ & -2.79932778 & -2.68458066 & 3.1151167 \\
\hline $\mathrm{O}$ & -1.46465744 & -3.38889462 & 6.0893113 \\
\hline $\mathrm{O}$ & -3.23282648 & -0.20665259 & 6.5678782 \\
\hline $\mathrm{H}$ & -0.19170465 & -1.43249557 & 3.93897717 \\
\hline $\mathrm{H}$ & 1.63432084 & -3.06665811 & 4.51643065 \\
\hline $\mathrm{H}$ & 1.71396128 & -1.64340640 & 5.58366578 \\
\hline $\mathrm{H}$ & 0.88726974 & -3.12674746 & 6.13306239 \\
\hline $\mathrm{H}$ & -3.44683501 & -2.32023418 & 5.1353638 \\
\hline $\mathrm{H}$ & -1.99109189 & -2.42783683 & 2.4260742 \\
\hline $\mathrm{H}$ & -2.80514453 & -3.77144687 & 3.2498409 \\
\hline $\mathrm{H}$ & -3.74779781 & -2.38544763 & 2.6560816 \\
\hline $\mathrm{H}$ & -2.35515325 & -0.13917657 & 3.5187531 \\
\hline $\mathrm{H}$ & -2.73409563 & 1.99091556 & 4.1371515 \\
\hline
\end{tabular}




$\begin{array}{llll}\mathrm{H} & -3.83182810 & 2.19227063 & 5.52932108 \\ \mathrm{H} & -2.08826811 & 2.17099360 & 5.78804196\end{array}$

\section{Doublet; Basis set B1; Model B; protonated Compound 0}

$\begin{array}{lrrr}\text { 100 } & & & \\ \mathrm{O} & -10.30702288 & 4.66098856 & -5.39164354 \\ \mathrm{C} & -11.11581968 & 5.51269509 & -5.00752342 \\ \mathrm{~N} & -12.34613919 & 5.16895444 & -4.54909364 \\ \mathrm{C} & -12.81692536 & 3.79448253 & -4.47365781 \\ \mathrm{C} & -10.77677475 & 6.99519699 & -5.03267728 \\ \mathrm{H} & -12.97495742 & 5.90654575 & -4.26516891 \\ \mathrm{H} & -13.70539470 & 3.65237679 & -5.09855510 \\ \mathrm{H} & -13.06002341 & 3.52230470 & -3.44071266 \\ \mathrm{H} & -12.01602279 & 3.15103789 & -4.83762629 \\ \mathrm{H} & -11.59139264 & 7.63972859 & -4.68928161 \\ \mathrm{H} & -10.50762410 & 7.27538615 & -6.05549113 \\ \mathrm{H} & -9.90004040 & 7.16816650 & -4.40038900 \\ \mathrm{C} & -4.16254197 & -1.43996669 & -0.87237538 \\ \mathrm{C} & -3.15745586 & -0.40531815 & -0.74650450 \\ \mathrm{~N} & -1.91978484 & -0.96799979 & -0.56639175 \\ \mathrm{C} & -2.11607986 & -2.32326933 & -0.56774352 \\ \mathrm{C} & -3.51698501 & -2.63067823 & -0.75266838 \\ \mathrm{C} & -3.44477351 & 0.95580213 & -0.77710663 \\ \mathrm{C} & -2.50669034 & 1.97958287 & -0.61047493 \\ \mathrm{~N} & -1.12929105 & 1.81754612 & -0.52349302 \\ \mathrm{C} & -0.59045847 & 3.08736391 & -0.35296374 \\ \mathrm{C} & -1.64866846 & 4.05950003 & -0.34784726 \\ \mathrm{C} & -2.82363910 & 3.37829580 & -0.50999959 \\ \mathrm{C} & 0.77759146 & 3.35400567 & -0.28537507 \\ \mathrm{C} & 1.79020663 & 2.39191860 & -0.25423481 \\ \mathrm{~N} & 1.62087468 & 1.02657007 & -0.26365200 \\ \mathrm{C} & 2.88544212 & 0.47976267 & -0.16006935 \\ \mathrm{C} & 3.86995518 & 1.53613872 & -0.09888133 \\ \mathrm{C} & 3.19565677 & 2.71632684 & -0.15997314 \\ \mathrm{Fe} & -0.14267241 & 0.02845866 & -0.39050815 \\ \mathrm{~S} & -0.37994868 & -0.07549552 & 1.90879294 \\ \mathrm{C} & 0.19482680 & 1.49394294 & 2.66143603 \\ \mathrm{C} & 3.19672412 & -0.88115456 & -0.10121436 \\ \mathrm{C} & 0.263975023 & -1.71409928 & -0.32847311 \\ \mathrm{~N} & & -2.96711351 & -0.31106588 \\ \mathrm{C} & & & \end{array}$




\begin{tabular}{|c|c|c|c|}
\hline $\mathrm{C}$ & 1.29720017 & -3.96452574 & -0.15488728 \\
\hline $\mathrm{C}$ & 2.48593803 & -3.30438209 & -0.07262114 \\
\hline $\mathrm{C}$ & -1.11350570 & -3.27052187 & -0.41857696 \\
\hline $\mathrm{H}$ & 4.23223651 & -1.18245776 & 0.00529533 \\
\hline $\mathrm{H}$ & 1.12236967 & -5.03240690 & -0.11368983 \\
\hline $\mathrm{H}$ & 4.93773305 & 1.37572010 & -0.0174069 \\
\hline $\mathrm{H}$ & 3.59540536 & 3.72237781 & -0.1373060 \\
\hline $\mathrm{H}$ & 1.08037541 & 4.39473306 & -0.2259210 \\
\hline $\mathrm{H}$ & -1.50539924 & 5.12736133 & -0.2400189 \\
\hline $\mathrm{H}$ & -3.82623151 & 3.78458745 & -0.5570158 \\
\hline $\mathrm{H}$ & -4.48262774 & 1.24955460 & -0.8957299 \\
\hline $\mathrm{H}$ & -5.21860923 & -1.25627035 & -1.024388 \\
\hline $\mathrm{H}$ & -3.93035300 & -3.63071951 & -0.7858179 \\
\hline $\mathrm{H}$ & -1.39228148 & -4.31880973 & -0.4013425 \\
\hline $\mathrm{H}$ & 3.47573637 & -3.72672681 & 0.04898940 \\
\hline $\mathrm{H}$ & 0.06305793 & 1.40447600 & 3.74490515 \\
\hline $\mathrm{H}$ & 1.25288535 & 1.66866295 & 2.45113897 \\
\hline $\mathrm{H}$ & -0.38471820 & 2.35024938 & 2.30685489 \\
\hline $\mathrm{O}$ & -0.07645141 & -0.05892623 & -2.34602419 \\
\hline $\mathrm{C}$ & -2.47387242 & -0.74085598 & -4.4889916 \\
\hline $\mathrm{C}$ & -3.13155759 & 0.59303948 & -4.3135897 \\
\hline $\mathrm{O}$ & -2.47234373 & 1.61720020 & -4.0531125 \\
\hline $\mathrm{O}$ & -4.42243538 & 0.58176979 & -4.4929995 \\
\hline $\mathrm{H}$ & -1.99533889 & -0.76447595 & -5.476551 \\
\hline $\mathrm{H}$ & -3.20366818 & -1.55035121 & -4.4380582 \\
\hline $\mathrm{H}$ & -1.69296407 & -0.88258608 & -3.7356218 \\
\hline $\mathrm{N}$ & -5.84653440 & 2.77322622 & -4.5978018 \\
\hline C & -5.40460215 & 3.97521147 & -5.1015820 \\
\hline $\mathrm{C}$ & -6.47453072 & 4.76374531 & -5.4488774 \\
\hline $\mathrm{N}$ & -7.59347580 & 4.01429432 & -5.1394546 \\
\hline $\mathrm{C}$ & -7.16745998 & 2.82852041 & -4.6389310 \\
\hline $\mathrm{C}$ & -6.51117656 & 6.13633760 & -6.0357646 \\
\hline $\mathrm{H}$ & -7.01206017 & 6.85700923 & -5.3758143 \\
\hline $\mathrm{H}$ & -5.49062769 & 6.49256221 & -6.2004322 \\
\hline $\mathrm{H}$ & -7.03288758 & 6.15748051 & -7.0010533 \\
\hline $\mathrm{H}$ & -8.56948492 & 4.29990525 & -5.2733154 \\
\hline $\mathrm{H}$ & -7.84134653 & 2.04370519 & -4.3261420 \\
\hline $\mathrm{H}$ & -4.89042438 & 1.50760448 & -4.4382200 \\
\hline $\mathrm{H}$ & -4.34872125 & 4.19282790 & -5.1829848 \\
\hline $\mathrm{O}$ & -0.18342258 & 1.31226729 & -2.9520531 \\
\hline $\mathrm{H}$ & -0.47619682 & 1.80183924 & -2.1198947 \\
\hline $\mathrm{H}$ & -1.06849473 & 1.31651864 & $-3.514571<$ \\
\hline $\mathrm{N}$ & -0.20047025 & -2.01624949 & 4.76761477 \\
\hline $\mathrm{C}$ & 1.07265944 & -2.46688961 & 5.2944990 \\
\hline $\mathrm{C}$ & -1.38689610 & -2.52893995 & 5.205097 \\
\hline
\end{tabular}




$\begin{array}{lrrr}\mathrm{C} & -2.63571579 & -2.01656208 & 4.44832221 \\ \mathrm{~N} & -2.72598782 & -0.56553609 & 4.33450114 \\ \mathrm{C} & -2.99011231 & 0.23272742 & 5.41120707 \\ \mathrm{C} & -2.95223765 & 1.73219444 & 5.13121624 \\ \mathrm{C} & -2.78398509 & -2.70144350 & 3.08719513 \\ \mathrm{O} & -1.48397341 & -3.38134097 & 6.08069335 \\ \mathrm{O} & -3.26182903 & -0.20738551 & 6.52204043 \\ \mathrm{H} & -0.19455588 & -1.42412728 & 3.94239077 \\ \mathrm{H} & 1.63391079 & -3.04938585 & 4.55148667 \\ \mathrm{H} & 1.69097231 & -1.61723652 & 5.60863759 \\ \mathrm{H} & 0.86550209 & -3.10106078 & 6.15808243 \\ \mathrm{H} & -3.45999186 & -2.32889525 & 5.09610218 \\ \mathrm{H} & -1.96818692 & -2.44478911 & 2.40697439 \\ \mathrm{H} & -2.78507713 & -3.78734506 & 3.22885205 \\ \mathrm{H} & -3.72850458 & -2.41142872 & 2.61431880 \\ \mathrm{H} & -2.38213930 & -0.14929019 & 3.47380404 \\ \mathrm{H} & -2.77886260 & 1.98177549 & 4.07978353 \\ \mathrm{H} & -3.90136073 & 2.17205687 & 5.45284577 \\ \mathrm{H} & -2.16228861 & 2.18585291 & 5.73943419\end{array}$

\section{Doublet; Basis set B1; Model B; Transition State 3}

$\begin{array}{llll}100 & & & \\ \mathrm{O} & -10.30292108 & 4.63693274 & -5.35497073 \\ \mathrm{C} & -11.09847006 & 5.50597835 & -4.98292539 \\ \mathrm{~N} & -12.32675383 & 5.18604104 & -4.50142891 \\ \mathrm{C} & -12.80888029 & 3.81848067 & -4.38508499 \\ \mathrm{C} & -10.74529228 & 6.98410580 & -5.05157534 \\ \mathrm{H} & -12.94494925 & 5.93658330 & -4.22858295 \\ \mathrm{H} & -13.70528327 & 3.66808798 & -4.99660680 \\ \mathrm{H} & -13.04302067 & 3.57536287 & -3.34284923 \\ \mathrm{H} & -12.01755684 & 3.15870367 & -4.74069940 \\ \mathrm{H} & -11.54787525 & 7.64559150 & -4.71198194 \\ \mathrm{H} & -10.49004960 & 7.23501022 & -6.08549640 \\ \mathrm{H} & -9.85671174 & 7.16394224 & -4.43808460 \\ \mathrm{C} & -4.19327248 & -1.41207003 & -0.86262614 \\ \mathrm{C} & -3.18827992 & -0.38091810 & -0.73761098 \\ \mathrm{~N} & -1.94887968 & -0.92018128 & -0.56334720 \\ \mathrm{C} & -2.14243755 & -2.26807400 & -0.57157892 \\ \mathrm{C} & -3.53781607 & -2.59632839 & -0.75056733 \\ \mathrm{C} & -3.44823096 & 0.96722841 & -0.77754281 \\ \mathrm{C} & -2.49896260 & 1.98418939 & -0.62830883\end{array}$




\begin{tabular}{|c|c|c|c|}
\hline $\mathrm{N}$ & -1.13683586 & 1.82373685 & -0.47849065 \\
\hline $\mathrm{C}$ & -0.59035331 & 3.08745943 & -0.38739310 \\
\hline $\mathrm{C}$ & -1.64062706 & 4.07195677 & -0.47678441 \\
\hline $\mathrm{C}$ & -2.81322212 & 3.39150514 & -0.62721897 \\
\hline $\mathrm{C}$ & 0.77496363 & 3.37212269 & -0.27124086 \\
\hline $\mathrm{C}$ & 1.79260494 & 2.43980222 & -0.19712180 \\
\hline $\mathrm{N}$ & 1.61334412 & 1.08260816 & -0.2249631 \\
\hline $\mathrm{C}$ & 2.87427078 & 0.54255728 & -0.1255522 \\
\hline $\mathrm{C}$ & 3.86326031 & 1.58916474 & -0.0331222 \\
\hline $\mathrm{C}$ & 3.19136959 & 2.77057850 & -0.07889585 \\
\hline $\mathrm{Fe}$ & -0.15517026 & 0.09927337 & -0.42474226 \\
\hline S & -0.39530574 & -0.07123699 & 1.92189387 \\
\hline $\mathrm{C}$ & 0.19469271 & 1.48453003 & 2.69122595 \\
\hline $\mathrm{C}$ & 3.17163105 & -0.80815585 & -0.10733250 \\
\hline $\mathrm{C}$ & 2.18912808 & -1.81859476 & -0.19095078 \\
\hline $\mathrm{N}$ & 0.82435729 & -1.64550046 & -0.3188168 \\
\hline $\mathrm{C}$ & 0.24067229 & -2.89251593 & -0.3305820 \\
\hline $\mathrm{C}$ & 1.27860699 & -3.89406250 & -0.21022060 \\
\hline $\mathrm{C}$ & 2.46966832 & -3.23727704 & -0.12924675 \\
\hline $\mathrm{C}$ & -1.13368462 & -3.18786168 & -0.4360897 \\
\hline $\mathrm{H}$ & 4.20692533 & -1.11694298 & -0.0146966 \\
\hline $\mathrm{H}$ & 1.10269890 & -4.96233979 & -0.19038622 \\
\hline $\mathrm{H}$ & 4.92887361 & 1.41945548 & 0.05477445 \\
\hline $\mathrm{H}$ & 3.58543845 & 3.77794698 & -0.0358526 \\
\hline $\mathrm{H}$ & 1.05316424 & 4.42119343 & -0.23561128 \\
\hline $\mathrm{H}$ & -1.48697442 & 5.14290261 & -0.4330881 \\
\hline $\mathrm{H}$ & -3.81171422 & 3.79544441 & -0.73617445 \\
\hline $\mathrm{H}$ & -4.47695160 & 1.28313025 & -0.9207451 \\
\hline $\mathrm{H}$ & -5.24936726 & -1.22816534 & -1.01159667 \\
\hline $\mathrm{H}$ & -3.93531793 & -3.60250757 & -0.7846034 \\
\hline $\mathrm{H}$ & -1.39938878 & -4.24056129 & -0.4307325 \\
\hline $\mathrm{H}$ & 3.46042260 & -3.66290165 & -0.0295072 \\
\hline $\mathrm{H}$ & 0.01452724 & 1.40504180 & 3.7685803 \\
\hline $\mathrm{H}$ & 1.26586499 & 1.62685017 & 2.52794036 \\
\hline $\mathrm{H}$ & -0.34405778 & 2.35527200 & 2.3090415 \\
\hline $\mathrm{O}$ & -0.01077958 & -0.10137665 & -2.1490861 \\
\hline $\mathrm{C}$ & -2.46375673 & -0.80328576 & $-4.414588 \mathrm{~s}$ \\
\hline $\mathrm{C}$ & -3.13087433 & 0.53309568 & -4.2433672 \\
\hline $\mathrm{O}$ & -2.49702337 & 1.57050707 & -4.0000093 \\
\hline $\mathrm{O}$ & -4.43002144 & 0.49623345 & -4.4116435 \\
\hline $\mathrm{H}$ & -2.00400708 & -0.83758367 & -5.4103625 \\
\hline $\mathrm{H}$ & -3.18700233 & -1.61643002 & -4.337001 \\
\hline $\mathrm{H}$ & -1.66884400 & -0.92801598 & -3.6743415 \\
\hline $\mathrm{N}$ & -5.84904384 & 2.72707834 & -4.5560836 \\
\hline C & -5.40162325 & 3.91270836 & -5.0948034 \\
\hline
\end{tabular}




$\begin{array}{lrrr}\mathrm{C} & -6.46748388 & 4.70271236 & -5.45327692 \\ \mathrm{~N} & -7.59016443 & 3.97088747 & -5.11471578 \\ \mathrm{C} & -7.16926587 & 2.79462598 & -4.58736374 \\ \mathrm{C} & -6.51200458 & 6.05934810 & -6.07714691 \\ \mathrm{H} & -7.00526875 & 6.79706324 & -5.43035820 \\ \mathrm{H} & -5.49416336 & 6.41226201 & -6.26487573 \\ \mathrm{H} & -7.04604139 & 6.05389070 & -7.03587212 \\ \mathrm{H} & -8.56431522 & 4.26180659 & -5.24678074 \\ \mathrm{H} & -7.84797508 & 2.02471500 & -4.24848598 \\ \mathrm{H} & -4.88617074 & 1.41879952 & -4.37160431 \\ \mathrm{H} & -4.34445346 & 4.11814515 & -5.19099284 \\ \mathrm{O} & -0.00347969 & 1.35909791 & -3.16293863 \\ \mathrm{H} & 0.02115061 & 2.01100234 & -2.43391638 \\ \mathrm{H} & -0.96990336 & 1.37696486 & -3.49846184 \\ \mathrm{~N} & -0.24486860 & -2.07764615 & 4.73672798 \\ \mathrm{C} & 1.01992849 & -2.56358858 & 5.25203116 \\ \mathrm{C} & -1.44026988 & -2.58142850 & 5.15927051 \\ \mathrm{C} & -2.67913373 & -2.02376566 & 4.41849099 \\ \mathrm{~N} & -2.74101600 & -0.56873424 & 4.34640210 \\ \mathrm{C} & -2.99140939 & 0.20372448 & 5.44512719 \\ \mathrm{C} & -2.92232430 & 1.70941223 & 5.20842552 \\ \mathrm{C} & -2.84277059 & -2.66654357 & 3.03916977 \\ \mathrm{O} & -1.55336498 & -3.45785182 & 6.00880502 \\ \mathrm{O} & -3.27106204 & -0.26294684 & 6.54297602 \\ \mathrm{H} & -0.22769510 & -1.45700280 & 3.93291390 \\ \mathrm{H} & 1.58212499 & -3.11427795 & 4.48600832 \\ \mathrm{H} & 1.64318568 & -1.73588874 & 5.61211689 \\ \mathrm{H} & 0.79986715 & -3.23646212 & 6.08251027 \\ \mathrm{H} & -3.50856898 & -2.33823902 & 5.05868826 \\ \mathrm{H} & -2.02485161 & -2.40352874 & 2.36405839 \\ \mathrm{H} & -2.86192276 & -3.75590108 & 3.14928320 \\ \mathrm{H} & -3.78364037 & -2.34727708 & 2.57806120 \\ \mathrm{H} & -2.39468804 & -0.13522860 & 3.49544952 \\ \mathrm{H} & -2.77225858 & 1.98598449 & 4.16014770 \\ \mathrm{H} & -3.84975069 & 2.16323053 & 5.57076798 \\ \mathrm{H} & -2.10323549 & 2.12483662 & 5.80565199\end{array}$

\section{Doublet; Basis set B1; Model B; Compound I}

100

$\begin{array}{llll}\mathrm{O} & -10.28013989 & 4.67864101 & -4.96027930 \\ \mathrm{C} & -10.97264840 & 5.56658240 & -4.45430786\end{array}$




\begin{tabular}{|c|c|c|c|}
\hline $\mathrm{N}$ & -12.15569015 & 5.28509889 & -3.84775419 \\
\hline $\mathrm{C}$ & -12.69930439 & 3.94019405 & -3.74412443 \\
\hline $\mathrm{C}$ & -10.54071487 & 7.02591121 & -4.49476332 \\
\hline $\mathrm{H}$ & -12.69267645 & 6.05021152 & -3.46551144 \\
\hline $\mathrm{H}$ & -13.66861430 & 3.87120960 & -4.25015177 \\
\hline $\mathrm{H}$ & -12.82330954 & 3.64896736 & -2.69516929 \\
\hline $\mathrm{H}$ & -11.99453047 & 3.26238367 & -4.22576516 \\
\hline $\mathrm{H}$ & -11.25817175 & 7.71151865 & -4.03380551 \\
\hline $\mathrm{H}$ & -10.38973105 & 7.31573353 & -5.53882701 \\
\hline $\mathrm{H}$ & -9.57866178 & 7.12361427 & -3.98191200 \\
\hline $\mathrm{C}$ & -3.92656048 & -1.71034107 & -0.96530533 \\
\hline $\mathrm{C}$ & -2.99789588 & -0.61816866 & -0.80384910 \\
\hline $\mathrm{N}$ & -1.71956032 & -1.11027092 & -0.63804224 \\
\hline $\mathrm{C}$ & -1.82689010 & -2.48721062 & -0.69458724 \\
\hline $\mathrm{C}$ & -3.20575048 & -2.86352938 & -0.89125350 \\
\hline $\mathrm{C}$ & -3.37219532 & 0.73619768 & -0.80333339 \\
\hline $\mathrm{C}$ & -2.44697375 & 1.77019956 & -0.62209591 \\
\hline $\mathrm{N}$ & -1.07299713 & 1.62913437 & -0.43576114 \\
\hline $\mathrm{C}$ & -0.56091996 & 2.90800981 & -0.33135869 \\
\hline $\mathrm{C}$ & -1.63480160 & 3.86927400 & -0.41694267 \\
\hline $\mathrm{C}$ & -2.78770936 & 3.17229040 & -0.59979778 \\
\hline $\mathrm{C}$ & 0.78861106 & 3.25436324 & -0.17744060 \\
\hline $\mathrm{C}$ & 1.84982531 & 2.34902690 & -0.13641114 \\
\hline $\mathrm{N}$ & 1.73932189 & 0.98298078 & -0.19740280 \\
\hline $\mathrm{C}$ & 3.03146947 & 0.49843649 & -0.12227225 \\
\hline $\mathrm{C}$ & 3.96464890 & 1.59152538 & -0.00084127 \\
\hline $\mathrm{C}$ & 3.23274841 & 2.73882570 & -0.00776995 \\
\hline $\mathrm{Fe}$ & 0.02794368 & -0.06347122 & -0.56034236 \\
\hline S & -0.54962611 & -0.01636099 & 1.98113669 \\
\hline $\mathrm{C}$ & 0.42895132 & 1.29392786 & 2.78702692 \\
\hline $\mathrm{C}$ & 3.39801749 & -0.84432917 & -0.14560854 \\
\hline $\mathrm{C}$ & 2.46203987 & -1.89299424 & -0.25708021 \\
\hline $\mathrm{N}$ & 1.09036334 & -1.76637654 & -0.36671017 \\
\hline $\mathrm{C}$ & 0.58045594 & -3.04356318 & -0.44705498 \\
\hline $\mathrm{C}$ & 1.65752704 & -4.00082929 & -0.37314255 \\
\hline $\mathrm{C}$ & 2.81647607 & -3.29149500 & -0.25958627 \\
\hline $\mathrm{C}$ & -0.77484308 & -3.39901771 & -0.59073269 \\
\hline $\mathrm{H}$ & 4.44889174 & -1.10131913 & -0.07336678 \\
\hline $\mathrm{H}$ & 1.53033697 & -5.07541991 & -0.40691882 \\
\hline $\mathrm{H}$ & 5.03827321 & 1.47720998 & 0.07726982 \\
\hline $\mathrm{H}$ & 3.58018606 & 3.76164762 & 0.06093642 \\
\hline $\mathrm{H}$ & 1.02517860 & 4.31164251 & -0.12556423 \\
\hline $\mathrm{H}$ & -1.50125361 & 4.94215930 & -0.36612520 \\
\hline $\mathrm{H}$ & -3.79088292 & 3.55828388 & -0.72703495 \\
\hline $\mathrm{H}$ & -4.41370330 & 0.99040801 & -0.96028826 \\
\hline
\end{tabular}




\begin{tabular}{|c|c|c|c|}
\hline $\mathrm{H}$ & -4.99127227 & -1.59171015 & -1.11870373 \\
\hline $\mathrm{H}$ & -3.56063521 & -3.88353066 & -0.96587483 \\
\hline $\mathrm{H}$ & -1.01268038 & -4.45602844 & -0.64250030 \\
\hline $\mathrm{H}$ & 3.82777573 & -3.67004770 & -0.18103426 \\
\hline $\mathrm{H}$ & 0.13742892 & 1.36039445 & 3.84035356 \\
\hline $\mathrm{H}$ & 1.50045116 & 1.09379118 & 2.70880144 \\
\hline $\mathrm{H}$ & 0.22097087 & 2.26032668 & 2.31296012 \\
\hline $\mathrm{O}$ & 0.19328935 & 0.00015457 & -2.17768331 \\
\hline C & -2.23282613 & -0.82166948 & -4.82509215 \\
\hline C & -3.07824160 & 0.43732407 & -4.79472262 \\
\hline $\mathrm{O}$ & -2.61711060 & 1.56157037 & -4.95122507 \\
\hline $\mathrm{O}$ & -4.37103352 & 0.20105354 & -4.5918985 \\
\hline $\mathrm{H}$ & -1.74553233 & -0.89551977 & -5.8031602 \\
\hline $\mathrm{H}$ & -2.83273982 & -1.71508646 & -4.6439221 \\
\hline $\mathrm{H}$ & -1.44255746 & -0.73993520 & -4.0707706 \\
\hline $\mathrm{N}$ & -5.87411176 & 2.48898470 & -4.7762702 \\
\hline C & -5.42037829 & 3.67765684 & -5.3109892 \\
\hline $\mathrm{C}$ & -6.47192268 & 4.54483957 & -5.5028919 \\
\hline $\mathrm{N}$ & -7.59024700 & 3.85461587 & -5.06542873 \\
\hline $\mathrm{C}$ & -7.18062612 & 2.63198691 & -4.64574676 \\
\hline C & -6.57125644 & 5.92614490 & -6.07049680 \\
\hline $\mathrm{H}$ & -6.97633874 & 6.64259423 & -5.3434453 \\
\hline $\mathrm{H}$ & -5.58047108 & 6.28188997 & -6.3673042 \\
\hline $\mathrm{H}$ & -7.21650482 & 5.95550964 & -6.9577348 \\
\hline $\mathrm{H}$ & -8.55270796 & 4.20198653 & -5.0729815 \\
\hline $\mathrm{H}$ & -7.86148738 & 1.88526082 & -4.26090618 \\
\hline $\mathrm{H}$ & -4.88464290 & 1.07699448 & -4.62440674 \\
\hline $\mathrm{H}$ & -4.36919369 & 3.81766842 & -5.5214364 \\
\hline $\mathrm{O}$ & -0.22718091 & 2.57099446 & -3.5652237 \\
\hline $\mathrm{H}$ & 0.08023899 & 1.72291393 & -3.20089822 \\
\hline $\mathrm{H}$ & -0.99946738 & 2.31407363 & -4.10270097 \\
\hline $\mathrm{N}$ & -0.70904694 & -1.74475205 & 4.9742704 \\
\hline $\mathrm{C}$ & 0.49808949 & -2.13120809 & 5.67799577 \\
\hline C & -1.93885007 & -2.24513897 & 5.2992084 \\
\hline $\mathrm{C}$ & -3.08713336 & -1.79739046 & 4.3640937 \\
\hline $\mathrm{N}$ & -3.15804845 & -0.35615140 & 4.1511079 \\
\hline $\mathrm{C}$ & -3.55263156 & 0.50333906 & 5.13955917 \\
\hline $\mathrm{C}$ & -3.48371516 & 1.98283591 & 4.7751057 \\
\hline $\mathrm{C}$ & -3.06227829 & -2.56811169 & 3.0408464 \\
\hline $\mathrm{O}$ & -2.12975974 & -3.04760024 & 6.2037488 \\
\hline $\mathrm{O}$ & -3.95735389 & 0.12469152 & 6.2314325 \\
\hline $\mathrm{H}$ & -0.62889869 & -1.15838142 & 4.1534548 \\
\hline $\mathrm{H}$ & 1.19280040 & -2.66706553 & 5.0183277 \\
\hline $\mathrm{H}$ & 1.01447383 & -1.25772476 & 6.09512524 \\
\hline $\mathrm{H}$ & 0.20049617 & -2.79263816 & 6.4933695 \\
\hline
\end{tabular}




$\begin{array}{llll}\mathrm{H} & -3.98558195 & -2.07559386 & 4.92216754 \\ \mathrm{H} & -2.16252263 & -2.35734229 & 2.45540327 \\ \mathrm{H} & -3.08746434 & -3.64262495 & 3.24921674 \\ \mathrm{H} & -3.93591953 & -2.31203147 & 2.43235532 \\ \mathrm{H} & -2.71216822 & 0.01721381 & 3.31954525 \\ \mathrm{H} & -3.09339113 & 2.17375501 & 3.77023090 \\ \mathrm{H} & -4.48795059 & 2.41166714 & 4.85356285 \\ \mathrm{H} & -2.85467579 & 2.49722664 & 5.50869436\end{array}$

\section{Doublet; Basis set B2; Model A; Fe+HOOH}

$\begin{array}{lccc}\mathrm{O} & -10.26155645 & 4.87152330 & -5.13513256 \\ \mathrm{C} & -10.83826656 & 5.80768655 & -4.56799125 \\ \mathrm{~N} & -11.97514256 & 5.61026327 & -3.84914019 \\ \mathrm{C} & -12.60869871 & 4.30950481 & -3.67778641 \\ \mathrm{C} & -10.29808631 & 7.23064188 & -4.66296320 \\ \mathrm{H} & -12.40721697 & 6.41234737 & -3.41076068 \\ \mathrm{H} & -13.63403411 & 4.32749933 & -4.06315143 \\ \mathrm{H} & -12.62644163 & 4.02340183 & -2.62016035 \\ \mathrm{H} & -12.02860234 & 3.57593865 & -4.23838122 \\ \mathrm{H} & -10.91104876 & 7.96933851 & -4.13646753 \\ \mathrm{H} & -10.23232222 & 7.50866290 & -5.71929535 \\ \mathrm{H} & -9.28517001 & 7.25109850 & -4.24840847 \\ \mathrm{C} & -4.18056959 & -1.61629546 & -0.81104567 \\ \mathrm{C} & -3.27723822 & -0.49778521 & -0.66864208 \\ \mathrm{~N} & -1.98883138 & -0.97744299 & -0.41068875 \\ \mathrm{C} & -2.08866195 & -2.36353437 & -0.37499470 \\ \mathrm{C} & -3.45648700 & -2.75678938 & -0.62469263 \\ \mathrm{C} & -3.67891142 & 0.85086778 & -0.78991117 \\ \mathrm{C} & -2.73027782 & 1.90035139 & -0.62920833 \\ \mathrm{~N} & -1.35911034 & 1.79052476 & -0.34835539 \\ \mathrm{C} & -0.85303331 & 3.09281312 & -0.29275697 \\ \mathrm{C} & -1.92631448 & 4.02463544 & -0.54496814 \\ \mathrm{C} & -3.06479331 & 3.29900111 & -0.75482581 \\ \mathrm{C} & 0.48845989 & 3.48926013 & -0.04804385 \\ \mathrm{C} & 1.52961348 & 2.55731661 & 0.13642914 \\ \mathrm{~N} & 1.41547145 & 1.16766260 & 0.14586960 \\ \mathrm{C} & 2.70631557 & 0.66987258 & 0.34089650 \\ \mathrm{C} & 3.63010168 & 1.77454789 & 0.45004280 \\ \mathrm{C} & 2.91116370 & 2.92852792 & 0.32955431 \\ \mathrm{Fe} & -0.32086806 & 0.10365489 & 0.06446314\end{array}$




\begin{tabular}{|c|c|c|c|}
\hline S & -0.73209530 & 0.02063288 & 2.24004087 \\
\hline $\mathrm{C}$ & -0.45097644 & 1.68218779 & 2.94484169 \\
\hline $\mathrm{C}$ & 3.10461128 & -0.68537172 & 0.41286882 \\
\hline $\mathrm{C}$ & 2.16749914 & -1.73119735 & 0.29466258 \\
\hline $\mathrm{N}$ & 0.79499981 & -1.61095044 & 0.07902963 \\
\hline C & 0.29413839 & -2.90596567 & 0.04575241 \\
\hline $\mathrm{C}$ & 1.37125406 & -3.85050956 & 0.23277590 \\
\hline $\mathrm{C}$ & 2.51965397 & -3.13026901 & 0.37927758 \\
\hline C & -1.04580509 & -3.28597621 & -0.14783113 \\
\hline $\mathrm{H}$ & 4.15056682 & -0.92212676 & 0.56820243 \\
\hline $\mathrm{H}$ & 1.25075145 & -4.92712223 & 0.24086746 \\
\hline $\mathrm{H}$ & 4.69845513 & 1.66744153 & 0.59558264 \\
\hline $\mathrm{H}$ & 3.27614019 & 3.94833008 & 0.35570842 \\
\hline $\mathrm{H}$ & 0.72167205 & 4.54775128 & -0.03451686 \\
\hline $\mathrm{H}$ & -1.81055016 & 5.10158410 & -0.57403969 \\
\hline $\mathrm{H}$ & -4.05458318 & 3.67088691 & -0.99104947 \\
\hline $\mathrm{H}$ & -4.70901246 & 1.09215047 & -1.02043345 \\
\hline $\mathrm{H}$ & -5.23599768 & -1.52479509 & -1.03697916 \\
\hline $\mathrm{H}$ & -3.80393744 & -3.78223433 & -0.6635718 \\
\hline $\mathrm{H}$ & -1.28796764 & -4.34302516 & -0.1447349 \\
\hline $\mathrm{H}$ & 3.52605927 & -3.50093651 & 0.53300146 \\
\hline $\mathrm{H}$ & -0.68324847 & 1.60766387 & 4.01376343 \\
\hline $\mathrm{H}$ & 0.58962583 & 1.99704439 & 2.83188993 \\
\hline $\mathrm{H}$ & -1.11025572 & 2.42745746 & 2.49248744 \\
\hline C & -2.30464230 & -0.85879845 & -5.3986570 \\
\hline $\mathrm{C}$ & -3.19776837 & 0.35905580 & -5.52147508 \\
\hline $\mathrm{O}$ & -2.83602704 & 1.42167321 & -6.0052213 \\
\hline $\mathrm{O}$ & -4.42785352 & 0.13817034 & -5.0465042 \\
\hline $\mathrm{H}$ & -1.31085224 & -0.63026912 & -5.7872069 \\
\hline $\mathrm{H}$ & -2.73793514 & -1.69746540 & -5.9556645 \\
\hline $\mathrm{H}$ & -2.23293394 & -1.16697321 & -4.3497774 \\
\hline $\mathrm{N}$ & -6.01666904 & 2.35208498 & -5.3474530 \\
\hline C & -5.52605463 & 3.50329110 & -5.9306728 \\
\hline $\mathrm{C}$ & -6.52431886 & 4.44607547 & -6.0309366 \\
\hline $\mathrm{N}$ & -7.64714163 & 3.84233854 & -5.4875435 \\
\hline $\mathrm{C}$ & -7.29223914 & 2.58969666 & -5.0976094 \\
\hline $\mathrm{C}$ & -6.55190836 & 5.83581840 & -6.5838562 \\
\hline $\mathrm{H}$ & -6.84511136 & 6.56447607 & -5.8163106 \\
\hline $\mathrm{H}$ & -5.55804755 & 6.11382550 & -6.9472935 \\
\hline $\mathrm{H}$ & -7.25568991 & 5.93028090 & -7.4211658 \\
\hline $\mathrm{H}$ & -8.57986012 & 4.25513024 & -5.4120917 \\
\hline $\mathrm{H}$ & -7.98751521 & 1.89527154 & -4.6458667 \\
\hline $\mathrm{H}$ & -5.00178194 & 0.96760748 & -5.1567140 \\
\hline $\mathrm{H}$ & -4.49090242 & 3.56281748 & -6.2385072 \\
\hline
\end{tabular}




\section{Doublet; Basis set B2; Model A; FeHOOH}

\begin{tabular}{|c|c|c|c|}
\hline $\mathrm{O}$ & -10.33625978 & 4.60637951 & -5.34919264 \\
\hline $\mathrm{C}$ & -11.16168794 & 5.48131002 & -5.05507118 \\
\hline $\mathrm{N}$ & -12.39192137 & 5.14381066 & -4.59648541 \\
\hline $\mathrm{C}$ & -12.80115317 & 3.75357471 & -4.42881402 \\
\hline $\mathrm{C}$ & -10.83314057 & 6.96187095 & -5.19047854 \\
\hline $\mathrm{H}$ & -13.04894909 & 5.87706583 & -4.37080975 \\
\hline $\mathrm{H}$ & -13.84391678 & 3.73504851 & -4.10504184 \\
\hline $\mathrm{H}$ & -12.18043925 & 3.25443297 & -3.67768408 \\
\hline $\mathrm{H}$ & -12.70384869 & 3.20945490 & -5.37301789 \\
\hline $\mathrm{H}$ & -11.66783222 & 7.62039070 & -4.92984248 \\
\hline $\mathrm{H}$ & -10.53005996 & 7.16241192 & -6.22269020 \\
\hline $\mathrm{H}$ & -9.98332973 & 7.19620282 & -4.54103751 \\
\hline $\mathrm{C}$ & -4.26256442 & -1.35883552 & -0.85182470 \\
\hline $\mathrm{C}$ & -3.24128974 & -0.33928614 & -0.77508409 \\
\hline $\mathrm{N}$ & -1.99746605 & -0.89162193 & -0.65395029 \\
\hline $\mathrm{C}$ & -2.21121280 & -2.24141967 & -0.63744699 \\
\hline $\mathrm{C}$ & -3.61689070 & -2.55237715 & -0.75493842 \\
\hline $\mathrm{C}$ & -3.48848192 & 1.01207249 & -0.82811827 \\
\hline $\mathrm{C}$ & -2.54349344 & 2.02963215 & -0.69033736 \\
\hline $\mathrm{N}$ & -1.18913768 & 1.86306647 & -0.52244243 \\
\hline $\mathrm{C}$ & -0.64612845 & 3.12728585 & -0.46413311 \\
\hline $\mathrm{C}$ & -1.69130791 & 4.11766313 & -0.58699744 \\
\hline $\mathrm{C}$ & -2.86388389 & 3.43786479 & -0.73259998 \\
\hline $\mathrm{C}$ & 0.70963796 & 3.41935014 & -0.31902595 \\
\hline $\mathrm{C}$ & 1.72569467 & 2.46752710 & -0.22990150 \\
\hline $\mathrm{N}$ & 1.54571305 & 1.09753626 & -0.31614931 \\
\hline $\mathrm{C}$ & 2.80705476 & 0.53514874 & -0.17913133 \\
\hline $\mathrm{C}$ & 3.79041234 & 1.57723546 & -0.02643125 \\
\hline $\mathrm{C}$ & 3.12340558 & 2.77002482 & -0.05668341 \\
\hline $\mathrm{Fe}$ & -0.23448345 & 0.11196020 & -0.36608941 \\
\hline $\mathrm{S}$ & -0.48124549 & -0.06453518 & 1.82808005 \\
\hline $\mathrm{C}$ & 0.00185636 & 1.51396224 & 2.61211023 \\
\hline $\mathrm{C}$ & 3.09310150 & -0.83074725 & -0.21085014 \\
\hline $\mathrm{C}$ & 2.11010650 & -1.82915103 & -0.32441008 \\
\hline $\mathrm{N}$ & 0.74526762 & -1.63666025 & -0.43696701 \\
\hline $\mathrm{C}$ & 0.16660333 & -2.88738703 & -0.46914681 \\
\hline $\mathrm{C}$ & 1.19783591 & -3.89845356 & -0.39587335 \\
\hline $\mathrm{C}$ & 2.39379089 & -3.24744088 & -0.31269880 \\
\hline $\mathrm{C}$ & -1.20725116 & -3.17556237 & -0.54466882 \\
\hline $\mathrm{H}$ & 4.12807724 & -1.14349287 & -0.11938407 \\
\hline $\mathrm{H}$ & 1.01613652 & -4.96639777 & -0.40919169 \\
\hline
\end{tabular}




\begin{tabular}{|c|c|c|c|}
\hline $\mathrm{H}$ & 4.85433215 & 1.40744628 & 0.08697164 \\
\hline $\mathrm{H}$ & 3.53258936 & 3.76960388 & 0.02634884 \\
\hline $\mathrm{H}$ & 0.99650193 & 4.46560086 & -0.26983595 \\
\hline $\mathrm{H}$ & -1.53030784 & 5.18883499 & -0.57676230 \\
\hline $\mathrm{H}$ & -3.86089159 & 3.83635483 & -0.87377914 \\
\hline $\mathrm{H}$ & -4.51864711 & 1.32828051 & -0.96687726 \\
\hline $\mathrm{H}$ & -5.32142340 & -1.16322905 & -0.96611313 \\
\hline $\mathrm{H}$ & -4.02935509 & -3.55367039 & -0.7695580 \\
\hline $\mathrm{H}$ & -1.48393965 & -4.22612435 & -0.54232330 \\
\hline $\mathrm{H}$ & 3.38633197 & -3.67613902 & -0.2434312 \\
\hline $\mathrm{H}$ & -0.16301878 & 1.39333888 & 3.68886140 \\
\hline $\mathrm{H}$ & 1.05718278 & 1.74308040 & 2.44109006 \\
\hline $\mathrm{H}$ & -0.61204928 & 2.34428026 & 2.2528675 \\
\hline $\mathrm{O}$ & -0.14338120 & 0.22805603 & -2.6511147 \\
\hline C & -2.43989588 & -0.75301822 & -4.4324374 \\
\hline $\mathrm{C}$ & -3.03095919 & 0.61003736 & -4.20734860 \\
\hline $\mathrm{O}$ & -2.36664118 & 1.57325474 & -3.8256498 \\
\hline $\mathrm{O}$ & -4.33605604 & 0.64216644 & -4.4344995 \\
\hline $\mathrm{H}$ & -1.42309546 & -0.67052572 & -4.820458 \\
\hline $\mathrm{H}$ & -3.06303830 & -1.34085164 & -5.1111528 \\
\hline $\mathrm{H}$ & -2.38268253 & -1.28084310 & -3.4744482 \\
\hline $\mathrm{N}$ & -5.82572144 & 2.87546026 & -4.3072942 \\
\hline $\mathrm{C}$ & -5.39357992 & 4.04268196 & -4.8988053 \\
\hline $\mathrm{C}$ & -6.46808145 & 4.78264001 & -5.33459439 \\
\hline $\mathrm{N}$ & -7.58238649 & 4.04142378 & -4.9870521 \\
\hline $\mathrm{C}$ & -7.14594094 & 2.90476497 & -4.3818581 \\
\hline $\mathrm{C}$ & -6.51496090 & 6.09160447 & -6.0515285 \\
\hline $\mathrm{H}$ & -7.04078973 & 6.86471805 & -5.4748868 \\
\hline $\mathrm{H}$ & -5.49590696 & 6.44801311 & -6.2294257 \\
\hline $\mathrm{H}$ & -7.01494296 & 6.00861165 & -7.0254898 \\
\hline $\mathrm{H}$ & -8.55911337 & 4.28834762 & -5.1709077 \\
\hline $\mathrm{H}$ & -7.81569354 & 2.13448603 & -4.0252161 \\
\hline $\mathrm{H}$ & -4.77456038 & 1.54197865 & -4.2626551 \\
\hline $\mathrm{H}$ & -4.33953752 & 4.27253571 & -4.9804007 \\
\hline $\mathrm{O}$ & 1.08652143 & 0.76740034 & -3.2057124 \\
\hline $\mathrm{H}$ & 1.52506258 & 1.05300450 & -2.3760179 \\
\hline $\mathrm{H}$ & -0.81562531 & 0.87741521 & -2.9882959 \\
\hline
\end{tabular}




\section{Doublet; Basis set B2; Model A; Compound 0}

\begin{tabular}{|c|c|c|c|}
\hline $\mathrm{O}$ & -10.19129959 & 4.67269502 & -5.20366005 \\
\hline $\mathrm{C}$ & -10.92509423 & 5.57870879 & -4.77231485 \\
\hline $\mathrm{N}$ & -12.11389781 & 5.29577978 & -4.19869454 \\
\hline $\mathrm{C}$ & -12.61301293 & 3.93014319 & -4.06256104 \\
\hline $\mathrm{C}$ & -10.51391430 & 7.03785858 & -4.83153368 \\
\hline $\mathrm{H}$ & -12.68846911 & 6.05798316 & -3.86771771 \\
\hline $\mathrm{H}$ & -13.61510237 & 3.96839697 & -3.63128208 \\
\hline $\mathrm{H}$ & -11.96085980 & 3.34407169 & -3.40727649 \\
\hline $\mathrm{H}$ & -12.65644663 & 3.43980136 & -5.03937234 \\
\hline $\mathrm{H}$ & -11.28778683 & 7.72299928 & -4.47275122 \\
\hline $\mathrm{H}$ & -10.26259652 & 7.29778641 & -5.86451730 \\
\hline $\mathrm{H}$ & -9.61455971 & 7.17905107 & -4.22222075 \\
\hline $\mathrm{C}$ & -4.17488574 & -1.50580473 & -0.92808665 \\
\hline $\mathrm{C}$ & -3.19545222 & -0.44082944 & -0.82189823 \\
\hline $\mathrm{N}$ & -1.94528808 & -0.97240290 & -0.63610683 \\
\hline $\mathrm{C}$ & -2.09919993 & -2.33266687 & -0.61856985 \\
\hline $\mathrm{C}$ & -3.49542109 & -2.67929064 & -0.79461953 \\
\hline $\mathrm{C}$ & -3.50556345 & 0.91834012 & -0.88628523 \\
\hline $\mathrm{C}$ & -2.57931662 & 1.95893339 & -0.72058477 \\
\hline $\mathrm{N}$ & -1.22133520 & 1.82140002 & -0.53983884 \\
\hline $\mathrm{C}$ & -0.70252447 & 3.09193340 & -0.42743574 \\
\hline $\mathrm{C}$ & -1.76757514 & 4.06455181 & -0.53598864 \\
\hline $\mathrm{C}$ & -2.92567928 & 3.36330947 & -0.71927524 \\
\hline $\mathrm{C}$ & 0.65326857 & 3.40778680 & -0.25950263 \\
\hline $\mathrm{C}$ & 1.69534503 & 2.47638047 & -0.17056555 \\
\hline $\mathrm{N}$ & 1.56102626 & 1.10933697 & -0.22916147 \\
\hline $\mathrm{C}$ & 2.82837031 & 0.58504612 & -0.06754678 \\
\hline $\mathrm{C}$ & 3.78490979 & 1.66186117 & 0.07808637 \\
\hline $\mathrm{C}$ & 3.08665363 & 2.83129326 & 0.01402773 \\
\hline $\mathrm{Fe}$ & -0.19683309 & 0.07460807 & -0.42236438 \\
\hline $\mathrm{S}$ & -0.45122442 & -0.08570868 & 1.85661372 \\
\hline $\mathrm{C}$ & -0.35149781 & 1.57940490 & 2.61047308 \\
\hline $\mathrm{C}$ & 3.16790512 & -0.77331128 & -0.01767564 \\
\hline $\mathrm{C}$ & 2.20624426 & -1.80467997 & -0.13222208 \\
\hline $\mathrm{N}$ & 0.84245761 & -1.66299072 & -0.31550470 \\
\hline $\mathrm{C}$ & 0.29661405 & -2.92646547 & -0.33087626 \\
\hline $\mathrm{C}$ & 1.35381831 & -3.90611541 & -0.16925315 \\
\hline $\mathrm{C}$ & 2.52505047 & -3.21747641 & -0.04931519 \\
\hline $\mathrm{C}$ & -1.06949766 & -3.25768472 & -0.46431058 \\
\hline $\mathrm{H}$ & 4.20678728 & -1.05018947 & 0.12508956 \\
\hline $\mathrm{H}$ & 1.20387859 & -4.97925035 & -0.14834007 \\
\hline
\end{tabular}




$\begin{array}{lrrr}\mathrm{H} & 4.85067041 & 1.52330190 & 0.21706032 \\ \mathrm{H} & 3.46215617 & 3.84497723 & 0.08968146 \\ \mathrm{H} & 0.91634868 & 4.45957111 & -0.19251108 \\ \mathrm{H} & -1.63377225 & 5.13830324 & -0.47372544 \\ \mathrm{H} & -3.93154777 & 3.75057965 & -0.83516166 \\ \mathrm{H} & -4.54616608 & 1.19379328 & -1.03237748 \\ \mathrm{H} & -5.23811121 & -1.35548045 & -1.07542644 \\ \mathrm{H} & -3.88444160 & -3.69054069 & -0.81211397 \\ \mathrm{H} & -1.32703370 & -4.31253296 & -0.45310552 \\ \mathrm{H} & 3.52329697 & -3.61618057 & 0.08888911 \\ \mathrm{H} & -0.47901186 & 1.45992360 & 3.69274813 \\ \mathrm{H} & 0.62080807 & 2.04733591 & 2.42517049 \\ \mathrm{H} & -1.13933192 & 2.24198194 & 2.23800395 \\ \mathrm{O} & -0.06424931 & -0.06853768 & -2.35931575 \\ \mathrm{C} & -2.34371342 & -0.78305927 & -4.63230961 \\ \mathrm{C} & -3.11979085 & 0.48101499 & -4.46487156 \\ \mathrm{O} & -2.49624964 & 1.56404780 & -4.06393166 \\ \mathrm{O} & -4.33068582 & 0.52616339 & -4.75014548 \\ \mathrm{H} & -1.74207703 & -0.70467703 & -5.54792257 \\ \mathrm{H} & -3.02543049 & -1.62945402 & -4.72567036 \\ \mathrm{H} & -1.64260874 & -0.92900096 & -3.79972607 \\ \mathrm{~N} & -5.79377509 & 2.63183093 & -4.91292451 \\ \mathrm{C} & -5.33482087 & 3.80177457 & -5.47083038 \\ \mathrm{C} & -6.41523600 & 4.61515925 & -5.68900191 \\ \mathrm{~N} & -7.52075138 & 3.90567555 & -5.24316222 \\ \mathrm{C} & -7.11361457 & 2.70833421 & -4.78924475 \\ \mathrm{C} & -6.48149174 & 5.97973943 & -6.28251260 \\ \mathrm{H} & -6.86861489 & 6.71760845 & -5.56902835 \\ \mathrm{H} & -5.47993904 & 6.29967053 & -6.58208198 \\ \mathrm{H} & -7.12379536 & 6.00330542 & -7.17110170 \\ \mathrm{H} & -8.50045230 & 4.23447814 & -5.26544744 \\ \mathrm{H} & -7.75709709 & 1.93641624 & -4.39555775 \\ \mathrm{H} & -5.16281713 & 1.77018219 & -4.72824921 \\ \mathrm{H} & -4.28368157 & 3.95887814 & -5.66035979 \\ \mathrm{O} & -0.08641832 & 1.17611167 & -3.16049174 \\ \mathrm{H} & 0.16855295 & 1.85732110 & -2.51177739 \\ \mathrm{H} & -1.54179084 & 1.38995791 & -3.70066330\end{array}$




\section{Doublet; Basis set B2; Model A; Transition State 2}

\begin{tabular}{|c|c|c|c|}
\hline $\mathrm{O}$ & -10.24491677 & 4.63495116 & -5.44506191 \\
\hline $\mathrm{C}$ & -11.08472878 & 5.44187236 & -5.01679245 \\
\hline $\mathrm{N}$ & -12.31576731 & 5.04366421 & -4.61524504 \\
\hline $\mathrm{C}$ & -12.77948022 & 3.66180389 & -4.65544054 \\
\hline $\mathrm{C}$ & -10.76424473 & 6.92442705 & -4.90515386 \\
\hline $\mathrm{H}$ & -12.95852837 & 5.75019669 & -4.28329667 \\
\hline $\mathrm{H}$ & -13.67741760 & 3.57812877 & -5.27668643 \\
\hline $\mathrm{H}$ & -13.00462317 & 3.30096190 & -3.64590729 \\
\hline $\mathrm{H}$ & -11.98699411 & 3.05057008 & -5.08765366 \\
\hline $\mathrm{H}$ & -11.60900785 & 7.53029596 & -4.56315434 \\
\hline $\mathrm{H}$ & -10.43786148 & 7.28976107 & -5.88367230 \\
\hline $\mathrm{H}$ & -9.93295692 & 7.05522545 & -4.20430359 \\
\hline $\mathrm{C}$ & -4.19316111 & -1.35117522 & -0.71108175 \\
\hline $\mathrm{C}$ & -3.16726203 & -0.32762924 & -0.65554067 \\
\hline $\mathrm{N}$ & -1.93119636 & -0.90939824 & -0.52933843 \\
\hline $\mathrm{C}$ & -2.14197554 & -2.26269502 & -0.49711735 \\
\hline $\mathrm{C}$ & -3.55792513 & -2.55094986 & -0.60323245 \\
\hline $\mathrm{C}$ & -3.43086992 & 1.04176846 & -0.69880519 \\
\hline $\mathrm{C}$ & -2.46063643 & 2.04631286 & -0.56943868 \\
\hline $\mathrm{N}$ & -1.09390381 & 1.85465509 & -0.47767652 \\
\hline $\mathrm{C}$ & -0.51990019 & 3.10909385 & -0.37840799 \\
\hline $\mathrm{C}$ & -1.55300240 & 4.11632314 & -0.40596084 \\
\hline $\mathrm{C}$ & -2.74707083 & 3.45997346 & -0.52560059 \\
\hline $\mathrm{C}$ & 0.85697682 & 3.35939587 & -0.32021716 \\
\hline $\mathrm{C}$ & 1.86006354 & 2.38348259 & -0.29445134 \\
\hline $\mathrm{N}$ & 1.66733747 & 1.02268400 & -0.32024532 \\
\hline $\mathrm{C}$ & 2.92145197 & 0.44959465 & -0.23643582 \\
\hline $\mathrm{C}$ & 3.92758446 & 1.48806543 & -0.17224076 \\
\hline $\mathrm{C}$ & 3.27372438 & 2.68341144 & -0.21062827 \\
\hline $\mathrm{Fe}$ & -0.13310161 & 0.06166503 & -0.39675143 \\
\hline $\mathrm{S}$ & -0.25028172 & -0.10239750 & 1.86743961 \\
\hline $\mathrm{C}$ & -0.05591437 & 1.55706152 & 2.61299559 \\
\hline $\mathrm{C}$ & 3.20911396 & -0.92021715 & -0.19498343 \\
\hline $\mathrm{C}$ & 2.20102450 & -1.91096836 & -0.25128900 \\
\hline $\mathrm{N}$ & 0.83551361 & -1.71297525 & -0.36845361 \\
\hline $\mathrm{C}$ & 0.23730715 & -2.95416889 & -0.33687547 \\
\hline $\mathrm{C}$ & 1.26013469 & -3.97419913 & -0.21996060 \\
\hline $\mathrm{C}$ & 2.46374814 & -3.33419104 & -0.17001561 \\
\hline $\mathrm{C}$ & -1.14641489 & -3.22985294 & -0.38935363 \\
\hline $\mathrm{H}$ & 4.24220925 & -1.23876006 & -0.10946889 \\
\hline $\mathrm{H}$ & 1.06738034 & -5.03974769 & -0.17919453 \\
\hline
\end{tabular}




$\begin{array}{lrrr}\mathrm{H} & 4.99379713 & 1.30767982 & -0.10360376 \\ \mathrm{H} & 3.69311777 & 3.68197147 & -0.17941186 \\ \mathrm{H} & 1.17224273 & 4.39795954 & -0.27873332 \\ \mathrm{H} & -1.37778305 & 5.18384070 & -0.34373556 \\ \mathrm{H} & -3.74121563 & 3.88802273 & -0.57904357 \\ \mathrm{H} & -4.46572123 & 1.35590750 & -0.79550916 \\ \mathrm{H} & -5.25414627 & -1.15581631 & -0.81213407 \\ \mathrm{H} & -3.98851331 & -3.54518626 & -0.59921733 \\ \mathrm{H} & -1.44637830 & -4.27272251 & -0.35487282 \\ \mathrm{H} & 3.45036270 & -3.77331293 & -0.08008158 \\ \mathrm{H} & -0.08207056 & 1.42925982 & 3.70128559 \\ \mathrm{H} & 0.90162303 & 2.00993129 & 2.33844076 \\ \mathrm{H} & -0.86625471 & 2.23242549 & 2.32169785 \\ \mathrm{O} & -0.13245598 & -0.03413645 & -2.39395995 \\ \mathrm{C} & -2.55622773 & -0.71929157 & -4.49820724 \\ \mathrm{C} & -3.19920912 & 0.62445193 & -4.34267753 \\ \mathrm{O} & -2.48684499 & 1.62908833 & -4.01340453 \\ \mathrm{O} & -4.44307988 & 0.70611686 & -4.59306389 \\ \mathrm{H} & -2.05494986 & -0.75131976 & -5.47535580 \\ \mathrm{H} & -3.30718726 & -1.51131724 & -4.47043740 \\ \mathrm{H} & -1.79207836 & -0.88394558 & -3.73117087 \\ \mathrm{~N} & -5.78336893 & 2.77494476 & -4.77692403 \\ \mathrm{C} & -5.34134418 & 4.01221246 & -5.18120271 \\ \mathrm{C} & -6.43185477 & 4.79632423 & -5.46068865 \\ \mathrm{~N} & -7.53892401 & 4.00247190 & -5.20914591 \\ \mathrm{C} & -7.10876589 & 2.78879104 & -4.80804751 \\ \mathrm{C} & -6.49807522 & 6.20321221 & -5.94903676 \\ \mathrm{H} & -6.99216644 & 6.86737164 & -5.22843282 \\ \mathrm{H} & -5.48539332 & 6.58271994 & -6.11099120 \\ \mathrm{H} & -7.04153966 & 6.27845916 & -6.89904382 \\ \mathrm{H} & -8.52314329 & 4.28117154 & -5.32670909 \\ \mathrm{H} & -7.75303532 & 1.95853715 & -4.56001847 \\ \mathrm{H} & -5.08126281 & 1.82883085 & -4.60604570 \\ \mathrm{H} & -4.28659068 & 4.23836329 & -5.23879777 \\ \mathrm{O} & -0.24734989 & 1.29113461 & -3.07913030 \\ \mathrm{H} & -0.29518890 & 1.89540968 & -2.30329259 \\ \mathrm{H} & -1.30163462 & 1.36608037 & -3.54422787\end{array}$




\section{Doublet; Basis set B2; Model A; protonated Compound 0}

$\begin{array}{lrrr}78 & & & \\ \mathrm{O} & -10.30708747 & 4.68732611 & -5.35605873 \\ \mathrm{C} & -11.10396305 & 5.54359769 & -4.94952683 \\ \mathrm{~N} & -12.33118446 & 5.20536922 & -4.47723327 \\ \mathrm{C} & -12.82232885 & 3.83528650 & -4.40236319 \\ \mathrm{C} & -10.73950244 & 7.02286443 & -4.96573417 \\ \mathrm{H} & -12.94040669 & 5.94741692 & -4.16005188 \\ \mathrm{H} & -13.75971057 & 3.73462745 & -4.95975539 \\ \mathrm{H} & -12.98898675 & 3.53861073 & -3.36070964 \\ \mathrm{H} & -12.06998556 & 3.18210529 & -4.84518452 \\ \mathrm{H} & -11.54001621 & 7.67590827 & -4.60381968 \\ \mathrm{H} & -10.48225034 & 7.30877557 & -5.99026790 \\ \mathrm{H} & -9.85185056 & 7.17435354 & -4.34305779 \\ \mathrm{C} & -4.14561682 & -1.45358491 & -0.83591174 \\ \mathrm{C} & -3.13648604 & -0.41634833 & -0.74706271 \\ \mathrm{~N} & -1.89556216 & -0.97444108 & -0.58627488 \\ \mathrm{C} & -2.08986452 & -2.33007611 & -0.55836002 \\ \mathrm{C} & -3.49569746 & -2.64280761 & -0.70887519 \\ \mathrm{C} & -3.42389384 & 0.94565357 & -0.78354015 \\ \mathrm{C} & -2.48843518 & 1.97376877 & -0.62113205 \\ \mathrm{~N} & -1.11202427 & 1.81411752 & -0.52518200 \\ \mathrm{C} & -0.57532152 & 3.08499791 & -0.36902968 \\ \mathrm{C} & -1.63405424 & 4.05960131 & -0.38419457 \\ \mathrm{C} & -2.80947212 & 3.37493551 & -0.54185285 \\ \mathrm{C} & 0.79246477 & 3.35458419 & -0.29273914 \\ \mathrm{C} & 1.81157642 & 2.39663527 & -0.24590672 \\ \mathrm{~N} & 1.64685970 & 1.03262351 & -0.25992295 \\ \mathrm{C} & 2.91065190 & 0.48739478 & -0.14946437 \\ \mathrm{C} & 3.89472056 & 1.54585820 & -0.07729401 \\ \mathrm{C} & 3.21717389 & 2.72643117 & -0.13986151 \\ \mathrm{Fe} & -0.11884925 & 0.02763685 & -0.37744949 \\ \mathrm{~S} & -0.32256057 & -0.12863646 & 1.86533859 \\ \mathrm{C} & -0.14532197 & 1.52984670 & 2.61609088 \\ \mathrm{C} & 3.22094891 & -0.87463498 & -0.09557625 \\ \mathrm{C} & 0.2359365738 & -1.88790649 & -0.17167468 \\ \mathrm{C} & -1.71886784 & -0.32898189 \\ \mathrm{C} & & -2.97172776 & -0.31248497 \\ \mathrm{C} & -33309848 & -3.96907147 & -0.16069946 \\ \mathrm{C} & -3.30490588 & -0.07772450 \\ \mathrm{C} & -3.27450659 & -0.41212973 \\ \mathrm{C} & -1.17384105 & 0.01417136 \\ \mathrm{C} & -5.03821175 & -0.12259441\end{array}$




$\begin{array}{lrrr}\mathrm{H} & 4.96282863 & 1.38793428 & 0.01162871 \\ \mathrm{H} & 3.61472988 & 3.73382055 & -0.11232079 \\ \mathrm{H} & 1.09121422 & 4.39747977 & -0.23851818 \\ \mathrm{H} & -1.49116562 & 5.12943069 & -0.29082644 \\ \mathrm{H} & -3.81261359 & 3.77961930 & -0.60148382 \\ \mathrm{H} & -4.46342738 & 1.23770422 & -0.89886299 \\ \mathrm{H} & -5.20506181 & -1.27230493 & -0.97058982 \\ \mathrm{H} & -3.90906962 & -3.64409865 & -0.71760269 \\ \mathrm{H} & -1.36206352 & -4.32343955 & -0.38345105 \\ \mathrm{H} & 3.51392694 & -3.72518278 & 0.04212001 \\ \mathrm{H} & -0.22345470 & 1.40200483 & 3.70168232 \\ \mathrm{H} & 0.82890656 & 1.97122316 & 2.38584717 \\ \mathrm{H} & -0.93467588 & 2.21231688 & 2.28706432 \\ \mathrm{O} & -0.02281040 & -0.04582469 & -2.40389753 \\ \mathrm{C} & -2.44488174 & -0.75774181 & -4.49019250 \\ \mathrm{C} & -3.11167053 & 0.57494342 & -4.37464667 \\ \mathrm{O} & -2.46242630 & 1.61328205 & -4.15231432 \\ \mathrm{O} & -4.40697869 & 0.54938632 & -4.55545013 \\ \mathrm{H} & -1.93825063 & -0.80943571 & -5.46319924 \\ \mathrm{H} & -3.17118770 & -1.57081596 & -4.43366606 \\ \mathrm{H} & -1.68104242 & -0.87383811 & -3.71417475 \\ \mathrm{~N} & -5.84130233 & 2.73465332 & -4.63827817 \\ \mathrm{C} & -5.39116205 & 3.93544516 & -5.14027389 \\ \mathrm{C} & -6.45825575 & 4.73857897 & -5.46521658 \\ \mathrm{~N} & -7.58207874 & 4.00030506 & -5.14126400 \\ \mathrm{C} & -7.16224393 & 2.80361806 & -4.65656928 \\ \mathrm{C} & -6.49591804 & 6.10654728 & -6.05820823 \\ \mathrm{H} & -6.98911333 & 6.83259662 & -5.39800803 \\ \mathrm{H} & -5.47525546 & 6.45966802 & -6.23305368 \\ \mathrm{H} & -7.02481364 & 6.12245226 & -7.02013782 \\ \mathrm{H} & -8.55653352 & 4.29261459 & -5.26497188 \\ \mathrm{H} & -7.83941011 & 2.02321363 & -4.33868140 \\ \mathrm{H} & -4.88491634 & 1.46887759 & -4.50890028 \\ \mathrm{H} & -4.33374106 & 4.14111142 & -5.23642875 \\ \mathrm{O} & -0.16052867 & 1.33542560 & -2.98277111 \\ \mathrm{H} & -0.45634195 & 1.80944985 & -2.14687021 \\ \mathrm{H} & -1.02378107 & 1.33275023 & -3.55059405\end{array}$




\section{Doublet; Basis set B2; Model A; Transition State 3}

\begin{tabular}{|c|c|c|c|}
\hline $\mathrm{O}$ & -10.34809495 & 4.68698325 & -5.34040948 \\
\hline $\mathrm{C}$ & -11.13461197 & 5.56863502 & -4.96855303 \\
\hline $\mathrm{N}$ & -12.36020003 & 5.24403636 & -4.49002725 \\
\hline $\mathrm{C}$ & -12.81120369 & 3.86018772 & -4.38876047 \\
\hline $\mathrm{C}$ & -10.76171773 & 7.04340426 & -5.02795689 \\
\hline $\mathrm{H}$ & -12.98402565 & 5.98338925 & -4.19914108 \\
\hline $\mathrm{H}$ & -13.84230173 & 3.85643634 & -4.02920863 \\
\hline $\mathrm{H}$ & -12.18291882 & 3.29773973 & -3.69055122 \\
\hline $\mathrm{H}$ & -12.76514815 & 3.37010315 & -5.36586598 \\
\hline $\mathrm{H}$ & -11.56430542 & 7.71074185 & -4.69825255 \\
\hline $\mathrm{H}$ & -10.48849854 & 7.29659512 & -6.05698290 \\
\hline $\mathrm{H}$ & -9.88245855 & 7.21173918 & -4.39753992 \\
\hline $\mathrm{C}$ & -4.14571105 & -1.47479597 & -0.80811498 \\
\hline $\mathrm{C}$ & -3.14464866 & -0.42985747 & -0.72933308 \\
\hline $\mathrm{N}$ & -1.90062978 & -0.98304696 & -0.57100019 \\
\hline $\mathrm{C}$ & -2.08074585 & -2.34386509 & -0.54043724 \\
\hline $\mathrm{C}$ & -3.48710378 & -2.65995389 & -0.68207420 \\
\hline $\mathrm{C}$ & -3.43103390 & 0.93398775 & -0.78796311 \\
\hline $\mathrm{C}$ & -2.48652781 & 1.95358866 & -0.64573620 \\
\hline $\mathrm{N}$ & -1.12507083 & 1.79355197 & -0.48484441 \\
\hline $\mathrm{C}$ & -0.58699479 & 3.06233577 & -0.41089047 \\
\hline $\mathrm{C}$ & -1.63903887 & 4.04637229 & -0.51898722 \\
\hline $\mathrm{C}$ & -2.80954769 & 3.36090549 & -0.66563073 \\
\hline $\mathrm{C}$ & 0.77366898 & 3.35924949 & -0.29366655 \\
\hline $\mathrm{C}$ & 1.80036901 & 2.40762436 & -0.20038751 \\
\hline $\mathrm{N}$ & 1.63993508 & 1.04164610 & -0.22627322 \\
\hline $\mathrm{C}$ & 2.90403709 & 0.49129214 & -0.11022351 \\
\hline $\mathrm{C}$ & 3.88092842 & 1.55263716 & -0.01047797 \\
\hline $\mathrm{C}$ & 3.20263406 & 2.73465095 & -0.06818450 \\
\hline $\mathrm{Fe}$ & -0.12522838 & 0.04593734 & -0.40810846 \\
\hline $\mathrm{S}$ & -0.30266155 & -0.17460846 & 1.87666440 \\
\hline $\mathrm{C}$ & -0.25035009 & 1.49409303 & 2.62513975 \\
\hline $\mathrm{C}$ & 3.22396299 & -0.87239837 & -0.08211166 \\
\hline $\mathrm{C}$ & 2.24466809 & -1.88933055 & -0.17064942 \\
\hline $\mathrm{N}$ & 0.87657428 & -1.72655254 & -0.30811295 \\
\hline $\mathrm{C}$ & 0.30479215 & -2.98214002 & -0.30909375 \\
\hline $\mathrm{C}$ & 1.35197013 & -3.97469523 & -0.18210595 \\
\hline $\mathrm{C}$ & 2.53878647 & -3.30591785 & -0.10129665 \\
\hline $\mathrm{C}$ & -1.07131727 & -3.29243939 & -0.40336250 \\
\hline $\mathrm{H}$ & 4.26282855 & -1.16489342 & 0.02231289 \\
\hline $\mathrm{H}$ & 1.18592924 & -5.04509207 & -0.15724585 \\
\hline
\end{tabular}




$\begin{array}{lrrr}\mathrm{H} & 4.94825932 & 1.39698273 & 0.09092276 \\ \mathrm{H} & 3.60060496 & 3.74125594 & -0.02361628 \\ \mathrm{H} & 1.05861431 & 4.40650986 & -0.26321796 \\ \mathrm{H} & -1.48587294 & 5.11844792 & -0.49099495 \\ \mathrm{H} & -3.80962666 & 3.75828889 & -0.78805502 \\ \mathrm{H} & -4.46623210 & 1.22851724 & -0.92887867 \\ \mathrm{H} & -5.20707859 & -1.30215258 & -0.93857724 \\ \mathrm{H} & -3.89609738 & -3.66310174 & -0.68705811 \\ \mathrm{H} & -1.34868934 & -4.34139472 & -0.38121162 \\ \mathrm{H} & 3.53342562 & -3.72258574 & 0.00375273 \\ \mathrm{H} & -0.32536367 & 1.36282746 & 3.71059076 \\ \mathrm{H} & 0.69165942 & 2.00383756 & 2.40096939 \\ \mathrm{H} & -1.08420523 & 2.11725417 & 2.28886324 \\ \mathrm{O} & 0.04035265 & -0.15604548 & -2.16669935 \\ \mathrm{C} & -2.43987616 & -0.75156873 & -4.50953742 \\ \mathrm{C} & -3.10846573 & 0.57647872 & -4.30941591 \\ \mathrm{O} & -2.47568125 & 1.60184839 & -4.02052862 \\ \mathrm{O} & -4.40978002 & 0.54817269 & -4.49745322 \\ \mathrm{H} & -1.90473015 & -0.73199106 & -5.46770386 \\ \mathrm{H} & -3.16812096 & -1.56432510 & -4.53094702 \\ \mathrm{H} & -1.69966942 & -0.92472137 & -3.72203056 \\ \mathrm{~N} & -5.87009203 & 2.76042838 & -4.55020381 \\ \mathrm{C} & -5.41782236 & 3.94809330 & -5.08232373 \\ \mathrm{C} & -6.48193219 & 4.74302008 & -5.43890199 \\ \mathrm{~N} & -7.60780586 & 4.01322078 & -5.10366235 \\ \mathrm{C} & -7.19065204 & 2.83017383 & -4.58190654 \\ \mathrm{C} & -6.51276484 & 6.09795667 & -6.06545934 \\ \mathrm{H} & -6.98644437 & 6.84629728 & -5.41578192 \\ \mathrm{H} & -5.49039519 & 6.43291565 & -6.26454629 \\ \mathrm{H} & -7.05611054 & 6.09684516 & -7.01939427 \\ \mathrm{H} & -8.58182918 & 4.29988702 & -5.24112804 \\ \mathrm{H} & -7.87185901 & 2.05900098 & -4.25011938 \\ \mathrm{H} & -4.88004626 & 1.45827162 & -4.41774663 \\ \mathrm{H} & -4.35982372 & 4.15241736 & -5.17659167 \\ \mathrm{O} & 0.04825009 & 1.29010304 & -3.17974680 \\ \mathrm{H} & 0.16456072 & 1.95597769 & -2.47396717 \\ \mathrm{H} & -0.91977552 & 1.36864230 & -3.47337710\end{array}$




\section{Doublet; Basis set B2; Model A; Compound I}

$\begin{array}{lrrr}78 & & & \\ \mathrm{O} & -10.28197995 & 4.78304457 & -5.12089661 \\ \mathrm{C} & -10.94529260 & 5.71394731 & -4.64605062 \\ \mathrm{~N} & -12.13420778 & 5.47354534 & -4.03889351 \\ \mathrm{C} & -12.68271166 & 4.12732024 & -3.91848439 \\ \mathrm{C} & -10.46011933 & 7.15575416 & -4.72331186 \\ \mathrm{H} & -12.66135385 & 6.25113408 & -3.66763274 \\ \mathrm{H} & -13.65899197 & 4.19219414 & -3.43338256 \\ \mathrm{H} & -12.02250084 & 3.49236323 & -3.31894829 \\ \mathrm{H} & -12.79699220 & 3.66959889 & -4.90581694 \\ \mathrm{H} & -11.15813708 & 7.87609208 & -4.28479389 \\ \mathrm{H} & -10.29375605 & 7.41471365 & -5.77349047 \\ \mathrm{H} & -9.49950522 & 7.23376696 & -4.20396727 \\ \mathrm{C} & -4.10124601 & -1.62413756 & -0.93178182 \\ \mathrm{C} & -3.14462976 & -0.55083926 & -0.79572300 \\ \mathrm{~N} & -1.88812248 & -1.05862870 & -0.57650219 \\ \mathrm{C} & -2.02560809 & -2.42715454 & -0.57704048 \\ \mathrm{C} & -3.40760338 & -2.78873982 & -0.79073361 \\ \mathrm{C} & -3.47662345 & 0.80215953 & -0.87768553 \\ \mathrm{C} & -2.56752697 & 1.85177754 & -0.73352577 \\ \mathrm{~N} & -1.21795269 & 1.73065885 & -0.47417039 \\ \mathrm{C} & -0.71700507 & 3.01044806 & -0.41840097 \\ \mathrm{C} & -1.78100620 & 3.96476481 & -0.63098879 \\ \mathrm{C} & -2.92342950 & 3.24925061 & -0.82829787 \\ \mathrm{C} & 0.61710650 & 3.36399696 & -0.18453504 \\ \mathrm{C} & 1.66028462 & 2.44454625 & -0.01705824 \\ \mathrm{~N} & 1.54780527 & 1.06540352 & -0.03168440 \\ \mathrm{C} & 2.83670610 & 0.56304732 & 0.10638740 \\ \mathrm{C} & 3.76544023 & 1.65921367 & 0.23949463 \\ \mathrm{C} & 3.04387240 & 2.81433753 & 0.16771665 \\ \mathrm{C} & -0.14945302 & 0.00537241 & -0.46033278 \\ \mathrm{Fe} & -0.14198287 & 0.00664474 & 2.12346749 \\ \mathrm{~S} & -0.71557238 & 1.61923932 & 2.72627457 \\ \mathrm{C} & 3.19914389 & -0.78606010 & 0.11138904 \\ \mathrm{C} & 2.25728944 & -1.83450754 & -0.01299689 \\ \mathrm{C} & 0.36632402 & -1.71009903 & -0.16775270 \\ \mathrm{C} & 1.43871413 & -3.94546201 & -0.10846965 \\ \mathrm{C} & 2.59939653 & -3.23746624 & 0.02361768 \\ \mathrm{H} & -3.34759857 & -0.41262889 \\ \mathrm{C} & -1.04627048 & 0.22203823 \\ \mathrm{H} & -5.02120560 & -0.12284990\end{array}$




$\begin{array}{lrrr}\mathrm{H} & 4.83535332 & 1.54493589 & 0.36367493 \\ \mathrm{H} & 3.40521900 & 3.83423925 & 0.21624605 \\ \mathrm{H} & 0.86779119 & 4.41951385 & -0.17267248 \\ \mathrm{H} & -1.64882634 & 5.03946072 & -0.64517799 \\ \mathrm{H} & -3.92038078 & 3.61550048 & -1.03913956 \\ \mathrm{H} & -4.51100375 & 1.05735897 & -1.08345357 \\ \mathrm{H} & -5.15777302 & -1.48508593 & -1.12324207 \\ \mathrm{H} & -3.77914619 & -3.80515925 & -0.83468889 \\ \mathrm{H} & -1.23363146 & -4.40524852 & -0.43729767 \\ \mathrm{H} & 3.60541083 & -3.62193540 & 0.14021860 \\ \mathrm{H} & -0.76282208 & 1.60002458 & 3.81960878 \\ \mathrm{H} & 0.00592530 & 2.38989215 & 2.42574563 \\ \mathrm{H} & -1.68989439 & 1.87891811 & 2.30400120 \\ \mathrm{O} & 0.10605948 & 0.02910937 & -2.06047671 \\ \mathrm{C} & -2.26570439 & -0.81772513 & -4.56678856 \\ \mathrm{C} & -3.10455595 & 0.43323544 & -4.69239434 \\ \mathrm{O} & -2.63087476 & 1.54513173 & -4.90568253 \\ \mathrm{O} & -4.41237188 & 0.21929023 & -4.54644767 \\ \mathrm{H} & -1.63670205 & -0.91500648 & -5.45807561 \\ \mathrm{H} & -2.88597492 & -1.70835080 & -4.44827564 \\ \mathrm{H} & -1.59228781 & -0.71582690 & -3.70802827 \\ \mathrm{~N} & -5.90516343 & 2.48786582 & -4.86031694 \\ \mathrm{C} & -5.41609397 & 3.65216867 & -5.41886013 \\ \mathrm{C} & -6.44540096 & 4.53940738 & -5.64195603 \\ \mathrm{~N} & -7.58501338 & 3.88775313 & -5.19795367 \\ \mathrm{C} & -7.21000500 & 2.66339021 & -4.74497459 \\ \mathrm{C} & -6.49690312 & 5.90221520 & -6.25498883 \\ \mathrm{H} & -6.87086721 & 6.65863931 & -5.55175342 \\ \mathrm{H} & -5.49361741 & 6.20879565 & -6.56649117 \\ \mathrm{H} & -7.14363494 & 5.92405516 & -7.14180666 \\ \mathrm{H} & -8.54062570 & 4.25203176 & -5.22670807 \\ \mathrm{H} & -7.91212821 & 1.94137175 & -4.35088145 \\ \mathrm{H} & -4.92812469 & 1.08955359 & -4.64589397 \\ \mathrm{H} & -4.35971571 & 3.76453544 & -5.62205804 \\ \mathrm{O} & 0.09372131 & 2.26345228 & -4.03068647 \\ \mathrm{H} & 0.21496619 & 1.56095587 & -3.36561329 \\ \mathrm{H} & & 2.08444394 & -4.40772977\end{array}$




\section{Doublet; Basis set B2; Model B; Fe+HOOH}

\begin{tabular}{|c|c|c|c|}
\hline $\mathrm{O}$ & -10.63283246 & 4.17496959 & -4.58274756 \\
\hline $\mathrm{C}$ & -11.52307087 & 4.98296384 & -4.28733436 \\
\hline $\mathrm{N}$ & -12.79853912 & 4.56465958 & -4.09538822 \\
\hline $\mathrm{C}$ & -13.18178473 & 3.16357233 & -4.23097194 \\
\hline $\mathrm{C}$ & -11.22371393 & 6.46671308 & -4.11501887 \\
\hline $\mathrm{H}$ & -13.50855799 & 5.24533818 & -3.86550359 \\
\hline $\mathrm{H}$ & -14.25945010 & 3.08158159 & -4.07463531 \\
\hline $\mathrm{H}$ & -12.66123901 & 2.54677820 & -3.49136818 \\
\hline $\mathrm{H}$ & -12.92956003 & 2.79146581 & -5.22857016 \\
\hline $\mathrm{H}$ & -12.11119875 & 7.06959209 & -3.89784752 \\
\hline $\mathrm{H}$ & -10.75426441 & 6.83987966 & -5.03029677 \\
\hline $\mathrm{H}$ & -10.50590701 & 6.58907025 & -3.29724846 \\
\hline $\mathrm{C}$ & -4.65753437 & -2.09466131 & 0.14328749 \\
\hline $\mathrm{C}$ & -3.72415789 & -1.04808870 & 0.48212520 \\
\hline $\mathrm{N}$ & -2.46300531 & -1.57100532 & 0.73326708 \\
\hline $\mathrm{C}$ & -2.61368496 & -2.93120927 & 0.56761804 \\
\hline $\mathrm{C}$ & -3.96995742 & -3.26566239 & 0.20011148 \\
\hline $\mathrm{C}$ & -4.06762449 & 0.29386509 & 0.54294234 \\
\hline $\mathrm{C}$ & -3.10056187 & 1.29311026 & 0.84334448 \\
\hline $\mathrm{N}$ & -1.72959519 & 1.12365082 & 1.07135694 \\
\hline $\mathrm{C}$ & -1.19806769 & 2.39591198 & 1.30670244 \\
\hline $\mathrm{C}$ & -2.25855763 & 3.37088965 & 1.20405126 \\
\hline $\mathrm{C}$ & -3.41379922 & 2.70035581 & 0.91979117 \\
\hline $\mathrm{C}$ & 0.15214477 & 2.72909889 & 1.57507857 \\
\hline $\mathrm{C}$ & 1.17616885 & 1.74520720 & 1.63449865 \\
\hline $\mathrm{N}$ & 1.04246082 & 0.36001115 & 1.49151752 \\
\hline $\mathrm{C}$ & 2.33358762 & -0.18506644 & 1.60348011 \\
\hline $\mathrm{C}$ & 3.27285868 & 0.88973436 & 1.80899027 \\
\hline $\mathrm{C}$ & 2.57028051 & 2.06218172 & 1.83221129 \\
\hline $\mathrm{Fe}$ & -0.73073563 & -0.61722122 & 1.33158022 \\
\hline $\mathrm{S}$ & -1.15048954 & -0.72326241 & 3.52525717 \\
\hline $\mathrm{C}$ & 0.45386754 & -0.88631955 & 4.39121375 \\
\hline $\mathrm{C}$ & 2.70403930 & -1.55943298 & 1.54421660 \\
\hline $\mathrm{C}$ & 1.70544010 & -2.54349654 & 1.34492570 \\
\hline $\mathrm{N}$ & 0.32595287 & -2.32717620 & 1.18761197 \\
\hline $\mathrm{C}$ & -0.25048169 & -3.57517128 & 0.97365620 \\
\hline $\mathrm{C}$ & 0.77821392 & -4.58719931 & 1.02069693 \\
\hline $\mathrm{C}$ & 1.96937650 & -3.95761880 & 1.24586240 \\
\hline $\mathrm{C}$ & -1.60697438 & -3.87097596 & 0.69044468 \\
\hline $\mathrm{H}$ & 3.74331329 & -1.84588004 & 1.64682247 \\
\hline $\mathrm{H}$ & 0.60239583 & -5.64742857 & 0.88388105 \\
\hline
\end{tabular}




\begin{tabular}{|c|c|c|c|}
\hline $\mathrm{H}$ & 4.34262631 & 0.75577645 & 1.91706028 \\
\hline $\mathrm{H}$ & 2.95903629 & 3.06535674 & 1.96060224 \\
\hline $\mathrm{H}$ & 0.41887017 & 3.76996271 & 1.71516440 \\
\hline $\mathrm{H}$ & -2.12349614 & 4.43941078 & 1.32193924 \\
\hline $\mathrm{H}$ & -4.40055447 & 3.11589474 & 0.75540552 \\
\hline $\mathrm{H}$ & -5.08482808 & 0.60412947 & 0.33648882 \\
\hline $\mathrm{H}$ & -5.69377479 & -1.92687185 & -0.12101200 \\
\hline $\mathrm{H}$ & -4.32410094 & -4.27021975 & 0.00448906 \\
\hline $\mathrm{H}$ & -1.85645485 & -4.91580535 & 0.53631876 \\
\hline $\mathrm{H}$ & 2.95267069 & -4.40501776 & 1.32821374 \\
\hline $\mathrm{H}$ & 0.23944177 & -1.02267268 & 5.45688143 \\
\hline $\mathrm{H}$ & 1.00535970 & -1.75823470 & 4.03252791 \\
\hline $\mathrm{H}$ & 1.06214323 & 0.01214162 & 4.26738007 \\
\hline $\mathrm{C}$ & -2.72878994 & -1.11534436 & -3.36088906 \\
\hline C & -3.50812440 & 0.18167376 & -3.37088278 \\
\hline $\mathrm{O}$ & -3.03191487 & 1.26379335 & -3.67803184 \\
\hline $\mathrm{O}$ & -4.78604147 & 0.00437498 & -3.01622349 \\
\hline $\mathrm{H}$ & -1.67163690 & -0.91526464 & -3.5442735 \\
\hline $\mathrm{H}$ & -3.11492061 & -1.78044136 & -4.14265698 \\
\hline $\mathrm{H}$ & -2.85452691 & -1.62977144 & -2.40342364 \\
\hline $\mathrm{N}$ & -6.26779015 & 2.26507840 & -3.29222096 \\
\hline $\mathrm{C}$ & -5.76821612 & 3.48763222 & -3.69427660 \\
\hline C & -6.79810719 & 4.31721910 & -4.07593598 \\
\hline $\mathrm{N}$ & -7.95134013 & 3.56884643 & -3.8940610 \\
\hline $\mathrm{C}$ & -7.57892959 & 2.34777785 & -3.4316037 \\
\hline $\mathrm{C}$ & -6.82876843 & 5.70677627 & -4.62184148 \\
\hline $\mathrm{H}$ & -7.38875624 & 6.39418587 & -3.97346531 \\
\hline $\mathrm{H}$ & -5.80929916 & 6.09463122 & -4.7115919 \\
\hline $\mathrm{H}$ & -7.28779640 & 5.74229071 & -5.61853125 \\
\hline $\mathrm{H}$ & -8.90248139 & 3.85363824 & -4.14331702 \\
\hline $\mathrm{H}$ & -8.28702334 & 1.55834439 & -3.2192408 \\
\hline $\mathrm{H}$ & -5.29725480 & 0.87976508 & -3.0890708 \\
\hline $\mathrm{H}$ & -4.70284477 & 3.67365795 & -3.7004913 \\
\hline $\mathrm{N}$ & -2.48524236 & -2.06248043 & 6.42293375 \\
\hline $\mathrm{C}$ & -1.54631141 & -2.64492024 & 7.3645863 \\
\hline $\mathrm{C}$ & -3.80057021 & -2.41535702 & 6.3777512 \\
\hline $\mathrm{O}$ & -4.30534703 & -3.27736297 & 7.0906926 \\
\hline C & -4.60772174 & -1.68865692 & 5.2699937 \\
\hline $\mathrm{C}$ & -4.27208905 & -2.45551137 & 3.9746250 \\
\hline $\mathrm{N}$ & -4.17354546 & -0.30291971 & 5.0682559 \\
\hline $\mathrm{C}$ & -4.64617061 & 0.73796669 & 5.8211635 \\
\hline $\mathrm{C}$ & -4.14314108 & 2.11972857 & 5.4278547 \\
\hline $\mathrm{O}$ & -5.42721087 & 0.57464322 & 6.75575696 \\
\hline $\mathrm{H}$ & -2.18823514 & -1.28963010 & 5.8441928 \\
\hline $\mathrm{H}$ & -0.70008418 & -3.10807559 & 6.8419551 \\
\hline
\end{tabular}




$\begin{array}{llll}\mathrm{H} & -1.16374367 & -1.89054579 & 8.06407823 \\ \mathrm{H} & -2.07570793 & -3.41325460 & 7.93164984 \\ \mathrm{H} & -5.66398043 & -1.71988929 & 5.53823925 \\ \mathrm{H} & -3.20610205 & -2.40317155 & 3.75093233 \\ \mathrm{H} & -4.55001405 & -3.50934940 & 4.08490611 \\ \mathrm{H} & -4.83188041 & -2.04773060 & 3.12544689 \\ \mathrm{H} & -3.56594710 & -0.10075784 & 4.28465404 \\ \mathrm{H} & -3.40156188 & 2.10706198 & 4.62285261 \\ \mathrm{H} & -4.99783911 & 2.72963724 & 5.11538099 \\ \mathrm{H} & -3.70662395 & 2.59724305 & 6.31111020\end{array}$

\section{Doublet; Basis set B2; Model A; FeHOOH}

$\begin{array}{llrl}100 & & & \\ \mathrm{O} & -10.54965826 & 4.09720438 & -4.51631529 \\ \mathrm{C} & -11.42838816 & 4.92099756 & -4.22705351 \\ \mathrm{~N} & -12.68026901 & 4.51251922 & -3.90750271 \\ \mathrm{C} & -13.05327184 & 3.10220043 & -3.88358145 \\ \mathrm{C} & -11.14281008 & 6.41607764 & -4.21417226 \\ \mathrm{H} & -13.37779191 & 5.20573057 & -3.67678160 \\ \mathrm{H} & -14.12171067 & 3.02906184 & -3.67022777 \\ \mathrm{H} & -12.49300076 & 2.56482536 & -3.11148553 \\ \mathrm{H} & -12.84281237 & 2.63561623 & -4.85041794 \\ \mathrm{H} & -12.02086194 & 7.02698738 & -3.98204020 \\ \mathrm{H} & -10.75457112 & 6.70895201 & -5.19451832 \\ \mathrm{H} & -10.36485374 & 6.62136252 & -3.47143997 \\ \mathrm{C} & -4.66215059 & -2.05490195 & 0.10760912 \\ \mathrm{C} & -3.73639613 & -0.99229685 & 0.42464303 \\ \mathrm{~N} & -2.48301748 & -1.51216096 & 0.66788999 \\ \mathrm{C} & -2.60231625 & -2.87449711 & 0.52620682 \\ \mathrm{C} & -3.96165007 & -3.22161665 & 0.17679022 \\ \mathrm{C} & -4.07411347 & 0.35634267 & 0.47791246 \\ \mathrm{C} & -3.16382499 & 1.37603110 & 0.77893729 \\ \mathrm{~N} & -1.81555896 & 1.21539597 & 1.01798435 \\ \mathrm{C} & -1.30513554 & 2.48093190 & 1.23263050 \\ \mathrm{C} & -2.36754969 & 3.45794730 & 1.13538938 \\ \mathrm{C} & -3.51270799 & 2.77609524 & 0.85223687 \\ \mathrm{C} & 0.03256581 & 2.80296320 & 1.47812958 \\ \mathrm{C} & 1.07842323 & 1.84909449 & 1.54496005 \\ \mathrm{~N} & 0.97471831 & 0.46983174 & 1.35371646 \\ \mathrm{C} & 2.25510169 & -0.06937961 & 1.52840648 \\ \mathrm{C} & 3.17307796 & 1.00685995 & 1.79845088\end{array}$




\begin{tabular}{|c|c|c|c|}
\hline $\mathrm{C}$ & 2.45682754 & 2.17504082 & 1.81028328 \\
\hline $\mathrm{Fe}$ & -0.77180037 & -0.53051194 & 1.13729323 \\
\hline S & -1.16192944 & -0.65206738 & 3.34891431 \\
\hline $\mathrm{C}$ & 0.43511778 & -0.81571426 & 4.22745869 \\
\hline $\mathrm{C}$ & 2.61412013 & -1.44051887 & 1.46744378 \\
\hline $\mathrm{C}$ & 1.66845488 & -2.44458691 & 1.25246121 \\
\hline $\mathrm{N}$ & 0.30958999 & -2.25227055 & 1.07360894 \\
\hline $\mathrm{C}$ & -0.23292629 & -3.50565178 & 0.90649940 \\
\hline $\mathrm{C}$ & 0.80384821 & -4.51089675 & 0.98455710 \\
\hline C & 1.97879653 & -3.85465965 & 1.19577658 \\
\hline $\mathrm{C}$ & -1.57415847 & -3.80417597 & 0.65963594 \\
\hline $\mathrm{H}$ & 3.65568816 & -1.70830764 & 1.60520820 \\
\hline $\mathrm{H}$ & 0.63714730 & -5.57623773 & 0.88205132 \\
\hline $\mathrm{H}$ & 4.23684169 & 0.88062651 & 1.96102913 \\
\hline $\mathrm{H}$ & 2.82918801 & 3.17776584 & 1.98298982 \\
\hline $\mathrm{H}$ & 0.29395861 & 3.84483009 & 1.62939223 \\
\hline $\mathrm{H}$ & -2.23400703 & 4.52606523 & 1.25677483 \\
\hline $\mathrm{H}$ & -4.50795248 & 3.16983449 & 0.68737216 \\
\hline $\mathrm{H}$ & -5.10097875 & 0.63905691 & 0.27083378 \\
\hline $\mathrm{H}$ & -5.70812165 & -1.90851343 & -0.13226226 \\
\hline $\mathrm{H}$ & -4.31571680 & -4.23128262 & 0.00813709 \\
\hline $\mathrm{H}$ & -1.83166233 & -4.85149370 & 0.53336511 \\
\hline $\mathrm{H}$ & 2.97318452 & -4.27068328 & 1.30242973 \\
\hline $\mathrm{H}$ & 0.21946369 & -0.91656079 & 5.29635382 \\
\hline $\mathrm{H}$ & 0.97670028 & -1.70435237 & 3.89538618 \\
\hline $\mathrm{H}$ & 1.05867657 & 0.06904210 & 4.08156788 \\
\hline $\mathrm{O}$ & -0.57994308 & -0.33012208 & -1.03491580 \\
\hline $\mathrm{C}$ & -2.56840666 & -1.09505250 & -3.41859648 \\
\hline $\mathrm{C}$ & -3.22672727 & 0.19244108 & -3.00504551 \\
\hline $\mathrm{O}$ & -2.65956667 & 1.04997566 & -2.32686705 \\
\hline $\mathrm{O}$ & -4.47856809 & 0.27447353 & -3.42583690 \\
\hline $\mathrm{H}$ & -1.48862188 & -0.96570257 & -3.50766623 \\
\hline $\mathrm{H}$ & -2.99027325 & -1.46250838 & -4.35765365 \\
\hline $\mathrm{H}$ & -2.75363542 & -1.85164607 & -2.64646394 \\
\hline $\mathrm{N}$ & -6.09076525 & 2.41518445 & -3.21334447 \\
\hline $\mathrm{C}$ & -5.65440727 & 3.64326886 & -3.66110633 \\
\hline $\mathrm{C}$ & -6.71218745 & 4.38458121 & -4.13508442 \\
\hline $\mathrm{N}$ & -7.82268084 & 3.58102183 & -3.95960129 \\
\hline $\mathrm{C}$ & -7.39911744 & 2.41008678 & -3.41224570 \\
\hline $\mathrm{C}$ & -6.72523136 & 5.75619327 & -4.73047506 \\
\hline $\mathrm{H}$ & -7.32390921 & 6.46214533 & -4.13918667 \\
\hline $\mathrm{H}$ & -5.70301054 & 6.14358210 & -4.77330407 \\
\hline $\mathrm{H}$ & -7.12699616 & 5.75748119 & -5.75219332 \\
\hline $\mathrm{H}$ & -8.78870692 & 3.81279751 & -4.21079315 \\
\hline $\mathrm{H}$ & -8.06899103 & 1.59159333 & -3.18768512 \\
\hline
\end{tabular}




$\begin{array}{lrrr}\mathrm{H} & -4.97643324 & 1.12365266 & -3.17082031 \\ \mathrm{H} & -4.60829547 & 3.91656324 & -3.62162717 \\ \mathrm{O} & 0.67916300 & 0.16675757 & -1.55887239 \\ \mathrm{H} & 1.09545074 & 0.46635478 & -0.72210649 \\ \mathrm{H} & -1.23637002 & 0.32010653 & -1.41096487 \\ \mathrm{~N} & -2.42467699 & -2.02718216 & 6.26201025 \\ \mathrm{C} & -1.44427562 & -2.61940659 & 7.15362373 \\ \mathrm{C} & -3.72994227 & -2.41438196 & 6.23838900 \\ \mathrm{O} & -4.19995024 & -3.28980631 & 6.95978017 \\ \mathrm{C} & -4.57237793 & -1.70827849 & 5.14377778 \\ \mathrm{C} & -4.23629914 & -2.47222549 & 3.84674645 \\ \mathrm{~N} & -4.17537968 & -0.31359951 & 4.93035578 \\ \mathrm{C} & -4.69948238 & 0.72548049 & 5.64811707 \\ \mathrm{C} & -4.19308635 & 2.11017471 & 5.26866375 \\ \mathrm{O} & -5.52882448 & 0.56363740 & 6.54157883 \\ \mathrm{H} & -2.13588689 & -1.29334721 & 5.63062771 \\ \mathrm{H} & -0.67573315 & -3.16870091 & 6.59423818 \\ \mathrm{H} & -0.95661887 & -1.85122154 & 7.76649672 \\ \mathrm{H} & -1.96773252 & -3.31694092 & 7.81060218 \\ \mathrm{H} & -5.62342384 & -1.76043671 & 5.42707739 \\ \mathrm{H} & -3.17679982 & -2.38783830 & 3.60307071 \\ \mathrm{H} & -4.48004407 & -3.53368319 & 3.96623986 \\ \mathrm{H} & -4.82205038 & -2.08418048 & 3.00661243 \\ \mathrm{H} & -3.51149037 & -0.11257487 & 4.19241699 \\ \mathrm{H} & -3.42713293 & 2.10127509 & 4.48693536 \\ \mathrm{H} & -5.04131678 & 2.71509810 & 4.93027854 \\ \mathrm{H} & -3.78599633 & 2.59021495 & 6.16475261\end{array}$

\section{Doublet; Basis set B2; Model B; Compound 0}

100

$\begin{array}{llll}\mathrm{O} & -10.30387113 & 4.24045115 & -4.01734621 \\ \mathrm{C} & -11.00298460 & 5.17047917 & -3.57725999 \\ \mathrm{~N} & -12.18340699 & 4.92704062 & -2.97161191 \\ \mathrm{C} & -12.71634190 & 3.57763769 & -2.80263613 \\ \mathrm{C} & -10.55506285 & 6.61705598 & -3.66047015 \\ \mathrm{H} & -12.72460634 & 5.70848805 & -2.62930338 \\ \mathrm{H} & -13.71791203 & 3.65106902 & -2.37492791 \\ \mathrm{H} & -12.08064656 & 2.99097965 & -2.13165883 \\ \mathrm{H} & -12.77017083 & 3.06667238 & -3.76801680 \\ \mathrm{H} & -11.30501367 & 7.32598067 & -3.29725246 \\ \mathrm{H} & -10.31421641 & 6.85923468 & -4.70024588\end{array}$




$\begin{array}{llcc}\mathrm{H} & -9.64285732 & 6.74200202 & -3.06674848 \\ \mathrm{C} & -4.55198671 & -2.19093087 & -0.02944248 \\ \mathrm{C} & -3.64504342 & -1.11153463 & 0.30456802 \\ \mathrm{~N} & -2.41634182 & -1.62150212 & 0.64476060 \\ \mathrm{C} & -2.51879521 & -2.98255489 & 0.53764623 \\ \mathrm{C} & -3.85491617 & -3.35223694 & 0.12093680 \\ \mathrm{C} & -3.99308190 & 0.23951447 & 0.28365256 \\ \mathrm{C} & -3.12310438 & 1.29267792 & 0.60238394 \\ \mathrm{~N} & -1.78505148 & 1.18237446 & 0.90923681 \\ \mathrm{C} & -1.31705080 & 2.45888502 & 1.12321267 \\ \mathrm{C} & -2.39702827 & 3.40693332 & 0.96014908 \\ \mathrm{C} & -3.51042116 & 2.68564157 & 0.63551495 \\ \mathrm{C} & 0.01046554 & 2.80587835 & 1.41482420 \\ \mathrm{C} & 1.06991765 & 1.90098493 & 1.54419525 \\ \mathrm{~N} & 0.97747364 & 0.53152371 & 1.43366906 \\ \mathrm{C} & 2.24241844 & 0.03487470 & 1.68551921 \\ \mathrm{C} & 3.15350377 & 1.13004128 & 1.93561663 \\ \mathrm{C} & 2.43086048 & 2.28350260 & 1.84791658 \\ \mathrm{Fe} & -0.72772773 & -0.53937885 & 1.05365190 \\ \mathrm{~S} & -1.20210625 & -0.72469170 & 3.36505628 \\ \mathrm{C} & -0.59700256 & 0.77349768 & 4.23693913 \\ \mathrm{C} & 2.61303504 & -1.31590115 & 1.73372126 \\ \mathrm{C} & 1.69179524 & -2.36499252 & 1.50599772 \\ \mathrm{~N} & 0.34645894 & -2.25029701 & 1.20441982 \\ \mathrm{C} & -0.15758004 & -3.52622602 & 1.08157074 \\ \mathrm{C} & 0.90729741 & -4.48432897 & 1.30927550 \\ \mathrm{C} & 2.04054671 & -3.77197182 & 1.56789277 \\ \mathrm{C} & -1.48631258 & -3.88696467 & 0.77421734 \\ \mathrm{H} & 3.64271940 & -1.57136927 & 1.95808582 \\ \mathrm{H} & -0.78711287 & -5.56055820 & 1.27340137 \\ \mathrm{H} & 4.20802994 & 1.01418642 & 2.15579343 \\ \mathrm{H} & 2.77144457 & 3.30327927 & 1.98201437 \\ \mathrm{H} & 0.23471546 & 3.86076800 & 1.54282339 \\ \mathrm{H} & -2.30193162 & 4.47950853 & 1.08179850 \\ \mathrm{H} & -4.51182934 & 3.05174900 & 0.44096601 \\ \mathrm{H} & -5.01679077 & 0.49508430 & 0.02668753 \\ \mathrm{H} & -5.58526806 & -2.05953062 & -0.32826275 \\ \mathrm{H} & -4.19686925 & -4.36936726 & -0.02809994 \\ \mathrm{H} & -1.70652951 & -4.94699268 & 0.69351069 \\ \mathrm{H} & -0.85057008 & 0.67855640 & 5.29922972 \\ \mathrm{H} & -1.06250986 & 1.68465784 & 3.85117868 \\ \mathrm{H} & -0.62271566 & -0.82592729 \\ \mathrm{H} & -1.26909235 & -3.39646326\end{array}$




\begin{tabular}{|c|c|c|c|}
\hline & 3.24230954 & .03031580 & \\
\hline & & & \\
\hline & & & \\
\hline & & -1.12108585 & \\
\hline & 3.07404704 & & \\
\hline & & 196649 & \\
\hline & -5.96403828 & & \\
\hline & -5.48598859 & 3.243150 & \\
\hline & -6.5 & & \\
\hline & -7.6 & & \\
\hline & -7.2 & & \\
\hline & -6.58 & 5.4 & \\
\hline & -6.9 & & \\
\hline & -5.57 & & \\
\hline & -7.2 & & \\
\hline & -8.6 & & \\
\hline & -7.9 & & \\
\hline & -5.3 & & \\
\hline & -4.4 & & \\
\hline & -0.3 & & \\
\hline & -0.0 & & -0. \\
\hline & & & \\
\hline & -2. & -2 . & \\
\hline & -1.4 & -2.8 & \\
\hline & -3.7 & -2.7 & \\
\hline & -4.1 & -3.6 & \\
\hline & -4.5 & -2.0 & \\
\hline & -4.1 & -2.8 & \\
\hline & -4.3 & -0.6 & \\
\hline & -5.0 & 0.3 & \\
\hline & -4.602690 & & \\
\hline & -6.0 & 0.1 & \\
\hline & -2.09 & -1.6 & \\
\hline & -1.20 & -3.892 & \\
\hline & -0.52 & -2.274 & \\
\hline & & -2.94150 & \\
\hline & -5.628371 & -2.23982 & \\
\hline & -3.07379199 & -2.652732 & \\
\hline & -4.27266194 & -3.9 & \\
\hline & & -2.507846 & \\
\hline & & -0.45000214 & 4.17200831 \\
\hline & & & \\
\hline & -5.47708300 & 2.306724 & \\
\hline & -4.21482934 & 2.22788760 & 5.8834 \\
\hline
\end{tabular}




\section{Doublet; Basis set B2; Model B; Transition State 2}

100

$\begin{array}{lccc}\mathrm{O} & -10.41623255 & 4.19262593 & -4.56471778 \\ \mathrm{C} & -11.27244794 & 4.98582987 & -4.14222202 \\ \mathrm{~N} & -12.51892941 & 4.56167231 & -3.83524774 \\ \mathrm{C} & -12.92928163 & 3.16924168 & -3.98929004 \\ \mathrm{C} & -10.96059403 & 6.45758967 & -3.92937798 \\ \mathrm{H} & -13.19516985 & 5.23423197 & -3.50215768 \\ \mathrm{H} & -13.98427748 & 3.08667234 & -3.72080312 \\ \mathrm{H} & -12.33900002 & 2.51842571 & -3.33653016 \\ \mathrm{H} & -12.79072444 & 2.84081135 & -5.02364542 \\ \mathrm{H} & -11.81954043 & 7.03949315 & -3.58145340 \\ \mathrm{H} & -10.60089439 & 6.88472987 & -4.87074978 \\ \mathrm{H} & -10.15570933 & 6.54703818 & -3.19221631 \\ \mathrm{C} & -4.67208169 & -2.00416607 & 0.17040434 \\ \mathrm{C} & -3.70118097 & -0.97473977 & 0.47877347 \\ \mathrm{~N} & -2.48267311 & -1.55369709 & 0.75348714 \\ \mathrm{C} & -2.65763809 & -2.90701270 & 0.63106491 \\ \mathrm{C} & -4.02852791 & -3.20098601 & 0.27288992 \\ \mathrm{C} & -3.99313691 & 0.39273564 & 0.50506829 \\ \mathrm{C} & -3.05267501 & 1.38855718 & 0.81291061 \\ \mathrm{~N} & -1.69241029 & 1.19302424 & 1.01350194 \\ \mathrm{C} & -1.13811862 & 2.43549103 & 1.26763317 \\ \mathrm{C} & -2.17271634 & 3.43756444 & 1.22726046 \\ \mathrm{C} & -3.34718445 & 2.79248589 & 0.94410515 \\ \mathrm{C} & 0.23028639 & 2.68122268 & 1.45076948 \\ \mathrm{C} & 1.24190929 & 1.73496523 & 1.48558154 \\ \mathrm{~N} & 1.06351060 & 0.38118800 & 1.38167813 \\ \mathrm{C} & 2.31243793 & -0.17111697 & 1.54675384 \\ \mathrm{C} & 3.30164112 & 0.86747733 & 1.73338589 \\ \mathrm{C} & 2.63694300 & 2.05467779 & 1.69296110 \\ \mathrm{Fe} & -0.72100196 & -0.58724151 & 1.09870922 \\ \mathrm{~S} & -1.05850579 & -0.80173674 & 3.39054717 \\ \mathrm{C} & -0.36839177 & 0.66315561 & 4.25234909 \\ \mathrm{C} & 2.59946778 & -1.52980101 & 1.56689048 \\ \mathrm{C} & 1.61793916 & -2.52599665 & 1.37410069 \\ \mathrm{~N} & 0.27072313 & -2.33762340 & 1.12841496 \\ \mathrm{C} & -0.30414797 & -3.58662176 & 1.03471865 \\ \mathrm{C} & -1.6614986569 & -4.59942187 & 1.20969030 \\ \mathrm{H} & -3.86882999 & 0.80431622 \\ \mathrm{H} & -1.84917457 & 1.73416769 \\ \mathrm{H} & -5.66741932 & 1.17778037\end{array}$




\begin{tabular}{|c|c|c|c|}
\hline $\mathrm{H}$ & 4.36016565 & 0.68959622 & 1.87903030 \\
\hline $\mathrm{H}$ & 3.03242208 & 3.05742013 & 1.79944904 \\
\hline $\mathrm{H}$ & 0.52015956 & 3.71995811 & 1.58446213 \\
\hline $\mathrm{H}$ & -2.01455050 & 4.49715195 & 1.38836419 \\
\hline $\mathrm{H}$ & -4.33365988 & 3.22629033 & 0.83206760 \\
\hline $\mathrm{H}$ & -5.01336292 & 0.70308103 & 0.30427338 \\
\hline $\mathrm{H}$ & -5.70899118 & -1.81697476 & -0.08149473 \\
\hline $\mathrm{H}$ & -4.42845700 & -4.19645348 & 0.12301460 \\
\hline $\mathrm{H}$ & -1.94460224 & -4.91393384 & 0.72555164 \\
\hline $\mathrm{H}$ & 2.87575854 & -4.37711676 & 1.58714239 \\
\hline $\mathrm{H}$ & -0.57752827 & 0.55135752 & 5.32242144 \\
\hline $\mathrm{H}$ & 0.71414527 & 0.72568897 & 4.11498628 \\
\hline $\mathrm{H}$ & -0.82617609 & 1.59345707 & 3.90614092 \\
\hline $\mathrm{O}$ & -0.58588004 & -0.58145616 & -0.8793857 \\
\hline C & -2.74756380 & -1.15688081 & -3.3873587 \\
\hline $\mathrm{C}$ & -3.41452882 & 0.16901932 & -3.17936560 \\
\hline $\mathrm{O}$ & -2.76167596 & 1.13838117 & -2.6884605 \\
\hline $\mathrm{O}$ & -4.63161093 & 0.25697566 & -3.5580792 \\
\hline $\mathrm{H}$ & -2.16811396 & -1.10967380 & -4.3194318 \\
\hline $\mathrm{H}$ & -3.48891024 & -1.95231280 & -3.485934 \\
\hline $\mathrm{H}$ & -2.05069841 & -1.38151151 & -2.5739619 \\
\hline $\mathrm{N}$ & -5.97929905 & 2.31550475 & -3.7151210 \\
\hline C & -5.52422542 & 3.58117728 & -4.00189888 \\
\hline $\mathrm{C}$ & -6.59518529 & 4.37885179 & -4.3190739 \\
\hline $\mathrm{N}$ & -7.70993657 & 3.56517404 & -4.2110577 \\
\hline $\mathrm{C}$ & -7.29773564 & 2.32956415 & -3.8543727 \\
\hline $\mathrm{C}$ & -6.62672737 & 5.81842936 & -4.7087627 \\
\hline $\mathrm{H}$ & -7.20156658 & 6.42489518 & -3.9971336 \\
\hline $\mathrm{H}$ & -5.60725084 & 6.21327280 & -4.7334221 \\
\hline $\mathrm{H}$ & -7.06846646 & 5.96432795 & -5.7024323 \\
\hline $\mathrm{H}$ & -8.68591226 & 3.84563529 & -4.3808240 \\
\hline $\mathrm{H}$ & -7.95559974 & 1.48460316 & -3.7140510 \\
\hline $\mathrm{H}$ & -5.24209503 & 1.32089792 & -3.5500711 \\
\hline $\mathrm{H}$ & -4.47169033 & 3.82154784 & -3.9594574 \\
\hline $\mathrm{O}$ & -0.63861969 & 0.78592525 & -1.4787787 \\
\hline $\mathrm{H}$ & -0.86000274 & 1.31653105 & -0.67171854 \\
\hline $\mathrm{H}$ & -1.57674131 & 0.85165181 & -2.0827124 \\
\hline $\mathrm{N}$ & -2.21856080 & -2.30272812 & 6.2841430 \\
\hline $\mathrm{C}$ & -1.21727817 & -3.02684081 & 7.0523857 \\
\hline $\mathrm{C}$ & -3.51665048 & -2.70833570 & 6.2772191 \\
\hline $\mathrm{O}$ & -3.94086235 & -3.61970262 & 6.9829213 \\
\hline $\mathrm{C}$ & -4.41160747 & -1.98665383 & 5.2364024 \\
\hline $\mathrm{C}$ & -4.09445722 & -2.71565325 & 3.9114228 \\
\hline $\mathrm{N}$ & -4.05205282 & -0.58244670 & 5.0297506 \\
\hline C & -4.64499221 & 0.44271720 & 5.7048678 \\
\hline
\end{tabular}




$\begin{array}{lrrr}\mathrm{C} & -4.16046724 & 1.83998080 & 5.33884430 \\ \mathrm{O} & -5.51670505 & 0.27198267 & 6.55825128 \\ \mathrm{H} & -1.89123302 & -1.71438629 & 5.52601358 \\ \mathrm{H} & -1.04306067 & -4.03515160 & 6.65286670 \\ \mathrm{H} & -0.27976013 & -2.46517707 & 7.02468319 \\ \mathrm{H} & -1.54877467 & -3.12664379 & 8.08959670 \\ \mathrm{H} & -5.45252290 & -2.06780537 & 5.54778244 \\ \mathrm{H} & -3.04632236 & -2.59669574 & 3.63782600 \\ \mathrm{H} & -4.30721151 & -3.78585701 & 4.01184126 \\ \mathrm{H} & -4.70989873 & -2.32045025 & 3.09658269 \\ \mathrm{H} & -3.35543007 & -0.37982982 & 4.31553958 \\ \mathrm{H} & -3.43861053 & 1.85489220 & 4.51638595 \\ \mathrm{H} & -5.02660291 & 2.45244148 & 5.06764086 \\ \mathrm{H} & -3.70224344 & 2.29484279 & 6.22410529\end{array}$

\section{Doublet; Basis set B2; Model B; protonated Compound 0}

100

$\begin{array}{llll}\mathrm{O} & -10.44356590 & 4.22689991 & -4.40566621 \\ \mathrm{C} & -11.24672422 & 5.08549199 & -4.01310515 \\ \mathrm{~N} & -12.48867607 & 4.73517529 & -3.60286474 \\ \mathrm{C} & -12.94306599 & 3.34849061 & -3.59757539 \\ \mathrm{C} & -10.87481137 & 6.56015265 & -3.96614323 \\ \mathrm{H} & -13.12237456 & 5.45704437 & -3.29014913 \\ \mathrm{H} & -13.99241474 & 3.32801002 & -3.29638038 \\ \mathrm{H} & -12.35409793 & 2.74945780 & -2.89542838 \\ \mathrm{H} & -12.84255626 & 2.91025727 & -4.59481983 \\ \mathrm{H} & -11.69520277 & 7.20733611 & -3.64043920 \\ \mathrm{H} & -10.54825866 & 6.87400254 & -4.96236275 \\ \mathrm{H} & -10.03085336 & 6.69094372 & -3.28075881 \\ \mathrm{C} & -4.61207992 & -2.06726068 & 0.06518435 \\ \mathrm{C} & -3.66649833 & -1.02017513 & 0.39098108 \\ \mathrm{~N} & -2.44452150 & -1.57394565 & 0.69744204 \\ \mathrm{C} & -2.59544012 & -2.93104929 & 0.58221835 \\ \mathrm{C} & -3.95100417 & -3.25185437 & 0.19230855 \\ \mathrm{C} & -3.98639143 & 0.34010442 & 0.41004752 \\ \mathrm{C} & -3.07979164 & 1.35701066 & 0.73829897 \\ \mathrm{~N} & -1.71392449 & 1.19206935 & 0.95106117 \\ \mathrm{C} & -1.19827719 & 2.44842684 & 1.23430253 \\ \mathrm{C} & -2.25668118 & 3.42137456 & 1.18899059 \\ \mathrm{C} & -3.41045598 & 2.74965509 & 0.88035999 \\ \mathrm{C} & 0.15960105 & 2.71854791 & 1.43399983\end{array}$




\begin{tabular}{|c|c|c|c|}
\hline $\mathrm{C}$ & 1.18718852 & 1.78965422 & 1.49464016 \\
\hline $\mathrm{N}$ & 1.03532703 & 0.43169503 & 1.40653429 \\
\hline $\mathrm{C}$ & 2.29102028 & -0.09591274 & 1.59690013 \\
\hline $\mathrm{C}$ & 3.25806837 & 0.96242270 & 1.78704844 \\
\hline $\mathrm{C}$ & 2.57275765 & 2.13666608 & 1.72074587 \\
\hline $\mathrm{Fe}$ & -0.71355627 & -0.57709273 & 1.09693829 \\
\hline S & -1.09189588 & -0.78928641 & 3.36955029 \\
\hline $\mathrm{C}$ & -0.44761158 & 0.69175787 & 4.23901656 \\
\hline $\mathrm{C}$ & 2.60093614 & -1.44866006 & 1.63246607 \\
\hline $\mathrm{C}$ & 1.64690415 & -2.46674707 & 1.42943155 \\
\hline $\mathrm{N}$ & 0.30340412 & -2.30616082 & 1.15168417 \\
\hline $\mathrm{C}$ & -0.24462739 & -3.56620408 & 1.05528309 \\
\hline $\mathrm{C}$ & 0.78964557 & -4.55779166 & 1.26365073 \\
\hline $\mathrm{C}$ & 1.95155055 & -3.88177506 & 1.49114729 \\
\hline $\mathrm{C}$ & -1.58939464 & -3.87240516 & 0.79089361 \\
\hline $\mathrm{H}$ & 3.62705149 & -1.74598437 & 1.82149936 \\
\hline $\mathrm{H}$ & 0.63168929 & -5.62910232 & 1.23673196 \\
\hline $\mathrm{H}$ & 4.31709588 & 0.80582243 & 1.95167288 \\
\hline $\mathrm{H}$ & 2.94841743 & 3.14766594 & 1.8201299 \\
\hline $\mathrm{H}$ & 0.43013053 & 3.76223572 & 1.5697489 \\
\hline $\mathrm{H}$ & -2.12913836 & 4.48292028 & 1.36313124 \\
\hline $\mathrm{H}$ & -4.40518984 & 3.16016450 & 0.75616336 \\
\hline $\mathrm{H}$ & -5.00960154 & 0.62759549 & 0.19064907 \\
\hline $\mathrm{H}$ & -5.64504868 & -1.89856959 & -0.2140180 \\
\hline $\mathrm{H}$ & -4.32930381 & -4.25531373 & 0.04003812 \\
\hline $\mathrm{H}$ & -1.85464410 & -4.92258477 & 0.7169082 \\
\hline $\mathrm{H}$ & 2.93540850 & -4.29045387 & 1.68770917 \\
\hline $\mathrm{H}$ & -0.66928816 & 0.57467867 & 5.30586399 \\
\hline $\mathrm{H}$ & 0.63498936 & 0.77788360 & 4.11674368 \\
\hline $\mathrm{H}$ & -0.92112388 & 1.61131319 & 3.88591303 \\
\hline $\mathrm{O}$ & -0.48234588 & -0.57408824 & -0.8867872 \\
\hline $\mathrm{C}$ & -2.60530674 & -1.20897953 & -3.4122913 \\
\hline $\mathrm{C}$ & -3.30914508 & 0.09559423 & -3.2102477 \\
\hline $\mathrm{O}$ & -2.70780681 & 1.10365846 & -2.7894869 \\
\hline $\mathrm{O}$ & -4.57146146 & 0.08899864 & -3.5376690 \\
\hline $\mathrm{H}$ & -1.98691731 & -1.13304939 & -4.3164127 \\
\hline $\mathrm{H}$ & -3.31558293 & -2.02707303 & -3.5447237 \\
\hline $\mathrm{H}$ & -1.93601764 & -1.41544101 & -2.5710358 \\
\hline $\mathrm{N}$ & -6.00781846 & 2.25310803 & -3.5734293 \\
\hline C & -5.53499346 & 3.47019168 & -4.0125685 \\
\hline $\mathrm{C}$ & -6.58613528 & 4.28539291 & -4.3585508 \\
\hline $\mathrm{N}$ & -7.72301407 & 3.53751702 & -4.1122839 \\
\hline $\mathrm{C}$ & -7.32727814 & 2.32451416 & -3.6505355 \\
\hline $\mathrm{C}$ & -6.59304639 & 5.67680965 & -4.8978389 \\
\hline $\mathrm{H}$ & -7.09187311 & 6.38244582 & -4.2202103 \\
\hline
\end{tabular}




$\begin{array}{lrrr}\mathrm{H} & -5.56410760 & 6.02180763 & -5.03618967 \\ \mathrm{H} & -7.09965120 & 5.73692939 & -5.86972152 \\ \mathrm{H} & -8.69274494 & 3.83548711 & -4.26551112 \\ \mathrm{H} & -8.01881553 & 1.53453417 & -3.39323535 \\ \mathrm{H} & -5.07376967 & 1.00344503 & -3.45253767 \\ \mathrm{H} & -4.47465880 & 3.67927826 & -4.05236883 \\ \mathrm{O} & -0.55349159 & 0.82017768 & -1.43425131 \\ \mathrm{H} & -0.91811059 & 1.27866764 & -0.61870176 \\ \mathrm{H} & -1.37428261 & 0.83360576 & -2.08086892 \\ \mathrm{~N} & -2.28436840 & -2.30155103 & 6.24403545 \\ \mathrm{C} & -1.28742812 & -3.00612941 & 7.03599647 \\ \mathrm{C} & -3.57543134 & -2.72992410 & 6.21149030 \\ \mathrm{O} & -3.99854743 & -3.64695755 & 6.91162410 \\ \mathrm{C} & -4.46130828 & -2.02716135 & 5.14995291 \\ \mathrm{C} & -4.10427258 & -2.75380210 & 3.83391074 \\ \mathrm{~N} & -4.12212180 & -0.61745457 & 4.94581318 \\ \mathrm{C} & -4.73115822 & 0.39666849 & 5.62456562 \\ \mathrm{C} & -4.26538398 & 1.80254055 & 5.26793700 \\ \mathrm{O} & -5.60064101 & 0.20760136 & 6.47547449 \\ \mathrm{H} & -1.95125908 & -1.70761658 & 5.49356778 \\ \mathrm{H} & -1.08988115 & -4.01356932 & 6.64535417 \\ \mathrm{H} & -0.35838231 & -2.43000070 & 7.02417196 \\ \mathrm{H} & -1.63795018 & -3.10596719 & 8.06690068 \\ \mathrm{H} & -5.50662707 & -2.12643887 & 5.44076944 \\ \mathrm{H} & -3.05211757 & -2.62092996 & 3.58334969 \\ \mathrm{H} & -4.30387197 & -3.82669158 & 3.93216924 \\ \mathrm{H} & -4.70757045 & -2.36974333 & 3.00465084 \\ \mathrm{H} & -3.42477421 & -0.40319798 & 4.23666819 \\ \mathrm{H} & -3.57422427 & 1.83579196 & 4.41994020 \\ \mathrm{H} & -5.14308044 & 2.41619847 & 5.04133194 \\ \mathrm{H} & -3.77521494 & 2.24259693 & 6.14373540\end{array}$

\section{Doublet; Basis set B2; Model B; Transition State 3}

100

$\begin{array}{llll}\mathrm{O} & -10.45067123 & 4.24890733 & -4.30056849 \\ \mathrm{C} & -11.21362041 & 5.14409743 & -3.91042610 \\ \mathrm{~N} & -12.44110469 & 4.84336243 & -3.42345313 \\ \mathrm{C} & -12.92441123 & 3.46972167 & -3.32970790 \\ \mathrm{C} & -10.80763926 & 6.61025911 & -3.95549935 \\ \mathrm{H} & -13.04195801 & 5.59392878 & -3.11342551 \\ \mathrm{H} & -13.95901973 & 3.48856634 & -2.98107162\end{array}$




\begin{tabular}{|c|c|c|c|}
\hline $\mathrm{H}$ & -12.31684295 & 2.89093252 & -2.62635921 \\
\hline $\mathrm{H}$ & -12.87921531 & 2.98216709 & -4.30790488 \\
\hline $\mathrm{H}$ & -11.59187799 & 7.29150791 & -3.61064701 \\
\hline $\mathrm{H}$ & -10.53774933 & 6.87018610 & -4.98374886 \\
\hline $\mathrm{H}$ & -9.91949274 & 6.75115009 & -3.33079035 \\
\hline $\mathrm{C}$ & -4.60630169 & -2.08587511 & 0.05398416 \\
\hline $\mathrm{C}$ & -3.67751977 & -1.02422776 & 0.37507777 \\
\hline $\mathrm{N}$ & -2.45424729 & -1.55780760 & 0.69867528 \\
\hline $\mathrm{C}$ & -2.58283923 & -2.92173172 & 0.59380432 \\
\hline $\mathrm{C}$ & -3.93079142 & -3.26031794 & 0.19729841 \\
\hline $\mathrm{C}$ & -3.99945433 & 0.33147790 & 0.36142145 \\
\hline $\mathrm{C}$ & -3.09187738 & 1.35152126 & 0.67110299 \\
\hline $\mathrm{N}$ & -1.74775622 & 1.19166305 & 0.96277300 \\
\hline $\mathrm{C}$ & -1.23064910 & 2.45667828 & 1.15748959 \\
\hline $\mathrm{C}$ & -2.27822336 & 3.43818131 & 0.99833621 \\
\hline $\mathrm{C}$ & -3.42191086 & 2.75572885 & 0.69665598 \\
\hline $\mathrm{C}$ & 0.11259066 & 2.75810534 & 1.40932532 \\
\hline $\mathrm{C}$ & 1.14428593 & 1.84476209 & 1.54168343 \\
\hline $\mathrm{N}$ & 1.00323464 & 0.48739388 & 1.45470467 \\
\hline $\mathrm{C}$ & 2.26092919 & -0.03484991 & 1.66297621 \\
\hline $\mathrm{C}$ & 3.21216140 & 1.03012337 & 1.87916040 \\
\hline $\mathrm{C}$ & 2.51960087 & 2.20028518 & 1.80170322 \\
\hline $\mathrm{Fe}$ & -0.72682577 & -0.51982212 & 1.06223758 \\
\hline S & -1.12805090 & -0.77296090 & 3.38464193 \\
\hline $\mathrm{C}$ & -0.53600152 & 0.73526769 & 4.24466374 \\
\hline $\mathrm{C}$ & 2.58854314 & -1.38396071 & 1.68012900 \\
\hline $\mathrm{C}$ & 1.65113456 & -2.41286433 & 1.46591063 \\
\hline $\mathrm{N}$ & 0.30495984 & -2.26787772 & 1.19745515 \\
\hline $\mathrm{C}$ & -0.22992179 & -3.53313271 & 1.08611444 \\
\hline $\mathrm{C}$ & 0.81788823 & -4.51259427 & 1.28677992 \\
\hline $\mathrm{C}$ & 1.97222181 & -3.82483873 & 1.51610940 \\
\hline $\mathrm{C}$ & -1.56960548 & -3.85426024 & 0.81450806 \\
\hline $\mathrm{H}$ & 3.61788000 & -1.66799117 & 1.87170363 \\
\hline $\mathrm{H}$ & 0.67238717 & -5.58544620 & 1.25360940 \\
\hline $\mathrm{H}$ & 4.26876048 & 0.88141731 & 2.06485190 \\
\hline $\mathrm{H}$ & 2.88601267 & 3.21353823 & 1.91101965 \\
\hline $\mathrm{H}$ & 0.36401553 & 3.81025710 & 1.50946472 \\
\hline $\mathrm{H}$ & -2.14149150 & 4.50799792 & 1.09874755 \\
\hline $\mathrm{H}$ & -4.40771561 & 3.15660026 & 0.49579961 \\
\hline $\mathrm{H}$ & -5.01381889 & 0.61660939 & 0.10305404 \\
\hline $\mathrm{H}$ & -5.63830953 & -1.93301381 & -0.23698470 \\
\hline $\mathrm{H}$ & -4.29540341 & -4.26975986 & 0.05170320 \\
\hline $\mathrm{H}$ & -1.82457812 & -4.90665618 & 0.73917710 \\
\hline $\mathrm{H}$ & 2.96099050 & -4.22318164 & 1.70885335 \\
\hline $\mathrm{H}$ & -0.80977265 & 0.64500933 & 5.30203315 \\
\hline
\end{tabular}




\begin{tabular}{|c|c|c|c|}
\hline $\mathrm{H}$ & 0.55095227 & 0.82768996 & 4.17529394 \\
\hline $\mathrm{H}$ & -0.99885905 & 1.64055673 & 3.84367086 \\
\hline $\mathrm{O}$ & -0.41618319 & -0.64593403 & -0.66998596 \\
\hline C & -2.55924693 & -1.24566173 & -3.42663143 \\
\hline $\mathrm{C}$ & -3.27864820 & 0.04525571 & -3.18402042 \\
\hline $\mathrm{O}$ & -2.70503324 & 1.04215803 & -2.72974263 \\
\hline $\mathrm{O}$ & -4.54630067 & 0.02095901 & -3.5233918 \\
\hline $\mathrm{H}$ & -1.92101848 & -1.12772539 & -4.3117093 \\
\hline $\mathrm{H}$ & -3.25907290 & -2.06372392 & -3.6058101 \\
\hline $\mathrm{H}$ & -1.90785331 & -1.48077839 & $-2.579420 €$ \\
\hline $\mathrm{N}$ & -6.01841413 & 2.20855704 & -3.5803024 \\
\hline C & -5.54265702 & 3.39199586 & -4.1023165 \\
\hline C & -6.59102282 & 4.21638986 & -4.4394134 \\
\hline $\mathrm{N}$ & -7.73044504 & 3.50929828 & -4.1019974 \\
\hline C & -7.33781561 & 2.31025810 & -3.5992080 \\
\hline $\mathrm{C}$ & -6.59064190 & 5.58767199 & -5.0328972 \\
\hline $\mathrm{H}$ & -6.97847430 & 6.34219985 & -4.3351950 \\
\hline $\mathrm{H}$ & -5.56701695 & 5.87287951 & -5.2933857 \\
\hline $\mathrm{H}$ & -7.19557288 & 5.64053796 & -5.9470996 \\
\hline $\mathrm{H}$ & -8.69944291 & 3.82154118 & -4.2249002 \\
\hline $\mathrm{H}$ & -8.03468501 & 1.55129341 & -3.2720136 \\
\hline $\mathrm{H}$ & -5.04776723 & 0.92128693 & -3.4313390 \\
\hline $\mathrm{H}$ & -4.48130550 & 3.57495760 & -4.2017527 \\
\hline $\mathrm{O}$ & -0.31451477 & 0.79387888 & -1.6054015 \\
\hline $\mathrm{H}$ & -0.32659617 & 1.45416590 & -0.8790438 \\
\hline $\mathrm{H}$ & -1.24258244 & 0.83940376 & -2.0304489 \\
\hline $\mathrm{N}$ & -2.31280810 & -2.34293879 & 6.2312398 \\
\hline C & -1.30966191 & -3.03345213 & 7.0279505 \\
\hline $\mathrm{C}$ & -3.59443131 & -2.79650253 & 6.18512987 \\
\hline $\mathrm{O}$ & -4.00326302 & -3.72996093 & 6.8719282 \\
\hline $\mathrm{C}$ & -4.48739994 & -2.09956965 & 5.1261841 \\
\hline $\mathrm{C}$ & -4.10676470 & -2.80556971 & 3.8058857 \\
\hline $\mathrm{N}$ & -4.17396283 & -0.68184990 & 4.9372266 \\
\hline $\mathrm{C}$ & -4.80245544 & 0.31495071 & 5.6237071 \\
\hline $\mathrm{C}$ & -4.36674217 & 1.73219737 & $5.2738560 €$ \\
\hline $\mathrm{O}$ & -5.66768819 & 0.10269651 & 6.4732948 \\
\hline $\mathrm{H}$ & -1.98379543 & -1.73100996 & 5.4932967 \\
\hline $\mathrm{H}$ & -1.10057984 & -4.03998710 & 6.6412371 \\
\hline $\mathrm{H}$ & -0.38720360 & -2.44692670 & 7.0151908 \\
\hline $\mathrm{H}$ & -1.66007803 & -3.13385271 & 8.0589840 \\
\hline $\mathrm{H}$ & -5.53259294 & -2.22215991 & 5.4085285 \\
\hline $\mathrm{H}$ & -3.05454244 & -2.65149912 & 3.5681248 \\
\hline $\mathrm{H}$ & -4.28837191 & -3.88279380 & 3.8906901 \\
\hline $\mathrm{H}$ & -4.70853587 & -2.42268500 & 2.9751278 \\
\hline $\mathrm{H}$ & -3.47850298 & -0.44842385 & 4.232044 \\
\hline
\end{tabular}




$\begin{array}{llll}\mathrm{H} & -3.65591246 & 1.78157632 & 4.44302972 \\ \mathrm{H} & -5.25504755 & 2.32056098 & 5.02221318 \\ \mathrm{H} & -3.91183191 & 2.18874536 & 6.16000203\end{array}$

\section{Doublet; Basis set B2; Model B; Compound I}

$\begin{array}{lccc}100 & & & \\ \mathrm{O} & -10.40333113 & 4.23010781 & -3.87035933 \\ \mathrm{C} & -11.07166935 & 5.13186897 & -3.34745478 \\ \mathrm{~N} & -12.25678202 & 4.85498889 & -2.75006919 \\ \mathrm{C} & -12.79480551 & 3.50046788 & -2.68713812 \\ \mathrm{C} & -10.59679256 & 6.57878912 & -3.35422926 \\ \mathrm{H} & -12.78188077 & 5.60891461 & -2.33030719 \\ \mathrm{H} & -13.78317928 & 3.54040080 & -2.22449828 \\ \mathrm{H} & -12.14390460 & 2.85040446 & -2.09327794 \\ \mathrm{H} & -12.88060064 & 3.07709720 & -3.69222428 \\ \mathrm{H} & -11.30067145 & 7.27205257 & -2.88282257 \\ \mathrm{H} & -10.43064024 & 6.88943596 & -4.39032365 \\ \mathrm{H} & -9.63770426 & 6.63891162 & -2.82962008 \\ \mathrm{C} & -4.37387685 & -2.34798925 & -0.10826314 \\ \mathrm{C} & -3.49677227 & -1.26351576 & 0.26128130 \\ \mathrm{~N} & -2.25088441 & -1.75135515 & 0.58770078 \\ \mathrm{C} & -2.32548677 & -3.11883418 & 0.43023945 \\ \mathrm{C} & -3.65160668 & -3.49814691 & 0.00554012 \\ \mathrm{C} & -3.88391720 & 0.08040580 & 0.29498469 \\ \mathrm{C} & -3.02691027 & 1.12430698 & 0.65404685 \\ \mathrm{~N} & -1.68791859 & 1.00044663 & 0.99940401 \\ \mathrm{C} & -1.22010618 & 2.28243464 & 1.21425940 \\ \mathrm{C} & -2.29817417 & 3.22802541 & 1.04354908 \\ \mathrm{C} & -3.40600312 & 2.51668467 & 0.69351674 \\ \mathrm{C} & 0.09946228 & 2.63944828 & 1.50908985 \\ \mathrm{C} & 1.17186256 & 1.75888797 & 1.61752729 \\ \mathrm{~N} & 1.09150190 & 0.39761450 & 1.50093877 \\ \mathrm{C} & 2.37541678 & -0.06787682 & 1.68883896 \\ \mathrm{C} & 3.27688694 & 1.03154178 & 1.93808235 \\ \mathrm{C} & 2.52831548 & 2.16876755 & 1.89406312 \\ \mathrm{Fe} & -0.55285532 & -0.67094395 & 0.91547720 \\ \mathrm{~S} & -1.32796671 & -0.68782328 & 3.46356700 \\ \mathrm{C} & -0.33149932 & 0.53408012 & 4.37371473 \\ \mathrm{C} & 2.75272226 & -1.40177329 & 1.66148744 \\ \mathrm{C} & 1.85962626 & -2.46567653 & 1.41391779\end{array}$




\begin{tabular}{|c|c|c|c|}
\hline $\mathrm{N}$ & 0.50876422 & -2.36333861 & 1.15259792 \\
\hline C & 0.04005844 & -3.64526529 & 0.95918389 \\
\hline $\mathrm{C}$ & 1.12478314 & -4.58413489 & 1.12180642 \\
\hline $\mathrm{C}$ & 2.24672253 & -3.85652297 & 1.39895825 \\
\hline $\mathrm{C}$ & -1.27767898 & -4.01466213 & 0.62734013 \\
\hline $\mathrm{H}$ & 3.79447880 & -1.65030759 & 1.83627351 \\
\hline $\mathrm{H}$ & 1.02960547 & -5.65897209 & 1.02892020 \\
\hline $\mathrm{H}$ & 4.33989964 & 0.92858221 & 2.11708445 \\
\hline $\mathrm{H}$ & 2.84772195 & 3.19485304 & 2.02656725 \\
\hline $\mathrm{H}$ & 0.30773106 & 3.69906422 & 1.62084912 \\
\hline $\mathrm{H}$ & -2.19602983 & 4.30079068 & 1.14940179 \\
\hline $\mathrm{H}$ & -4.39515772 & 2.88814752 & 0.45691466 \\
\hline $\mathrm{H}$ & -4.90020474 & 0.32927399 & 0.00922908 \\
\hline $\mathrm{H}$ & -5.40401071 & -2.22536559 & -0.41772607 \\
\hline $\mathrm{H}$ & -3.97072301 & -4.51547245 & -0.18410060 \\
\hline $\mathrm{H}$ & -1.47982759 & -5.07223506 & 0.49060408 \\
\hline $\mathrm{H}$ & 3.25155097 & -4.21871840 & 1.57846304 \\
\hline $\mathrm{H}$ & -0.65579928 & 0.56319306 & 5.41971599 \\
\hline $\mathrm{H}$ & 0.73393212 & 0.29729493 & 4.32145867 \\
\hline $\mathrm{H}$ & -0.48940454 & 1.53150688 & 3.94497543 \\
\hline $\mathrm{O}$ & -0.22241125 & -0.51423473 & -0.6615798 \\
\hline $\mathrm{C}$ & -2.31464482 & -1.27152752 & -3.53477488 \\
\hline C & -3.18049968 & -0.03247209 & -3.5903878 \\
\hline $\mathrm{O}$ & -2.72471304 & 1.10246626 & -3.6929907 \\
\hline $\mathrm{O}$ & -4.48554276 & -0.28659176 & -3.5119077 \\
\hline $\mathrm{H}$ & -1.62676389 & -1.26400134 & -4.38659330 \\
\hline $\mathrm{H}$ & -2.91417979 & -2.18388270 & -3.5455196 \\
\hline $\mathrm{H}$ & -1.70351467 & -1.24055525 & -2.6250893 \\
\hline $\mathrm{N}$ & -6.01512072 & 1.95905620 & -3.71541994 \\
\hline $\mathrm{C}$ & -5.53225469 & 3.15693794 & -4.2040853 \\
\hline $\mathrm{C}$ & -6.56831261 & 4.04428315 & -4.3921438 \\
\hline $\mathrm{N}$ & -7.70591841 & 3.35834063 & -3.9982073 \\
\hline $\mathrm{C}$ & -7.32354409 & 2.11533264 & -3.60728038 \\
\hline $\mathrm{C}$ & -6.61874736 & 5.43875164 & -4.9305887 \\
\hline $\mathrm{H}$ & -7.00432830 & 6.15437193 & -4.1919716 \\
\hline $\mathrm{H}$ & -5.61286077 & 5.76482555 & -5.2122989 \\
\hline $\mathrm{H}$ & -7.25485859 & 5.50692147 & -5.8226037 \\
\hline $\mathrm{H}$ & -8.66502374 & 3.71602808 & -4.0124581 \\
\hline $\mathrm{H}$ & -8.02308174 & 1.36639681 & -3.2618461 \\
\hline $\mathrm{H}$ & -5.02328461 & 0.57770529 & -3.5647856 \\
\hline $\mathrm{H}$ & -4.47477970 & 3.29262203 & -4.3860138 \\
\hline $\mathrm{O}$ & -0.31437322 & 1.99372891 & -2.2536460 \\
\hline $\mathrm{H}$ & -0.17390976 & 1.18321585 & -1.7307850 \\
\hline $\mathrm{H}$ & -1.07154172 & 1.77576018 & -2.8309341 \\
\hline $\mathrm{N}$ & -2.86228791 & -1.96741183 & 6.2884761 \\
\hline
\end{tabular}




$\begin{array}{llll}\mathrm{C} & -1.99149124 & -2.49722806 & 7.32585142 \\ \mathrm{C} & -4.12762047 & -2.43541269 & 6.11730236 \\ \mathrm{O} & -4.62357306 & -3.29833821 & 6.83634509 \\ \mathrm{C} & -4.86471947 & -1.85520268 & 4.88323915 \\ \mathrm{C} & -4.31726447 & -2.67264560 & 3.69350160 \\ \mathrm{~N} & -4.53602216 & -0.45168071 & 4.62503890 \\ \mathrm{C} & -5.24029122 & 0.58508746 & 5.17026955 \\ \mathrm{C} & -4.76525174 & 1.97961802 & 4.78479899 \\ \mathrm{O} & -6.19402223 & 0.41706132 & 5.92793210 \\ \mathrm{H} & -2.50263187 & -1.28094803 & 5.64057631 \\ \mathrm{H} & -1.76559950 & -3.55670814 & 7.15444553 \\ \mathrm{H} & -1.05774480 & -1.92877021 & 7.32666165 \\ \mathrm{H} & -2.46811668 & -2.40815296 & 8.30750434 \\ \mathrm{H} & -5.93829534 & -1.96727319 & 5.03297513 \\ \mathrm{H} & -3.23637872 & -2.56195322 & 3.60111526 \\ \mathrm{H} & -4.53742836 & -3.73575448 & 3.83991963 \\ \mathrm{H} & -4.78358818 & -2.35528178 & 2.75525891 \\ \mathrm{H} & -3.76346369 & -0.25354182 & 3.99732018 \\ \mathrm{H} & -3.97051748 & 1.98406316 & 4.03180044 \\ \mathrm{H} & -5.61969217 & 2.54980092 & 4.40656337 \\ \mathrm{H} & -4.40645921 & 2.48920893 & 5.68611500\end{array}$

\section{Quartet; Basis set B2; Model A; Fe+HOOH}

74

$\begin{array}{llll}\mathrm{O} & -10.28636211 & 4.47791229 & -4.92928880 \\ \mathrm{C} & -10.98886550 & 5.40385925 & -4.50323733 \\ \mathrm{~N} & -12.14365114 & 5.14521042 & -3.84087706 \\ \mathrm{C} & -12.60740677 & 3.78380054 & -3.59723745 \\ \mathrm{C} & -10.59094040 & 6.86042073 & -4.70338386 \\ \mathrm{H} & -12.70139081 & 5.91850235 & -3.50737504 \\ \mathrm{H} & -13.57470280 & 3.83149314 & -3.09256507 \\ \mathrm{H} & -11.89706446 & 3.23917993 & -2.96683115 \\ \mathrm{H} & -12.71623142 & 3.24138193 & -4.54124347 \\ \mathrm{H} & -11.32151960 & 7.57178492 & -4.30513610 \\ \mathrm{H} & -10.46059372 & 7.04515724 & -5.77408680 \\ \mathrm{H} & -9.62692792 & 7.03413524 & -4.21425248 \\ \mathrm{C} & -3.96218763 & -1.31415020 & -0.72755026 \\ \mathrm{C} & -2.99072482 & -0.25200897 & -0.67658536 \\ \mathrm{~N} & -1.72253293 & -0.71443794 & -0.51448297 \\ \mathrm{C} & -1.87026582 & -2.06506113 & -0.53207522\end{array}$




\begin{tabular}{|c|c|c|c|}
\hline $\mathrm{C}$ & -3.25045650 & -2.46841878 & -0.63119807 \\
\hline C & -3.26110349 & 1.06077613 & -0.88821859 \\
\hline C & -2.35081807 & 2.11210057 & -0.84777282 \\
\hline $\mathrm{N}$ & -1.00004850 & 2.01123877 & -0.59852953 \\
\hline C & -0.51146489 & 3.29746649 & -0.59465749 \\
\hline $\mathrm{C}$ & -1.57780340 & 4.22968422 & -0.85784840 \\
\hline $\mathrm{C}$ & -2.71437308 & 3.49424174 & -1.02815970 \\
\hline $\mathrm{C}$ & 0.81489383 & 3.65818738 & -0.38709874 \\
\hline C & 1.87350030 & 2.79602634 & -0.23518688 \\
\hline $\mathrm{N}$ & 1.75754815 & 1.43593696 & -0.26521784 \\
\hline C & 3.04387712 & 0.97133133 & -0.17268637 \\
\hline $\mathrm{C}$ & 3.98417057 & 2.05784467 & -0.05904384 \\
\hline $\mathrm{C}$ & 3.24929204 & 3.20362949 & -0.0948819 \\
\hline $\mathrm{Fe}$ & 0.00833979 & 0.36705466 & -0.16867036 \\
\hline S & -0.19287630 & 0.46758618 & 2.20827985 \\
\hline $\mathrm{C}$ & -0.41942582 & -1.26312986 & 2.77087194 \\
\hline $\mathrm{C}$ & 3.38153024 & -0.35824903 & -0.2332625 \\
\hline $\mathrm{C}$ & 2.45208152 & -1.40177636 & -0.3614343 \\
\hline $\mathrm{N}$ & 1.07126928 & -1.27368596 & -0.4007823 \\
\hline $\mathrm{C}$ & 0.55261615 & -2.54715309 & -0.53565812 \\
\hline C & 1.63271208 & -3.49698651 & -0.5938494 \\
\hline $\mathrm{C}$ & 2.80053332 & -2.79222170 & -0.4821367 \\
\hline $\mathrm{C}$ & -0.80435600 & -2.90111420 & -0.5881879 \\
\hline $\mathrm{H}$ & 4.43120700 & -0.63255993 & -0.18869883 \\
\hline $\mathrm{H}$ & 1.50646510 & -4.56720335 & -0.7040685 \\
\hline $\mathrm{H}$ & 5.05730894 & 1.93958065 & 0.02512207 \\
\hline $\mathrm{H}$ & 3.58757376 & 4.23120740 & -0.04740616 \\
\hline $\mathrm{H}$ & 1.03185721 & 4.72314786 & -0.38012310 \\
\hline $\mathrm{H}$ & -1.45557745 & 5.30412029 & -0.9181183 \\
\hline $\mathrm{H}$ & -3.71381171 & 3.83986692 & -1.2603840 \\
\hline $\mathrm{H}$ & -4.29188682 & 1.33659041 & -1.0935694 \\
\hline $\mathrm{H}$ & -5.02665133 & -1.17024306 & -0.8590909 \\
\hline $\mathrm{H}$ & -3.59625900 & -3.49418565 & -0.6548191 \\
\hline $\mathrm{H}$ & -1.00180573 & -3.96785470 & -0.6670104 \\
\hline $\mathrm{H}$ & 3.81398474 & -3.17468553 & -0.4854325 \\
\hline $\mathrm{H}$ & -0.49084574 & -1.25953176 & 3.8637347 \\
\hline $\mathrm{H}$ & -1.33844231 & -1.69415853 & 2.36194755 \\
\hline $\mathrm{H}$ & 0.42906125 & -1.88800053 & 2.47651699 \\
\hline $\mathrm{C}$ & -2.12840773 & -0.84825677 & -4.2523733 \\
\hline $\mathrm{C}$ & -2.90892653 & 0.43726791 & -4.4492675 \\
\hline $\mathrm{O}$ & -2.42223060 & 1.48365582 & -4.8477201 \\
\hline $\mathrm{O}$ & -4.20835450 & 0.27625123 & -4.1698283 \\
\hline $\mathrm{H}$ & -2.24951437 & -1.47118497 & -5.1479550 \\
\hline $\mathrm{H}$ & -2.50650784 & -1.41407815 & -3.39851622 \\
\hline $\mathrm{H}$ & -1.06742516 & -0.62422188 & -4.1256513 \\
\hline
\end{tabular}




$\begin{array}{llll}\mathrm{N} & -5.77948819 & 2.45985648 & -4.63956765 \\ \mathrm{C} & -5.37443977 & 3.60384742 & -5.29617429 \\ \mathrm{C} & -6.45911852 & 4.41198931 & -5.55307526 \\ \mathrm{~N} & -7.54707444 & 3.73291062 & -5.02980519 \\ \mathrm{C} & -7.08888035 & 2.56924331 & -4.49895935 \\ \mathrm{C} & -6.59699700 & 5.71796398 & -6.26552513 \\ \mathrm{H} & -7.09404348 & 6.47633869 & -5.64592629 \\ \mathrm{H} & -5.60844424 & 6.10328769 & -6.53363373 \\ \mathrm{H} & -7.17818077 & 5.61738213 & -7.19194313 \\ \mathrm{H} & -8.52265541 & 4.04096878 & -5.05388349 \\ \mathrm{H} & -7.73660181 & 1.84138075 & -4.02966821 \\ \mathrm{H} & -4.72952825 & 1.12663767 & -4.34770008 \\ \mathrm{H} & -4.33280350 & 3.76019452 & -5.54267013\end{array}$

\section{Quartet; Basis set B2; Model A; FeHOOH}

78

$\begin{array}{llll}\mathrm{O} & -10.38058307 & 4.48577756 & -5.16879131 \\ \mathrm{C} & -11.19287320 & 5.34456199 & -4.81219871 \\ \mathrm{~N} & -12.39930311 & 5.00629816 & -4.28691672 \\ \mathrm{C} & -12.83171885 & 3.63008920 & -4.10159108 \\ \mathrm{C} & -10.88833362 & 6.82899886 & -4.95470389 \\ \mathrm{H} & -13.03412985 & 5.74755508 & -4.02727503 \\ \mathrm{H} & -13.73811452 & 3.42456975 & -4.68174083 \\ \mathrm{H} & -13.03034455 & 3.42247998 & -3.04428760 \\ \mathrm{H} & -12.02776285 & 2.98252123 & -4.45143030 \\ \mathrm{H} & -11.69985012 & 7.48046540 & -4.61666574 \\ \mathrm{H} & -10.67438073 & 7.04187508 & -6.00635086 \\ \mathrm{H} & -9.98610246 & 7.06090656 & -4.38002258 \\ \mathrm{C} & -4.07786446 & -1.27761930 & -0.92090434 \\ \mathrm{C} & -3.08181530 & -0.23860022 & -0.81589503 \\ \mathrm{~N} & -1.82605679 & -0.74506459 & -0.70958517 \\ \mathrm{C} & -2.00704353 & -2.08975154 & -0.71666470 \\ \mathrm{C} & -3.39780487 & -2.45062848 & -0.85107422 \\ \mathrm{C} & -3.33798499 & 1.09758829 & -0.79020568 \\ \mathrm{C} & -2.39125400 & 2.11562128 & -0.65853640 \\ \mathrm{~N} & -1.03057776 & 1.95662884 & -0.53243315 \\ \mathrm{C} & -0.48370084 & 3.21921743 & -0.47412981 \\ \mathrm{C} & -1.53482413 & 4.20048928 & -0.54992271 \\ \mathrm{C} & -2.71080017 & 3.51934638 & -0.66616045 \\ \mathrm{C} & 0.87250413 & 3.53204400 & -0.38733160\end{array}$




\begin{tabular}{|c|c|c|c|}
\hline $\mathrm{C}$ & 1.92188818 & 2.64889713 & -0.36350857 \\
\hline $\mathrm{N}$ & 1.76292609 & 1.29264287 & -0.45073733 \\
\hline C & 3.03140065 & 0.77902450 & -0.36671380 \\
\hline $\mathrm{C}$ & 4.00796623 & 1.83122208 & -0.25671056 \\
\hline $\mathrm{C}$ & 3.31286384 & 3.00294722 & -0.25309180 \\
\hline $\mathrm{Fe}$ & -0.04818544 & 0.27319088 & -0.34772629 \\
\hline S & -0.27261888 & 0.33815147 & 2.05335395 \\
\hline $\mathrm{C}$ & 1.10713201 & -0.64244997 & 2.76154677 \\
\hline $\mathrm{C}$ & 3.29799780 & -0.56869615 & -0.36903196 \\
\hline C & 2.32545795 & -1.57593654 & -0.42604731 \\
\hline $\mathrm{N}$ & 0.95326133 & -1.40206496 & -0.4715452 \\
\hline $\mathrm{C}$ & 0.38863746 & -2.66008703 & -0.51587206 \\
\hline $\mathrm{C}$ & 1.43192263 & -3.65068465 & -0.4884656 \\
\hline $\mathrm{C}$ & 2.62264497 & -2.98348746 & -0.43755821 \\
\hline $\mathrm{C}$ & -0.97397262 & -2.96771551 & -0.6080758 \\
\hline $\mathrm{H}$ & 4.33370537 & -0.89028944 & -0.3175193 \\
\hline $\mathrm{H}$ & 1.26562502 & -4.72015021 & -0.5186235 \\
\hline $\mathrm{H}$ & 5.07731508 & 1.67768005 & -0.1872023 \\
\hline $\mathrm{H}$ & 3.69028421 & 4.01498666 & $-0.1821784 \subseteq$ \\
\hline $\mathrm{H}$ & 1.11213346 & 4.59076382 & -0.33809840 \\
\hline $\mathrm{H}$ & -1.37832461 & 5.27163129 & -0.53088381 \\
\hline $\mathrm{H}$ & -3.71145678 & 3.91903209 & -0.7681961 \\
\hline $\mathrm{H}$ & -4.36977644 & 1.42171739 & -0.8911152 \\
\hline $\mathrm{H}$ & -5.14044417 & -1.10555008 & -1.030417 \\
\hline $\mathrm{H}$ & -3.77411920 & -3.46488439 & -0.8860676 \\
\hline $\mathrm{H}$ & -1.21178889 & -4.02835229 & -0.6202352 \\
\hline $\mathrm{H}$ & 3.62170208 & -3.40053341 & -0.4153922 \\
\hline $\mathrm{H}$ & 1.01168069 & -0.62498908 & 3.8518417 \\
\hline $\mathrm{H}$ & 1.07351451 & -1.68177415 & 2.4227761 \\
\hline $\mathrm{H}$ & 2.07532326 & -0.21092825 & 2.49041133 \\
\hline $\mathrm{O}$ & 0.07317664 & 0.54860146 & -3.0571269 \\
\hline $\mathrm{C}$ & -2.37428871 & -0.78798807 & -4.4055045 \\
\hline $\mathrm{C}$ & -3.02139465 & 0.55587419 & -4.1797515 \\
\hline $\mathrm{O}$ & -2.39311016 & 1.58648682 & -3.9492893 \\
\hline $\mathrm{O}$ & -4.34049129 & 0.49907189 & -4.2968707 \\
\hline $\mathrm{H}$ & -2.18911576 & -0.90165643 & -5.4817017 \\
\hline $\mathrm{H}$ & -3.04396736 & -1.59353750 & -4.096576 \\
\hline $\mathrm{H}$ & -1.41584411 & -0.85902881 & -3.89337860 \\
\hline $\mathrm{N}$ & -5.83997742 & 2.75350628 & -4.3863683 \\
\hline $\mathrm{C}$ & -5.44443570 & 3.92796947 & -4.9870797 \\
\hline $\mathrm{C}$ & -6.54215562 & 4.66919275 & -5.3564099 \\
\hline $\mathrm{N}$ & -7.63367164 & 3.91858225 & -4.9610379 \\
\hline C & -7.16117241 & 2.78036972 & -4.3914194 \\
\hline $\mathrm{C}$ & -6.64581673 & 5.99039751 & -6.0475719 \\
\hline $\mathrm{H}$ & -7.13011320 & 6.75262950 & -5.422310 \\
\hline
\end{tabular}




$\begin{array}{lrrr}\mathrm{H} & -5.64531707 & 6.35448483 & -6.29727625 \\ \mathrm{H} & -7.21845963 & 5.91926779 & -6.98125556 \\ \mathrm{H} & -8.61803204 & 4.17055396 & -5.08562065 \\ \mathrm{H} & -7.80861338 & 2.00593179 & -4.00439017 \\ \mathrm{H} & -4.78229061 & 1.40862364 & -4.22734542 \\ \mathrm{H} & -4.39658026 & 4.16099457 & -5.11839974 \\ \mathrm{O} & 1.24202157 & 1.32416023 & -3.43687177 \\ \mathrm{H} & 1.62444286 & 1.45426018 & -2.54709095 \\ \mathrm{H} & -0.66825994 & 1.13409279 & -3.35304910\end{array}$

\section{Quartet; Basis set B2; Model A; Transition State 1}

78

$\begin{array}{llll}\mathrm{O} & -10.25569247 & 4.47041389 & -5.06600706 \\ \mathrm{C} & -11.03449618 & 5.33221002 & -4.63255000 \\ \mathrm{~N} & -12.20384472 & 5.00139410 & -4.03892115 \\ \mathrm{C} & -12.66274765 & 3.63015045 & -3.86741394 \\ \mathrm{C} & -10.71613835 & 6.81174088 & -4.71812559 \\ \mathrm{H} & -12.80645095 & 5.74798887 & -3.72315933 \\ \mathrm{H} & -13.61287771 & 3.47205336 & -4.38836979 \\ \mathrm{H} & -12.79370458 & 3.39601477 & -2.80562228 \\ \mathrm{H} & -11.90655125 & 2.97075278 & -4.29270589 \\ \mathrm{H} & -11.52507624 & 7.45590695 & -4.36223148 \\ \mathrm{H} & -10.49072342 & 7.06686810 & -5.75792684 \\ \mathrm{H} & -9.81949831 & 7.01826404 & -4.12389492 \\ \mathrm{C} & -4.11893760 & -1.33304591 & -0.94551251 \\ \mathrm{C} & -3.10717506 & -0.28981820 & -0.87023773 \\ \mathrm{~N} & -1.88009162 & -0.83906271 & -0.76297011 \\ \mathrm{C} & -2.04090659 & -2.17990512 & -0.74184872 \\ \mathrm{C} & -3.44996602 & -2.51873560 & -0.86169412 \\ \mathrm{C} & -3.31440775 & 1.08081932 & -0.85608219 \\ \mathrm{C} & -2.38335741 & 2.10819707 & -0.71152543 \\ \mathrm{~N} & -1.02801289 & 1.97308956 & -0.59854966 \\ \mathrm{C} & -0.49176942 & 3.22729094 & -0.49761029 \\ \mathrm{C} & -1.55754314 & 4.20315581 & -0.53520339 \\ \mathrm{C} & -2.72497836 & 3.51191692 & -0.67296037 \\ \mathrm{C} & 0.85976275 & 3.55499042 & -0.38260268 \\ \mathrm{C} & 1.95504709 & 2.70458688 & -0.37499334 \\ \mathrm{~N} & 1.84254286 & 1.36126559 & -0.54064686 \\ \mathrm{C} & 3.08031794 & 0.83686885 & -0.33895680 \\ \mathrm{C} & 4.04139827 & 1.89815488 & -0.10453166 \\ \mathrm{C} & 3.33774924 & 3.06660648 & -0.12722502\end{array}$




\begin{tabular}{|c|c|c|c|}
\hline $\mathrm{Fe}$ & -0.04189330 & 0.26934879 & -0.57280309 \\
\hline S & -0.30204096 & 0.40793387 & 1.81819441 \\
\hline $\mathrm{C}$ & 1.01983242 & -0.56651772 & 2.63492187 \\
\hline $\mathrm{C}$ & 3.30917869 & -0.52917757 & -0.27549598 \\
\hline $\mathrm{C}$ & 2.32925931 & -1.54937514 & -0.35563966 \\
\hline $\mathrm{N}$ & 0.95715038 & -1.41693447 & -0.46658140 \\
\hline $\mathrm{C}$ & 0.39845645 & -2.68141455 & -0.4891859 \\
\hline $\mathrm{C}$ & 1.46759559 & -3.64888151 & -0.3867361 \\
\hline $\mathrm{C}$ & 2.64379579 & -2.95886331 & -0.3026965 \\
\hline C & -0.96557802 & -3.04180326 & -0.6050477 \\
\hline $\mathrm{H}$ & 4.33151649 & -0.86951822 & -0.1347240 \\
\hline $\mathrm{H}$ & 1.32678492 & -4.72315111 & -0.38280598 \\
\hline $\mathrm{H}$ & 5.09983411 & 1.75687950 & 0.07842744 \\
\hline $\mathrm{H}$ & 3.70455702 & 4.07354284 & 0.03143499 \\
\hline $\mathrm{H}$ & 1.07062496 & 4.61657235 & -0.27107666 \\
\hline $\mathrm{H}$ & -1.41429773 & 5.27461398 & -0.4658421 \\
\hline $\mathrm{H}$ & -3.73309403 & 3.90363432 & -0.73744680 \\
\hline $\mathrm{H}$ & -4.34913135 & 1.41071454 & -0.93632066 \\
\hline $\mathrm{H}$ & -5.18694367 & -1.17101144 & -1.03247903 \\
\hline $\mathrm{H}$ & -3.85888018 & -3.52208243 & -0.86734930 \\
\hline $\mathrm{H}$ & -1.16554429 & -4.11026576 & -0.5932302 \\
\hline $\mathrm{H}$ & 3.64558355 & -3.36260864 & -0.21792864 \\
\hline $\mathrm{H}$ & 0.84995614 & -0.51652038 & 3.71622018 \\
\hline $\mathrm{H}$ & 0.99977165 & -1.61603633 & 2.3275248 \\
\hline $\mathrm{H}$ & 2.01049520 & -0.15359377 & 2.42153480 \\
\hline $\mathrm{O}$ & -0.09086105 & 0.21812256 & -2.5556066 \\
\hline $\mathrm{C}$ & -2.34933231 & -0.81386589 & -4.4977528 \\
\hline $\mathrm{C}$ & -3.03422816 & 0.50090524 & -4.3085507 \\
\hline $\mathrm{O}$ & -2.33762433 & 1.53879332 & -3.89803025 \\
\hline $\mathrm{O}$ & -4.23217313 & 0.62854716 & -4.6021556 \\
\hline $\mathrm{H}$ & -1.95487827 & -0.84531360 & -5.5222436 \\
\hline $\mathrm{H}$ & -3.07197531 & -1.62280410 & -4.3811787 \\
\hline $\mathrm{H}$ & -1.50673412 & -0.92091398 & -3.807804 \\
\hline $\mathrm{N}$ & -5.77719159 & 2.66864838 & -4.7898484 \\
\hline $\mathrm{C}$ & -5.38997813 & 3.85312849 & -5.3673698 \\
\hline $\mathrm{C}$ & -6.51410959 & 4.60428873 & -5.5851182 \\
\hline $\mathrm{N}$ & -7.57469722 & 3.84360377 & -5.1213230 \\
\hline $\mathrm{C}$ & -7.09854432 & 2.67770155 & -4.6545359 \\
\hline $\mathrm{C}$ & -6.62637795 & 5.97125400 & -6.1719671 \\
\hline $\mathrm{H}$ & -7.05616559 & 6.68617343 & -5.4599958 \\
\hline $\mathrm{H}$ & -5.63301089 & 6.33185407 & -6.4490399 \\
\hline $\mathrm{H}$ & -7.25291826 & 5.97877961 & -7.0717653 \\
\hline $\mathrm{H}$ & -8.57035855 & 4.12624942 & -5.1319160 \\
\hline $\mathrm{H}$ & -7.69559483 & 1.87971566 & -4.2416700 \\
\hline $\mathrm{H}$ & -5.09575456 & 1.85062304 & -4.588120 \\
\hline
\end{tabular}




$\begin{array}{lrrr}\mathrm{H} & -4.35171305 & 4.06683265 & -5.56954589 \\ \mathrm{O} & 0.45107829 & 1.40198252 & -3.23268983 \\ \mathrm{H} & 1.14031124 & 1.67312107 & -2.59524243 \\ \mathrm{H} & -1.44590420 & 1.28532911 & -3.52907259\end{array}$

\section{Quartet; Basis set B2; Model A; Compound 0}

78

\begin{tabular}{llll}
$\mathrm{O}$ & -10.26935036 & 4.57340712 & -5.10107870 \\
$\mathrm{C}$ & -11.00992817 & 5.44816286 & -4.61995681 \\
$\mathrm{~N}$ & -12.18586229 & 5.12179254 & -4.04271072 \\
$\mathrm{C}$ & -12.66197931 & 3.74382146 & -3.95916072 \\
$\mathrm{C}$ & -10.62099642 & 6.91484078 & -4.61989330 \\
$\mathrm{H}$ & -12.76683011 & 5.85980182 & -3.67061190 \\
$\mathrm{H}$ & -13.65596363 & 3.74703326 & -3.50790950 \\
$\mathrm{H}$ & -11.98867214 & 3.13920671 & -3.34332774 \\
$\mathrm{H}$ & -12.71710643 & 3.29704167 & -4.95610304 \\
$\mathrm{H}$ & -11.40612068 & 7.57294778 & -4.23567427 \\
$\mathrm{H}$ & -10.37174267 & 7.21949237 & -5.64097961 \\
$\mathrm{H}$ & -9.72522039 & 7.04540444 & -4.00284940 \\
$\mathrm{C}$ & -4.06023279 & -1.46877063 & -0.89003959 \\
$\mathrm{C}$ & -3.09552291 & -0.38335186 & -0.79956457 \\
$\mathrm{~N}$ & -1.84395418 & -0.89739060 & -0.65991698 \\
$\mathrm{C}$ & -1.95205640 & -2.25047289 & -0.65362607 \\
$\mathrm{C}$ & -3.34912626 & -2.63155564 & -0.79596145 \\
$\mathrm{C}$ & -3.37234452 & 0.98880727 & -0.81948024 \\
$\mathrm{C}$ & -2.42200621 & 2.01127714 & -0.67993254 \\
$\mathrm{~N}$ & -1.05262698 & 1.87050754 & -0.57014221 \\
$\mathrm{C}$ & -0.51669800 & 3.13209417 & -0.42734171 \\
$\mathrm{C}$ & -1.58337272 & 4.10504778 & -0.45774423 \\
$\mathrm{C}$ & -2.75294338 & 3.41430798 & -0.61544639 \\
$\mathrm{C}$ & 0.83979487 & 3.46326362 & -0.30438081 \\
$\mathrm{C}$ & 1.93309247 & 2.60406188 & -0.26584104 \\
$\mathrm{~N}$ & 1.83067545 & 1.25238534 & -0.33513480 \\
$\mathrm{C}$ & 3.09333471 & 0.75380392 & -0.22372319 \\
$\mathrm{C}$ & 4.04844438 & 1.84011164 & -0.09793106 \\
$\mathrm{C}$ & 3.32437322 & 2.99700813 & -0.12450416 \\
$\mathrm{Fe}$ & -0.01189918 & 0.17930093 & -0.47215662 \\
$\mathrm{~S}$ & -0.36857537 & 0.16859354 & 1.93141665 \\
$\mathrm{C}$ & 1.24937928 & -0.12417118 & 2.73971412 \\
$\mathrm{C}$ & 3.37554717 & -0.60867849 & -0.19314408 \\
$\mathrm{C}$ & 2.40774740 & -1.62914404 & -0.28577992 \\
& & & \\
\hline & & &
\end{tabular}




\begin{tabular}{|c|c|c|c|}
\hline $\mathrm{N}$ & 1.03655490 & -1.49328369 & -0.41290884 \\
\hline $\mathrm{C}$ & 0.49297046 & -2.76175536 & -0.43328499 \\
\hline $\mathrm{C}$ & 1.55982727 & -3.73114013 & -0.32908721 \\
\hline $\mathrm{C}$ & 2.73246889 & -3.03671044 & -0.23886907 \\
\hline $\mathrm{C}$ & -0.86138530 & -3.11702069 & -0.53894918 \\
\hline $\mathrm{H}$ & 4.41016118 & -0.92268628 & -0.09018675 \\
\hline $\mathrm{H}$ & 1.41697261 & -4.80519793 & -0.32486545 \\
\hline $\mathrm{H}$ & 5.12082460 & 1.72179287 & 0.00444465 \\
\hline $\mathrm{H}$ & 3.68388240 & 4.01654118 & -0.04920890 \\
\hline $\mathrm{H}$ & 1.05324880 & 4.52681212 & -0.22390031 \\
\hline $\mathrm{H}$ & -1.44382942 & 5.17549926 & -0.36381699 \\
\hline $\mathrm{H}$ & -3.75997495 & 3.81088283 & -0.67392804 \\
\hline $\mathrm{H}$ & -4.41063759 & 1.29646849 & -0.91142910 \\
\hline $\mathrm{H}$ & -5.13171493 & -1.34867559 & -1.00344634 \\
\hline $\mathrm{H}$ & -3.72373266 & -3.64844783 & -0.81645735 \\
\hline $\mathrm{H}$ & -1.07267929 & -4.18330721 & -0.53836849 \\
\hline $\mathrm{H}$ & 3.73731540 & -3.43149935 & -0.14610802 \\
\hline $\mathrm{H}$ & 1.09808534 & -0.11259637 & 3.82532933 \\
\hline $\mathrm{H}$ & 1.66612396 & -1.09662118 & 2.45677041 \\
\hline $\mathrm{H}$ & 1.97262968 & 0.65615646 & 2.48204860 \\
\hline $\mathrm{O}$ & 0.00896379 & 0.10376458 & -2.56706181 \\
\hline $\mathrm{C}$ & -2.35473269 & -0.77420580 & -4.67594209 \\
\hline $\mathrm{C}$ & -3.17343361 & 0.46800339 & -4.54169561 \\
\hline $\mathrm{O}$ & -2.58482892 & 1.58124468 & -4.17626384 \\
\hline $\mathrm{O}$ & -4.38829505 & 0.46711638 & -4.81938959 \\
\hline $\mathrm{H}$ & -1.81510400 & -0.73037921 & -5.63186200 \\
\hline $\mathrm{H}$ & -3.00511466 & -1.65031323 & -4.67923168 \\
\hline $\mathrm{H}$ & -1.59922686 & -0.84114188 & -3.88119593 \\
\hline $\mathrm{N}$ & -5.83758390 & 2.59000396 & -4.96115955 \\
\hline $\mathrm{C}$ & -5.40350474 & 3.79201387 & -5.47043530 \\
\hline $\mathrm{C}$ & -6.49952078 & 4.59724241 & -5.63883603 \\
\hline $\mathrm{N}$ & -7.58812817 & 3.84969679 & -5.21263342 \\
\hline $\mathrm{C}$ & -7.15662264 & 2.64030354 & -4.81820361 \\
\hline $\mathrm{C}$ & -6.60868878 & 5.98356758 & -6.17436983 \\
\hline $\mathrm{H}$ & -7.00267696 & 6.68109545 & -5.42507287 \\
\hline $\mathrm{H}$ & -5.62075879 & 6.34185012 & -6.47599513 \\
\hline $\mathrm{H}$ & -7.26582689 & 6.02572598 & -7.05128918 \\
\hline $\mathrm{H}$ & -8.57274454 & 4.16357609 & -5.20792515 \\
\hline $\mathrm{H}$ & -7.78229401 & 1.84131826 & -4.45063041 \\
\hline $\mathrm{H}$ & -5.20003730 & 1.72373311 & -4.80757039 \\
\hline $\mathrm{H}$ & -4.35718447 & 3.97417394 & -5.66419424 \\
\hline $\mathrm{O}$ & -0.16701271 & 1.36067459 & -3.32575493 \\
\hline $\mathrm{H}$ & -0.04446814 & 2.03979962 & -2.63670005 \\
\hline
\end{tabular}




\section{Quartet; Basis set B2; Model A; Transition State 2}

$\begin{array}{lrrr}78 & & & \\ \mathrm{O} & -10.26067079 & 4.57728057 & -5.19264119 \\ \mathrm{C} & -11.06254038 & 5.39016540 & -4.70312122 \\ \mathrm{~N} & -12.27566164 & 4.98973901 & -4.26423799 \\ \mathrm{C} & -12.71878068 & 3.60104834 & -4.34893715 \\ \mathrm{C} & -10.71331408 & 6.85874485 & -4.54049538 \\ \mathrm{H} & -12.90688607 & 5.67897769 & -3.88065058 \\ \mathrm{H} & -13.75233510 & 3.54470230 & -4.00205244 \\ \mathrm{H} & -12.09297268 & 2.95639579 & -3.72349668 \\ \mathrm{H} & -12.66209281 & 3.24452279 & -5.38148173 \\ \mathrm{H} & -11.54458397 & 7.46520706 & -4.16832134 \\ \mathrm{H} & -10.38789314 & 7.25772211 & -5.50609676 \\ \mathrm{H} & -9.87544925 & 6.94962988 & -3.84067005 \\ \mathrm{C} & -4.02048326 & -1.48705649 & -0.81061372 \\ \mathrm{C} & -3.02026492 & -0.44064799 & -0.72211463 \\ \mathrm{~N} & -1.77546438 & -0.99422038 & -0.63690587 \\ \mathrm{C} & -1.93854759 & -2.34263956 & -0.66674296 \\ \mathrm{C} & -3.34673486 & -2.67374575 & -0.76911105 \\ \mathrm{C} & -3.27219179 & 0.92932729 & -0.70847974 \\ \mathrm{C} & -2.29715332 & 1.92667484 & -0.57327121 \\ \mathrm{~N} & -0.92747435 & 1.74461305 & -0.51714830 \\ \mathrm{C} & -0.35320192 & 2.98962309 & -0.37412392 \\ \mathrm{C} & -1.39189652 & 3.99130329 & -0.34521396 \\ \mathrm{C} & -2.58598685 & 3.33551176 & -0.47096702 \\ \mathrm{C} & 1.01937968 & 3.26902555 & -0.31185143 \\ \mathrm{C} & 2.07094443 & 2.36712646 & -0.33889098 \\ \mathrm{~N} & 1.91436560 & 1.01696473 & -0.40382508 \\ \mathrm{C} & 3.16960669 & 0.48393707 & -0.37450127 \\ \mathrm{C} & 4.16257756 & 1.53540564 & -0.29684340 \\ \mathrm{C} & 3.47610548 & 2.71413656 & -0.27568537 \\ \mathrm{Fe} & 0.06312366 & 0.01429079 & -0.48631856 \\ \mathrm{~S} & -0.22363001 & -0.13257122 & 1.95050797 \\ \mathrm{C} & 0.75071464 & 1.20876195 & 2.73412399 \\ \mathrm{C} & 3.42834527 & -0.88021330 & -0.39362582 \\ \mathrm{C} & 2.43175717 & -1.86973997 & -0.47840763 \\ \mathrm{~N} & 1.06133536 & -1.68986621 & -0.56599218 \\ \mathrm{C} & 0.48223340 & -2.94255781 & -0.57843715 \\ \mathrm{C} & & -3.94484702 & -0.52104979\end{array}$




$\begin{array}{lrrr}\mathrm{C} & 2.71673531 & -3.28723372 & -0.46118247 \\ \mathrm{C} & -0.88512263 & -3.25295873 & -0.62053737 \\ \mathrm{H} & 4.45896255 & -1.21765958 & -0.34224458 \\ \mathrm{H} & 1.34725817 & -5.01396389 & -0.52196060 \\ \mathrm{H} & 5.23402652 & 1.37841191 & -0.26168588 \\ \mathrm{H} & 3.86833822 & 3.72259731 & -0.21947110 \\ \mathrm{H} & 1.27996322 & 4.32196630 & -0.23275654 \\ \mathrm{H} & -1.21906040 & 5.05585206 & -0.24046656 \\ \mathrm{H} & -3.58271068 & 3.76046893 & -0.48753870 \\ \mathrm{H} & -4.30610066 & 1.25747258 & -0.76827657 \\ \mathrm{H} & -5.08890046 & -1.32327237 & -0.88821193 \\ \mathrm{H} & -3.75112278 & -3.67837825 & -0.80664560 \\ \mathrm{H} & -1.13608738 & -4.31022353 & -0.62836361 \\ \mathrm{H} & 3.71202281 & -3.71154596 & -0.40392151 \\ \mathrm{H} & 0.73455938 & 1.06498062 & 3.82052131 \\ \mathrm{H} & 1.79178453 & 1.19786125 & 2.39626432 \\ \mathrm{H} & 0.32012356 & 2.19133940 & 2.50997156 \\ \mathrm{O} & 0.01033784 & 0.07138917 & -2.66750803 \\ \mathrm{C} & -2.44196841 & -0.76795517 & -4.61707952 \\ \mathrm{C} & -3.20163209 & 0.51169850 & -4.46096657 \\ \mathrm{O} & -2.56452705 & 1.58501866 & -4.11526228 \\ \mathrm{O} & -4.43254843 & 0.53573499 & -4.71099567 \\ \mathrm{H} & -1.96236117 & -0.76343780 & -5.60571018 \\ \mathrm{H} & -3.12344023 & -1.61938932 & -4.56794806 \\ \mathrm{H} & -1.64350286 & -0.85299337 & -3.86925560 \\ \mathrm{~N} & -5.79922596 & 2.66661841 & -4.82845721 \\ \mathrm{C} & -5.35510304 & 3.91629089 & -5.19384157 \\ \mathrm{C} & -6.45187119 & 4.71614878 & -5.38734543 \\ \mathrm{~N} & -7.55425160 & 3.91632858 & -5.12294492 \\ \mathrm{C} & -7.12556811 & 2.68425766 & -4.79773247 \\ \mathrm{C} & -6.55711641 & 6.13927584 & -5.81605882 \\ \mathrm{H} & -7.03934792 & 6.76245045 & -5.05277487 \\ \mathrm{H} & -5.55833071 & 6.54642399 & -5.99584102 \\ \mathrm{H} & -7.13338703 & 6.23890444 & -6.74389466 \\ \mathrm{H} & -8.54191223 & 4.20991718 & -5.18039869 \\ \mathrm{H} & -7.76114196 & 1.84570087 & -4.55673030 \\ \mathrm{H} & -4.15343910 & 1.77061187 & -4.69822907 \\ \mathrm{H} & -27118811 & 1.38066240 & -3.31326055 \\ \mathrm{H} & & & \\ \mathrm{H} & -1.98395784 & 1.42225557 & -3.75269686\end{array}$




\section{Quartet; Basis set B2; Model A; protonated Compound 0}

78

\begin{tabular}{|c|c|c|c|}
\hline $\mathrm{O}$ & -10.25092876 & 4.62349710 & -5.04167018 \\
\hline $\mathrm{C}$ & -10.98994789 & 5.47411962 & -4.52779011 \\
\hline $\mathrm{N}$ & -12.17406249 & 5.13532833 & -3.95624103 \\
\hline C & -12.68356342 & 3.77128543 & -3.89314814 \\
\hline C & -10.60008164 & 6.94701758 & -4.51917928 \\
\hline $\mathrm{H}$ & -12.73848560 & 5.87306967 & -3.55626768 \\
\hline $\mathrm{H}$ & -13.66193838 & 3.70536299 & -4.38107906 \\
\hline $\mathrm{H}$ & -12.77651188 & 3.44002434 & -2.85286879 \\
\hline $\mathrm{H}$ & -11.97875403 & 3.12318874 & -4.41466382 \\
\hline $\mathrm{H}$ & -11.34898244 & 7.59661259 & -4.05497149 \\
\hline $\mathrm{H}$ & -10.43745562 & 7.27211425 & -5.55139386 \\
\hline $\mathrm{H}$ & -9.65377233 & 7.05844128 & -3.98008332 \\
\hline $\mathrm{C}$ & -3.99417118 & -1.55871514 & -1.01604815 \\
\hline C & -3.02155097 & -0.49669982 & -0.87752080 \\
\hline $\mathrm{N}$ & -1.76438006 & -1.01754014 & -0.74173170 \\
\hline $\mathrm{C}$ & -1.90898173 & -2.37168558 & -0.78424503 \\
\hline $\mathrm{C}$ & -3.30015848 & -2.73050421 & -0.95058288 \\
\hline $\mathrm{C}$ & -3.32418838 & 0.85689367 & -0.85337002 \\
\hline $\mathrm{C}$ & -2.39287182 & 1.88470602 & -0.67275280 \\
\hline $\mathrm{N}$ & -1.01210323 & 1.73805940 & -0.61034628 \\
\hline $\mathrm{C}$ & -0.48301999 & 2.99975064 & -0.39177298 \\
\hline C & -1.55091309 & 3.96293519 & -0.34922847 \\
\hline $\mathrm{C}$ & -2.72300892 & 3.27649300 & -0.52523640 \\
\hline $\mathrm{C}$ & 0.87949590 & 3.29765321 & -0.30128790 \\
\hline $\mathrm{C}$ & 1.93660444 & 2.40622553 & -0.30142008 \\
\hline $\mathrm{N}$ & 1.80552309 & 1.05033782 & -0.34953569 \\
\hline $\mathrm{C}$ & 3.07661663 & 0.55098737 & -0.29717404 \\
\hline $\mathrm{C}$ & 4.04275493 & 1.62294067 & -0.21507367 \\
\hline $\mathrm{C}$ & 3.33014258 & 2.78468819 & -0.21891272 \\
\hline $\mathrm{Fe}$ & 0.01662602 & 0.00708917 & -0.45919955 \\
\hline S & -0.36348648 & -0.13241367 & 1.94744024 \\
\hline $\mathrm{C}$ & 0.57261200 & 1.21372991 & 2.76924086 \\
\hline $\mathrm{C}$ & 3.37934538 & -0.79918608 & -0.30965157 \\
\hline $\mathrm{C}$ & 2.41952180 & -1.81987290 & -0.42038724 \\
\hline $\mathrm{N}$ & 1.04966785 & -1.67131621 & -0.54942142 \\
\hline $\mathrm{C}$ & 0.50259153 & -2.93683123 & -0.59024520 \\
\hline C & 1.56330053 & -3.91467136 & -0.50216565 \\
\hline $\mathrm{C}$ & 2.73881762 & -3.22910339 & -0.40042252 \\
\hline $\mathrm{C}$ & -0.85659701 & -3.26802534 & -0.69277780 \\
\hline $\mathrm{H}$ & 4.41949068 & -1.10078120 & -0.23463051 \\
\hline $\mathrm{H}$ & 1.41381596 & -4.98748828 & -0.51618022 \\
\hline $\mathrm{H}$ & 5.11619177 & 1.48782245 & -0.16373681 \\
\hline
\end{tabular}




$\begin{array}{lrrr}\mathrm{H} & 3.69535069 & 3.80335077 & -0.17108201 \\ \mathrm{H} & 1.12780367 & 4.35198863 & -0.20676500 \\ \mathrm{H} & -1.41570771 & 5.02794389 & -0.20423399 \\ \mathrm{H} & -3.73019294 & 3.67415232 & -0.55191156 \\ \mathrm{H} & -4.36698754 & 1.14737233 & -0.94020799 \\ \mathrm{H} & -5.05956232 & -1.40996194 & -1.14274806 \\ \mathrm{H} & -3.67781705 & -3.74391803 & -1.01148149 \\ \mathrm{H} & -1.09437724 & -4.32783970 & -0.71573607 \\ \mathrm{H} & 3.74154649 & -3.63002636 & -0.31480615 \\ \mathrm{H} & 0.54366011 & 1.05056405 & 3.85205787 \\ \mathrm{H} & 1.61744486 & 1.22792630 & 2.44443655 \\ \mathrm{H} & 0.12442102 & 2.19018331 & 2.55473387 \\ \mathrm{O} & 0.15809498 & 0.12165975 & -2.77514637 \\ \mathrm{C} & -2.28980417 & -0.79941185 & -4.69583206 \\ \mathrm{C} & -3.04692597 & 0.48815240 & -4.60198277 \\ \mathrm{O} & -2.48034050 & 1.57417042 & -4.37926562 \\ \mathrm{O} & -4.33623291 & 0.38415836 & -4.80549673 \\ \mathrm{H} & -1.88684044 & -0.88726326 & -5.71406027 \\ \mathrm{H} & -2.94435488 & -1.65464352 & -4.51351344 \\ \mathrm{H} & -1.44442520 & -0.80029431 & -3.99893597 \\ \mathrm{~N} & -5.77638171 & 2.57504789 & -4.82963319 \\ \mathrm{C} & -5.35163879 & 3.78814935 & -5.32743730 \\ \mathrm{C} & -6.42924114 & 4.62082696 & -5.51499330 \\ \mathrm{~N} & -7.53070973 & 3.88641565 & -5.10997973 \\ \mathrm{C} & -7.09051501 & 2.66567776 & -4.71463555 \\ \mathrm{C} & -6.53278889 & 6.00601598 & -6.05648689 \\ \mathrm{H} & -6.93392596 & 6.71190862 & -5.31680050 \\ \mathrm{H} & -5.54172128 & 6.36492987 & -6.35032936 \\ \mathrm{H} & -7.18089187 & 6.05021970 & -6.94129691 \\ \mathrm{H} & -8.50622661 & 4.19960011 & -5.12767335 \\ \mathrm{H} & -7.74842847 & 1.88427226 & -4.36047788 \\ \mathrm{H} & -4.84218127 & 1.28976071 & -4.75633044 \\ \mathrm{H} & -4.30396724 & 3.97859758 & -5.51578532 \\ \mathrm{H} & -0.17074987 & 1.51425635 & -3.22607318 \\ & -0.46449382 & 1.89702055 & -2.35544630 \\ \mathrm{H} & -1.04123857 & 1.45487294 & -3.77662173\end{array}$




\section{Quartet; Basis set B2; Model A; Transition State 3}

\begin{tabular}{|c|c|c|c|}
\hline $\mathrm{O}$ & -10.28948427 & 4.61524939 & -5.07683776 \\
\hline $\mathrm{C}$ & -11.02974157 & 5.47789745 & -4.58446195 \\
\hline $\mathrm{N}$ & -12.21902637 & 5.13481278 & -4.03305978 \\
\hline $\mathrm{C}$ & -12.68386845 & 3.75249474 & -3.99030289 \\
\hline $\mathrm{C}$ & -10.63792594 & 6.94868119 & -4.57157616 \\
\hline $\mathrm{H}$ & -12.80559650 & 5.85927964 & -3.64393135 \\
\hline $\mathrm{H}$ & -13.68294234 & 3.73563303 & -3.54983912 \\
\hline $\mathrm{H}$ & -12.01161981 & 3.13594824 & -3.38491752 \\
\hline $\mathrm{H}$ & -12.72416640 & 3.33070986 & -4.99902512 \\
\hline $\mathrm{H}$ & -11.40457837 & 7.60134667 & -4.14220731 \\
\hline $\mathrm{H}$ & -10.43276903 & 7.26780122 & -5.59803915 \\
\hline $\mathrm{H}$ & -9.71438323 & 7.06395390 & -3.99471271 \\
\hline $\mathrm{C}$ & -4.00114817 & -1.54283203 & -0.96983704 \\
\hline $\mathrm{C}$ & -3.02743875 & -0.48171230 & -0.84077652 \\
\hline $\mathrm{N}$ & -1.76943319 & -1.00464225 & -0.70643612 \\
\hline $\mathrm{C}$ & -1.91631028 & -2.35981844 & -0.74713716 \\
\hline $\mathrm{C}$ & -3.30863074 & -2.71571179 & -0.90458963 \\
\hline $\mathrm{C}$ & -3.32798101 & 0.87258674 & -0.83422782 \\
\hline $\mathrm{C}$ & -2.39093069 & 1.89948910 & -0.67447916 \\
\hline $\mathrm{N}$ & -1.01473700 & 1.74834075 & -0.59578686 \\
\hline $\mathrm{C}$ & -0.48227489 & 3.01009974 & -0.40266319 \\
\hline $\mathrm{C}$ & -1.54652979 & 3.98106546 & -0.38810596 \\
\hline $\mathrm{C}$ & -2.71898983 & 3.29651493 & -0.55868345 \\
\hline $\mathrm{C}$ & 0.88027851 & 3.30828038 & -0.30123389 \\
\hline $\mathrm{C}$ & 1.93522241 & 2.41334133 & -0.28309112 \\
\hline $\mathrm{N}$ & 1.80165974 & 1.05647298 & -0.32323954 \\
\hline $\mathrm{C}$ & 3.07318531 & 0.55510958 & -0.28163643 \\
\hline $\mathrm{C}$ & 4.04018927 & 1.62577690 & -0.20457648 \\
\hline $\mathrm{C}$ & 3.32904830 & 2.78894001 & -0.20684317 \\
\hline $\mathrm{Fe}$ & 0.01000529 & 0.01770375 & -0.43055739 \\
\hline $\mathrm{S}$ & -0.38580875 & -0.11328018 & 1.96702694 \\
\hline $\mathrm{C}$ & 0.72781426 & 1.07061463 & 2.81785238 \\
\hline $\mathrm{C}$ & 3.37693454 & -0.79588846 & -0.30657407 \\
\hline $\mathrm{C}$ & 2.41520421 & -1.81454448 & -0.42330185 \\
\hline $\mathrm{N}$ & 1.04348197 & -1.66409846 & -0.54215424 \\
\hline $\mathrm{C}$ & 0.49493021 & -2.93022431 & -0.58354404 \\
\hline $\mathrm{C}$ & 1.55602446 & -3.90852334 & -0.51012202 \\
\hline $\mathrm{C}$ & 2.73284898 & -3.22418145 & -0.41440554 \\
\hline $\mathrm{C}$ & -0.86562070 & -3.26032910 & -0.67100304 \\
\hline $\mathrm{H}$ & 4.41733314 & -1.09844234 & -0.24062711 \\
\hline $\mathrm{H}$ & 1.40562286 & -4.98116265 & -0.52764570 \\
\hline
\end{tabular}




$\begin{array}{lrrr}\mathrm{H} & 5.11380499 & 1.48933868 & -0.16041161 \\ \mathrm{H} & 3.69636598 & 3.80708103 & -0.16504792 \\ \mathrm{H} & 1.13025344 & 4.36325983 & -0.22073296 \\ \mathrm{H} & -1.40641656 & 5.04822814 & -0.26560032 \\ \mathrm{H} & -3.72475790 & 3.69614391 & -0.60283411 \\ \mathrm{H} & -4.36968719 & 1.16454419 & -0.92725391 \\ \mathrm{H} & -5.06666332 & -1.39282301 & -1.09370122 \\ \mathrm{H} & -3.68790380 & -3.72870125 & -0.96218620 \\ \mathrm{H} & -1.10550061 & -4.31946852 & -0.69609677 \\ \mathrm{H} & 3.73580551 & -3.62644164 & -0.33801043 \\ \mathrm{H} & 0.64367221 & 0.91579506 & 3.89896393 \\ \mathrm{H} & 1.77019405 & 0.92049924 & 2.52149577 \\ \mathrm{H} & 0.44340737 & 2.10437340 & 2.59361205 \\ \mathrm{O} & 0.18662386 & 0.05247469 & -2.70828633 \\ \mathrm{C} & -2.31033494 & -0.78961240 & -4.67725969 \\ \mathrm{C} & -3.06756463 & 0.49831779 & -4.57773063 \\ \mathrm{O} & -2.50048388 & 1.58613265 & -4.37479522 \\ \mathrm{O} & -4.36217902 & 0.38941915 & -4.75316963 \\ \mathrm{H} & -1.87302513 & -0.85431419 & -5.68277380 \\ \mathrm{H} & -2.97127419 & -1.64712879 & -4.53446652 \\ \mathrm{H} & -1.48639971 & -0.80682163 & -3.95598095 \\ \mathrm{~N} & -5.79913674 & 2.59103409 & -4.79032063 \\ \mathrm{C} & -5.36860014 & 3.80663168 & -5.27770896 \\ \mathrm{C} & -6.44529165 & 4.63554680 & -5.48770820 \\ \mathrm{~N} & -7.55228265 & 3.89605695 & -5.10740820 \\ \mathrm{C} & -7.11580393 & 2.67678815 & -4.70352923 \\ \mathrm{C} & -6.54714203 & 6.01939254 & -6.03423104 \\ \mathrm{H} & -6.98028930 & 6.72125382 & -5.30907807 \\ \mathrm{H} & -5.55153255 & 6.38961554 & -6.29718371 \\ \mathrm{H} & -7.16629716 & 6.05417636 & -6.93999632 \\ \mathrm{H} & -8.52906765 & 4.20385775 & -5.14424238 \\ \mathrm{H} & -7.77850064 & 1.89265314 & -4.36470049 \\ \mathrm{H} & -4.86232224 & 1.29612300 & -4.70919781 \\ \mathrm{H} & -4.31781679 & 4.00076166 & -5.44386761 \\ \mathrm{O} & -0.12940311 & 1.59955815 & -3.31058223 \\ \mathrm{H} & -0.38130476 & 1.96101793 & -2.42641295 \\ \mathrm{H} & -1.02442581 & 1.49599861 & -3.80084997 \\ & & & \\ & & & \end{array}$




\section{Quartet; Basis set B2; Model A; Compound I}

\begin{tabular}{|c|c|c|c|}
\hline $\mathrm{O}$ & -10.28762344 & 4.69491427 & -4.91502280 \\
\hline $\mathrm{C}$ & -10.94475644 & 5.60206505 & -4.38841406 \\
\hline $\mathrm{N}$ & -12.10353244 & 5.32635145 & -3.73935923 \\
\hline $\mathrm{C}$ & -12.62491611 & 3.96836072 & -3.63136326 \\
\hline $\mathrm{C}$ & -10.48527998 & 7.05297852 & -4.44674919 \\
\hline $\mathrm{H}$ & -12.62554892 & 6.08481735 & -3.32395944 \\
\hline $\mathrm{H}$ & -13.58712015 & 4.00563305 & -3.11615130 \\
\hline $\mathrm{H}$ & -11.93627931 & 3.33223289 & -3.06587589 \\
\hline $\mathrm{H}$ & -12.76110493 & 3.53022572 & -4.62468882 \\
\hline $\mathrm{H}$ & -11.17733701 & 7.75073365 & -3.96454525 \\
\hline $\mathrm{H}$ & -10.36195192 & 7.34062163 & -5.49536636 \\
\hline $\mathrm{H}$ & -9.50730535 & 7.13378801 & -3.96129582 \\
\hline $\mathrm{C}$ & -3.92785237 & -1.70675763 & -1.02072799 \\
\hline $\mathrm{C}$ & -3.00798426 & -0.60446939 & -0.86366244 \\
\hline $\mathrm{N}$ & -1.72714893 & -1.08845862 & -0.69130245 \\
\hline $\mathrm{C}$ & -1.81963602 & -2.46411333 & -0.73761597 \\
\hline $\mathrm{C}$ & -3.19652468 & -2.85431840 & -0.93587551 \\
\hline $\mathrm{C}$ & -3.39014546 & 0.74581927 & -0.86960720 \\
\hline $\mathrm{C}$ & -2.47919833 & 1.78985390 & -0.67483932 \\
\hline $\mathrm{N}$ & -1.10806540 & 1.66294172 & -0.46780793 \\
\hline $\mathrm{C}$ & -0.60399577 & 2.94651469 & -0.35821588 \\
\hline $\mathrm{C}$ & -1.68408962 & 3.89819074 & -0.46824052 \\
\hline $\mathrm{C}$ & -2.83112562 & 3.18882939 & -0.66769470 \\
\hline $\mathrm{C}$ & 0.74083360 & 3.30116025 & -0.19848424 \\
\hline $\mathrm{C}$ & 1.81086848 & 2.40791103 & -0.14548921 \\
\hline $\mathrm{N}$ & 1.71640001 & 1.04119215 & -0.20078471 \\
\hline $\mathrm{C}$ & 3.01013195 & 0.56566326 & -0.11668213 \\
\hline $\mathrm{C}$ & 3.93419763 & 1.66881725 & 0.00741531 \\
\hline $\mathrm{C}$ & 3.19148382 & 2.81115784 & -0.00994441 \\
\hline $\mathrm{Fe}$ & 0.01250176 & -0.02210426 & -0.58816966 \\
\hline $\mathrm{S}$ & -0.63871058 & 0.04955596 & 1.92212476 \\
\hline $\mathrm{C}$ & 0.42312965 & 1.24252703 & 2.78370248 \\
\hline $\mathrm{C}$ & 3.38586492 & -0.77424899 & -0.13360168 \\
\hline $\mathrm{C}$ & 2.46243632 & -1.83210078 & -0.25791870 \\
\hline $\mathrm{N}$ & 1.09153483 & -1.72117362 & -0.37732687 \\
\hline $\mathrm{C}$ & 0.59121731 & -3.00131420 & -0.47018789 \\
\hline $\mathrm{C}$ & 1.67847284 & -3.94960430 & -0.39456215 \\
\hline $\mathrm{C}$ & 2.83110787 & -3.22876540 & -0.26697775 \\
\hline $\mathrm{C}$ & -0.75889218 & -3.36608303 & -0.62404930 \\
\hline $\mathrm{H}$ & 4.43886789 & -1.02226184 & -0.05312768 \\
\hline $\mathrm{H}$ & 1.56274883 & -5.02567561 & -0.43943481 \\
\hline
\end{tabular}




$\begin{array}{lrrr}\mathrm{H} & 5.00907860 & 1.56460791 & 0.09044774 \\ \mathrm{H} & 3.53018480 & 3.83783377 & 0.05352392 \\ \mathrm{H} & 0.96899217 & 4.36129651 & -0.15115155 \\ \mathrm{H} & -1.56064010 & 4.97325777 & -0.42535935 \\ \mathrm{H} & -3.83474906 & 3.56676207 & -0.81794608 \\ \mathrm{H} & -4.43264621 & 0.99122794 & -1.03755182 \\ \mathrm{H} & -4.99325898 & -1.59809982 & -1.18100734 \\ \mathrm{H} & -3.54228939 & -3.87833826 & -1.00718595 \\ \mathrm{H} & -0.98806769 & -4.42557532 & -0.67697233 \\ \mathrm{H} & 3.84622004 & -3.59808034 & -0.18568803 \\ \mathrm{H} & 0.16799623 & 1.25233765 & 3.84799950 \\ \mathrm{H} & 1.48149911 & 1.00581888 & 2.64539680 \\ \mathrm{H} & 0.23873908 & 2.24446187 & 2.37518271 \\ \mathrm{O} & 0.20985201 & 0.04284969 & -2.19567830 \\ \mathrm{C} & -2.19133010 & -0.80479139 & -4.87686347 \\ \mathrm{C} & -3.04428697 & 0.44315549 & -4.90825214 \\ \mathrm{O} & -2.58558831 & 1.56889247 & -5.07621648 \\ \mathrm{O} & -4.34394769 & 0.20780301 & -4.73219320 \\ \mathrm{H} & -1.56899515 & -0.83482624 & -5.77723502 \\ \mathrm{H} & -2.80062022 & -1.70838896 & -4.81110733 \\ \mathrm{H} & -1.51311790 & -0.75271575 & -4.01689389 \\ \mathrm{~N} & -5.86836716 & 2.46739603 & -4.89382237 \\ \mathrm{C} & -5.42122825 & 3.65415162 & -5.44003370 \\ \mathrm{C} & -6.47303298 & 4.52880900 & -5.59815070 \\ \mathrm{~N} & -7.58296420 & 3.84603531 & -5.12636964 \\ \mathrm{C} & -7.17011050 & 2.61704100 & -4.72170451 \\ \mathrm{C} & -6.57482390 & 5.90346216 & -6.17709827 \\ \mathrm{H} & -6.94669183 & 6.63414727 & -5.44618102 \\ \mathrm{H} & -5.58875676 & 6.24216604 & -6.50961749 \\ \mathrm{H} & -7.24719164 & 5.92911111 & -7.04456748 \\ \mathrm{H} & -8.54473884 & 4.19428892 & -5.10688313 \\ \mathrm{H} & -7.84370652 & 1.87303029 & -4.31877136 \\ \mathrm{H} & -4.87088483 & 1.07683244 & -4.76853687 \\ \mathrm{H} & -4.37571633 & 3.79064857 & -5.68118687 \\ \mathrm{O} & 0.07606569 & 2.35449201 & -4.06383927 \\ \mathrm{H} & 0.21699058 & 1.62105031 & -3.43695235 \\ \mathrm{H} & -0.78190851 & 2.15143706 & -4.48411874\end{array}$




\section{Quartet; Basis set B2; Model B; Fe+HOOH}

\begin{tabular}{|c|c|c|c|}
\hline $\mathrm{O}$ & -10.15667184 & 4.64601556 & -4.79409331 \\
\hline $\mathrm{C}$ & -10.75309158 & 5.59262087 & -4.26394140 \\
\hline $\mathrm{N}$ & -11.84357124 & 5.37548276 & -3.48813488 \\
\hline $\mathrm{C}$ & -12.36178888 & 4.03552116 & -3.23526530 \\
\hline $\mathrm{C}$ & -10.28952354 & 7.03113338 & -4.45874512 \\
\hline $\mathrm{H}$ & -12.30620818 & 6.16595182 & -3.06155780 \\
\hline $\mathrm{H}$ & -13.31404220 & 4.12485093 & -2.70828149 \\
\hline $\mathrm{H}$ & -11.66401069 & 3.45319300 & -2.62366380 \\
\hline $\mathrm{H}$ & -12.51633050 & 3.50657940 & -4.17979766 \\
\hline $\mathrm{H}$ & -10.93497406 & 7.76816503 & -3.97015974 \\
\hline $\mathrm{H}$ & -10.25175929 & 7.24612381 & -5.53095799 \\
\hline $\mathrm{H}$ & -9.27471243 & 7.13256664 & -4.06043629 \\
\hline $\mathrm{C}$ & -4.09165271 & -1.59837074 & -1.05811945 \\
\hline $\mathrm{C}$ & -3.15827305 & -0.51618434 & -0.89508682 \\
\hline $\mathrm{N}$ & -1.88394621 & -0.97632004 & -0.69071719 \\
\hline $\mathrm{C}$ & -2.00331287 & -2.33867377 & -0.72842766 \\
\hline $\mathrm{C}$ & -3.36672328 & -2.74501237 & -0.94687164 \\
\hline $\mathrm{C}$ & -3.48752498 & 0.81032637 & -0.96797564 \\
\hline $\mathrm{C}$ & -2.59996084 & 1.87438012 & -0.82616441 \\
\hline $\mathrm{N}$ & -1.24427475 & 1.78061428 & -0.58228784 \\
\hline $\mathrm{C}$ & -0.77664055 & 3.07905098 & -0.50935796 \\
\hline $\mathrm{C}$ & -1.86103918 & 4.00281318 & -0.71715605 \\
\hline $\mathrm{C}$ & -2.98683868 & 3.25789275 & -0.91936706 \\
\hline $\mathrm{C}$ & 0.54722573 & 3.45462243 & -0.29265493 \\
\hline $\mathrm{C}$ & 1.60068308 & 2.56839623 & -0.16521316 \\
\hline $\mathrm{N}$ & 1.48368116 & 1.19651043 & -0.19998705 \\
\hline $\mathrm{C}$ & 2.77078877 & 0.71941783 & -0.08937528 \\
\hline $\mathrm{C}$ & 3.70682724 & 1.80530363 & 0.02945147 \\
\hline $\mathrm{C}$ & 2.97759690 & 2.95745360 & -0.01623672 \\
\hline $\mathrm{Fe}$ & -0.22680660 & 0.11128281 & -0.20561927 \\
\hline $\mathrm{S}$ & -0.62784179 & -0.02266352 & 2.18649740 \\
\hline $\mathrm{C}$ & 0.14131827 & 1.48617458 & 2.90166406 \\
\hline $\mathrm{C}$ & 3.12581057 & -0.61693680 & -0.10825561 \\
\hline $\mathrm{C}$ & 2.23617796 & -1.67256314 & -0.24633757 \\
\hline $\mathrm{N}$ & 0.86906658 & -1.55511434 & -0.38200366 \\
\hline $\mathrm{C}$ & 0.38852757 & -2.84244073 & -0.47003729 \\
\hline $\mathrm{C}$ & 1.47395375 & -3.78640037 & -0.39646336 \\
\hline $\mathrm{C}$ & 2.62068371 & -3.06043427 & -0.26120733 \\
\hline $\mathrm{C}$ & -0.94477998 & -3.20070744 & -0.61502405 \\
\hline $\mathrm{H}$ & 4.17949531 & -0.86003534 & -0.00902947 \\
\hline $\mathrm{H}$ & 1.35814812 & -4.86179062 & -0.44872393 \\
\hline
\end{tabular}




\begin{tabular}{|c|c|c|c|}
\hline $\mathrm{H}$ & 4.77906973 & 1.68708912 & 0.12385599 \\
\hline $\mathrm{H}$ & 3.32705807 & 3.98122656 & 0.03288211 \\
\hline $\mathrm{H}$ & 0.76822277 & 4.51705445 & -0.25773600 \\
\hline $\mathrm{H}$ & -1.75844345 & 5.08090807 & -0.72033188 \\
\hline $\mathrm{H}$ & -3.99313791 & 3.60027791 & -1.12521826 \\
\hline $\mathrm{H}$ & -4.52333783 & 1.06450068 & -1.17074901 \\
\hline $\mathrm{H}$ & -5.14933440 & -1.47451215 & -1.25069387 \\
\hline $\mathrm{H}$ & -3.69989176 & -3.77265266 & -1.01839718 \\
\hline $\mathrm{H}$ & -1.16173774 & -4.26476621 & -0.65628997 \\
\hline $\mathrm{H}$ & 3.63955041 & -3.41778275 & -0.17862192 \\
\hline $\mathrm{H}$ & -0.06836657 & 1.50380264 & 3.97643397 \\
\hline $\mathrm{H}$ & 1.22487599 & 1.47964225 & 2.75638473 \\
\hline $\mathrm{H}$ & -0.27515151 & 2.39064840 & 2.44994492 \\
\hline $\mathrm{C}$ & -2.05820461 & -0.91796726 & -4.78147892 \\
\hline C & -2.92402240 & 0.28808017 & -5.09150536 \\
\hline $\mathrm{O}$ & -2.54236056 & 1.25366908 & -5.7345205 \\
\hline $\mathrm{O}$ & -4.15194801 & 0.18081041 & -4.56889335 \\
\hline $\mathrm{H}$ & -1.13935171 & -0.87160298 & -5.36859226 \\
\hline $\mathrm{H}$ & -2.59916934 & -1.84485376 & -4.99815200 \\
\hline $\mathrm{H}$ & -1.80778353 & -0.92438898 & -3.71445695 \\
\hline $\mathrm{N}$ & -5.78028377 & 2.35194209 & -5.06270349 \\
\hline $\mathrm{C}$ & -5.37943447 & 3.49605444 & -5.72264283 \\
\hline C & -6.43273824 & 4.37746900 & -5.83272124 \\
\hline $\mathrm{N}$ & -7.49808611 & 3.74219422 & -5.2165263 \\
\hline $\mathrm{C}$ & -7.05848712 & 2.53340088 & -4.77743908 \\
\hline $\mathrm{C}$ & -6.54826811 & 5.72959149 & -6.46501707 \\
\hline $\mathrm{H}$ & -6.88782098 & 6.49092843 & -5.75009776 \\
\hline $\mathrm{H}$ & -5.57182394 & 6.04482090 & -6.84532498 \\
\hline $\mathrm{H}$ & -7.25182794 & 5.72989580 & -7.30797167 \\
\hline $\mathrm{H}$ & -8.45061978 & 4.10510783 & -5.12585862 \\
\hline $\mathrm{H}$ & -7.69844326 & 1.82724679 & -4.2661273 \\
\hline $\mathrm{H}$ & -4.70704544 & 1.00148498 & -4.7833644 \\
\hline $\mathrm{H}$ & -4.36279076 & 3.60145111 & -6.0768920 \\
\hline $\mathrm{N}$ & -0.64095934 & -1.97841455 & 5.04069883 \\
\hline $\mathrm{C}$ & 0.57286004 & -2.45844521 & 5.68091032 \\
\hline $\mathrm{C}$ & -1.86092456 & -2.47659767 & 5.37714763 \\
\hline $\mathrm{C}$ & -3.05843733 & -1.94971691 & 4.55038632 \\
\hline $\mathrm{N}$ & -3.13475485 & -0.49484237 & 4.4493571 \\
\hline $\mathrm{C}$ & -3.46237883 & 0.30693204 & $5.5065840 €$ \\
\hline $\mathrm{C}$ & -3.39958272 & 1.80525893 & 5.2355223 \\
\hline $\mathrm{C}$ & -3.12957663 & -2.61151593 & 3.1698982 \\
\hline $\mathrm{O}$ & -2.01303153 & -3.34689536 & 6.2322732 \\
\hline $\mathrm{O}$ & -3.81811772 & -0.13347680 & 6.5968899 \\
\hline $\mathrm{H}$ & -0.55929079 & -1.36782946 & 4.2327088 \\
\hline $\mathrm{H}$ & 0.72523366 & -3.53000030 & 5.5002328 \\
\hline
\end{tabular}




$\begin{array}{lrrr}\mathrm{H} & 1.42300711 & -1.90054389 & 5.27980692 \\ \mathrm{H} & 0.51912244 & -2.30590597 & 6.76398635 \\ \mathrm{H} & -3.92427441 & -2.26781080 & 5.13881988 \\ \mathrm{H} & -2.28344741 & -2.33539604 & 2.53397059 \\ \mathrm{H} & -3.12769365 & -3.70039061 & 3.28811617 \\ \mathrm{H} & -4.05434675 & -2.31945756 & 2.65967790 \\ \mathrm{H} & -2.71826903 & -0.07011650 & 3.62478281 \\ \mathrm{H} & -3.00584468 & 2.05686923 & 4.24520007 \\ \mathrm{H} & -4.40817684 & 2.22199344 & 5.33184245 \\ \mathrm{H} & -2.77624330 & 2.27540562 & 6.00294869\end{array}$

\section{Quartet; Basis set B2; Model B; FeHOOH}

100

$\begin{array}{llll}\mathrm{O} & -10.31953661 & 4.52459843 & -5.24698195 \\ \mathrm{C} & -11.12922549 & 5.38576403 & -4.88890839 \\ \mathrm{~N} & -12.34428272 & 5.05161593 & -4.38268745 \\ \mathrm{C} & -12.79210771 & 3.67720119 & -4.22064723 \\ \mathrm{C} & -10.81014853 & 6.86905574 & -5.00542528 \\ \mathrm{H} & -12.97577503 & 5.79524902 & -4.12144631 \\ \mathrm{H} & -13.69479741 & 3.48924516 & -4.81227376 \\ \mathrm{H} & -13.00325476 & 3.45733565 & -3.16829912 \\ \mathrm{H} & -11.99144101 & 3.02624677 & -4.57164641 \\ \mathrm{H} & -11.62332111 & 7.52253709 & -4.67552892 \\ \mathrm{H} & -10.57279476 & 7.09423397 & -6.04944086 \\ \mathrm{H} & -9.91838326 & 7.08646919 & -4.40901448 \\ \mathrm{C} & -4.19472920 & -1.34448137 & -0.92880403 \\ \mathrm{C} & -3.17035269 & -0.33795566 & -0.81956838 \\ \mathrm{~N} & -1.93035837 & -0.87474893 & -0.68728440 \\ \mathrm{C} & -2.15256390 & -2.21447187 & -0.70758863 \\ \mathrm{C} & -3.54903610 & -2.53631791 & -0.84903573 \\ \mathrm{C} & -3.38945572 & 0.99978018 & -0.86035826 \\ \mathrm{C} & -2.44924495 & 2.01500082 & -0.73457323 \\ \mathrm{~N} & -1.09917123 & 1.85260720 & -0.54941171 \\ \mathrm{C} & -0.55859445 & 3.11795313 & -0.50354104 \\ \mathrm{C} & -1.60161636 & 4.10041119 & -0.65090012 \\ \mathrm{C} & -2.77092355 & 3.41718261 & -0.79893722 \\ \mathrm{C} & 0.79037616 & 3.41370341 & -0.35705574 \\ \mathrm{C} & 1.80943175 & 2.48143551 & -0.26936838 \\ \mathrm{~N} & 1.62807977 & 1.11651741 & -0.35050897 \\ \mathrm{C} & 2.89047179 & 0.56656345 & -0.24322402 \\ \mathrm{C} & 3.87420918 & 1.60282963 & -0.10018224\end{array}$




\begin{tabular}{|c|c|c|c|}
\hline $\mathrm{C}$ & 3.20324718 & 2.79201483 & -0.11573104 \\
\hline $\mathrm{Fe}$ & -0.15740178 & 0.13105247 & -0.34761766 \\
\hline $\mathrm{S}$ & -0.48199111 & -0.05030393 & 2.09731139 \\
\hline $\mathrm{C}$ & 0.30798432 & 1.44494037 & 2.81842856 \\
\hline $\mathrm{C}$ & 3.15876619 & -0.79032623 & -0.28700810 \\
\hline $\mathrm{C}$ & 2.17677729 & -1.77900757 & -0.40450271 \\
\hline $\mathrm{N}$ & 0.80904979 & -1.57828949 & -0.48797215 \\
\hline $\mathrm{C}$ & 0.22570485 & -2.82635065 & -0.55376026 \\
\hline $\mathrm{C}$ & 1.25274467 & -3.83584781 & -0.52170410 \\
\hline $\mathrm{C}$ & 2.45184058 & -3.19190884 & -0.43407042 \\
\hline $\mathrm{C}$ & -1.14214734 & -3.11682175 & -0.63727525 \\
\hline $\mathrm{H}$ & 4.19160732 & -1.11458024 & -0.21702022 \\
\hline $\mathrm{H}$ & 1.06714624 & -4.90151076 & -0.56483625 \\
\hline $\mathrm{H}$ & 4.93907889 & 1.43278123 & -0.00557965 \\
\hline $\mathrm{H}$ & 3.60697841 & 3.79337574 & -0.03767087 \\
\hline $\mathrm{H}$ & 1.06623822 & 4.46294573 & -0.31506312 \\
\hline $\mathrm{H}$ & -1.44177516 & 5.17107503 & -0.65263013 \\
\hline $\mathrm{H}$ & -3.76709558 & 3.81028494 & -0.95466466 \\
\hline $\mathrm{H}$ & -4.41389056 & 1.33059614 & -1.00879424 \\
\hline $\mathrm{H}$ & -5.24967404 & -1.14001216 & -1.05349705 \\
\hline $\mathrm{H}$ & -3.95102170 & -3.54017463 & -0.88750448 \\
\hline $\mathrm{H}$ & -1.39726663 & -4.17245961 & -0.66637965 \\
\hline $\mathrm{H}$ & 3.44281346 & -3.62565752 & -0.39111117 \\
\hline $\mathrm{H}$ & 0.14493167 & 1.43443804 & 3.90092126 \\
\hline $\mathrm{H}$ & 1.38444419 & 1.45116468 & 2.62738026 \\
\hline $\mathrm{H}$ & -0.13159058 & 2.35795795 & 2.40721371 \\
\hline $\mathrm{O}$ & 0.04291515 & 0.41517718 & -3.00648581 \\
\hline $\mathrm{C}$ & -2.38341240 & -0.84169873 & -4.31933393 \\
\hline $\mathrm{C}$ & -3.00647510 & 0.52302821 & -4.16076457 \\
\hline $\mathrm{O}$ & -2.35092908 & 1.54693054 & -3.97206030 \\
\hline $\mathrm{O}$ & -4.32455591 & 0.48995068 & -4.27451587 \\
\hline $\mathrm{H}$ & -2.00024484 & -0.93083813 & -5.34365570 \\
\hline $\mathrm{H}$ & -3.12408371 & -1.62733152 & -4.15909153 \\
\hline $\mathrm{H}$ & -1.53693532 & -0.97120581 & -3.64677801 \\
\hline $\mathrm{N}$ & -5.80821189 & 2.73880358 & -4.44089131 \\
\hline $\mathrm{C}$ & -5.39586436 & 3.91893086 & -5.01813797 \\
\hline $\mathrm{C}$ & -6.48255982 & 4.67503654 & -5.38976973 \\
\hline $\mathrm{N}$ & -7.58470317 & 3.92857386 & -5.01922770 \\
\hline $\mathrm{C}$ & -7.12958751 & 2.77730859 & -4.46174024 \\
\hline $\mathrm{C}$ & -6.55214339 & 6.01558459 & -6.04924769 \\
\hline $\mathrm{H}$ & -7.01906170 & 6.77307795 & -5.40548763 \\
\hline $\mathrm{H}$ & -5.54158953 & 6.35891050 & -6.28711729 \\
\hline $\mathrm{H}$ & -7.12333323 & 5.98278379 & -6.98577407 \\
\hline $\mathrm{H}$ & -8.56596486 & 4.19263604 & -5.14929376 \\
\hline $\mathrm{H}$ & -7.78892313 & 2.00255652 & -4.09623078 \\
\hline
\end{tabular}




$\begin{array}{lrrr}\mathrm{H} & -4.75853770 & 1.40830036 & -4.24685955 \\ \mathrm{H} & -4.34482482 & 4.14544385 & -5.13471893 \\ \mathrm{O} & 1.26438924 & 1.11160220 & -3.37039538 \\ \mathrm{H} & 1.62113117 & 1.26635829 & -2.47493414 \\ \mathrm{H} & -0.65634669 & 1.02809144 & -3.34929018 \\ \mathrm{~N} & -0.49109802 & -2.07700138 & 4.90159641 \\ \mathrm{C} & 0.73767631 & -2.60948132 & 5.45578406 \\ \mathrm{C} & -1.71807627 & -2.53245360 & 5.28732837 \\ \mathrm{C} & -2.91289414 & -1.91977488 & 4.51700930 \\ \mathrm{~N} & -2.91186882 & -0.46311998 & 4.45387186 \\ \mathrm{C} & -3.15973851 & 0.31099860 & 5.55240795 \\ \mathrm{C} & -2.99937785 & 1.81252859 & 5.33488614 \\ \mathrm{C} & -3.06025303 & -2.54542600 & 3.12634940 \\ \mathrm{O} & -1.89068338 & -3.41011133 & 6.12538381 \\ \mathrm{O} & -3.50408629 & -0.15026292 & 6.63373205 \\ \mathrm{H} & -0.42806428 & -1.43732611 & 4.11520328 \\ \mathrm{H} & 1.31471939 & -3.15928741 & 4.70030519 \\ \mathrm{H} & 1.36923774 & -1.80874692 & 5.86010784 \\ \mathrm{H} & 0.46533734 & -3.29345391 & 6.26134481 \\ \mathrm{H} & -3.77327161 & -2.20477123 & 5.12907939 \\ \mathrm{H} & -2.20998557 & -2.31337462 & 2.47884751 \\ \mathrm{H} & -3.12853501 & -3.63375199 & 3.22598948 \\ \mathrm{H} & -3.97225114 & -2.18280531 & 2.63982630 \\ \mathrm{H} & -2.50169602 & -0.03859627 & 3.62667960 \\ \mathrm{H} & -2.79151250 & 2.08858531 & 4.29626143 \\ \mathrm{H} & -3.91436007 & 2.31423316 & 5.66416284 \\ \mathrm{H} & -2.18394026 & 2.17664743 & 5.96939182\end{array}$

\section{Quartet; Basis set B2; Model B; Transition State 1}

100

$\begin{array}{llll}\mathrm{O} & -10.19272405 & 4.54270315 & -5.12365782 \\ \mathrm{C} & -10.96638357 & 5.41668609 & -4.70398977 \\ \mathrm{~N} & -12.15663522 & 5.10542973 & -4.14383526 \\ \mathrm{C} & -12.64915587 & 3.74277695 & -3.99695713 \\ \mathrm{C} & -10.61721733 & 6.88985948 & -4.76778141 \\ \mathrm{H} & -12.75281569 & 5.86182689 & -3.83925083 \\ \mathrm{H} & -13.58793032 & 3.60989597 & -4.54471369 \\ \mathrm{H} & -12.81397856 & 3.50312033 & -2.94121047 \\ \mathrm{H} & -11.89613581 & 3.07060091 & -4.40757144 \\ \mathrm{H} & -11.42008993 & 7.54649574 & -4.42135515 \\ \mathrm{H} & -10.36611796 & 7.15151809 & -5.80012729\end{array}$




\begin{tabular}{|c|c|c|c|}
\hline $\mathrm{H}$ & -9.72885091 & 7.07254251 & -4.15364202 \\
\hline C & -4.22305578 & -1.41189043 & -1.02783773 \\
\hline $\mathrm{C}$ & -3.19216041 & -0.40162395 & -0.89375823 \\
\hline $\mathrm{N}$ & -1.97728474 & -0.97115255 & -0.7408340 \\
\hline $\mathrm{C}$ & -2.18196157 & -2.30821669 & -0.75879124 \\
\hline $\mathrm{C}$ & -3.58659266 & -2.61357446 & -0.9367532 \\
\hline $\mathrm{C}$ & -3.37976812 & 0.96206764 & -0.8981861 \\
\hline $\mathrm{C}$ & -2.44924065 & 1.97806925 & -0.7167519 \\
\hline $\mathrm{N}$ & -1.10058652 & 1.83138850 & -0.54614121 \\
\hline $\mathrm{C}$ & -0.56716334 & 3.08890007 & -0.4473235 \\
\hline $\mathrm{C}$ & -1.62396120 & 4.06982990 & -0.5352324 \\
\hline C & -2.78733514 & 3.38209129 & -0.70676202 \\
\hline C & 0.78028122 & 3.40415372 & -0.29619551 \\
\hline C & 1.84094837 & 2.50561368 & -0.2422372 \\
\hline $\mathrm{N}$ & 1.69108639 & 1.15188310 & -0.3603083 \\
\hline $\mathrm{C}$ & 2.93149011 & 0.60253131 & -0.18531242 \\
\hline $\mathrm{C}$ & 3.91030936 & 1.64803205 & 0.01234260 \\
\hline $\mathrm{C}$ & 3.23224681 & 2.83258581 & -0.02344227 \\
\hline $\mathrm{Fe}$ & -0.14864687 & 0.10467634 & -0.52100323 \\
\hline S & -0.52106988 & -0.01643218 & 1.98429773 \\
\hline $\mathrm{C}$ & 0.28015896 & 1.44868559 & 2.75293877 \\
\hline $\mathrm{C}$ & 3.16990307 & -0.76683824 & -0.15636468 \\
\hline $\mathrm{C}$ & 2.17348991 & -1.76577949 & -0.27161942 \\
\hline $\mathrm{N}$ & 0.80572209 & -1.60482569 & -0.4321442 \\
\hline $\mathrm{C}$ & 0.22479173 & -2.86061072 & -0.4743477 \\
\hline $\mathrm{C}$ & 1.27279171 & -3.84721452 & -0.34102040 \\
\hline $\mathrm{C}$ & 2.45770975 & -3.18161774 & -0.2187840 \\
\hline $\mathrm{C}$ & -1.14292558 & -3.19975706 & -0.6185555 \\
\hline $\mathrm{H}$ & 4.18922677 & -1.11221984 & -0.01687082 \\
\hline $\mathrm{H}$ & 1.10977095 & -4.91802901 & -0.34064648 \\
\hline $\mathrm{H}$ & 4.96968700 & 1.48775193 & 0.17080237 \\
\hline $\mathrm{H}$ & 3.62641797 & 3.83390792 & 0.09985738 \\
\hline $\mathrm{H}$ & 1.02267256 & 4.46005521 & -0.21056270 \\
\hline $\mathrm{H}$ & -1.47555525 & 5.14085062 & -0.47372939 \\
\hline $\mathrm{H}$ & -3.79130656 & 3.77400740 & -0.8140184 \\
\hline $\mathrm{H}$ & -4.40550492 & 1.30326367 & -1.0271656 \\
\hline $\mathrm{H}$ & -5.28034157 & -1.21607619 & -1.1568599 \\
\hline $\mathrm{H}$ & -4.01142253 & -3.60885722 & -0.9766675 \\
\hline $\mathrm{H}$ & -1.36500429 & -4.26302211 & -0.62452307 \\
\hline $\mathrm{H}$ & 3.44788252 & -3.60423266 & -0.1004466 \\
\hline $\mathrm{H}$ & 0.16019318 & 1.38754743 & 3.8403198 \\
\hline $\mathrm{H}$ & 1.34964976 & 1.47795431 & 2.5251727 \\
\hline $\mathrm{H}$ & -0.17657005 & 2.38081119 & 2.40641602 \\
\hline $\mathrm{O}$ & -0.14165654 & 0.12588786 & -2.5258665 \\
\hline C & -2.38284869 & -0.87641511 & -4.3913141 \\
\hline
\end{tabular}




\begin{tabular}{|c|c|c|c|}
\hline $\mathrm{C}$ & -3.05134685 & 0.45126683 & -4.25133320 \\
\hline $\mathrm{O}$ & -2.33258159 & 1.49988661 & -3.90022171 \\
\hline $\mathrm{O}$ & -4.25170551 & 0.58100134 & -4.52574611 \\
\hline $\mathrm{H}$ & -2.03547091 & -0.96970393 & -5.42925913 \\
\hline $\mathrm{H}$ & -3.10700470 & -1.67028136 & -4.2014445 \\
\hline $\mathrm{H}$ & -1.51330342 & -0.95537654 & -3.733384 \\
\hline $\mathrm{N}$ & -5.76589314 & 2.64754888 & -4.7599590 \\
\hline $\mathrm{C}$ & -5.33995983 & 3.82624621 & -5.3223449 \\
\hline $\mathrm{C}$ & -6.44285309 & 4.60283129 & -5.5563847 \\
\hline $\mathrm{N}$ & -7.52910182 & 3.86345905 & $-5.117748 \mathrm{~s}$ \\
\hline $\mathrm{C}$ & -7.08939421 & 2.68473229 & -4.6498043 \\
\hline C & -6.52145680 & 5.97215248 & -6.13963208 \\
\hline $\mathrm{H}$ & -6.93855175 & 6.69473836 & -5.4278129 \\
\hline $\mathrm{H}$ & -5.51968917 & 6.31198533 & -6.4127911 \\
\hline $\mathrm{H}$ & -7.14451076 & 5.99487643 & -7.0414797 \\
\hline $\mathrm{H}$ & -8.51897568 & 4.16839031 & -5.1483951 \\
\hline $\mathrm{H}$ & -7.71221018 & 1.89771659 & -4.2542383 \\
\hline $\mathrm{H}$ & -5.10841910 & 1.81924118 & -4.5481773 \\
\hline $\mathrm{H}$ & -4.29340405 & 4.01630846 & -5.5041339 \\
\hline $\mathrm{O}$ & 0.45987253 & 1.29284539 & -3.17524280 \\
\hline $\mathrm{H}$ & 1.12193917 & 1.55695644 & -2.50883741 \\
\hline $\mathrm{H}$ & -1.44289271 & 1.24172463 & -3.5352470 \\
\hline $\mathrm{N}$ & -0.44765439 & -1.93911342 & 4.8599727 \\
\hline $\mathrm{C}$ & 0.80340778 & -2.37994509 & 5.4431879 \\
\hline $\mathrm{C}$ & -1.64596278 & -2.46761376 & 5.2407683 \\
\hline $\mathrm{C}$ & -2.86479801 & -1.96867259 & 4.4263630 \\
\hline $\mathrm{N}$ & -2.96263057 & -0.51825647 & 4.3113244 \\
\hline $\mathrm{C}$ & -3.28575950 & 0.27385997 & 5.37490186 \\
\hline $\mathrm{C}$ & -3.16332917 & 1.77372196 & 5.11924408 \\
\hline $\mathrm{C}$ & -2.93608579 & -2.64698672 & 3.0543234 \\
\hline $\mathrm{O}$ & -1.77536221 & -3.31759494 & 6.1163680 \\
\hline $\mathrm{O}$ & -3.67103145 & -0.16366520 & 6.45413669 \\
\hline $\mathrm{H}$ & -0.40877799 & -1.36171942 & 4.0204368 \\
\hline $\mathrm{H}$ & 1.40176742 & -2.95826042 & 4.72608282 \\
\hline $\mathrm{H}$ & 1.40163710 & -1.52525564 & 5.78210559 \\
\hline $\mathrm{H}$ & 0.56409289 & -3.01501094 & 6.29794546 \\
\hline $\mathrm{H}$ & -3.71634285 & -2.29159905 & 5.0317677 \\
\hline $\mathrm{H}$ & -2.09405255 & -2.36680931 & 2.4159354 \\
\hline $\mathrm{H}$ & -2.92353522 & -3.73428086 & 3.1864501 \\
\hline $\mathrm{H}$ & -3.86245915 & -2.36869625 & 2.5400902 \\
\hline $\mathrm{H}$ & -2.49474461 & -0.10027373 & 3.5070732 \\
\hline $\mathrm{H}$ & -2.91314795 & 2.02454467 & 4.0838917 \\
\hline $\mathrm{H}$ & -4.10793075 & 2.25509263 & 5.3912929 \\
\hline $\mathrm{H}$ & -2.38948118 & 2.18119354 & 5.7791037 \\
\hline
\end{tabular}




\section{Quartet; Basis set B2; Model B; Compound 0}

100

$\begin{array}{lccc}\mathrm{O} & -10.18260296 & 4.58749630 & -5.06729991 \\ \mathrm{C} & -10.90688761 & 5.46060963 & -4.55795904 \\ \mathrm{~N} & -12.08280170 & 5.14498115 & -3.97463885 \\ \mathrm{C} & -12.58849218 & 3.77806113 & -3.88851598 \\ \mathrm{C} & -10.49855617 & 6.92201688 & -4.53815806 \\ \mathrm{H} & -12.62512730 & 5.88551837 & -3.55151453 \\ \mathrm{H} & -13.67394895 & 3.80947133 & -3.77123213 \\ \mathrm{H} & -12.14937240 & 3.24486789 & -3.03733063 \\ \mathrm{H} & -12.33791121 & 3.24193973 & -4.80590088 \\ \mathrm{H} & -11.26933985 & 7.58399898 & -4.13230903 \\ \mathrm{H} & -10.26033108 & 7.24053084 & -5.55765775 \\ \mathrm{H} & -9.59243066 & 7.03064061 & -3.93207811 \\ \mathrm{C} & -4.08989792 & -1.52807626 & -1.00913790 \\ \mathrm{C} & -3.11178688 & -0.46985357 & -0.86378388 \\ \mathrm{~N} & -1.86497466 & -0.99278075 & -0.69022866 \\ \mathrm{C} & -2.01304888 & -2.34341046 & -0.72472958 \\ \mathrm{C} & -3.40270647 & -2.70163493 & -0.91703428 \\ \mathrm{C} & -3.38693665 & 0.88503809 & -0.89078083 \\ \mathrm{C} & -2.45820694 & 1.91780829 & -0.72217193 \\ \mathrm{~N} & -1.09716995 & 1.78433166 & -0.56620699 \\ \mathrm{C} & -0.58023713 & 3.05179948 & -0.41952412 \\ \mathrm{C} & -1.65000827 & 4.01811339 & -0.48905559 \\ \mathrm{C} & -2.80793303 & 3.31656139 & -0.67796487 \\ \mathrm{C} & 0.77198758 & 3.37553253 & -0.25874847 \\ \mathrm{C} & 1.83122568 & 2.47216311 & -0.19062819 \\ \mathrm{~N} & 1.70683369 & 1.10990048 & -0.24896853 \\ \mathrm{C} & 2.97397804 & 0.59644944 & -0.13575842 \\ \mathrm{C} & 3.93167113 & 1.67219361 & -0.00303346 \\ \mathrm{C} & 3.22264587 & 2.83789591 & -0.03743983 \\ \mathrm{Fe} & -0.07283906 & 0.06568836 & -0.48184836 \\ \mathrm{~S} & -0.52199719 & -0.02454710 & 2.04933271 \\ \mathrm{C} & 0.40744524 & 1.35576867 & 2.83271999 \\ \mathrm{C} & 3.28557391 & -0.76357279 & -0.13532702 \\ \mathrm{C} & 2.31972126 & -1.77894140 & -0.26919239 \\ \mathrm{C} & 0.95129936 & -1.62708959 & -0.43237154 \\ \mathrm{~N} & 0.39839444 & -2.89129462 & -0.47675425 \\ \mathrm{C} & 1.45372188 & -3.87076183 & -0.35441391 \\ \mathrm{C} & 2.62992103 & -3.18914598 & -0.22976715 \\ \mathrm{H} & -0.95966753 & -3.23161225 & -0.60809642 \\ \mathrm{H} & -3210814 & -1.06365738 & -0.02315483 \\ \mathrm{H} & -4.94312454 & -0.35814725\end{array}$




\begin{tabular}{|c|c|c|c|}
\hline $\mathrm{H}$ & 5.00149471 & 1.53977286 & 0.10429120 \\
\hline $\mathrm{H}$ & 3.59386911 & 3.85312179 & 0.03508231 \\
\hline $\mathrm{H}$ & 1.01413694 & 4.43182088 & -0.18031950 \\
\hline $\mathrm{H}$ & -1.52023594 & 5.08981607 & -0.39727705 \\
\hline $\mathrm{H}$ & -3.81653869 & 3.70146304 & -0.77046067 \\
\hline $\mathrm{H}$ & -4.42367422 & 1.18485538 & -1.01982184 \\
\hline $\mathrm{H}$ & -5.15272410 & -1.37696291 & -1.15457160 \\
\hline $\mathrm{H}$ & -3.78252595 & -3.71471380 & -0.96974288 \\
\hline $\mathrm{H}$ & -1.18721900 & -4.29376304 & -0.62556019 \\
\hline $\mathrm{H}$ & 3.62738804 & -3.59499989 & -0.11181142 \\
\hline $\mathrm{H}$ & 0.28395534 & 1.30743679 & 3.92084277 \\
\hline $\mathrm{H}$ & 1.47553345 & 1.29140685 & 2.60358784 \\
\hline $\mathrm{H}$ & 0.03553244 & 2.32635978 & 2.48815949 \\
\hline $\mathrm{O}$ & 0.04969782 & 0.06744674 & -2.57939836 \\
\hline $\mathrm{C}$ & -2.30419305 & -0.82338221 & -4.56517304 \\
\hline C & -3.14216890 & 0.41056072 & -4.52017654 \\
\hline $\mathrm{O}$ & -2.56742061 & 1.55275306 & -4.20744487 \\
\hline $\mathrm{O}$ & -4.35055984 & 0.38638798 & -4.81203448 \\
\hline $\mathrm{H}$ & -1.69809662 & -0.79791669 & -5.48126671 \\
\hline $\mathrm{H}$ & -2.94432986 & -1.70627022 & -4.59199557 \\
\hline $\mathrm{H}$ & -1.59602510 & -0.85981640 & -3.7263476 \\
\hline $\mathrm{N}$ & -5.78933314 & 2.53249661 & -4.99495506 \\
\hline C & -5.34051235 & 3.73563845 & -5.4899642 \\
\hline $\mathrm{C}$ & -6.42613792 & 4.55897068 & -5.6348025 \\
\hline $\mathrm{N}$ & -7.52183938 & 3.82121378 & -5.2083330 \\
\hline C & -7.10636313 & 2.60006239 & -4.83728916 \\
\hline $\mathrm{C}$ & -6.52872240 & 5.95464483 & -6.1442474 \\
\hline $\mathrm{H}$ & -6.89408481 & 6.64332271 & -5.3725929 \\
\hline $\mathrm{H}$ & -5.54414379 & 6.30568488 & -6.4648687 \\
\hline $\mathrm{H}$ & -7.20746498 & 6.02207304 & -7.0027288 \\
\hline $\mathrm{H}$ & -8.50234615 & 4.15033840 & -5.1911372 \\
\hline $\mathrm{H}$ & -7.74044515 & 1.80544864 & -4.4746126 \\
\hline $\mathrm{H}$ & -5.16748628 & 1.66334255 & -4.84307453 \\
\hline $\mathrm{H}$ & -4.29345677 & 3.90424855 & -5.6910476 \\
\hline $\mathrm{O}$ & -0.13207092 & 1.31748466 & -3.3388815 \\
\hline $\mathrm{H}$ & 0.04062197 & 2.00276990 & -2.6680137 \\
\hline $\mathrm{H}$ & -1.60012476 & 1.43631453 & -3.86221928 \\
\hline $\mathrm{N}$ & -0.58382494 & -1.92447786 & 4.9403602 \\
\hline $\mathrm{C}$ & 0.62323118 & -2.41537896 & 5.5829875 \\
\hline C & -1.80837677 & -2.40576194 & 5.2805526 \\
\hline $\mathrm{C}$ & -2.99256953 & -1.88121305 & 4.4326861 \\
\hline $\mathrm{N}$ & -3.04767874 & -0.42715163 & 4.3047577 \\
\hline C & -3.37267670 & 0.39792962 & 5.3418932 \\
\hline $\mathrm{C}$ & -3.20492069 & 1.88750948 & 5.06261322 \\
\hline $\mathrm{C}$ & -3.05371147 & -2.56636960 & 3.0632392 \\
\hline
\end{tabular}




$\begin{array}{lrrr}\mathrm{O} & -1.97758259 & -3.25139254 & 6.15973149 \\ \mathrm{O} & -3.80900608 & -0.00501354 & 6.41994262 \\ \mathrm{H} & -0.48998776 & -1.36088245 & 4.09402047 \\ \mathrm{H} & 0.75714174 & -3.49289014 & 5.42051304 \\ \mathrm{H} & 1.48072135 & -1.87986666 & 5.16690316 \\ \mathrm{H} & 0.57876139 & -2.24333664 & 6.66373428 \\ \mathrm{H} & -3.86974308 & -2.17642078 & 5.01608358 \\ \mathrm{H} & -2.19549126 & -2.31032234 & 2.43614718 \\ \mathrm{H} & -3.06951104 & -3.65317949 & 3.20202540 \\ \mathrm{H} & -3.96693233 & -2.27002576 & 2.53497350 \\ \mathrm{H} & -2.53287992 & -0.03224183 & 3.51567165 \\ \mathrm{H} & -2.77611395 & 2.10228226 & 4.07855936 \\ \mathrm{H} & -4.18404670 & 2.37312598 & 5.14119279 \\ \mathrm{H} & -2.56244445 & 2.31864682 & 5.83766584\end{array}$

\title{
Quartet; Basis set B2; Model B; Transition State 2
}

\author{
100
}

$\begin{array}{llll}\mathrm{O} & -10.22357143 & 4.57202121 & -5.15198157 \\ \mathrm{C} & -10.98455532 & 5.40919386 & -4.64600856 \\ \mathrm{~N} & -12.16774176 & 5.05036596 & -4.08955489 \\ \mathrm{C} & -12.65879003 & 3.67864142 & -4.03543473 \\ \mathrm{C} & -10.62058153 & 6.88694072 & -4.62225936 \\ \mathrm{H} & -12.75064982 & 5.77851655 & -3.69894592 \\ \mathrm{H} & -13.61780725 & 3.59395170 & -4.55764615 \\ \mathrm{H} & -12.78435362 & 3.35488712 & -2.99648472 \\ \mathrm{H} & -11.92622603 & 3.03780867 & -4.52652335 \\ \mathrm{H} & -11.39329113 & 7.52146128 & -4.17713030 \\ \mathrm{H} & -10.43496264 & 7.21961005 & -5.64816664 \\ \mathrm{H} & -9.69225527 & 7.01359757 & -4.05563903 \\ \mathrm{C} & -4.11146797 & -1.47572392 & -1.01313795 \\ \mathrm{C} & -3.12100339 & -0.43271135 & -0.86134766 \\ \mathrm{~N} & -1.87789334 & -0.97219062 & -0.68727035 \\ \mathrm{C} & -2.05057157 & -2.32354712 & -0.71970999 \\ \mathrm{C} & -3.44282759 & -2.65895003 & -0.91745475 \\ \mathrm{C} & -3.39696273 & 0.92146356 & -0.86707579 \\ \mathrm{C} & -2.46339243 & 1.94670893 & -0.68147164 \\ \mathrm{~N} & -1.09485709 & 1.79686562 & -0.56872588 \\ \mathrm{C} & -0.56459178 & 3.06049189 & -0.39169062 \\ \mathrm{C} & -1.62798020 & 4.03253866 & -0.40821702 \\ \mathrm{C} & -2.79688958 & 3.34492073 & -0.58956766 \\ \mathrm{C} & 0.79790527 & 3.35468978 & -0.27897140\end{array}$




\begin{tabular}{|c|c|c|c|}
\hline $\mathrm{C}$ & 1.83580415 & 2.42769030 & -0.24116067 \\
\hline $\mathrm{N}$ & 1.68835809 & 1.06437251 & -0.27377003 \\
\hline $\mathrm{C}$ & 2.95435096 & 0.53733118 & -0.18435126 \\
\hline $\mathrm{C}$ & 3.92820337 & 1.60078124 & -0.09662310 \\
\hline $\mathrm{C}$ & 3.23467389 & 2.77465880 & -0.13394842 \\
\hline $\mathrm{Fe}$ & -0.09191542 & 0.04794964 & -0.44727420 \\
\hline S & -0.50268738 & -0.03768966 & 2.05302793 \\
\hline $\mathrm{C}$ & 0.47904743 & 1.31190412 & 2.82513435 \\
\hline $\mathrm{C}$ & 3.25630599 & -0.82286643 & -0.16617605 \\
\hline $\mathrm{C}$ & 2.27975129 & -1.82967773 & -0.28154212 \\
\hline $\mathrm{N}$ & 0.91301392 & -1.65985006 & -0.4487222 \\
\hline $\mathrm{C}$ & 0.34286011 & -2.91838531 & -0.4691703 \\
\hline $\mathrm{C}$ & 1.38442017 & -3.90984766 & -0.3346596 \\
\hline $\mathrm{C}$ & 2.57030497 & -3.24277554 & -0.2225799 \\
\hline $\mathrm{C}$ & -1.02104076 & -3.23621773 & -0.5931773 \\
\hline $\mathrm{H}$ & 4.29145289 & -1.12959502 & -0.0608984 \\
\hline $\mathrm{H}$ & 1.21619313 & -4.97985964 & -0.32340317 \\
\hline $\mathrm{H}$ & 4.99838284 & 1.45337662 & -0.01776842 \\
\hline $\mathrm{H}$ & 3.61971135 & 3.78623418 & -0.0912579 \\
\hline $\mathrm{H}$ & 1.06706169 & 4.40445010 & -0.2047850 \\
\hline $\mathrm{H}$ & -1.48957242 & 5.10085227 & -0.2947360 \\
\hline $\mathrm{H}$ & -3.80237431 & 3.74246839 & -0.6541933 \\
\hline $\mathrm{H}$ & -4.43372519 & 1.22369471 & -0.9847478 \\
\hline $\mathrm{H}$ & -5.17054514 & -1.30586045 & -1.1634295 \\
\hline $\mathrm{H}$ & -3.83596976 & -3.66667809 & -0.9720047 \\
\hline $\mathrm{H}$ & -1.27203584 & -4.29281369 & -0.6014565 \\
\hline $\mathrm{H}$ & 3.56244618 & -3.66070745 & -0.1024194 \\
\hline $\mathrm{H}$ & 0.36212235 & 1.26484889 & 3.91363657 \\
\hline $\mathrm{H}$ & 1.54160545 & 1.21088992 & 2.58585825 \\
\hline $\mathrm{H}$ & 0.13519859 & 2.29377576 & 2.48486485 \\
\hline $\mathrm{O}$ & -0.00228154 & 0.12307287 & -2.6595704 \\
\hline $\mathrm{C}$ & -2.36088686 & -0.79001187 & -4.5561759 \\
\hline $\mathrm{C}$ & -3.08183996 & 0.51565553 & -4.4991837 \\
\hline $\mathrm{O}$ & -2.47306333 & 1.59380437 & -4.2369832 \\
\hline $\mathrm{O}$ & -4.34033029 & 0.49227901 & -4.7695358 \\
\hline $\mathrm{H}$ & -1.93888833 & -0.89919258 & -5.5649543 \\
\hline $\mathrm{H}$ & -3.05343583 & -1.61674126 & -4.3864745 \\
\hline $\mathrm{H}$ & -1.53016557 & -0.80620446 & -3.8411601 \\
\hline $\mathrm{N}$ & -5.73330339 & 2.60348122 & -4.9143095 \\
\hline C & -5.32219560 & 3.82752759 & -5.3946029 \\
\hline $\mathrm{C}$ & -6.41389018 & 4.63981745 & -5.5876478 \\
\hline $\mathrm{N}$ & -7.50589534 & 3.88113505 & -5.2051387 \\
\hline $\mathrm{C}$ & -7.05277057 & 2.66605482 & -4.8152596 \\
\hline $\mathrm{C}$ & -6.50267732 & 6.03911543 & -6.1026457 \\
\hline $\mathrm{H}$ & -6.91983542 & 6.73085776 & -5.3594766 \\
\hline
\end{tabular}




$\begin{array}{lrrr}\mathrm{H} & -5.50272760 & 6.39587961 & -6.36580812 \\ \mathrm{H} & -7.12796013 & 6.10019258 & -7.00237507 \\ \mathrm{H} & -8.48850180 & 4.18023018 & -5.22560457 \\ \mathrm{H} & -7.69834030 & 1.86832286 & -4.47662526 \\ \mathrm{H} & -4.87632188 & 1.44699854 & -4.76790825 \\ \mathrm{H} & -4.27629438 & 4.04104942 & -5.56471428 \\ \mathrm{O} & -0.22752534 & 1.46533301 & -3.26029762 \\ \mathrm{H} & -0.35504169 & 2.01380996 & -2.45524001 \\ \mathrm{H} & -1.30685186 & 1.47074131 & -3.78472589 \\ \mathrm{~N} & -0.54815197 & -1.93883194 & 4.94356266 \\ \mathrm{C} & 0.68636532 & -2.41367298 & 5.54311312 \\ \mathrm{C} & -1.77234274 & -2.41169586 & 5.30816201 \\ \mathrm{C} & -2.96012526 & -1.87026771 & 4.47417456 \\ \mathrm{~N} & -2.99648066 & -0.41565454 & 4.34785638 \\ \mathrm{C} & -3.29824186 & 0.41175248 & 5.39221930 \\ \mathrm{C} & -3.10872371 & 1.89944577 & 5.11860903 \\ \mathrm{C} & -3.04682716 & -2.55361320 & 3.10535673 \\ \mathrm{O} & -1.94983238 & -3.25607053 & 6.18517101 \\ \mathrm{O} & -3.72045501 & 0.00864108 & 6.47552315 \\ \mathrm{H} & -0.48035166 & -1.36168731 & 4.10547051 \\ \mathrm{H} & 1.26414858 & -3.02347742 & 4.83561232 \\ \mathrm{H} & 1.30822741 & -1.57081544 & 5.86791839 \\ \mathrm{H} & 0.42954921 & -3.02589541 & 6.40981034 \\ \mathrm{H} & -3.83304186 & -2.15276623 & 5.06964878 \\ \mathrm{H} & -2.19271313 & -2.30818168 & 2.46824739 \\ \mathrm{H} & -3.0741155 & -3.64023795 & 3.24290069 \\ \mathrm{H} & -3.96296802 & -2.24575942 & 2.58895078 \\ \mathrm{H} & -2.50083928 & -0.02212400 & 3.54793144 \\ \mathrm{H} & -2.78644073 & 2.11975601 & 4.09586847 \\ \mathrm{H} & -4.05260091 & 2.41712723 & 5.31917938 \\ \mathrm{H} & -2.36420701 & 2.29265093 & 5.81970407\end{array}$

Quartet; Basis set B2; Model B; protonated Compound 0

103

$\begin{array}{llll}\mathrm{O} & -10.24378426 & 4.60128199 & -5.12037885 \\ \mathrm{C} & -10.99523484 & 5.45666953 & -4.63179730 \\ \mathrm{~N} & -12.18379904 & 5.12139101 & -4.06886542 \\ \mathrm{C} & -12.68793194 & 3.75610837 & -3.98763715 \\ \mathrm{C} & -10.61360736 & 6.93149340 & -4.64068944 \\ \mathrm{H} & -12.75676985 & 5.86284421 & -3.68879955 \\ \mathrm{H} & -13.65408068 & 3.67364054 & -4.49695785\end{array}$




\begin{tabular}{|c|c|c|c|}
\hline $\mathrm{H}$ & -12.80411210 & 3.44834547 & -2.94258375 \\
\hline $\mathrm{H}$ & -11.96775510 & 3.10047373 & -4.47764090 \\
\hline $\mathrm{H}$ & -11.37565713 & 7.58432581 & -4.20343136 \\
\hline $\mathrm{H}$ & -10.43111140 & 7.24014918 & -5.67462890 \\
\hline $\mathrm{H}$ & -9.67958369 & 7.05766011 & -4.08357257 \\
\hline C & -4.07825728 & -1.50441653 & -1.03646962 \\
\hline $\mathrm{C}$ & -3.09369324 & -0.45792199 & -0.88038775 \\
\hline $\mathrm{N}$ & -1.84701203 & -0.98883329 & -0.70117289 \\
\hline $\mathrm{C}$ & -2.01554667 & -2.34166555 & -0.73809736 \\
\hline $\mathrm{C}$ & -3.40431546 & -2.68399278 & -0.94045000 \\
\hline $\mathrm{C}$ & -3.38173738 & 0.89188507 & -0.88025784 \\
\hline $\mathrm{C}$ & -2.46246261 & 1.92703224 & -0.69591609 \\
\hline $\mathrm{N}$ & -1.08798638 & 1.78730599 & -0.59889059 \\
\hline $\mathrm{C}$ & -0.57153832 & 3.05766343 & -0.40038295 \\
\hline $\mathrm{C}$ & -1.64381654 & 4.01627905 & -0.40096097 \\
\hline C & -2.80746044 & 3.31859061 & -0.58519460 \\
\hline C & 0.78756895 & 3.35456351 & -0.29040983 \\
\hline $\mathrm{C}$ & 1.82797084 & 2.43046476 & -0.24732926 \\
\hline $\mathrm{N}$ & 1.68791196 & 1.06594831 & -0.26816497 \\
\hline $\mathrm{C}$ & 2.95823521 & 0.54735970 & -0.19083424 \\
\hline $\mathrm{C}$ & 3.92593809 & 1.61628043 & -0.11225055 \\
\hline $\mathrm{C}$ & 3.22508426 & 2.78571462 & -0.14929119 \\
\hline $\mathrm{Fe}$ & -0.07508268 & 0.03524791 & -0.43156554 \\
\hline S & -0.50217601 & -0.03948127 & 2.04523200 \\
\hline $\mathrm{C}$ & 0.44631595 & 1.33305343 & 2.81816186 \\
\hline $\mathrm{C}$ & 3.26973019 & -0.80957210 & -0.18081181 \\
\hline $\mathrm{C}$ & 2.30424627 & -1.82539953 & -0.29217479 \\
\hline $\mathrm{N}$ & 0.93654850 & -1.66452548 & -0.45182150 \\
\hline $\mathrm{C}$ & 0.37625237 & -2.92675206 & -0.47994538 \\
\hline $\mathrm{C}$ & 1.42457618 & -3.91127158 & -0.35072592 \\
\hline C & 2.60604136 & -3.23594025 & -0.23906280 \\
\hline C & -0.98525151 & -3.24951939 & -0.60930826 \\
\hline $\mathrm{H}$ & 4.30870203 & -1.10652348 & -0.08497221 \\
\hline $\mathrm{H}$ & 1.26394986 & -4.98241776 & -0.34449878 \\
\hline $\mathrm{H}$ & 4.99747499 & 1.47547745 & -0.04188727 \\
\hline $\mathrm{H}$ & 3.60401834 & 3.79978083 & -0.11459377 \\
\hline $\mathrm{H}$ & 1.05584367 & 4.40455607 & -0.21564417 \\
\hline $\mathrm{H}$ & -1.51670591 & 5.08471085 & -0.27686744 \\
\hline $\mathrm{H}$ & -3.81673402 & 3.70738323 & -0.64082541 \\
\hline $\mathrm{H}$ & -4.42117382 & 1.18374450 & -1.00032530 \\
\hline $\mathrm{H}$ & -5.13650409 & -1.33821289 & -1.19467333 \\
\hline $\mathrm{H}$ & -3.79109865 & -3.69372627 & -1.00007505 \\
\hline $\mathrm{H}$ & -1.23163142 & -4.30712352 & -0.62488675 \\
\hline $\mathrm{H}$ & 3.60160280 & -3.64679050 & -0.12385556 \\
\hline $\mathrm{H}$ & 0.32302173 & 1.28516339 & 3.90574672 \\
\hline
\end{tabular}




\begin{tabular}{|c|c|c|c|}
\hline $\mathrm{H}$ & 1.51196711 & 1.25321336 & 2.58533945 \\
\hline $\mathrm{H}$ & 0.08226987 & 2.30564747 & 2.47302666 \\
\hline $\mathrm{O}$ & 0.11548739 & 0.12525392 & -2.68165853 \\
\hline C & -2.31823451 & -0.80469920 & -4.54898298 \\
\hline $\mathrm{C}$ & -3.05123662 & 0.49896502 & -4.50827800 \\
\hline $\mathrm{O}$ & -2.45982650 & 1.58117318 & -4.31556426 \\
\hline $\mathrm{O}$ & -4.33645778 & 0.41491454 & -4.71779239 \\
\hline $\mathrm{H}$ & -1.87788839 & -0.92056455 & -5.5488034 \\
\hline $\mathrm{H}$ & -2.99943193 & -1.64102596 & -4.3783623 \\
\hline $\mathrm{H}$ & -1.49844278 & -0.81262287 & -3.8236431 \\
\hline $\mathrm{N}$ & -5.76722158 & 2.58198690 & -4.8287519 \\
\hline C & -5.34231779 & 3.78995939 & -5.33773386 \\
\hline $\mathrm{C}$ & -6.42238761 & 4.61299174 & -5.5511884 \\
\hline $\mathrm{N}$ & -7.52510047 & 3.87851347 & -5.15132812 \\
\hline C & -7.08429794 & 2.66630608 & -4.73300522 \\
\hline $\mathrm{C}$ & -6.51160781 & 5.99273677 & -6.1109681 \\
\hline $\mathrm{H}$ & -6.93193036 & 6.70687041 & -5.3904580 \\
\hline $\mathrm{H}$ & -5.51284941 & 6.34720133 & -6.3828860 \\
\hline $\mathrm{H}$ & -7.13577073 & 6.02602195 & -7.0132077 \\
\hline $\mathrm{H}$ & -8.50311500 & 4.18530792 & $-5.186670 \subseteq$ \\
\hline $\mathrm{H}$ & -7.74253088 & 1.88606193 & -4.3772797 \\
\hline $\mathrm{H}$ & -4.84753219 & 1.33013492 & -4.7015233 \\
\hline $\mathrm{H}$ & -4.29377475 & 3.98461055 & -5.5163366 \\
\hline $\mathrm{O}$ & -0.16641315 & 1.50066006 & -3.1974351 \\
\hline $\mathrm{H}$ & -0.43273889 & 1.94681451 & -2.3516080 \\
\hline $\mathrm{H}$ & -1.05928473 & 1.44694807 & -3.7365451 \\
\hline $\mathrm{N}$ & -0.56473343 & -1.92731353 & 4.9441586 \\
\hline C & 0.66410957 & -2.39335001 & 5.5620196 \\
\hline $\mathrm{C}$ & -1.79188220 & -2.40138865 & 5.29890082 \\
\hline $\mathrm{C}$ & -2.97401799 & -1.86765291 & 4.45258500 \\
\hline $\mathrm{N}$ & -3.01742254 & -0.41421228 & 4.3170620 \\
\hline $\mathrm{C}$ & -3.33248446 & 0.41796156 & 5.35368072 \\
\hline $\mathrm{C}$ & -3.16654978 & 1.90602123 & 5.06727537 \\
\hline $\mathrm{C}$ & -3.05075816 & -2.56143598 & 3.0880097 \\
\hline $\mathrm{O}$ & -1.97507731 & -3.24301990 & 6.1769967 도 \\
\hline $\mathrm{O}$ & -3.75044774 & 0.01749355 & 6.43859972 \\
\hline $\mathrm{H}$ & -0.48998735 & -1.35044424 & 4.1073566 \\
\hline $\mathrm{H}$ & 1.24651889 & -3.01801807 & 4.87137400 \\
\hline $\mathrm{H}$ & 1.28464607 & -1.54530839 & 5.8754126 \\
\hline $\mathrm{H}$ & 0.39952336 & -2.98836252 & 6.4382327 \\
\hline $\mathrm{H}$ & -3.85069124 & -2.15037346 & 5.0426129 \\
\hline $\mathrm{H}$ & -2.19576754 & -2.31593340 & 2.4518937 \\
\hline $\mathrm{H}$ & -3.07222471 & -3.64703197 & 3.2336856 \\
\hline $\mathrm{H}$ & -3.96708351 & -2.26326490 & 2.5662009 \\
\hline $\mathrm{H}$ & -2.52945180 & -0.02151568 & $3.512947 €$ \\
\hline
\end{tabular}




$\begin{array}{llll}\mathrm{H} & -2.82751802 & 2.12173932 & 4.04883875 \\ \mathrm{H} & -4.12495050 & 2.40649759 & 5.24140261 \\ \mathrm{H} & -2.44589438 & 2.32221997 & 5.77975893\end{array}$

\section{Quartet; Basis set B2; Model B; Transition State 3}

100

$\begin{array}{lccc}\mathrm{O} & -10.27756490 & 4.59802145 & -5.02878058 \\ \mathrm{C} & -11.00949179 & 5.47783291 & -4.55432511 \\ \mathrm{~N} & -12.19698365 & 5.15706209 & -3.98645888 \\ \mathrm{C} & -12.66994848 & 3.77932533 & -3.90269730 \\ \mathrm{C} & -10.60881983 & 6.94618860 & -4.58306257 \\ \mathrm{H} & -12.77538552 & 5.89518725 & -3.61080462 \\ \mathrm{H} & -13.67226258 & 3.78194443 & -3.46941363 \\ \mathrm{H} & -12.00581460 & 3.17826959 & -3.27307426 \\ \mathrm{H} & -12.70530922 & 3.32655412 & -4.89796091 \\ \mathrm{H} & -11.36748315 & 7.61438430 & -4.16346001 \\ \mathrm{H} & -10.41174811 & 7.23768309 & -5.61929578 \\ \mathrm{H} & -9.67921704 & 7.07054362 & -4.01796537 \\ \mathrm{C} & -4.03094236 & -1.57975231 & -0.98951389 \\ \mathrm{C} & -3.05750247 & -0.52511620 & -0.83312090 \\ \mathrm{~N} & -1.79814148 & -1.04934668 & -0.66546854 \\ \mathrm{C} & -1.96064455 & -2.40500621 & -0.71792442 \\ \mathrm{C} & -3.34770178 & -2.75392051 & -0.91053808 \\ \mathrm{C} & -3.36669717 & 0.81637592 & -0.85704878 \\ \mathrm{C} & -2.45413360 & 1.86702002 & -0.68263903 \\ \mathrm{~N} & -1.10364615 & 1.73361808 & -0.49383364 \\ \mathrm{C} & -0.58348177 & 2.99987953 & -0.38132260 \\ \mathrm{C} & -1.65165581 & 3.97176427 & -0.49032987 \\ \mathrm{C} & -2.80701012 & 3.26984194 & -0.67911739 \\ \mathrm{C} & 0.77467088 & 3.31251782 & -0.21863951 \\ \mathrm{C} & 1.81782926 & 2.39153229 & -0.13530733 \\ \mathrm{~N} & 1.69347827 & 1.02320642 & -0.17625202 \\ \mathrm{C} & 2.96762637 & 0.51976815 & -0.10135390 \\ \mathrm{C} & 3.92247617 & 1.59702214 & 0.01743555 \\ \mathrm{C} & 3.20933018 & 2.75923801 & -0.00504327 \\ \mathrm{Fe} & -0.04629378 & -0.02904001 & -0.50535750 \\ \mathrm{~S} & -0.51270825 & -0.02632731 & 1.92116733 \\ \mathrm{C} & 0.39282562 & 1.33175974 & 2.74489064 \\ \mathrm{C} & 3.30162347 & -0.83470711 & -0.14552140 \\ \mathrm{C} & 2.34922065 & -1.85677847 & -0.29449182 \\ \mathrm{~N} & 0.97367939 & -1.71000974 & -0.43886637\end{array}$




\begin{tabular}{|c|c|c|c|}
\hline $\mathrm{C}$ & 0.43214815 & -2.97986461 & -0.50158358 \\
\hline C & 1.49652832 & -3.95350694 & -0.41181660 \\
\hline $\mathrm{C}$ & 2.66856145 & -3.26595853 & -0.28958659 \\
\hline $\mathrm{C}$ & -0.92795810 & -3.31269662 & -0.62416816 \\
\hline $\mathrm{H}$ & 4.34470259 & -1.11958644 & -0.05874342 \\
\hline $\mathrm{H}$ & 1.35111570 & -5.02662932 & -0.43590807 \\
\hline $\mathrm{H}$ & 4.99449342 & 1.46563335 & 0.09856895 \\
\hline $\mathrm{H}$ & 3.57600373 & 3.77670600 & 0.05483736 \\
\hline $\mathrm{H}$ & 1.03533015 & 4.36547299 & -0.16152542 \\
\hline $\mathrm{H}$ & -1.51959647 & 5.04545372 & -0.4329621 \\
\hline $\mathrm{H}$ & -3.81073161 & 3.65306657 & -0.8150520 \\
\hline $\mathrm{H}$ & -4.40546118 & 1.09140120 & -1.0139512 \\
\hline $\mathrm{H}$ & -5.09068154 & -1.41886540 & -1.1427280 \\
\hline $\mathrm{H}$ & -3.72513591 & -3.76653077 & -0.979872 \\
\hline $\mathrm{H}$ & -1.16916043 & -4.37068266 & -0.664012 \\
\hline $\mathrm{H}$ & 3.67025485 & -3.66712598 & -0.1941254 \\
\hline $\mathrm{H}$ & 0.16835858 & 1.28454948 & 3.8167802 \\
\hline $\mathrm{H}$ & 1.47193522 & 1.24102298 & 2.6005579 \\
\hline $\mathrm{H}$ & 0.05843309 & 2.30308445 & 2.36858800 \\
\hline $\mathrm{O}$ & 0.13494208 & 0.17869101 & -2.2295092 \\
\hline $\mathrm{C}$ & -2.27210190 & -0.81516945 & -4.5481270 \\
\hline $\mathrm{C}$ & -3.03853216 & 0.47400702 & -4.4422650 \\
\hline $\mathrm{O}$ & -2.48768498 & 1.56100496 & -4.2232420 \\
\hline $\mathrm{O}$ & -4.33510514 & 0.34572591 & -4.62903917 \\
\hline $\mathrm{H}$ & -1.75689896 & -0.83520653 & -5.5170715 \\
\hline $\mathrm{H}$ & -2.93750130 & -1.67851338 & -4.4884637 \\
\hline $\mathrm{H}$ & -1.50741522 & -0.86582286 & -3.7669902 \\
\hline $\mathrm{N}$ & -5.79734783 & 2.55499383 & -4.7078133 \\
\hline C & -5.36247391 & 3.75730201 & -5.2246489 \\
\hline $\mathrm{C}$ & -6.43525479 & 4.58658743 & -5.4554453 \\
\hline $\mathrm{N}$ & -7.54537457 & 3.86149474 & -5.0576544 \\
\hline $\mathrm{C}$ & -7.11380155 & 2.65017664 & -4.6241445 \\
\hline $\mathrm{C}$ & -6.52512367 & 5.96019435 & -6.0326665 \\
\hline $\mathrm{H}$ & -6.95838266 & 6.67997134 & -5.3254231 \\
\hline $\mathrm{H}$ & -5.52527153 & 6.31782484 & -6.2968902 \\
\hline $\mathrm{H}$ & -7.13815677 & 5.97972841 & -6.9430272 \\
\hline $\mathrm{H}$ & -8.52077702 & 4.17348742 & -5.0989201 \\
\hline $\mathrm{H}$ & -7.78133107 & 1.87820417 & -4.2672090 \\
\hline $\mathrm{H}$ & -4.83985372 & 1.24498372 & -4.5970431 \\
\hline $\mathrm{H}$ & -4.31099824 & 3.94353311 & -5.3955997 \\
\hline $\mathrm{O}$ & 0.06580235 & 1.48286190 & -3.3792735 \\
\hline $\mathrm{H}$ & 0.16098118 & 2.21608358 & -2.7457312 \\
\hline $\mathrm{H}$ & -0.89375811 & 1.50491822 & -3.6835101 \\
\hline $\mathrm{N}$ & -0.61829333 & -1.81925261 & 4.8785091 \\
\hline $\mathrm{C}$ & 0.57849727 & -2.30902614 & 5.5445141 \\
\hline
\end{tabular}




$\begin{array}{lrrr}\mathrm{C} & -1.85332878 & -2.28572571 & 5.21501571 \\ \mathrm{C} & -3.02297946 & -1.79111299 & 4.33112804 \\ \mathrm{~N} & -3.07475088 & -0.34294252 & 4.14912436 \\ \mathrm{C} & -3.41555350 & 0.51967063 & 5.15308730 \\ \mathrm{C} & -3.29817606 & 1.99979878 & 4.81021748 \\ \mathrm{C} & -3.07027666 & -2.53047209 & 2.99017522 \\ \mathrm{O} & -2.03523446 & -3.09737742 & 6.11969445 \\ \mathrm{O} & -3.81756447 & 0.14496337 & 6.25237462 \\ \mathrm{H} & -0.50995757 & -1.30739513 & 4.00815077 \\ \mathrm{H} & 0.74847066 & -3.37487279 & 5.34309486 \\ \mathrm{H} & 1.43806401 & -1.73474634 & 5.18864324 \\ \mathrm{H} & 0.48235028 & -2.18192521 & 6.62683795 \\ \mathrm{H} & -3.90790494 & -2.06267428 & 4.91451024 \\ \mathrm{H} & -2.20319198 & -2.30911771 & 2.36149319 \\ \mathrm{H} & -3.09373454 & -3.61026667 & 3.17264724 \\ \mathrm{H} & -3.97478736 & -2.25257904 & 2.43823632 \\ \mathrm{H} & -2.62324683 & 0.03213781 & 3.31811891 \\ \mathrm{H} & -2.93424103 & 2.18880031 & 3.79503831 \\ \mathrm{H} & -4.28151200 & 2.46693490 & 4.92995389 \\ \mathrm{H} & -2.62158803 & 2.47423939 & 5.52909564\end{array}$

\section{Quartet; Basis set B2; Model B; Compound I}

100

$\begin{array}{llll}\mathrm{O} & -10.28411055 & 4.68598856 & -4.94562404 \\ \mathrm{C} & -10.98141430 & 5.56618201 & -4.42508445 \\ \mathrm{~N} & -12.16167722 & 5.27209136 & -3.81923034 \\ \mathrm{C} & -12.71129092 & 3.92582188 & -3.72650970 \\ \mathrm{C} & -10.54324806 & 7.02641270 & -4.44821099 \\ \mathrm{H} & -12.69233269 & 6.03227130 & -3.41570968 \\ \mathrm{H} & -13.69995746 & 3.88183260 & -4.19612967 \\ \mathrm{H} & -12.79572547 & 3.61248833 & -2.67987659 \\ \mathrm{H} & -12.03575196 & 3.24908246 & -4.25045127 \\ \mathrm{H} & -11.25994621 & 7.70724938 & -3.97783537 \\ \mathrm{H} & -10.39351807 & 7.32936938 & -5.48899404 \\ \mathrm{H} & -9.58155806 & 7.11388589 & -3.93252996 \\ \mathrm{C} & -3.93949272 & -1.71085216 & -0.98039399 \\ \mathrm{C} & -3.01252146 & -0.61775258 & -0.80717792 \\ \mathrm{~N} & -1.73572616 & -1.10980432 & -0.63286354 \\ \mathrm{C} & -1.83906715 & -2.48629514 & -0.69542049 \\ \mathrm{C} & -3.21680823 & -2.86457699 & -0.90473612 \\ \mathrm{C} & -3.38397320 & 0.73780948 & -0.80592753\end{array}$




\begin{tabular}{|c|c|c|c|}
\hline C & -2.46432449 & 1.77455348 & -0.60923882 \\
\hline $\mathrm{N}$ & -1.09082277 & 1.63583948 & -0.42000041 \\
\hline C & -0.57914918 & 2.91426006 & -0.30610370 \\
\hline $\mathrm{C}$ & -1.65401559 & 3.87569778 & -0.39338739 \\
\hline $\mathrm{C}$ & -2.80742354 & 3.17670272 & -0.58472951 \\
\hline $\mathrm{C}$ & 0.77222317 & 3.25840764 & -0.15955651 \\
\hline $\mathrm{C}$ & 1.83764705 & 2.35690625 & -0.11731313 \\
\hline $\mathrm{N}$ & 1.72961278 & 0.99127199 & -0.17105605 \\
\hline $\mathrm{C}$ & 3.02083099 & 0.50624686 & -0.09956567 \\
\hline C & 3.95536150 & 1.60082299 & 0.01456194 \\
\hline $\mathrm{C}$ & 3.22192001 & 2.74945255 & 0.00417848 \\
\hline $\mathrm{Fe}$ & 0.01257686 & -0.05869104 & -0.53489599 \\
\hline S & -0.55486854 & -0.01685828 & 1.99365825 \\
\hline C & 0.42418526 & 1.29769505 & 2.78900881 \\
\hline $\mathrm{C}$ & 3.38468101 & -0.83829345 & -0.12206797 \\
\hline $\mathrm{C}$ & 2.45220050 & -1.89086247 & -0.23679574 \\
\hline $\mathrm{N}$ & 1.08129425 & -1.76778246 & -0.34620093 \\
\hline $\mathrm{C}$ & 0.57194885 & -3.04331080 & -0.43612839 \\
\hline C & 1.65064209 & -4.00137708 & -0.36735181 \\
\hline $\mathrm{C}$ & 2.81012064 & -3.28998460 & -0.24783481 \\
\hline C & -0.78429663 & -3.39564745 & -0.58774655 \\
\hline $\mathrm{H}$ & 4.43640737 & -1.09453125 & -0.05060938 \\
\hline $\mathrm{H}$ & 1.52567185 & -5.07635036 & -0.40956079 \\
\hline $\mathrm{H}$ & 5.02994004 & 1.48812055 & 0.08741357 \\
\hline $\mathrm{H}$ & 3.56982997 & 3.77308242 & 0.06431362 \\
\hline $\mathrm{H}$ & 1.00820005 & 4.31673068 & -0.11162196 \\
\hline $\mathrm{H}$ & -1.52240092 & 4.94923568 & -0.34137855 \\
\hline $\mathrm{H}$ & -3.81023390 & 3.56262423 & -0.71845569 \\
\hline $\mathrm{H}$ & -4.42504554 & 0.99206506 & -0.97020574 \\
\hline $\mathrm{H}$ & -5.00320360 & -1.59297765 & -1.14442513 \\
\hline $\mathrm{H}$ & -3.57004167 & -3.88512320 & -0.98648494 \\
\hline $\mathrm{H}$ & -1.02134559 & -4.45314459 & -0.64617248 \\
\hline $\mathrm{H}$ & 3.82223192 & -3.66823093 & -0.17251541 \\
\hline $\mathrm{H}$ & 0.15321468 & 1.35848673 & 3.84847264 \\
\hline $\mathrm{H}$ & 1.49613097 & 1.10878881 & 2.68826795 \\
\hline $\mathrm{H}$ & 0.19691077 & 2.26488758 & 2.32458700 \\
\hline $\mathrm{O}$ & 0.20016164 & 0.00422216 & -2.14481435 \\
\hline C & -2.22669878 & -0.81406188 & -4.82487915 \\
\hline $\mathrm{C}$ & -3.06253467 & 0.44669424 & -4.82538884 \\
\hline $\mathrm{O}$ & -2.58759393 & 1.56823308 & -4.97675195 \\
\hline $\mathrm{O}$ & -4.36398187 & 0.22647861 & -4.65135999 \\
\hline $\mathrm{H}$ & -1.72360919 & -0.90402107 & -5.79415174 \\
\hline $\mathrm{H}$ & -2.83415501 & -1.70269518 & -4.64233612 \\
\hline $\mathrm{H}$ & -1.44718676 & -0.73010925 & -4.05963222 \\
\hline $\mathrm{N}$ & -5.86483210 & 2.48963481 & -4.81001767 \\
\hline
\end{tabular}




\begin{tabular}{|c|c|c|c|}
\hline $\mathrm{C}$ & -5.41424621 & 3.68398901 & -5.33697839 \\
\hline $\mathrm{C}$ & -6.46857710 & 4.55266495 & -5.51035794 \\
\hline $\mathrm{N}$ & -7.58360377 & 3.85867532 & -5.06820737 \\
\hline $\mathrm{C}$ & -7.17139371 & 2.62942859 & -4.66514918 \\
\hline $\mathrm{C}$ & -6.56517598 & 5.93196133 & -6.07976158 \\
\hline $\mathrm{H}$ & -6.96616225 & 6.65192314 & -5.35388019 \\
\hline $\mathrm{H}$ & -5.57253762 & 6.28219737 & -6.37902031 \\
\hline $\mathrm{H}$ & -7.21129656 & 5.95925565 & -6.96678842 \\
\hline $\mathrm{H}$ & -8.54817804 & 4.20084948 & -5.06924420 \\
\hline $\mathrm{H}$ & -7.84829470 & 1.87722875 & -4.2835527 \\
\hline $\mathrm{H}$ & -4.88401648 & 1.10276515 & -4.6872358 \\
\hline $\mathrm{H}$ & -4.36532702 & 3.82966688 & -5.5567937 \\
\hline $\mathrm{O}$ & -0.02225777 & 2.44355220 & -3.8296876 \\
\hline $\mathrm{H}$ & 0.15534923 & 1.65427907 & -3.2859106 \\
\hline $\mathrm{H}$ & -0.84437175 & 2.22354578 & -4.3092409 \\
\hline $\mathrm{N}$ & -0.70571562 & -1.75102881 & 4.9838919 \\
\hline $\mathrm{C}$ & 0.50979285 & -2.12797242 & 5.68463900 \\
\hline $\mathrm{C}$ & -1.93577948 & -2.24883576 & 5.30434221 \\
\hline $\mathrm{C}$ & -3.08828068 & -1.80083816 & 4.3735721 \\
\hline $\mathrm{N}$ & -3.16521101 & -0.35752786 & 4.16118137 \\
\hline C & -3.55716035 & 0.51473044 & 5.1396604 \\
\hline $\mathrm{C}$ & -3.48231434 & 1.99050618 & 4.76863588 \\
\hline $\mathrm{C}$ & -3.07666416 & -2.56941567 & 3.0485578 \\
\hline $\mathrm{O}$ & -2.12880036 & -3.05798820 & 6.20725500 \\
\hline $\mathrm{O}$ & -3.96921829 & 0.14713601 & 6.23664228 \\
\hline $\mathrm{H}$ & -0.62797234 & -1.16338154 & 4.1620925 \\
\hline $\mathrm{H}$ & 1.19426540 & -2.67569237 & 5.0242787 \\
\hline $\mathrm{H}$ & 1.02775471 & -1.24420932 & 6.07679655 \\
\hline $\mathrm{H}$ & 0.22850636 & -2.77436877 & 6.51810555 \\
\hline $\mathrm{H}$ & -3.98462317 & -2.08056437 & 4.9349255 \\
\hline $\mathrm{H}$ & -2.19750902 & -2.33907783 & $2.4397226^{\prime}$ \\
\hline $\mathrm{H}$ & -3.07559747 & -3.64501388 & 3.2546152 \\
\hline $\mathrm{H}$ & -3.97217302 & -2.32944998 & 2.46511090 \\
\hline $\mathrm{H}$ & -2.71396029 & 0.01527745 & 3.3310586 \\
\hline $\mathrm{H}$ & -3.07568028 & 2.17350869 & 3.7684798 \\
\hline $\mathrm{H}$ & -4.48880445 & 2.41853920 & 4.82661174 \\
\hline $\mathrm{H}$ & -2.86454843 & 2.50862290 & 5.5096304 \\
\hline
\end{tabular}




\section{Appendix B: Supplement to Ch. 4}

\section{Structures}

\section{${ }^{2} \mathbf{R}-\mathbf{A}$}

$\begin{array}{lrrr}\mathrm{C} & -4.05965173 & -4.22751100 & -3.09559911 \\ \mathrm{H} & -5.12301723 & -4.17175111 & -3.31261557 \\ \mathrm{C} & -3.12976565 & -3.66127010 & -3.97080489 \\ \mathrm{H} & -3.46793906 & -3.16914530 & -4.87932950 \\ \mathrm{C} & -3.60688758 & -4.88787822 & -1.94987284 \\ \mathrm{H} & -4.31828192 & -5.34945674 & -1.26960836 \\ \mathrm{C} & -1.76320118 & -3.73628665 & -3.69758233 \\ \mathrm{H} & -1.05320563 & -3.32202707 & -4.40545154 \\ \mathrm{C} & -2.24105531 & -4.96566653 & -1.68148021 \\ \mathrm{H} & -1.89556949 & -5.48918053 & -0.79244519 \\ \mathrm{C} & -1.29017877 & -4.37257595 & -2.53518667 \\ \mathrm{C} & 0.14150898 & -4.47859298 & -2.19121732 \\ \mathrm{H} & 0.40999804 & -5.40330262 & -1.67940107 \\ \mathrm{C} & 1.12448188 & -3.57898355 & -2.37537710 \\ \mathrm{H} & 2.11624213 & -3.87475724 & -2.03166605 \\ \mathrm{C} & 1.04729009 & -2.18976462 & -2.93955543 \\ \mathrm{H} & 1.54616060 & -1.48270995 & -2.26477606 \\ \mathrm{H} & 1.56643115 & -2.12728191 & -3.90738172 \\ \mathrm{H} & 0.01987185 & -1.84732161 & -3.08229346 \\ \mathrm{O} & 1.93873150 & 0.30553604 & -0.44150423 \\ \mathrm{Fe} & 0.87729982 & 0.98085981 & 0.58372181 \\ \mathrm{~N} & -0.72587486 & 0.67157628 & -0.59050983 \\ \mathrm{~N} & 1.07909505 & 2.83172662 & -0.19160535 \\ \mathrm{~N} & 2.27557137 & 1.42768219 & 1.98050454 \\ \mathrm{~N} & 0.49360424 & -0.74542178 & 1.55990274 \\ \mathrm{C} & -1.51271223 & -0.44898000 & -0.62808791 \\ \mathrm{C} & -2.48475286 & -0.34734963 & -1.69058189 \\ \mathrm{C} & -2.27034538 & 0.85163226 & -2.29849480 \\ \mathrm{C} & -1.17036650 & 1.47772938 & -1.60496830 \\ \mathrm{C} & 0.39784692 & 3.34943763 & -1.26630778 \\ \mathrm{C} & 0.92477592 & 4.64670773 & -1.61388332 \\ \mathrm{C} & 1.93806988 & 4.90408886 & -0.74199032 \\ \mathrm{C} & 2.02858458 & 3.76316731 & 0.13662760 \\ \mathrm{C} & 3.05960782 & 2.55760453 & 2.02840294 \\ \mathrm{C} & 4.04372670 & 2.43731924 & 3.07626358\end{array}$




$\begin{array}{lrrr}\mathrm{C} & 3.86606369 & 1.21412084 & 3.64540030 \\ \mathrm{C} & 2.76883927 & 0.58483241 & 2.95378202 \\ \mathrm{C} & 1.22224193 & -1.29526370 & 2.58239085 \\ \mathrm{C} & 0.71259932 & -2.60421636 & 2.91384823 \\ \mathrm{C} & -0.33425866 & -2.83965882 & 2.07753767 \\ \mathrm{C} & -0.45868361 & -1.67790243 & 1.23099974 \\ \mathrm{C} & -1.40096815 & -1.54247092 & 0.22241418 \\ \mathrm{H} & -2.08717450 & -2.36790066 & 0.06262325 \\ \mathrm{C} & -0.65150198 & 2.72622380 & -1.92553544 \\ \mathrm{H} & -1.10276405 & 3.25549494 & -2.75926896 \\ \mathrm{C} & 2.94503165 & 3.64701809 & 1.17723303 \\ \mathrm{H} & 3.63963320 & 4.46878182 & 1.32303493 \\ \mathrm{C} & 2.29037259 & -0.68484665 & 3.22908703 \\ \mathrm{H} & 2.77852065 & -1.24219541 & 4.02253550 \\ \mathrm{H} & -2.79700559 & 1.28743429 & -3.13800097 \\ \mathrm{H} & -3.21406503 & -1.10992109 & -1.93111383 \\ \mathrm{H} & 0.55604513 & 5.26512694 & -2.42232505 \\ \mathrm{H} & 2.57472353 & 5.77786608 & -0.68521826 \\ \mathrm{H} & 4.77529786 & 3.19621597 & 3.32315584 \\ \mathrm{H} & 4.41956079 & 0.76230877 & 4.45866793 \\ \mathrm{H} & 1.11301366 & -3.24149663 & 3.69193407 \\ \mathrm{H} & -0.97268504 & -3.71186552 & 2.02158179 \\ \mathrm{~S} & -0.56819292 & 1.95306037 & 2.54593416 \\ \mathrm{C} & -0.41169722 & 3.76223240 & 2.56603845 \\ \mathrm{H} & -1.01940512 & 4.16863910 & 3.37987414 \\ \mathrm{H} & 0.63643033 & 4.02709668 & 2.75291484 \\ \mathrm{H} & -0.71122784 & 4.20003365 & 1.61036102\end{array}$

\section{${ }^{2}$ TS1-A}

62

$\begin{array}{lrrr}\mathrm{C} & 0.49994733 & -6.21568428 & -1.75723859 \\ \mathrm{H} & 0.37947922 & -7.29381671 & -1.69465994 \\ \mathrm{C} & -0.36972707 & -5.45255605 & -2.54140086 \\ \mathrm{H} & -1.16895026 & -5.93943249 & -3.09457511 \\ \mathrm{C} & 1.52931815 & -5.57715117 & -1.05875460 \\ \mathrm{H} & 2.21473051 & -6.15764048 & -0.44633263 \\ \mathrm{C} & -0.22292713 & -4.07105094 & -2.62903737 \\ \mathrm{H} & -0.90296433 & -3.51291306 & -3.25834640 \\ \mathrm{C} & 1.68021223 & -4.19827757 & -1.14539078 \\ \mathrm{H} & 2.47803822 & -3.70760255 & -0.59306178 \\ \mathrm{C} & 0.80657162 & -3.40046511 & -1.92551594\end{array}$




\begin{tabular}{|c|c|c|c|}
\hline $\mathrm{C}$ & 1.06046262 & -1.97345247 & -1.96309625 \\
\hline $\mathrm{H}$ & 1.98016222 & -1.68040185 & -1.46173146 \\
\hline $\mathrm{C}$ & 0.29578588 & -0.91361479 & -2.43145770 \\
\hline $\mathrm{H}$ & 0.82531774 & 0.03172120 & -2.48213175 \\
\hline C & -0.93968865 & -0.99037052 & -3.28998591 \\
\hline $\mathrm{H}$ & -1.35990285 & 0.00980088 & -3.42353129 \\
\hline $\mathrm{H}$ & -0.70962462 & -1.40078285 & -4.28328701 \\
\hline $\mathrm{H}$ & -1.70981022 & -1.61241525 & -2.8261566 \\
\hline $\mathrm{O}$ & -0.65376620 & -0.36304414 & -0.7776428 \\
\hline $\mathrm{Fe}$ & -0.24475553 & 0.82794728 & 0.37181286 \\
\hline $\mathrm{N}$ & -1.75869949 & 2.02167295 & -0.22130070 \\
\hline $\mathrm{N}$ & 1.04563329 & 1.88461247 & -0.75338742 \\
\hline $\mathrm{N}$ & 1.31335475 & -0.19389489 & 1.15592030 \\
\hline $\mathrm{N}$ & -1.50145800 & -0.09822302 & 1.66809653 \\
\hline C & -3.06123614 & 1.97263132 & 0.19278837 \\
\hline C & -3.85539276 & 2.93869662 & -0.53158116 \\
\hline $\mathrm{C}$ & -3.01242982 & 3.56529564 & -1.3967624 \\
\hline $\mathrm{C}$ & -1.70455316 & 2.98583404 & -1.19038887 \\
\hline $\mathrm{C}$ & 0.72313910 & 2.87896781 & -1.64716176 \\
\hline $\mathrm{C}$ & 1.91085581 & 3.35572380 & -2.31424193 \\
\hline $\mathrm{C}$ & 2.95781090 & 2.64715105 & -1.80716026 \\
\hline $\mathrm{C}$ & 2.40782103 & 1.73236291 & -0.83627474 \\
\hline $\mathrm{C}$ & 2.64296228 & -0.07924069 & 0.82625840 \\
\hline $\mathrm{C}$ & 3.42106611 & -1.06620006 & 1.53670135 \\
\hline $\mathrm{C}$ & 2.54382380 & -1.79481644 & 2.28126782 \\
\hline $\mathrm{C}$ & 1.23082336 & -1.24658764 & 2.0405230 \\
\hline $\mathrm{C}$ & -1.20051365 & -1.16397530 & 2.47024059 \\
\hline $\mathrm{C}$ & -2.38619912 & -1.62340783 & 3.1598290 \\
\hline $\mathrm{C}$ & -3.40504193 & -0.81353537 & 2.7659041 \\
\hline $\mathrm{C}$ & -2.84294148 & 0.12998812 & 1.82515160 \\
\hline $\mathrm{C}$ & -3.56860135 & 1.10743762 & 1.15790678 \\
\hline $\mathrm{H}$ & -4.62800173 & 1.18313271 & 1.38443326 \\
\hline $\mathrm{C}$ & -0.55390980 & 3.38179934 & -1.8639745 \\
\hline $\mathrm{H}$ & -0.65777058 & 4.16840477 & -2.6056382 \\
\hline $\mathrm{C}$ & 3.15906402 & 0.82597004 & -0.0931293 \\
\hline $\mathrm{H}$ & 4.23169710 & 0.80996409 & -0.26269009 \\
\hline $\mathrm{C}$ & 0.06685467 & -1.71283528 & 2.6333654 \\
\hline $\mathrm{H}$ & 0.15860261 & -2.56018519 & 3.3061259 \\
\hline $\mathrm{H}$ & -3.23551289 & 4.35046801 & -2.1080866 \\
\hline $\mathrm{H}$ & -4.91600097 & 3.09985356 & -0.3857866 \\
\hline $\mathrm{H}$ & 1.92618284 & 4.13761686 & -3.0630454 \\
\hline $\mathrm{H}$ & 4.00867298 & 2.72575028 & -2.0557362 \\
\hline $\mathrm{H}$ & 4.49524310 & -1.17831148 & 1.4590074 \\
\hline $\mathrm{H}$ & 2.75121523 & -2.62543261 & 2.9440289 \\
\hline $\mathrm{H}$ & -2.41296913 & -2.45227874 & 3.855751 \\
\hline
\end{tabular}




$\begin{array}{llll}\mathrm{H} & -4.44442537 & -0.84009558 & 3.06763771 \\ \mathrm{~S} & 0.19031981 & 2.25294449 & 2.44629945 \\ \mathrm{C} & 1.74641964 & 3.16823341 & 2.22967507 \\ \mathrm{H} & 1.93314601 & 3.76801731 & 3.12574052 \\ \mathrm{H} & 2.57630292 & 2.46147833 & 2.11128521 \\ \mathrm{H} & 1.71251795 & 3.81147070 & 1.34617134\end{array}$

$\begin{array}{lrrr}{ }^{2} \text { I*-A } & & & \\ & & & \\ 62 & & & \\ \mathrm{C} & -0.33678630 & -5.91370161 & -0.97292900 \\ \mathrm{H} & -0.69477657 & -6.93606207 & -0.88779667 \\ \mathrm{C} & -1.20942364 & -4.91225752 & -1.41535903 \\ \mathrm{H} & -2.27136826 & -5.12488121 & -1.50706976 \\ \mathrm{C} & 1.02090291 & -5.61460347 & -0.79362780 \\ \mathrm{H} & 1.69133421 & -6.38107043 & -0.41331749 \\ \mathrm{C} & -0.74648376 & -3.63030833 & -1.68113180 \\ \mathrm{H} & -1.44551058 & -2.85929387 & -1.97966388 \\ \mathrm{C} & 1.48350537 & -4.32677026 & -1.00203161 \\ \mathrm{H} & 2.52155425 & -4.07856690 & -0.79975193 \\ \mathrm{C} & 0.62527330 & -3.29463599 & -1.49087313 \\ \mathrm{C} & 1.18512883 & -2.02109074 & -1.71704896 \\ \mathrm{H} & 2.23818179 & -1.92902629 & -1.45599454 \\ \mathrm{C} & 0.52186451 & -0.74823404 & -2.15454818 \\ \mathrm{H} & 1.24263338 & 0.06896794 & -2.17682151 \\ \mathrm{C} & -0.30058307 & -0.80516760 & -3.44472455 \\ \mathrm{H} & -0.68268380 & 0.19588095 & -3.66096529 \\ \mathrm{H} & 0.27656332 & -1.18917803 & -4.29416626 \\ \mathrm{H} & -1.15596824 & -1.47114319 & -3.30983500 \\ \mathrm{O} & -0.42483171 & -0.36073600 & -1.13955856 \\ \mathrm{Fe} & -0.15699554 & 0.81232170 & 0.21674191 \\ \mathrm{~N} & -1.76245338 & 1.87820488 & -0.36213462 \\ \mathrm{~N} & 1.07075996 & 2.06972133 & -0.76880137 \\ \mathrm{~N} & 1.45150326 & -0.17302021 & 0.95843032 \\ \mathrm{~N} & -1.37232306 & -0.35659675 & 1.35709271 \\ \mathrm{C} & -3.07640447 & 1.62711180 & -0.08133822 \\ \mathrm{C} & -3.93030820 & 2.56344179 & -0.78149726 \\ \mathrm{C} & -3.10672951 & 3.38072372 & -1.49202816 \\ \mathrm{C} & -1.75448708 & 2.94331881 & -1.22220813 \\ \mathrm{C} & 0.69468765 & 3.14594144 & -1.53150302 \\ \mathrm{C} & 1.85669459 & 3.77447655 & -2.11322945 \\ \mathrm{C} & 2.94218652 & 3.07097451 & -1.68444175\end{array}$




$\begin{array}{lrrr}\mathrm{C} & 2.44142811 & 2.01295162 & -0.84038811 \\ \mathrm{C} & 2.77509843 & 0.06239164 & 0.65316315 \\ \mathrm{C} & 3.60869848 & -0.94953767 & 1.25537323 \\ \mathrm{C} & 2.78175179 & -1.77377974 & 1.95911207 \\ \mathrm{C} & 1.43730151 & -1.29638798 & 1.75506885 \\ \mathrm{C} & -1.00153396 & -1.43427549 & 2.11280125 \\ \mathrm{C} & -2.16675258 & -2.08368390 & 2.67414430 \\ \mathrm{C} & -3.24400640 & -1.35629924 & 2.27786275 \\ \mathrm{C} & -2.73865240 & -0.31084120 & 1.41222123 \\ \mathrm{C} & -3.53698338 & 0.62226500 & 0.76357338 \\ \mathrm{H} & -4.61039335 & 0.54161611 & 0.90695257 \\ \mathrm{C} & -0.61948868 & 3.55752858 & -1.74486074 \\ \mathrm{H} & -0.78156772 & 4.34406329 & -2.47630066 \\ \mathrm{C} & 3.23652803 & 1.07190396 & -0.18740270 \\ \mathrm{H} & 4.30895575 & 1.13061316 & -0.35011007 \\ \mathrm{C} & 0.30486289 & -1.86074524 & 2.32493879 \\ \mathrm{H} & 0.44805531 & -2.74337418 & 2.94071020 \\ \mathrm{H} & -3.37909028 & 4.20164164 & -2.14366431 \\ \mathrm{H} & -4.99563099 & 2.66240156 & -0.61404480 \\ \mathrm{H} & 1.84525473 & 4.60689042 & -2.80589259 \\ \mathrm{H} & 3.99469133 & 3.21641064 & -1.89350272 \\ \mathrm{H} & 4.68517321 & -0.98818981 & 1.14253105 \\ \mathrm{H} & 3.06132681 & -2.64505548 & 2.53817239 \\ \mathrm{H} & -2.13642858 & -2.94344298 & 3.33117638 \\ \mathrm{H} & -4.29213369 & -1.52892709 & 2.48769814 \\ \mathrm{~S} & 0.13207803 & 2.03913146 & 2.34756216 \\ \mathrm{C} & 1.63710251 & 3.05293136 & 2.28490156 \\ \mathrm{H} & 1.77495669 & 3.53001934 & 3.26018424 \\ \mathrm{H} & 2.50920144 & 2.40790001 & 2.12428192 \\ \mathrm{H} & 1.59955777 & 3.79471591 & 1.48315954\end{array}$

\section{${ }^{2} \mathbf{I}-\mathbf{A}$}

62

$\begin{array}{lrrr}\mathrm{C} & -0.58095443 & -5.72796185 & -0.52660513 \\ \mathrm{H} & -1.01122498 & -6.63476500 & -0.10961097 \\ \mathrm{C} & -1.13528687 & -4.48377185 & -0.20889260 \\ \mathrm{H} & -1.99035366 & -4.42077574 & 0.45746304 \\ \mathrm{C} & 0.53557829 & -5.81813761 & -1.37786666 \\ \mathrm{H} & 0.95906570 & -6.78942047 & -1.61657489 \\ \mathrm{C} & -0.58870695 & -3.31617160 & -0.72691599 \\ \mathrm{H} & -0.98779848 & -2.33647650 & -0.49697522\end{array}$




\begin{tabular}{|c|c|c|c|}
\hline $\mathrm{C}$ & 1.08800038 & -4.66865329 & -1.90817098 \\
\hline $\mathrm{H}$ & 1.94773301 & -4.72780870 & -2.57099832 \\
\hline C & 0.53637271 & -3.38498975 & -1.60361597 \\
\hline $\mathrm{C}$ & 1.10329079 & -2.24041839 & -2.19227155 \\
\hline $\mathrm{H}$ & 1.95488349 & -2.41170525 & -2.85214684 \\
\hline $\mathrm{C}$ & 0.70820455 & -0.81746083 & -2.07883957 \\
\hline $\mathrm{H}$ & 1.67910069 & -0.32695730 & -1.82122471 \\
\hline C & 0.40398559 & -0.28485816 & -3.53236966 \\
\hline $\mathrm{H}$ & 0.24643172 & 0.79202986 & -3.44937579 \\
\hline $\mathrm{H}$ & 1.22250804 & -0.48179067 & -4.23359133 \\
\hline $\mathrm{H}$ & -0.51428922 & -0.75007105 & -3.90163043 \\
\hline $\mathrm{O}$ & -0.29087647 & -0.49110389 & -1.2281768 \\
\hline $\mathrm{Fe}$ & -0.16693843 & 0.79555372 & 0.2540157 \\
\hline $\mathrm{N}$ & -1.81050369 & 1.72404075 & -0.46967156 \\
\hline $\mathrm{N}$ & 1.03317867 & 2.03464585 & -0.79148375 \\
\hline $\mathrm{N}$ & 1.46903421 & -0.16008648 & 0.95408340 \\
\hline $\mathrm{N}$ & -1.37768317 & -0.44428283 & 1.30951489 \\
\hline $\mathrm{C}$ & -3.12047312 & 1.42003458 & -0.22559569 \\
\hline C & -3.99043931 & 2.33434896 & -0.9337329 \\
\hline $\mathrm{C}$ & -3.18227937 & 3.19957501 & -1.6020064 \\
\hline $\mathrm{C}$ & -1.82078251 & 2.80756082 & -1.3041945 \\
\hline $\mathrm{C}$ & 0.62593650 & 3.08739628 & -1.5693896 \\
\hline $\mathrm{C}$ & 1.77199660 & 3.75514167 & -2.1431969 \\
\hline $\mathrm{C}$ & 2.87543789 & 3.08346237 & -1.7110378 \\
\hline $\mathrm{C}$ & 2.40102874 & 2.01237057 & -0.8658596 \\
\hline $\mathrm{C}$ & 2.78160974 & 0.08926469 & 0.6457659 \\
\hline $\mathrm{C}$ & 3.64545347 & -0.82442094 & 1.35816753 \\
\hline $\mathrm{C}$ & 2.83504611 & -1.62122604 & 2.10821791 \\
\hline $\mathrm{C}$ & 1.47677347 & -1.20041930 & 1.84764302 \\
\hline $\mathrm{C}$ & -0.97079103 & -1.42585185 & 2.1700384 \\
\hline $\mathrm{C}$ & -2.11802881 & -2.08144735 & 2.7605519 \\
\hline $\mathrm{C}$ & -3.22237317 & -1.48203400 & 2.2361177 \\
\hline $\mathrm{C}$ & -2.74709558 & -0.45732540 & 1.3332059 \\
\hline $\mathrm{C}$ & -3.56604458 & 0.39915953 & 0.6074750 \\
\hline $\mathrm{H}$ & -4.63962691 & 0.27738213 & 0.7176611 \\
\hline C & -0.69578048 & 3.44783137 & -1.8123838 \\
\hline $\mathrm{H}$ & -0.86350715 & 4.30229505 & -2.46166808 \\
\hline $\mathrm{C}$ & 3.22109987 & 1.08913889 & -0.2182682 \\
\hline $\mathrm{H}$ & 4.29236784 & 1.17859183 & -0.3742675 \\
\hline C & 0.35144160 & -1.78687749 & 2.4162496 \\
\hline $\mathrm{H}$ & 0.51953924 & -2.60028793 & 3.1161648 \\
\hline $\mathrm{H}$ & -3.46071581 & 4.02987512 & -2.2388346 \\
\hline $\mathrm{H}$ & -5.07244799 & 2.30548976 & -0.9042826 \\
\hline $\mathrm{H}$ & 1.72124204 & 4.62014909 & -2.7926415 \\
\hline $\mathrm{H}$ & 3.91675730 & 3.28549703 & -1.9289167 \\
\hline
\end{tabular}




$\begin{array}{lrll}\mathrm{H} & 4.72624597 & -0.83393438 & 1.29076272 \\ \mathrm{H} & 3.11411175 & -2.42257990 & 2.78100236 \\ \mathrm{H} & -2.06577299 & -2.88667380 & 3.48291406 \\ \mathrm{H} & -4.26520190 & -1.69213250 & 2.43861459 \\ \mathrm{~S} & -0.22046678 & 2.20431348 & 2.06954815 \\ \mathrm{C} & 1.44489393 & 2.90887435 & 2.34451843 \\ \mathrm{H} & 1.37995257 & 3.55268841 & 3.22807316 \\ \mathrm{H} & 2.18679726 & 2.12844892 & 2.53776205 \\ \mathrm{H} & 1.77536161 & 3.51329461 & 1.49504468\end{array}$

${ }^{2}$ TS2-A

62

$\begin{array}{lrrr}\mathrm{C} & -0.71724496 & -5.64014813 & -0.92020420 \\ \mathrm{H} & -1.18798427 & -6.60787900 & -0.76957802 \\ \mathrm{C} & -1.50033838 & -4.52733173 & -1.26267215 \\ \mathrm{H} & -2.57678069 & -4.63428785 & -1.36110035 \\ \mathrm{C} & 0.66475805 & -5.50189695 & -0.74547524 \\ \mathrm{H} & 1.26703269 & -6.36017308 & -0.46155812 \\ \mathrm{C} & -0.91397838 & -3.28623306 & -1.45092598 \\ \mathrm{H} & -1.51293701 & -2.40528764 & -1.64682925 \\ \mathrm{C} & 1.26226868 & -4.26232895 & -0.92536607 \\ \mathrm{H} & 2.33381897 & -4.14700709 & -0.78630801 \\ \mathrm{C} & 0.49578980 & -3.13008898 & -1.31914418 \\ \mathrm{C} & 1.18621601 & -1.92409242 & -1.60351334 \\ \mathrm{H} & 2.23872830 & -1.91311013 & -1.32532037 \\ \mathrm{C} & 0.61864818 & -0.67408121 & -2.20610618 \\ \mathrm{H} & 1.42585510 & 0.06977245 & -2.24594483 \\ \mathrm{C} & 0.06323713 & -0.83040038 & -3.63002007 \\ \mathrm{H} & -0.36900425 & 0.12864836 & -3.93425705 \\ \mathrm{H} & 0.85566845 & -1.09901932 & -4.33992926 \\ \mathrm{H} & -0.71921825 & -1.59325294 & -3.67713393 \\ \mathrm{O} & -0.38268949 & -0.31086277 & -1.30086887 \\ \mathrm{Fe} & -0.11012425 & 0.78301597 & 0.23586919 \\ \mathrm{~N} & -1.77179326 & 1.78042967 & -0.34182225 \\ \mathrm{~N} & 1.05979253 & 2.08882529 & -0.75919197 \\ \mathrm{~N} & 1.53952527 & -0.24402866 & 0.78196716 \\ \mathrm{~N} & -1.28947366 & -0.51816646 & 1.26121633 \\ \mathrm{C} & -3.07197582 & 1.47782524 & -0.04967520 \\ \mathrm{C} & -3.96225572 & 2.43600951 & -0.67034759 \\ \mathrm{C} & -3.17592940 & 3.32390374 & -1.33447743 \\ \mathrm{C} & -1.80638251 & 2.90482670 & -1.12024029\end{array}$




$\begin{array}{lrrr}\mathrm{C} & 0.63008647 & 3.18223315 & -1.46499775 \\ \mathrm{C} & 1.75888902 & 3.88765885 & -2.02832552 \\ \mathrm{C} & 2.87581369 & 3.20343396 & -1.65578673 \\ \mathrm{C} & 2.42785287 & 2.08223803 & -0.86263440 \\ \mathrm{C} & 2.85183102 & 0.05981195 & 0.49479528 \\ \mathrm{C} & 3.72974350 & -0.89607946 & 1.12365869 \\ \mathrm{C} & 2.93645179 & -1.76735808 & 1.81206633 \\ \mathrm{C} & 1.57203405 & -1.34951877 & 1.60606132 \\ \mathrm{C} & -0.86211912 & -1.58801897 & 1.99592966 \\ \mathrm{C} & -1.99250914 & -2.27552295 & 2.58503203 \\ \mathrm{C} & -3.10604106 & -1.60352779 & 2.18831342 \\ \mathrm{C} & -2.65351912 & -0.50712527 & 1.35845238 \\ \mathrm{C} & -3.49151574 & 0.41711020 & 0.74599779 \\ \mathrm{H} & -4.55999913 & 0.30569867 & 0.90571136 \\ \mathrm{C} & -0.69707171 & 3.56477645 & -1.63503491 \\ \mathrm{H} & -0.88192107 & 4.45562757 & -2.22832757 \\ \mathrm{C} & 3.26852588 & 1.13483320 & -0.28422812 \\ \mathrm{H} & 4.33700982 & 1.25451923 & -0.43868449 \\ \mathrm{C} & 0.46395965 & -1.97966300 & 2.15783868 \\ \mathrm{H} & 0.65086758 & -2.84874623 & 2.78175170 \\ \mathrm{H} & -3.47367379 & 4.18642028 & -1.91746535 \\ \mathrm{H} & -5.04202792 & 2.41644540 & -0.59165998 \\ \mathrm{H} & 1.68798484 & 4.78606696 & -2.62865200 \\ \mathrm{H} & 3.91062894 & 3.42468796 & -1.88559146 \\ \mathrm{H} & 4.81034327 & -0.88289376 & 1.05226640 \\ \mathrm{H} & 3.23686593 & -2.61205984 & 2.41959045 \\ \mathrm{H} & -1.92166683 & -3.15281107 & 3.21582513 \\ \mathrm{H} & -4.14167476 & -1.81167209 & 2.42654277 \\ \mathrm{~S} & -0.03648668 & 1.97395822 & 2.21401267 \\ \mathrm{C} & 1.56575492 & 2.83902398 & 2.37101655 \\ \mathrm{H} & 1.56473356 & 3.37345868 & 3.32605988 \\ \mathrm{H} & 2.40096991 & 2.13187197 & 2.36781182 \\ \mathrm{H} & 1.71066797 & 3.55711901 & 1.55890195\end{array}$

${ }^{2}$ E-A

62

$\begin{array}{llll}\mathrm{C} & -2.03445844 & -5.21214978 & -2.43683144 \\ \mathrm{H} & -2.69070602 & -5.99640374 & -2.80446561 \\ \mathrm{C} & -2.38054646 & -3.87027138 & -2.60549319 \\ \mathrm{H} & -3.31056720 & -3.60637717 & -3.10222862 \\ \mathrm{C} & -0.84397919 & -5.54283832 & -1.78431229\end{array}$




\begin{tabular}{|c|c|c|c|}
\hline $\mathrm{H}$ & -0.57066841 & -6.58505087 & -1.64223478 \\
\hline $\mathrm{C}$ & -1.53988821 & -2.86019491 & -2.13365566 \\
\hline $\mathrm{H}$ & -1.81052598 & -1.81554306 & -2.24710645 \\
\hline $\mathrm{C}$ & -0.00568976 & -4.53585937 & -1.30748554 \\
\hline $\mathrm{H}$ & 0.91656882 & -4.79706320 & -0.79270501 \\
\hline C & -0.34148825 & -3.18654428 & -1.48659150 \\
\hline $\mathrm{C}$ & 0.58897446 & -2.13780992 & -0.97395467 \\
\hline $\mathrm{H}$ & 1.01688198 & -2.34501835 & 0.00642564 \\
\hline $\mathrm{C}$ & 1.36836895 & -1.21237811 & -1.83568334 \\
\hline $\mathrm{H}$ & 2.28404983 & -0.81831555 & -1.39743103 \\
\hline $\mathrm{C}$ & 1.30622369 & -1.18874866 & -3.33876939 \\
\hline $\mathrm{H}$ & 1.35223879 & -0.15438795 & -3.69729358 \\
\hline $\mathrm{H}$ & 2.16536905 & -1.72951255 & -3.75349612 \\
\hline $\mathrm{H}$ & 0.38986036 & -1.65004398 & -3.71463865 \\
\hline $\mathrm{O}$ & 0.18681255 & -0.76061355 & -1.13240674 \\
\hline $\mathrm{Fe}$ & 0.08485583 & 0.82953908 & 0.49494653 \\
\hline $\mathrm{N}$ & -1.66054554 & 1.31160934 & -0.38768997 \\
\hline $\mathrm{N}$ & 1.08629416 & 2.02763679 & -0.77237405 \\
\hline $\mathrm{N}$ & 1.84785288 & 0.19212980 & 1.23482836 \\
\hline $\mathrm{N}$ & -0.89446765 & -0.53484274 & 1.61100913 \\
\hline $\mathrm{C}$ & -2.90938891 & 0.81936369 & -0.10655378 \\
\hline $\mathrm{C}$ & -3.90613951 & 1.49075260 & -0.90893593 \\
\hline $\mathrm{C}$ & -3.24418368 & 2.40184501 & -1.67156304 \\
\hline $\mathrm{C}$ & -1.84207320 & 2.28121677 & -1.34074799 \\
\hline $\mathrm{C}$ & 0.52513953 & 2.89521356 & -1.67772277 \\
\hline $\mathrm{C}$ & 1.55589195 & 3.64344943 & -2.35732636 \\
\hline $\mathrm{C}$ & 2.74816821 & 3.21830270 & -1.85523276 \\
\hline $\mathrm{C}$ & 2.44601588 & 2.21589244 & -0.86214315 \\
\hline $\mathrm{C}$ & 3.11093380 & 0.62379917 & 0.89569933 \\
\hline $\mathrm{C}$ & 4.09836854 & -0.01301590 & 1.73218561 \\
\hline $\mathrm{C}$ & 3.42068207 & -0.82470365 & 2.59100807 \\
\hline $\mathrm{C}$ & 2.01921150 & -0.69206230 & 2.27451206 \\
\hline $\mathrm{C}$ & -0.34753813 & -1.31786981 & 2.59829272 \\
\hline $\mathrm{C}$ & -1.37803547 & -2.09008417 & 3.25349855 \\
\hline $\mathrm{C}$ & -2.55406259 & -1.76321520 & 2.65326467 \\
\hline $\mathrm{C}$ & -2.24410819 & -0.78524289 & 1.63702565 \\
\hline $\mathrm{C}$ & -3.18899014 & -0.16831626 & 0.82849523 \\
\hline $\mathrm{H}$ & -4.22679574 & -0.46072934 & 0.95606638 \\
\hline $\mathrm{C}$ & -0.83352288 & 3.02556712 & -1.93715830 \\
\hline $\mathrm{H}$ & -1.12903280 & 3.75767700 & -2.68274593 \\
\hline $\mathrm{C}$ & 3.39766283 & 1.55623179 & -0.09394539 \\
\hline $\mathrm{H}$ & 4.44085398 & 1.80718321 & -0.26120488 \\
\hline $\mathrm{C}$ & 1.00227060 & -1.38943392 & 2.91631395 \\
\hline $\mathrm{H}$ & 1.28940106 & -2.05695393 & 3.72329251 \\
\hline $\mathrm{H}$ & -3.64821169 & 3.09499347 & -2.39871408 \\
\hline
\end{tabular}




$\begin{array}{lrrr}\mathrm{H} & -4.96782514 & 1.28087891 & -0.87592716 \\ \mathrm{H} & 1.37347621 & 4.39000947 & -3.12021941 \\ \mathrm{H} & 3.74642717 & 3.54535849 & -2.11807458 \\ \mathrm{H} & 5.16602729 & 0.15367994 & 1.66257319 \\ \mathrm{H} & 3.81848212 & -1.46311554 & 3.36998327 \\ \mathrm{H} & -1.20427869 & -2.78818546 & 4.06275971 \\ \mathrm{H} & -3.54796943 & -2.13533057 & 2.86742496 \\ \mathrm{~S} & -0.23699936 & 2.39456516 & 2.03849510 \\ \mathrm{C} & 1.32565871 & 3.30913001 & 2.29162937 \\ \mathrm{H} & 1.12766492 & 4.05823905 & 3.06556272 \\ \mathrm{H} & 2.12672321 & 2.64997873 & 2.63573940 \\ \mathrm{H} & 1.64411273 & 3.82058440 & 1.37976248\end{array}$

\section{${ }^{2} \mathbf{R}-\mathbf{B}$}

160

$\begin{array}{lrrr}\mathrm{C} & -4.80803611 & -2.82162247 & -2.91793404 \\ \mathrm{H} & -5.84839970 & -2.52084122 & -3.00740391 \\ \mathrm{C} & -3.85627735 & -2.34923724 & -3.82519371 \\ \mathrm{H} & -4.15645228 & -1.68345366 & -4.63039657 \\ \mathrm{C} & -4.41367654 & -3.70887948 & -1.91232985 \\ \mathrm{H} & -5.14772003 & -4.10317130 & -1.21411307 \\ \mathrm{C} & -2.52101596 & -2.74177937 & -3.71964582 \\ \mathrm{H} & -1.80312979 & -2.39549798 & -4.45540075 \\ \mathrm{C} & -3.08013624 & -4.10367631 & -1.81191437 \\ \mathrm{H} & -2.78370522 & -4.80635222 & -1.03577779 \\ \mathrm{C} & -2.09995339 & -3.61197137 & -2.69693701 \\ \mathrm{C} & -0.70255465 & -4.05850605 & -2.53140355 \\ \mathrm{H} & -0.60170689 & -5.07729120 & -2.15613943 \\ \mathrm{C} & 0.43581012 & -3.36993868 & -2.73099008 \\ \mathrm{H} & 1.36335598 & -3.90944825 & -2.53740744 \\ \mathrm{C} & 0.61966226 & -1.93642804 & -3.13851031 \\ \mathrm{H} & 1.32650055 & -1.43985107 & -2.46191875 \\ \mathrm{H} & 1.04472485 & -1.86516962 & -4.15030097 \\ \mathrm{H} & -0.31346286 & -1.36863380 & -3.12260518 \\ \mathrm{O} & 2.13346343 & 0.12343458 & -0.45222132 \\ \mathrm{Fe} & 1.37410155 & 0.82815365 & 0.79246073 \\ \mathrm{~N} & -0.39552499 & 0.95870763 & -0.16364491 \\ \mathrm{~N} & 1.80908067 & 2.70662464 & 0.21059653 \\ \mathrm{~N} & 2.98212725 & 0.84060952 & 2.00434997 \\ \mathrm{~N} & 0.78000916 & -0.91223178 & 1.61426745 \\ \mathrm{C} & -1.31517965 & -0.05194396 & -0.32156645\end{array}$




\begin{tabular}{|c|c|c|c|}
\hline $\mathrm{C}$ & -2.35007872 & 0.34681876 & -1.24541186 \\
\hline C & -2.05112123 & 1.61344756 & -1.64501454 \\
\hline $\mathrm{C}$ & -0.81959565 & 1.97674643 & -0.98921768 \\
\hline $\mathrm{C}$ & 1.09893585 & 3.48993685 & -0.66281867 \\
\hline $\mathrm{C}$ & 1.80874818 & 4.71562741 & -0.93460171 \\
\hline $\mathrm{C}$ & 2.96701362 & 4.66197920 & -0.22089723 \\
\hline $\mathrm{C}$ & 2.95807786 & 3.40696759 & 0.48836057 \\
\hline $\mathrm{C}$ & 3.96421808 & 1.79388679 & 2.05908196 \\
\hline C & 5.00125927 & 1.40090767 & 2.98287167 \\
\hline C & 4.63210617 & 0.19371773 & 3.49030075 \\
\hline C & 3.37548825 & -0.14736713 & 2.86823733 \\
\hline $\mathrm{C}$ & 1.48158946 & -1.68599785 & 2.50648045 \\
\hline $\mathrm{C}$ & 0.81813833 & -2.95074890 & 2.70745146 \\
\hline $\mathrm{C}$ & -0.28286703 & -2.94543480 & 1.90739289 \\
\hline $\mathrm{C}$ & -0.29713301 & -1.67294850 & 1.22935452 \\
\hline $\mathrm{C}$ & -1.26724737 & -1.28353091 & 0.31611596 \\
\hline $\mathrm{H}$ & -2.04704981 & -1.99901819 & 0.07484393 \\
\hline $\mathrm{C}$ & -0.13840678 & 3.16335996 & -1.20950147 \\
\hline $\mathrm{H}$ & -0.60592024 & 3.88938698 & -1.86572749 \\
\hline $\mathrm{C}$ & 3.96374144 & 2.98631121 & 1.34656664 \\
\hline $\mathrm{H}$ & 4.81381232 & 3.64802258 & 1.48110509 \\
\hline $\mathrm{C}$ & 2.67771453 & -1.32383922 & 3.11048322 \\
\hline $\mathrm{H}$ & 3.11576532 & -2.02800976 & 3.81108597 \\
\hline $\mathrm{H}$ & -2.59343289 & 2.25624921 & -2.32566285 \\
\hline $\mathrm{H}$ & -3.18018810 & -0.28044246 & -1.54435170 \\
\hline $\mathrm{H}$ & 1.45590900 & 5.49819508 & -1.59399964 \\
\hline $\mathrm{H}$ & 3.76211114 & 5.39441771 & -0.16672529 \\
\hline $\mathrm{H}$ & 5.88477268 & 1.98565220 & 3.20397811 \\
\hline $\mathrm{H}$ & 5.14862443 & -0.42174052 & 4.21538296 \\
\hline $\mathrm{H}$ & 1.16836587 & -3.73140586 & 3.37031869 \\
\hline $\mathrm{H}$ & -1.02579107 & -3.72118137 & 1.77504025 \\
\hline S & -0.04123244 & 1.91192143 & 2.92079664 \\
\hline $\mathrm{C}$ & -0.58991827 & 3.59380833 & 2.46882425 \\
\hline $\mathrm{H}$ & -0.33134447 & 4.22417834 & 3.3299467 \\
\hline $\mathrm{H}$ & -0.01770829 & 3.95870664 & 1.6149398 \\
\hline $\mathrm{H}$ & -2.31175845 & 3.32351721 & 1.20688037 \\
\hline $\mathrm{C}$ & -2.09488024 & 3.78188783 & 2.16867478 \\
\hline $\mathrm{C}$ & -2.96416585 & 3.18324423 & 3.28841946 \\
\hline $\mathrm{N}$ & -3.78908343 & 2.15041595 & 2.9849078 \\
\hline C & -4.67298639 & 1.57790361 & 4.0218089 \\
\hline C & -3.94715293 & 1.00715690 & 5.24711377 \\
\hline $\mathrm{N}$ & -2.76260851 & 0.38892249 & 5.0129986 \\
\hline C & -2.01538216 & -0.28388366 & 6.0765785 \\
\hline $\mathrm{C}$ & -1.44840658 & 0.71813661 & 7.1065593 \\
\hline $\mathrm{N}$ & -0.76983430 & 1.78227587 & 6.5899126 \\
\hline
\end{tabular}




\begin{tabular}{|c|c|c|c|}
\hline $\mathrm{C}$ & -0.25375133 & 2.83412947 & 7.46294213 \\
\hline $\mathrm{C}$ & -1.36574393 & 3.57115570 & 8.24073827 \\
\hline $\mathrm{N}$ & -2.50977277 & 3.89511457 & 7.56782306 \\
\hline $\mathrm{C}$ & -3.57097811 & 4.57324557 & 8.30884618 \\
\hline $\mathrm{C}$ & -4.17177714 & 3.73245458 & 9.46427793 \\
\hline $\mathrm{N}$ & -4.32936924 & 2.40851707 & 9.20986589 \\
\hline $\mathrm{N}$ & -2.34778206 & 5.20738505 & 2.00385445 \\
\hline $\mathrm{C}$ & -2.73570386 & 5.71439227 & 0.79708178 \\
\hline $\mathrm{C}$ & -3.00393070 & 7.23544592 & 0.71788183 \\
\hline $\mathrm{N}$ & -2.30281127 & 8.03382621 & 1.74259251 \\
\hline C & -2.90181053 & 8.21664942 & 2.95711616 \\
\hline $\mathrm{C}$ & -2.14890759 & 9.02861675 & 4.02730727 \\
\hline $\mathrm{N}$ & -2.98145243 & 9.24514784 & 5.18447356 \\
\hline $\mathrm{C}$ & -3.45131933 & 10.49215657 & 5.4822475 \\
\hline $\mathrm{C}$ & -4.29754475 & 10.57129511 & 6.743335 \\
\hline $\mathrm{H}$ & -4.36540586 & 9.61887487 & 7.2759287 \\
\hline $\mathrm{H}$ & -5.30436079 & 10.90779172 & 6.473959 \\
\hline $\mathrm{H}$ & -3.86970587 & 11.32861189 & 7.4075971 \\
\hline $\mathrm{O}$ & -3.21067380 & 11.48293171 & $4.793735 \varepsilon$ \\
\hline $\mathrm{H}$ & -3.22363926 & 8.43542469 & 5.7628951 \\
\hline $\mathrm{H}$ & -1.89726629 & 10.01701178 & 3.628868 \\
\hline $\mathrm{C}$ & -0.87253461 & 8.27076655 & 4.46483725 \\
\hline $\mathrm{H}$ & -0.30331675 & 8.89770149 & 5.15682011 \\
\hline $\mathrm{H}$ & -1.15121725 & 7.35449400 & 4.9973767 \\
\hline $\mathrm{H}$ & -0.22419428 & 7.99987639 & 3.62626100 \\
\hline $\mathrm{O}$ & -3.96170964 & 7.62697022 & 3.1978642 \\
\hline $\mathrm{C}$ & -1.25199700 & 8.89045667 & 1.15282242 \\
\hline $\mathrm{H}$ & -1.58380675 & 9.93667259 & 1.1338719 \\
\hline $\mathrm{H}$ & -0.32405729 & 8.84064484 & 1.72769537 \\
\hline $\mathrm{C}$ & -1.09499772 & 8.33193531 & -0.26851198 \\
\hline $\mathrm{H}$ & -0.72127455 & 9.08674543 & -0.96618696 \\
\hline $\mathrm{H}$ & -0.38597323 & 7.49470297 & -0.2653078 \\
\hline $\mathrm{C}$ & -2.50621197 & 7.82976681 & -0.6109158 \\
\hline $\mathrm{H}$ & -3.14853435 & 8.66999740 & -0.89709594 \\
\hline $\mathrm{H}$ & -2.53169391 & 7.08578172 & -1.4081054 \\
\hline $\mathrm{H}$ & -4.08352705 & 7.35947065 & 0.84941746 \\
\hline $\mathrm{O}$ & -2.86416192 & 5.01605238 & -0.2088805 \\
\hline $\mathrm{H}$ & -2.45128523 & 5.76843104 & 2.83994449 \\
\hline $\mathrm{O}$ & -2.85826205 & 3.62051832 & 4.44479777 \\
\hline $\mathrm{C}$ & -4.17979128 & 1.70651205 & 1.6236701 \\
\hline $\mathrm{H}$ & -4.20357388 & 2.54586076 & 0.9258802 \\
\hline $\mathrm{H}$ & -3.46113510 & 0.97098749 & 1.2476016 \\
\hline C & -5.56430743 & 1.07973209 & 1.8366792 \\
\hline $\mathrm{H}$ & -6.33934837 & 1.85320984 & 1.7823198 \\
\hline $\mathrm{H}$ & -5.79267595 & 0.32216753 & 1.0814250 \\
\hline
\end{tabular}




\begin{tabular}{|c|c|c|c|}
\hline $\mathrm{C}$ & -5.48090906 & 0.50967247 & 3.25898412 \\
\hline $\mathrm{H}$ & -4.93116264 & -0.43897882 & 3.25875932 \\
\hline $\mathrm{H}$ & -6.45363482 & 0.33544784 & 3.72478188 \\
\hline $\mathrm{H}$ & -5.33202744 & 2.36010676 & 4.41410724 \\
\hline $\mathrm{O}$ & -4.48653052 & 1.04848355 & 6.35001541 \\
\hline $\mathrm{H}$ & -2.30641955 & 0.52931102 & 4.11688656 \\
\hline $\mathrm{H}$ & -2.72470585 & -0.88691181 & 6.64903185 \\
\hline $\mathrm{C}$ & -0.91845116 & -1.17433667 & 5.49286162 \\
\hline $\mathrm{H}$ & -1.34649311 & -1.93510519 & 4.83205212 \\
\hline $\mathrm{H}$ & -0.18729380 & -0.59147350 & 4.92358552 \\
\hline $\mathrm{H}$ & -0.39102695 & -1.68001823 & 6.30749031 \\
\hline $\mathrm{O}$ & -1.54249696 & 0.50262534 & 8.30869720 \\
\hline $\mathrm{H}$ & -0.86122918 & 1.97880812 & 5.59968575 \\
\hline $\mathrm{H}$ & 0.33135984 & 2.35584714 & 8.25118169 \\
\hline $\mathrm{C}$ & 0.61523702 & 3.84072316 & 6.69373395 \\
\hline $\mathrm{H}$ & 0.00063491 & 4.31195267 & 5.91229075 \\
\hline $\mathrm{H}$ & 0.87516753 & 4.63888300 & 7.40122947 \\
\hline $\mathrm{C}$ & 1.90764505 & 3.28017134 & 6.06457180 \\
\hline $\mathrm{H}$ & 1.63015019 & 2.49851326 & 5.34346393 \\
\hline $\mathrm{C}$ & 2.84779907 & 2.65366930 & 7.10481115 \\
\hline $\mathrm{H}$ & 3.76679377 & 2.29551482 & 6.62529775 \\
\hline $\mathrm{H}$ & 3.13274758 & 3.38659428 & 7.87090155 \\
\hline $\mathrm{H}$ & 2.38847792 & 1.80023945 & 7.61437362 \\
\hline $\mathrm{C}$ & 2.62868382 & 4.39126856 & 5.28578894 \\
\hline $\mathrm{H}$ & 3.51846700 & 4.00022632 & 4.77839426 \\
\hline $\mathrm{H}$ & 1.97762950 & 4.83466729 & 4.52204156 \\
\hline $\mathrm{H}$ & 2.95098253 & 5.19754155 & 5.95768660 \\
\hline $\mathrm{O}$ & -1.17045184 & 3.90779623 & 9.40178051 \\
\hline $\mathrm{H}$ & -2.64656122 & 3.63087205 & 6.59839491 \\
\hline $\mathrm{H}$ & -3.15595816 & 5.46323878 & 8.78568812 \\
\hline $\mathrm{C}$ & -4.72655901 & 4.98276250 & 7.39523042 \\
\hline $\mathrm{H}$ & -5.54266918 & 5.32716361 & 8.04368467 \\
\hline $\mathrm{H}$ & -5.10463643 & 4.11167538 & 6.84531379 \\
\hline $\mathrm{O}$ & -3.52560142 & 6.96207950 & 6.69090511 \\
\hline $\mathrm{C}$ & -4.40344562 & 6.12563466 & 6.44292050 \\
\hline $\mathrm{H}$ & -4.93002755 & 6.86362569 & 4.61424690 \\
\hline $\mathrm{H}$ & -5.79429916 & 5.43308957 & 5.10378577 \\
\hline $\mathrm{O}$ & -4.58673842 & 4.29942804 & 10.47036695 \\
\hline $\mathrm{H}$ & -3.91716864 & 2.01137307 & 8.37499011 \\
\hline $\mathrm{C}$ & -4.83346521 & 1.48405987 & 10.20733361 \\
\hline $\mathrm{H}$ & -5.17084367 & 2.07001740 & 11.06363619 \\
\hline $\mathrm{H}$ & -5.67463568 & 0.90487971 & 9.80786203 \\
\hline $\mathrm{H}$ & -4.04749806 & 0.78961814 & 10.52695134 \\
\hline $\mathrm{N}$ & -5.19517573 & 6.20686333 & 5.34887472 \\
\hline
\end{tabular}




\section{${ }^{2}$ TS1-B}

160

\begin{tabular}{|c|c|c|c|}
\hline $\mathrm{C}$ & -2.45839534 & -5.63936697 & -2.38338919 \\
\hline $\mathrm{H}$ & -3.23804349 & -6.36802860 & -2.58686846 \\
\hline $\mathrm{C}$ & -2.05226276 & -4.74783723 & -3.38216463 \\
\hline $\mathrm{H}$ & -2.51446629 & -4.78800343 & -4.36461614 \\
\hline $\mathrm{C}$ & -1.84925162 & -5.59298604 & -1.12545665 \\
\hline $\mathrm{H}$ & -2.15417664 & -6.28553295 & -0.34585485 \\
\hline $\mathrm{C}$ & -1.05478366 & -3.81203421 & -3.13274245 \\
\hline $\mathrm{H}$ & -0.74634077 & -3.15005423 & -3.93063778 \\
\hline C & -0.84894120 & -4.66285584 & -0.87273470 \\
\hline $\mathrm{H}$ & -0.37668833 & -4.62717535 & 0.10558082 \\
\hline C & -0.42992377 & -3.73878057 & -1.86335106 \\
\hline $\mathrm{C}$ & 0.62406905 & -2.81752294 & -1.50159183 \\
\hline $\mathrm{H}$ & 1.07405412 & -3.02108061 & -0.53311532 \\
\hline $\mathrm{C}$ & 1.10913925 & -1.68514797 & -2.13452936 \\
\hline $\mathrm{H}$ & 2.00436077 & -1.26346080 & -1.68927129 \\
\hline $\mathrm{C}$ & 0.76414799 & -1.16159383 & -3.49811840 \\
\hline $\mathrm{H}$ & 1.17934320 & -0.15914364 & -3.62662773 \\
\hline $\mathrm{H}$ & 1.18552755 & -1.80607998 & -4.28300253 \\
\hline $\mathrm{H}$ & -0.31692751 & -1.09890505 & -3.64594598 \\
\hline $\mathrm{O}$ & -0.02336500 & -0.25920540 & -1.08688327 \\
\hline $\mathrm{Fe}$ & 0.19509306 & 0.57428687 & 0.32942854 \\
\hline $\mathrm{N}$ & -0.10837928 & 2.29838532 & -0.68391749 \\
\hline $\mathrm{N}$ & 2.18097398 & 0.80207698 & 0.11209616 \\
\hline $\mathrm{N}$ & 0.51061295 & -1.03711836 & 1.49286960 \\
\hline $\mathrm{N}$ & -1.78634677 & 0.45288101 & 0.70784598 \\
\hline $\mathrm{C}$ & -1.31845961 & 2.85802133 & -1.00236443 \\
\hline $\mathrm{C}$ & -1.12551586 & 4.00622559 & -1.85956718 \\
\hline $\mathrm{C}$ & 0.21393259 & 4.12617125 & -2.06148913 \\
\hline $\mathrm{C}$ & 0.83970757 & 3.04372195 & -1.33818006 \\
\hline $\mathrm{C}$ & 2.82207160 & 1.75176020 & -0.64769959 \\
\hline $\mathrm{C}$ & 4.24776802 & 1.54083346 & -0.60629412 \\
\hline $\mathrm{C}$ & 4.46366477 & 0.45876327 & 0.19302242 \\
\hline $\mathrm{C}$ & 3.16964104 & 0.00149358 & 0.63328522 \\
\hline $\mathrm{C}$ & 1.72120190 & -1.58024997 & 1.84458593 \\
\hline $\mathrm{C}$ & 1.52384624 & -2.74605302 & 2.67296157 \\
\hline $\mathrm{C}$ & 0.17871491 & -2.90032136 & 2.81721345 \\
\hline $\mathrm{C}$ & -0.44469008 & -1.82562253 & 2.08187226 \\
\hline C & -2.43412757 & -0.56825718 & 1.35261069 \\
\hline $\mathrm{C}$ & -3.86416455 & -0.38488521 & 1.26783479 \\
\hline $\mathrm{C}$ & -4.07052678 & 0.75558595 & 0.55528512 \\
\hline $\mathrm{C}$ & -2.76687227 & 1.26538774 & 0.19922518 \\
\hline
\end{tabular}




\begin{tabular}{|c|c|c|c|}
\hline $\mathrm{C}$ & -2.55752628 & 2.39170473 & -0.58426069 \\
\hline $\mathrm{H}$ & -3.43551638 & 2.93176956 & -0.92554242 \\
\hline $\mathrm{C}$ & 2.20436183 & 2.79631542 & -1.32642737 \\
\hline $\mathrm{H}$ & 2.83979155 & 3.48627863 & -1.87157480 \\
\hline $\mathrm{C}$ & 2.96303736 & -1.10294468 & 1.44923665 \\
\hline $\mathrm{H}$ & 3.84033793 & -1.63874492 & 1.79760879 \\
\hline C & -1.81684692 & -1.62715747 & 2.0036806 \\
\hline $\mathrm{H}$ & -2.45584028 & -2.34996689 & 2.5010477 \\
\hline $\mathrm{H}$ & 0.75026116 & 4.87375199 & -2.63033533 \\
\hline $\mathrm{H}$ & -1.92420087 & 4.62721383 & -2.24530100 \\
\hline $\mathrm{H}$ & 4.97531055 & 2.15320410 & -1.12387307 \\
\hline $\mathrm{H}$ & 5.40470714 & -0.00020854 & 0.4680647 \\
\hline $\mathrm{H}$ & 2.32112175 & -3.35108264 & 3.08541200 \\
\hline $\mathrm{H}$ & -0.35770520 & -3.65356247 & 3.3801344 \\
\hline $\mathrm{H}$ & -4.59724319 & -1.05256779 & 1.7020269 \\
\hline $\mathrm{H}$ & -5.00934899 & 1.21761865 & 0.27758477 \\
\hline S & 0.31046681 & 1.95461702 & 2.49323881 \\
\hline $\mathrm{C}$ & 1.64897031 & 3.20059898 & 2.33511503 \\
\hline $\mathrm{H}$ & 2.23497884 & 3.13951968 & 3.26010770 \\
\hline $\mathrm{H}$ & 2.31098264 & 2.93676748 & 1.51095244 \\
\hline $\mathrm{H}$ & 0.81296156 & 4.78253643 & 1.13807212 \\
\hline $\mathrm{C}$ & 1.20414407 & 4.66826712 & 2.14592162 \\
\hline $\mathrm{C}$ & 0.16352825 & 5.07468363 & 3.20279585 \\
\hline $\mathrm{N}$ & -1.08043733 & 5.41926632 & 2.7857759 \\
\hline $\mathrm{C}$ & -2.09833205 & 5.84693580 & 3.7656076 \\
\hline $\mathrm{C}$ & -2.43912471 & 4.80436060 & 4.83833202 \\
\hline $\mathrm{N}$ & -2.44996482 & 3.50650944 & 4.44341262 \\
\hline $\mathrm{C}$ & -2.85080064 & 2.43262591 & 5.35383778 \\
\hline $\mathrm{C}$ & -1.82976143 & 2.22631197 & 6.49495627 \\
\hline $\mathrm{N}$ & -0.52449388 & 2.11792519 & 6.12305537 \\
\hline $\mathrm{C}$ & 0.52798143 & 1.99333199 & 7.12825126 \\
\hline $\mathrm{C}$ & 0.60630019 & 3.21795305 & 8.06499966 \\
\hline $\mathrm{N}$ & 0.48630151 & 4.45907188 & 7.50722037 \\
\hline $\mathrm{C}$ & 0.54701076 & 5.61559247 & 8.39775405 \\
\hline C & -0.60394530 & 5.67649139 & 9.4344518 \\
\hline $\mathrm{N}$ & -1.82524486 & 5.29435473 & 8.9823719 \\
\hline $\mathrm{N}$ & 2.38921234 & 5.51600589 & 2.2203404 \\
\hline $\mathrm{C}$ & 2.82241349 & 6.21794681 & 1.13476577 \\
\hline C & 4.08372566 & 7.09733636 & 1.3105235 \\
\hline $\mathrm{N}$ & 4.97924179 & 6.67419387 & 2.40487107 \\
\hline C & 4.74759964 & 7.14728111 & 3.66567261 \\
\hline $\mathrm{C}$ & 5.66016342 & 6.66027000 & 4.80675708 \\
\hline $\mathrm{N}$ & 5.36917034 & 7.36624407 & 6.03007581 \\
\hline C & 6.26256508 & 8.25658659 & 6.55181928 \\
\hline C & 5.82427371 & 8.91195924 & 7.8524365 \\
\hline
\end{tabular}




\begin{tabular}{|c|c|c|c|}
\hline $\mathrm{H}$ & 4.86981468 & 8.53063071 & 8.22516582 \\
\hline $\mathrm{H}$ & 5.74539817 & 9.99301198 & 7.69521819 \\
\hline $\mathrm{H}$ & 6.59987867 & 8.74997500 & 8.60748849 \\
\hline $\mathrm{O}$ & 7.34381490 & 8.51311098 & 6.02322918 \\
\hline $\mathrm{H}$ & 4.46414957 & 7.20023100 & 6.48026593 \\
\hline $\mathrm{H}$ & 6.70502827 & 6.87413771 & 4.55862954 \\
\hline $\mathrm{C}$ & 5.45004789 & 5.14524199 & 5.04165775 \\
\hline $\mathrm{H}$ & 6.16586686 & 4.79603273 & 5.79111797 \\
\hline $\mathrm{H}$ & 4.44273423 & 4.96804466 & 5.43514263 \\
\hline $\mathrm{H}$ & 5.57616292 & 4.54779541 & 4.13380992 \\
\hline $\mathrm{O}$ & 3.75042760 & 7.84850383 & 3.87060704 \\
\hline $\mathrm{C}$ & 6.25828035 & 6.13501466 & 1.89799113 \\
\hline $\mathrm{H}$ & 7.06573385 & 6.86056600 & 2.06191937 \\
\hline $\mathrm{H}$ & 6.53162339 & 5.20569686 & 2.40398618 \\
\hline C & 5.98926080 & 5.93253305 & 0.39989703 \\
\hline $\mathrm{H}$ & 6.90789756 & 5.98293180 & -0.19171869 \\
\hline $\mathrm{H}$ & 5.53263084 & 4.94924177 & 0.23178029 \\
\hline $\mathrm{C}$ & 4.98463790 & 7.04593986 & 0.06477626 \\
\hline $\mathrm{H}$ & 5.50451602 & 8.00377400 & -0.04942571 \\
\hline $\mathrm{H}$ & 4.39798154 & 6.85833262 & -0.83538838 \\
\hline $\mathrm{H}$ & 3.72203370 & 8.10883096 & 1.51991669 \\
\hline $\mathrm{O}$ & 2.26100381 & 6.17145557 & 0.03974624 \\
\hline $\mathrm{H}$ & 2.76218214 & 5.72489434 & 3.13723737 \\
\hline $\mathrm{O}$ & 0.46261349 & 5.03788306 & 4.40648724 \\
\hline $\mathrm{C}$ & -1.48416104 & 5.74440372 & 1.39611356 \\
\hline $\mathrm{H}$ & -0.66114658 & 6.19979618 & 0.84160482 \\
\hline $\mathrm{H}$ & -1.78638047 & 4.83219208 & 0.87162842 \\
\hline $\mathrm{C}$ & -2.66842174 & 6.70233256 & 1.5847759 \\
\hline $\mathrm{H}$ & -2.30592931 & 7.73070403 & 1.6996468 \\
\hline $\mathrm{H}$ & -3.35446542 & 6.68234609 & 0.7327059 \\
\hline $\mathrm{C}$ & -3.31199143 & 6.21637963 & 2.89036925 \\
\hline $\mathrm{H}$ & -3.92016971 & 5.32274878 & 2.7068094 \\
\hline $\mathrm{H}$ & -3.94524612 & 6.96153433 & 3.37762646 \\
\hline $\mathrm{H}$ & -1.73455445 & 6.72081339 & 4.3173839 \\
\hline $\mathrm{O}$ & -2.77627280 & 5.17668248 & 5.9599828 \\
\hline $\mathrm{H}$ & -1.99448948 & 3.25459559 & 3.57018227 \\
\hline $\mathrm{H}$ & -3.76088666 & 2.75645151 & 5.8646462 \\
\hline $\mathrm{C}$ & -3.10883020 & 1.13590536 & $4.5869153 \subseteq$ \\
\hline $\mathrm{H}$ & -3.89703427 & 1.27772407 & $3.8403185^{\prime}$ \\
\hline $\mathrm{H}$ & -2.20682551 & 0.78533551 & 4.07564766 \\
\hline $\mathrm{H}$ & -3.42865413 & 0.35822997 & 5.28759888 \\
\hline $\mathrm{O}$ & -2.20587477 & 2.10734854 & 7.6559478 \\
\hline $\mathrm{H}$ & -0.26399496 & 2.30442009 & 5.16071478 \\
\hline $\mathrm{H}$ & 0.25713807 & 1.17810167 & 7.8030171 \\
\hline $\mathrm{C}$ & 1.90181812 & 1.72438754 & 6.4966575 \\
\hline
\end{tabular}




$\begin{array}{lrrc}\mathrm{H} & 2.14238200 & 2.54952257 & 5.81038358 \\ \mathrm{H} & 2.63680072 & 1.77680877 & 7.31062671 \\ \mathrm{C} & 2.06273818 & 0.37861970 & 5.75961091 \\ \mathrm{H} & 1.34658257 & 0.35125597 & 4.92729310 \\ \mathrm{C} & 1.78930470 & -0.82859394 & 6.66871017 \\ \mathrm{H} & 1.94424327 & -1.76571670 & 6.12012614 \\ \mathrm{H} & 2.46311135 & -0.83165653 & 7.53560456 \\ \mathrm{H} & 0.76131844 & -0.83611561 & 7.04586351 \\ \mathrm{C} & 3.47418366 & 0.29162180 & 5.15861505 \\ \mathrm{H} & 3.60338239 & -0.63839229 & 4.59244438 \\ \mathrm{H} & 3.67093762 & 1.12677647 & 4.47531795 \\ \mathrm{H} & 4.23952377 & 0.31459779 & 5.94573206 \\ \mathrm{O} & 0.84910965 & 3.05521330 & 9.25454787 \\ \mathrm{H} & 0.30469975 & 4.57801971 & 6.51626856 \\ \mathrm{H} & 1.46390944 & 5.56079364 & 8.98772544 \\ \mathrm{C} & 0.52541359 & 6.93132610 & 7.61913519 \\ \mathrm{H} & 0.40206754 & 7.73761769 & 8.35388827 \\ \mathrm{H} & -0.34937073 & 6.96773733 & 6.95786138 \\ \mathrm{O} & 2.90310456 & 6.78433989 & 7.19529868 \\ \mathrm{C} & 1.80220365 & 7.22966761 & 6.84636891 \\ \mathrm{H} & 2.45384585 & 8.20184821 & 5.17485041 \\ \mathrm{H} & 0.73791527 & 8.28694574 & 5.45044447 \\ \mathrm{O} & -0.39805802 & 6.17621371 & 10.53651863 \\ \mathrm{H} & -1.90053832 & 4.84327455 & 8.07906126 \\ \mathrm{C} & -2.98922651 & 5.24828078 & 9.84606968 \\ \mathrm{H} & -2.71706357 & 5.71021385 & 10.79638321 \\ \mathrm{H} & -3.82458869 & 5.79982583 & 9.39819123 \\ \mathrm{H} & -3.30420392 & 4.21254019 & 10.02039520 \\ \mathrm{~N} & 1.65991215 & 8.08126642 & 5.80465768\end{array}$




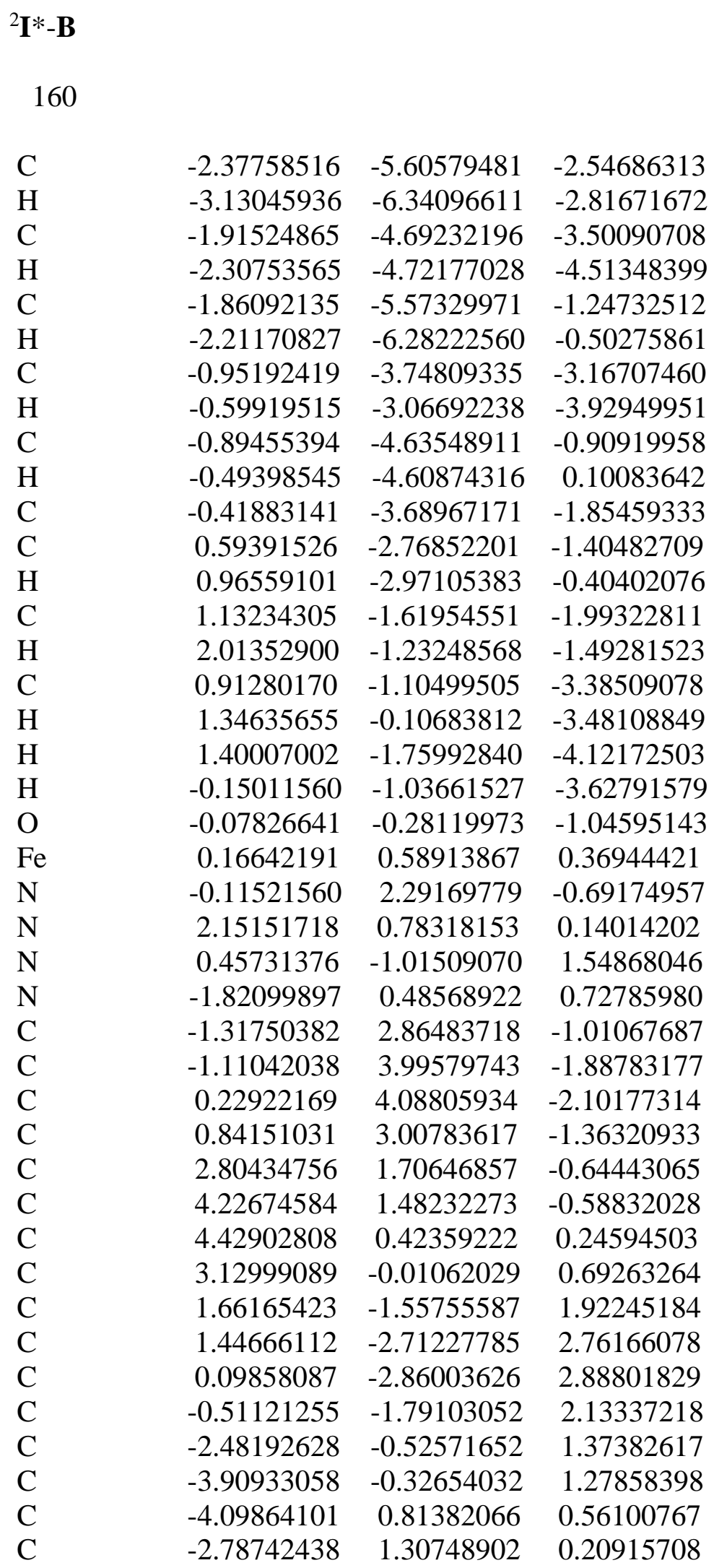




\begin{tabular}{|c|c|c|c|}
\hline $\mathrm{C}$ & -2.56281381 & 2.42445563 & -0.58331281 \\
\hline $\mathrm{H}$ & -3.43302604 & 2.97394756 & -0.92913867 \\
\hline $\mathrm{C}$ & 2.20223456 & 2.73993359 & -1.35205429 \\
\hline $\mathrm{H}$ & 2.84713761 & 3.40645666 & -1.91472575 \\
\hline $\mathrm{C}$ & 2.91037483 & -1.09518533 & 1.53156357 \\
\hline $\mathrm{H}$ & 3.78112784 & -1.62823671 & 1.89934761 \\
\hline C & -1.88110998 & -1.58576466 & 2.0378117 \\
\hline $\mathrm{H}$ & -2.53051587 & -2.29872867 & 2.5357855 \\
\hline $\mathrm{H}$ & 0.77415718 & 4.81642272 & -2.68713179 \\
\hline $\mathrm{H}$ & -1.90096295 & 4.62437274 & -2.27809500 \\
\hline $\mathrm{H}$ & 4.96305692 & 2.07252433 & -1.11917485 \\
\hline $\mathrm{H}$ & 5.36484740 & -0.03402952 & 0.5404657 \\
\hline $\mathrm{H}$ & 2.23530047 & -3.31579921 & 3.1925943 \\
\hline $\mathrm{H}$ & -0.44845854 & -3.60455941 & 3.4525212 \\
\hline $\mathrm{H}$ & -4.65251031 & -0.98378937 & 1.7116878 \\
\hline $\mathrm{H}$ & -5.03060040 & 1.28562395 & 0.2764777 \\
\hline S & 0.30132293 & 1.94191022 & 2.48480555 \\
\hline $\mathrm{C}$ & 1.63869112 & 3.19135211 & 2.30845528 \\
\hline $\mathrm{H}$ & 2.24011902 & 3.13162133 & 3.22300404 \\
\hline $\mathrm{H}$ & 2.28512127 & 2.93167417 & 1.47150926 \\
\hline $\mathrm{H}$ & 0.78934439 & 4.77506193 & 1.12332932 \\
\hline $\mathrm{C}$ & 1.18567830 & 4.65791561 & 2.12875118 \\
\hline $\mathrm{C}$ & 0.14860553 & 5.05831729 & 3.19129732 \\
\hline $\mathrm{N}$ & -1.09701320 & 5.40526028 & 2.7813465 \\
\hline $\mathrm{C}$ & -2.11035026 & 5.83066888 & 3.7665629 \\
\hline $\mathrm{C}$ & -2.44140311 & 4.78961064 & 4.84406065 \\
\hline $\mathrm{N}$ & -2.44884150 & 3.49026747 & 4.4544384 \\
\hline $\mathrm{C}$ & -2.84013771 & 2.41798980 & 5.37096179 \\
\hline $\mathrm{C}$ & -1.81066484 & 2.21840105 & 6.5057459 \\
\hline $\mathrm{N}$ & -0.50759931 & 2.11538415 & 6.1270317 \\
\hline $\mathrm{C}$ & 0.55048580 & 1.99285828 & 7.12654299 \\
\hline $\mathrm{C}$ & 0.62742720 & 3.21747946 & 8.06289204 \\
\hline $\mathrm{N}$ & 0.49808192 & 4.45788530 & 7.50633202 \\
\hline $\mathrm{C}$ & 0.55953694 & 5.61425768 & 8.39703029 \\
\hline C & -0.58591568 & 5.66948185 & 9.44020200 \\
\hline $\mathrm{N}$ & -1.80869115 & 5.28401361 & 8.99475054 \\
\hline $\mathrm{N}$ & 2.36789133 & 5.51002131 & 2.2007729 \\
\hline $\mathrm{C}$ & 2.79087005 & 6.22246916 & 1.11856936 \\
\hline C & 4.05400115 & 7.10055129 & 1.2900754 \\
\hline $\mathrm{N}$ & 4.95870209 & 6.67067069 & 2.3740087 \\
\hline C & 4.73673539 & 7.13321888 & $3.6403030 €$ \\
\hline $\mathrm{C}$ & 5.65420354 & 6.63282316 & $4.7715921 \mathrm{~s}$ \\
\hline $\mathrm{N}$ & 5.37525064 & 7.33166423 & 6.00182997 \\
\hline C & 6.27620489 & 8.21440711 & 6.5232208 \\
\hline C & 5.84631022 & 8.86951214 & 7.8267154 \\
\hline
\end{tabular}




\begin{tabular}{|c|c|c|c|}
\hline $\mathrm{H}$ & 4.88932071 & 8.49524387 & 8.20000793 \\
\hline $\mathrm{H}$ & 5.77667035 & 9.95169821 & 7.67281214 \\
\hline $\mathrm{H}$ & 6.62213611 & 8.69856547 & 8.57959497 \\
\hline $\mathrm{O}$ & 7.35822674 & 8.46398314 & 5.99264543 \\
\hline $\mathrm{H}$ & 4.46642229 & 7.17937288 & 6.44932230 \\
\hline $\mathrm{H}$ & 6.69866679 & 6.84245424 & 4.51835518 \\
\hline $\mathrm{C}$ & 5.43698885 & 5.11734832 & 4.99672080 \\
\hline $\mathrm{H}$ & 6.15514771 & 4.75845058 & 5.73935660 \\
\hline $\mathrm{H}$ & 4.43094122 & 4.94271093 & 5.39490788 \\
\hline $\mathrm{H}$ & 5.55393899 & 4.52583531 & 4.08370400 \\
\hline $\mathrm{O}$ & 3.74462314 & 7.83736308 & 3.85822098 \\
\hline C & 6.23078085 & 6.12836702 & 1.85392839 \\
\hline $\mathrm{H}$ & 7.04304813 & 6.84896906 & 2.01596563 \\
\hline $\mathrm{H}$ & 6.50369141 & 5.19455718 & 2.35196975 \\
\hline $\mathrm{C}$ & 5.94883685 & 5.93636301 & 0.35671207 \\
\hline $\mathrm{H}$ & 6.86286594 & 5.98758394 & -0.24195247 \\
\hline $\mathrm{H}$ & 5.48770816 & 4.95559029 & 0.18612302 \\
\hline $\mathrm{C}$ & 4.94480499 & 7.05482638 & 0.03676075 \\
\hline $\mathrm{H}$ & 5.46649778 & 8.01189837 & -0.07575083 \\
\hline $\mathrm{H}$ & 4.35033862 & 6.87411198 & -0.85970289 \\
\hline $\mathrm{H}$ & 3.69494859 & 8.11107273 & 1.50804290 \\
\hline $\mathrm{O}$ & 2.21872766 & 6.18818270 & 0.02871577 \\
\hline $\mathrm{H}$ & 2.75323332 & 5.70422633 & 3.11555278 \\
\hline $\mathrm{O}$ & 0.45450345 & 5.02389704 & 4.3933683 \\
\hline $\mathrm{C}$ & -1.51073513 & 5.72432330 & 1.39346544 \\
\hline $\mathrm{H}$ & -0.69327301 & 6.18231904 & 0.8327854 \\
\hline $\mathrm{H}$ & -1.81189557 & 4.80916794 & 0.8737635 \\
\hline $\mathrm{C}$ & -2.69821432 & 6.67736653 & 1.58670372 \\
\hline $\mathrm{H}$ & -2.33987221 & 7.70787828 & 1.69537322 \\
\hline $\mathrm{H}$ & -3.38986290 & 6.65092607 & 0.73932104 \\
\hline $\mathrm{C}$ & -3.33105308 & 6.19334917 & 2.89824887 \\
\hline $\mathrm{H}$ & -3.93723231 & 5.29697088 & 2.72171074 \\
\hline $\mathrm{H}$ & -3.96357674 & 6.93793942 & 3.3873161 \\
\hline $\mathrm{H}$ & -1.74688110 & 6.70695970 & 4.31477347 \\
\hline $\mathrm{O}$ & -2.77596239 & 5.16565996 & 5.9653090 \\
\hline $\mathrm{H}$ & -1.99289719 & 3.23624547 & 3.58186718 \\
\hline $\mathrm{H}$ & -3.74812550 & 2.74003964 & 5.8864605 \\
\hline $\mathrm{C}$ & -3.09748772 & 1.11814887 & 4.6096089 \\
\hline $\mathrm{H}$ & -3.89037231 & 1.25490004 & $3.8670833 \subseteq$ \\
\hline $\mathrm{H}$ & -2.19694646 & 0.77025708 & 4.0939192 \\
\hline $\mathrm{H}$ & -3.40980609 & 0.34105132 & 5.3143645 \\
\hline $\mathrm{O}$ & -2.17886472 & 2.10537932 & 7.67021136 \\
\hline $\mathrm{H}$ & -0.25274676 & 2.27929846 & 5.15891177 \\
\hline $\mathrm{H}$ & 0.28763023 & 1.17608488 & 7.80271160 \\
\hline $\mathrm{C}$ & 1.92307519 & 1.73298001 & 6.4882681 \\
\hline
\end{tabular}




\begin{tabular}{lrrl}
$\mathrm{H}$ & 2.14588827 & 2.55010317 & 5.78679384 \\
$\mathrm{H}$ & 2.66368719 & 1.80832560 & 7.29527754 \\
$\mathrm{C}$ & 2.09876832 & 0.37806675 & 5.77180330 \\
$\mathrm{H}$ & 1.37357559 & 0.32415561 & 4.94865754 \\
$\mathrm{C}$ & 1.85884955 & -0.81790614 & 6.70500491 \\
$\mathrm{H}$ & 2.02572404 & -1.76169088 & 6.17146749 \\
$\mathrm{H}$ & 2.54235034 & -0.79261702 & 7.56394548 \\
$\mathrm{H}$ & 0.83564279 & -0.83864577 & 7.09444282 \\
$\mathrm{C}$ & 3.50499587 & 0.30670554 & 5.15643799 \\
$\mathrm{H}$ & 3.64768035 & -0.63283556 & 4.60963160 \\
$\mathrm{H}$ & 3.67612255 & 1.12996643 & 4.45227642 \\
$\mathrm{H}$ & 4.27836086 & 0.36306779 & 5.93399212 \\
$\mathrm{O}$ & 0.87879615 & 3.05601336 & 9.25094645 \\
$\mathrm{H}$ & 0.30577770 & 4.57588084 & 6.51723954 \\
$\mathrm{H}$ & 1.48005935 & 5.56270507 & 8.98153552 \\
$\mathrm{C}$ & 0.52856301 & 6.92936033 & 7.61775290 \\
$\mathrm{H}$ & 0.40867728 & 7.73636665 & 8.35218928 \\
$\mathrm{H}$ & -0.35111673 & 6.96225992 & 6.96287330 \\
$\mathrm{O}$ & 2.90522802 & 6.79832908 & 7.18650721 \\
$\mathrm{C}$ & 1.79918448 & 7.22858264 & 6.83532361 \\
$\mathrm{H}$ & 2.43456229 & 8.18269609 & 5.14766964 \\
$\mathrm{H}$ & 0.71880499 & 8.24634763 & 5.42414146 \\
$\mathrm{O}$ & -0.37577978 & 6.16882155 & 10.54171918 \\
$\mathrm{H}$ & -1.88608765 & 4.82869118 & 8.09378115 \\
$\mathrm{C}$ & -2.96550613 & 5.22757656 & 9.86749902 \\
$\mathrm{H}$ & -2.69262305 & 5.69934082 & 10.81276551 \\
$\mathrm{H}$ & -3.81125674 & 5.76476160 & 9.42187190 \\
$\mathrm{H}$ & -3.26527873 & 4.18885625 & 10.05107795 \\
$\mathrm{~N}$ & 1.64416711 & 8.06387272 & 5.78210864 \\
& & & \\
\hline & & & \\
& & &
\end{tabular}


160

\begin{tabular}{|c|c|c|c|}
\hline $\mathrm{C}$ & -3.15191294 & -2.91520812 & -4.32468356 \\
\hline $\mathrm{H}$ & -3.86630334 & -3.08973812 & -5.12450157 \\
\hline $\mathrm{C}$ & -2.47902940 & -1.69510707 & -4.23828124 \\
\hline $\mathrm{H}$ & -2.67135716 & -0.91449378 & -4.96948859 \\
\hline $\mathrm{C}$ & -2.90918049 & -3.90862597 & -3.37273781 \\
\hline $\mathrm{H}$ & -3.43424658 & -4.85827695 & -3.42860415 \\
\hline $\mathrm{C}$ & -1.55988687 & -1.46774973 & -3.21215449 \\
\hline $\mathrm{H}$ & -1.04613923 & -0.51532353 & -3.13167907 \\
\hline C & -1.99652572 & -3.68164841 & -2.34333381 \\
\hline $\mathrm{H}$ & -1.81604965 & -4.45475266 & -1.59945528 \\
\hline C & -1.30707154 & -2.46371446 & -2.26021115 \\
\hline $\mathrm{C}$ & -0.32642020 & -2.26974407 & -1.15235692 \\
\hline $\mathrm{H}$ & -0.63386205 & -2.67998614 & -0.19171360 \\
\hline $\mathrm{C}$ & 1.13782385 & -2.10376022 & -1.33874277 \\
\hline $\mathrm{H}$ & 1.75532516 & -2.39154984 & -0.48921581 \\
\hline $\mathrm{C}$ & 1.83484663 & -2.08774938 & -2.67099598 \\
\hline $\mathrm{H}$ & 2.63915651 & -1.34384819 & -2.66351225 \\
\hline $\mathrm{H}$ & 2.28363888 & -3.06981592 & -2.86159803 \\
\hline $\mathrm{H}$ & 1.14719403 & -1.85581843 & -3.48756120 \\
\hline $\mathrm{O}$ & 0.28999290 & -0.96568597 & -1.03443951 \\
\hline $\mathrm{Fe}$ & 0.39902047 & 0.33599515 & 0.78026944 \\
\hline $\mathrm{N}$ & 0.12895730 & 1.81796860 & -0.54134197 \\
\hline $\mathrm{N}$ & 2.38296190 & 0.42471309 & 0.43274610 \\
\hline $\mathrm{N}$ & 0.66468038 & -1.29986406 & 1.93943386 \\
\hline $\mathrm{N}$ & -1.59228149 & 0.07547543 & 0.94564497 \\
\hline $\mathrm{C}$ & -1.07635575 & 2.35013677 & -0.92801059 \\
\hline $\mathrm{C}$ & -0.86822050 & 3.42042734 & -1.87609483 \\
\hline $\mathrm{C}$ & 0.47501199 & 3.52431288 & -2.06559003 \\
\hline $\mathrm{C}$ & 1.08857169 & 2.51205758 & -1.24071808 \\
\hline $\mathrm{C}$ & 3.05193786 & 1.28464159 & -0.40722129 \\
\hline $\mathrm{C}$ & 4.47374495 & 1.05421035 & -0.33032024 \\
\hline $\mathrm{C}$ & 4.66056219 & 0.05300535 & 0.57404824 \\
\hline C & 3.35242478 & -0.33504053 & 1.04333568 \\
\hline $\mathrm{C}$ & 1.87235336 & -1.79692945 & 2.37208476 \\
\hline C & 1.66274118 & -2.88180654 & 3.30059852 \\
\hline C & 0.31620174 & -3.03565813 & 3.42962087 \\
\hline $\mathrm{C}$ & -0.29812137 & -2.04143215 & 2.58467392 \\
\hline $\mathrm{C}$ & -2.26555006 & -0.87425032 & 1.67759932 \\
\hline $\mathrm{C}$ & -3.69105185 & -0.69434452 & 1.54167874 \\
\hline $\mathrm{C}$ & -3.87390145 & 0.37820546 & 0.72390732 \\
\hline C & -2.55999700 & 0.84995094 & 0.35448575 \\
\hline
\end{tabular}




\begin{tabular}{|c|c|c|c|}
\hline $\mathrm{C}$ & -2.32659150 & 1.91331310 & -0.50772146 \\
\hline $\mathrm{H}$ & -3.19456389 & 2.42403235 & -0.91408434 \\
\hline $\mathrm{C}$ & 2.45323844 & 2.26605693 & -1.18841877 \\
\hline $\mathrm{H}$ & 3.09877370 & 2.89420234 & -1.79370377 \\
\hline $\mathrm{C}$ & 3.12217526 & -1.35465552 & 1.95892436 \\
\hline $\mathrm{H}$ & 3.99050325 & -1.85871458 & 2.37160487 \\
\hline $\mathrm{C}$ & -1.66909057 & -1.86169959 & 2.45048763 \\
\hline $\mathrm{H}$ & -2.32093744 & -2.53287567 & 3.00084686 \\
\hline $\mathrm{H}$ & 1.01857519 & 4.22084627 & -2.68949786 \\
\hline $\mathrm{H}$ & -1.65941081 & 4.00378306 & -2.33013263 \\
\hline $\mathrm{H}$ & 5.21810375 & 1.59635685 & -0.89980329 \\
\hline $\mathrm{H}$ & 5.59001662 & -0.39602121 & 0.90058149 \\
\hline $\mathrm{H}$ & 2.45398448 & -3.44408778 & 3.77990944 \\
\hline $\mathrm{H}$ & -0.22594902 & -3.74664751 & 4.03988431 \\
\hline $\mathrm{H}$ & -4.43883503 & -1.31882327 & 2.0136493 \\
\hline $\mathrm{H}$ & -4.80411202 & 0.81389317 & 0.38173638 \\
\hline S & 0.47605122 & 1.67014804 & 2.63450532 \\
\hline $\mathrm{C}$ & 1.70563140 & 3.00920693 & 2.3547189 \\
\hline $\mathrm{H}$ & 2.32957682 & 3.03724196 & 3.25499990 \\
\hline $\mathrm{H}$ & 2.34598149 & 2.75511621 & 1.51222101 \\
\hline $\mathrm{H}$ & 0.70811112 & 4.45999995 & 1.11718524 \\
\hline $\mathrm{C}$ & 1.13606589 & 4.42613576 & 2.11595171 \\
\hline $\mathrm{C}$ & 0.09792073 & 4.81075414 & 3.18480363 \\
\hline $\mathrm{N}$ & -1.17841621 & 5.04220192 & 2.78519830 \\
\hline $\mathrm{C}$ & -2.19897788 & 5.46279860 & 3.76639135 \\
\hline $\mathrm{C}$ & -2.43567657 & 4.48043014 & 4.91986333 \\
\hline $\mathrm{N}$ & -2.36111495 & 3.15963145 & 4.62115320 \\
\hline C & -2.66842934 & 2.12702148 & 5.61217937 \\
\hline $\mathrm{C}$ & -1.61257641 & 2.07604360 & 6.73885279 \\
\hline $\mathrm{N}$ & -0.31095937 & 2.03675066 & 6.33910359 \\
\hline $\mathrm{C}$ & 0.77066129 & 2.06388181 & 7.32064252 \\
\hline $\mathrm{C}$ & 0.78024547 & 3.35552241 & 8.16527889 \\
\hline $\mathrm{N}$ & 0.55438853 & 4.54165712 & 7.52622059 \\
\hline $\mathrm{C}$ & 0.55819517 & 5.75934641 & 8.33365614 \\
\hline $\mathrm{C}$ & -0.56366890 & 5.81286077 & 9.40195874 \\
\hline $\mathrm{N}$ & -1.76778630 & 5.31773316 & 9.01809868 \\
\hline $\mathrm{N}$ & 2.25236628 & 5.36513616 & 2.10980179 \\
\hline C & 2.59492823 & 6.04532976 & 0.97758863 \\
\hline $\mathrm{C}$ & 3.79202275 & 7.02194903 & 1.06473282 \\
\hline $\mathrm{N}$ & 4.75251269 & 6.72210741 & 2.1440326 \\
\hline $\mathrm{C}$ & 4.52429694 & 7.23979519 & 3.38774878 \\
\hline $\mathrm{C}$ & 5.50256512 & 6.87929226 & 4.52085780 \\
\hline $\mathrm{N}$ & 5.19634942 & 7.62675380 & 5.71549985 \\
\hline $\mathrm{C}$ & 6.03683721 & 8.60584641 & 6.16107433 \\
\hline C & 5.58539263 & 9.30280193 & 7.4352565 \\
\hline
\end{tabular}




\begin{tabular}{|c|c|c|c|}
\hline $\mathrm{H}$ & 4.67047756 & 8.87746313 & 7.85632366 \\
\hline $\mathrm{H}$ & 5.42511687 & 10.36474922 & 7.22035118 \\
\hline $\mathrm{H}$ & 6.38936898 & 9.23965252 & 8.17516643 \\
\hline $\mathrm{O}$ & 7.08408513 & 8.90543090 & 5.58867413 \\
\hline $\mathrm{H}$ & 4.31147989 & 7.43258424 & 6.19323159 \\
\hline $\mathrm{H}$ & 6.52161427 & 7.15338624 & 4.22860434 \\
\hline C & 5.41047009 & 5.36755814 & 4.83758251 \\
\hline $\mathrm{H}$ & 6.16915975 & 5.11042745 & 5.58204318 \\
\hline $\mathrm{H}$ & 4.42937647 & 5.13890916 & 5.26909777 \\
\hline $\mathrm{H}$ & 5.55704689 & 4.73444732 & 3.95730286 \\
\hline $\mathrm{O}$ & 3.48467473 & 7.87669960 & 3.59105333 \\
\hline C & 6.05064094 & 6.25110782 & 1.61858672 \\
\hline $\mathrm{H}$ & 6.80747756 & 7.03995841 & 1.71904007 \\
\hline $\mathrm{H}$ & 6.40728338 & 5.37033750 & 2.15850542 \\
\hline C & 5.74899213 & 5.95470701 & 0.14232797 \\
\hline $\mathrm{H}$ & 6.64144774 & 6.04297420 & -0.48381750 \\
\hline $\mathrm{H}$ & 5.36269882 & 4.93337562 & 0.03723803 \\
\hline $\mathrm{C}$ & 4.65289575 & 6.97274748 & -0.20940263 \\
\hline $\mathrm{H}$ & 5.09499902 & 7.95917727 & -0.38883984 \\
\hline $\mathrm{H}$ & 4.05313203 & 6.69666883 & -1.07754071 \\
\hline $\mathrm{H}$ & 3.36238436 & 8.01344403 & 1.23754167 \\
\hline $\mathrm{O}$ & 1.99765880 & 5.90983770 & -0.08978359 \\
\hline $\mathrm{H}$ & 2.64219483 & 5.64106743 & 3.00152953 \\
\hline $\mathrm{O}$ & 0.42904607 & 4.85448531 & 4.3799150 \\
\hline C & -1.63498486 & 5.26547554 & 1.38982547 \\
\hline $\mathrm{H}$ & -0.86063675 & 5.75318423 & 0.79414270 \\
\hline $\mathrm{H}$ & -1.87642626 & 4.30692015 & 0.91912768 \\
\hline C & -2.88498316 & 6.14019279 & 1.55825043 \\
\hline $\mathrm{H}$ & -2.60081286 & 7.19807574 & 1.60464981 \\
\hline $\mathrm{H}$ & -3.58732739 & 6.01832002 & 0.72835108 \\
\hline $\mathrm{C}$ & -3.45819895 & 5.68464140 & 2.90660137 \\
\hline $\mathrm{H}$ & -3.99902494 & 4.73772613 & 2.79194810 \\
\hline $\mathrm{H}$ & -4.13584470 & 6.40673989 & 3.3680994 \\
\hline $\mathrm{H}$ & -1.88539178 & 6.39728109 & 4.24495814 \\
\hline $\mathrm{O}$ & -2.76406599 & 4.90901273 & 6.02351843 \\
\hline $\mathrm{H}$ & -1.95487494 & 2.87734619 & 3.73453621 \\
\hline $\mathrm{H}$ & -3.59014395 & 2.42028939 & 6.1209748 \\
\hline C & -2.84473998 & 0.76452494 & 4.9434653 \\
\hline $\mathrm{H}$ & -3.65053903 & 0.79798708 & 4.20265837 \\
\hline $\mathrm{H}$ & -1.92694917 & 0.44049467 & 4.4432760 \\
\hline $\mathrm{H}$ & -3.10036059 & 0.01921430 & 5.7030277 \\
\hline $\mathrm{O}$ & -1.95474313 & 2.00306440 & 7.9134885 \\
\hline $\mathrm{H}$ & -0.08886956 & 2.21064632 & 5.36584452 \\
\hline $\mathrm{H}$ & 0.57576624 & 1.28221099 & 8.05799271 \\
\hline C & 2.14546742 & 1.85028274 & 6.6704941 \\
\hline
\end{tabular}




$\begin{array}{lrrl}\mathrm{H} & 2.30642037 & 2.63708953 & 5.91882034 \\ \mathrm{H} & 2.89347203 & 2.01851465 & 7.45633249 \\ \mathrm{C} & 2.39186303 & 0.46858985 & 6.03010249 \\ \mathrm{H} & 1.66238042 & 0.32494440 & 5.22164602 \\ \mathrm{C} & 2.22856649 & -0.68373827 & 7.03214097 \\ \mathrm{H} & 2.44424582 & -1.64534776 & 6.55064803 \\ \mathrm{H} & 2.91693994 & -0.57114430 & 7.87999139 \\ \mathrm{H} & 1.21139235 & -0.74002688 & 7.43350699 \\ \mathrm{C} & 3.79401687 & 0.44141745 & 5.40211644 \\ \mathrm{H} & 3.98201656 & -0.51509279 & 4.90058999 \\ \mathrm{H} & 3.91554662 & 1.23677575 & 4.65656691 \\ \mathrm{H} & 4.57000025 & 0.57778642 & 6.16692448 \\ \mathrm{O} & 1.06188009 & 3.29739926 & 9.35593816 \\ \mathrm{H} & 0.34771693 & 4.57858648 & 6.53396894 \\ \mathrm{H} & 1.49289944 & 5.80846489 & 8.89548393 \\ \mathrm{C} & 0.42313066 & 7.01259160 & 7.46902336 \\ \mathrm{H} & 0.26108960 & 7.85823544 & 8.15003871 \\ \mathrm{H} & -0.46818781 & 6.94231869 & 6.83277764 \\ \mathrm{O} & 2.79024678 & 6.97120713 & 6.96843496 \\ \mathrm{C} & 1.65616063 & 7.34106526 & 6.63916440 \\ \mathrm{H} & 2.19818692 & 8.25918631 & 4.90066637 \\ \mathrm{H} & 0.49005956 & 8.28205581 & 5.23894241 \\ \mathrm{O} & -0.36176096 & 6.40082064 & 10.46019471 \\ \mathrm{H} & -1.83590651 & 4.80064767 & 8.15022902 \\ \mathrm{C} & -2.89935868 & 5.25057069 & 9.92270293 \\ \mathrm{H} & -2.63072445 & 5.79039113 & 10.83206222 \\ \mathrm{H} & -3.78395162 & 5.71524818 & 9.47077206 \\ \mathrm{H} & -3.13709068 & 4.20977855 & 10.17272974 \\ \mathrm{~N} & 1.43324131 & 8.13125476 & 5.56371384\end{array}$

${ }^{4} \mathbf{R}-\mathbf{A}$

62

$\begin{array}{llll}\mathrm{C} & 2.61048996 & -4.91041708 & -1.26929258 \\ \mathrm{H} & 3.20751491 & -5.40419691 & -0.50655286 \\ \mathrm{C} & 1.81223083 & -3.81304571 & -0.93942650 \\ \mathrm{H} & 1.79309600 & -3.44045414 & 0.08181430 \\ \mathrm{C} & 2.64799894 & -5.35945075 & -2.59190515 \\ \mathrm{H} & 3.27431245 & -6.20549754 & -2.86443925 \\ \mathrm{C} & 1.04417607 & -3.17428459 & -1.91457466 \\ \mathrm{H} & 0.46705201 & -2.29615687 & -1.64317787 \\ \mathrm{C} & 1.88473755 & -4.72052673 & -3.56664634\end{array}$




\begin{tabular}{|c|c|c|c|}
\hline $\mathrm{H}$ & 1.91936236 & -5.07369071 & -4.59520608 \\
\hline C & 1.05347685 & -3.62965450 & -3.24724169 \\
\hline $\mathrm{C}$ & 0.26819638 & -2.99740861 & -4.32393718 \\
\hline $\mathrm{H}$ & 0.73365011 & -3.05072991 & -5.30888108 \\
\hline $\mathrm{C}$ & -0.94039329 & -2.41184769 & -4.25372670 \\
\hline $\mathrm{H}$ & -1.33172141 & -1.99791033 & -5.18355239 \\
\hline C & -1.84537753 & -2.27874761 & -3.0608074 \\
\hline $\mathrm{H}$ & -2.89442892 & -2.36786355 & -3.36969947 \\
\hline $\mathrm{H}$ & -1.64299586 & -3.04418665 & -2.30539832 \\
\hline $\mathrm{H}$ & -1.73148966 & -1.30387861 & -2.567723 \\
\hline $\mathrm{O}$ & -0.51346333 & -0.14854199 & -0.642615 \\
\hline $\mathrm{Fe}$ & -0.37156145 & 0.90897668 & 0.5809953 \\
\hline $\mathrm{N}$ & -1.52937425 & 2.30428664 & -0.2989480 \\
\hline $\mathrm{N}$ & 1.26637950 & 1.79461791 & -0.19288121 \\
\hline $\mathrm{N}$ & 0.81344068 & -0.26687122 & 1.71787491 \\
\hline $\mathrm{N}$ & -1.98113036 & 0.20956422 & 1.5818762 \\
\hline $\mathrm{C}$ & -2.88998055 & 2.42284509 & -0.2030795 \\
\hline $\mathrm{C}$ & -3.36552215 & 3.47113861 & -1.07362786 \\
\hline $\mathrm{C}$ & -2.27221074 & 3.97886296 & -1.70533425 \\
\hline $\mathrm{C}$ & -1.13231887 & 3.24293726 & -1.2136586 \\
\hline $\mathrm{C}$ & 1.29019168 & 2.79599519 & -1.13344233 \\
\hline $\mathrm{C}$ & 2.64623852 & 3.05461909 & -1.55350903 \\
\hline $\mathrm{C}$ & 3.44143267 & 2.19385308 & -0.86155841 \\
\hline $\mathrm{C}$ & 2.56941829 & 1.40960456 & -0.0208294 \\
\hline $\mathrm{C}$ & 2.18561233 & -0.36300157 & 1.6521233 \\
\hline $\mathrm{C}$ & 2.65211436 & -1.42253936 & 2.5121095 \\
\hline $\mathrm{C}$ & 1.54972043 & -1.98495863 & 3.0795955 \\
\hline $\mathrm{C}$ & 0.40578607 & -1.26674297 & 2.5766613 \\
\hline $\mathrm{C}$ & -2.01691042 & -0.85117701 & 2.44892652 \\
\hline $\mathrm{C}$ & -3.37570787 & -1.12730868 & 2.84902380 \\
\hline $\mathrm{C}$ & -4.16263092 & -0.21598805 & 2.21585074 \\
\hline $\mathrm{C}$ & -3.28493275 & 0.60902306 & $1.4211158 \mathrm{~s}$ \\
\hline $\mathrm{C}$ & -3.71164610 & 1.64599983 & 0.60525113 \\
\hline $\mathrm{H}$ & -4.77673041 & 1.85491081 & 0.58002327 \\
\hline $\mathrm{C}$ & 0.17922844 & 3.47569019 & -1.60951647 \\
\hline $\mathrm{H}$ & 0.34737110 & 4.25197680 & -2.3496426 \\
\hline $\mathrm{C}$ & 3.00577503 & 0.41594648 & 0.8495630 \\
\hline $\mathrm{H}$ & 4.07262190 & 0.21807753 & 0.88606860 \\
\hline $\mathrm{C}$ & -0.90793447 & -1.55228870 & 2.9069175 \\
\hline $\mathrm{H}$ & -1.08417758 & -2.37577775 & 3.5918068 \\
\hline $\mathrm{H}$ & -2.22279452 & 4.77694311 & -2.4350244 \\
\hline $\mathrm{H}$ & -4.40262266 & 3.76372211 & -1.1766553 \\
\hline $\mathrm{H}$ & 2.93312702 & 3.80022799 & -2.28398597 \\
\hline $\mathrm{H}$ & 4.51747136 & 2.08424344 & -0.90550049 \\
\hline $\mathrm{H}$ & 3.69160625 & -1.69461554 & 2.6437438 \\
\hline
\end{tabular}




$\begin{array}{lrll}\mathrm{H} & 1.49791638 & -2.81128386 & 3.77695451 \\ \mathrm{H} & -3.66842208 & -1.91425443 & 3.53220557 \\ \mathrm{H} & -5.23756394 & -0.09938002 & 2.26788440 \\ \mathrm{~S} & -0.11602760 & 2.32351787 & 2.77347873 \\ \mathrm{C} & 1.52119263 & 3.10884688 & 2.79221508 \\ \mathrm{H} & 1.62597999 & 3.70276418 & 3.70495310 \\ \mathrm{H} & 2.29139334 & 2.32757044 & 2.79940150 \\ \mathrm{H} & 1.67459432 & 3.73451697 & 1.90929553\end{array}$

\section{${ }^{4}$ TS1-A}

62

$\begin{array}{lrrr}\mathrm{C} & -0.52033163 & -6.24922958 & -1.57778445 \\ \mathrm{H} & -0.92854537 & -7.25618794 & -1.57197681 \\ \mathrm{C} & -1.05325511 & -5.27616708 & -2.42861200 \\ \mathrm{H} & -1.88126332 & -5.52704162 & -3.08673672 \\ \mathrm{C} & 0.54300624 & -5.91206985 & -0.73443766 \\ \mathrm{H} & 0.96693126 & -6.65739973 & -0.06628491 \\ \mathrm{C} & -0.53640945 & -3.98425136 & -2.44587859 \\ \mathrm{H} & -0.97251419 & -3.25673237 & -3.11661826 \\ \mathrm{C} & 1.06009166 & -4.62251797 & -0.74693912 \\ \mathrm{H} & 1.88436325 & -4.36573969 & -0.08595979 \\ \mathrm{C} & 0.54034836 & -3.61744591 & -1.60166110 \\ \mathrm{C} & 1.16623774 & -2.31320831 & -1.54856638 \\ \mathrm{H} & 1.97201322 & -2.24536739 & -0.82171587 \\ \mathrm{C} & 0.88034127 & -1.12251422 & -2.21206892 \\ \mathrm{H} & 1.63569057 & -0.35519284 & -2.08086622 \\ \mathrm{C} & 0.02307126 & -0.95429044 & -3.44120445 \\ \mathrm{H} & 0.04882196 & 0.08635204 & -3.77405097 \\ \mathrm{H} & 0.38189231 & -1.59026770 & -4.26185562 \\ \mathrm{H} & -1.02117320 & -1.20512990 & -3.23825950 \\ \mathrm{O} & -0.36889910 & -0.16154016 & -1.01470779 \\ \mathrm{Fe} & -0.17261402 & 0.88177592 & 0.31024602 \\ \mathrm{~N} & -1.78942858 & 1.92540851 & -0.25960035 \\ \mathrm{~N} & 1.02248996 & 2.14297292 & -0.72741655 \\ \mathrm{~N} & 1.49293172 & -0.08527751 & 0.99612090 \\ \mathrm{~N} & -1.31257890 & -0.23782068 & 1.52551410 \\ \mathrm{C} & -3.08539310 & 1.71044403 & 0.12189577 \\ \mathrm{C} & -3.96689902 & 2.64101201 & -0.54875686 \\ \mathrm{C} & -3.18220041 & 3.42091543 & -1.33834447 \\ \mathrm{C} & -1.82112445 & 2.96988327 & -1.14594721 \\ \mathrm{C} & 0.60780056 & 3.15508042 & -1.55423905 \\ \mathrm{C} & 1.74370591 & 3.76866216 & -2.19866331\end{array}$




$\begin{array}{lrrr}\mathrm{C} & 2.85037349 & 3.10796307 & -1.75692404 \\ \mathrm{C} & 2.38914988 & 2.08960773 & -0.84495984 \\ \mathrm{C} & 2.79911782 & 0.17304596 & 0.66365355 \\ \mathrm{C} & 3.67587542 & -0.76995501 & 1.31973175 \\ \mathrm{C} & 2.88092759 & -1.60019612 & 2.05010393 \\ \mathrm{C} & 1.51972207 & -1.16183289 & 1.84494330 \\ \mathrm{C} & -0.90259565 & -1.29823234 & 2.29610571 \\ \mathrm{C} & -2.03780985 & -1.90139753 & 2.95330297 \\ \mathrm{C} & -3.13561233 & -1.19545256 & 2.56969820 \\ \mathrm{C} & -2.67405419 & -0.16432992 & 1.67076016 \\ \mathrm{C} & -3.50410615 & 0.74575231 & 1.02914506 \\ \mathrm{H} & -4.56705015 & 0.68753761 & 1.24262360 \\ \mathrm{C} & -0.71315772 & 3.54380312 & -1.75296807 \\ \mathrm{H} & -0.89037732 & 4.36994510 & -2.43510925 \\ \mathrm{C} & 3.22163950 & 1.18565600 & -0.19048090 \\ \mathrm{H} & 4.28856503 & 1.26939880 & -0.37517025 \\ \mathrm{C} & 0.40576693 & -1.73916308 & 2.44025261 \\ \mathrm{H} & 0.57294500 & -2.59358355 & 3.08891488 \\ \mathrm{H} & -3.47560015 & 4.23429899 & -1.98992313 \\ \mathrm{H} & -5.04072357 & 2.67765223 & -0.41583010 \\ \mathrm{H} & 1.68528244 & 4.59464884 & -2.89624356 \\ \mathrm{H} & 3.88720393 & 3.27923216 & -2.01767139 \\ \mathrm{H} & 4.75446623 & -0.77920295 & 1.22508287 \\ \mathrm{H} & 3.17321703 & -2.42906773 & 2.68250071 \\ \mathrm{H} & -1.98188247 & -2.75369388 & 3.61845197 \\ \mathrm{H} & -4.16936965 & -1.34799904 & 2.85268656 \\ \mathrm{~S} & 0.15511530 & 2.18809889 & 2.36606362 \\ \mathrm{C} & 1.52425528 & 3.37061973 & 2.16941587 \\ \mathrm{H} & 1.59889236 & 3.96216724 & 3.08730764 \\ \mathrm{H} & 2.46549338 & 2.82571892 & 2.03508225 \\ \mathrm{H} & 1.37206019 & 4.02844617 & 1.31056094\end{array}$

\section{${ }^{4} \mathbf{I}-\mathbf{A}$}

62

$\begin{array}{lrrr}\mathrm{C} & -3.25745838 & -1.70270452 & 1.90548451 \\ \mathrm{C} & -2.71444368 & -0.57078422 & 1.19046722 \\ \mathrm{~N} & -1.34626957 & -0.59035890 & 1.25361661 \\ \mathrm{C} & -1.00655367 & -1.70008386 & 1.98163512 \\ \mathrm{C} & -2.19884734 & -2.40786580 & 2.38671542 \\ \mathrm{C} & -3.47877868 & 0.36502338 & 0.50884059 \\ \mathrm{C} & -2.96691196 & 1.40656167 & -0.25330968\end{array}$




\begin{tabular}{|c|c|c|c|}
\hline $\mathrm{N}$ & -1.63844378 & 1.71185757 & -0.40486278 \\
\hline $\mathrm{C}$ & -1.57839264 & 2.75738872 & -1.29329829 \\
\hline $\mathrm{C}$ & -2.91003389 & 3.12630857 & -1.70824628 \\
\hline $\mathrm{C}$ & -3.77048576 & 2.29734374 & -1.05518622 \\
\hline $\mathrm{C}$ & -0.42043952 & 3.40131215 & -1.70628355 \\
\hline $\mathrm{C}$ & 0.85721161 & 3.10895329 & -1.24492989 \\
\hline $\mathrm{N}$ & 1.17746802 & 2.10287602 & -0.36664701 \\
\hline $\mathrm{C}$ & 2.52916381 & 2.20172031 & -0.13731413 \\
\hline $\mathrm{C}$ & 3.07386361 & 3.30414326 & -0.88941620 \\
\hline $\mathrm{C}$ & 2.04109605 & 3.85858038 & -1.58436597 \\
\hline $\mathrm{Fe}$ & -0.08130198 & 0.73884119 & 0.41121475 \\
\hline S & -0.24251214 & 1.97954099 & 2.35244259 \\
\hline $\mathrm{C}$ & -0.55992951 & 3.72191910 & 1.91218859 \\
\hline $\mathrm{C}$ & 3.28001736 & 1.34276383 & 0.65508041 \\
\hline $\mathrm{C}$ & 2.78324838 & 0.22109290 & 1.30457256 \\
\hline $\mathrm{N}$ & 1.47359547 & -0.19079127 & 1.30106715 \\
\hline $\mathrm{C}$ & 1.42864970 & -1.35158874 & 2.02848970 \\
\hline C & 2.75149415 & -1.69107423 & 2.49361242 \\
\hline $\mathrm{C}$ & 3.58889129 & -0.71182499 & 2.05388338 \\
\hline $\mathrm{C}$ & 0.28260622 & -2.07565256 & 2.32901023 \\
\hline $\mathrm{H}$ & 4.34304024 & 1.54343934 & 0.74302941 \\
\hline $\mathrm{H}$ & 2.98765505 & -2.56482813 & 3.08747848 \\
\hline $\mathrm{H}$ & 4.11624924 & 3.59645908 & -0.88347226 \\
\hline $\mathrm{H}$ & 2.06072822 & 4.70298622 & -2.26167030 \\
\hline $\mathrm{H}$ & -0.52516152 & 4.22330906 & -2.40730664 \\
\hline $\mathrm{H}$ & -3.13823325 & 3.92271016 & -2.40523688 \\
\hline $\mathrm{H}$ & -4.85131913 & 2.26811356 & -1.10971103 \\
\hline $\mathrm{H}$ & -4.55757161 & 0.24951793 & 0.53839995 \\
\hline $\mathrm{H}$ & -4.31389280 & -1.91695284 & 2.00564010 \\
\hline $\mathrm{H}$ & -2.20287121 & -3.32103027 & 2.96794913 \\
\hline $\mathrm{H}$ & 0.40109048 & -2.98125530 & 2.91492499 \\
\hline $\mathrm{H}$ & 4.65619582 & -0.61649116 & 2.20809399 \\
\hline $\mathrm{H}$ & -0.63648864 & 4.27748947 & 2.85229488 \\
\hline $\mathrm{H}$ & 0.26082003 & 4.13617138 & 1.32077284 \\
\hline $\mathrm{H}$ & -1.49485439 & 3.82706344 & 1.35621138 \\
\hline $\mathrm{O}$ & 0.03242079 & -0.31551236 & -1.04912552 \\
\hline $\mathrm{C}$ & 1.15905592 & -0.71107867 & -1.80657312 \\
\hline $\mathrm{C}$ & 1.50554257 & -2.16729403 & -1.61249802 \\
\hline $\mathrm{C}$ & 0.62182965 & -3.27458489 & -1.59072482 \\
\hline $\mathrm{C}$ & 1.16168635 & -4.59424384 & -1.52191613 \\
\hline $\mathrm{C}$ & 0.34326473 & -5.71308533 & -1.51939662 \\
\hline $\mathrm{C}$ & -1.05030634 & -5.57073145 & -1.58424400 \\
\hline $\mathrm{C}$ & -1.60723531 & -4.28595412 & -1.64560769 \\
\hline $\mathrm{C}$ & -0.79986495 & -3.15677613 & -1.64497067 \\
\hline C & 0.86844168 & -0.38795493 & -3.28993985 \\
\hline
\end{tabular}




$\begin{array}{lrrr}\mathrm{H} & 0.00643664 & -0.96142724 & -3.64370923 \\ \mathrm{H} & 1.73429569 & -0.63982371 & -3.91341861 \\ \mathrm{H} & 0.65236298 & 0.67989116 & -3.39962948 \\ \mathrm{H} & 2.03447086 & -0.12295276 & -1.50353623 \\ \mathrm{H} & 2.57074622 & -2.38986361 & -1.57490573 \\ \mathrm{H} & 2.24201826 & -4.71319997 & -1.47618461 \\ \mathrm{H} & 0.78616723 & -6.70489151 & -1.46984564 \\ \mathrm{H} & -1.69155885 & -6.44792709 & -1.58351801 \\ \mathrm{H} & -2.68731282 & -4.16794184 & -1.68834576 \\ \mathrm{H} & -1.24023034 & -2.16711004 & -1.66624726\end{array}$

${ }^{4}$ TS2-A

62

$\begin{array}{lrrr}\mathrm{C} & -2.70009082 & -1.79338094 & 2.73282558 \\ \mathrm{C} & -2.32133660 & -0.78185312 & 1.77291328 \\ \mathrm{~N} & -0.96814043 & -0.58242762 & 1.81096058 \\ \mathrm{C} & -0.47537155 & -1.43993567 & 2.75655871 \\ \mathrm{C} & -1.55402572 & -2.20562865 & 3.33919386 \\ \mathrm{C} & -3.20764340 & -0.13889874 & 0.91953466 \\ \mathrm{C} & -2.85230641 & 0.78977732 & -0.04931743 \\ \mathrm{~N} & -1.58251990 & 1.24819605 & -0.29874590 \\ \mathrm{C} & -1.68383950 & 2.11622388 & -1.35683228 \\ \mathrm{C} & -3.05780902 & 2.20982975 & -1.78751194 \\ \mathrm{C} & -3.78217879 & 1.39332442 & -0.97294220 \\ \mathrm{C} & -0.63181226 & 2.82737643 & -1.91857852 \\ \mathrm{C} & 0.68310077 & 2.78420041 & -1.47346507 \\ \mathrm{~N} & 1.15538831 & 2.00339829 & -0.44465663 \\ \mathrm{C} & 2.49666290 & 2.28819997 & -0.32490286 \\ \mathrm{C} & 2.87600665 & 3.27544186 & -1.30313767 \\ \mathrm{C} & 1.75531506 & 3.57725553 & -2.01866062 \\ \mathrm{Fe} & 0.09820711 & 0.71392681 & 0.66483274 \\ \mathrm{~S} & -0.26209412 & 2.33563547 & 2.39632363 \\ \mathrm{C} & -0.70693858 & 3.91384893 & 1.58862313 \\ \mathrm{C} & 3.37752912 & 1.69496440 & 0.56981547 \\ \mathrm{C} & 3.04253367 & 0.69688534 & 1.47527466 \\ \mathrm{~N} & 1.79100888 & 0.15758482 & 1.63447398 \\ \mathrm{C} & 1.90096972 & -0.79454921 & 2.61519684 \\ \mathrm{C} & 3.26659183 & -0.86938456 & 3.07685584 \\ \mathrm{C} & 3.97325363 & 0.05922301 & 2.37467956 \\ \mathrm{C} & 0.85762535 & -1.55437421 & 3.12950856 \\ \mathrm{H} & 4.41110119 & 2.02572270 & 0.54668186\end{array}$




$\begin{array}{lrrr}\mathrm{H} & 3.61917295 & -1.54336751 & 3.84743886 \\ \mathrm{H} & 3.87624689 & 3.67334632 & -1.41860448 \\ \mathrm{H} & 1.64788351 & 4.27592787 & -2.83877763 \\ \mathrm{H} & -0.85855108 & 3.48918333 & -2.74856751 \\ \mathrm{H} & -3.40616027 & 2.82969738 & -2.60408000 \\ \mathrm{H} & -4.84758746 & 1.20116617 & -0.98555097 \\ \mathrm{H} & -4.25801091 & -0.40130517 & 0.99762781 \\ \mathrm{H} & -3.71382335 & -2.13391990 & 2.90140325 \\ \mathrm{H} & -1.43072494 & -2.95289756 & 4.11294760 \\ \mathrm{H} & 1.09945524 & -2.27218277 & 3.90719153 \\ \mathrm{H} & 5.02564908 & 0.30284267 & 2.44691247 \\ \mathrm{H} & -0.87125292 & 4.65769315 & 2.37475766 \\ \mathrm{H} & 0.09629994 & 4.26465177 & 0.93400946 \\ \mathrm{H} & -1.62481315 & 3.81235433 & 1.00220519 \\ \mathrm{O} & 0.31694926 & -0.76225860 & -0.59396959 \\ \mathrm{C} & 1.45199750 & -1.23120165 & -1.28359570 \\ \mathrm{C} & 1.03800741 & -2.67041168 & -1.28633220 \\ \mathrm{C} & 0.02394683 & -3.30661801 & -2.06234041 \\ \mathrm{C} & -0.05219053 & -4.72578810 & -2.02704137 \\ \mathrm{C} & -0.96505114 & -5.41914563 & -2.81018880 \\ \mathrm{C} & -1.85636258 & -4.72063325 & -3.63275568 \\ \mathrm{C} & -1.82974408 & -3.31967069 & -3.65105873 \\ \mathrm{C} & -0.91202419 & -2.61827745 & -2.88178167 \\ \mathrm{C} & 1.74059262 & -0.57860521 & -2.63902195 \\ \mathrm{H} & 0.88847306 & -0.63181968 & -3.31994738 \\ \mathrm{H} & 2.59060326 & -1.08276107 & -3.11630958 \\ \mathrm{H} & 2.00260174 & 0.47259376 & -2.49908107 \\ \mathrm{H} & 2.35360257 & -1.13060761 & -0.66107964 \\ \mathrm{H} & 1.48923895 & -3.28713452 & -0.51047290 \\ \mathrm{H} & 0.63634464 & -5.27175067 & -1.38665580 \\ \mathrm{H} & -0.99150069 & -6.50494491 & -2.77607642 \\ \mathrm{H} & -2.57766308 & -5.26109814 & -4.23939278 \\ \mathrm{H} & -2.54403751 & -2.77338619 & -4.26131739 \\ \mathrm{H} & -0.94254004 & -1.53661417 & -2.84958399 \\ & & & \\ & & & \\ & & & \end{array}$

${ }^{4}$ E-A

62

$\begin{array}{llll}\mathrm{C} & -1.88721088 & -5.52644496 & -2.55849362 \\ \mathrm{H} & -2.49004633 & -6.35929481 & -2.91053806 \\ \mathrm{C} & -2.27144048 & -4.21352812 & -2.83725450 \\ \mathrm{H} & -3.17848864 & -4.02076920 & -3.40435679\end{array}$




\begin{tabular}{|c|c|c|c|}
\hline C & -0.72746110 & -5.76429130 & -1.81624406 \\
\hline $\mathrm{H}$ & -0.42498881 & -6.78306207 & -1.58834575 \\
\hline $\mathrm{C}$ & -1.49871955 & -3.14187032 & -2.38554259 \\
\hline $\mathrm{H}$ & -1.79859593 & -2.11804817 & -2.58443294 \\
\hline $\mathrm{C}$ & 0.04220982 & -4.69466569 & -1.36025509 \\
\hline $\mathrm{H}$ & 0.94017163 & -4.88375914 & -0.77562859 \\
\hline $\mathrm{C}$ & -0.33030484 & -3.37414687 & -1.64887290 \\
\hline $\mathrm{C}$ & 0.52122164 & -2.25214927 & -1.15257170 \\
\hline $\mathrm{H}$ & 0.91772841 & -2.39668195 & -0.14640452 \\
\hline C & 1.29017917 & -1.33303453 & -2.02967863 \\
\hline $\mathrm{H}$ & 2.17315958 & -0.87480986 & -1.58164753 \\
\hline C & 1.30038182 & -1.40151681 & -3.53413363 \\
\hline $\mathrm{H}$ & 1.29667789 & -0.38956217 & -3.95561614 \\
\hline $\mathrm{H}$ & 2.21063005 & -1.90690861 & -3.87896287 \\
\hline $\mathrm{H}$ & 0.43454392 & -1.94363253 & -3.92208436 \\
\hline $\mathrm{O}$ & 0.06809574 & -0.91033357 & -1.39624359 \\
\hline $\mathrm{Fe}$ & 0.04686984 & 0.99935888 & 0.63853111 \\
\hline $\mathrm{N}$ & -1.72009937 & 1.46902806 & -0.20951056 \\
\hline $\mathrm{N}$ & 0.99292075 & 2.15127772 & -0.72002659 \\
\hline $\mathrm{N}$ & 1.83315883 & 0.25477299 & 1.21536737 \\
\hline $\mathrm{N}$ & -0.87748732 & -0.43614338 & 1.71548331 \\
\hline $\mathrm{C}$ & -2.95972234 & 0.99043255 & 0.13635537 \\
\hline C & -3.98573334 & 1.66784424 & -0.61726314 \\
\hline $\mathrm{C}$ & -3.35389258 & 2.56444717 & -1.42330554 \\
\hline $\mathrm{C}$ & -1.94149034 & 2.43157483 & -1.16527978 \\
\hline $\mathrm{C}$ & 0.40551791 & 3.01793008 & -1.61275773 \\
\hline $\mathrm{C}$ & 1.41456120 & 3.75275424 & -2.33234174 \\
\hline C & 2.62130496 & 3.32137705 & -1.87021204 \\
\hline C & 2.35033266 & 2.32541560 & -0.86437596 \\
\hline $\mathrm{C}$ & 3.08063570 & 0.68813390 & 0.82266514 \\
\hline $\mathrm{C}$ & 4.10387240 & 0.00128830 & 1.56808510 \\
\hline $\mathrm{C}$ & 3.46720584 & -0.84951040 & 2.42157506 \\
\hline $\mathrm{C}$ & 2.05435442 & -0.68757395 & 2.19562325 \\
\hline $\mathrm{C}$ & -0.29372419 & -1.28553098 & 2.62687780 \\
\hline $\mathrm{C}$ & -1.30184449 & -2.06340216 & 3.30261720 \\
\hline C & -2.50313845 & -1.67726785 & 2.79193875 \\
\hline $\mathrm{C}$ & -2.23024440 & -0.65995890 & 1.80766206 \\
\hline C & -3.20686918 & -0.00076980 & 1.07538105 \\
\hline $\mathrm{H}$ & -4.24098005 & -0.27870380 & 1.25272131 \\
\hline C & -0.95919279 & 3.16214366 & -1.81705117 \\
\hline $\mathrm{H}$ & -1.28160314 & 3.89049295 & -2.55448113 \\
\hline $\mathrm{C}$ & 3.32912143 & 1.64856648 & -0.14895337 \\
\hline $\mathrm{H}$ & 4.36555659 & 1.89217770 & -0.36099065 \\
\hline $\mathrm{C}$ & 1.06850933 & -1.40284700 & 2.86177937 \\
\hline $\mathrm{H}$ & 1.38856760 & -2.11624879 & 3.61474311 \\
\hline
\end{tabular}




$\begin{array}{lrrr}\mathrm{H} & -3.78739559 & 3.25515202 & -2.13531264 \\ \mathrm{H} & -5.04647822 & 1.47036287 & -0.52830644 \\ \mathrm{H} & 1.21011002 & 4.49606754 & -3.09244617 \\ \mathrm{H} & 3.61157379 & 3.63893456 & -2.17163595 \\ \mathrm{H} & 5.16767471 & 0.16106379 & 1.44500479 \\ \mathrm{H} & 3.90138262 & -1.53191662 & 3.14127063 \\ \mathrm{H} & -1.09914944 & -2.80616762 & 4.06364630 \\ \mathrm{H} & -3.49185035 & -2.03653368 & 3.04759161 \\ \mathrm{~S} & -0.15889389 & 2.69181163 & 2.36659990 \\ \mathrm{C} & 1.47975352 & 3.50103220 & 2.51180946 \\ \mathrm{H} & 1.41207244 & 4.27479019 & 3.28279985 \\ \mathrm{H} & 2.25195402 & 2.78284896 & 2.80351201 \\ \mathrm{H} & 1.77226664 & 3.97222124 & 1.56860668\end{array}$

${ }^{4} \mathbf{R}-\mathrm{B}$

160

$\begin{array}{lrrr}\mathrm{C} & 2.91300044 & -4.59702892 & -2.14101868 \\ \mathrm{H} & 3.21001179 & -5.49168049 & -1.59977358 \\ \mathrm{C} & 1.82633052 & -3.83605711 & -1.70437847 \\ \mathrm{H} & 1.27853388 & -4.13011962 & -0.81244027 \\ \mathrm{C} & 3.62248655 & -4.19198073 & -3.27461313 \\ \mathrm{H} & 4.47619868 & -4.76968117 & -3.62004911 \\ \mathrm{C} & 1.43823052 & -2.68793055 & -2.39717980 \\ \mathrm{H} & 0.61955616 & -2.08846493 & -2.01325756 \\ \mathrm{C} & 3.23741009 & -3.04500377 & -3.96545867 \\ \mathrm{H} & 3.79399530 & -2.73574639 & -4.84761638 \\ \mathrm{C} & 2.12831441 & -2.28012350 & -3.55521665 \\ \mathrm{C} & 1.76991834 & -1.07957425 & -4.33303953 \\ \mathrm{H} & 2.61295883 & -0.60524608 & -4.83675356 \\ \mathrm{C} & 0.55738392 & -0.53269091 & -4.53422891 \\ \mathrm{H} & 0.52992804 & 0.36686220 & -5.14987102 \\ \mathrm{C} & -0.78042651 & -1.02076884 & -4.05168586 \\ \mathrm{H} & -1.55319623 & -0.80924881 & -4.80073607 \\ \mathrm{H} & -0.77807816 & -2.09749685 & -3.85607531 \\ \mathrm{H} & -1.08446845 & -0.52396550 & -3.12011014 \\ \mathrm{O} & -0.98337946 & -0.60028743 & -0.53335403 \\ \mathrm{Fe} & -0.67710921 & 0.24816787 & 0.81233729 \\ \mathrm{~N} & -1.39072474 & 1.98261480 & 0.08152517 \\ \mathrm{~N} & 1.17685677 & 0.78647322 & 0.24114770 \\ \mathrm{~N} & 0.09608630 & -1.32154671 & 1.81248751 \\ \mathrm{~N} & -2.47659533 & -0.12612882 & 1.63655244\end{array}$




\begin{tabular}{|c|c|c|c|}
\hline C & -2.71190172 & 2.36012952 & 0.01134249 \\
\hline $\mathrm{C}$ & -2.84680702 & 3.57507314 & -0.75455384 \\
\hline $\mathrm{C}$ & -1.59433445 & 3.93434146 & -1.14854189 \\
\hline $\mathrm{C}$ & -0.69684537 & 2.92438046 & -0.64700635 \\
\hline $\mathrm{C}$ & 1.52925743 & 1.87538662 & -0.51390813 \\
\hline C & 2.93574560 & 1.83747684 & -0.83153248 \\
\hline $\mathrm{C}$ & 3.43229626 & 0.70302716 & -0.2671787 \\
\hline $\mathrm{C}$ & 2.32961016 & 0.05505699 & 0.39818651 \\
\hline $\mathrm{C}$ & 1.39204722 & -1.76187639 & 1.77601568 \\
\hline C & 1.53629222 & -2.97647566 & 2.54252886 \\
\hline C & 0.30610412 & -3.26535782 & 3.04749526 \\
\hline C & -0.58392576 & -2.22974802 & 2.5805431 \\
\hline C & -2.82343181 & -1.21067649 & 2.4053065 \\
\hline C & -4.24564773 & -1.22515655 & 2.6437956 \\
\hline C & -4.76257957 & -0.14825252 & 1.9914732 \\
\hline C & -3.65375935 & 0.52839217 & 1.3653701 \\
\hline $\mathrm{C}$ & -3.76930509 & 1.67849751 & 0.5971170 \\
\hline $\mathrm{H}$ & -4.76662990 & 2.08158612 & 0.4490582 \\
\hline $\mathrm{C}$ & 0.66273365 & 2.88820017 & -0.91276946 \\
\hline $\mathrm{H}$ & 1.07913152 & 3.71207755 & -1.4820073 \\
\hline C & 2.43543198 & -1.12975531 & 1.1109023 \\
\hline $\mathrm{H}$ & 3.41255462 & -1.59995413 & 1.1542742 \\
\hline C & -1.94241488 & -2.17934721 & 2.8659731 \\
\hline $\mathrm{H}$ & -2.35316458 & -2.97592050 & 3.4785735 \\
\hline $\mathrm{H}$ & -1.28520714 & 4.79363886 & -1.7284993 \\
\hline $\mathrm{H}$ & -3.78604929 & 4.07535788 & -0.9538987 \\
\hline $\mathrm{H}$ & 3.45402208 & 2.58324341 & -1.4203925 \\
\hline $\mathrm{H}$ & 4.44510505 & 0.32256534 & -0.2915665 \\
\hline $\mathrm{H}$ & 2.46406517 & -3.51909128 & 2.6692492 \\
\hline $\mathrm{H}$ & 0.01073282 & -4.09539770 & 3.6760845 \\
\hline $\mathrm{H}$ & -4.76450558 & -1.97384553 & 3.2283050 \\
\hline $\mathrm{H}$ & -5.79485815 & 0.17139559 & 1.92887746 \\
\hline S & -0.29625117 & 1.69959610 & 3.12246158 \\
\hline $\mathrm{C}$ & 0.92696163 & 3.01545677 & 2.79694984 \\
\hline $\mathrm{H}$ & 1.63406573 & 2.96592234 & 3.63574913 \\
\hline $\mathrm{H}$ & 1.48568189 & 2.79255702 & 1.88706994 \\
\hline $\mathrm{H}$ & -0.14720922 & 4.56919492 & 1.76588780 \\
\hline $\mathrm{C}$ & 0.39287839 & 4.46360076 & 2.70375015 \\
\hline C & -0.49886382 & 4.80594944 & 3.9104419 \\
\hline $\mathrm{N}$ & -1.80527341 & 5.09628059 & 3.6890775 \\
\hline C & -2.68178717 & 5.47495424 & 4.8173890 \\
\hline $\mathrm{C}$ & -2.81271508 & 4.41619733 & 5.9199912 \\
\hline $\mathrm{N}$ & -2.82739485 & 3.12163902 & 5.5145649 \\
\hline $\mathrm{C}$ & -3.04497080 & 2.01800386 & 6.4510053 \\
\hline C & -1.85903157 & 1.84510797 & 7.4255332 \\
\hline
\end{tabular}




\begin{tabular}{|c|c|c|c|}
\hline $\mathrm{N}$ & -0.62142916 & 1.80370030 & 6.85439144 \\
\hline $\mathrm{C}$ & 0.58053745 & 1.71983769 & 7.68093587 \\
\hline $\mathrm{C}$ & 0.75353303 & 2.93482938 & 8.61816807 \\
\hline $\mathrm{N}$ & 0.49308613 & 4.17657585 & 8.11145673 \\
\hline $\mathrm{C}$ & 0.63561704 & 5.32357112 & 9.00571203 \\
\hline $\mathrm{C}$ & -0.34548130 & 5.31475326 & 10.20591808 \\
\hline $\mathrm{N}$ & -1.60248626 & 4.87925694 & 9.93631622 \\
\hline $\mathrm{N}$ & 1.53675128 & 5.36137621 & 2.61522706 \\
\hline $\mathrm{C}$ & 1.76582942 & 6.09206655 & 1.48466565 \\
\hline C & 2.98886968 & 7.03830328 & 1.48492163 \\
\hline $\mathrm{N}$ & 4.05579897 & 6.65762561 & 2.43131392 \\
\hline $\mathrm{C}$ & 3.98484323 & 7.11072859 & 3.71862600 \\
\hline $\mathrm{C}$ & 5.09035287 & 6.68692577 & 4.70330160 \\
\hline $\mathrm{N}$ & 4.93957698 & 7.36782013 & 5.96529455 \\
\hline $\mathrm{C}$ & 5.83872753 & 8.31777695 & 6.35741369 \\
\hline $\mathrm{C}$ & 5.56359838 & 8.93322844 & 7.72057590 \\
\hline $\mathrm{H}$ & 4.71122846 & 8.47918090 & 8.23312500 \\
\hline $\mathrm{H}$ & 5.38037019 & 10.00567622 & 7.59478034 \\
\hline $\mathrm{H}$ & 6.45858655 & 8.82806470 & 8.34158880 \\
\hline $\mathrm{O}$ & 6.80279195 & 8.65416449 & 5.67117764 \\
\hline $\mathrm{H}$ & 4.13208796 & 7.12931625 & 6.54827962 \\
\hline $\mathrm{H}$ & 6.06827343 & 6.97438251 & 4.30322174 \\
\hline $\mathrm{C}$ & 5.02346816 & 5.16035832 & 4.94807923 \\
\hline $\mathrm{H}$ & 5.86620396 & 4.86142813 & 5.57761994 \\
\hline $\mathrm{H}$ & 4.10133023 & 4.91253281 & 5.48572062 \\
\hline $\mathrm{H}$ & 5.05365366 & 4.57468796 & 4.02450950 \\
\hline $\mathrm{O}$ & 2.98150419 & 7.73718804 & 4.07929379 \\
\hline $\mathrm{C}$ & 5.27934513 & 6.20837381 & 1.73320295 \\
\hline $\mathrm{H}$ & 6.05247094 & 6.98575120 & 1.78768715 \\
\hline $\mathrm{H}$ & 5.68438829 & 5.29700216 & 2.17961311 \\
\hline $\mathrm{C}$ & 4.80601380 & 5.99656125 & 0.28831124 \\
\hline $\mathrm{H}$ & 5.62274431 & 6.10704087 & -0.43060377 \\
\hline $\mathrm{H}$ & 4.39054237 & 4.98749991 & 0.17475724 \\
\hline $\mathrm{C}$ & 3.69822602 & 7.04789735 & 0.11985795 \\
\hline $\mathrm{H}$ & 4.13728422 & 8.03636038 & -0.05505988 \\
\hline $\mathrm{H}$ & 2.99858959 & 6.83074988 & -0.68824921 \\
\hline $\mathrm{H}$ & 2.60629772 & 8.02616760 & 1.7607095 \\
\hline $\mathrm{O}$ & 1.05121020 & 6.01357690 & 0.48495995 \\
\hline $\mathrm{H}$ & 2.01639247 & 5.60207358 & 3.47348591 \\
\hline $\mathrm{O}$ & -0.02190284 & 4.76170207 & 5.0548987 \\
\hline $\mathrm{C}$ & -2.42641497 & 5.41585467 & 2.3799585 \\
\hline $\mathrm{H}$ & -1.71598059 & 5.91247287 & 1.7161276 \\
\hline $\mathrm{H}$ & -2.76481365 & 4.49649145 & 1.89054529 \\
\hline $\mathrm{C}$ & -3.60883302 & 6.31853910 & 2.75737778 \\
\hline $\mathrm{H}$ & -3.27724468 & 7.36092219 & 2.8301676 \\
\hline
\end{tabular}




\begin{tabular}{|c|c|c|c|}
\hline $\mathrm{H}$ & -4.41330931 & 6.27393603 & 2.01748574 \\
\hline C & -4.02747510 & 5.79610770 & 4.13819386 \\
\hline $\mathrm{H}$ & -4.61871961 & 4.87829441 & 4.03668172 \\
\hline $\mathrm{H}$ & -4.61122882 & 6.50982190 & 4.72409958 \\
\hline $\mathrm{H}$ & -2.27461259 & 6.36168680 & 5.31537895 \\
\hline $\mathrm{O}$ & -2.98410935 & 4.76647418 & 7.08473125 \\
\hline $\mathrm{H}$ & -2.52421410 & 2.90192168 & 4.57096473 \\
\hline $\mathrm{H}$ & -3.88546460 & 2.29061718 & 7.09420127 \\
\hline C & -3.35371538 & 0.72115792 & 5.70286870 \\
\hline $\mathrm{H}$ & -4.24889009 & 0.83426812 & 5.08270495 \\
\hline $\mathrm{H}$ & -2.52082784 & 0.41998999 & 5.05939871 \\
\hline $\mathrm{H}$ & -3.53229048 & -0.08030416 & 6.42622862 \\
\hline $\mathrm{O}$ & -2.05101674 & 1.67367948 & 8.62302462 \\
\hline $\mathrm{H}$ & -0.52402085 & 2.09882329 & 5.88950746 \\
\hline $\mathrm{H}$ & 0.45134587 & 0.88476311 & 8.37262726 \\
\hline C & 1.84456261 & 1.51389761 & 6.83273116 \\
\hline $\mathrm{H}$ & 1.95106723 & 2.36642325 & 6.14546262 \\
\hline $\mathrm{H}$ & 2.69874641 & 1.56658394 & 7.52026109 \\
\hline C & 1.91723987 & 0.19529368 & 6.03485132 \\
\hline $\mathrm{H}$ & 1.06371153 & 0.16224493 & 5.34311161 \\
\hline $\mathrm{C}$ & 1.84417393 & -1.04527265 & 6.93729557 \\
\hline $\mathrm{H}$ & 1.92837492 & -1.96067664 & 6.3392367 \\
\hline $\mathrm{H}$ & 2.66074281 & -1.04762578 & 7.6710553 \\
\hline $\mathrm{H}$ & 0.90000688 & -1.09855263 & 7.48932545 \\
\hline $\mathrm{C}$ & 3.19916804 & 0.17169321 & 5.18827610 \\
\hline $\mathrm{H}$ & 3.24452573 & -0.73310106 & 4.57079680 \\
\hline $\mathrm{H}$ & 3.25282233 & 1.03674755 & 4.51559250 \\
\hline $\mathrm{H}$ & 4.09258956 & 0.18922147 & 5.82610472 \\
\hline $\mathrm{O}$ & 1.18357872 & 2.76659682 & 9.75227275 \\
\hline $\mathrm{H}$ & 0.16046206 & 4.30182034 & 7.1617372 \\
\hline $\mathrm{H}$ & 1.63314689 & 5.30445921 & 9.4486775 \\
\hline $\mathrm{C}$ & 0.43733310 & 6.64700400 & 8.26616379 \\
\hline $\mathrm{H}$ & 0.39359349 & 7.43683485 & 9.02734723 \\
\hline $\mathrm{H}$ & -0.52969804 & 6.65281165 & 7.74755935 \\
\hline $\mathrm{O}$ & 2.71938596 & 6.59029479 & 7.4633082 \\
\hline C & 1.56571108 & 7.01194083 & 7.31180483 \\
\hline $\mathrm{H}$ & 1.91386427 & 8.05463985 & 5.59185509 \\
\hline $\mathrm{H}$ & 0.26576360 & 8.09751954 & 6.1539808 \\
\hline $\mathrm{O}$ & 0.00344361 & 5.80803920 & 11.2739088 \\
\hline $\mathrm{H}$ & -1.79479188 & 4.44309665 & 9.0434142 \\
\hline $\mathrm{C}$ & -2.61930573 & 4.76686898 & 10.9645405 \\
\hline $\mathrm{H}$ & -2.22348443 & 5.21995109 & 11.874740 \\
\hline $\mathrm{H}$ & -3.53345933 & 5.29255040 & $10.663605 \varepsilon$ \\
\hline $\mathrm{H}$ & -2.86411966 & 3.71554260 & 11.1566086 \\
\hline $\mathrm{N}$ & 1.23589561 & 7.88948828 & 6.33642385 \\
\hline
\end{tabular}




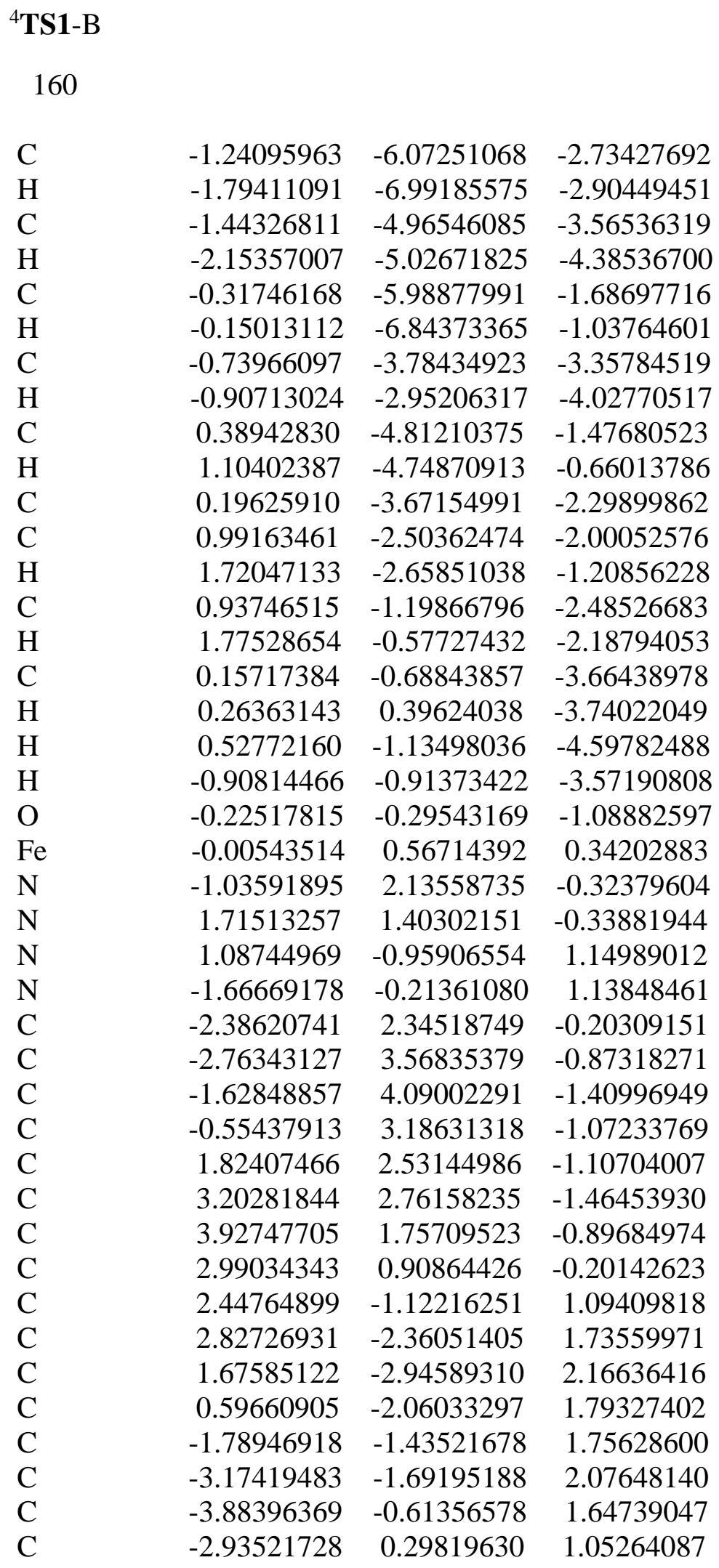




\begin{tabular}{|c|c|c|c|}
\hline $\mathrm{C}$ & -3.27579804 & 1.50029569 & 0.44697909 \\
\hline $\mathrm{H}$ & -4.32432873 & 1.78225307 & 0.44790311 \\
\hline $\mathrm{C}$ & 0.76523437 & 3.36871161 & -1.45296494 \\
\hline $\mathrm{H}$ & 0.99445735 & 4.24914259 & -2.04455531 \\
\hline $\mathrm{C}$ & 3.33776895 & -0.25552801 & 0.47293862 \\
\hline $\mathrm{H}$ & 4.39050845 & -0.51892730 & 0.49904528 \\
\hline $\mathrm{C}$ & -0.74749477 & -2.29746811 & 2.06208501 \\
\hline $\mathrm{H}$ & -0.99984950 & -3.22376370 & 2.56862927 \\
\hline $\mathrm{H}$ & -1.50401931 & 5.00806844 & -1.96833358 \\
\hline $\mathrm{H}$ & -3.77178071 & 3.95996348 & -0.91734924 \\
\hline $\mathrm{H}$ & 3.55419575 & 3.58947786 & -2.06726091 \\
\hline $\mathrm{H}$ & 4.99625088 & 1.58999664 & -0.93780756 \\
\hline $\mathrm{H}$ & 3.84430084 & -2.71779443 & 1.83470288 \\
\hline $\mathrm{H}$ & 1.55190322 & -3.87984794 & 2.69957390 \\
\hline $\mathrm{H}$ & -3.53594586 & -2.58657838 & 2.56697860 \\
\hline $\mathrm{H}$ & -4.95088763 & -0.44033565 & 1.70729414 \\
\hline S & 0.48663867 & 1.67025963 & 2.53710628 \\
\hline $\mathrm{C}$ & 1.23998424 & 3.32133902 & 2.24865935 \\
\hline $\mathrm{H}$ & 2.12391911 & 3.39555671 & 2.89061191 \\
\hline $\mathrm{H}$ & 1.56657877 & 3.40154751 & 1.21249092 \\
\hline $\mathrm{H}$ & -0.43084616 & 4.58551105 & 1.76050354 \\
\hline $\mathrm{C}$ & 0.31662859 & 4.52616963 & 2.54742416 \\
\hline $\mathrm{C}$ & -0.33565693 & 4.41553610 & 3.93543205 \\
\hline $\mathrm{N}$ & -1.68853250 & 4.31384415 & 3.99560685 \\
\hline $\mathrm{C}$ & -2.37031625 & 4.24440072 & 5.30340622 \\
\hline $\mathrm{C}$ & -1.95948388 & 3.05606309 & 6.18216247 \\
\hline $\mathrm{N}$ & -1.70029045 & 1.88785423 & 5.54329503 \\
\hline C & -1.39306225 & 0.65956149 & 6.27794259 \\
\hline $\mathrm{C}$ & -0.02368418 & 0.73964125 & 6.98987921 \\
\hline $\mathrm{N}$ & 1.02798966 & 1.15767837 & 6.23344519 \\
\hline $\mathrm{C}$ & 2.34697672 & 1.34187518 & 6.83402118 \\
\hline $\mathrm{C}$ & 2.35324534 & 2.41751334 & 7.94124094 \\
\hline $\mathrm{N}$ & 1.65650275 & 3.57100809 & 7.71893038 \\
\hline $\mathrm{C}$ & 1.66465126 & 4.58407588 & 8.77151067 \\
\hline $\mathrm{C}$ & 1.00541106 & 4.12362228 & 10.09680398 \\
\hline $\mathrm{N}$ & -0.10951200 & 3.36059337 & 9.96684998 \\
\hline $\mathrm{N}$ & 1.11513790 & 5.74245155 & 2.44331260 \\
\hline C & 0.87730513 & 6.65843018 & 1.46044667 \\
\hline $\mathrm{C}$ & 1.76364419 & 7.92663681 & 1.43782296 \\
\hline $\mathrm{N}$ & 3.07851299 & 7.76787700 & 2.08910777 \\
\hline $\mathrm{C}$ & 3.17779753 & 7.99398167 & 3.43310147 \\
\hline $\mathrm{C}$ & 4.54426419 & 7.78075810 & 4.11051639 \\
\hline $\mathrm{N}$ & 4.50201068 & 8.20269706 & 5.48883711 \\
\hline $\mathrm{C}$ & 5.18521343 & 9.31016910 & 5.90127385 \\
\hline C & 5.05766014 & 9.62281174 & 7.3842223 \\
\hline
\end{tabular}




\begin{tabular}{|c|c|c|c|}
\hline $\mathrm{H}$ & 4.47807589 & 8.87526496 & 7.93246693 \\
\hline $\mathrm{H}$ & 4.58446893 & 10.60374971 & 7.50002101 \\
\hline $\mathrm{H}$ & 6.06046543 & 9.69363311 & 7.81691690 \\
\hline $\mathrm{O}$ & 5.85978651 & 10.00578209 & 5.14284710 \\
\hline $\mathrm{H}$ & 3.92256933 & 7.66541267 & 6.14058575 \\
\hline $\mathrm{H}$ & 5.30099978 & 8.39692216 & 3.61381395 \\
\hline C & 4.93296082 & 6.28392635 & 4.05740279 \\
\hline $\mathrm{H}$ & 5.94181434 & 6.16110017 & 4.46127779 \\
\hline $\mathrm{H}$ & 4.24933117 & 5.70183175 & 4.68564266 \\
\hline $\mathrm{H}$ & 4.90966371 & 5.86933458 & 3.04519627 \\
\hline $\mathrm{O}$ & 2.15249326 & 8.24235757 & 4.07740950 \\
\hline C & 4.18871907 & 7.80496440 & 1.11453662 \\
\hline $\mathrm{H}$ & 4.72148874 & 8.76225512 & 1.18492467 \\
\hline $\mathrm{H}$ & 4.90933685 & 7.00347969 & 1.29534859 \\
\hline C & 3.47753693 & 7.66313773 & -0.23891197 \\
\hline $\mathrm{H}$ & 4.05189412 & 8.11480014 & -1.05281138 \\
\hline $\mathrm{H}$ & 3.33318938 & 6.60183803 & -0.47609310 \\
\hline $\mathrm{C}$ & 2.11903520 & 8.34058507 & -0.00052079 \\
\hline $\mathrm{H}$ & 2.22461830 & 9.43007642 & -0.05087857 \\
\hline $\mathrm{H}$ & 1.34105750 & 8.03321124 & -0.70045385 \\
\hline $\mathrm{H}$ & 1.19818011 & 8.70116468 & 1.96513458 \\
\hline $\mathrm{O}$ & 0.00324992 & 6.51056870 & 0.60668862 \\
\hline $\mathrm{H}$ & 1.70495291 & 5.98705711 & 3.22807095 \\
\hline $\mathrm{O}$ & 0.37183444 & 4.37683499 & 4.95299519 \\
\hline $\mathrm{C}$ & -2.64184340 & 4.59882078 & 2.89339280 \\
\hline $\mathrm{H}$ & -2.26054910 & 5.38056565 & 2.2333180 \\
\hline $\mathrm{H}$ & -2.80409778 & 3.69517248 & 2.29578774 \\
\hline $\mathrm{C}$ & -3.91982378 & 5.02491034 & 3.62894558 \\
\hline $\mathrm{H}$ & -3.88800088 & 6.09909670 & 3.84599973 \\
\hline $\mathrm{H}$ & -4.81954677 & 4.82924868 & 3.03810768 \\
\hline $\mathrm{C}$ & -3.86603441 & 4.21741426 & 4.93252283 \\
\hline $\mathrm{H}$ & -4.18374665 & 3.18320736 & 4.75462813 \\
\hline $\mathrm{H}$ & -4.48478400 & 4.62708605 & 5.73434818 \\
\hline $\mathrm{H}$ & -2.12946179 & 5.13624520 & 5.89238713 \\
\hline $\mathrm{O}$ & -1.96839044 & 3.17355946 & 7.40506797 \\
\hline $\mathrm{H}$ & -1.57032201 & 1.89671113 & 4.53636480 \\
\hline $\mathrm{H}$ & -2.11412817 & 0.57345705 & 7.0945415 \\
\hline $\mathrm{C}$ & -1.48468089 & -0.55954462 & 5.36093117 \\
\hline $\mathrm{H}$ & -2.48676413 & -0.64349650 & 4.9269364 \\
\hline $\mathrm{H}$ & -0.75686997 & -0.50469914 & 4.54544340 \\
\hline $\mathrm{H}$ & -1.27935503 & -1.46499112 & 5.94044166 \\
\hline $\mathrm{O}$ & 0.09231348 & 0.38623804 & 8.15822477 \\
\hline $\mathrm{H}$ & 0.86514482 & 1.49954893 & 5.29263101 \\
\hline $\mathrm{H}$ & 2.60654390 & 0.42710900 & 7.3715472 \\
\hline $\mathrm{C}$ & 3.42171581 & 1.66475444 & 5.78576226 \\
\hline
\end{tabular}




$\begin{array}{crrc}\mathrm{H} & 3.12136820 & 2.57046642 & 5.23889180 \\ \mathrm{H} & 4.33117732 & 1.92793485 & 6.34166506 \\ \mathrm{C} & 3.75755807 & 0.54369024 & 4.78061592 \\ \mathrm{H} & 2.85447395 & 0.31631298 & 4.19834527 \\ \mathrm{C} & 4.22234179 & -0.74702705 & 5.47108988 \\ \mathrm{H} & 4.48828119 & -1.50771899 & 4.72692040 \\ \mathrm{H} & 5.10700686 & -0.56506226 & 6.09527992 \\ \mathrm{H} & 3.44451456 & -1.17484170 & 6.11203156 \\ \mathrm{C} & 4.83001127 & 1.04606219 & 3.80138580 \\ \mathrm{H} & 5.06878304 & 0.28190138 & 3.05226485 \\ \mathrm{H} & 4.49656495 & 1.94385330 & 3.26673332 \\ \mathrm{H} & 5.75881135 & 1.29668329 & 4.33066036 \\ \mathrm{O} & 3.03706564 & 2.24887113 & 8.94351107 \\ \mathrm{H} & 1.11478773 & 3.70715874 & 6.87227367 \\ \mathrm{H} & 2.69909101 & 4.81785936 & 9.03049901 \\ \mathrm{C} & 0.95241174 & 5.86368187 & 8.33261268 \\ \mathrm{H} & 0.85030222 & 6.49668275 & 9.22372739 \\ \mathrm{H} & -0.06390594 & 5.63453561 & 7.98825328 \\ \mathrm{O} & 2.93558894 & 6.62696822 & 7.17443407 \\ \mathrm{C} & 1.70484903 & 6.68187548 & 7.29288943 \\ \mathrm{H} & 1.37437058 & 7.99281096 & 5.76513163 \\ \mathrm{H} & -0.06164422 & 7.43628704 & 6.57630807 \\ \mathrm{O} & 1.42289331 & 4.56456335 & 11.16348526 \\ \mathrm{H} & -0.35433247 & 2.99346425 & 9.05551862 \\ \mathrm{C} & -0.80787834 & 2.81241250 & 11.11353286 \\ \mathrm{H} & -0.38008745 & 3.26308029 & 12.01041999 \\ \mathrm{H} & -1.87786098 & 3.04689386 & 11.06197168 \\ \mathrm{H} & -0.68781494 & 1.72342512 & 11.15979463 \\ \mathrm{~N} & 0.94264182 & 7.53189148 & 6.56668307 \\ & & & \\ & & & \end{array}$

${ }^{4}$ I*-B

160

$\begin{array}{llll}\mathrm{C} & -1.71586768 & -5.57517410 & -3.94527969 \\ \mathrm{H} & -2.27122474 & -6.35044592 & -4.46550901 \\ \mathrm{C} & -1.76490851 & -4.24845417 & -4.39212482 \\ \mathrm{H} & -2.36804460 & -3.99358274 & -5.25973838 \\ \mathrm{C} & -0.94114604 & -5.89140124 & -2.82031659 \\ \mathrm{H} & -0.89576438 & -6.91658814 & -2.46164750 \\ \mathrm{C} & -1.05622756 & -3.24823343 & -3.74093226 \\ \mathrm{H} & -1.12010579 & -2.23598712 & -4.11388932 \\ \mathrm{C} & -0.23172393 & -4.90045940 & -2.16181953\end{array}$




\begin{tabular}{|c|c|c|c|}
\hline $\mathrm{H}$ & 0.36957125 & -5.15483991 & -1.29219801 \\
\hline $\mathrm{C}$ & -0.25603955 & -3.53735564 & -2.59532041 \\
\hline $\mathrm{C}$ & 0.52973333 & -2.60933432 & -1.86574863 \\
\hline $\mathrm{H}$ & 1.03589929 & -3.02601723 & -0.99711824 \\
\hline $\mathrm{C}$ & 0.72610925 & -1.12159836 & -1.97926992 \\
\hline $\mathrm{H}$ & 1.76245606 & -0.91778088 & -1.68037136 \\
\hline $\mathrm{C}$ & 0.49464800 & -0.43705575 & -3.33178960 \\
\hline $\mathrm{H}$ & 0.87100529 & 0.58857497 & -3.27966057 \\
\hline $\mathrm{H}$ & 1.02196976 & -0.96237143 & -4.13603609 \\
\hline $\mathrm{H}$ & -0.56982990 & -0.38458511 & -3.57210187 \\
\hline $\mathrm{O}$ & -0.14988123 & -0.50872935 & -1.01843332 \\
\hline $\mathrm{Fe}$ & 0.12284501 & 0.40112978 & 0.50278259 \\
\hline $\mathrm{N}$ & -1.11270016 & 1.85911283 & -0.12573634 \\
\hline $\mathrm{N}$ & 1.68775556 & 1.38251771 & -0.29982621 \\
\hline $\mathrm{N}$ & 1.38497816 & -1.02244437 & 1.20939224 \\
\hline $\mathrm{N}$ & -1.41381877 & -0.52700924 & 1.4084043 \\
\hline $\mathrm{C}$ & -2.47225037 & 1.90769549 & 0.0438805 \\
\hline $\mathrm{C}$ & -3.01444797 & 3.05519466 & -0.64467900 \\
\hline $\mathrm{C}$ & -1.96681986 & 3.69476450 & -1.2332504 \\
\hline $\mathrm{C}$ & -0.78285475 & 2.93453405 & -0.9148968 \\
\hline $\mathrm{C}$ & 1.63729182 & 2.52287014 & -1.06870712 \\
\hline $\mathrm{C}$ & 2.96411374 & 2.88360310 & -1.49706669 \\
\hline $\mathrm{C}$ & 3.81570428 & 1.95560680 & -0.97553991 \\
\hline $\mathrm{C}$ & 3.01235274 & 1.01707599 & -0.2359972 \\
\hline $\mathrm{C}$ & 2.75067285 & -1.05099319 & 1.0740649 \\
\hline $\mathrm{C}$ & 3.28421624 & -2.23321460 & 1.70703010 \\
\hline $\mathrm{C}$ & 2.22497685 & -2.91548135 & 2.22277028 \\
\hline $\mathrm{C}$ & 1.04215607 & -2.14852341 & 1.9115542 \\
\hline $\mathrm{C}$ & -1.38061191 & -1.74606974 & 2.04263300 \\
\hline $\mathrm{C}$ & -2.71331963 & -2.13554044 & 2.4316654 \\
\hline $\mathrm{C}$ & -3.55036075 & -1.14337038 & 2.0206984 \\
\hline $\mathrm{C}$ & -2.73267273 & -0.14848712 & 1.3707859 \\
\hline $\mathrm{C}$ & -3.23356858 & 0.98895038 & 0.7534899 \\
\hline $\mathrm{H}$ & -4.30628792 & 1.14943120 & 0.7928272 \\
\hline $\mathrm{C}$ & 0.49073308 & 3.25071432 & -1.3630423 \\
\hline $\mathrm{H}$ & 0.60200263 & 4.14684104 & -1.96352138 \\
\hline $\mathrm{C}$ & 3.51688307 & -0.10389815 & 0.4109305 \\
\hline $\mathrm{H}$ & 4.59003646 & -0.26090064 & 0.3750014 \\
\hline $\mathrm{C}$ & -0.24560619 & -2.50411152 & 2.28814918 \\
\hline $\mathrm{H}$ & -0.37253513 & -3.43902732 & 2.8242115 \\
\hline $\mathrm{H}$ & -1.97186563 & 4.59838229 & -1.8281848 \\
\hline $\mathrm{H}$ & -4.06411573 & 3.31973639 & -0.6653847 \\
\hline $\mathrm{H}$ & 3.19709443 & 3.74033290 & -2.11638667 \\
\hline $\mathrm{H}$ & 4.89106935 & 1.89159306 & -1.0823101 \\
\hline $\mathrm{H}$ & 4.33509505 & -2.49004000 & 1.7443629 \\
\hline
\end{tabular}




\begin{tabular}{|c|c|c|c|}
\hline $\mathrm{H}$ & 2.22630110 & -3.84616032 & 2.77566043 \\
\hline $\mathrm{H}$ & -2.95795676 & -3.05484323 & 2.94818216 \\
\hline $\mathrm{H}$ & -4.62639535 & -1.08360705 & 2.12270760 \\
\hline S & 0.55342990 & 1.56752245 & 2.62060820 \\
\hline $\mathrm{C}$ & 1.31152256 & 3.20857758 & 2.29849805 \\
\hline $\mathrm{H}$ & 2.17057223 & 3.28862110 & 2.97401962 \\
\hline $\mathrm{H}$ & 1.68766920 & 3.25295919 & 1.27814011 \\
\hline $\mathrm{H}$ & -0.33063641 & 4.46898545 & 1.71886287 \\
\hline $\mathrm{C}$ & 0.39459696 & 4.43096292 & 2.52771445 \\
\hline $\mathrm{C}$ & -0.29619591 & 4.37844126 & 3.90148907 \\
\hline $\mathrm{N}$ & -1.65030921 & 4.29235794 & 3.93391299 \\
\hline $\mathrm{C}$ & -2.36469537 & 4.29749146 & 5.22680035 \\
\hline $\mathrm{C}$ & -1.98520670 & 3.15174924 & 6.17355372 \\
\hline $\mathrm{N}$ & -1.72252083 & 1.95151860 & 5.59898881 \\
\hline $\mathrm{C}$ & -1.44638139 & 0.75355177 & 6.39331364 \\
\hline $\mathrm{C}$ & -0.09325057 & 0.85591964 & 7.13236985 \\
\hline $\mathrm{N}$ & 0.97720228 & 1.23246806 & 6.37933184 \\
\hline $\mathrm{C}$ & 2.28731857 & 1.43099037 & 6.99431147 \\
\hline $\mathrm{C}$ & 2.28145236 & 2.55321915 & 8.05397878 \\
\hline $\mathrm{N}$ & 1.59855263 & 3.70219546 & 7.77173055 \\
\hline $\mathrm{C}$ & 1.59554898 & 4.75462175 & 8.78622685 \\
\hline $\mathrm{C}$ & 0.90547172 & 4.34468249 & 10.11265033 \\
\hline $\mathrm{N}$ & -0.21207733 & 3.58574279 & 9.98216983 \\
\hline $\mathrm{N}$ & 1.20898240 & 5.63399674 & 2.39896762 \\
\hline $\mathrm{C}$ & 0.99986447 & 6.50911728 & 1.37225222 \\
\hline $\mathrm{C}$ & 1.88713584 & 7.77490535 & 1.32415923 \\
\hline $\mathrm{N}$ & 3.18189487 & 7.64161193 & 2.01992078 \\
\hline $\mathrm{C}$ & 3.24001712 & 7.92164385 & 3.35520109 \\
\hline $\mathrm{C}$ & 4.59256212 & 7.76967679 & 4.07398770 \\
\hline $\mathrm{N}$ & 4.50344489 & 8.24319159 & 5.43289232 \\
\hline $\mathrm{C}$ & 5.14765519 & 9.38285455 & 5.81952432 \\
\hline $\mathrm{C}$ & 4.97282510 & 9.74883411 & 7.28521866 \\
\hline $\mathrm{H}$ & 4.40297102 & 9.00500133 & 7.84850057 \\
\hline $\mathrm{H}$ & 4.46634354 & 10.71776683 & 7.35040985 \\
\hline $\mathrm{H}$ & 5.96152169 & 9.86789119 & 7.73923766 \\
\hline $\mathrm{O}$ & 5.82358272 & 10.06624252 & 5.05148181 \\
\hline $\mathrm{H}$ & 3.92356358 & 7.71085891 & 6.08857555 \\
\hline $\mathrm{H}$ & 5.34825975 & 8.38286880 & 3.57219418 \\
\hline $\mathrm{C}$ & 5.01499268 & 6.28043557 & 4.09377601 \\
\hline $\mathrm{H}$ & 6.03012064 & 6.19956320 & 4.49310939 \\
\hline $\mathrm{H}$ & 4.34946160 & 5.71874602 & 4.75852599 \\
\hline $\mathrm{H}$ & 4.99011264 & 5.81209501 & 3.10548651 \\
\hline $\mathrm{O}$ & 2.19015830 & 8.16203168 & 3.96290753 \\
\hline $\mathrm{C}$ & 4.32059959 & 7.65251384 & 1.07773840 \\
\hline $\mathrm{H}$ & 4.84184092 & 8.61755859 & 1.12498575 \\
\hline
\end{tabular}




\begin{tabular}{|c|c|c|c|}
\hline $\mathrm{H}$ & 5.04255626 & 6.86650797 & 1.31234215 \\
\hline $\mathrm{C}$ & 3.65130314 & 7.44951659 & -0.28880866 \\
\hline $\mathrm{H}$ & 4.24765234 & 7.87055399 & -1.10328808 \\
\hline $\mathrm{H}$ & 3.51814476 & 6.37842386 & -0.48492386 \\
\hline $\mathrm{C}$ & 2.28468521 & 8.13130070 & -0.11870061 \\
\hline $\mathrm{H}$ & 2.39024014 & 9.21811002 & -0.21022735 \\
\hline $\mathrm{H}$ & 1.52832970 & 7.79374592 & -0.82839241 \\
\hline $\mathrm{H}$ & 1.30778559 & 8.56974583 & 1.80437033 \\
\hline $\mathrm{O}$ & 0.15520135 & 6.32186858 & 0.49658161 \\
\hline $\mathrm{H}$ & 1.75118199 & 5.92494683 & 3.20275341 \\
\hline $\mathrm{O}$ & 0.38563212 & 4.37461148 & 4.93855704 \\
\hline C & -2.57461243 & 4.51967172 & 2.79460078 \\
\hline $\mathrm{H}$ & -2.17384898 & 5.26170828 & 2.10145136 \\
\hline $\mathrm{H}$ & -2.72807754 & 3.58479721 & 2.24523123 \\
\hline C & -3.86656458 & 4.99386798 & 3.47351772 \\
\hline $\mathrm{H}$ & -3.82989134 & 6.07813070 & 3.63181858 \\
\hline $\mathrm{H}$ & -4.75393177 & 4.77395156 & 2.87276855 \\
\hline $\mathrm{C}$ & -3.85118263 & 4.26095293 & 4.82118934 \\
\hline $\mathrm{H}$ & -4.17315839 & 3.22055046 & 4.69422533 \\
\hline $\mathrm{H}$ & -4.48647330 & 4.71997824 & 5.58231554 \\
\hline $\mathrm{H}$ & -2.12971632 & 5.21664417 & 5.77452015 \\
\hline $\mathrm{O}$ & -2.01759123 & 3.33455275 & 7.38788508 \\
\hline $\mathrm{H}$ & -1.58491087 & 1.90886924 & 4.59395527 \\
\hline $\mathrm{H}$ & -2.18696984 & 0.70650565 & 7.19577417 \\
\hline $\mathrm{C}$ & -1.52440973 & -0.50372182 & 5.52855876 \\
\hline $\mathrm{H}$ & -2.52146290 & -0.61120777 & 5.0887710 \\
\hline $\mathrm{H}$ & -0.78946565 & -0.47960882 & 4.71780316 \\
\hline $\mathrm{H}$ & -1.32148845 & -1.38273672 & 6.14811905 \\
\hline $\mathrm{O}$ & -0.00312437 & 0.54727198 & 8.31530617 \\
\hline $\mathrm{H}$ & 0.82625782 & 1.56462885 & 5.43382606 \\
\hline $\mathrm{H}$ & 2.53219877 & 0.53688673 & 7.57156568 \\
\hline $\mathrm{C}$ & 3.37814665 & 1.70738943 & 5.94923796 \\
\hline $\mathrm{H}$ & 3.08841968 & 2.59200757 & 5.3633415 \\
\hline $\mathrm{H}$ & 4.28194496 & 1.99006503 & 6.50472269 \\
\hline $\mathrm{C}$ & 3.72063693 & 0.54498751 & 4.99487728 \\
\hline $\mathrm{H}$ & 2.81414949 & 0.27421093 & 4.43654510 \\
\hline $\mathrm{C}$ & 4.21576013 & -0.70307097 & 5.7408301 \\
\hline $\mathrm{H}$ & 4.46375035 & -1.50233932 & 5.0320395 \\
\hline $\mathrm{H}$ & 5.11833449 & -0.48069722 & 6.32508969 \\
\hline $\mathrm{H}$ & 3.46283787 & -1.09767665 & 6.4310996 \\
\hline $\mathrm{C}$ & 4.77160800 & 1.01427258 & 3.97691537 \\
\hline $\mathrm{H}$ & 5.01603855 & 0.21527739 & 3.2673718 \\
\hline $\mathrm{H}$ & 4.41462159 & 1.87569556 & 3.3992775 \\
\hline $\mathrm{H}$ & 5.70095307 & 1.31082852 & 4.4809590 \\
\hline $\mathrm{O}$ & 2.93788610 & 2.41874211 & 9.0794354 \\
\hline
\end{tabular}




$\begin{array}{lrrc}\mathrm{H} & 1.08951006 & 3.82298655 & 6.90291903 \\ \mathrm{H} & 2.62620884 & 4.99139646 & 9.05710226 \\ \mathrm{C} & 0.90063295 & 6.02120699 & 8.28544870 \\ \mathrm{H} & 0.77340517 & 6.68439264 & 9.15116573 \\ \mathrm{H} & -0.10565156 & 5.78335998 & 7.91804085 \\ \mathrm{O} & 2.91668536 & 6.70827364 & 7.13702559 \\ \mathrm{C} & 1.68697032 & 6.80177796 & 7.24171637 \\ \mathrm{H} & 1.40940443 & 8.07463555 & 5.67476241 \\ \mathrm{H} & -0.04810849 & 7.59941908 & 6.49620512 \\ \mathrm{O} & 1.30496047 & 4.81559271 & 11.17318690 \\ \mathrm{H} & -0.43503278 & 3.19183358 & 9.07655800 \\ \mathrm{C} & -0.92911798 & 3.06257237 & 11.12919182 \\ \mathrm{H} & -0.50575846 & 3.52149294 & 12.02401138 \\ \mathrm{H} & -1.99569173 & 3.30949712 & 11.06420654 \\ \mathrm{H} & -0.82238666 & 1.97314027 & 11.19082094 \\ \mathrm{~N} & 0.95903970 & 7.66281968 & 6.49273593\end{array}$

${ }^{4}$ E-B

160

$\begin{array}{lrrr}\mathrm{C} & -2.92800187 & -4.75815844 & -3.51754458 \\ \mathrm{H} & -3.71130886 & -5.37032441 & -3.95604294 \\ \mathrm{C} & -3.08178001 & -3.37305235 & -3.43599848 \\ \mathrm{H} & -3.98965436 & -2.90371021 & -3.80578226 \\ \mathrm{C} & -1.76454263 & -5.35507181 & -3.02505314 \\ \mathrm{H} & -1.63920705 & -6.43310829 & -3.07981826 \\ \mathrm{C} & -2.07639674 & -2.58412785 & -2.87326846 \\ \mathrm{H} & -2.19436038 & -1.50805804 & -2.79620564 \\ \mathrm{C} & -0.76181582 & -4.56917668 & -2.45835986 \\ \mathrm{H} & 0.13975078 & -5.03879631 & -2.07043750 \\ \mathrm{C} & -0.90396075 & -3.17616538 & -2.38688465 \\ \mathrm{C} & 0.19577405 & -2.36571006 & -1.78369999 \\ \mathrm{H} & 0.65299813 & -2.81128250 & -0.89921942 \\ \mathrm{C} & 1.04105776 & -1.40604005 & -2.53725582 \\ \mathrm{H} & 2.03383045 & -1.21859971 & -2.12596362 \\ \mathrm{C} & 0.88565606 & -1.10672618 & -4.00422441 \\ \mathrm{H} & 1.03057406 & -0.03568717 & -4.18717590 \\ \mathrm{H} & 1.64514073 & -1.65185259 & -4.57730939 \\ \mathrm{H} & -0.10117199 & -1.39429170 & -4.37456872 \\ \mathrm{O} & -0.00869269 & -0.94562944 & -1.65814310 \\ \mathrm{Fe} & 0.37096961 & 0.36093386 & 0.60772877 \\ \mathrm{~N} & -1.25767736 & 1.35962034 & -0.03156109 \\ \mathrm{~N} & 1.53134216 & 1.58841482 & -0.48055027 \\ \mathrm{~N} & 1.96597836 & -0.83527836 & 0.92941150\end{array}$




\begin{tabular}{|c|c|c|c|}
\hline $\mathrm{N}$ & -0.81555483 & -1.03518000 & 1.42766533 \\
\hline $\mathrm{C}$ & -2.57220686 & 1.04885348 & 0.22268843 \\
\hline $\mathrm{C}$ & -3.44090201 & 2.04615163 & -0.35044275 \\
\hline $\mathrm{C}$ & -2.63801757 & 2.97083595 & -0.94827873 \\
\hline $\mathrm{C}$ & -1.27988266 & 2.53258063 & -0.74983200 \\
\hline $\mathrm{C}$ & 1.13834332 & 2.74546449 & -1.11952709 \\
\hline $\mathrm{C}$ & 2.27957281 & 3.41495840 & -1.68268741 \\
\hline $\mathrm{C}$ & 3.37027164 & 2.64780655 & -1.39464607 \\
\hline $\mathrm{C}$ & 2.89796986 & 1.51520860 & -0.64391517 \\
\hline $\mathrm{C}$ & 3.27534183 & -0.57993142 & 0.58701812 \\
\hline $\mathrm{C}$ & 4.14057222 & -1.59876031 & 1.12543210 \\
\hline $\mathrm{C}$ & 3.34748018 & -2.46598870 & 1.81370684 \\
\hline $\mathrm{C}$ & 1.99555064 & -1.98332692 & 1.68881135 \\
\hline $\mathrm{C}$ & -0.41995668 & -2.17063963 & 2.10072997 \\
\hline $\mathrm{C}$ & -1.56833320 & -2.87804381 & 2.60226987 \\
\hline $\mathrm{C}$ & -2.66747701 & -2.16975357 & 2.21804909 \\
\hline $\mathrm{C}$ & -2.19224788 & -1.02295259 & 1.49009515 \\
\hline $\mathrm{C}$ & -3.01650121 & -0.05762363 & 0.93236712 \\
\hline $\mathrm{H}$ & -4.08780363 & -0.17929505 & 1.05644350 \\
\hline $\mathrm{C}$ & -0.16634428 & 3.20244015 & -1.23435892 \\
\hline $\mathrm{H}$ & -0.32410470 & 4.14570951 & -1.74511663 \\
\hline $\mathrm{C}$ & 3.71664143 & 0.50756357 & -0.15116514 \\
\hline $\mathrm{H}$ & 4.78080397 & 0.58252935 & -0.35039868 \\
\hline $\mathrm{C}$ & 0.88939212 & -2.60743636 & 2.24577554 \\
\hline $\mathrm{H}$ & 1.05666066 & -3.51462914 & 2.81716034 \\
\hline $\mathrm{H}$ & -2.92186761 & 3.86529044 & -1.48780844 \\
\hline $\mathrm{H}$ & -4.52212041 & 2.02281869 & -0.29947866 \\
\hline $\mathrm{H}$ & 2.23354960 & 4.34740915 & -2.22986374 \\
\hline $\mathrm{H}$ & 4.40490599 & 2.81910533 & -1.66341668 \\
\hline $\mathrm{H}$ & 5.21447562 & -1.62740706 & 0.99262623 \\
\hline $\mathrm{H}$ & 3.63563710 & -3.35390337 & 2.36163553 \\
\hline $\mathrm{H}$ & -1.52457714 & -3.80168943 & 3.16484421 \\
\hline $\mathrm{H}$ & -3.71061740 & -2.39461804 & 2.39962993 \\
\hline S & 0.62131936 & 1.58125797 & 2.79993337 \\
\hline $\mathrm{C}$ & 1.49233827 & 3.16648523 & 2.44816938 \\
\hline $\mathrm{H}$ & 2.28956396 & 3.26698592 & 3.19249049 \\
\hline $\mathrm{H}$ & 1.95457342 & 3.13521655 & 1.46197653 \\
\hline $\mathrm{H}$ & -0.06229445 & 4.42724057 & 1.65972644 \\
\hline C & 0.59325809 & 4.41932587 & 2.52674995 \\
\hline $\mathrm{C}$ & -0.20676299 & 4.44324418 & 3.83904302 \\
\hline $\mathrm{N}$ & -1.56043835 & 4.38215307 & 3.76705513 \\
\hline $\mathrm{C}$ & -2.36909908 & 4.41912985 & 5.00106002 \\
\hline $\mathrm{C}$ & -2.08677016 & 3.28098866 & 5.99261237 \\
\hline $\mathrm{N}$ & -1.80553432 & 2.06662932 & 5.45545997 \\
\hline $\mathrm{C}$ & -1.60479246 & 0.88584497 & 6.2968009 \\
\hline
\end{tabular}




$\begin{array}{lrrr}\mathrm{C} & -0.30702541 & 0.97300051 & 7.13150475 \\ \mathrm{~N} & 0.82407356 & 1.30819624 & 6.45366768 \\ \mathrm{C} & 2.09012537 & 1.48966051 & 7.15872066 \\ \mathrm{C} & 2.03447752 & 2.63469126 & 8.19298334 \\ \mathrm{~N} & 1.40042093 & 3.78865987 & 7.83022662 \\ \mathrm{C} & 1.33712365 & 4.87421855 & 8.80533423 \\ \mathrm{C} & 0.53759332 & 4.52508777 & 10.08630010 \\ \mathrm{~N} & -0.58160725 & 3.78124066 & 9.89600201 \\ \mathrm{~N} & 1.44161951 & 5.60073704 & 2.40856164 \\ \mathrm{C} & 1.34043947 & 6.43246079 & 1.33307587 \\ \mathrm{C} & 2.26703561 & 7.67156189 & 1.30411502 \\ \mathrm{~N} & 3.49708200 & 7.53349241 & 2.10816081 \\ \mathrm{C} & 3.46244429 & 7.86880124 & 3.43211984 \\ \mathrm{C} & 4.74291554 & 7.67519874 & 4.26596855 \\ \mathrm{~N} & 4.57443846 & 8.21147611 & 5.59394159 \\ \mathrm{C} & 5.25111818 & 9.32980121 & 5.98696881 \\ \mathrm{C} & 4.98867702 & 9.76303363 & 7.42110342 \\ \mathrm{H} & 4.35059498 & 9.06566763 & 7.97057291 \\ \mathrm{H} & 4.51744174 & 10.75180619 & 7.41174410 \\ \mathrm{H} & 5.94702288 & 9.86535192 & 7.93948318 \\ \mathrm{O} & 6.01812087 & 9.94659370 & 5.24834368 \\ \mathrm{H} & 3.92919917 & 7.73248363 & 6.22946499 \\ \mathrm{H} & 5.56853276 & 8.22671525 & 3.80420156 \\ \mathrm{C} & 5.08053727 & 6.16881950 & 4.37536430 \\ \mathrm{H} & 6.04402491 & 6.05257115 & 4.87963905 \\ \mathrm{H} & 4.32264610 & 5.66382401 & 4.98458805 \\ \mathrm{H} & 5.13220066 & 5.66763283 & 3.40426421 \\ \mathrm{O} & 2.38470614 & 8.19765963 & 3.94122768 \\ \mathrm{C} & 4.70549799 & 7.45254676 & 1.26135870 \\ \mathrm{H} & 5.26512516 & 8.39579312 & 1.31038529 \\ \mathrm{H} & 5.37075457 & 6.64856371 & 1.58589445 \\ \mathrm{C} & 4.13844237 & 7.21561087 & -0.14583868 \\ \mathrm{H} & 4.81421136 & 7.57800289 & -0.92590444 \\ \mathrm{H} & 3.97720944 & 6.14291553 & -0.30938913 \\ \mathrm{C} & 2.79038143 & 7.95235535 & -0.11447386 \\ \mathrm{H} & & & \\ \mathrm{H} & & & \end{array}$




\begin{tabular}{|c|c|c|c|}
\hline $\mathrm{H}$ & -2.51745268 & 3.59268506 & 2.03974727 \\
\hline $\mathrm{C}$ & -3.72504783 & 5.07123297 & 3.10878129 \\
\hline $\mathrm{H}$ & -3.68563475 & 6.16112112 & 3.22116110 \\
\hline $\mathrm{H}$ & -4.56581047 & 4.83122800 & 2.45125488 \\
\hline $\mathrm{C}$ & -3.82271353 & 4.39656945 & 4.48394028 \\
\hline $\mathrm{H}$ & -4.15838212 & 3.35858484 & 4.37558333 \\
\hline $\mathrm{H}$ & -4.50219372 & 4.89912316 & 5.17633657 \\
\hline $\mathrm{H}$ & -2.16091332 & 5.34331650 & 5.55073554 \\
\hline $\mathrm{O}$ & -2.21978568 & 3.47775095 & 7.19830746 \\
\hline $\mathrm{H}$ & -1.51777178 & 2.01656808 & 4.48154526 \\
\hline $\mathrm{H}$ & -2.40136008 & 0.87659504 & 7.04466219 \\
\hline $\mathrm{C}$ & -1.65705261 & -0.39268942 & 5.45932876 \\
\hline $\mathrm{H}$ & -2.62494690 & -0.48494139 & 4.95554857 \\
\hline $\mathrm{H}$ & -0.86777685 & -0.41075095 & 4.7009354 \\
\hline $\mathrm{H}$ & -1.52277759 & -1.26066600 & 6.11227324 \\
\hline $\mathrm{O}$ & -0.31515602 & 0.70131670 & 8.32749278 \\
\hline $\mathrm{H}$ & 0.76562043 & 1.56149698 & 5.47244146 \\
\hline $\mathrm{H}$ & 2.27057586 & 0.60434251 & 7.77328484 \\
\hline C & 3.26174331 & 1.71085659 & 6.19007192 \\
\hline $\mathrm{H}$ & 3.04708936 & 2.59636810 & 5.57441384 \\
\hline $\mathrm{H}$ & 4.13455325 & 1.96696306 & 6.8049577 \\
\hline $\mathrm{C}$ & 3.62355457 & 0.52208958 & 5.2754391 \\
\hline $\mathrm{H}$ & 2.74533516 & 0.27585963 & 4.66355945 \\
\hline $\mathrm{C}$ & 4.02346090 & -0.72985035 & 6.07041708 \\
\hline $\mathrm{H}$ & 4.30958510 & -1.54254036 & 5.3912950 \\
\hline $\mathrm{H}$ & 4.88052289 & -0.52460048 & 6.72520018 \\
\hline $\mathrm{H}$ & 3.20595406 & -1.09834855 & 6.69902010 \\
\hline $\mathrm{C}$ & 4.75226235 & 0.93536682 & 4.31806750 \\
\hline $\mathrm{H}$ & 5.00011115 & 0.11993796 & 3.62827949 \\
\hline $\mathrm{H}$ & 4.46592419 & 1.80620214 & 3.71569395 \\
\hline $\mathrm{H}$ & 5.66348566 & 1.19656837 & 4.8724921 \\
\hline $\mathrm{O}$ & 2.61591632 & 2.51458712 & 9.26434537 \\
\hline $\mathrm{H}$ & 0.94860505 & 3.88219063 & 6.92674608 \\
\hline $\mathrm{H}$ & 2.34804997 & 5.10458375 & 9.14737488 \\
\hline $\mathrm{C}$ & 0.70799460 & 6.13277447 & 8.20605722 \\
\hline $\mathrm{H}$ & 0.51537686 & 6.82547100 & 9.03562754 \\
\hline $\mathrm{H}$ & -0.26503736 & 5.89419836 & 7.7589023 \\
\hline $\mathrm{O}$ & 2.82708368 & 6.78216522 & 7.23152230 \\
\hline $\mathrm{C}$ & 1.59245169 & 6.86923740 & 7.20933728 \\
\hline $\mathrm{H}$ & 1.46340528 & 8.08363372 & 5.57253446 \\
\hline $\mathrm{H}$ & -0.06369055 & 7.60889191 & 6.25610357 \\
\hline $\mathrm{O}$ & 0.86105295 & 5.02956337 & 11.15761097 \\
\hline $\mathrm{H}$ & -0.74971175 & 3.35981560 & 8.99095802 \\
\hline C & -1.41321235 & 3.34002922 & 10.99880080 \\
\hline $\mathrm{H}$ & -1.06582228 & 3.84588476 & 11.9010233 \\
\hline
\end{tabular}




$\begin{array}{lrll}\mathrm{H} & -2.46345379 & 3.59770165 & 10.81687877 \\ \mathrm{H} & -1.33580434 & 2.25484968 & 11.13622797 \\ \mathrm{~N} & 0.93780874 & 7.68538211 & 6.35140550\end{array}$

${ }^{2}$ TS1*-A

$\begin{array}{lrrr}\mathrm{C} & -0.09430445 & -6.21778372 & -1.56005032 \\ \mathrm{H} & -0.38365815 & -7.26113859 & -1.46896857 \\ \mathrm{C} & -0.67560536 & -5.40954114 & -2.54193551 \\ \mathrm{H} & -1.41496110 & -5.82754618 & -3.22000509 \\ \mathrm{C} & 0.86737594 & -5.67517279 & -0.70218119 \\ \mathrm{H} & 1.32917788 & -6.29550017 & 0.06139295 \\ \mathrm{C} & -0.31293251 & -4.07209546 & -2.66568351 \\ \mathrm{H} & -0.76490995 & -3.47682359 & -3.44797071 \\ \mathrm{C} & 1.23692710 & -4.34135062 & -0.82598142 \\ \mathrm{H} & 1.98265642 & -3.92108407 & -0.15588218 \\ \mathrm{C} & 0.64999993 & -3.49759218 & -1.80139110 \\ \mathrm{C} & 1.10705685 & -2.12463863 & -1.85299564 \\ \mathrm{H} & 1.97774962 & -1.92485920 & -1.23303729 \\ \mathrm{C} & 0.57943041 & -1.00910024 & -2.48294449 \\ \mathrm{H} & 1.20432603 & -0.12293833 & -2.44633490 \\ \mathrm{C} & -0.56775919 & -0.94994637 & -3.45184432 \\ \mathrm{H} & -0.84796486 & 0.09100068 & -3.62986927 \\ \mathrm{H} & -0.29422123 & -1.39802334 & -4.41824047 \\ \mathrm{H} & -1.44543840 & -1.47279686 & -3.06363134 \\ \mathrm{O} & -0.46273627 & -0.30019410 & -0.86846641 \\ \mathrm{Fe} & -0.17116699 & 0.83922208 & 0.33107227 \\ \mathrm{~N} & -1.76105015 & 1.94136382 & -0.25592290 \\ \mathrm{~N} & 1.05292280 & 2.01204460 & -0.74938235 \\ \mathrm{~N} & 1.44322037 & -0.12702224 & 1.06898811 \\ \mathrm{~N} & -1.37259714 & -0.18775007 & 1.59029102 \\ \mathrm{C} & -3.06231815 & 1.79428355 & 0.14273002 \\ \mathrm{C} & -3.90474520 & 2.75093244 & -0.53949072 \\ \mathrm{C} & -3.09256106 & 3.47722424 & -1.35210141 \\ \mathrm{C} & -1.75303631 & 2.96748335 & -1.16150268 \\ \mathrm{C} & 0.67542582 & 3.03694919 & -1.58375308 \\ \mathrm{C} & 1.83431065 & 3.61022688 & -2.22164205 \\ \mathrm{C} & 2.91778956 & 2.92274512 & -1.76454243 \\ \mathrm{C} & 2.42252575 & 1.92587997 & -0.84869219 \\ \mathrm{C} & 2.75662951 & 0.05125126 & 0.71402017 \\ \mathrm{C} & 3.59177755 & -0.90940076 & 1.39546950 \\ \mathrm{C} & 1.42409960 & -1.17874630 & 1.95021967 \\ \mathrm{C} & & & \end{array}$




$\begin{array}{lccc}\mathrm{C} & -1.00114468 & -1.23211985 & 2.39453845 \\ \mathrm{C} & -2.15754634 & -1.77748628 & 3.06715151 \\ \mathrm{C} & -3.22892657 & -1.04621556 & 2.65866229 \\ \mathrm{C} & -2.72863277 & -0.05966557 & 1.72946931 \\ \mathrm{C} & -3.52185355 & 0.86823362 & 1.06835264 \\ \mathrm{H} & -4.58502382 & 0.86440806 & 1.28777445 \\ \mathrm{C} & -0.62598310 & 3.47552475 & -1.79125207 \\ \mathrm{H} & -0.76902015 & 4.29744042 & -2.48608691 \\ \mathrm{C} & 3.22182535 & 1.00883394 & -0.17869689 \\ \mathrm{H} & 4.29130362 & 1.04817425 & -0.36165019 \\ \mathrm{C} & 0.29372335 & -1.70194995 & 2.56218545 \\ \mathrm{H} & 0.43595253 & -2.53962872 & 3.23804544 \\ \mathrm{H} & -3.35311938 & 4.29063825 & -2.01735806 \\ \mathrm{H} & -4.97422737 & 2.84032639 & -0.39698496 \\ \mathrm{H} & 1.80536972 & 4.43371968 & -2.92392835 \\ \mathrm{H} & 3.96169306 & 3.06492875 & -2.01423242 \\ \mathrm{H} & 4.66796211 & -0.97244374 & 1.29417004 \\ \mathrm{H} & 3.02094054 & -2.49093100 & 2.82306683 \\ \mathrm{H} & -2.13106620 & -2.60829644 & 3.76074043 \\ \mathrm{H} & -4.26776043 & -1.15099338 & 2.94477541 \\ \mathrm{~S} & 0.04825750 & 2.34286717 & 2.31738643 \\ \mathrm{C} & 1.66314363 & 3.19084353 & 2.25590631 \\ \mathrm{H} & 1.70214429 & 3.85260539 & 3.12915869 \\ \mathrm{H} & 2.49489471 & 2.48349852 & 2.32709035 \\ \mathrm{H} & 1.77749100 & 3.79719083 & 1.35352332\end{array}$




\section{Appendix C: Computational and Submission Scripts}

\section{C1: NWCHEM Single Point Calculation}

START Example \#Example is the name of the job.

PERMANENT_DIR ./perm \#Subdirectory to store checkpoint files. MEMORY total $4 \mathrm{gb}$

ECHO

\#

geometry noautosym nocenter print xyz

$\begin{array}{llll}\mathrm{O} & -10.28013989 & 4.67864101 & -4.96027930 \\ \mathrm{C} & -10.97264840 & 5.56658240 & -4.45430786 \\ \mathrm{~N} & -12.15569015 & 5.28509889 & -3.84775419 \\ \mathrm{C} & -12.69930439 & 3.94019405 & -3.74412443 \\ \mathrm{C} & -10.54071487 & 7.02591121 & -4.49476332 \\ \mathrm{H} & -12.69267645 & 6.05021152 & -3.46551144 \\ \mathrm{H} & -13.66861430 & 3.87120960 & -4.25015177 \\ \mathrm{H} & -12.82330954 & 3.64896736 & -2.69516929 \\ \mathrm{H} & -11.99453047 & 3.26238367 & -4.22576516 \\ \mathrm{H} & -11.25817175 & 7.71151865 & -4.03380551 \\ \mathrm{H} & -10.38973105 & 7.31573353 & -5.53882701 \\ \mathrm{H} & -9.57866178 & 7.12361427 & -3.98191200 \\ \mathrm{C} & -3.92656048 & -1.71034107 & -0.96530533 \\ \mathrm{C} & -2.99789588 & -0.61816866 & -0.80384910 \\ \mathrm{~N} & -1.71956032 & -1.11027092 & -0.63804224 \\ \mathrm{C} & -1.82689010 & -2.48721062 & -0.69458724 \\ \mathrm{C} & -3.20575048 & -2.86352938 & -0.89125350 \\ \mathrm{C} & -3.37219532 & 0.73619768 & -0.80333339 \\ \mathrm{C} & -2.44697375 & 1.77019956 & -0.62209591 \\ \mathrm{~N} & -1.07299713 & 1.62913437 & -0.43576114 \\ \mathrm{C} & -0.56091996 & 2.90800981 & -0.33135869 \\ \mathrm{C} & -1.63480160 & 3.86927400 & -0.41694267 \\ \mathrm{C} & -2.78770936 & 3.17229040 & -0.59979778 \\ \mathrm{C} & 0.78861106 & 3.25436324 & -0.17744060 \\ \mathrm{C} & 1.84982531 & 2.34902690 & -0.13641114 \\ \mathrm{~N} & 1.73932189 & 0.98298078 & -0.19740280 \\ \mathrm{C} & 3.03146947 & 0.49843649 & -0.12227225 \\ \mathrm{C} & 3.96464890 & 1.59152538 & -0.00084127 \\ \mathrm{C} & 3.23274841 & 2.73882570 & -0.00776995 \\ \mathrm{Fe} & 0.02794368 & -0.06347122 & -0.56034236 \\ \mathrm{~S} & -0.54962611 & -0.01636099 & 1.98113669\end{array}$




\begin{tabular}{|c|c|c|c|}
\hline $\mathrm{C}$ & 0.42895132 & 1.29392786 & 2.78702692 \\
\hline $\mathrm{C}$ & 3.39801749 & -0.84432917 & -0.14560854 \\
\hline $\mathrm{C}$ & 2.46203987 & -1.89299424 & -0.25708021 \\
\hline $\mathrm{N}$ & 1.09036334 & -1.76637654 & -0.36671017 \\
\hline $\mathrm{C}$ & 0.58045594 & -3.04356318 & -0.44705498 \\
\hline $\mathrm{C}$ & 1.65752704 & -4.00082929 & -0.37314255 \\
\hline $\mathrm{C}$ & 2.81647607 & -3.29149500 & -0.25958627 \\
\hline $\mathrm{C}$ & -0.77484308 & -3.39901771 & -0.5907326 \\
\hline $\mathrm{H}$ & 4.44889174 & -1.10131913 & -0.07336678 \\
\hline $\mathrm{H}$ & 1.53033697 & -5.07541991 & -0.4069188 \\
\hline $\mathrm{H}$ & 5.03827321 & 1.47720998 & 0.07726982 \\
\hline $\mathrm{H}$ & 3.58018606 & 3.76164762 & 0.06093642 \\
\hline $\mathrm{H}$ & 1.02517860 & 4.31164251 & -0.12556423 \\
\hline $\mathrm{H}$ & -1.50125361 & 4.94215930 & -0.36612520 \\
\hline $\mathrm{H}$ & -3.79088292 & 3.55828388 & -0.7270349 \\
\hline $\mathrm{H}$ & -4.41370330 & 0.99040801 & -0.9602882 \\
\hline $\mathrm{H}$ & -4.99127227 & -1.59171015 & -1.1187037 \\
\hline $\mathrm{H}$ & -3.56063521 & -3.88353066 & -0.9658748 \\
\hline $\mathrm{H}$ & -1.01268038 & -4.45602844 & -0.64250030 \\
\hline $\mathrm{H}$ & 3.82777573 & -3.67004770 & -0.18103426 \\
\hline $\mathrm{H}$ & 0.13742892 & 1.36039445 & 3.84035356 \\
\hline $\mathrm{H}$ & 1.50045116 & 1.09379118 & 2.70880144 \\
\hline $\mathrm{H}$ & 0.22097087 & 2.26032668 & 2.31296012 \\
\hline $\mathrm{O}$ & 0.19328935 & 0.00015457 & -2.1776833 \\
\hline $\mathrm{C}$ & -2.23282613 & -0.82166948 & -4.8250921 \\
\hline $\mathrm{C}$ & -3.07824160 & 0.43732407 & -4.7947226 \\
\hline $\mathrm{O}$ & -2.61711060 & 1.56157037 & -4.95122507 \\
\hline $\mathrm{O}$ & -4.37103352 & 0.20105354 & -4.5918985 \\
\hline $\mathrm{H}$ & -1.74553233 & -0.89551977 & -5.8031602 \\
\hline $\mathrm{H}$ & -2.83273982 & -1.71508646 & -4.64392217 \\
\hline $\mathrm{H}$ & -1.44255746 & -0.73993520 & $-4.070770 €$ \\
\hline $\mathrm{N}$ & -5.87411176 & 2.48898470 & -4.7762702 \\
\hline $\mathrm{C}$ & -5.42037829 & 3.67765684 & -5.3109892 \\
\hline $\mathrm{C}$ & -6.47192268 & 4.54483957 & -5.5028919 \\
\hline $\mathrm{N}$ & -7.59024700 & 3.85461587 & -5.0654287 \\
\hline $\mathrm{C}$ & -7.18062612 & 2.63198691 & -4.64574676 \\
\hline $\mathrm{C}$ & -6.57125644 & 5.92614490 & -6.0704968 \\
\hline $\mathrm{H}$ & -6.97633874 & 6.64259423 & -5.34344536 \\
\hline $\mathrm{H}$ & -5.58047108 & 6.28188997 & -6.3673042 \\
\hline $\mathrm{H}$ & -7.21650482 & 5.95550964 & $-6.957734 \varepsilon$ \\
\hline $\mathrm{H}$ & -8.55270796 & 4.20198653 & -5.0729815 \\
\hline $\mathrm{H}$ & -7.86148738 & 1.88526082 & -4.2609061 \\
\hline $\mathrm{H}$ & -4.88464290 & 1.07699448 & -4.6244067 \\
\hline $\mathrm{H}$ & -4.36919369 & 3.81766842 & -5.5214364 \\
\hline $\mathrm{O}$ & -0.22718091 & 2.57099446 & -3.5652237 \\
\hline
\end{tabular}




\begin{tabular}{|c|c|c|c|}
\hline $\mathrm{H}$ & 0.08023899 & 1.72291393 & -3.20089822 \\
\hline $\mathrm{H}$ & -0.99946738 & 2.31407363 & -4.10270097 \\
\hline bqH & -0.30533029 & 6.39043169 & -3.46447214 \\
\hline bqH & -1.30187651 & 5.84914350 & -4.65744550 \\
\hline $\mathrm{bqH}$ & -0.30938721 & 5.80142272 & -4.26851399 \\
\hline $\mathrm{N}$ & -0.70904694 & -1.74475205 & 4.97427042 \\
\hline $\mathrm{C}$ & 0.49808949 & -2.13120809 & 5.67799577 \\
\hline $\mathrm{C}$ & -1.93885007 & -2.24513897 & 5.29920849 \\
\hline $\mathrm{C}$ & -3.08713336 & -1.79739046 & 4.36409379 \\
\hline $\mathrm{N}$ & -3.15804845 & -0.35615140 & 4.15110794 \\
\hline $\mathrm{C}$ & -3.55263156 & 0.50333906 & 5.13955917 \\
\hline $\mathrm{C}$ & -3.48371516 & 1.98283591 & 4.77510574 \\
\hline $\mathrm{C}$ & -3.06227829 & -2.56811169 & 3.04084649 \\
\hline $\mathrm{O}$ & -2.12975974 & -3.04760024 & 6.20374889 \\
\hline $\mathrm{O}$ & -3.95735389 & 0.12469152 & 6.23143256 \\
\hline $\mathrm{H}$ & -0.62889869 & -1.15838142 & 4.15345488 \\
\hline $\mathrm{H}$ & 1.19280040 & -2.66706553 & 5.01832779 \\
\hline $\mathrm{H}$ & 1.01447383 & -1.25772476 & 6.09512524 \\
\hline $\mathrm{H}$ & 0.20049617 & -2.79263816 & 6.49336954 \\
\hline $\mathrm{H}$ & -3.98558195 & -2.07559386 & 4.92216754 \\
\hline $\mathrm{H}$ & -2.16252263 & -2.35734229 & 2.45540327 \\
\hline $\mathrm{H}$ & -3.08746434 & -3.64262495 & 3.24921674 \\
\hline $\mathrm{H}$ & -3.93591953 & -2.31203147 & 2.43235532 \\
\hline $\mathrm{H}$ & -2.71216822 & 0.01721381 & 3.31954525 \\
\hline $\mathrm{H}$ & -3.09339113 & 2.17375501 & 3.77023090 \\
\hline $\mathrm{H}$ & -4.48795059 & 2.41166714 & 4.85356285 \\
\hline $\mathrm{H}$ & -2.85467579 & 2.49722664 & 5.50869436 \\
\hline \multicolumn{4}{|l|}{ end } \\
\hline \multicolumn{4}{|c|}{ BASIS spherical } \\
\hline \multicolumn{4}{|c|}{ * library 6-31G* except Fe } \\
\hline \multicolumn{4}{|c|}{ bqH library H 6-31G } \\
\hline \multicolumn{4}{|c|}{ Fe library LANL2DZ_ecp } \\
\hline \multicolumn{4}{|c|}{ END } \\
\hline \multicolumn{4}{|c|}{ ECP } \\
\hline \multicolumn{4}{|c|}{ Fe library LANL2DZ_ecp } \\
\hline \multicolumn{4}{|c|}{ END } \\
\hline \multicolumn{4}{|c|}{ \#\#\#\#\#\#\#\#\#\#\#\#\#\#\#\#\#\#\#\#\#\#\#\#\#\#\#\#\#\#\#\#\#\#\#\#\#\#\#\# } \\
\hline \multicolumn{4}{|c|}{ DFT } \\
\hline \multicolumn{4}{|c|}{ CONVERGENCE energy $1 \mathrm{e}-7$} \\
\hline \multicolumn{4}{|c|}{ CONVERGENCE density $1 \mathrm{e}-6$} \\
\hline \multicolumn{4}{|c|}{ CONVERGENCE gradient 5e-6 } \\
\hline \multicolumn{4}{|c|}{ vectors output SampleSP.mos } \\
\hline $\begin{array}{l}\text { semic } \\
\text { iterati }\end{array}$ & msize 8000000 & 0 filesize 0 & \\
\hline
\end{tabular}


xc b3lyp

odft

mult 2

\# grid lebedev 16018 H 9014 C 10017 N 10017 O 10017 S 12518 Fe 16018

grid lebedev 12518 H 75 11 C 9014 N 9014 O 9014 S 9014 Fe 12518

\#grid lebedev 9014 H 7511 C 7511

grid ssf euler

END

title "SampleSP"

task dft

\section{C2. NWCHEM Geometry Optimization Job}

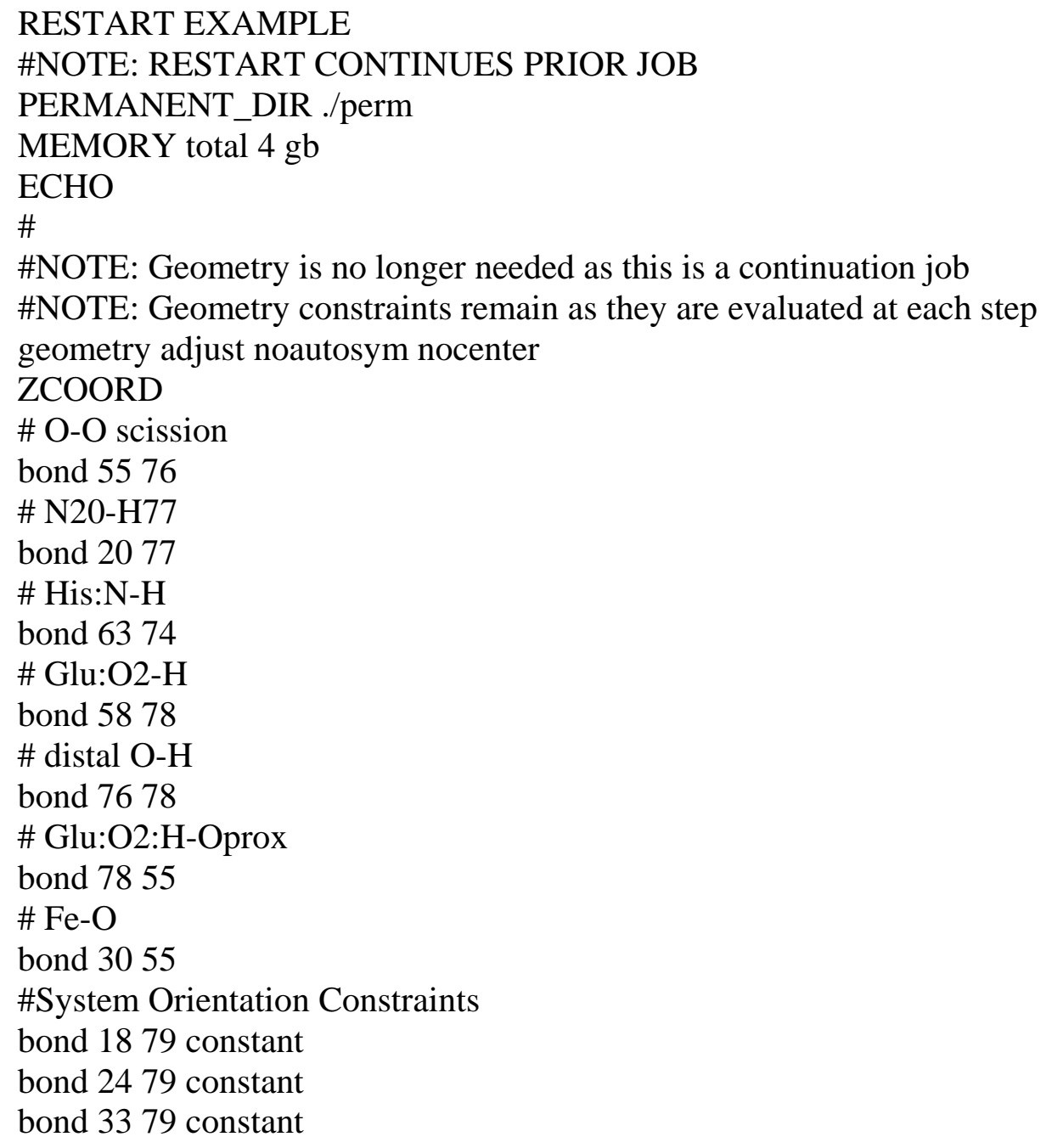


bond 3979 constant

bond 5679 constant

bond 6879 constant

angle 187980 constant

angle 247980 constant

angle 337980 constant

angle 397980 constant

angle 567980 constant

angle 687980 constant

torsion 18798081 constant

torsion 24798081 constant

torsion 33798081 constant

torsion 39798081 constant

torsion 56798081 constant

torsion 68798081 constant

angle 686580 constant

\#fix ghost atoms as well

bond 5680 constant

bond 5681 constant

bond 6880 constant

bond 6881 constant

bond 8018 constant

bond 8118 constant

angle 811824 constant

angle 801824 constant

angle 811824 constant

torsion 79182433 constant

torsion 80182433 constant

torsion 81182433 constant

\#

bond 8079 constant

bond 8179 constant

bond 8081 constant

\#

\#fix orientation of ASP

angle 26667 constant

angle 36667 constant

angle 56667 constant

torsion 526667 constant

torsion 326667 constant

\#

\#fix prox region

\#

torsion 15303182 constant

torsion 30318284 constant 
torsion 31828485 constant

torsion 82848586 constant

torsion 84858687 constant

angle 303182 constant

bond 31863.41 constant

bond 31823.46 constant

\#

end

end

\#

\#NOTE: Basis set data is no longer needed as this is a continuation job

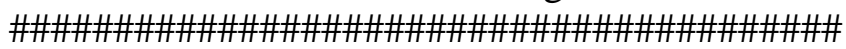

\#NOTE: CONVERGENCE DATA LEFT AS IT IS EVALUATED AT EACH STEP

DFT

CONVERGENCE energy $1 \mathrm{e}-7$

CONVERGENCE density 1e-6

CONVERGENCE gradient 5e-6

vectors input SampleSP.mos output SampleOpt.mos

semidirect memsize 80000000 filesize 0

iterations 2000

xc b3lyp

odft

mult 2

\# grid lebedev 16018 H 90 14 C 10017 N 10017 O 10017 S 12518 Fe 16018

grid lebedev 12518 H 7511 C 9014 N 9014 O 9014 S 9014 Fe 12518

\#grid lebedev 9014 H 7511 C 7511

grid ssf euler

END

driver

\#GMAX 0.0015

\#GRMS 0.0010

\#XMAX 0.0024

\#XRMS 0.0018

\# trust 0.1

default; maxiter 200; eprec 1e-6; xyz ./XYZ/ExampleOPT-1

END

title "SampleJob"

task dft optimize 


\section{C3. NWCHEM Saddle Point Optimization}




torsion 39798081 constant torsion 56798081 constant torsion 68798081 constant angle 686580 constant \#fix ghost atoms as well bond 5680 constant bond 5681 constant bond 6880 constant bond 6881 constant bond 8018 constant bond 8118 constant angle 811824 constant angle 801824 constant angle 811824 constant torsion 79182433 constant torsion 80182433 constant torsion 81182433 constant \#

bond 8079 constant bond 8179 constant bond 8081 constant \#

\#fix orientation of ASP angle 26667 constant angle 36667 constant angle 56667 constant torsion 526667 constant torsion 326667 constant \#

\#fix prox region

\#

torsion 15303182 constant torsion 30318284 constant torsion 31828485 constant torsion 82848586 constant torsion 84858687 constant angle 303182 constant bond 31863.41 constant bond 31823.46 constant \#

end

end

\#\#NOTE: Basis set data is no longer needed as this is a continuation job

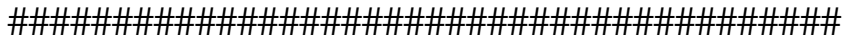

\#NOTE: CONVERGENCE DATA LEFT AS IT IS EVALUATED AT EACH STEP 


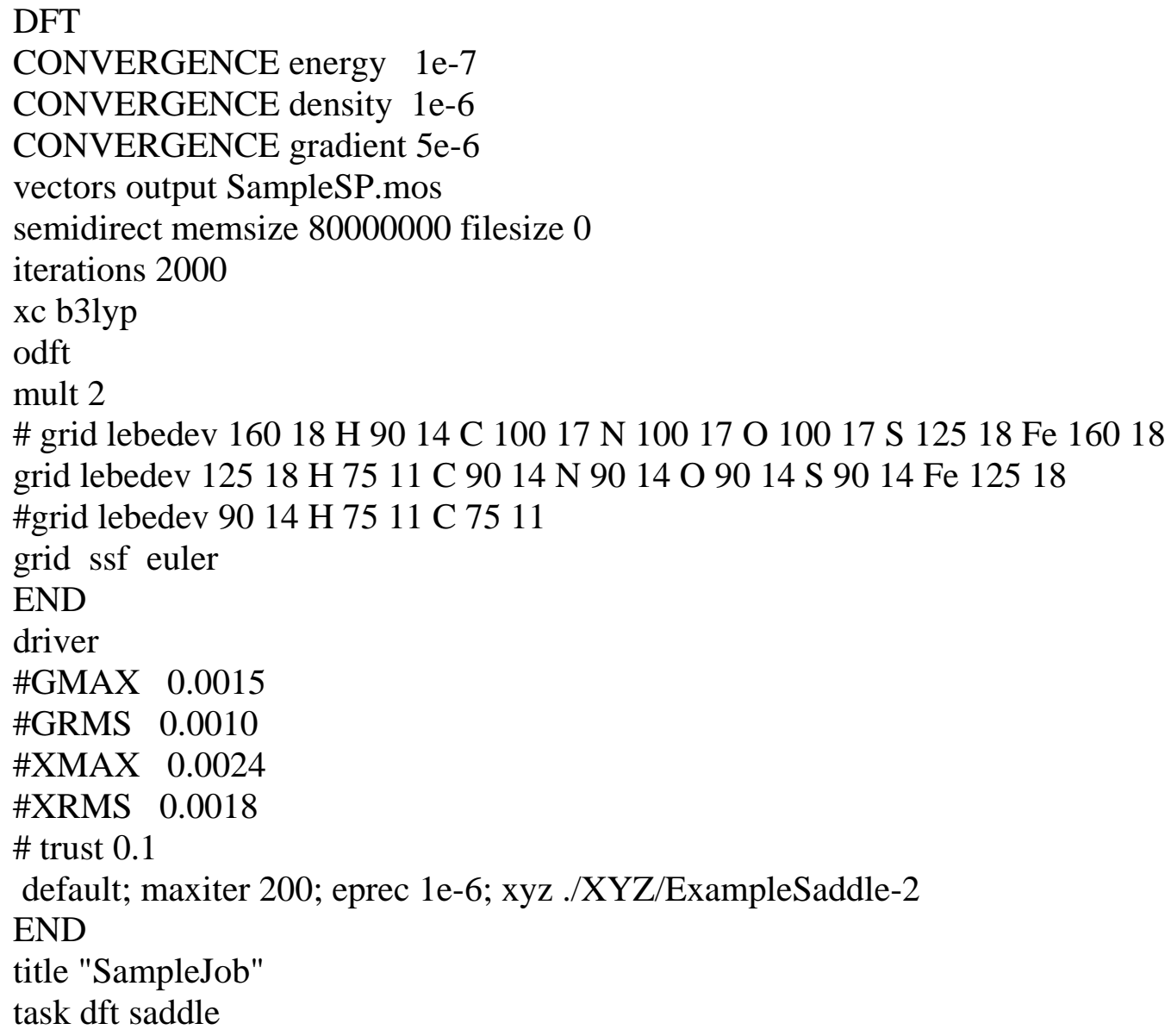

\section{C4. NWCHEM Frequency Calculation}

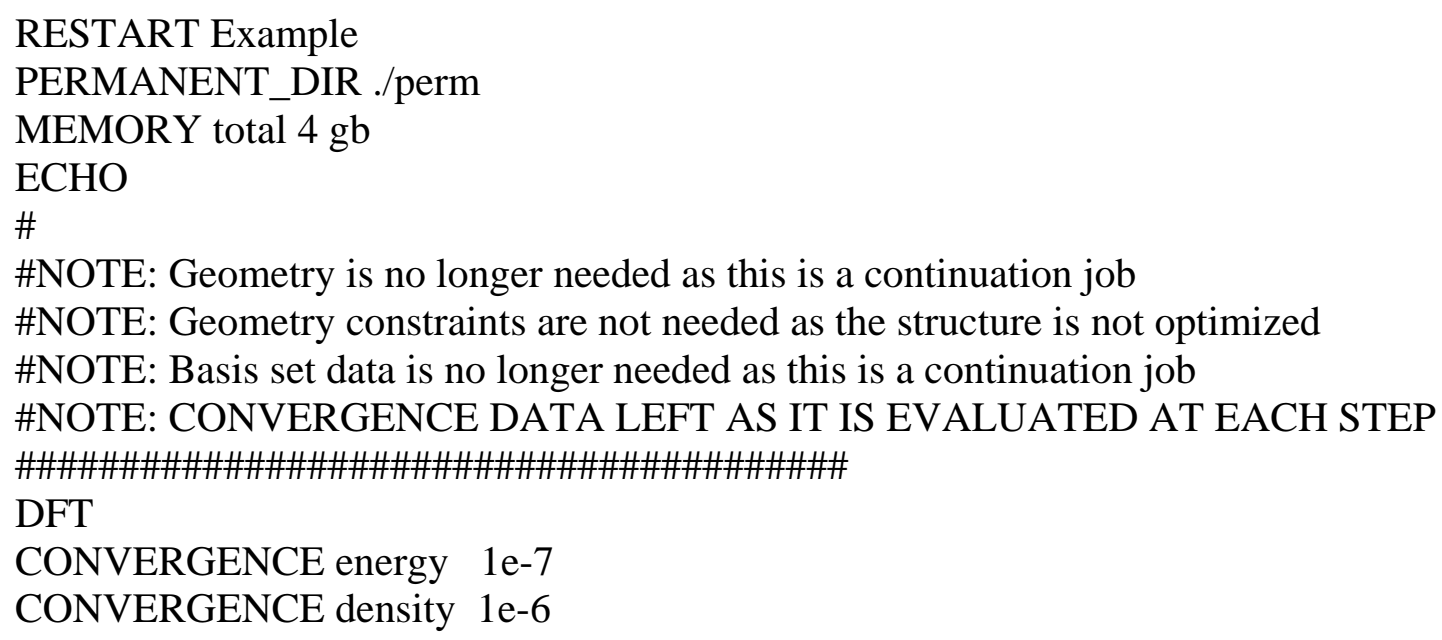


CONVERGENCE gradient 5e-6

\# direct

\#vectors output

semidirect memsize 80000000 filesize 0

iterations 2000

xc b3lyp

odft

mult 2

\# grid lebedev 16018 H 9014 C 10017 N 10017 O 10017 S 12518 Fe 16018

grid lebedev 12518 H 7511 C 9014 N 9014 O 9014 S 9014 Fe 12518

\#grid lebedev 9014 H 75 11 C 7511

grid ssf euler

END

hessian

print "hess_follow"

end

freq

animate .03

end

title "SampleJob"

task dft hessian numerical

task dft freq numerical

\section{C5. NWCHEM Mulliken Job}

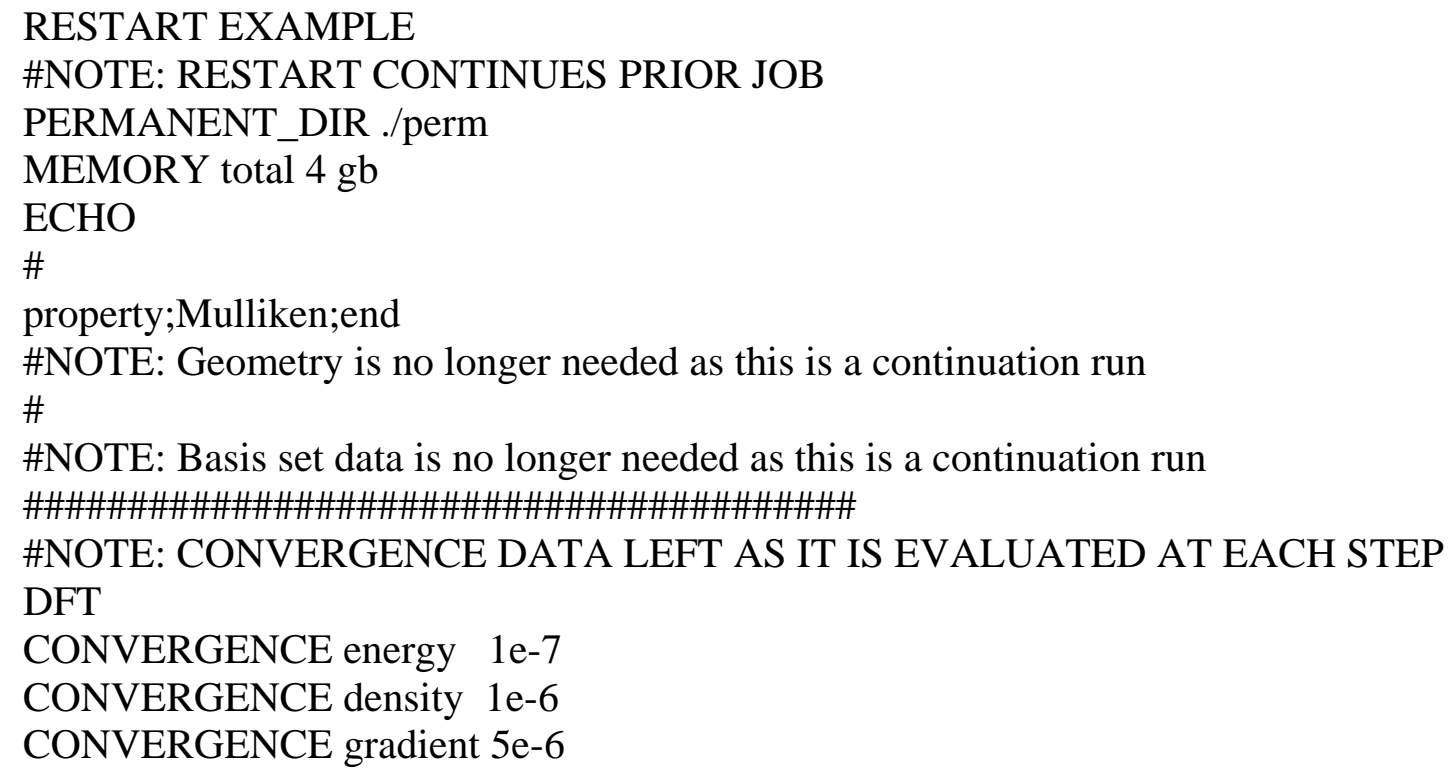


\#NOTE: vectors not included/not necessary/RESTART file/vectors unchanged semidirect memsize 80000000 filesize 0

iterations 2000

xc b3lyp

odft

mult 2

\# grid lebedev 16018 H 9014 C 10017 N 10017 O 10017 S 12518 Fe 16018

grid lebedev 12518 H 7511 C 9014 N 9014 O 9014 S 9014 Fe 12518

\#grid lebedev 9014 H 7511 C 7511

grid ssf euler

END

driver

\#GMAX 0.0015

\#GRMS 0.0010

\#XMAX 0.0024

\#XRMS 0.0018

\# trust 0.1

default; maxiter 200; eprec 1e-6; xyz ./XYZ/ExampleOPT-1

END

title "cpd1towards0withprox"

task dft

task property

\section{C6. NWCHEM NBO file generator (*.gen)}

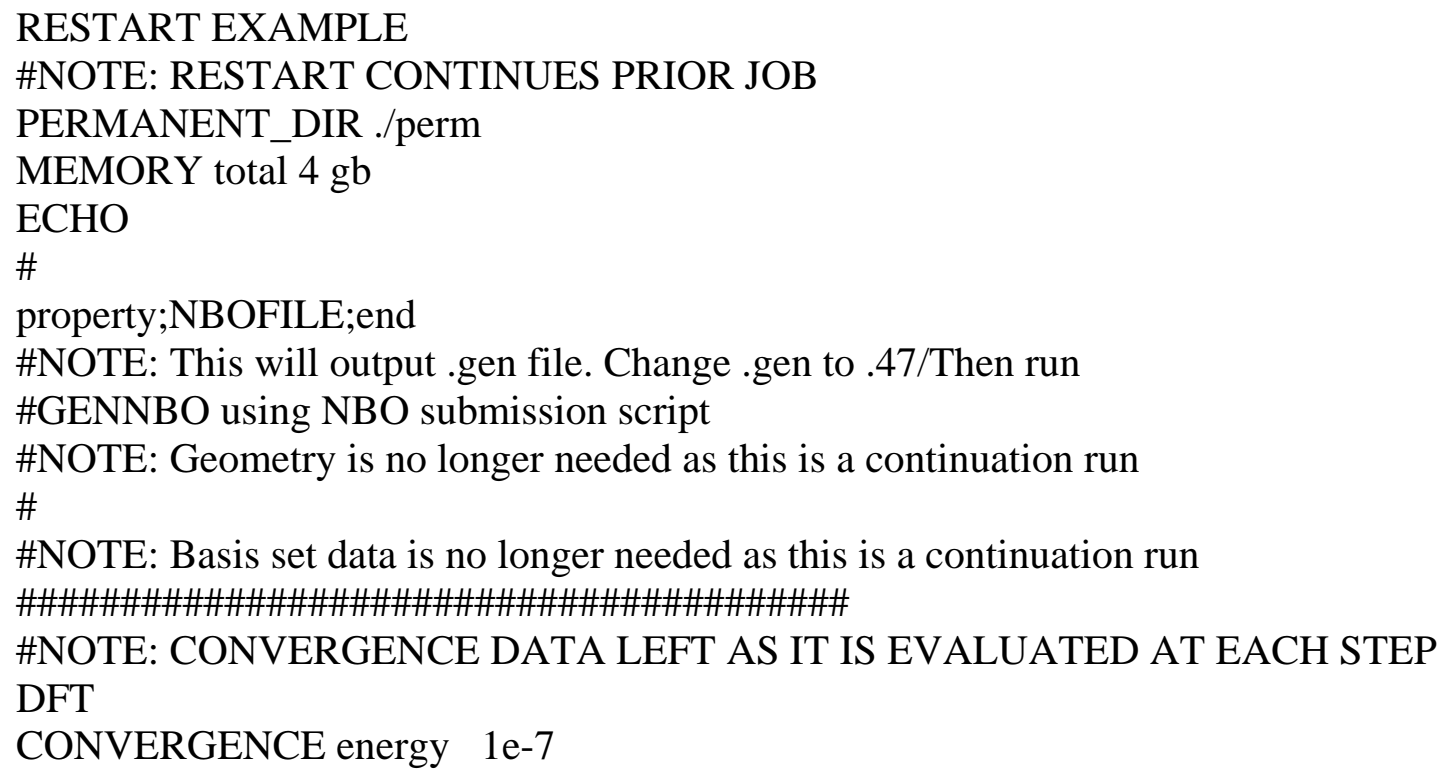


CONVERGENCE density $1 \mathrm{e}-6$

CONVERGENCE gradient 5e-6

\#NOTE: vectors not included/not necessary/RESTART file/vectors unchanged semidirect memsize 80000000 filesize 0

iterations 2000

xc b3lyp

odft

mult 2

\# grid lebedev 16018 H 9014 C 10017 N 10017 O 10017 S 12518 Fe 16018

grid lebedev 12518 H 75 11 C 9014 N 9014 O 9014 S 9014 Fe 12518

\#grid lebedev 9014 H 75 11 C 7511

grid ssf euler

END

driver

\#GMAX 0.0015

\#GRMS 0.0010

\#XMAX 0.0024

\#XRMS 0.0018

\# trust 0.1

default; maxiter 200; eprec 1e-6; xyz ./XYZ/ExampleOPT-1

END

title "cpd1towards0withprox"

task dft

task property

\section{C7. Gaussian Single Point Calculation Job}

$\% \mathrm{CHK}=$ SampleFeHOOH

$\% \mathrm{MEM}=1 \mathrm{~GB}$

\#UB3LYP/gen GFInput scf=(tight,XQC) nosymm int=(grid=ultrafine)

SP energy calculation of doublet FeHOOH ! Title

02

$\begin{array}{llll}\text { O } & -10.27708700 & 4.49546998 & -5.17994838\end{array}$

$\begin{array}{lllll}\text { C } & & -11.10259823 & 5.36078734 & -4.87105973\end{array}$

$\begin{array}{lllll}\mathrm{N} & & -12.32409659 & 5.03316780 & -4.37645351\end{array}$

$\begin{array}{lllll}\text { C } & -12.76239344 & 3.66109586 & -4.17337375\end{array}$

$\begin{array}{lllll}\text { C } & -10.79681172 & 6.84194599 & -5.03871064\end{array}$

$\begin{array}{lllll}\mathrm{H} & -12.96835148 & 5.77938441 & -4.15713229\end{array}$

$\begin{array}{llll}\mathrm{H} & -13.65155787 & 3.44313113 & -4.77509187\end{array}$

$\begin{array}{llll}\mathrm{H} & -12.99208286 & 3.47721518 & -3.11802546\end{array}$ 


\begin{tabular}{|c|c|c|c|}
\hline $\mathrm{H}$ & -11.94910971 & 3.00580601 & -4.48511702 \\
\hline $\mathrm{H}$ & -11.61689026 & 7.49903396 & -4.73407438 \\
\hline $\mathrm{H}$ & -10.55877804 & 7.03285538 & -6.08950504 \\
\hline $\mathrm{H}$ & -9.90873395 & 7.08821091 & -4.44821909 \\
\hline $\mathrm{C}$ & -4.24624691 & -1.33075699 & -0.89914580 \\
\hline $\mathrm{C}$ & -3.20767717 & -0.33475028 & -0.78729114 \\
\hline $\mathrm{N}$ & -1.97687871 & -0.88384939 & -0.63779145 \\
\hline $\mathrm{C}$ & -2.21499911 & -2.22143452 & -0.66379503 \\
\hline $\mathrm{C}$ & -3.61666228 & -2.52884768 & -0.81103680 \\
\hline $\mathrm{C}$ & -3.40957730 & 1.00353394 & -0.87532924 \\
\hline $\mathrm{C}$ & -2.47682439 & 2.02840348 & -0.73452080 \\
\hline $\mathrm{N}$ & -1.13042484 & 1.88212072 & -0.52841523 \\
\hline $\mathrm{C}$ & -0.60495935 & 3.15564495 & -0.46848024 \\
\hline $\mathrm{C}$ & -1.65887098 & 4.12713518 & -0.62935772 \\
\hline $\mathrm{C}$ & -2.81506078 & 3.42953721 & -0.80446380 \\
\hline $\mathrm{C}$ & 0.74211136 & 3.45848879 & -0.29811869 \\
\hline $\mathrm{C}$ & 1.75271674 & 2.49194228 & -0.20312035 \\
\hline $\mathrm{N}$ & 1.57139573 & 1.11672680 & -0.29532867 \\
\hline $\mathrm{C}$ & 2.83101915 & 0.54270877 & -0.14980367 \\
\hline $\mathrm{C}$ & 3.81040004 & 1.58325356 & 0.02067861 \\
\hline $\mathrm{C}$ & 3.14907296 & 2.77859040 & -0.01092542 \\
\hline $\mathrm{Fe}$ & -0.20035162 & 0.13201885 & -0.37362003 \\
\hline S & -0.46184634 & -0.00427837 & 1.85762887 \\
\hline $\mathrm{C}$ & 0.27540144 & 1.47676989 & 2.64243131 \\
\hline $\mathrm{C}$ & 3.11701142 & -0.82889761 & -0.18916661 \\
\hline $\mathrm{C}$ & 2.12297897 & -1.81333356 & -0.32945549 \\
\hline $\mathrm{N}$ & 0.75719239 & -1.60503106 & -0.44677619 \\
\hline $\mathrm{C}$ & 0.16340453 & -2.84827559 & -0.52377718 \\
\hline $\mathrm{C}$ & 1.18532907 & -3.86717392 & -0.47245444 \\
\hline $\mathrm{C}$ & 2.38609888 & -3.23281480 & -0.35564527 \\
\hline $\mathrm{C}$ & -1.21277460 & -3.13463609 & -0.61580579 \\
\hline $\mathrm{H}$ & 4.14860831 & -1.14756959 & -0.09258205 \\
\hline $\mathrm{H}$ & 0.99416622 & -4.93192515 & -0.51945484 \\
\hline $\mathrm{H}$ & 4.87257768 & 1.41251491 & 0.14394352 \\
\hline $\mathrm{H}$ & 3.56575625 & 3.77377316 & 0.08138553 \\
\hline $\mathrm{H}$ & 1.03331040 & 4.50218117 & -0.24425599 \\
\hline $\mathrm{H}$ & -1.51335407 & 5.19998263 & -0.62353887 \\
\hline $\mathrm{H}$ & -3.81272369 & 3.81063001 & -0.98038712 \\
\hline $\mathrm{H}$ & -4.42932456 & 1.33636202 & -1.05236848 \\
\hline $\mathrm{H}$ & -5.29742625 & -1.11377794 & -1.03513510 \\
\hline $\mathrm{H}$ & -4.02917985 & -3.52837555 & -0.85359758 \\
\hline $\mathrm{H}$ & -1.47227312 & -4.18890568 & -0.65394116 \\
\hline $\mathrm{H}$ & 3.37181883 & -3.67609182 & -0.28924438 \\
\hline $\mathrm{H}$ & 0.04995589 & 1.41357442 & 3.71237752 \\
\hline $\mathrm{H}$ & 1.35941062 & 1.50082980 & 2.51022504 \\
\hline
\end{tabular}




\begin{tabular}{|c|c|c|c|}
\hline $\mathrm{H}$ & -0.15966955 & 2.39554998 & 2.24294399 \\
\hline $\mathrm{O}$ & -0.11086718 & 0.22173625 & -2.59870225 \\
\hline C & -2.36880205 & -0.81664983 & -4.28222251 \\
\hline $\mathrm{C}$ & -2.96889640 & 0.54947371 & -4.09252359 \\
\hline $\mathrm{O}$ & -2.29861573 & 1.53092345 & -3.77005099 \\
\hline $\mathrm{O}$ & -4.27543144 & 0.55911173 & -4.28285540 \\
\hline $\mathrm{H}$ & -1.42273692 & -0.73662146 & -4.82247498 \\
\hline $\mathrm{H}$ & -3.05605411 & -1.47311306 & -4.81986347 \\
\hline $\mathrm{H}$ & -2.14566566 & -1.26091670 & -3.30908541 \\
\hline $\mathrm{N}$ & -5.76818357 & 2.78754101 & -4.22189856 \\
\hline C & -5.35345463 & 3.94396650 & -4.84334953 \\
\hline $\mathrm{C}$ & -6.43788207 & 4.67689805 & -5.26230590 \\
\hline $\mathrm{N}$ & -7.54193578 & 3.94180779 & -4.87576029 \\
\hline C & -7.08950568 & 2.81735760 & -4.26412662 \\
\hline $\mathrm{C}$ & -6.49563791 & 5.98397722 & -5.98376477 \\
\hline $\mathrm{H}$ & -7.01758976 & 6.75719426 & -5.4041627 \\
\hline $\mathrm{H}$ & -5.48036832 & 6.34282710 & -6.17377588 \\
\hline $\mathrm{H}$ & -7.00670939 & 5.89885770 & -6.95142803 \\
\hline $\mathrm{H}$ & -8.52282967 & 4.19198663 & -5.03445079 \\
\hline $\mathrm{H}$ & -7.75026401 & 2.05499840 & -3.8759135 \\
\hline $\mathrm{H}$ & -4.71133563 & 1.46662116 & -4.1423616 \\
\hline $\mathrm{H}$ & -4.30218496 & 4.17201619 & -4.9548981 \\
\hline $\mathrm{O}$ & 1.12144701 & 0.77248227 & -3.13574791 \\
\hline $\mathrm{H}$ & 1.53728950 & 1.06086592 & -2.29551723 \\
\hline $\mathrm{H}$ & -0.79012668 & 0.85312698 & -2.9656906 \\
\hline $\mathrm{N}$ & -0.39503662 & -2.00727572 & 4.67811430 \\
\hline $\mathrm{C}$ & 0.85356454 & -2.51860167 & 5.20918719 \\
\hline $\mathrm{C}$ & -1.60672375 & -2.48059381 & 5.09220484 \\
\hline $\mathrm{C}$ & -2.82461404 & -1.90326917 & 4.3323063 \\
\hline $\mathrm{N}$ & -2.85244061 & -0.44748977 & 4.2485957 \\
\hline $\mathrm{C}$ & -3.09570620 & 0.34026275 & 5.3388648 \\
\hline $\mathrm{C}$ & -3.01660628 & 1.84231157 & 5.08460584 \\
\hline $\mathrm{C}$ & -2.98348753 & -2.55358528 & 2.95593448 \\
\hline $\mathrm{O}$ & -1.74728427 & -3.34461355 & 5.94917762 \\
\hline $\mathrm{O}$ & -3.37425216 & -0.11388410 & 6.44162491 \\
\hline $\mathrm{H}$ & -0.35796189 & -1.40143722 & 3.86612915 \\
\hline $\mathrm{H}$ & 1.39809795 & -3.11180928 & 4.46234803 \\
\hline $\mathrm{H}$ & 1.50226450 & -1.70058174 & 5.54489903 \\
\hline $\mathrm{H}$ & 0.61091336 & -3.15792424 & 6.0595492 \\
\hline $\mathrm{H}$ & -3.66853403 & -2.19571282 & 4.96406507 \\
\hline $\mathrm{H}$ & -2.14689179 & -2.32265205 & 2.2913502 \\
\hline $\mathrm{H}$ & -3.03430018 & -3.64078678 & 3.0753060 \\
\hline $\mathrm{H}$ & -3.90735023 & -2.21378038 & 2.47585516 \\
\hline $\mathrm{H}$ & -2.52938609 & -0.02276040 & 3.38578597 \\
\hline $\mathrm{H}$ & -2.83804506 & 2.10588910 & 4.0372540 \\
\hline
\end{tabular}




$\begin{array}{llll}\mathrm{H} & -3.95266501 & 2.30298795 & 5.41487181 \\ \mathrm{H} & -2.21401837 & 2.26346771 & 5.69957441\end{array}$

H C N S O

6-31G*

$* * * *$

$\mathrm{Fe}$

LANL2DZ

$* * * *$

$\mathrm{Fe}$

LANL2DZ

C8. Continuation Gaussian Single Point Calculation Job

$\% \mathrm{CHK}=$ SampleFeHOOH

$\%$ MEM=1GB

\#UB3LYP/gen GFInput guess=read pseudo=read scf=(tight,XQC) nosymm int $=$ (grid=ultrafine) geom=checkpoint

Continued SP energy calculation of doublet FeHOOH ! Title

02

H C N S O

6-31G*

****

$\mathrm{Fe}$

LANL2DZ

*****

Fe

LANL2DZ 


\section{C9. Gaussian Solvation Script}

$\% \mathrm{CHK}=$ SampleFeHOOHsolv

$\% \mathrm{MEM}=1 \mathrm{~GB}$

\#UB3LYP/gen GFInput guess=read pseudo=read scf=(tight,XQC)

$\mathrm{SCRF}=($ solvent $=$ chlorobenzene $)$ nosymm int $=($ grid $=$ ultrafine $)$ geom $=$ checkpoint

Solvation ! Title

02

$\mathrm{H}$ C N S O

6-31G*

$* * * *$

$\mathrm{Fe}$

LANL2DZ

$* * * *$

$\mathrm{Fe}$

LANL2DZ

\section{C10. Gaussian 09 Frequency Calculation}

$\% \mathrm{CHK}=$ SampleFreq

$\% \mathrm{MEM}=1 \mathrm{~GB}$

\#UB3LYP/gen GFInput guess=read pseudo=read scf=(tight,XQC) nosymm

int $=($ grid=ultrafine $)$ freq geom=checkpoint

Frequency Calculation ! Title

02

H C N S O

6-31G*

$* * * *$

$\mathrm{Fe}$

LANL2DZ

$* * * *$ 
$\mathrm{Fe}$

LANL2DZ

\section{C11. Gaussian 09 Stability Check}

$\% \mathrm{CHK}=$ SampleStabilityCheck

$\% \mathrm{MEM}=1 \mathrm{~GB}$

\#UB3LYP/gen GFInput guess=read pseudo=read scf=(tight,XQC) nosymm

int $=$ (grid=ultrafine) stable test geom=checkpoint

Stability Check ! Title

02

H C N S O

6-31G*

$* * * *$

$\mathrm{Fe}$

LANL2DZ

$* * * *$

$\mathrm{Fe}$

LANL2DZ

\section{C12. Gaussian 09 NPA job}

$\% \mathrm{CHK}=\mathrm{FeHOOH}-\mathrm{NPA}$

$\% \mathrm{MEM}=256 \mathrm{MB}$

\#UB3LYP/gen GFInput guess=read pseudo=read scf=(tight,XQC) pop=npa nosymm int $=$ (grid=ultrafine) geom $=$ checkpoint

SP energy calculation of doublet Shaik

02

H C N S O 
6-31G*

****

$\mathrm{Fe}$

LANL2DZ

$* * * *$

Fe

LANL2DZ

\title{
C13. NWChem submission script
}

\author{
\# BSUB -a intelmpi \\ \# BSUB -q normal \\ \# BSUB -n 32 \\ \#BSUB -R "rusage[mem=2048]" \\ \# BSUB -oo "sample.log" \\ \# BSUB -eo "sample.err" \\ . \$MODULESHOME/../global/profile.modules \\ module load nwchem/6.1.1/intel-mkl-scpk \\ mpirun.lsf nwchem sample.nw
}

\section{C14. Gaussian 09 submission script}

\author{
\# BSUB -q normal \\ \# BSUB -n 1 \\ \# BSUB -eo "sample.err" \\ . \$MODULESHOME/../global/profile.modules \\ module load gaussian/09 \\ g09 sample.com sample.log
}

\section{C15. CHARMM Topology File (selected segments of file) \\ ! This section defines the atom type and mass

$\begin{array}{llll}\text { MASS } & 1 \mathrm{H} & 1.00800 \mathrm{H} ! \text { polar } \mathrm{H} \\ \text { MASS } & 2 \mathrm{HC} & 1.00800 \mathrm{H} ! \mathrm{N}-\text { ter } \mathrm{H} \\ \text { MASS } & 3 \mathrm{HA} & 1.00800 \mathrm{H} ! \text { nonpolar H }\end{array}$




$\begin{array}{llllll}\text { MASS } & 4 \mathrm{HT} & 1.00800 \mathrm{H} ! \text { TIPS3P WATER HYDROGEN } \\ \text { MASS } & 5 \mathrm{HP} & 1.00800 \mathrm{H} ! \text { aromatic H } \\ \text { MASS } & 6 \mathrm{HB} & 1.00800 \mathrm{H} ! \text { backbone H } \\ \text { MASS } & 7 \mathrm{HR} 1 & 1.00800 \mathrm{H} ! \text { his he1, }(+) \text { his HG, HD2 } \\ \text { MASS } & 8 \mathrm{HR} 2 & 1.00800 \mathrm{H} !(+) \text { his HE1 } \\ \text { MASS } & 9 \mathrm{HR} 3 & 1.00800 \mathrm{H} ! \text { neutral his HG, HD2 } \\ \text { MASS } & 10 \mathrm{HS} & 1.00800 \mathrm{H} ! \text { thiol hydrogen } \\ \text { MASS } & 11 \mathrm{HE} 1 & 1.00800 \mathrm{H} ! \text { for alkene; RHC=CR } \\ \text { MASS } & 12 \mathrm{HE2} & 1.00800 \mathrm{H} ! \text { for alkene; H2C=CR } \\ \text { MASS } & 13 \mathrm{HA1} & 1.00800 \mathrm{H} ! \text { alkane, CH, new LJ params }\end{array}$

! This section declare the atom types that will be referenced in adjoining residues

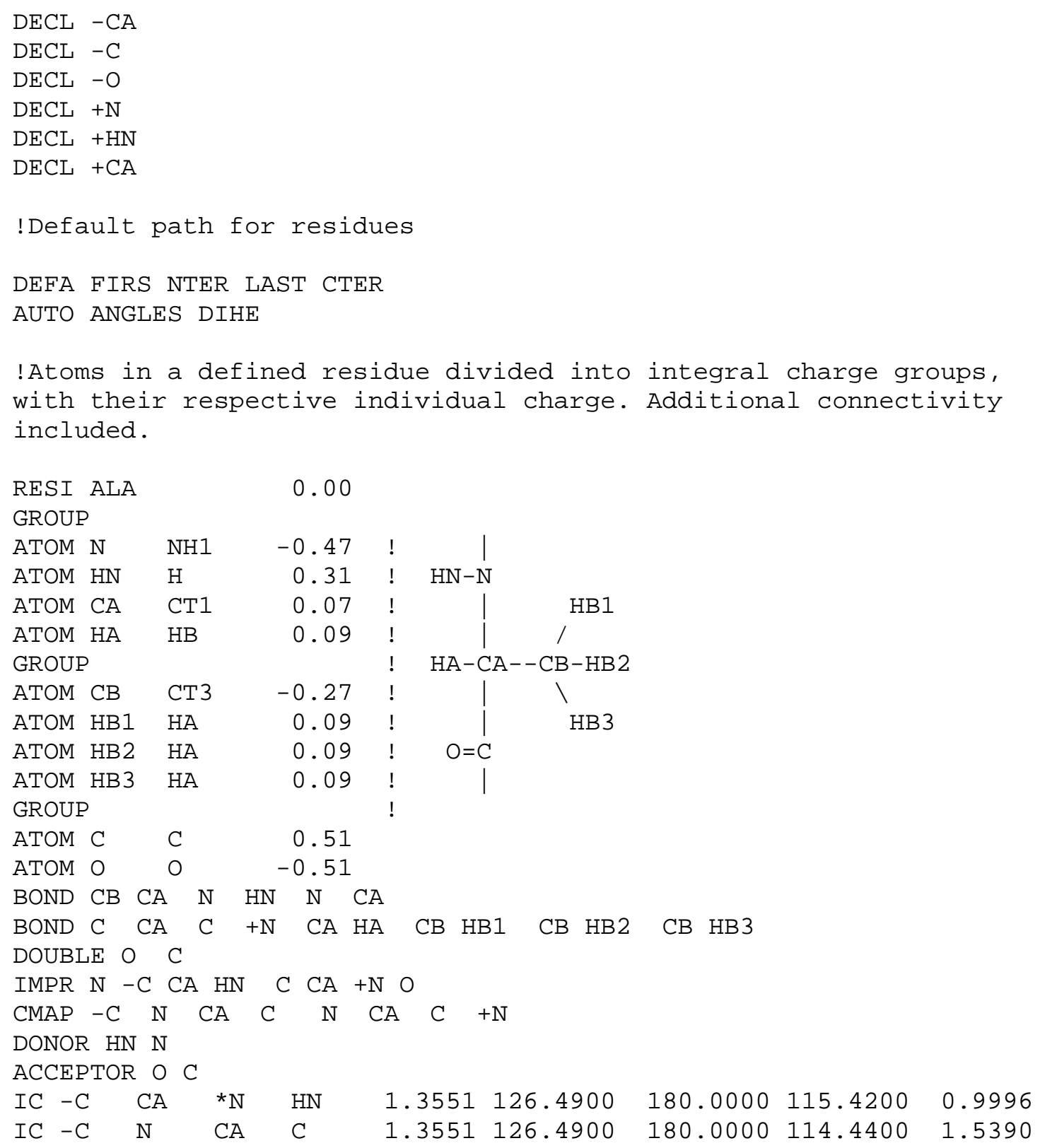




\begin{tabular}{|c|c|c|c|c|c|c|c|c|}
\hline IC $\mathrm{N}$ & $C A$ & C & $+\mathrm{N}$ & 1.4592 & 114.4400 & $180.0 \odot \odot \odot$ & 116.8400 & 1.3558 \\
\hline $\mathrm{IC}+\mathrm{N}$ & CA & ${ }^{*} \mathrm{C}$ & 0 & 1.3558 & 116.8400 & $180.0 \odot \odot \odot$ & 122.5200 & 1. 2297 \\
\hline IC CA & C & $+\mathrm{N}$ & $+\mathrm{CA}$ & 1.5390 & 116.8400 & $180.0 \odot \odot \odot$ & 126.7700 & 1.4613 \\
\hline IC & C & ${ }^{*} \mathrm{CA}$ & CB & 1. 4592 & 114.4400 & 123.2300 & 111.0900 & 1.5461 \\
\hline IC & C & ${ }^{*} \mathrm{CA}$ & $\mathrm{HA}$ & 1.4592 & 114.4400 & -120.4500 & 106.3900 & 1.0840 \\
\hline IC & CA & CB & HB1 & 1.5390 & 111.0900 & 177.2500 & 109.6000 & 1.1109 \\
\hline HB1 & CA & ${ }^{*} \mathrm{CB}$ & HB2 & 1.1109 & 109.6000 & 119.1300 & 111.0500 & 1.1119 \\
\hline IC HB1 & CA & ${ }^{*} \mathrm{CB}$ & HB3 & 1.1109 & 109.6000 & -119.5800 & 111.6100 & 1.1114 \\
\hline
\end{tabular}

\section{C16. CHARMM Parameter File (selected segments of file)}

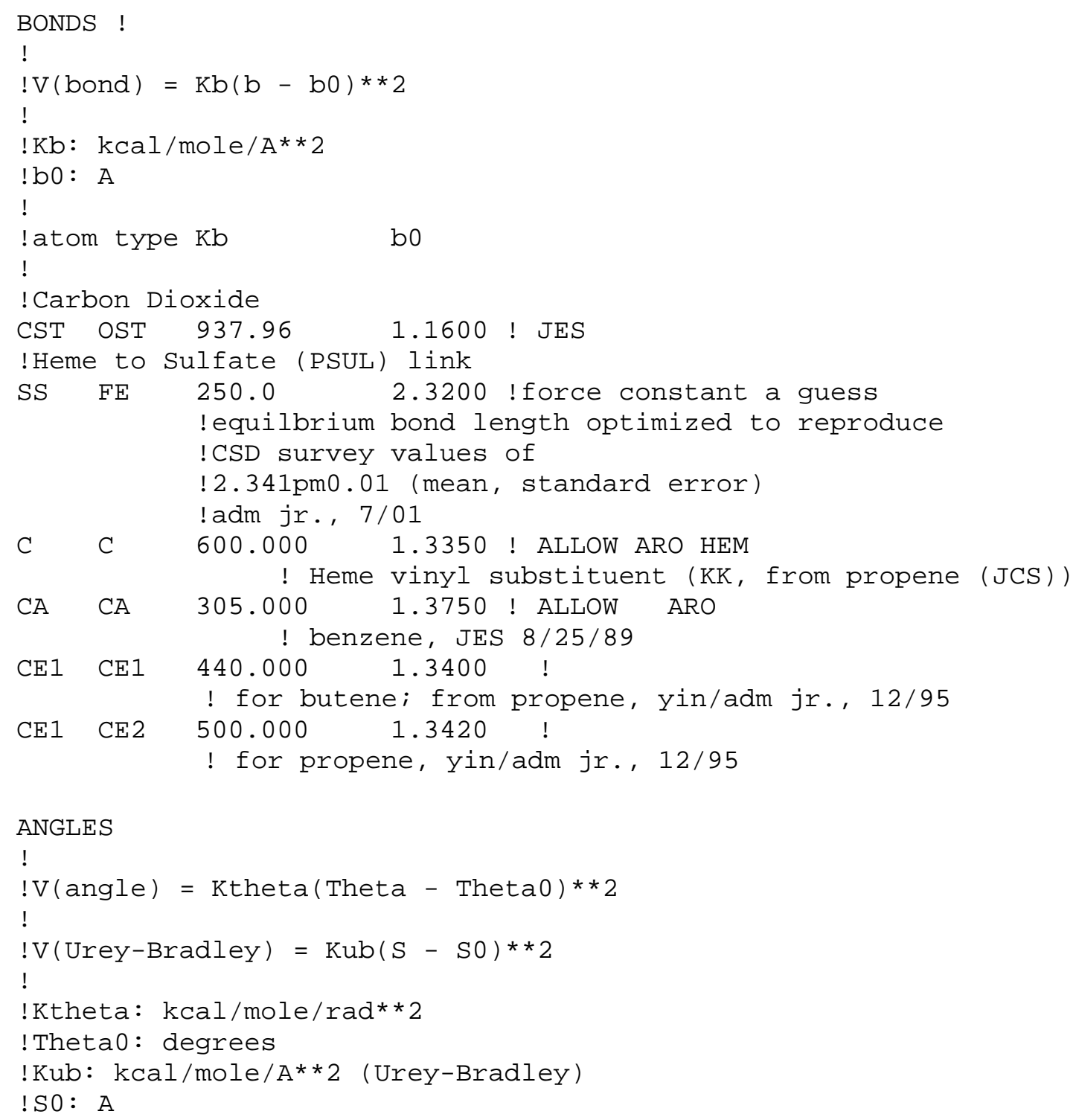




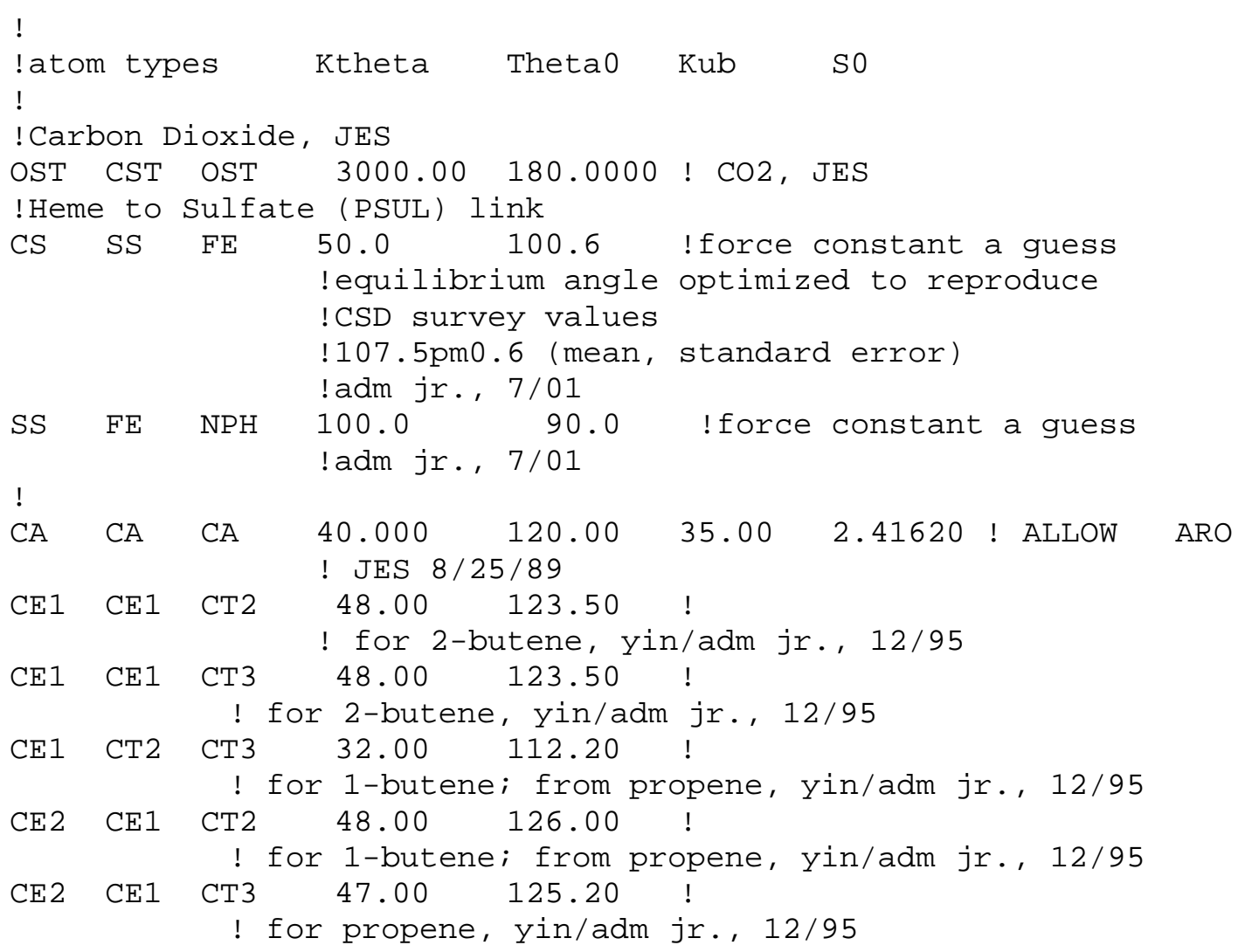




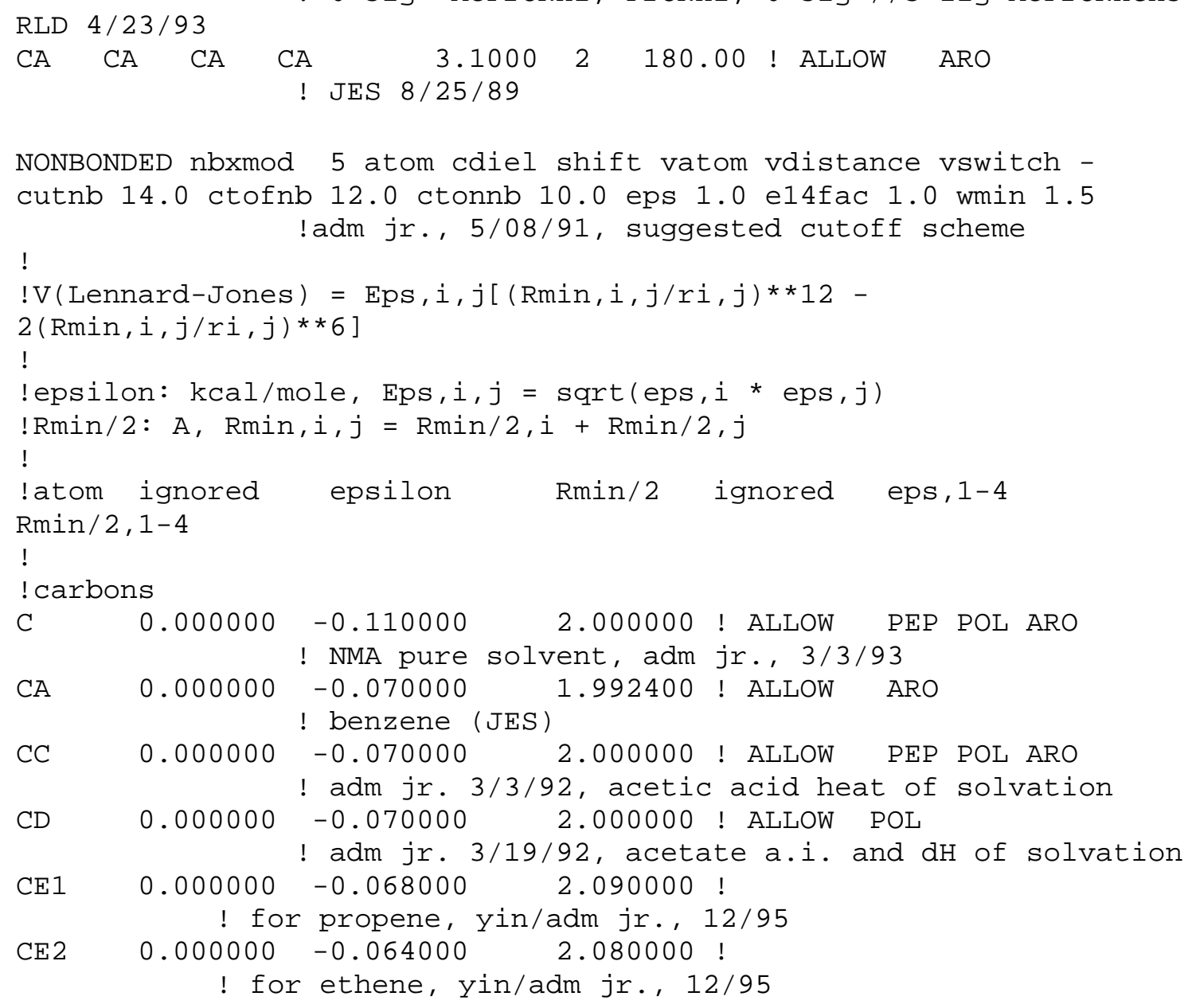

\section{C17. CHARMM Coordinate File (selected segments of file)}

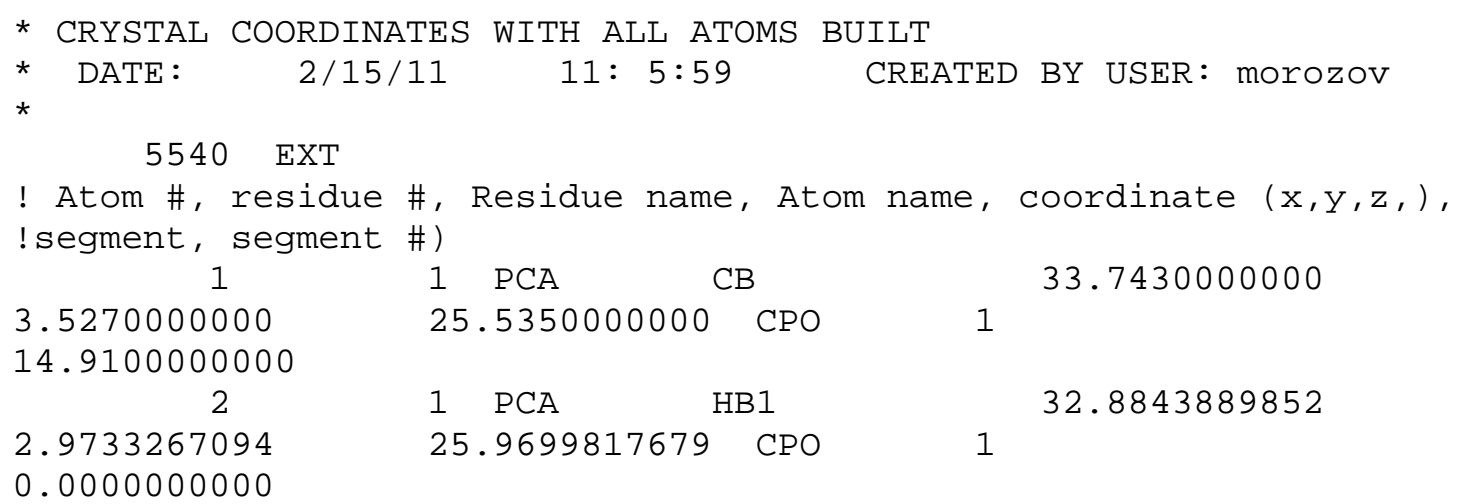




\begin{tabular}{|c|c|c|c|}
\hline 3 & $1 \quad \mathrm{PCA} \quad \mathrm{HB} 2$ & & 33.9393423444 \\
\hline $\begin{array}{l}\text { 4. } 4292330678 \\
\odot .0 \odot \odot \odot \odot \odot \odot \odot \odot \odot ~\end{array}$ & $26.1517668126 \quad$ СРО & 1 & \\
\hline 4 & 1 PCA $\quad$ CG & & $33.5 \odot \odot \odot \odot \odot \odot \odot \odot \odot$ \\
\hline $\begin{array}{l}3.8850 \odot \odot \odot \odot \odot \odot \\
14.590 \odot \odot \odot \odot \odot \odot \odot\end{array}$ & $24.0780 \odot \odot \odot \odot \odot \odot \quad$ СРО & 1 & \\
\hline 5 & $1 \quad \mathrm{PCA} \quad \mathrm{HG1}$ & & 34.1814676269 \\
\hline $\begin{array}{l}\text { 4. } 6969277166 \\
\odot .0 \odot \odot \odot \odot \odot \odot \odot \odot \odot ~\end{array}$ & $23.7462816280 \quad$ CPO & 1 & \\
\hline 6 & 1 PCA & & 32.4481098032 \\
\hline 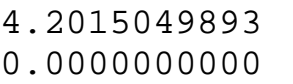 & $23.9136423794 \quad$ CPO & 1 & \\
\hline 7 & $1 \quad \mathrm{PCA} \quad \mathrm{CD}$ & & $33.79800000 \odot \odot$ \\
\hline $\begin{array}{l}2.5880 \odot \odot \odot \odot \odot \odot \\
13.9700 \odot \odot \odot \odot \odot \odot\end{array}$ & $23.32500 \odot \odot \odot \odot \odot \quad$ CPO & 1 & \\
\hline $\begin{array}{c}8 \\
2.5830 \odot \odot \odot \odot \odot \odot \\
16.40 \odot \odot \odot \odot \odot \odot \odot \odot\end{array}$ & 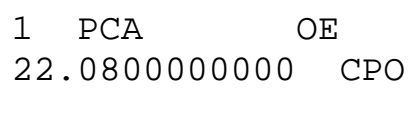 & 1 & 33.8210000000 \\
\hline 9 & 1 PCA & & $34.934000000 \odot$ \\
\hline $\begin{array}{l}2.0370 \odot \odot \odot \odot \odot \odot \\
13.450 \odot \odot \odot \odot \odot \odot \odot\end{array}$ & $24.07700000 \odot \odot \quad$ СРО & 1 & \\
\hline 10 & 1 PCA & & 35.5975768546 \\
\hline $\begin{array}{l}\text { 1. } 3608775913 \\
\odot .0 \odot \odot \odot \odot \odot \odot \odot \odot \odot ~\end{array}$ & $23.7799094434 \quad$ CРO & 1 & \\
\hline
\end{tabular}

\section{C18. CHARMM Protein Structure File (selected segments of file)}

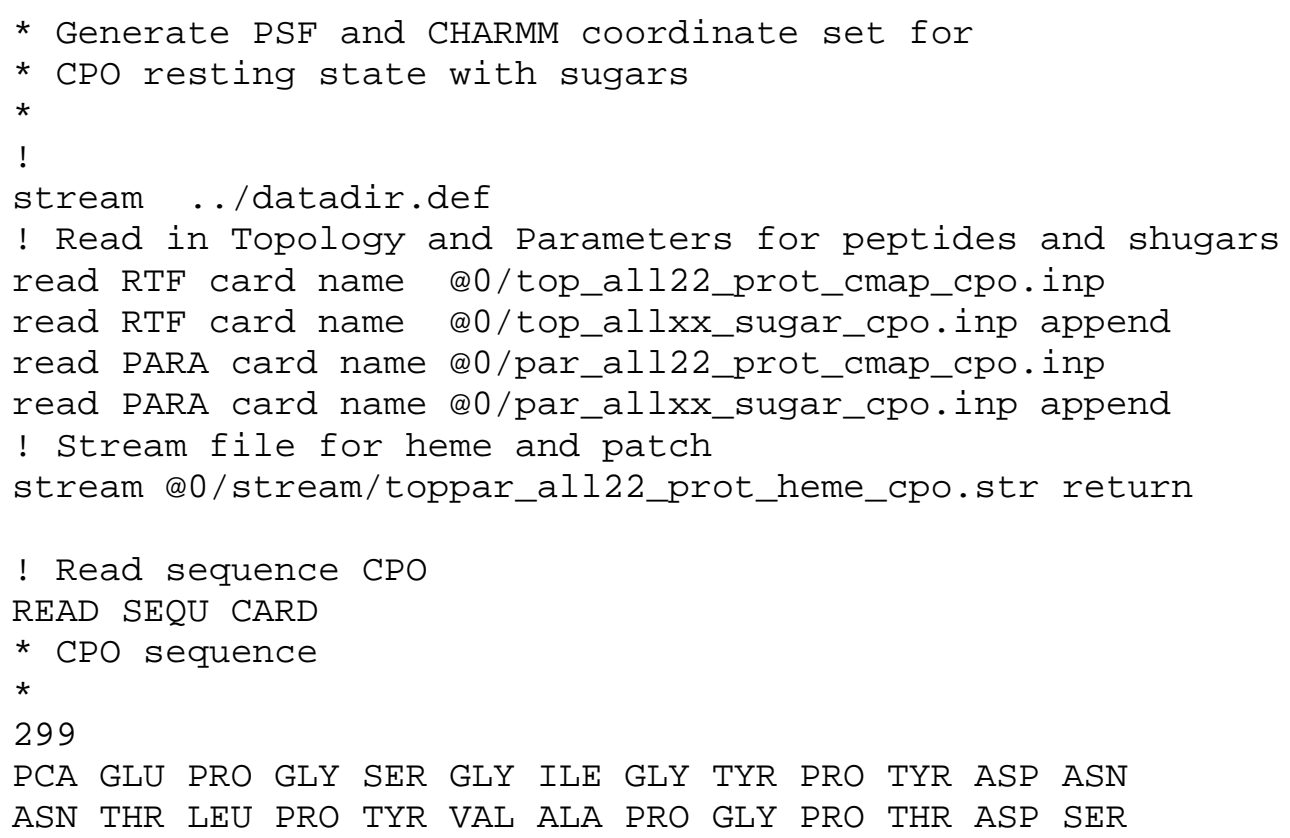


ARG ALA PRO CYS PRO ALA LEU ASN ALA LEU ALA ASN HSP GLY TYR ILE PRO HSP ASP GLY ARG ALA ILE SER ARG GLU THR LEU GLN ASN ALA PHE LEU ASN HSP MET GLY ILE ALA ASN SER VAL ILE GLU LEU ALA LEU THR ASN ALA PHE VAL VAL CYS GLU TYR VAL THR GLY SER ASP CYS GLY ASP SER LEU VAL ASN LEU THR LEU LEU ALA GLU PRO HSP ALA PHE GLU HSP ASP HSP SER PHE SER ARG LYS ASP TYR LYS GLN GLY VAL ALA ASN SER ASN ASP PHE ILE ASP ASN ARG ASN PHE ASP ALA GLU THR PHE GLN THR SER LEU ASP VAL VAL ALA GLY LYS THR HSP PHE ASP TYR ALA ASP MET ASN GLU ILE ARG LEU GLN ARG GLU SER LEU SER ASN GLU LEU ASP PHE PRO GLY TRP PHE THR GLU SER LYS PRO ILE GLN ASN VAL GLU SER GLY PHE ILE PHE ALA LEU VAL SER ASP PHE ASN LEU PRO ASP ASN ASP GLU ASN PRO LEU VAL ARG ILE ASP TRP TRP LYS TYR TRP PHE THR ASN GLU SER PHE PRO TYR HSP LEU GLY TRP HSP PRO PRO SER PRO ALA ARG GLU ILE GLU PHE VAL THR SER ALA SER SER ALA VAL LEU ALA ALA SER VAL THR SER THR PRO SER SER LEU PRO SER GLY ALA ILE GLY PRO GLY ALA GLU ALA VAL PRO LEU SER PHE ALA SER THR MET THR PRO PHE LEU LEU ALA THR ASN ALA PRO TYR TYR ALA GLN ASP PRO THR LEU GLY PRO ASN ASP

GENErate CPO SETUp first none last CTER

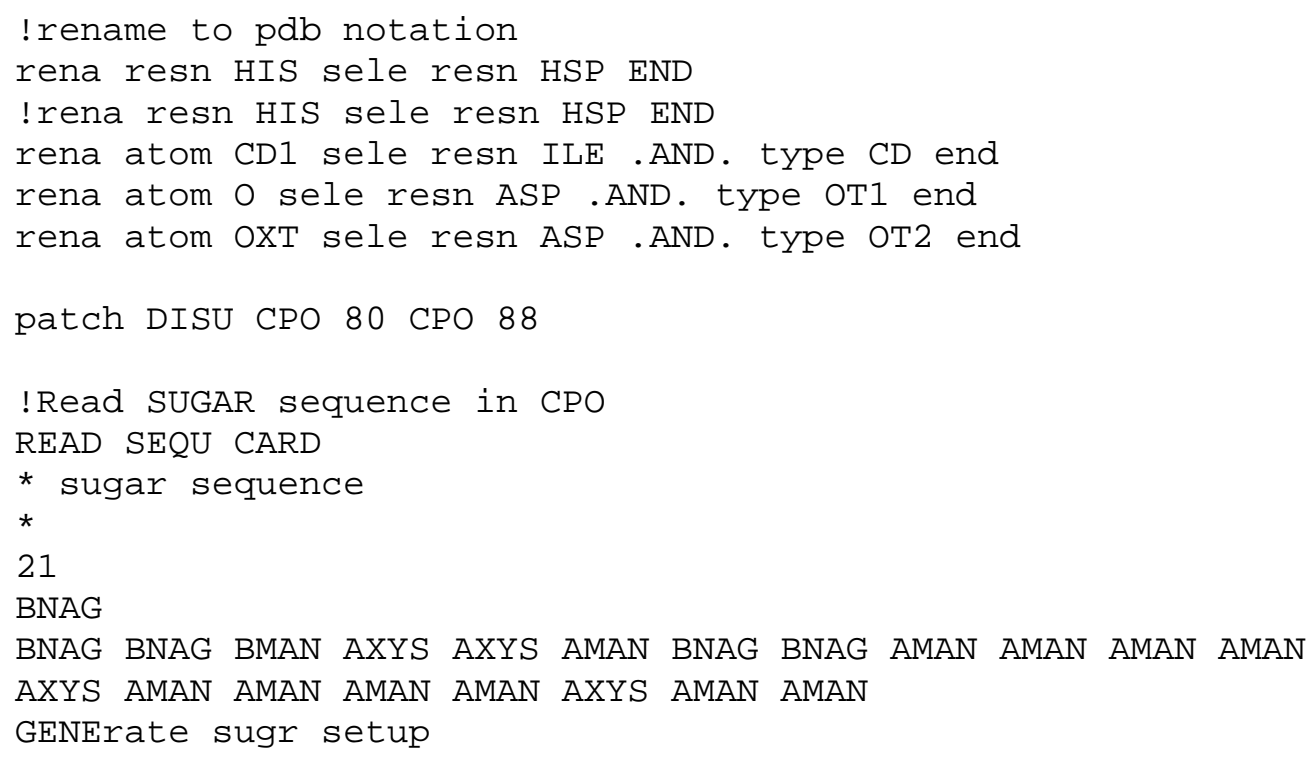




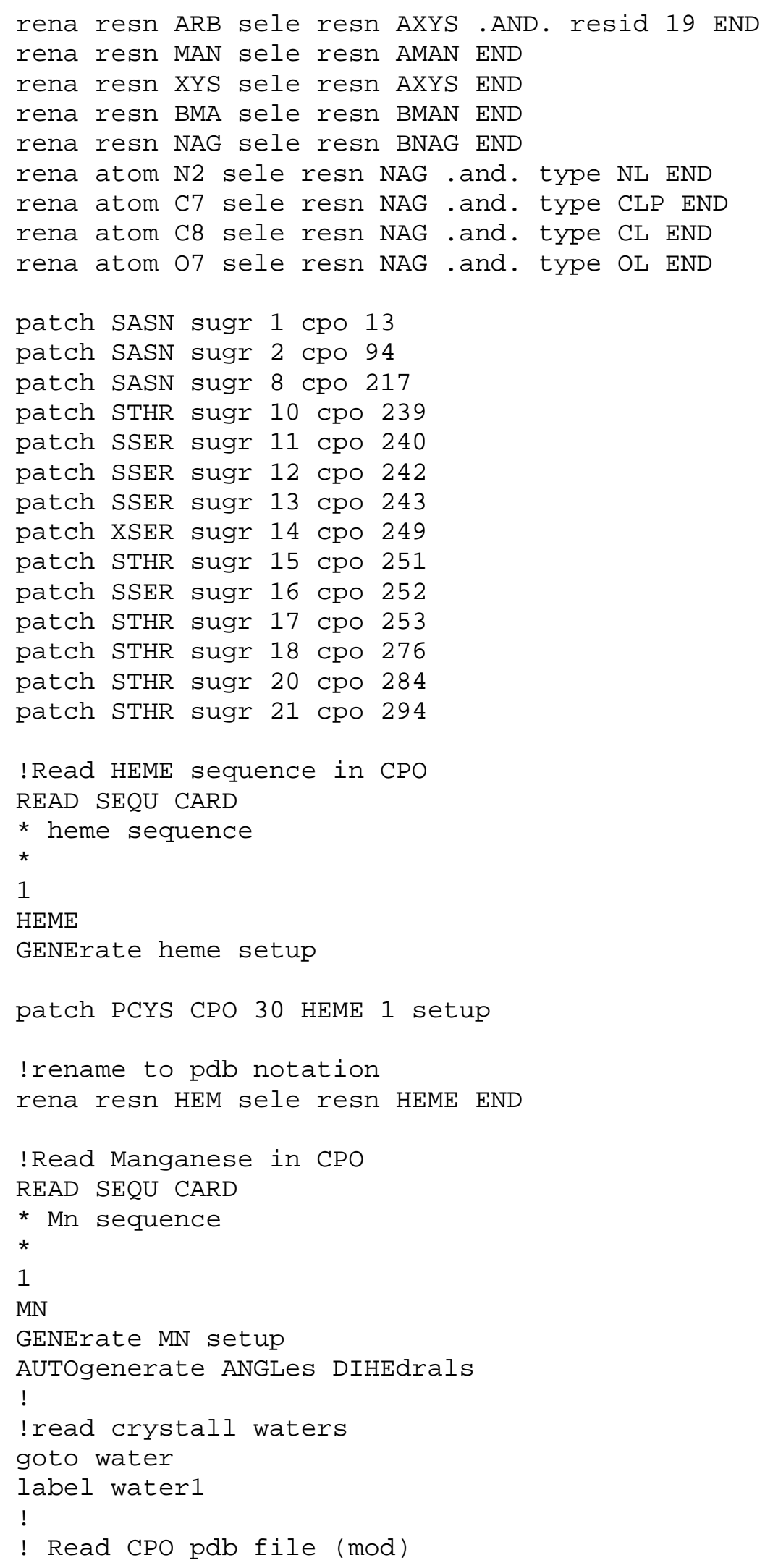









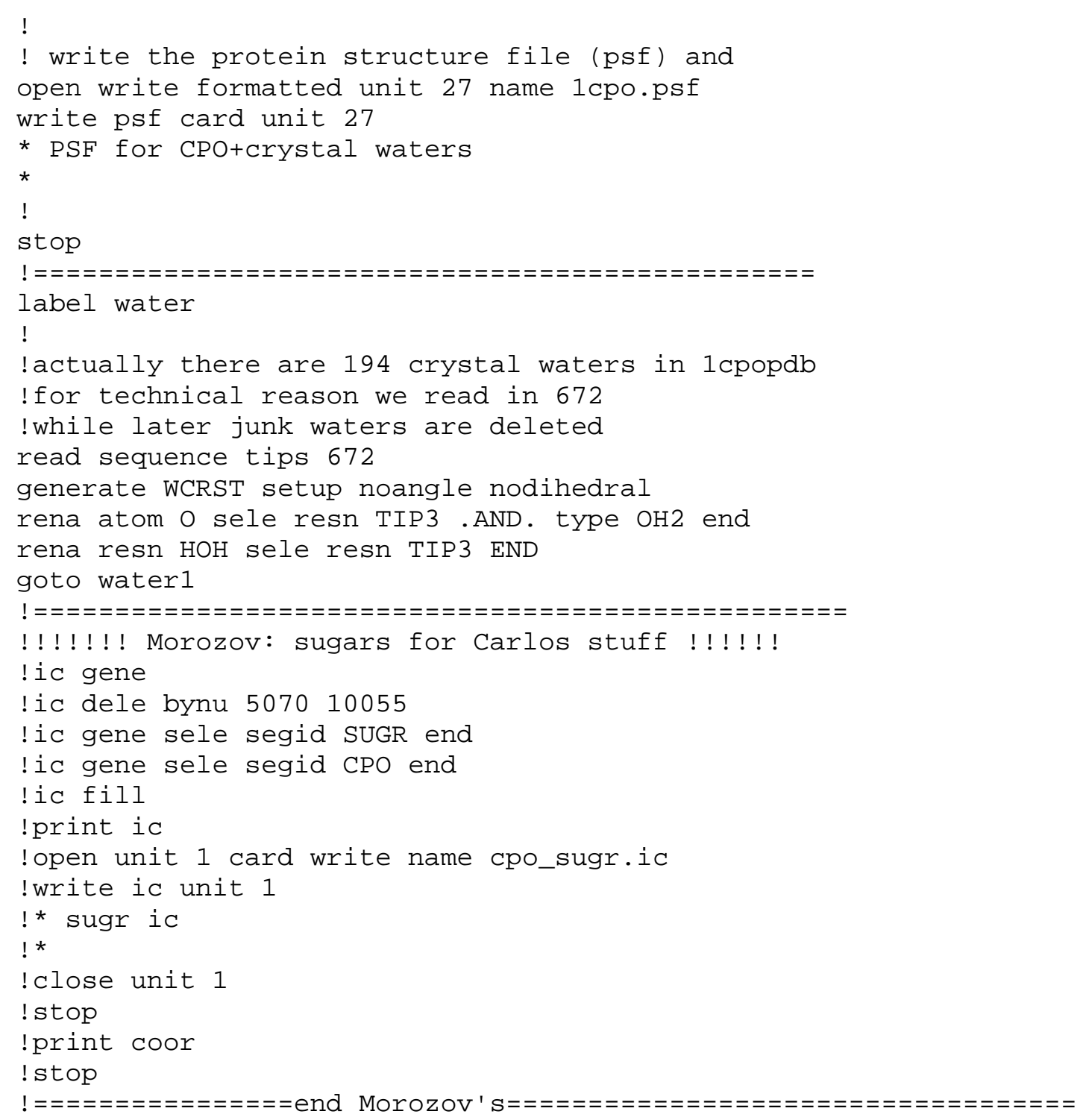

\section{C19. CHARMM Minimization Script}

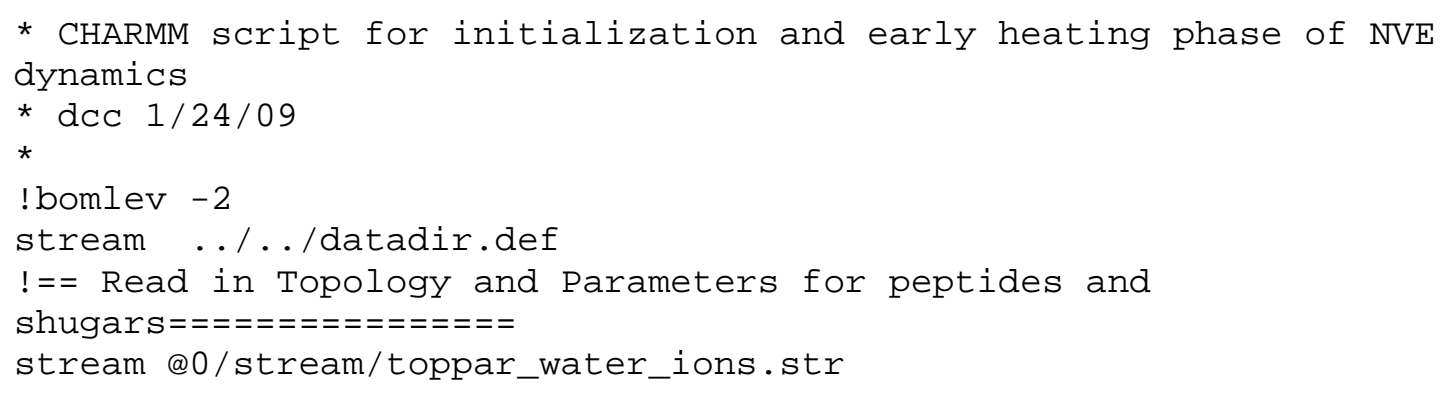




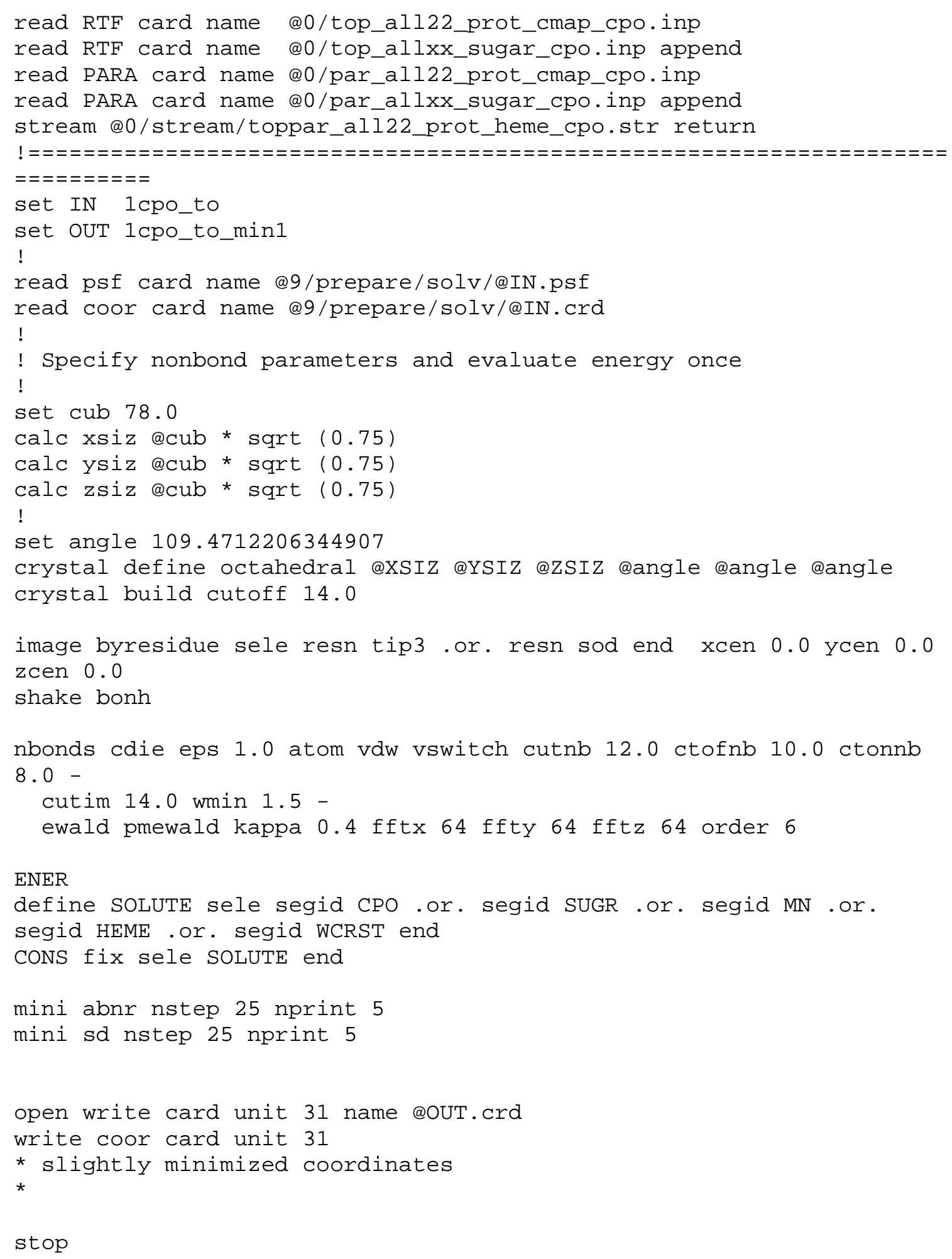




\section{C20. CHARMM Solvation Script}

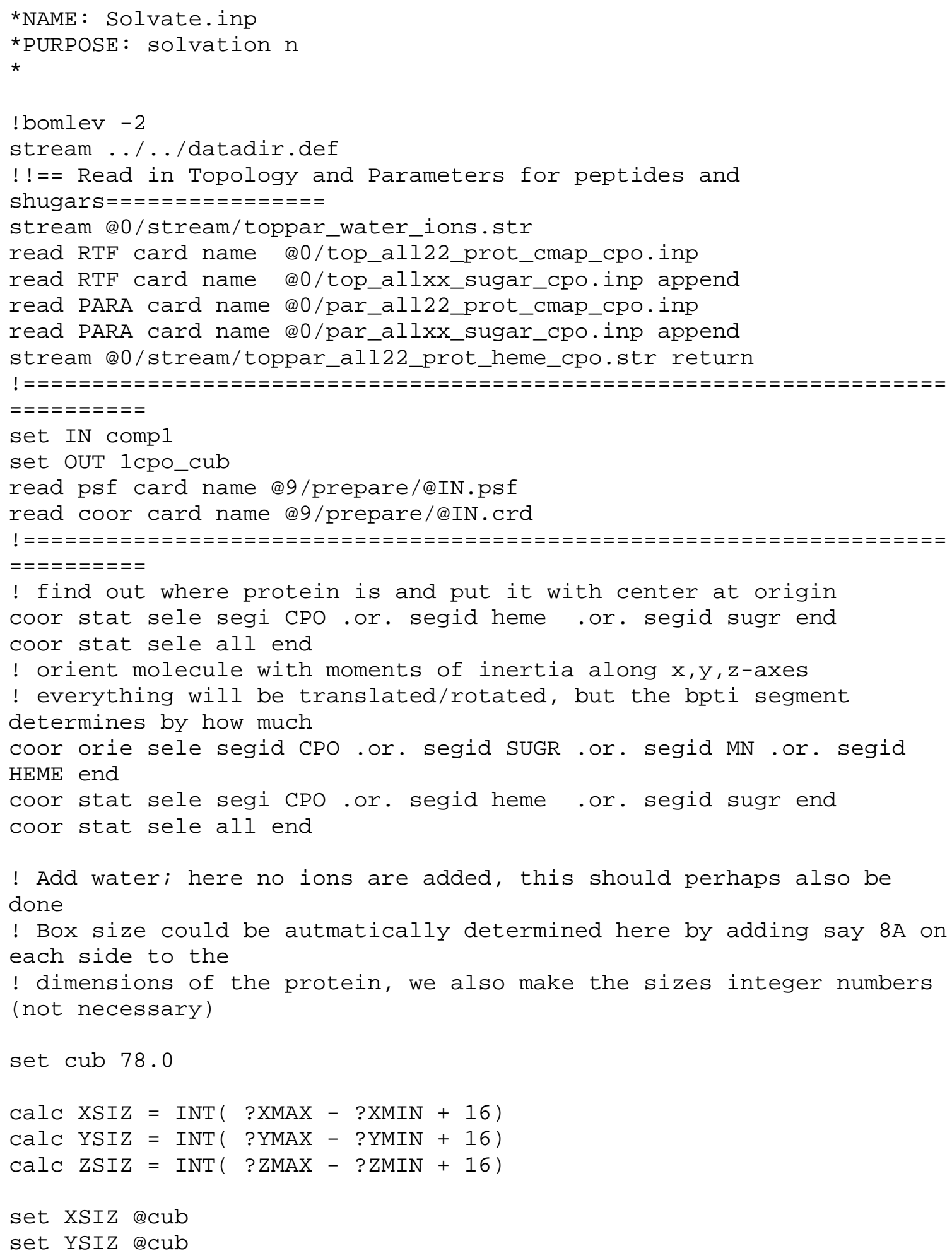




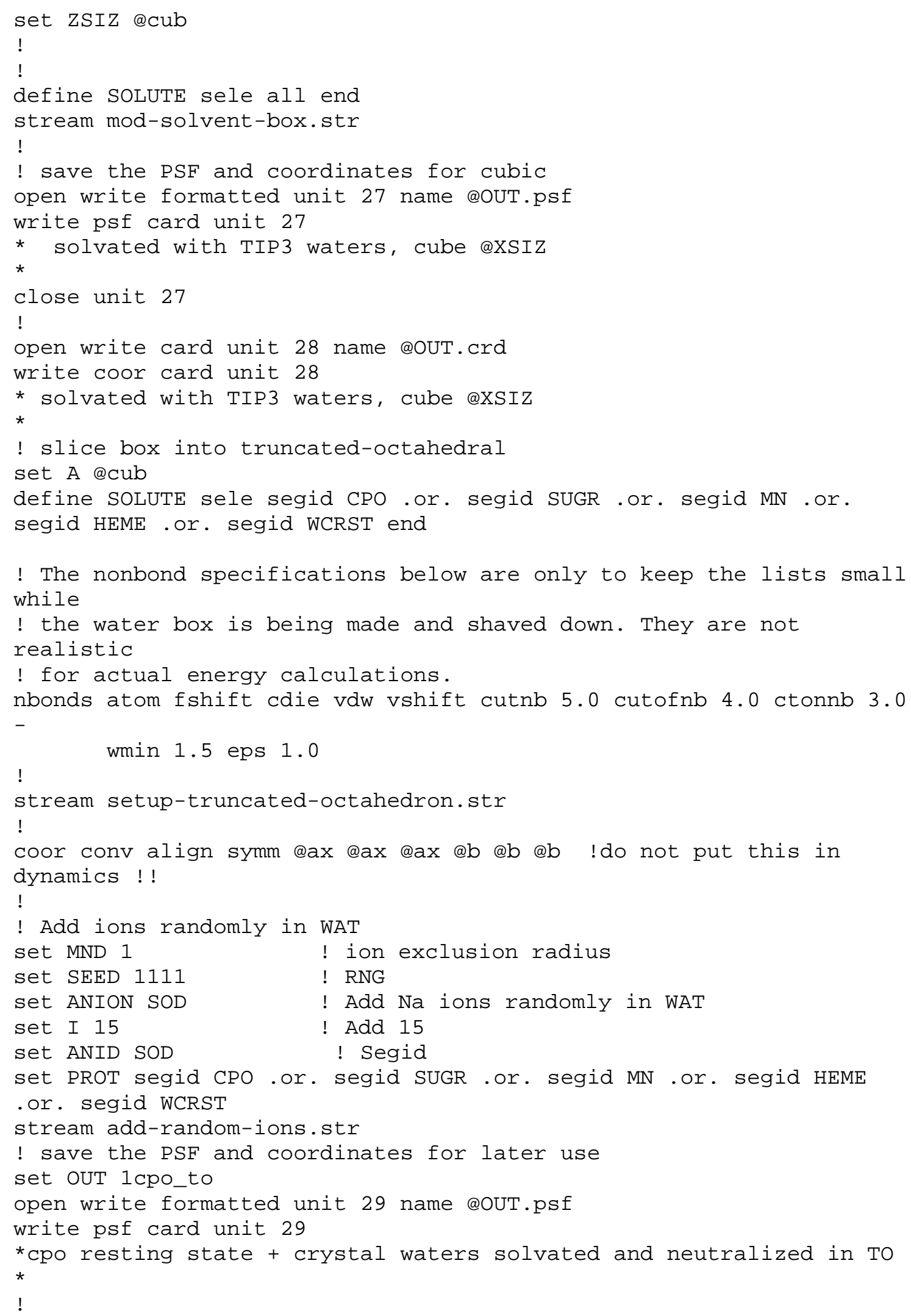


open write formatted unit 30 name @OUT.crd

write coor card unit 30

${ }^{*}$ cpo resting state + crystal waters solvated and neutralized in T0

*

!

stop

\section{C21. CHARMM Dynamics - Heating Script}

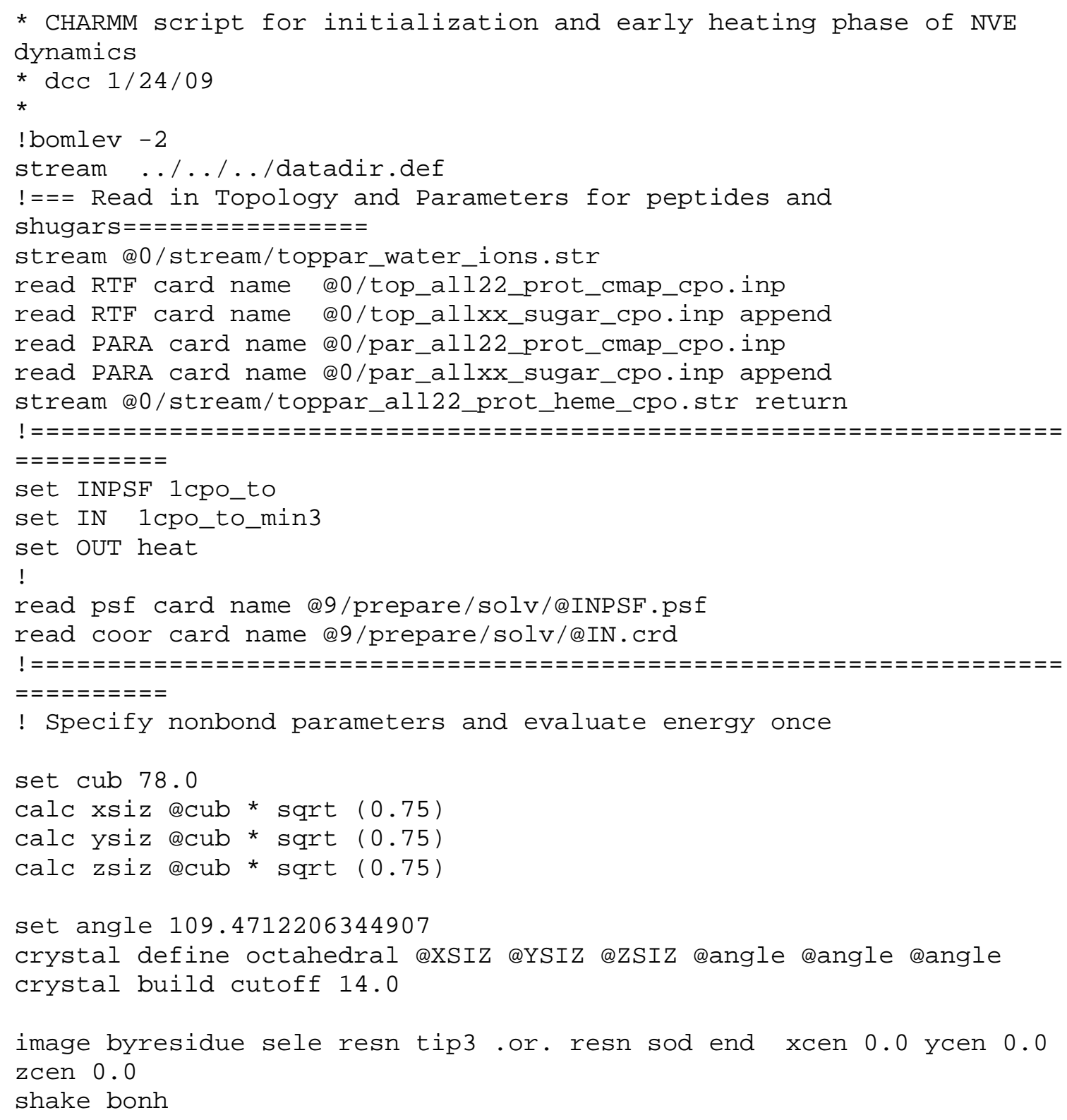


nbonds cdie eps 1.0 atom vdw vswitch cutnb 12.0 ctofnb 10.0 ctonnb $8.0-$

cutim 14.0 wmin 1.5

ewald pmewald kappa 0.4 fftx 64 ffty 64 fftz 64 order 6

ener

! Open files for writing dynamics data.

open write card unit 31 name @oUT.rst

open write file unit 41 name @ouT.trj

set st 10000

set $\mathrm{fr} 1000$

! Dynamics command with options described

DYNAMICS -

- ! algorithm

LEAP - ! use leapfrog verlet algorithm

VERLET - !

- ! start/restart/initialization info

START - ! START for initial run; RESTART to pick up from previous run

IASORS 1 - ! specifies scaling $(\odot)$ or assigning (other value) of velocities

IASVEL 1 - ! species method of assigning velocities during heating

ISCVEL $\odot$ - ! specifies method of scaling velocities ( $\odot=$ same factor)

ECHEck 9999. - !max variation of energy step-to-step

- ! time step info

NSTEP @st - ! number of steps

TIMESTEP 0.001 - ! timestep in picoseconds

IPRFRQ@fr - ! frequency for calculating averages and

fluctuations

NTRFRQ $=0$ )

- ! (must be at least as large as NTRFRQ unless

- ! heating info

IHTFRQ @fr - ! heating frequency

FIRSTT 50.0 - ! initial temperature

FINALT 298.0 - ! final temperature

TEMINC 24.8 - !temperature increment

- ! equilibration info

IEQFRQ 50000 - ! frequency for assigning or scaling velocities (during equil.)

! ICHECW $\odot$ - ! every IEQFREQ steps, always change velocities

$(\odot)$

(other value)

- ! or change only if temperature is outside window

! TWINDH 10.0 - ! upper temperature window limit

! TWINDL -10.0 - ! lower temperature window limit 


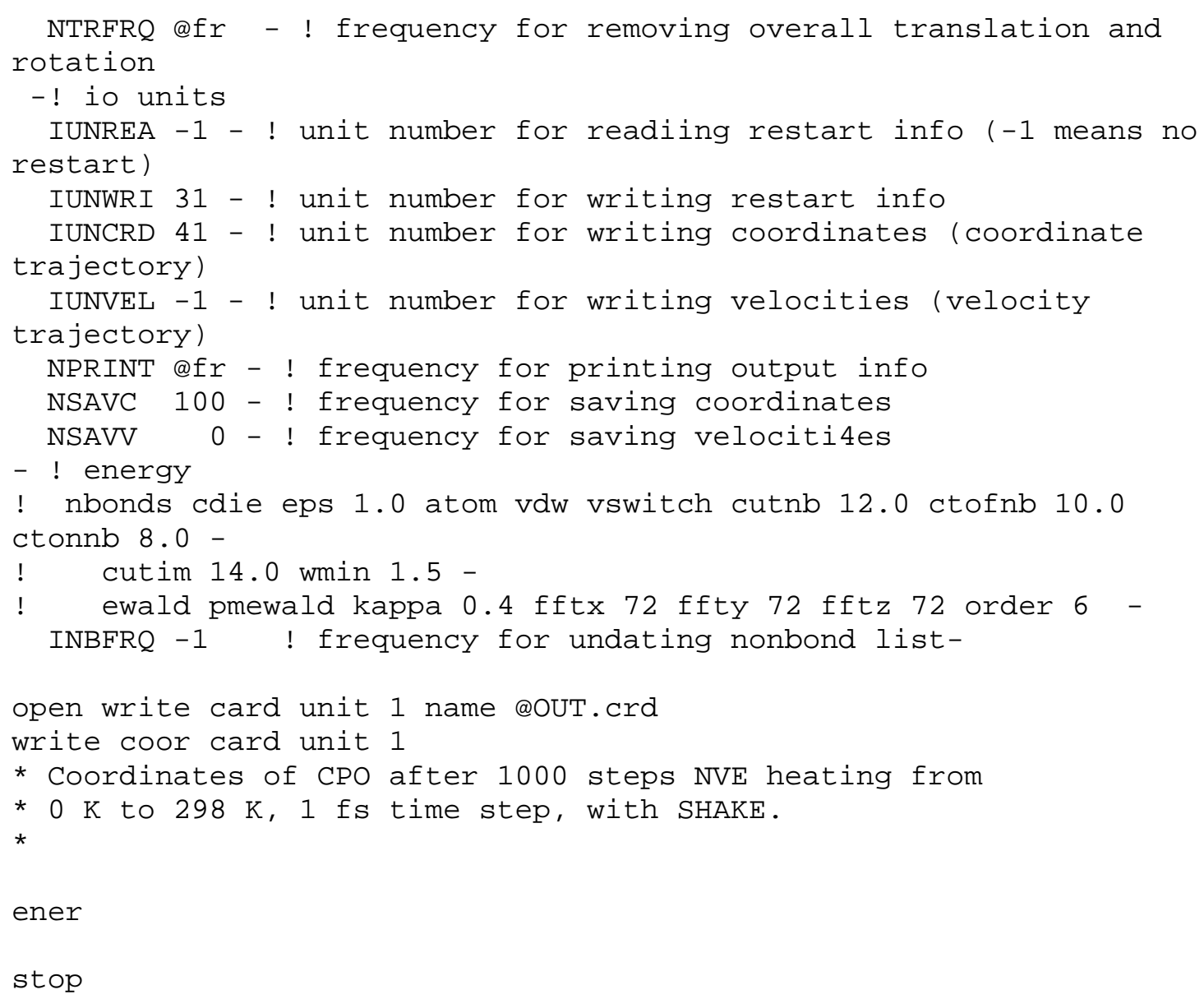

\section{C22. CHARMM Dynamics - Equilibration Script}

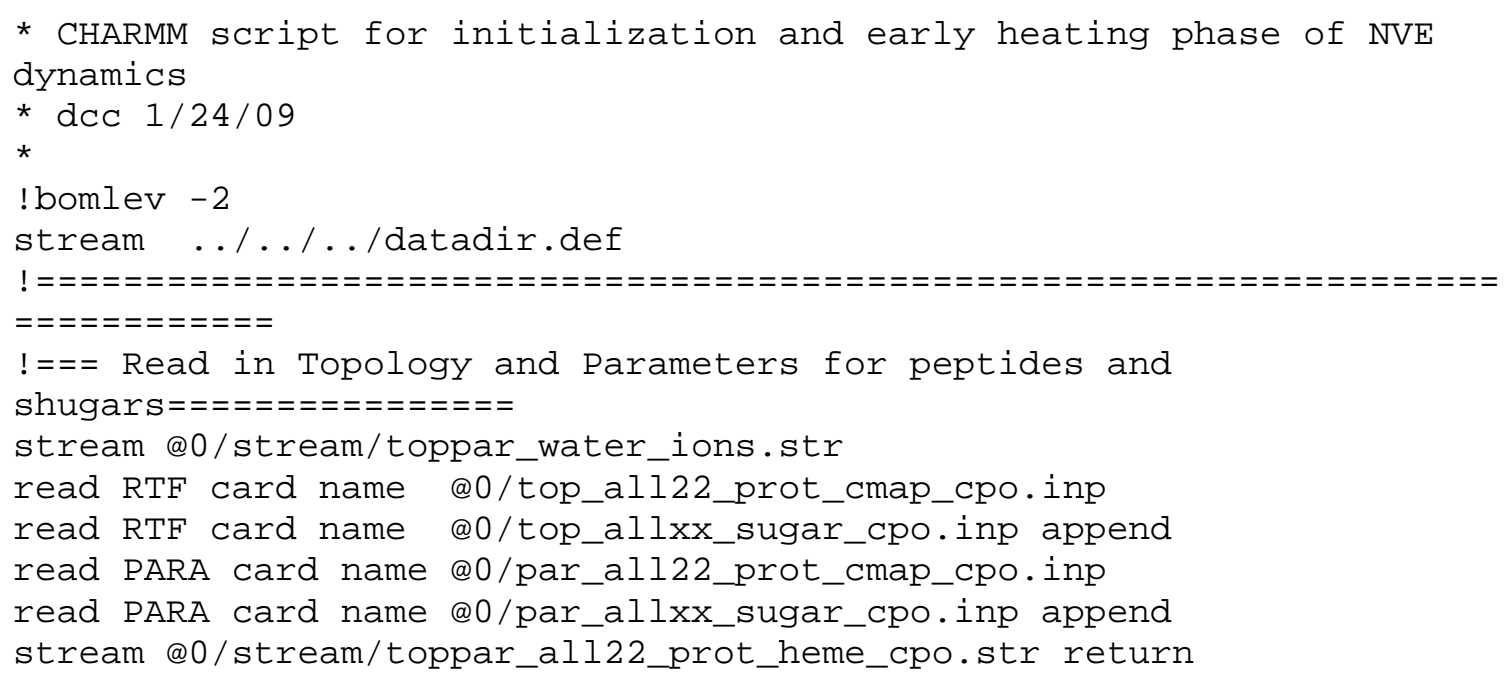




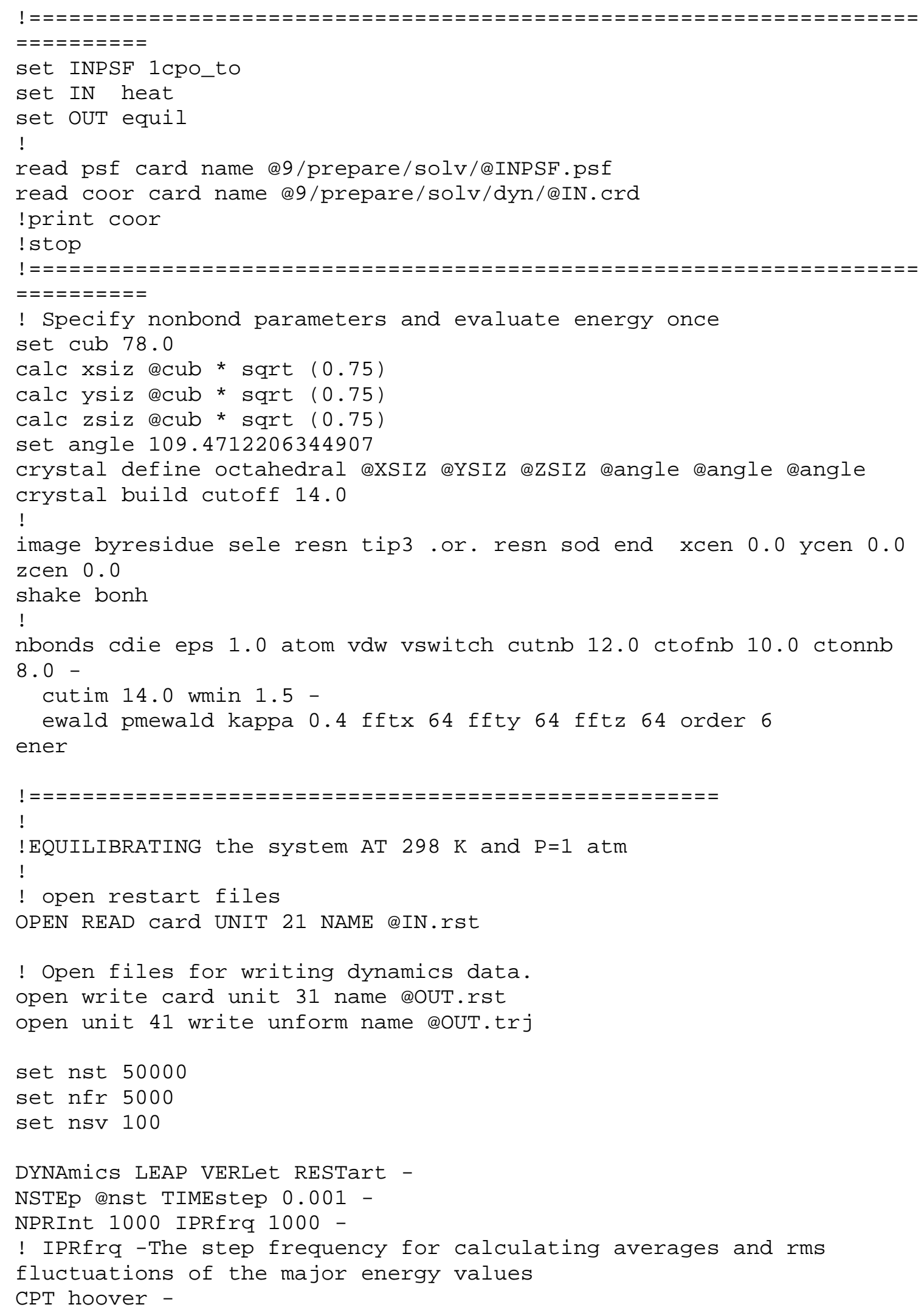




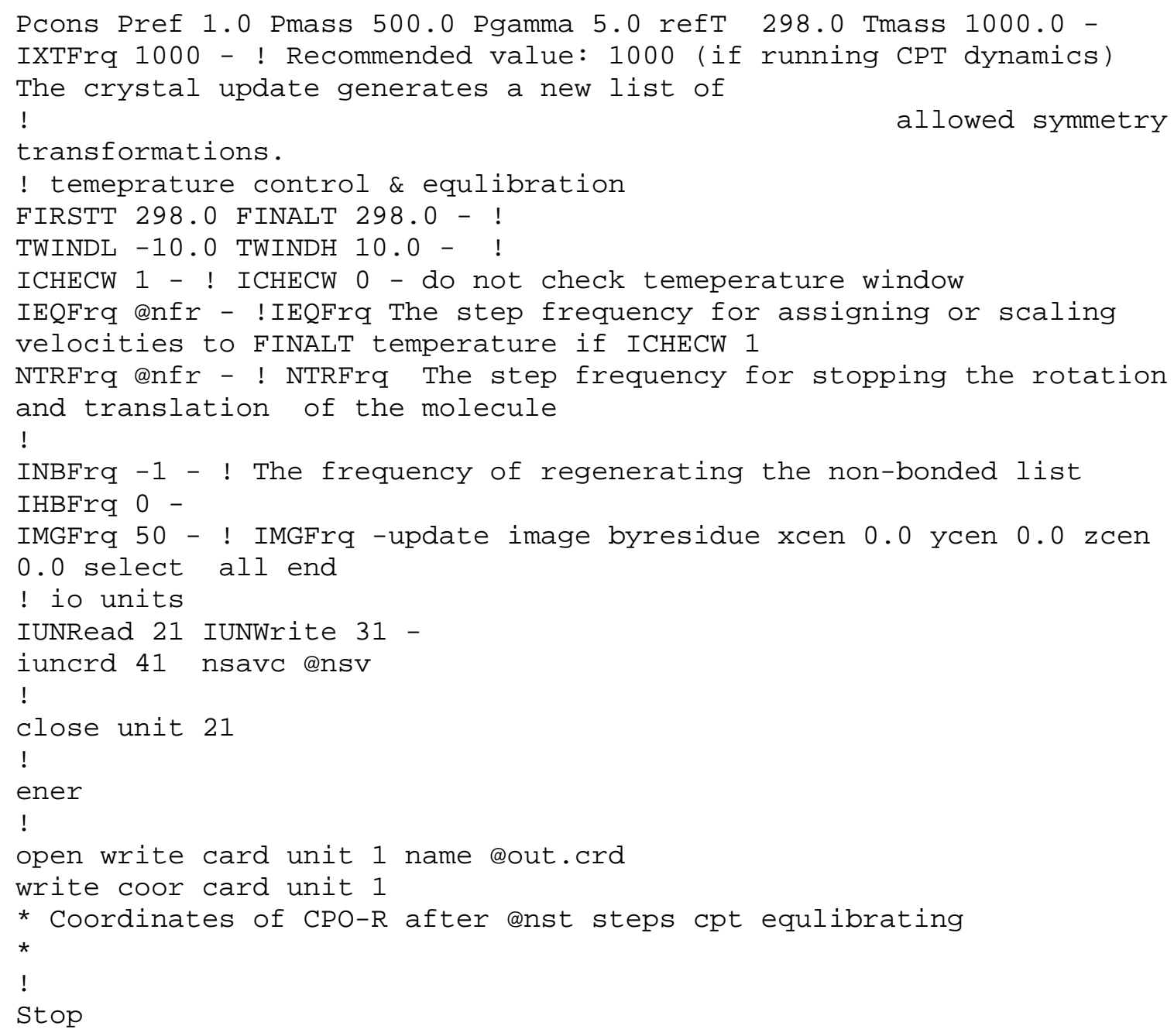


VITA

ARMANDO D. PARDILLO

$1998-2003$

Bachelor of Arts: Business Administration: Finance

Florida International University

Miami, Florida, USA

$2003-2004$

Cost Accountant

World Fuel Services

Miami, Florida, USA

$2004-2005$

Ark Lending

Loan Officer

Lauderdale by the Sea, Florida, USA

2005- 2006

Premier Mortgage Capital

Office Manager

Orlando, Florida, USA

$2007-2009$

Bachelor of Science: Chemistry

Florida International University

Miami, Florida, USA

$2010-2015$

Teaching Assistant

Florida International University

Miami, Florida, USA

\section{PUBLICATIONS AND PRESENTATIONS}

Pardillo, Armando D.; Morozov, Alexander N.; Chatfield, David C. "Proximal Pocket Hydrogen Bonds Significantly Influence the Mechanism of Chloroperoxidase Compound I Formation,” Journal of Physical Chemistry B (2015), Ahead of Print.

Morozov, Alexander N.; Pardillo, Armando D.; Chatfield, David C. “ChloroperoxidaseCatalyzed Epoxidation of Cis- $\beta$-Methylstyrene: $\mathrm{NH} \bullet \bullet$ S hydrogen bonds and Proximal Helix Dipole Change the Catalytic Mechanism and Significantly Stabilize the Reaction Barrier.” Journal of Physical Chemistry B (2015), (under peer review - corrections submitted).

Pardillo, Armando D.; Morozov, Oleksandr; Chatfield, David C. "DFT Study Elucidates Proximal Pocket Hydrogen Bond Influence on Mechanism of Compound I Formation in Chloroperoxidase,” 249th ACS National Meeting \& Exposition, Denver, CO, United States, March 22-26, 2015 (2015), BIOL-182. 
Pardillo, Armando D.; Morozov, Alexander N.; Chatfield, David C. "DFT study shows proximal-pocket hydrogen bonds significantly influence mechanism of Compound I formation in chloroperoxidase,” 247th ACS National Meeting \& Exposition, Dallas, TX, United States, March 16-20, 2014 (2014), COMP-196.

By Chatfield, David C.; Pardillo, Armando D.; Zhang, Rui; Wang, Xiaotang; D'Cunha, Cassian; Morozov, Alexander N. "Reactivity of chloroperoxidase, a promising bioengineering target, revealed through simulation,” 247th ACS National Meeting \& Exposition, Dallas, TX, United States, March 16-20, 2014 (2014), COMP-42.

Morozov, Alexander N.; Pardillo, Armando D.; Chatfield, David C. "Versatility of chloroperoxidase catalytic function: Apoenzyme tunes reactivity of the active center," 245th ACS National Meeting \& Exposition, New Orleans, LA, United States, April 7-11, 2013 (2013), COMP-280.

Pardillo, Armando; Morozov, Alexander N.; Chatfield, David C. "Comparitive quantum chemical study of thiolate, thiol, and hydrogen bonded thiol and thiolate proximal heme ligands on the rate determining step of CPO activation to Compound I," 243rd ACS National Meeting \& Exposition, San Diego, CA, United States, March 25-29, 2012 (2012), COMP-409.

Padron, Alejandro; Pardillo, Armando; D'Cunha, Cassian; Chatfield, David C. "MD study of wildtype and mutant dehaloperoxidase: Insight into halophenol ligand stabilization and allosteric role of His55,” 241st ACS National Meeting \& Exposition, Anaheim, CA, United States, March 27-31, 2011 (2011), COMP-211.

Chatfield, David C.; Pardillo, Armando "Molecular dynamics study of gate-keeperresidue switch in hybrid globin-peroxidase, DHP,” 237th ACS National Meeting, Salt Lake City, UT, United States, March 22-26, 2009 (2009), PHYS-294. 